DEPARTMENT OF COMMERCE

\title{
Technologic Papers
}

OF THE

\section{Bureau OF Standards}

S. W. STRATTON, DIRECTOR

\section{No. 170 \\ PYROMETRIC PRACTICE}

BY

PAUL. D. FOOTE, Physicist

C. O. FAIRCHILD, Physicist

T. R. HARRISON, Associate Physicist

Bureau of Standards

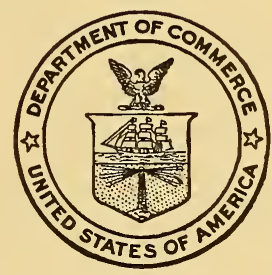

PRICE, 60 CENTS

Sold only by the Superintendent of Documents, Government Printing Urifice Washington, D. C.

WASHINGTON

GOVERNMENT PRINTING OFFICE

1921 



\title{
PYROMETRIC PRACTICE ${ }^{a}$
}

\author{
By Paul D. Foote, C. O. Fairchild, and T. R. Harrison
}

\section{CONTENTS}

I. Temperature scale $\ldots \ldots \ldots \ldots \ldots \ldots \ldots \ldots \ldots \ldots \ldots \ldots \ldots \ldots \ldots \ldots \ldots$

II. High-temperature thermometry $\ldots \ldots \ldots \ldots \ldots \ldots \ldots \ldots \ldots \ldots \ldots \ldots$ Io

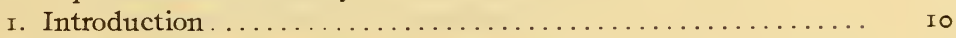

2. High-temperature mercurial thermometers.............. II

3. Industrial thermometers. ..................... I2

4. Emergent-stem error of mercury in glass thermometers....... I4

5. Other sources of error in use of high-temperature mercurial thermometers.............................. I6

6. Filling high-temperature thermometers under pressure........ I6

7 . Improper annealing . . . . . . .

8. Indicating and recording thermometers................ I7

(a) Pressure thermometers...................... I7

(I) Liquids used in vapor-pressure thermometers... I8

(2) Materials and pressures employed in liquid- and gas-filled thermometers................ I8

(3) Principles underlying the action of pressure

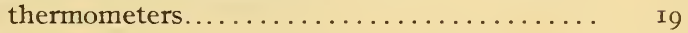

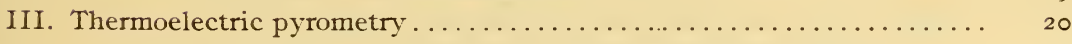

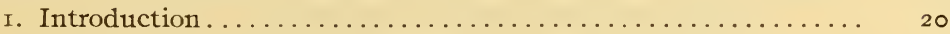

2. Metals used for thermocouples...................... 2 I

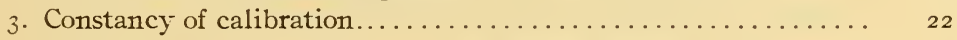

4. Reproducibility of couples....................... 23

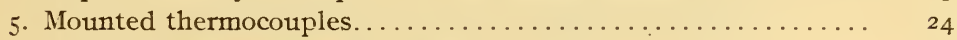

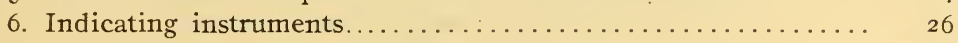

(a) Galvanometer method ....................... ${ }_{2} 6$

(I) Resistance of indicating instruments............ 28

(2) Use of portable test set.............. 33

(3) Galvanometer with variable series resistance.... 38

(4) Harrison-Foote method ................ 39

(b) Potentiometer method ..................... 43

(c) Semïpotentiometer method ................... ${ }_{4} 6$

(I) Northrup's method ................ 46

(2) Northrup's method applied to continuously deflecting instruments................. 47

a Throughout this paper important technical features of different instruments are considered with a view to making available to the users of such instruments information which is essential to determining the adaptability of the several instruments to the various applications of high temperature measurement, as well as to secure the most efficient use of the instruments when installed. It is to be emphasized that no attempt is made to designate the best instrument for any given purpose, as this depends upon so many factors, such as accuracy required, cost, adaptability to working conditions, that it is necessary for the user to decide with the aid of the technical information given which particular instrument will most satisfactorily meet his needs. 
III. Thermoelectric pyrometry-Continued

6. Indicating instruments-Continued

Page

(I) Deflection potentiometer graduated to read temperature directly................

(e) Temperature of the cold junctions of thermocouples....

(I) Compensating leads. . . . . . . . . . . .

(2) Potentiometric compensation methods.........

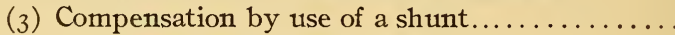

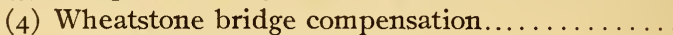

(f) Methods employed in correcting for variations in the temperature-emf relation of different couples of the

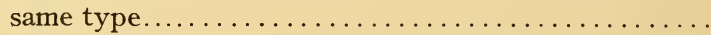

(I) Compensation by series resistance.......... $6_{5}$

(2) Compensation by shunt resistance.......... 69

(3) Compensation by shunt and series resistance... 70

(4) Summary statement on reproducibility of

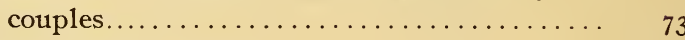

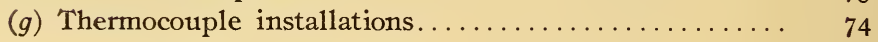

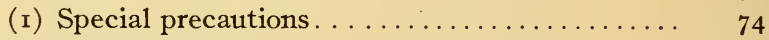

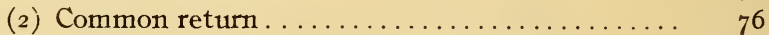

(3) Wiring diagrams of thermocouple installations. . 78

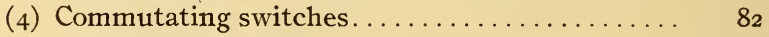

(5) Use of junction box............... $8_{3}$

(6) Determination of temperature of buried cold junction .............................. 87

(7) Depth of immersion of couples........... 88

(8) Couples purposely insufficiently immersed . . . 89

(9) Protecting tubes for thermocouples......... 89

IV. General theory of optical and radiation pyrometry ............ 94

I. Optical pyrometer temperature scale . . . . . . . . . . . . 96

2. Radiation pyrometer temperature scale................ 97

V. Optical pyrometry . . . . . . . . . . . . . . . . . . . . $9^{8}$

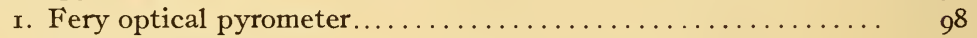

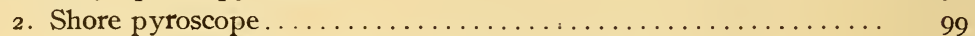

(a) Directions for use of Shore pyroscope............ 99

3. Wanner pyrometer.......................... Iо

4. Scimatco pyrometer......................... Iо3

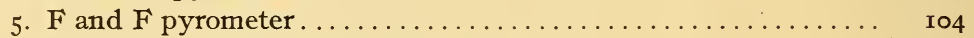

6. Disappearing filament optical pyrometer............ Io5

7. General use of optical pyrometers . . . . . . . . . . . . .

8. Black-body and nonblack-body conditions . . . . . . . . . I

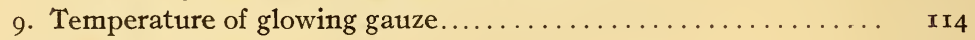

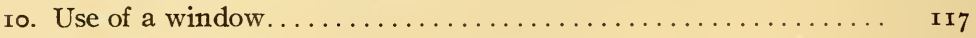

II. Flames and smoke . . . . . . . . . . $\ldots \ldots \ldots \ldots \ldots \ldots \ldots$ I 7

I2. Method of sighting into a closed tube $\ldots \ldots \ldots \ldots \ldots \ldots \ldots \ldots$ r 8

VI. Radiation pyrometry . . . . . . . . . . .

I. Thwing radiation pyrometer...................... I

2. Foster radiation pyrometer................ I2 I

3. Fery radiation pyrometer . . . . . . $\ldots \ldots \ldots \ldots \ldots \ldots \ldots \ldots \ldots \ldots$

4. Errors to which radiation pyrometers are subject. . . . . . . I25

5. Advantages and disadvantages of radiation pyrometers....... 125

6. Black-body and nonblack-body conditions ............. 126 
VII. Resistance thermometry...................... I 27

I. Three-lead Wheatstone bridge method ................ I30

2. Four-lead Wheatstone bridge method ................. I I

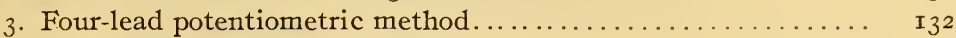

4. Deflection-galvanometer method ................. I $3^{2}$

5. Summary statement on resistance thermometers . . . . . . . I 33

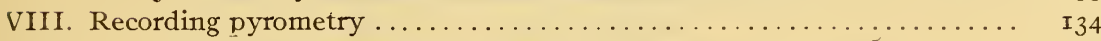

I. Forms of temperature records................... I35

2. General types of thermocouple recorders for temperature-time

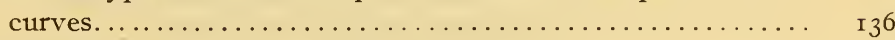

3. Record charts ...................... I 36

4. Thermocouple recorders operating on the galvanometric principle $\mathrm{I}_{3} 8$

(a) Industrial types of recorders . . . . . . . .

(I) The circular-chart, single-point recorder...... I42

(2) Roll-chart recorders................. I 44

5. Thermocouple recorder operating on the potentiometric principle ${ }_{5} 6$

6 . Transformation point indicators and recorders........... I6 I

(a) The Brown transformation point recorder......... $\mathrm{I}_{2}$

(b) The Leeds \& Northrup transformation-point indicator. $\quad$ I $6_{3}$

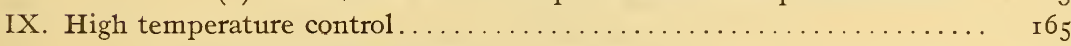

I. Devices used for control. ....................... I69

(a) Automatic alarm...................... r69

(b) Manual signaling .................... I69

(c) Automatic signaling................. I 70

(d) Automatic temperature control.............. I 74

2. Interesting examples of the utility of recorders in furnace control ................................

X. Melting-point methods at high temperatures.............. I 87

I. Introduction $\ldots \ldots \ldots \ldots \ldots \ldots \ldots \ldots \ldots \ldots \ldots \ldots \ldots \ldots \ldots \ldots \ldots \ldots \ldots \ldots \ldots$

2. Use of thermoelectric pyrometers .................. I89

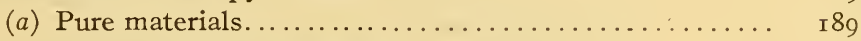

(I) General discussion................... I 89

(2) Precision work with pure metals......... I92

(3) Crucibles and protecting tubes.......... I93

(4) Table of melting points............. I94

(5) Construction of furnace.............. I95

(6) General precautions............... I96

(b) Metals with impurities, and alloys............ I97

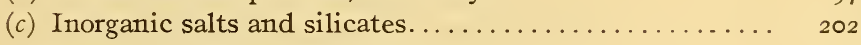

(I) Table of melting points............ 206

(d) Technical melting-point determinations........... 206

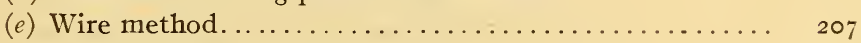

3. Use of resistance thermometers.................... 209

4. Use of optical pyrometers........................ 2 г

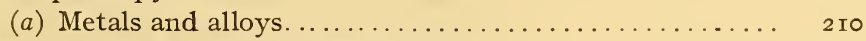

(I) Table of melting points............ 2 I 2

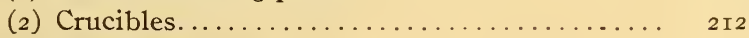

(3) Furnaces.......................... $2 \mathrm{I}_{3}$

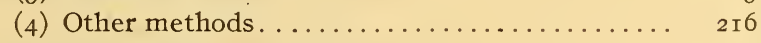

(5) Micropyrometer................. 2 r6 
$\mathrm{X}$. Melting-point methods at high temperatures-Continued

4. Use of optical pyrometers-Continued

(b) Refractory materials.

Page

2 I 7

217

(2) Fire bricks and ceramic materials.......... 2 I8

(3) Meaning of the melting point............ $2 x 9$

(4) Conditions affecting observed values of the melting point.

(5) Method of melting-point determinationsampling, grinding, and molding.

(6) Time and rate of heating.

(7) Type of furnace and conditions existing therein.

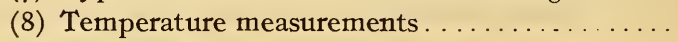

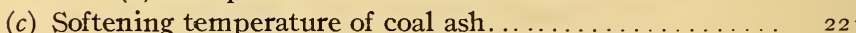

(d) Table of melting points of refractory materials

XI. Standardization of pyrometers......................... 225

I. Standardization of high-temperature thermometers . . . . . . 225

2 Standardization of thermocouples................... 226

(a) Standardization of rare-metal couples . . . . . . . 227

(x) Annealing......................... 227

(2) Homogeneity test................. 228

(3) Laboratory potentiometer.............. 232

(4) Cold-junction ice box................ 233

(5) Calibration of primary standards.......... 234

(6) Secondary or comparison calibration of rare metal couples.................... 239

(b) Standardization of base-metal couples............ 242

(x) Use of muffle furnace ................ 242

(2) Use of molten-metal bath................ 243

(3) Single-point calibration or check point....... 244

(4) Checking thermocouples in fixed installations. 244

(c) Cold-junction correction..................... 246

(d) Method of making thermocouple junctions.......... 247

3. Use of standardized couples with various types of measuring instrument ................................. ${ }_{24} 8$

(a) Galvanometer adjusted to read zero on open circuit, or potentiometer without cold-junction compensator....

(b) Galvanometer adjusted to read cold junction temperature or equivalent emf on open circuit.............. 249

(c) Potentiometer with cold-junction compensator....... $249^{\circ}$

(r) Main scale and manually operated compensator graduated in emf .....................

(2) Main scale and manually operated compensator graduated in temperature................

(3) Main scale graduated in temperature, manually operated compensator graduated in emf......

(4) Main scale graduated in emf and manually operated compensator graduated in temperature. .

(5) Scale graduated in emf and automatic compensator.............................

(6) Scale graduated in temperature and automatic compensator .......................

\section{0}

20

$2 \mathrm{I}$

2 I

22

3

5

5

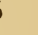

7

33

39

42


XI. Standardization of pyrometers-Continued

4. Standardization of thermoelectric instruments........... 253

(a) Laboratory standard potentiometer............... $\quad 253$

(b) Adjustment of instruments for calibration........... 253

(c) Galvanometers......................... ${ }_{253}$

(d) Portable and recording potentiometers........... 255

(e) Potentiometer with compensator graduated in temper-

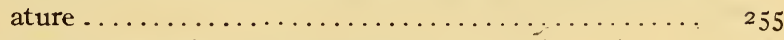

(f) Potentiometer with compensator graduated in emf..... 255

(g) Potentiometer with automatic compensator.......... 255

(h) Instruments employing the Harrison-Foote method.... 256

5. Calibration of couple and indicator as a unit............. ${ }_{257}$

(a) Galvanometric instruments.................. 257

(b) Potentiometers.......................... 257

6. Standardization of optical pyrometers................. $\quad 25^{8}$

(a) Secondary standardization.................. 259

(I) Comparison source................ 259

(2) Disappearing-filament pyrometer.......... 260

(3) Wanner pyrometer.................. $26_{3}$

(4) Fery optical and F and F pyrometer........ 266

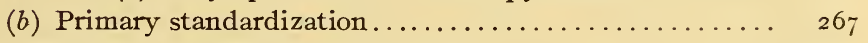

7. Standardization of radiation pyrometers................ 273

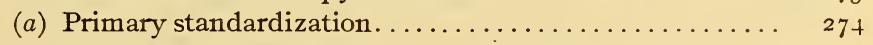

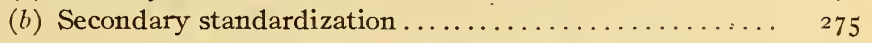

(I) Computation of standardization data ....... 276

8. Standardization of resistance thermometers.............. 278

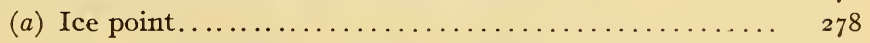

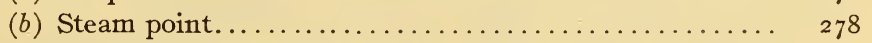

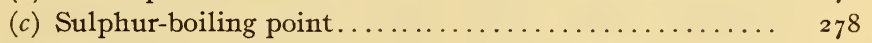

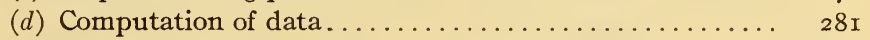

(e) Nickel coils............................ $28 \mathrm{r}$

(f) Thermometers with indicating instruments........ $28 \mathrm{r}$

XII. Industrial applications of pyrometry....................... 282

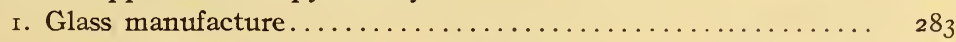

(a) Temperature measurements in glass melting........ 283

(b) General principles of annealing................. 285

(c) Temperature measurements in annealing ovens...... 286

2. Rotary Portland cement kilns.................... 287

3. Ceramic industries............................... 289

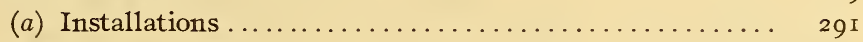

4. By-product coke industry ....................... $\quad 293$

(a) Locations for installing thermocouples............ 293

(b) Measurements with the optical pyrometer.......... 294

(c) Experimental measurements in oven proper......... 295

5. Steel manufacture............................... ${ }_{29} 6$

(a) Blast furnace........................... ${ }_{29} 6$

(b) Bessemer converter..................... 296

(c) Open-hearth furnace......................... ${ }_{29} 6$

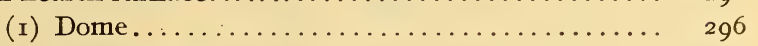

(2) Slag pocket.................... 296

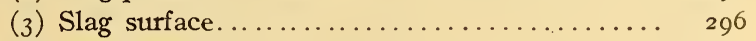

(4) Metal bath..................... 297 
XII. Industrial applications of pyrometry-Continued 5. Steel manufacture-Continued

(d) Tapping and teeming . . . . . . . . . . . . . . . . 297

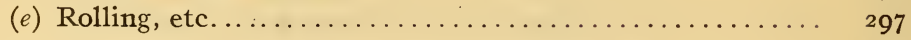

6. Temperatures of metal surfaces.................... ${ }_{29} 98$

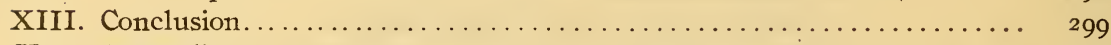

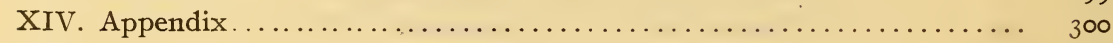

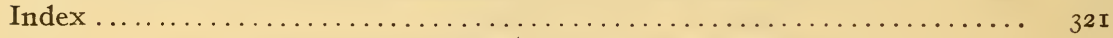

\section{TEMPERATURE SCALE}

The ideal temperature scale is known as the thermodynamic scale. Kelvin has defined this scale to be such that "the absolute values of two temperatures are to one another in the proportion of the heat taken in to the heat rejected in a reversible thermodynamic engine working with a source and refrigerator at the higher and lower of the temperatures, respectively." The size of the unit degree is given by arbitrarily defining the temperature interval from the freezing point to the boiling point of pure water under standard conditions as equivalent to $100^{\circ} \mathrm{C}$.

No thermometer or pyrometer uses this principle of temperature measurement, but scales defined by other means are supposedly in agreement with the thermodynamic scale or differ from this scale by small, known amounts.

Primarily, the high-temperature scale from $100^{\circ} \mathrm{C}$ to about ${ }^{1} 500^{\circ} \mathrm{C}$ is defined by the expansion of nitrogen in the constantvolume gas thermometer. If an "ideal" gas existed and were employed in the gas thermometer, the temperature scale would agree throughout this range with the thermodynamic scale. Such a gas does not exist, but the corrections which are necessary to apply to the nitrogen-gas thermometer scale to make this scale agree with that of an ideal gas are fairly well known. These corrections are very small, amounting in the above case to only $0.05^{\circ}$ at $200^{\circ} \mathrm{C}, 0.3^{\circ}$ at $600^{\circ} \mathrm{C}$, and $\mathrm{I}^{\circ}$ at $1200^{\circ} \mathrm{C}$.

The gas thermometer is very difficult and inconvenient to operate and is not employed even for precision work except as a fundamental standard instrument. It would not be possible to calibrate all other types of pyrometer directly by comparison with the gas thermometer. Accordingly, a series of so-called fixed points have been adopted, defined by the melting or boiling points of several of the chemical elements and compounds. The temperatures at which the phenomena of melting or boiling of these materials occur have been determined up to $1500^{\circ} \mathrm{C}$ by the gas thermometer. In these primary experiments a thermo- 
couple or resistance thermometer usually served as a transfer instrument, since, except in a few instances, it was not possible to place the bulb of the gas thermometer directly into the melting or boiling chemical element. Having obtained by means of the gas thermometer a number of fixed points which could be corrected to conform to the true thermodynamic temperature scale, it was found that the temperature scale defined by other forms of pyrometer agreed with the thermodynamic scale as closely as this scale could be determined by the gas thermometer and that the precision possible in other pyrometers is far greater than that of which the gas thermometer is capable.

Thus, it has been found that the scale defined by the platinum resistance thermometer calibrated as described later, in terms of the melting point of ice, $0^{\circ} \mathrm{C}$, the boiling points of water $100^{\circ} \mathrm{C}$, and of sulphur, $444.6^{\circ} \mathrm{C}$, at standard atmospheric pressure, represents the true thermodynamic scale (as closely as we have any means of determining this agreement) up to $\mathrm{I} \mathrm{IOO}^{\circ} \mathrm{C}$, the highest temperature at which the resistance thermometer can be operated. It has been also found that the temperature scale defined by the rare-metal thermocouple ( $\mathrm{Pt}, 90$ per cent $\mathrm{Pt}$-Io per cent $\mathrm{Rh}$ ) calibrated at three fixed points, such as the freezing points of zinc, antimony, and copper, agrees with the thermodynamic

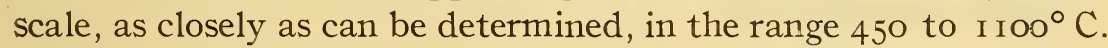

The gas thermometer has never been used with high accuracy at a temperature greater than $1550^{\circ} \mathrm{C}$. Above this range the temperature scale must be defined by means of the Stefan-Boltzmann or Wien-Planck radiation laws. These laws have a theoretical significance, and experimental evidence is such that the scales defined by these two laws are in mutual agreement and that they represent the ideal thermodynamic scale.

In order to obtain as consistent a reproducible temperature scale as is at present possible, this Bureau has provisionally adopted the following procedure: In the interval -40 to $450^{\circ} \mathrm{C}$ the temperature scale is defined by the platinum resistance thermometer calibrated in ice, steam, and sulphur vapor $\left(444.6^{\circ} \mathrm{C}\right)$. The scale from $45^{\circ}$ to $\mathrm{I} \mathrm{IO0^{ \circ }}$ is defined by the fixed points copper $1083^{\circ} \mathrm{C}$, antimony $630^{\circ} \mathrm{C}$, and zinc $419.4^{\circ} \mathrm{C}$, and interpolation between these points is based on the temperature scale defined by the rare-metal thermocouple (Pt, 9o per cent Pt-Io per cent Rh) calibrated at these three standard temperatures, using a parabolic interpolation formula. The temperature 
scale ${ }^{1}$ above $\mathrm{IIOO}^{\circ} \mathrm{C}$ is based on the extrapolation of Wien's law, using the fiducial point $\mathrm{m}$. p. gold $=\mathrm{IO} 63^{\circ} \mathrm{C}$, and $\mathrm{c}_{2}=\mathrm{I} 435^{\circ}$. On the basis of the scale defined above, the following table represents the melting and boiling points of several important pure substances, the accuracy being possibly $0 . \mathrm{I}^{\circ}$ at $500^{\circ} \mathrm{C}$, I ${ }^{\circ}$ at

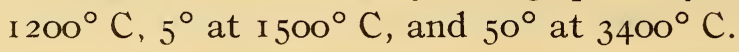

TABLE 1.-Standard Fixed Points

\begin{tabular}{|c|c|c|c|}
\hline Freezing or melting points: & ${ }^{\circ} \mathrm{C}$ & Freezing or melting points & ${ }^{\circ} \mathrm{C}$ \\
\hline Tin ...... & 231.9 & Gold................... & 1063 \\
\hline Lead.. & 327.4 & Copper.......... & .1083 \\
\hline Zinc..... & 419.4 & Nickel.......... & .1452 \\
\hline Antimony.............. & 630.0 & Palladium... & .1550 \\
\hline Aluminum (0.997 pure) & 658.7 & Platinum... & .1755 \\
\hline Copper-silver eutectic.. & 779.0 & Alumina....... & . 2050 \\
\hline Silver.. & 960.5 & Tungsten..... & 3400 \\
\hline \multicolumn{4}{|l|}{ Boiling points: } \\
\hline \multicolumn{4}{|c|}{$+0.058 \quad(a P-760)$} \\
\hline \multirow{2}{*}{\multicolumn{4}{|c|}{$\begin{array}{r}.305 .9+.063(P-760) \\
.444 .6+.091 \quad(P-760)\end{array}$}} \\
\hline & & $\therefore .$. & $-760)$ \\
\hline
\end{tabular}

a $P$ denotes pressure in $\mathrm{mm} H g$.

The above table applies for materials of the highest possible purity. The presence of impurities will, in general, lower the freezing or melting points and raise the boiling points. Methods for using the above materials for the standardization of pyrometers are discussed later.

\section{HIGH-TEMPERATURE THERMOMETRY ${ }^{2}$}

\section{INTRODUCTION}

High-temperature thermometry is concerned with the measurement of temperature in the range $\mathrm{I} O \mathrm{oo}$ to $55^{\circ} \mathrm{C}$. The lower arbitrary limit corresponds to the temperature of boiling water at normal atmospheric pressure, the upper limit $\left(55^{\circ} \mathrm{C}\right)$ is approximately the highest temperature to which a mercury-in-glass thermometer may be safely subjected. ${ }^{3}$

The following table gives a brief classification of the various types of high-temperature thermometers:

\footnotetext{
${ }^{1}$ For a more complete discussion of the temperature scale see Waidner, Mueller, and Foote, Standard Scale of Temperature, Chicago, Pyrometry Symposium.

'This section is a synopsis of a paper by Wilhelm, Chicago, Pyrometry Symposium.

: Quartz glass thermometers filled with mercury under pressure have been constructed to measure temperatures up to $70^{\circ} \mathrm{C}$. Reports as to their behavior, however, have not been promising. Special glasses have been recently produced which offer some possibilities for use even above $600^{\circ} \mathrm{C}$, but mercurial thermometry above $500^{\circ} \mathrm{C}$ is still in the development stage.
} 
TABLE 2.-Classification of Thermometers

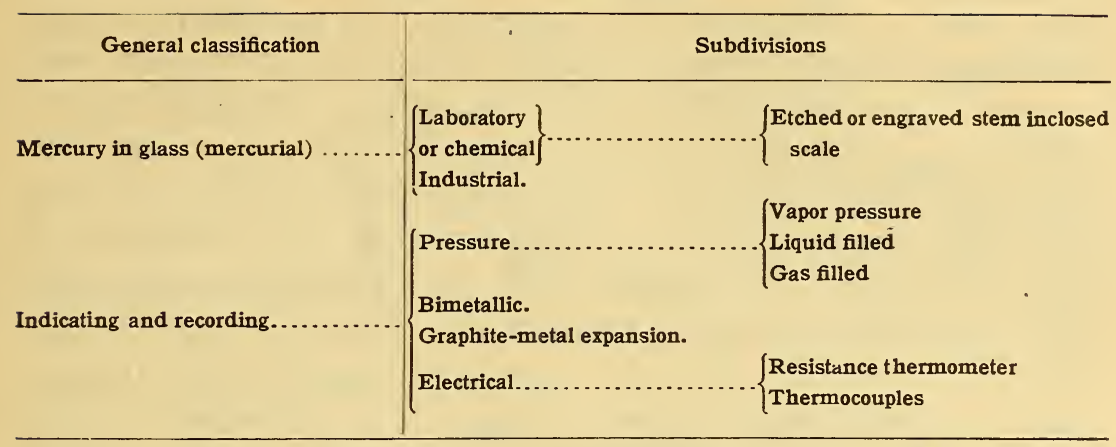

\section{HIGH-TEMPERATURE MERCURIAL THERMOMETERS}

In the laboratory the so-called engraved or etched-stem thermometer is more generally used than the inclosed-scale (einschluss) type, probably because of the preference of American manufacturers for the former. For some classes of work, and especially under high temperature conditions, the inclosed-scale thermometer has certain advantages over the etchedscale type, in that the coloring matter in the graduation lines can not be removed by solvents, the thermometer may be repaired if only the outer tubing is broken, and parallax may be avoided by a simple procedure. The disadvantages, however, may offset the good features for the scale may become loose, and this, as well as the fact that the scale and capillary tubing may not be in close contact, may introduce uncertain errors. The computation of the correction for emer-

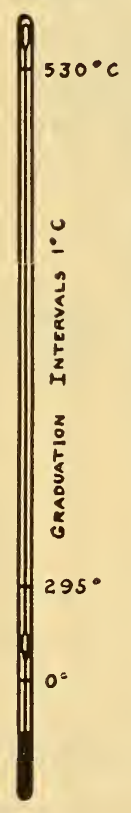

(a)

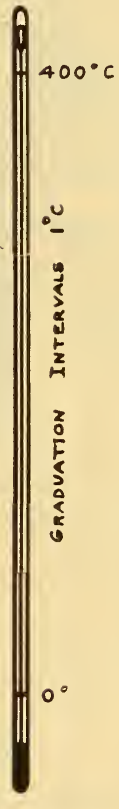

(b)

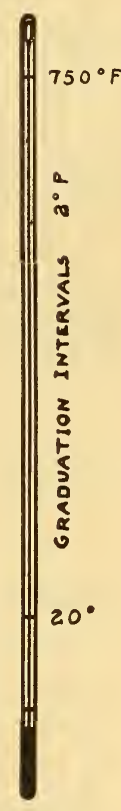

(c)
FIG. x.-Types of high-temperature mercury-in-glass thermometers gent stem is also less reliable because of the uncertainty as to the actual temperature inside the glass tubing.

Fig. I shows characteristic types of etched-stem, high-temperature laboratory thermometers. The first instrument $(a)$ is the 
type used as a standard at the Bureau of Standards in the range 300 to $530^{\circ} \mathrm{C}$. The second thermometer $(b)$ is a continuous scale instrument used for making distillation tests and graduated from o to $400^{\circ} \mathrm{C}$ in $\mathrm{I}^{\circ}$ intervals, for total immersion. The third instrument $(c)$ is a 20 to $750^{\circ} \mathrm{F}$ thermometer divided into $2^{\circ}$ intervals for partial immersion, and is used in oil testing or other industrial work. For convenience in re-standardizing, it is desirable to have the ice-point graduations $\left(0^{\circ} \mathrm{C}, 32^{\circ} \mathrm{F}\right)$ marked on the stem.

If it is necessary to use a thermometer for partial immersionthat is, with the bulb and only a part of the stem heated-it is advisable to use the continuous-scale type $(b)$ or $(c)$ rather than the standard type $(a)$, especially if the enlargement in the capillary shown between the o and $295^{\circ}$ marks projects out into the air, which is much cooler than the bulb, as otherwise large and uncertain errors may be introduced.

It is not advisable to graduate thermometers to be used in the range 200 to $550^{\circ} \mathrm{C}$ into intervals smaller than $\mathrm{I}$ or $2^{\circ} \mathrm{C}$, as the maximum obtainable accuracy is from 0.1 to $0.5^{\circ} \mathrm{C}$. The following table illustrates the accuracy which may be expected of highgrade thermometers used with total immersion:

TABLE 3.-Accuracy of High-Grade Thermometers

\begin{tabular}{|c|c|c|c|c|c|c|c|}
\hline Scale range & $\begin{array}{c}\text { Sub- } \\
\text { division }\end{array}$ & $\begin{array}{l}\text { Probable } \\
\text { accuracy } \\
\text { unstand- } \\
\text { ardized }\end{array}$ & $\begin{array}{l}\text { Maximum } \\
\text { accuracy } \\
\text { standard- } \\
\text { ized }\end{array}$ & Scale range & $\begin{array}{c}\text { Sub- } \\
\text { division }\end{array}$ & $\begin{array}{l}\text { Probable } \\
\text { accuracy } \\
\text { unstand- } \\
\text { ardized }\end{array}$ & $\begin{array}{c}\text { Maximum } \\
\text { accuracy } \\
\text { standard- } \\
\text { ized }\end{array}$ \\
\hline \multirow[t]{4}{*}{$\begin{array}{c}{ }^{\circ} \mathbf{C} \\
100 \text { to } 200\end{array}$} & ${ }_{5}^{\circ} \mathrm{C}$ & ${ }^{\circ} \mathrm{C}_{2}$ & ${ }^{\circ} \mathrm{C}_{1}$ & 300 to 400 & ${ }^{\circ} \mathrm{C}$ & ${ }^{\circ} \mathrm{C}_{3}$ & ${ }^{\circ} \mathrm{C}_{2}$ \\
\hline & 2 & 1 & 0.5 & & 2 & 3 & 1 \\
\hline & 1 & 1 & .2 & & 1 & 2 & .5 \\
\hline & 0.2 er 0.5 & 0.5 & .1 & 400 to 500 & 5 & $\cdot 5$ & 2 \\
\hline \multirow[t]{3}{*}{200 to 300} & 5 & 2 & 1 & & 1 or 2 & 2 & 1 \\
\hline & 2 & 2 & .5 & & & & \\
\hline & 5 or 1 & 1 & .2 & & & & \\
\hline
\end{tabular}

\section{INDUSTRIAL THERMOMETERS}

The use of the chemical or laboratory type of mercurial thermometer is restricted to the laboratory, since unprotected glass thermometers are too frail to withstand the rough usage of the plant and shop and are comparatively difficult to read. To meet the requirement of the plant, there has been developed what is generally known as the industrial type of mercurial thermometer. As shown in Fig. 2, it is characterized by a heavy metal back and protecting tube for the bulb, large and distinct figures and gradua- 
tion marks, and threaded connections for attaching the instrument readily and firmly to some part of the apparatus. This same general design of instrument is used for many different operations, covering ranges of temperature from -30 to $55^{\circ} \mathrm{C}$.

It will be noted that the bulb does not come into direct contact. with the substance the temperature of which is to be measured and that it is surrounded by large metal parts, which extend into a region of different temperature. These two peculiarities in the construction of an industrial thermometer must be taken into consideration in their use and calibration. Since the bulb is not in direct contact with the heated substance, the time required to take up changes in temperature is greater than for a bare bulb. The "lag" can be reduced by filling the space between the bulb and the outer wall of the casing with a good conducting medium, the most satisfactory substance for temperatures below $200^{\circ} \mathrm{C}$ being mercury. The lag of an instrument read in steam at $100^{\circ} \mathrm{C}$ with mercury surrounding the bulb has been found $1 / 2$ to $1 / 3$ as great as when powdered graphite, oil, or air was used.

This lag, however, may not be

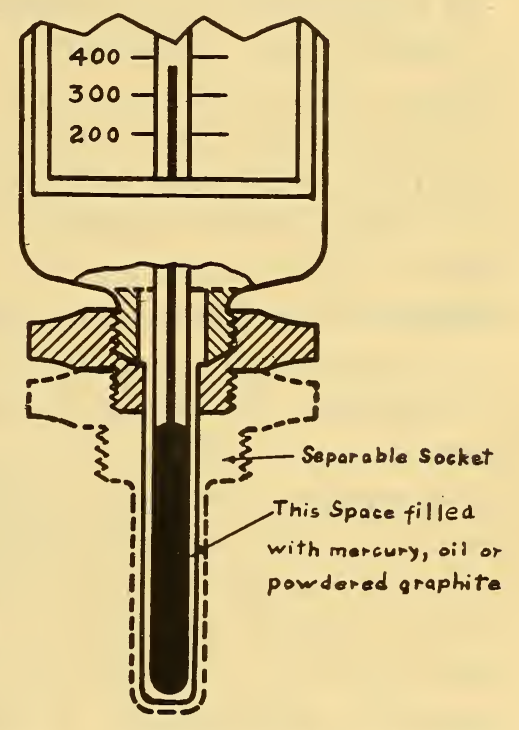

FIG. 2.-Industrial type of mercury-inglass thermometer as great a source of error as the actual discrepancy in the readings of the thermometers when used to measure the same temperature; but, under different conditions as regards construction of the protecting case surrounding the bulb, the material into which the bulb is immersed-that is, whether liquid, vapor, or gas-the rate of flow of the material past the bulb, and the exterior conditions to which the protecting stem and casing are subjected. No data, however, are available in regard to the precise effects of these various factors, even for definite types of thermometers. 


\section{EMERGENT-STEM ERROR OF MERCURY-IN-GLASS THERMOMETERS}

It is common practice to graduate and standardize thermometers for the condition that the bulb and the part of the stem containing the mercury are at the same temperature. This process is called graduation and standardization for total immersion. Some manufacturers, however, point part of their stock thermometers for other conditions of immersion, such as 3 inches, believing that this condition has a wider application than that of total immersion.

In the actual use of a thermometer it is quite often impossible to observe the conditions of total immersion of the mercury column, and many instruments must be used with the bulb only, or the bulb and a very small portion of the stem, heated. The remainder of the stem, containing the mercury column, is usually at a temperature considerably lower than the bulb, and the original calibration of the instrument, if for total immersion, will not hold. The thermometer reads too low under these conditions.

It is possible to compute the approximate correction to apply to the reading to reduce it to standard conditions by means of the formula ${ }^{4} S=a n(T-t)$, where $S$ is the correction to be applied to the reading, $a$ is a factor representing the relative expansion of mercury in glass, $n$ is the number of degrees of the mercury emergent from the bath, $T$ is the temperature of the bulb or bath, and $t$ is the average temperature of the emergent mercury column. The value of $a$ is about o.ooor 6 for centigrade temperatures and 0.00009 for Fahrenheit. $T$ can be approximated by using the reading of the thermometer, and, if a higher degree of accuracy is desired, a second approximation may be made by adding the correction first found to the reading and using this value of $T$ to obtain a second correction. The value of $t$ can be measured roughly by an auxiliary thermometer the bulb of which is placed about three-fourths the distance down the exposed mercury column. A more precise method is to use a "faden" or thread thermometer, which is designed to give the average temperature of a given length of the mercury column. The theory of stem correction and the use of faden thermometers is discussed by Buckingham. ${ }^{5}$

In processes for which the immersion of the thermometer is definitely known thermometers may be previously graduated for

\footnotetext{
${ }^{4}$ Rimbach, Zeit. f. Inst., 10, 1890 , gives stem-correction tables which have been widely published. Their application is limited, however, as the data were obtained using special thermometers in distillation apparatus.

${ }^{5}$ B. S. Sci. Papers, No. I70: I9xi.
} 
the required immersion. Partial-immersion thermometers are subject to error unless the conditions under which the thermometer is used exactly correspond closely to those of pointing or standardization. Thus, changes in the room temperature or temperature conditions surrounding the stem may introduce errors of several degrees. However, when high accuracy is not desired, it is probably more satisfactory to use these partialimmersion instruments than to attempt a stem correction with a total-immersion thermometer. Thermometers so graduated should be marked, preferably, with a line around the stem indicating the depth of immersion and also with a statement to this effect on the stem.

Several processes in which thermometers calibrated for total immersion are used under partial-immersion conditions have been investigated at the Bureau of Standards and the following stem-correction data obtained: ${ }^{6}$

TABLE 4.--Stem-Correction Data for Cleveland Open-Cup Flash and Fire-Point Tester Thermometer

[Range-20 to $760^{\circ} \mathrm{F}$ in $2^{\circ}$ intervals, length about 15 in
\begin{tabular}{|c|c|c|c|}
\hline $\begin{array}{c}\text { Ther- } \\
\text { mometer } \\
\text { reading }\end{array}$ & $\begin{array}{c}\text { Degrees } \\
\text { of } \\
\text { mercury } \\
\text { column } \\
\text { emergent }\end{array}$ & $\begin{array}{c}\text { Mean } \\
\text { tempera- } \\
\text { ture of } \\
\text { emergent } \\
\text { mercury } \\
\text { column }\end{array}$ & $\begin{array}{c}\text { Stem } \\
\text { correction }\end{array}$ \\
\hline${ }^{\circ} \mathrm{F}$ & ${ }^{\circ} \mathrm{F}$ & ${ }^{\circ} \mathrm{F}$ & ${ }^{\circ} \mathrm{F}$ \\
200 & 208 & 174 & 0.5 \\
300 & 308 & 177 & 3.5 \\
400 & 408 & 177 & 8.5 \\
500 & 508 & 187 & 15.0 \\
600 & 608 & 203 & 23.0 \\
\hline
\end{tabular}

TARLE 5.-Stem-Correction Data for Distillation Flask Used in Distillation of Creosote Oil

[Dimensions of flask: Diameter bulb, $8 \mathrm{~cm}$; diameter neck, $2 \mathrm{~cm}$; height neck, $15 \mathrm{~cm}$; distance bottom - of outlet tube to top, $8 \mathrm{~cm}$. Thermometer range, 0 to $400^{\circ} \mathrm{C}$ in $1^{\circ}$ intervals; length, $40 \mathrm{~cm}$ l

\begin{tabular}{|c|c|c|c|}
\hline $\begin{array}{c}\text { Ther- } \\
\text { mometer } \\
\text { reading }\end{array}$ & $\begin{array}{c}\text { Degrees of } \\
\text { mercury } \\
\text { column } \\
\text { above } \\
\text { bulb }\end{array}$ & $\begin{array}{c}\text { Mean } \\
\text { tempera- } \\
\text { ture of } \\
\text { mercury } \\
\text { column }\end{array}$ & $\begin{array}{c}\text { Stem } \\
\text { correction }\end{array}$ \\
\cline { 1 - 3 } & ${ }^{\circ} \mathrm{C}$ & ${ }^{\circ} \mathrm{C}$ & ${ }^{\circ} \mathrm{C}$ \\
${ }^{\circ} \mathrm{C}$ & 208 & 59 & .4 .7 \\
200 & 258 & 111 & 6.0 \\
250 & 308 & 98 & 10.5 \\
300 & 358 & 95 & 15.5 \\
350 & & 95 \\
\hline
\end{tabular}

${ }^{6}$ Wilhelm, B. S. Tech. Papers, No. 49. 
TABLE 6.-Stem-Correction Data for Pensky-Martin Flash-Point Apparatus

Thermometer used up to $150^{\circ} \mathrm{C}$, range 40 to $160^{\circ} \mathrm{C}$ in $1^{\circ}$ intervals; scale length, $9.5 \mathrm{~cm}$; thermometer used from 200 to $300^{\circ}$, range 200 to $360^{\circ}$ in $1^{\circ}$ intervals; scale length, $12 \mathrm{~cm}$ ]

\begin{tabular}{|c|c|c|c|}
\hline $\begin{array}{c}\text { Ther- } \\
\text { mometer } \\
\text { reading }\end{array}$ & $\begin{array}{c}\text { Degrees } \\
\text { of } \\
\text { mercury } \\
\text { column } \\
\text { emergent }\end{array}$ & $\begin{array}{c}\text { Mean } \\
\text { tempera- } \\
\text { ture of } \\
\text { emergent } \\
\text { mercury } \\
\text { column }\end{array}$ & $\begin{array}{c}\text { Stem } \\
\text { correction }\end{array}$ \\
\cline { 1 - 2 } & ${ }^{\circ} \mathrm{C}$ & ${ }^{\circ} \mathrm{C}$ & ${ }^{\circ} \mathrm{C}$ \\
50 & 30 & 35 & 0.1 \\
100 & 80 & 45 & .7 \\
150 & 130 & 55 & 2.0 \\
200 & 10 & 75 & .2 \\
250 & 60 & 85 & 1.6 \\
300 & 110 & 100 & 3.5 \\
\hline
\end{tabular}

Empirical methods are occasionally employed for certain more or less standard operations, such as distillation, in which a totalimmersion thermometer is specified, to be used without regard to the emergent-stem correction.

\section{OTHER SOURCES OF ERROR IN USE OF HIGH-TEMPERATURE MER- CURIAL THERMOMETERS}

Aside from the error arising from the failure to observe the proper condition of immersion, there are two common sources of error which may be attributed to actual defects in the manufacture of the instrument. These are insufficient pressure above the mercury to prevent breaking of the mercury column or evaporation at the surface of the mercury and improper or insufficient annealing for use at high temperatures. This latter defect may result in a rise of the reading with continued heating amounting to as much as $30^{\circ} \mathrm{C}$ in extreme cases.

\section{FILLING HIGH-TEMPERATURE THERMOMETERS UNDER PRESSURE}

Mercury boils at $357^{\circ} \mathrm{C}$ at atmospheric pressure and evaporates fairly rapidly at much lower temperatures. It is accordingly desirable for thermometers used above $100^{\circ} \mathrm{C}$ to fill the capillary above the mercury with nitrogen under a pressure of from 2 to 20 atmospheres, depending upon the temperature range and construction of the stem. Failure to fill high-temperature thermometers under the sufficient pressure may be sometimes detected by the broken appearance of the mercury column and by drops of mercury which have distilled from the top, but often the column breaks in a part of the stem not visible and the defect is not readily noticed. 


\section{IMPROPER ANNEALING}

Improper annealing can be detected only by repeated calibration of the instruments from time to time. Laboratories usually nave facilities for checking thermometers either at the ice or steam point or for comparing them with a thermometer known to be reliable.

\section{INDICATING AND RECORDING THERMOMETERS}

The term "indicating" is usually employed to designate thermometers of the dial-and-pointer type, rather than those constructed of mercury and glass. Indicating thermometers may or may not be distance reading; that is, so constructed as to allow

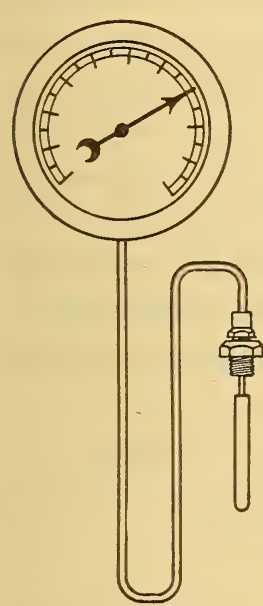

FIG. 3.-Distancereading indicating thermometer the indicator to be placed at some distance from the bulb. A distance-reading indicating thermometer is shown in Fig. 3. A recording thermometer employs a mechanism for making a continuous record of temperature on a suitable chart, as shown in Fig. 4.

Indicating and recording thermometers may be divided into three general classes-electrical, pressure, and bimetallic. Electrical thermometers or pyrometers are considered later. Pressure thermometers comprise a bulb containing a liquid or gas or both connected by means of

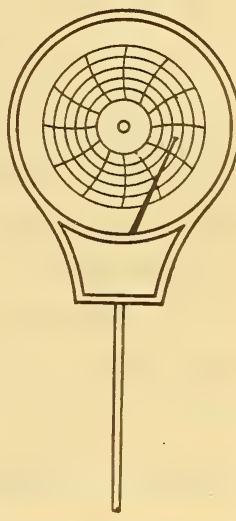

FIG. 4.-Recording thermometer capillary tubing to some form of pressure gage. Bimetallic thermometers utilize the turning moment produced by heating a strip consisting of two metals brazed together, and having different coefficients of expansion. Graphite-metal thermometers make use of the linear differential expansion of rods of graphite and a suitable metal. The accuracy and adaptability of these types of instrument have not been sufficiently investigated to allow a more detailed discussion.

(a) PRESSURE THERMOMETERS.-Pressure thermometers may be subdivided into vapor-pressure, liquid-filled, and gas-filled. The thermal expansion of a liquid or gas is approximately linear with temperature; hence these thermometers have a linear tem$8513^{\circ}-21-2$ 
pera,iure scale. The change in vapor pressure, with temperature, however, is not linear, and the temperature scale of a vaporpressure thermometer becomes more open at higher temperatures. Otherwise, the three types of pressure thermometers are similar in outward appearance.

(I) Liquid Used in Vapor-Pressure Thermometers. -The volatile liquid used in a vapor-pressure thermometer must possess certain well-defined characteristics. It should be stable, readily obtained, and purified, should not act upon the metals with which it is in contact, must have a sufficiently high pressure at the lower temperatures to insure a readable temperature scale, and its critical temperature must be higher than the highest temperature to be measured. The vapor pressures of the liquids available have been determined over wide ranges, and it is usually possible by making use of such data to select the most suitable liquid for a given range of temperature or, if a liquid be given, to predict the temperature range within which it can be used and the pressures which will be developed. In general, the temperature and pressure range for a given liquid will be included between its boiling point at a pressure of one atmosphere and its critical temperature. Although these limits are very arbitrary, they are convenient for reference.

The following table gives the boiling points at a pressure of I atmosphere and the critical temperátures and pressures for various liquids suggested for use in vapor-pressure thermometers.

TABLE 7.-Boiling Points and Critical Data of Liquids Suggested for Use in VaporPressure Thermometers

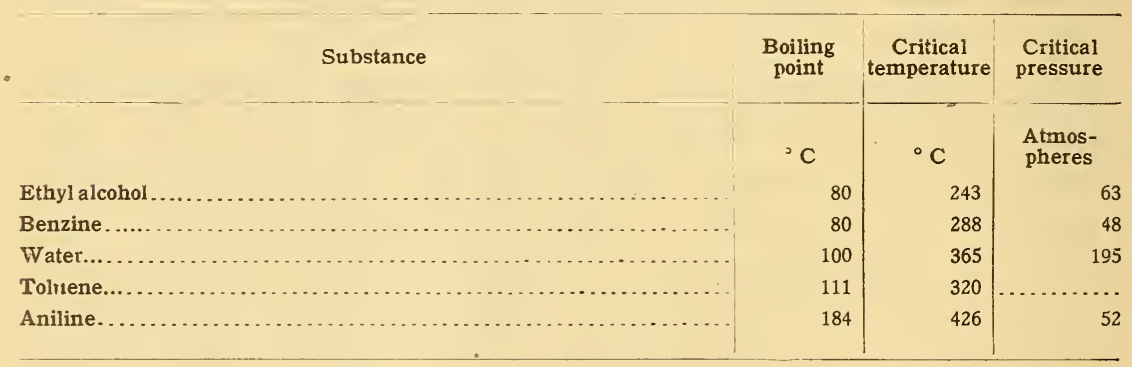

(2) Materials and Pressures Employed in Liquid and Gas-filled Thermometers.-Pure materials chemically inactive upon the bulb are requisite, and the highest temperature measured should not exceed the critical temperature of the liquid. Alcohol, which has been used in liquid-filled thermometers, is not satisfactory above $200^{\circ} \mathrm{C}$. Aniline has been suggested for temperatures below 
$400^{\circ} \mathrm{C}$. Mercury, however, is used almost exclusively for temperatures up to 500 or $600^{\circ} \mathrm{C}$. Gas-filled thermometers using preferably nitrogen are not satisfactory much above $500^{\circ} \mathrm{C}$, since the metal bulbs become permeable to the gas at high temperatures. The initial pressure and the pressure range of a liquid or gas filled thermometer varies according to the pressure range of the gage and the temperature range.

(3) Principles Underlying the Action of Pressure Thermometers.The action of the vapor-pressure thermometer depends upon the fact that the pressure inside the thermometer is determined solely by the temperature of a free surface of the liquid. It follows, therefore, that the thermometer must be so constructed that one free surface is always in the bulb. If this condition is fulfilled the reading of the instrument is not sensibly affected by changes in the temperature of the gage and capillary. This is a decided advantage over other types, when the connecting tubing and gage are subjected to considerable changes in temperature. If the vapor-pressure thermometer is not filled properly - that is, the proportion of liquid is too great or too small as compared with the volume of the bulb, capillary, and gage-very large and uncertain errors may be introduced. As an ex-

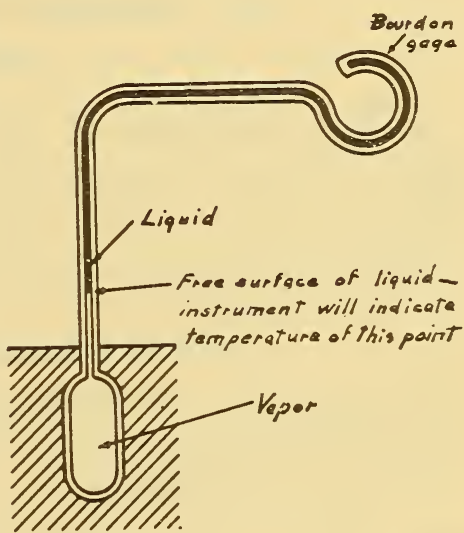

Not Sufficiont Liquid Bulb Hotian Than Tubing and Gaga

Fig. 5.-Vapor-pressure thermometer with insufficient liquid ample, consider the condition where the bulb is much hotter than the capillary. The liquid is condensed in the capillary, and there should be sufficient liquid to entirely fill the capillary and partly fill the bulb. If the liquid fills the gage and only partly fills the capillary, and there is no liquid in the bulb, the capillary will contain the free surface of the liquid and the temperature indicated will be that corresponding to this portion of the capillary as shown in Fig. 5. This temperature, which may be several hundred degrees lower than that of the bulb, will be indicated instead of the true temperature of the bulb. 
Gas and liquid filled thermometers operate on the principle of thermal expansion. They are entirely filled with either the liquid or the gas. The expansion of the liquid or gas in the bulb is transmitted through capillary tubing to the pressure gage. These instruments are subject to error if the gage and capillary are at a different temperature from that at which they were calibrated. This error may be made negligible in many instances by reducing the volume of the capillary and gage as compared with that of the bulb or by using a compensator in the lead. Such a compensator, however, will not eliminate errors due to the heating or cooling of the capillary alone.

\section{THERMOELECTRIC PYROMETRY}

\section{INTRODUCTION}

Seebeck discovered in I82 I that if in a closed circuit of two metals the two junctions of the metals are at different temperatures an electric current will flow in the circuit. For example, if the ends of an iron and of a copper wire are fused together and one of the junctions is heated (within certain temperature limits), positive current will flow in the direction copper to iron at the hot junction or iron to copper at the cold junction. Two causes contribute to the production of the emf which actuates the current. An emf exists between two different metals placed in contact the magnitude of which depends upon the temperature and upon the metals used. Also, if a wire of homogeneous material is heated at one end, an emf is developed between the hot and cold ends of the wire the magnitude of which depends upon the metal and upon the differences in temperature of the ends. These two emfs are known as the Peltier emf and the Thomson emf, respectively. The total emf acting in the copper-iron circuit described above is the sum of the Peltier emf at each junction and the Thomson emf over each wire, consideration being given, of course, to the algebraic signs of these four emfs. The total emf acting in a circuit of two dissimilar metals thus depends upon the temperatures of the two junctions. If the temperature of one junction is fixed at that of the room or of melting ice, etc., the temperature of the other junction can be determined by measuring the emf developed in the circuit. This is the basic principle of thermoelectric pyrometry. In general, the thermoelectric circuit consists of more than two different metals, copper and manganin being introduced by the instrument employed for measuring the emf. However, it 
may be shown that the insertion of a different metal in the circuit produces no effect upon the total emf if both ends of the introduced metal are at the same temperature. The emfs developed by thermocouples are small, usually a few thousandths of a volt. To measure such small emfs, special types of sensitive voltmeters (millivoltmeters) or indicators are required. For any particular type of couple these instruments may be graduated to read temperature directly instead of emf.

A simple thermoelectric pyrometer consists of three parts: (a) The thermocouple of two different metals or alloys having a fused junction (the hot junction), which is inserted into the furnace, and the cold junctions, which protrude from the furnace and are maintained at some fixed temperature, such as that of the room or of melting ice; (b) two lead wires, usually of copper running from the cold junctions of the thermocouple to the indicator; (c) the indicator, which may be a millivoltmeter, a potentiometer, or a special type of instrument embodying both of these principles, and may be graduated to read either emf, temperature, or both.

\section{METALS USED FOR THERMOCOUPLES}

Although any two dissimilar metals might be employed for a thermocouple, certain combinations are unsatisfactory because of the very small emfs which can be developed and because of the fact that with some combinations the emf may first increase, then decrease, become zero, and change sign as the temperature increases. Obviously, desirable properties for a thermocouple are:

I. Capability for resisting corrosion and oxidation.

2. The development of relatively large emfs.

3. A temperature-emf relation such that the emf increases continuously with increasing temperature over the temperature range employed.

For general work at higher temperatures several different types of couples are employed in this country: Up to $360^{\circ} \mathrm{C}$ for extreme precision or to $500^{\circ} \mathrm{C}$ for a precision of 5 or $10^{\circ} \mathrm{C}$ the couple may consist of one wire of copper and the other wire of constantan. Iron-constantan or nichrome-constantan may be employed for technical processes below $900^{\circ} \mathrm{C}$. For operation below i IOo ${ }^{\circ} \mathrm{C}$ special patented alloys of chromium and nickel and of aluminum and nickel, "chromel-alumel" or nichrome-alumel are very satisfactory even for continuous service. For the temperature range

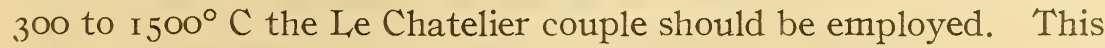


couple consists of one wire of platinum and the other wire an alloy containing 90 per cent platinum and ro per cent rhodium. Other alloys and metals may be employed for special work, but the above combinations are sufficient for almost all technical processes carried on in the temperature range o to $1500^{\circ} \mathrm{C}$. No satisfactory couple has been developed for operation above $1500^{\circ} \mathrm{C}$. There are several metals and numerous alloys which melt at much higher temperatures, but all so far known are subject to serious disadvantages which make their application useless commercially. For example, iridium, 90 per cent iridium-1o per cent ruthenium, may be used up to $2000^{\circ} \mathrm{C}$, but the couple is so costly as to be prohibitive, is so fragile and brittle that a slight jar will fracture it, and the iridium rapidly volatilizes even at $\mathrm{r} 200^{\circ} \mathrm{C}$. Tungstenmolybdenum could be employed possibly up to $2400^{\circ} \mathrm{C}$, but both of these metals oxidize so readily that extreme care must be taken by use of hydrogen to prevent oxidation. A satisfactory method for thus protecting such a couple for technical purposes has never been developed. The peculiar fact that nickel is readily oxidized by heating in air and soon becomes so brittle as to be useless, but when alloyed with chromium or aluminum the material resists oxidation and does not crystallize so rapidly, suggests the possibility that certain alloys of tungsten may be developed which would not exhibit the property of rapid oxidation characteristic of the pure metal. Such alloys might prove of great value in thermoelectric pyrometry.

\section{CONSTANCY OF CALIBRATION}

A further essential property of a thermocouple is constancy of calibration. Alterations in the calibration may be due to various causes. Inhomogeneity may develop on account of contamination by fumes from the furnace, metallic vapors, by oxidation, etc. Contamination may be usually prevented by the use of suitable protecting tubes surrounding the couple, and the effect of contamination may be minimized by using wire of large cross section. Different furnace conditions and different types of couples require different methods of protection against contamination. Each process must be considered individually. For example, a platinum couple is usually protected against contamination by means of refractory porcelain tubes; but, if the atmosphere surrounding the platinum is reducing, the use of porcelain may do more harm than good. In this case the reducing atmosphere changes the silica of the porcelain into silicon, which readily attacks the plati- 
num. The emf of some couples gradually decreases with use. The (platinum, platinum 90 per cent-iridium ro per cent) couple has not proved very satisfactory for this reason, although it develops a much larger emf than the platinum-rhodium couple. The iridium gradually distills from the alloy wire, especially above I $200^{\circ} \mathrm{C}$, requiring frequent recalibration of the couple.

\section{REPRODUCIBILITY OF COUPLES}

When thermocouples are employed in the laboratory for scientific purposes, it is desirable, although not of serious importance, that the calibration or temperature-emf relation of couples of the same type be exactly similar. However, in the industrial plant this question of reproducibility is of considerable moment. The indicating instruments are usually graduated in degrees of temperature, and the graduation applies for one definite temperatureemf relation only. If the temperature-emf relations of various couples of the same type are not similar, corrections must be applied to the readings of the indicator, and these corrections will be different for each couple. When several couples are operated with one indicator, and when the process is such as to require a frequent renewal of couples, the applying of these corrections becomes very troublesome. For extreme precision it is always necessary to apply such corrections, but for most industrial processes thermocouples which are sufficiently reproducible can be secured, so that the corrections are negligibly small and may be omitted. Thus the temperature-emf relations of different homogeneous and uncontaminated chromel-alumel or iron-constantan couples should not vary in general by more than 10 or $15^{\circ} \mathrm{C}$ and of platinum, platinum-rhodium couples by more than 2 or $3^{\circ} \mathrm{C}$. In fact, specifications for chromel-alumel and iron-constantan couples may be written as closely as $\pm 15^{\circ} \mathrm{F}$ and in certain instances specifications to $\pm 5^{\circ} \mathrm{F}$ are being satisfied. There are no industrial processes carried out under such accurate temperature control that the variations in the calibration of different new platinum, platinum-rhodium thermocouples of the same type warrant consideration. The variations in the calibration of different basemetal couples are frequently corrected by use of series or shunt resistance, but most methods so far devised are rather unsatisfactory, and some of the compensation methods employed may derelop after continued use larger errors than those arising from the irreproducibility of the couple, as will be shown later. The 
above statements in regard to reproducibility of different couples of the same type apply only for new couples. After a couple has been used for some time, especially a base-metal couple, or has become contaminated in any manner, the calibration may change considerably. All thermocouples should be checked frequently as installed to determine whether the calibration has altered.

\section{MOUNTED THERMOCOUPLES}

The question of properly mounting and protecting a thermocouple is of great importance. The type of protection necessary. depends upon the particular industrial process for which a couple is employed and will be considered in some detail later. In the laboratory if it is desired to measure the temperature of a perfectly clean porcelain platinum-wound furnace containing no material such as iron, etc., which could contaminate platinum, a rare-metal couple may be used without any protection whatever. For most laboratory experiments, however, a rare-metal couple requires protection. The usual rare-metal couple consists of wires $0.5 \mathrm{~mm}$ or preferably $0.6 \mathrm{~mm}$ in diameter and from 50 to I $25 \mathrm{~cm}$ in length. Wires as small as o.I $\mathrm{mm}$, and even less, are frequently used for special research work. One or both of the wires are insulated by threading them through small porcelain or quartz tubes. In measuring temperatures below about $1400^{\circ} \mathrm{C}$, two-hole porcelain tubes are very useful for insulating both wires, but for higher temperatures separate tubes should be used. The hot junction of the couple is made by fusing the two wires in an arc or oxygen-illuminating gas flame. The couple and insulating tube are inserted in a small outside protecting tube of porcelain, glazed on the outside only, or of fused silica, hemispherically closed at one end. On the open end of the protecting tube may be mounted the head of the couple, which serves as a handle and as a support for rigidly holding the wires of the couple. The couple wires frequently extend beyond the head of the couple, so that their ends may be maintained at some controlled coldjunction temperature. Usually the wires terminate in binding posts on the couple head, in which case the cold-junction temperature may be controlled by water jackets, or may be allowed to remain that of the surroundings, or the couple may be fitted with one of the various devices discussed later for the elimination of cold-junction errors.

Base-metal couples for laboratory use may be constructed in much the same manner described above and may be made of wire 
as small as No. 20 or of very much smaller wire for certain types of research at lower temperatures. Small wires, however, are readily oxidized completely at high temperatures, so that for con-

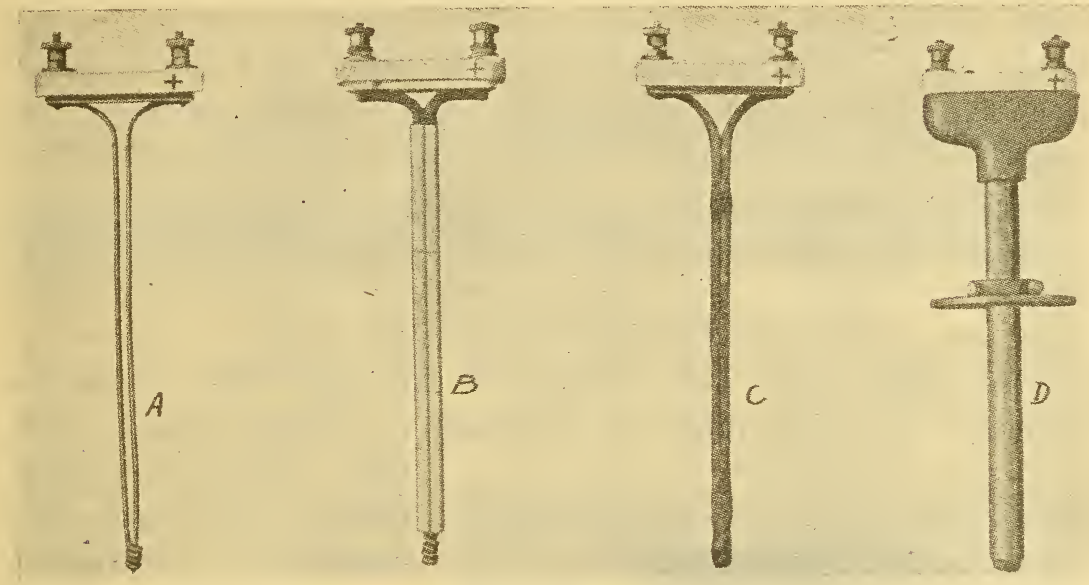

FIG. 6.-Types of commercial thermocouples

A, bure couple; B, couple with porcelain insulators; C, couple with asbestos insulation; D, ordinary mounting of base-metal couple in an iron protecting tube

tinuous operation in industrial installations the couples are constructed of No. 8 or No. 6 wire, or of still larger wire when there is danger of contamination. The hot junction is fused as in the case of rare-metal couples, and usually the two wires are twisted

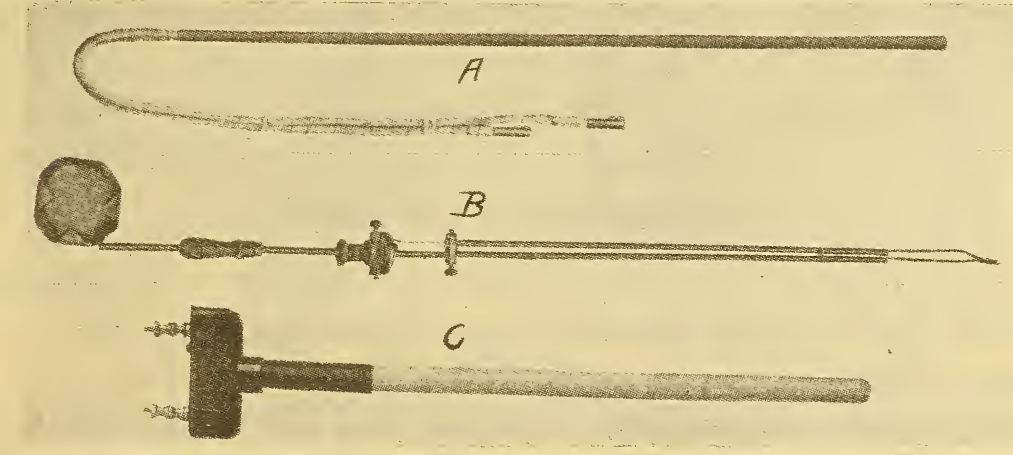

FIG. 7.-Types of commercial thermocouples

A, Iron-constantan couple in iron protecting tube for use below $900^{\circ} \mathrm{C}$. B, extensible chromel-alumel couplefor molten brass. The exposed junction which is immersed in the molten brass without protection is renewed from the magazine of reserve wire. C, porcelain or fused silica protecting tube and mounting for rare-metal couples

for a few turns at the hot junction in order to give greater mechanical strength to the joint. The two wires are insulated by fire-clay insulating tubes or by asbestos sleeving or cord, and are 
connected to a suitable couple head, forming the cold junction. For severe use it is necessary to incase the couple in a protecting tube of steel, chromel, porcelain, fire clay, etc. A different form of base-metal couple consists of an outer tube of iron and an inner wire or rod of constantan. The two are fused at one end into a neat joint, forming the hot junction, and are insulated from each other up to the couple head or cold junction. The couple is thus mechanically stronger than couples formed of two wires, and, when used without an additional protecting tube, is somewhat less liable to contamination than the bare two-wire couple of the same type, since the constantan, which is probably more susceptible to contamination than the iron, is protected by the iron tube. However, couples of this type must be further protected by outer tubes, if subjected to severe furnace condi-

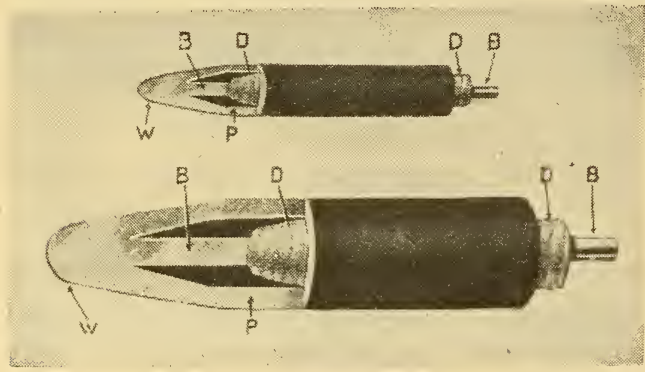

FIG. 8.-Types of commercial thermocouples

End section showing the outer tube $P$, which is one element, welded to the other element $\mathrm{B}$ at $\mathrm{W}$. The inner rod is insu-

lated from the tube by asbestos cord ous shapes; but, before bending, one should examine the couple and temporarily remove porcelain tubes or insulators liable to be broken by the process.

\section{INDICATING INSTRUMENTS}

The indicating instruments which are connected to the thermocouple are of three general types-those operating upon the galvanometric principle, as an ordinary voltmeter, those operating upon the potentiometric principle, and those operating upon a combination of these two principles. The first two types of instrument have been made automatically recording, as will be discussed elsewhere.

(a) Galvanometer Method.-Galvanometers for measuring the emf developed by a thermocouple usually operate on the d'Arsonval principle, having a moving coil mounted between the poles of a permanent magnet. The deflection of the pointer 
or boom attached to the coil is proportional to the thermoelectric current passing through the coil, and the current is proportional to the emf developed by the couple. Different methods for mounting the coil are employed. The coil may be suspended both above and below by phosphor-bronze suspensions, and,

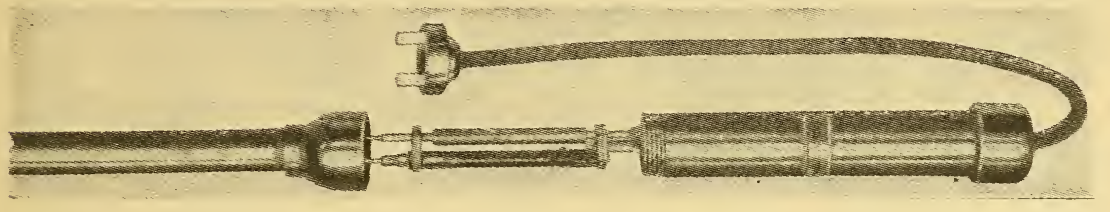

FIG. 9.-Types of commercial thermocouples

Interior of thermocouple head showing the cold-junction compensator described later under Fig. 37

although many foreign instruments of this type have proved very delicate, in one of the latest forms the American instrument, using the double-suspension galvanometer, may be subjected to severe handling without any injury whatever. The mounting

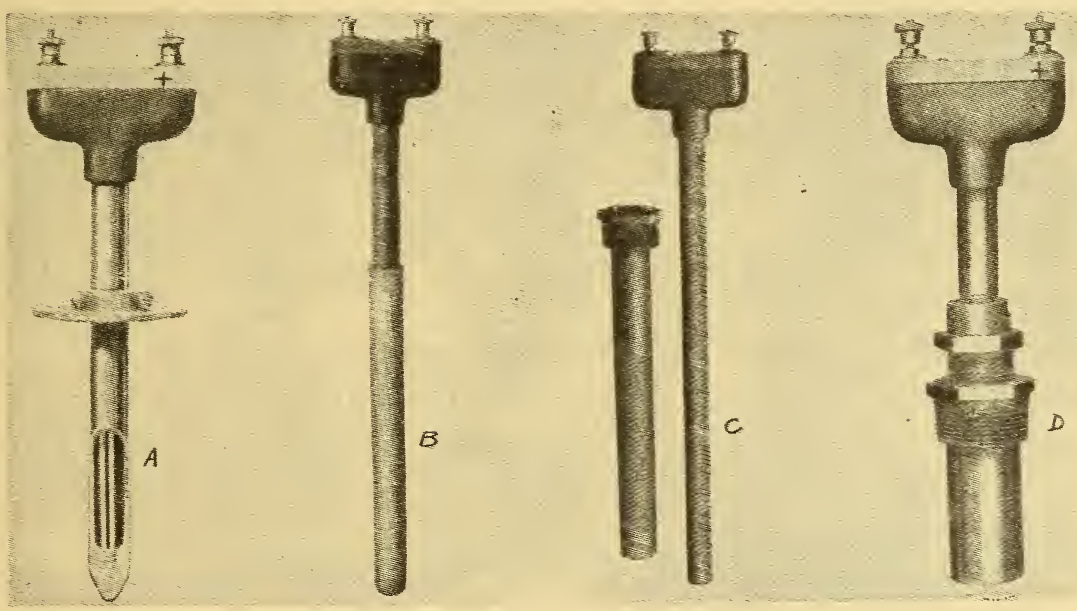

FIG. I0.-Types of commercial thermocouples showing special mountings for base-metal couples

A, cross section of the end of a thermocouple showing the hot junction welded to the end of the protecting tube to reduce thermal lag. A couple of this type is useful in the measurement of rapidly changing temperatures. B, couple protected by nichrome tube. C, couple with extra protecting sheath. D, couple for pipes and mains. The protecting tube is threaded into the main

consisting of an upper suspension and a lower pivot has been used extensively.

A unipivot system is employed by one English and one American manufacturer. The pivot is at the center of the coil, and the movement is balanced so that the center of gravity of the whole 
moving system is at the point of the pivot. In all pivot instruments the pivots must fit in well-made jewel bearings in order to reduce friction to a minimum. The advantages claimed for the unipivot system are (I) the axis of rotation of the coil passes through the pivot point thereby minimizing undesirable effects of friction; (2) the pivot and jewel may be separated during clamping; and (3) a heavier coil may be employed.

The scale of the instrument may be graduated to read emf or temperature. By use of a series resistance mounted inside the galvanometer case and an extra binding-post terminal, two scale ranges may be obtained, one for base-metal couples of a certain type and the other for the rare-metal Le Chatelier couple. For precision work the scale should have a mirror placed parallel to the scale and extending throughout its length for use in eliminating the error in reading due to parallax. While obtaining a reading the observer should use one eye only, and he should move his head to the position where the pointer and its reflected image in the mirror coincide.

Indicators may be obtained in either the switchboard type or portable type. The former is desirable for permanent installations. Many switchboard instruments have a scale ruled on ground glass and illuminated from the rear by an electric lamp. The black enameled graduations upon the scale thus appear distinct against a bright background. Paper scales illuminated from the front are frequently employed. Usually high precision is not required of an instrument of the switchboard type, so that the graduations can be coarse and readable at some distance. However, it is possible to obtain highly accurate switchboard indicators, in which case the scale should have a mirror to permit readings without parallax.

If the instrument is mounted in a dusty room, it should be protected by an outside case of steel or wood having a glass front, as the presence of dirt filtering into the interior of the galvanometer may seriously affect its calibration. Both the wall type and the portable indicator, especially the latter, must be sufficiently robust to withstand reasonably severe usage.

(I) Resistance of Indicating Instruments.-When operated at the highest safe-working temperatures, most base-metal couples develop a maximum emf of less than 70 millivolts, and the Le Chatelier couple develops an emf of about 16 millivolts. In order to measure such small emfs accurately, a very sensitive indicator 
or millivoltmeter is required. On the other hand, the instrument must be robust and able to withstand rough handling, to which it is necessarily more or less subjected. These conditions of mechanical robustness and of high accuracy as a pyrometer indicator are difficult to satisfy. The deflection of the pointer of the millivoltmeter is approximately proportional to the current flowing through the coil, so that, if the resistance of the total thermoelectric circuit is very low, relatively large currents are obtained, with a resulting high torque or turning moment on the movable coil. Thus when the current is high, the construction of the indicator may be made very robust. Relatively strong springs, against which the turning moment of the coil carrying the current is balanced, may be employed and less attention need be given to the friction of the pivots in their bearings. The torque may be made high without greatly increasing the resistance of the circuit by using a large number of turns of copper wire in the coil Copper possesses a large temperature coefficient of resistance, so that ordinarily the calibration of an instrument having its entire electrical circuit of copper would be affected by the temperature. However, by use of shunt and series resistances of a certain type it is possible to reduce these errors due to temperature changes to an amount of negligible importance. Other satisfactory methods for reducing this error are also employed. Thus it is not difficult to construct a sufficiently sensitive millivoltmeter of low resistance. The objection, however, from the pyrometric standpoint, to such an instrument used as a simple galvanometer has led to the development of indicators of considerable resistance, as will appear immediately. These are made by placing a high resistance of zero temperature coefficient, such as manganin, in series with the coil and, by increasing the number of turns on the moving coil, to compensate for the decrease in sensitivity arising from the increased resistance. The so-called swamping resistance of zero temperature coefficient may be so proportioned relative to the copper that, due account being taken of the temperature coefficient of elasticity of the springs, the instrument as a whole possesses a negligible temperature coefficient. The use of high resistance greatly diminishes the magnitude of the current flowing through the coil, and thus decreases the torque-producing deflection. Hence, attention must be given to the elimination of bearing friction as far as possible. Thus the instrument is necessarily more delicate than an indicator of low resistance and of the same type. The ad- 
vantage of an instrument having a high resistance is demonstrated by the following discussion.

When a thermocouple in a furnace is connected to a millivoltmeter, the emf of the couple causes a current to flow around the circuit, which in accordance with Ohm's law is equal to $\frac{e}{R}$ where $e$ is the emf developed by the couple and $R$ is the total resistance of the circuit. Thus, if the temperature of the furnace remains constant, $e$ is constant, but the deflection of the instrument will be altered by changes in the resistance $R$. Hence, any variation in $R$ which produces a change in the reading of the instrument would be interpreted as a change in the temperature of the furnace, although actually this is constant. The total resistance of the circuit may be divided into three parts- $R_{\mathrm{g}}$ the resistance of the millivoltmeter, $R_{\mathrm{L}}$ the resistance of the line, and $R_{\mathrm{c}}$ the resistance of the couple. If these resistances are properly proportioned, the effect upon the reading of the indicator of any changes likely to occur in the resistance $R$ can be reduced to practically zero. This condition is realized when $R_{\mathrm{g}}$, the resistance of the galvanometer, is sufficiently high compared with $R_{\mathrm{c}}+R_{\mathrm{L}}$, the resistance of the external circuit. Suppose that the indicator has an emf scale graduated to read the potential difference at its terminals. The relation between the reading of the instrument $e_{\mathrm{o}}$ and the true emf, $e$, of the couple to which it is connected is given by the following equation:

$$
e_{\mathrm{o}}=\frac{R_{\mathrm{g}}}{R_{\mathrm{g}}+R_{\mathrm{c}}+R_{\mathrm{L}}} e .
$$

Thus, if $R_{\mathrm{g}}$ is very large compared to $R_{\mathrm{c}}+R_{\mathrm{L}}$, the ratio $\frac{R_{\mathrm{g}}}{R_{\mathrm{g}}+R_{\mathrm{c}}+R_{\mathrm{L}}}$ is practically $\mathrm{I}$, and the reading of the galvanometer gives the true emf of the couple.

Robust indicators are now obtainable having a resistance of 300 to 1200 ohms. As a particular example of the behavior of such an instrument, values of the factors occurring in the above equation will be considered for an actual thermocouple installation.

Galvanometer resistance $=R_{\mathrm{g}}=300 \mathrm{ohms}$.

Couple resistance $\quad=R_{\mathrm{c}}=\mathrm{I}$ ohm.

Line resistance $\quad=R_{\mathrm{L}}=\mathrm{I}$ ohm.

Whence,

$$
e_{\mathrm{o}}=\frac{R_{\mathrm{g}}}{R_{\mathrm{g}}+R_{\mathrm{c}}+R_{\mathrm{L}}} e=\frac{300}{300+\mathrm{I}+\mathrm{I}} e=0.993 e .
$$


Thus, if the galvanometer is graduated to read potential differences at its terminals, the reading of the instrument gives the true emf of the couple to within 0.7 per cent. Instruments having a resistance as low as Io ohms, or even less, are in extensive use. Suppose a galvanometer ro ohms in resistance, calibrated to read potential differences at its terminals, is used in the circuit described above.

$$
\epsilon_{\mathrm{o}}=\frac{R_{\mathrm{g}}}{R_{\mathrm{g}}+R_{\mathrm{c}}+R_{\mathrm{L}}} e=\frac{10}{10+\mathrm{I}+\mathrm{I}} e=0.83 e .
$$

Thus, if the galvanometer is graduated to read potential differences at its terminals, the emfs indicated by the instrument would be 17 per cent less than the true emf of the couple. Such large errors are compensated for by arbitrarily graduating the scale to read the emf of the couple when the resistance of the couple and line has a certain fixed value; in the above example, 2 ohms. The instrument will accordingly read correctly for this external resistance, but will read in error if the external resistance is altered. Bad contacts, deterioration of the couple from oxidation, change in depth of immersion, temperature coefficient of the copper lead wires, etc., may at any time alter the resistance of the external circuit. Let us compare the behavior of the $300-0 h m$ instrument and the io-ohm instrument, assuming both instruments are compensatingly graduated to read correctly for an external resistance of 2 ohms, when for one of the several reasons cited above the external resistance varies slightly. The relation between the potential drop $e_{0}$ across the terminals of the galvanometer and the emf, $e$, of the couple is as follows, where the total line resistance $R^{\prime}=R_{\mathrm{c}}+R_{\mathrm{L}}$ :

$$
e_{\mathrm{o}}=\frac{R_{\mathrm{g}}}{R_{\mathbf{g}}+R^{\prime}} e .
$$

Hence, $e_{\mathrm{o}}$ is always less than $e$. Suppose, however, for a certain line resistance, $R^{\prime}=R^{\prime}$ o, the scale of the indicator is arbitrarily graduated so that the reading $e^{\prime}$ on the galvanometer equals the true emf, $e$, of the couple. The relation between the scale reading and the potential drop across the terminals of the instrument must be accordingly:

$$
e^{\prime}=\frac{R_{\mathrm{g}}+R_{\mathrm{o}}^{\prime}}{R_{\mathrm{g}}} e_{\mathrm{o}}=F e_{\mathrm{o}},
$$

where $F$ is a constant. The graduations on the scale are such that for any deflection of the pointer the scale reading is a con- 
stant $F$ times the potential drop across the galvanometer terminals. On substituting this value of $e_{0}$ in the above equation we obtain.

$$
e^{\prime}=F e_{\mathrm{o}}=F \frac{R_{\mathbf{g}}}{R_{\mathbf{g}}+R^{\prime}} e .
$$

The following table illustrates the per cent error in the indicated emf, $e^{\prime}$, computed from the above equation, when the line resistance $R^{\prime}$ has the values I, 2, 3, and 4 ohms, respectively, when $R_{g}$, the resistances of the indicators are 300 and Io ohms, respectively, and $R_{0}^{\prime}$, the normal line resistance, is 2 ohms.

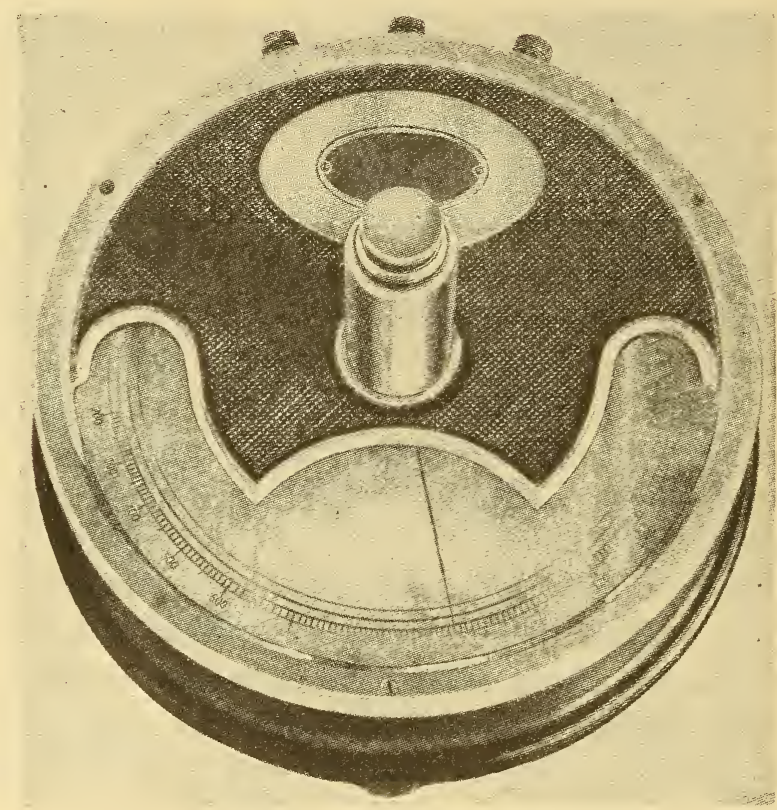

FIG. II.-Types of indicating galvanometers

In the instrument illustrated the moving element is supported by a phosphor-bronze filament and by one pivot. Most of the weight of the moving coil is carried by the suspension. The instrument has a very high resistance and a very long scale, the pointer swinging through an arc of nearly $180^{\circ}$. These indicators are equipped to use the device illustrated by Fig. 37 for cold-junction compensation

TABLE 8.-Error Due to Change in Line Resistance

\begin{tabular}{|c|c|c|c|c|}
\hline \multirow{2}{*}{ Line resistance (ohms) } & \multicolumn{2}{|c|}{$\begin{array}{l}\text { Error in indicator } \\
\text { reading }\end{array}$} & \multicolumn{2}{|c|}{$\begin{array}{l}\text { Error in degrees at } \\
1000^{\circ} \mathrm{C}\end{array}$} \\
\hline & 300 ohms & 10 ohms & $300 \mathrm{ohms}$ & $10 \mathrm{ohms}$ \\
\hline & Per cent & Per cent & ${ }^{\circ} \mathrm{C}$ & ${ }^{\circ} \mathrm{C}$ \\
\hline 1. & +0.33 & +9.1 & +3.3 & +91.0 \\
\hline 2. & \pm 0.00 & \pm 0.0 & \pm 0.0 & \pm 0.0 \\
\hline 3. & -.33 & -7.7 & -3.3 & -77.0 \\
\hline $4 \ldots \ldots \ldots \ldots$ & -.66 & -14.3 & -6.6 & -143.0 \\
\hline
\end{tabular}


Thus when botlı instruments read correctly for an external resistance of 2 ohms, if the external resistance is increased by an ohm, the indicator of low resistance is in error by 7.7 per cent, or about $77^{\circ} \mathrm{C}$ at $1000^{\circ} \mathrm{C}$, while the instrument of high resistance still reads practically correct. Considerations such as the above emphasize the importance of using a galvanometer having a resistance of 300 ohms or more. In actual operation the line resistance may change by several ohms on account of bad contacts and deterioration of the thermocouple.

It must not be inferred from the above discussion that all indicators of high resistance are necessarily superior to all indicators of low resistance Excellent workmanship and mechanical details, robustness, etc., frequently lead to the choice of certain instruments having a resistance less than Ioo ohms, especially in the case of recording millivoltmeters; but, nevertheless, the consideration of the resistance of the instrument is a matter of extreme importance, and a simple galvanometric indicator of low resistance is always subject to the large errors arising

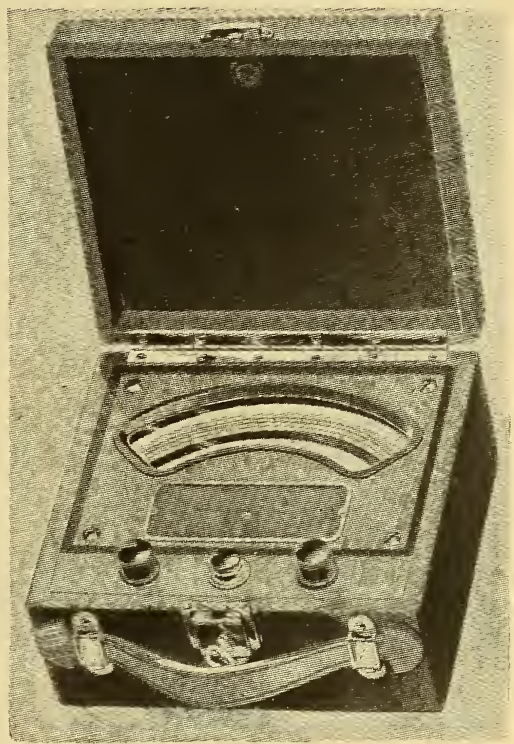

FIG. 12.-Types of indicating galvanometers

These portable indicators are made in two types, one having a resistance of about 6 ohms per millivolt and the other about 20 ohms per millivolt

from small changes in the resistance of the circuit. This statement does not apply to the compensated galvanometer, or to semipotentiometric instruments described later. Figs. I I to is represent typical indicating galvanometers of American manufacture.

(2) Use of Portable Test Set.-On account of the errors which may be introduced in the reading of a galvanometer when the resistance of the line or couple varies, it is important to have some means for measuring this resistance occasionally. This is, of course, especially necessary when the resistance of the galvanometer is low. Every plant requiring a large thermocouple installation with simple galvanometric indicators should have a $8513^{\circ}-21-3$ 


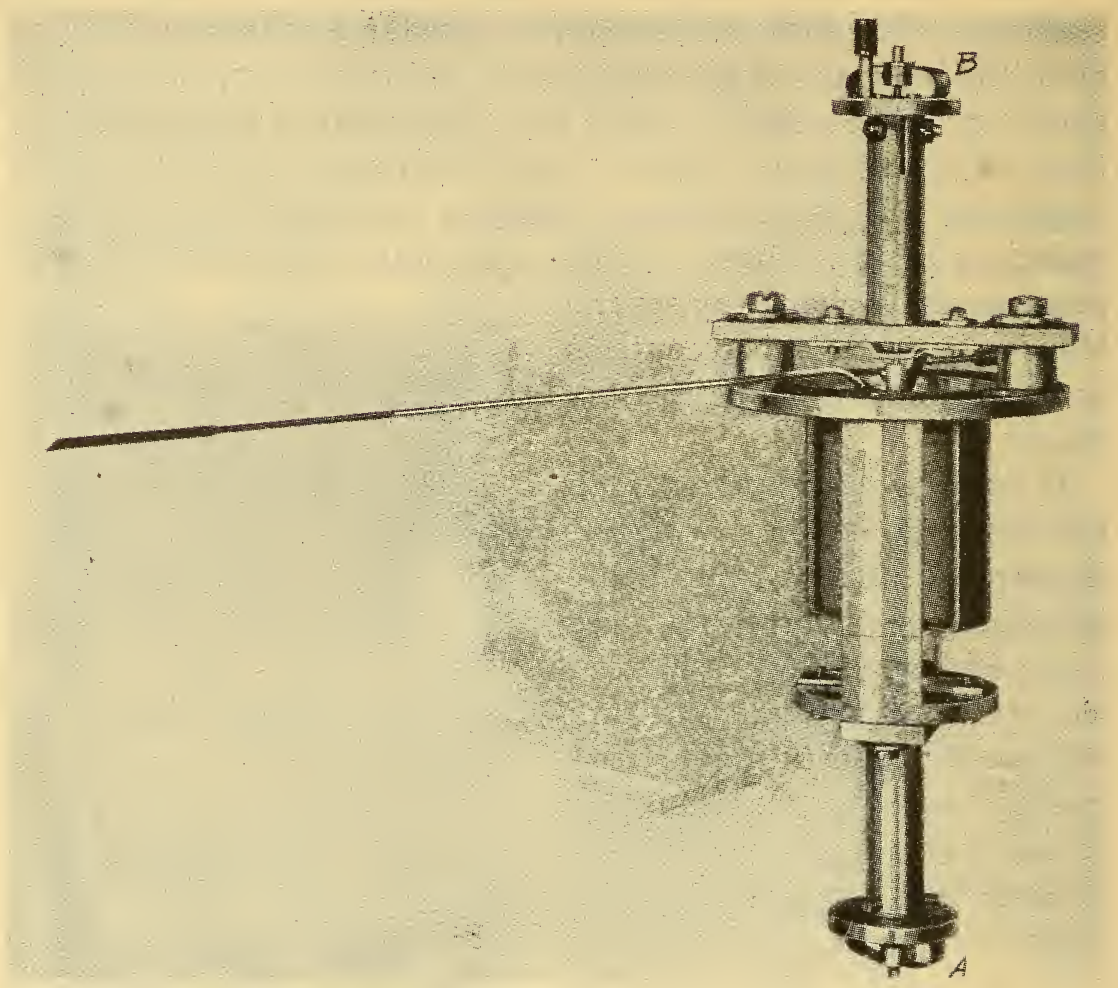

Fig. 13.-Types of indicating galvanometers

Moving element of a double suspension instrument in which the suspensions are kept under tension by the springs $\mathrm{A}$ and $\mathrm{B}$. The tension is sufficient to maintain axial alignment of the coil without precise leveling. The instrument has a high resistance, about 50 ohms per mi11ivc't

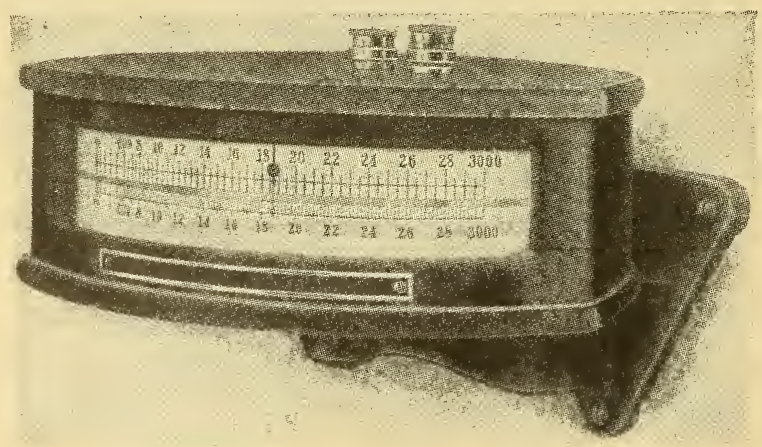

FIG. I4.-Types of indicating galvanometers

The wall-type instruments shown can be obtained with resistances as high as $550 \mathrm{ohms}$ for basemetal couples and 200 ohms for rare-metal couples 
portable Wheatstone bridge or "test" set for this purpose. Fig. 20 illustrates a simple and inexpensive instrument. Operating directions are mounted in the cover of the instrument for con-

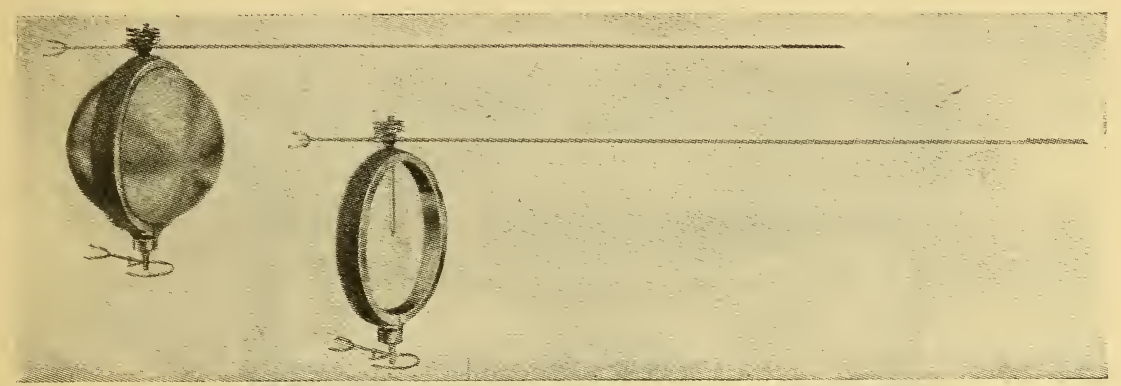

FIG. I5.-Single-pivot movement used in indicators shown in Fig. I4

The pivot is at the center of gravity of the moving system

venient reference. Disconnect the pyrometer indicator from the circuit and connect the two line wires to the $X$ terminals of the

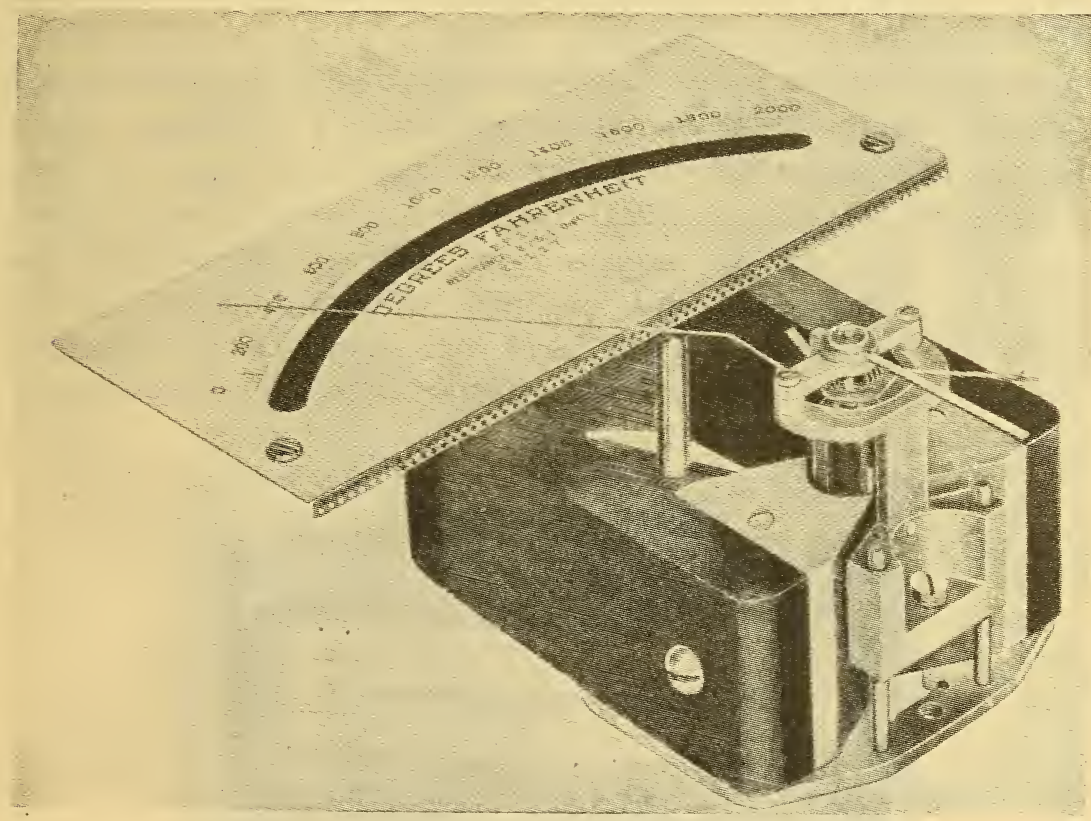

FIG. I6.-Types of indicating galvanometers

In this instrument the moving element is shown partially removed from the magnet. These indicators have resistances approximately $\mathrm{I}_{5}$ to $30 \mathrm{ohms}$ per millivolt

test set. Note the measured resistance, reverse the + and lead wires at $X$ and obtain a new setting. The mean of the two observations is the resistance of the circuit. The two settings 
will be different because of the emf developed by the couple. In measuring the resistance of the line care must be taken, if two indicators or an indicator and recorder are operated in parallel

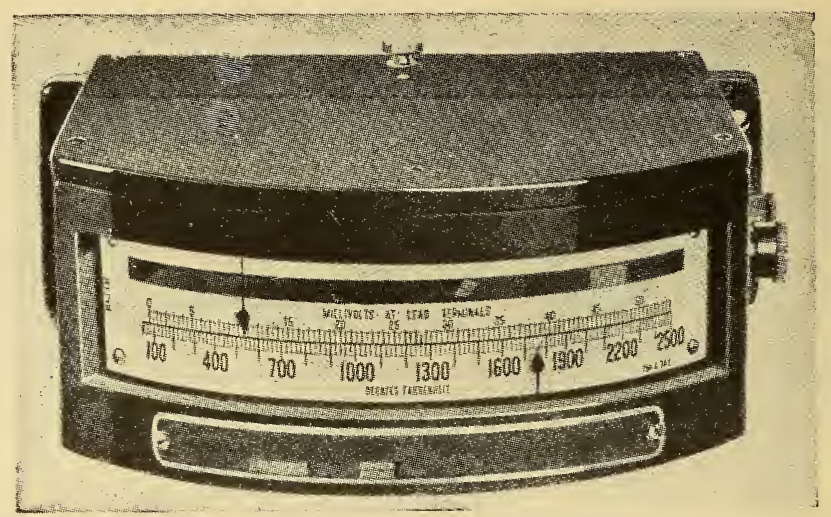

FIG. 17.-Types of indicating galvanometers

A wall-type indicator which is made in two general types, "high resistance" and "low resistance." The former has a resistance of from I50 to $300 \mathrm{ohms}$ and the latter about $8 \mathrm{ohms}$, for use with chromel-alumel couples

on the same couple, to see that both instruments are disconnected from the circuit during the measurement of the resistance; otherwise the instrument left connected acts as a shunt upon the line. If the resistance of the line and couple is found to be much higher than that for which the indicator was designed, short-circuit the line at the cold junction and determine whether the fault is in the couple or the line. If in the former, it usually means that the couple is broken or requires replacement. By making occasional

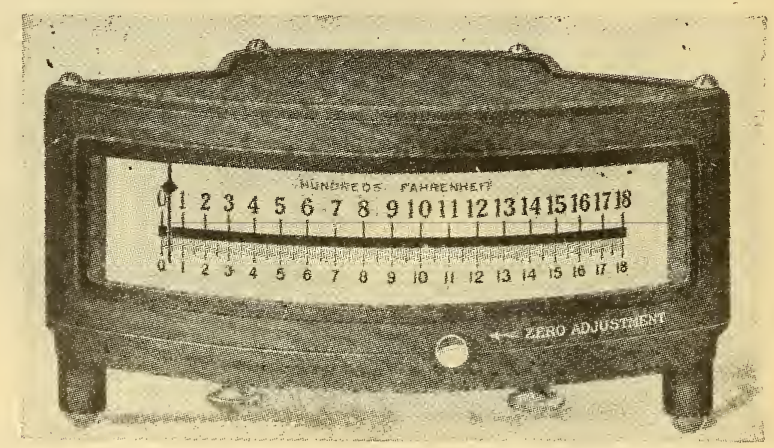

FIG. 18.- Types of indicating galvanometers

A wall-type indicator made with a resistance of approximately ro ohms per millivolt

observations of this kind serious faults may be detected long before they would be suspected from the resulting low values in the indicated temperatures. 


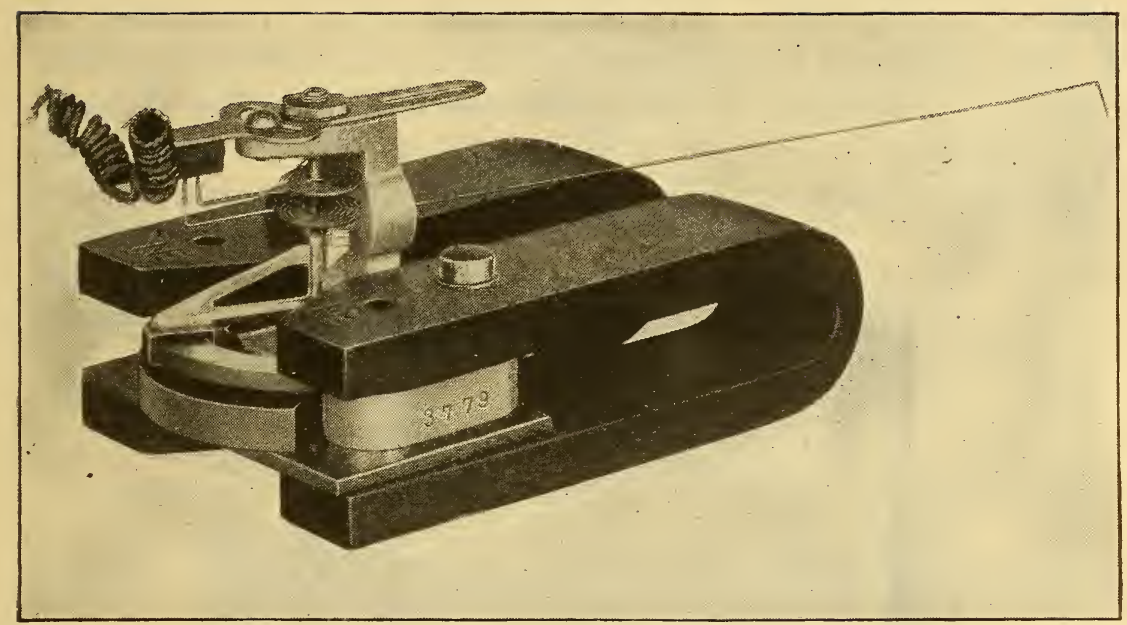

FIG. I9.-Magnet and moving element of an indicating galvanometer which is made with a resistance of approximately $20 \mathrm{ohms}$ per millivolt

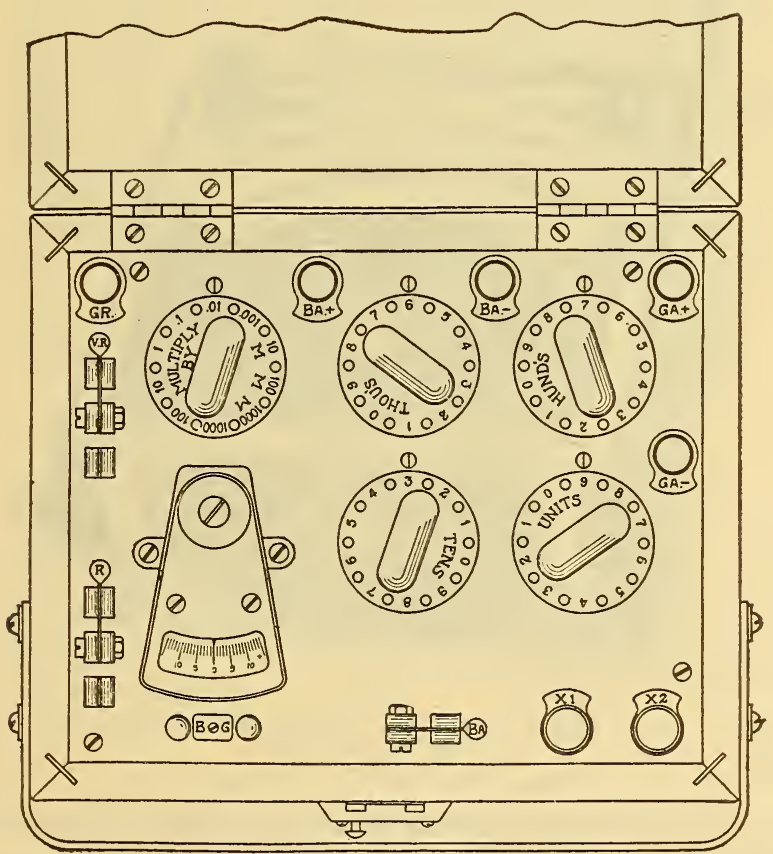

FIG. 20.- "Test set" or Wheatstone bridge for measuring line resistance. 
(3) Galvanometer with Variable Series Resistance.-As shown above, galvanometers, especially those of low resistance, are usually calibrated to read correctly for a definite line resistance. Suppose an indicator is desired for operation on a line of which the resistance changes from practically zero to ro ohms. The instrument is calibrated to read correctly for a line resistance of

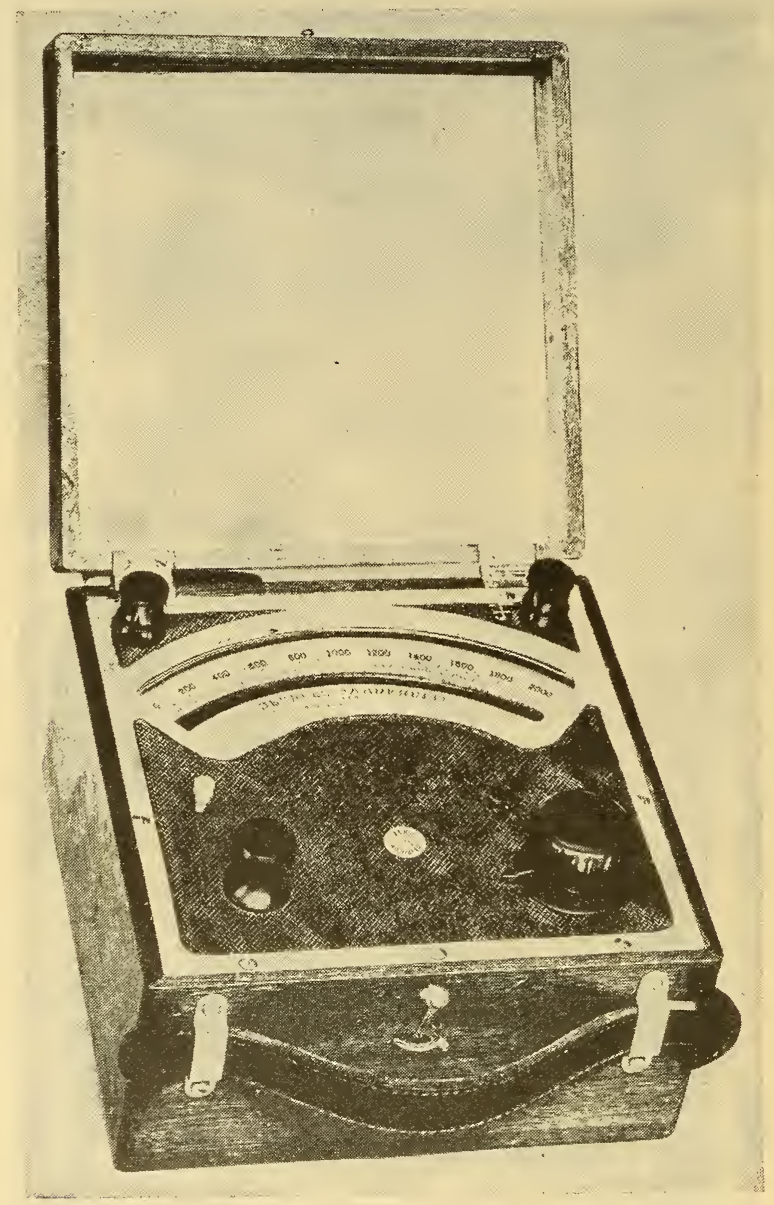

FIG. 21.-Type of indicating galvanometer embodying Harrison-Foote method

Io ohms. Located in the case of the galvanometer and in series with the line is a variable resistance from zero to ro ohms. As the resistance of the line increases the variable resistance is decreased by manual adjustment, so that the sum of the line resistance and the variable series resistance always equals io ohms. The dial of the variable resistance is graduated to read the amount 
of resistance cut out of the circuit. Hence it should be set at the resistance of the line and couple. This value may be determined by means of a test set as discussed above. Thus the method is of great value for precision work with a galvanometric indicator. The principal objection to it, which applies to all galvanometric indicators so far described when used for accurate measurements, is the necessity of measuring the resistance of the line and couple, this latter measurement requiring the use of a test set or similar device.

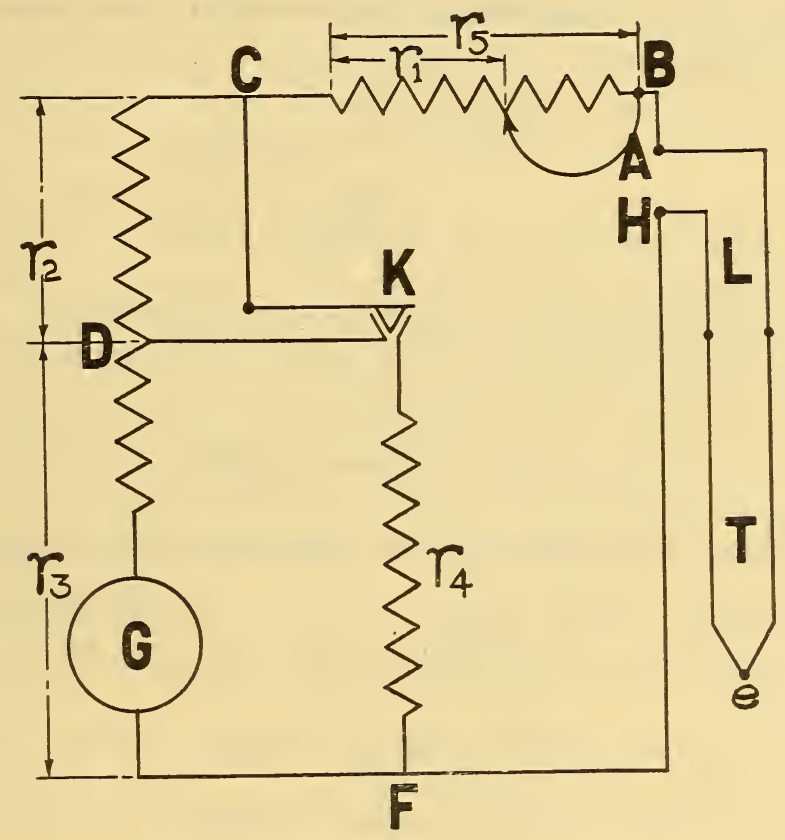

Fig. 2I a.-Wiring diagram for Fig. 2I

(4) Harrison-Foote Method. ${ }^{7}$-In this method the necessity of using a separate instrument to measure the line resistance has been eliminated. The method is employed in the Brown Improved Heatmeter shown in Fig. 2I. The circuit CDGF, Fig. 2ra, is an ordinary millivoltmeter in which $G$ represents the moving coil. In series with this is an adjustable rheostat $C B$. The maximum value $r_{5}$ of this resistance is chosen equal to the maximum value of the resistance of the line and couple likely to occur in practice. A convenient value is $\mathrm{I}_{5} \mathrm{ohms}$. ITith the slide of the rheostat set for the maximum resistance, $r_{1}=r_{5}$, the instrument is calibrated in terms of the potential drop across $A H$. Hence when

${ }^{7}$ See Harrison and Foote, Jour. A. I. E. E., Feb. 20, 1920. 
the instrument is connected through the line of resistance $L$ to the couple of resistance $T$ the rheostat $C B$ must be adjusted until $r_{1}+L+T=r_{5}$. When this adjustment is made, the scale reading gives correctly the emf $e$ of the couple or the correct temperature if the scale is graduated in degrees. This adjustment is made in the following manner: By depressing the key $K$, a portion of the galvanometer resistance $r_{2}$ is short-circuited and the rest of the resistance $r_{3}$, containing the moving coil, is shunted by the resistance $r_{4}$. If $e^{\prime}$ represents the potential drop across $D F$ when the key is open and $e^{\prime \prime}$ represents the potential drop when the key is closed, we obtain:

$$
e^{\prime \prime}=\frac{e^{\prime}=\frac{e r_{3}}{L+T+r_{1}+r_{2}+r_{3}}}{e r_{3} r_{4}}
$$

If $r_{1}$ is so adjusted that these two potential drops and hence the deflections of the indicator are the same, we have on equating ( $\mathrm{I}$ ) and (2):

$$
L+T+r_{1}=\frac{r_{2} r_{4}}{r_{3}}=\text { a constant }
$$

If, now, in the construction of the instrument $\frac{r_{2} r_{4}}{r_{3}}$ is made equal to $r_{5}$, the above setting makes $L+T+r_{1}=r_{5}$ the adjustment required in order that the reading of the scale give the true emf of the couple.

The ease with which the proper setting can be obtained is greatly improved by making $\frac{r_{3}}{r_{4}}$ equal to from 5 to Io. Suppose $\frac{r_{3}}{r_{4}}$ is made equal to 9 . Then, if $r_{1}$ is improperly adjusted and the instrument reads in error by say $\delta e$ when the key $K$ is open, on depressing the key the reading is changed by $9 \delta e$. If now $r_{1}$ is readjusted with the key depressed until the reading takes its initial value, the error with the key open is reduced to $\frac{\delta e}{\mathrm{IO}}$. The process for operating the instrument is accordingly as follows:

I. Read the instrument with the key open.

2. Close the key and adjust the rheostat $r_{1}$ until the instrument reads approximately the same as in $\mathrm{I}$. 
3. Repeat I and 2, if necessary.

When $\frac{r_{3}}{r_{4}}=9$ it is rarely necessary to make a second adjustment. In position I the instrument acts as an ordinary galvanometer. The single setting in position 2 reduces the error in the ordinary galvanometer by the factor $\frac{1}{10}$, which is usually sufficient. The adjustment for the proper external resistance, if desired, can be made with ro times the precision necessary. Thus, if the gaivanometer can be read to $\frac{1}{10}$ of a scale division, the line resistance can be set for an error less than $\frac{1}{100}$ of a scale division, which is at least ro times the accuracy possible with any indicating instrument. Hence, the factor of variable line resistance which may give rise to very serious errors is easily and accurately controlled by a simple mechanical adjustment.

The device is readily applicable to multiple installations of different line resistance. For multiple-point recorders and indicators as many resistances $B C$ may be employed as there are couples. These may be inexpensive rheostats having a resistance of approximately I 5 ohms each located in each couple line between the couple and the selective switch. These rheostats may be adjusted in the manner described whenever convenient or necessary. The following illustrates a suitable proportioning of resistance for a 300-ohm indicator:

$$
\begin{aligned}
r_{2} & =\mathrm{I} 35 \text { ohms. } \\
r_{3} & =\mathrm{I} 50 \text { ohms. } \\
r_{4} & =\mathrm{I} 5 \mathrm{O} / 9 \mathrm{ohms} . \\
r_{5} & =\mathrm{I} 5 \text { ohms. } \\
r_{2}+r_{3}+r_{5} & =300 \text { ohms. }
\end{aligned}
$$

If the simple indicator has the proper ratio of manganin to copper, its temperature coefficient is practically zero. In that case the shunt $r_{4}$ should have the same manganin to copper ratio as the portion of the galvanometer resistance comprised by $r_{3}$. The complete instrument will have, accordingly, a zero temperature coefficient. If the simple indicator does not have a zero temperature coefficient, it is possible by increasing the proportion of manganin in $r_{4}$ to compensate for the temperature coefficient of the resistance $r_{3}$ so that the complete instrument has a zero temperature coefficient.

Fig. $2 \mathrm{I} b$ shows another wiring diagram for an instrument which accomplishes the same results as the one already described. For many purposes this is the more desirable arrangement, since 
resistance at the contact point in the switch can produce no error, and if the instrument is properly damped with the key open it will be also properly damped with the key closed.

When the variable resistance $r_{1}$ is so adjusted that the position of the pointer is unchanged by closing key $K$, the following relation exists between the resistances:

$$
r_{5}=\frac{r_{2} r_{4}}{r_{6}}, \text { where } r_{5} \text { is defined as before. }
$$

This adjustment is, therefore, independent of the value of $r_{3}$, which may be altered slightly, when desired, for calibrating the

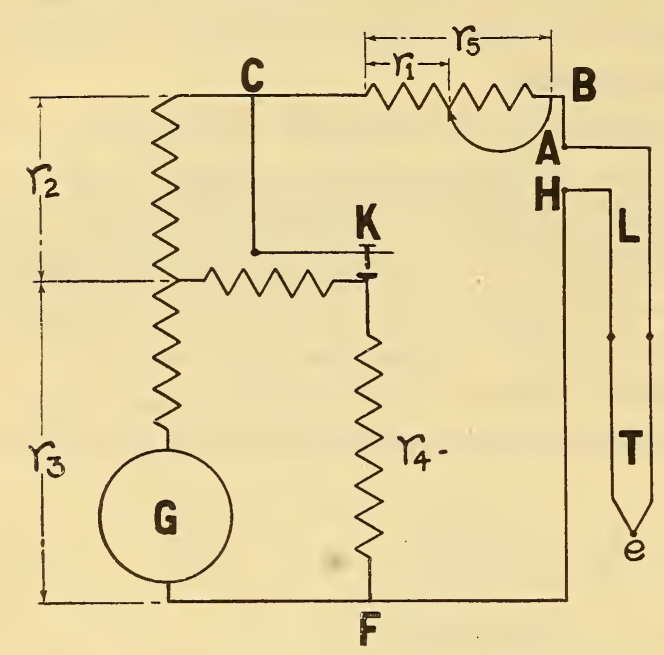

FIG. 2Ib.-A nother wiring diagram employing the Harrison-Foote method instrument. It is also independent of the resistance of branch $C K$, and therefore of resistance in the key. ${ }^{8}$

When resistances $r_{2}$ and $r_{4}$ are made of manganin and $r_{6}$ of copper or other material with high temperature coefficient $\beta$, it is seen that as $r_{6}$ increases $r_{5}$ must be decreased if the instrument is to read the same with the key open or closed.

Let $\alpha=$ temperature coefficient for current when the instrument is kept adjusted at all temperatures.

Let $R_{\mathbf{g}}=$ resistance of galvanometer coil and springs.

Let $\gamma=$ ratio of temperature coefficients of resistance of $R_{\mathrm{g}}$ and $\gamma_{6}$.

Let $i^{\prime}=$ current through galvanometer required for full scale deflection.

Let $e^{\prime}=$ desired emf reading corresponding to full scale deflection.

Let $S=\frac{e^{\prime}}{i^{\prime}}$.

Let $R_{\mathrm{d}}=$ total resistance of galvanometer circuit necessary for critical damping.

Let $K=$ multiplication factor for errors (represented by $\frac{r_{3}}{r_{4}}$ in foregoing discussion). 
The following relations exist between the various characteristic constants and resistances:

$$
\begin{aligned}
& r_{3}=\left[\frac{\alpha}{\beta}-\frac{r_{5}}{S}+\frac{R_{\mathrm{g}} \gamma}{R_{\mathrm{d}}}\right] \frac{S R_{\mathrm{d}}}{S-R_{\mathrm{d}}} \\
& r_{2}=\frac{S}{R_{\mathrm{d}}}\left(S-r_{3}\right)-r_{5} \\
& K=\frac{r_{2}}{r_{5}} \frac{S-R_{\mathrm{d}}}{S-r_{3}} \\
& r_{4}=r_{5} \frac{R_{\mathrm{d}}}{S-R_{\mathrm{d}}} \\
& r_{6}=\frac{r_{2} r_{4}}{r_{5}}
\end{aligned}
$$

For zero temperature coefficient, $\gamma$ is made equal to the combined temperature coefficients of the springs and magnet; that is, $\gamma$ is taken about -0.0002 .

If a very low range instrument is being designed, and it is not readily possible to realize a zero temperature coefficient, a larger negative value is chosen for $\gamma . \quad R_{\mathrm{g}}$ can never be larger than $r_{3}$.

For a multiple range instrument $r_{3}$ may be made of a number of resistances, any of which may be short-circuited by a suitable arrangement. Without additional wiring, however, the instrument would not be critically damped for more than one range.

Since compensation is made for all variations of resistance, this instrument may be used as the galvanometer of a simple type of potentiometer, and an accurately compensated deflection potentiometer will be obtained. ${ }^{9}$

(b) Potentiometer Method.-The most accurate method for measuring the emf of a thermocouple is by use of a potentiometer. The fundamental principle of this instrument is illustrated by Fig. 22. A constant current from the battery $B$ flows through the slide wire resistance $a b c$. One wire of the couple $T$ is connected to the movable contact $b$ and the other wire, in series with a sensitive galvanometer, is connected to $a$. The contact $b$ is moved until the galvanometer reads zero, thus showing that no current is flowing through the thermocouple circuit. When this balance or zero setting is made, the true emf of the couple is equal to the potential drop across $a b$. This is obtained from Ohm's law, $e=i r$, where $i$ is the current flowing through the resistance $r=a b$. If the current is always adjusted to a definite value the

${ }^{9}$ Harrison and Foote, Jour. A. I. E. E. Feb. $20,1920$. 
slide wire may be graduated to read millivolts or temperature directly. Various devices are employed for this purpose. An ammeter will serve, but the usual method is to obtain a preliminary setting by replacing the thermocouple by a constant known source of emf, such as a standard cell. The galvanometer $G$ is always used as a zero instrument in a strictly potentiometric circuit. Hence, it requires no calibrated scale, and no attention need be given to the constancy of its sensitivity, provided it is always sufficiently sensitive to serve its purpose. These requirements are easily met, and the entire potentiometer, galvanometer, battery, standard cell, slide wires, etc., as constructed are mounted in a case not much larger than that of a millivoltmeter. The instrument is as mechanically robust as indicators of equal sensitivity operating on the galvanometric principle.

There are several important advantages in the potentiometer

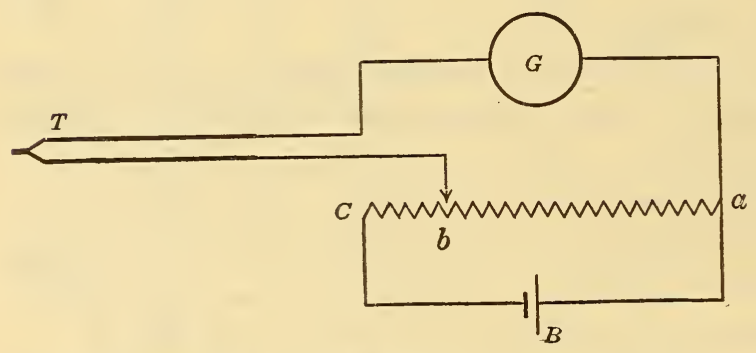

FIG. 22.-Simple wiring diagram for potentiometric indicator method. The emf or temperature scale is easily made very open, thus permittingaccurate readings. The calibration of the scale is in no way dependent upon the constancy of magnets, springs, or jewel

bearings, nor upon the level of the instrument. From the pyrometric standpoint, however, the greatest advantage is the complete elimination of any error due to ordinary changes in the resistance of the couple or of the lead wires. Thus if a potentiometer is correctly balanced and the resistance of the thermocouple is then greatly increased, the balance is unchanged. However, excessive resistance in the couple circuit reduces the sensitivity of a setting. Thus if the resistance is very high, there appears to be a considerable range over which the balance is satisfactory. This range can be decreased by increasing the sensitivity of the galvanometer, but for obvious reasons this is already made as high as is consistent with sufficient ruggedness.

The sensitivity of the galvanometer also determines the proper length of the emf scale. Thus there is little advantage gained in making the scale or slide wire of such length that a millimeter deflection on the galvanometer scale corresponds to more than a 
few millimeters on the emf scale. The emf scale of one type of portable instrument has a length of $40 \mathrm{~cm}$, while in another the scale is $250 \mathrm{~cm}$ long.

The only objections to the potentiometer are slightly greater initial cost and the fact that usually a manual adjustment must be made to obtain a setting. In the potentiometric recording instrument, however, all the various manipulations may be performed automatically, even to the balancing against the standard cell.

Fig. 23 illustrates the Leeds \& Northrup Co. portable potentiom-

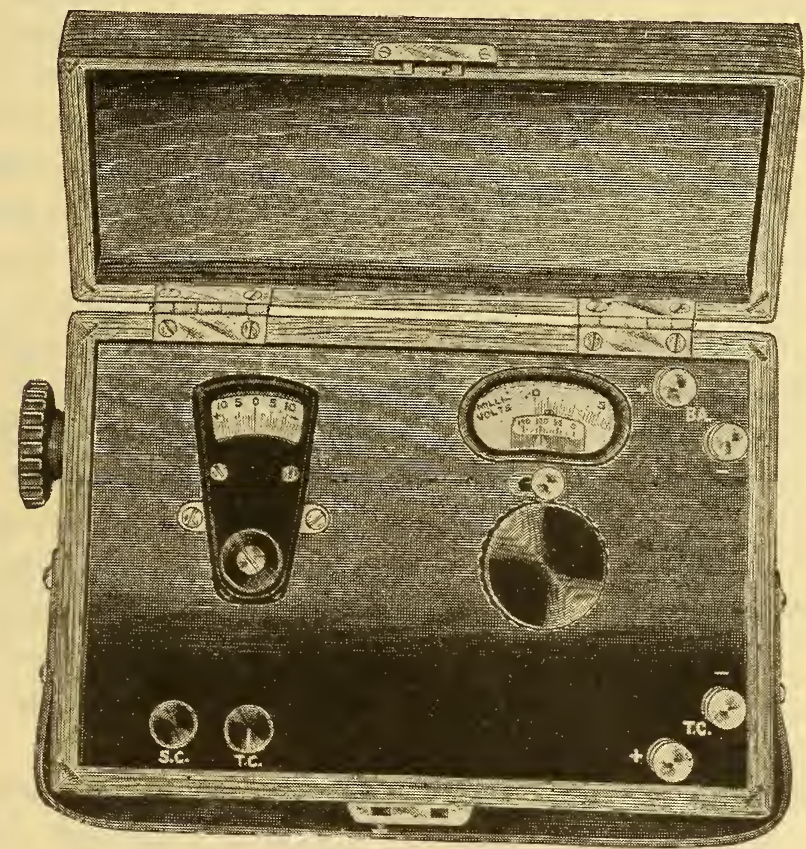

Fig. 23. - Type of portable potentiometer

eter and Fig. 24 shows the wiring diagram. The galvanometer should be adjusted to read zero on open circuit. At intervals of a few hours the setting on the standard cell should be made. This is done by depressing the key S.C. and adjusting the resistance $R R^{\prime}$ by turning the knurled head on the left of the case until the galvanometer reads zero. The thermocouple is connected to the terminals T.C., and the emf or temperature is obtained directly from the dial scale by depressing the key marked $T . C$. and turning the main dial until the galvanometer indicates zero. The key T.C. and the key S.C. should never be depressed 
at the same time. These instruments can be made in any scale range or with multiple scale ranges adapted to various types of couple.

The Brown portable potentiometer has a scale length of 96 inches, graduated in I/50 millivolt intervals. The galvanometer, which is of the pivot type, has a higher sensitivity than the suspension instruments usually employed in portable potentiometers.

(c) Semipotentiometer Method.- It is possible by means of a single galvanometer or millivoltmeter to measure the emf of a couple potentiometrically. Thus, in Fig. 22, by using a shunted galvanometer first in the main circuit $a b c B$ as an ammeter, the initial setting for a standard current is obtained. Then the instrument without the shunt is placed in the position $G$ and the

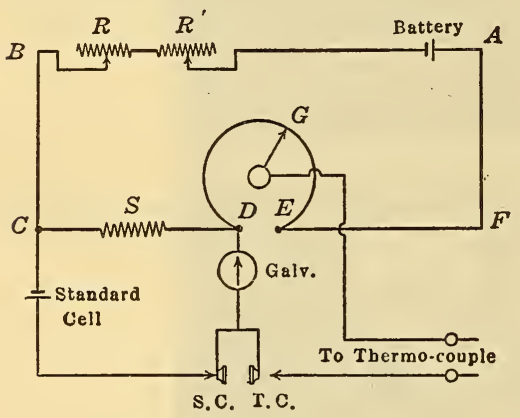

FIG. 24.-Wiring diagram of potentiometer shown in Fig. 23 contact $b$ is moved along the graduated slide wire until a zero setting is obtained. The objection to this method is that, if the millivoltmeter is sufficiently sensitive to be used as a zero instrument, it may not be reliable as an ammeter, and if reliable as an ammeter it may not possess sufficient sensitivity when used as a detector or null instrument. The semipotentiometer method, however, in other forms has proven of value in thermoelectric pyrometry.

(I) Northrup's Method.-Fig. 25 shows a wiring diagram of the pyrovolter, an instrument based on Northrup's method. In position (a) the dry cell $B$ contained in the case of the instrument sends a current through the variable resistance $R$ and the fixed resistances $C$ and $S$. The resistance $C$ of copper is equal in value to the resistance of the copper coil of the moving element of the galvanometer $G$. The couple $T$ is connected in series with the moving coil of the galvanometer across the resistance $S$. The resistance $R$ is adjusted until the galvanometer reads zero by turning the knurled head on the lower right-hand corner of the instrument. The key on the lower left-hand corner is then depressed, which throws the thermocouple and the resistance $C$ out of the circuit and replaces $C$ by the galvanometer $G$ of equiva- 
lent resistance, Fig. $25(b)$. The galvanometer is now deflected by an amount proportional to the current flowing through it, which in turn is proportional to the potential drop across $S$. The scale of the instrument is graduated to read the potential drop over $S$, and, since this potential difference was made equal to the emf of the couple by the initial setting for zero deflection, the galvanometer indicates directly the true emf of the couple. The initial setting is not altered by introducing re-

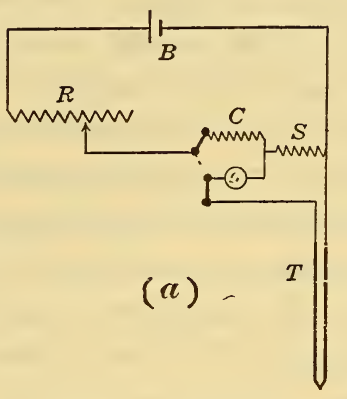

FIG. 25.-Wiring diagrcm illustrating Northrup's method

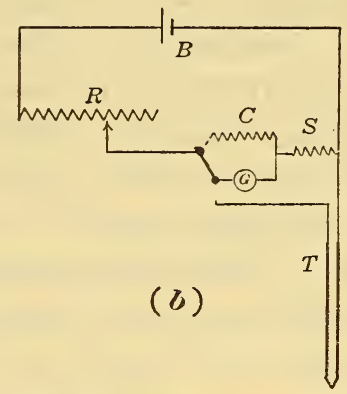

$T$

sistance into the thermocouple circuit, so that the instrument is really a form of potentiometer. The scale may be graduated to indicate temperature for any particular type of couple, and the instrument may be obtained with several different scale ranges.

(2) Northrup's Method Applied to Continuously Deflecting Instruments.-This instrument is the ordinary pyrovolter with the

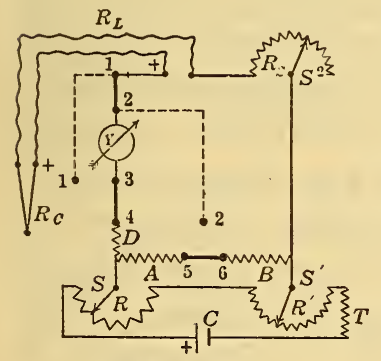

Position No.1

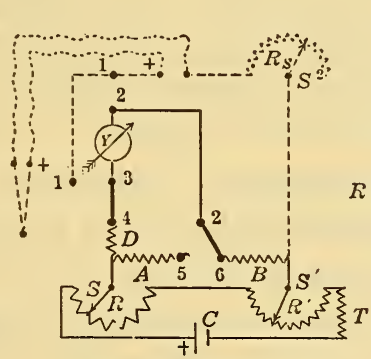

Position No.2

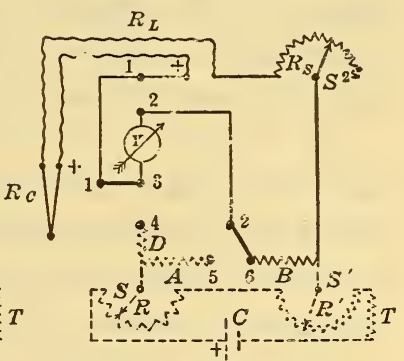

Position No.3

FIG. 26.-Wiring diagram illustrating continuously deflecting instrument embodying a method described in the text

addition of an extra key and an adjustable resistance. After the emf of the couple has been determined by the pyrovolter method just described, the galvanometer in series with this resistance is connected directly to the thermocouple terminals. The resistance is then adjusted until the reading of the instrument is the same as that determined by means of the pyrovolter.

Figure 26 is the wiring diagram of another instrument known as the Brown precision heatmeter which, making use of somewhat 
differently arranged circuits, employs a principle very similar to that of the Northrup continuously deflecting pyrovolter. By means of suitable switches the electrical connections are thrown successively into the three positions illustrated, position No. 3 being the final working position, in which the couple is connected directly to the millivoltmeter through a definite line resistance. In position No. I the emf of the couple is balanced against the potential drop between $S$ and $S^{\prime}$ by varying $R$ and $R^{\prime}$ until the galvanometer $Y$ indicates zero. In position No. 2, the thermocouple circuit is cut out and the galvanometer is connected to the points $S$ and $S^{\prime}$. The scale of the galvanometer is so divided as to read the potential differences across $S S^{\prime}$. This potential difference is not altered by switching from position No. I to position No. 2, since the resistance $D$ is so chosen that $A=Y+D$. The total resistance of the galvanometer circuit in position No. 2 is $D+Y+B$. In position No. 3 the resistance $\mathrm{R}_{\mathrm{s}}$ is adjusted until the total resistance of the galvanometer circuit is equal to that of position No. 2, viz.:

$$
D+Y+B=R_{\mathrm{c}}+R_{\mathrm{L}}+R_{\mathrm{s}}+B+Y
$$

This adjustment is obtained when the reading of the galvanometer is not altered by switching from position No. 2 to positioil No. 3 . Thus with $R_{\mathrm{s}}$ properly adjusted, the reading of the indicator in position No. 3 gives directly the true emf or the temperature of the couple as long as the line resistance $R_{\mathrm{L}}+R_{\mathrm{c}}$ remains unchanged.

(d) Deflection Potentiometer Method.-A potentiometer is ordinarily used as a null instrument. The emf of the couple is exactly balanced by the potential drop over a resistance through which a current from a battery is flowing. No current flows through the couple circuit when the proper setting is obtained. This condition is indicated by zero deflection of the galvanometer in series with the couple. The objection sometimes raised against the ordinary potentiometer for industrial installations is that it requires a manual adjustment of a dial or slide wire every time an observation is made. This objection is practically eliminated in the deflection potentiometer, which may be constructed so that it embodies the accuracy of the ordinary potentiometer and the convenience of the galvanometer indicator.

In the deflection potentiometer, part of the emf of the couple is balanced against the potenial drop over a resistance through which a current is flowing, and the remaining portion of the emf is indicated by the deflection of the galvanometer in series with 
the couple. For example, the instrument may be constructed with a dial of say 16 points, representing potential differences from $\mathrm{o}$ to $\mathrm{I} 5$ millivolts, and a galvanometer which gives full scale deflection on I millivolt. The dial is set to the approximate emf developed by the couple, and the dial reading combined with the galvanometer reading gives the true emf of the couple. If the couple developed an emf of 13.58 millivolts, the value $I_{3}$ is obtained from the dial and the value 0.58 is read from the galvanometer scale. Usually the scale is graduated both ways with zero at the center, so that + or - deflections may be readily shown. Thus, in the above example, if the dial had been set at I 4 the pointer would have read -0.42 , and the final reading would be $14.00-0.42=13.58$, as before. To avoid the necessity of thus subtracting a number from the dial setting, the galvanometer scale may be graduated from left to right as follows:

$$
\text { o, } 10,-80,90,0,10,20-100
$$

When the pointer swings to the left, the next lower dial reading is used. Hence in the above example, with the dial set at I4, the galvanometer would read 58 on the left part of the scale. Thus the correct value is 13.58 as before. In many industrial processes the temperature of the couple will vary only slightly during several hours, so that a new dial.setting is infrequently required. Thus the method for obtaining the emf of the couple is nearly as simple as when an ordinary galvanometric indicator is used.

The theory of the deflection potentiometer has been developed in detail by Brooks. ${ }^{10}$ As applied to emf measurements the simple theory may be deduced as follows: It has been shown in the case of an ordinary potentiometer that if an emf $e^{\prime}$ is exactly balanced against the potential drop in a resistance wire of a potentiometer, the value of this emf will be given by the equation

$$
e^{\prime}=\frac{E r_{1}}{r_{1}+r_{2}}
$$

where $E$ is the emf of the battery used to furnish the current in the resistance wire, $r_{1}$ is the resistance of this wire, and $r_{2}$ is all other resistance in the battery circuit. Usually the value of $e^{\prime}$ is indicated by figures on the dials or slide wire of the potentiometer. If the emf $e^{\prime}$ changes to a new value $e$ and the potentiometer

${ }^{10}$ B. S. Sci. Papers, Nos. 33, 79, 172, and 173 . $8513^{\circ}-21-4$ 
remains adjusted as before, a current will flow through the galvanometer and thermocouple, causing the galvanometer to deflect. The currents now flowing through $r_{1}$ and $r_{2}$ are unequal and are different from the original value. The currents flowing in the different branches of the circuit are indicated by Fig. 27, in which $T$ represents a thermocouple, $G$ the galvanometer, $a b c B$ the poten-

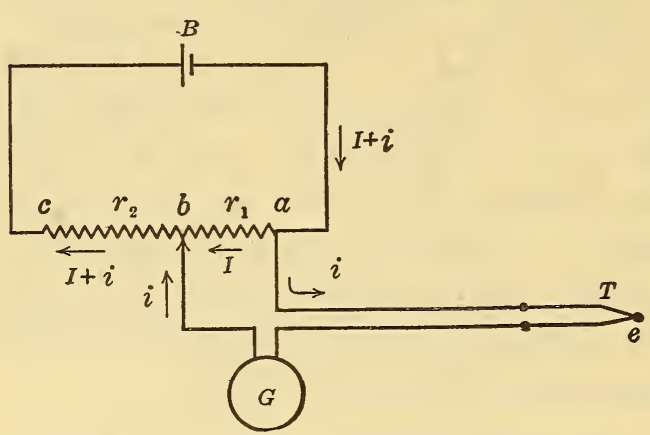

FIG. 27.-The unbalanced potentiometer circuit tiometer, $R$ the resistance of the galvanometer and thermocouple, $e$ the emf of the couple, and $B$ the battery. $I$ represents the current flowing from $a$ to $b$ through $r_{1}$ and $i$ the current flowing through the galvanometer. Since the sum of the emfs and potential drops around any closed circuit must equal zero, the following two equations may be written:

$$
\begin{gathered}
E=\left(r_{1}+r_{2}\right) I+r_{2} i \\
e=r_{1} I-R i
\end{gathered}
$$

Substituting the value of $I$ from equation (2) in equation (3) and subtracting this value of $e$ from the value of $e^{\prime}$ given by equation (I) we obtain

$$
e^{\prime}-e=i\left(R+\frac{r_{1} r_{2}}{r_{1}+r_{2}}\right)
$$

or

$$
i=\frac{e^{\prime}-e}{R+\frac{r_{1} r_{2}}{r_{1}+r_{2}}} .
$$

Hence when a potentiometer is not balanced a current will flow through the galvanometer equal to the difference between the setting of the potentiometer and the emf of the thermocouple, divided by the total resistance $R+r_{1} r_{2} /\left(r_{1}+r_{2}\right)$ in the galvanometer circuit.

Representing the resistance within the potentiometer $r_{1} r_{2} /\left(r_{1}+r_{2}\right)$ by $r_{\mathrm{p}}$, the resistance of the galvanometer circuit will equal $R+r_{\mathrm{p}}$. If the galvanometer is to indicate correctly at all values of $e^{\prime}$ the unbalanced emf $e^{\prime}-e$, its sensitivity must remain constant. This requires that $R+r_{\mathrm{p}}$ remain constant. The value of $r_{\mathrm{p}}$ will change as point $b$ is moved nearer to $a$ or $c$, thus altering the values of $r_{1}$ and $r_{2}$. Hence, in order that $R+r_{\mathrm{p}}$ remain constant it is necessary 
to put in the galvanometer circuit a variable resistance which compensates for these changes in $r_{\mathrm{p}}$.

In instruments of low range, suitable for thermocouples, $r_{1}$ is made small compared with $r_{2}$. Since $r_{p}=\frac{r_{1} r_{2}}{\left(r_{1}+r_{2}\right)}$, if $r_{2}$ is sufficiently large compared with $r_{1}$, we may neglect the term $r_{1}$ in the denominator, and the above equation reduces to $r_{\mathrm{p}}=r_{1}$. For such an instrument the compensating resistance in series with the galvanometer is decreased by the value of $r_{1}$ at any dial setting.

Instruments of this type have been designed by Brooks and by White. The compensating resistance is mounted as an integral part of the dial, so that turning the dial changes the emf setting and at the same time adjusts the compensating resistance in the galvanometer circuit to its proper value.

Fig. 28 illustrates the wiring diagram of a deflection potentiometer for thermocouples, known as the Taylor Instrument Co.'s "range control board." The galvanometer $G$ is provided with two scales, in the ranges zero to $500^{\circ} \mathrm{C}$ and $45^{\circ}$ to $950^{\circ} \mathrm{C}$, respectively. The galvanometer circuit is connected at fixed points a

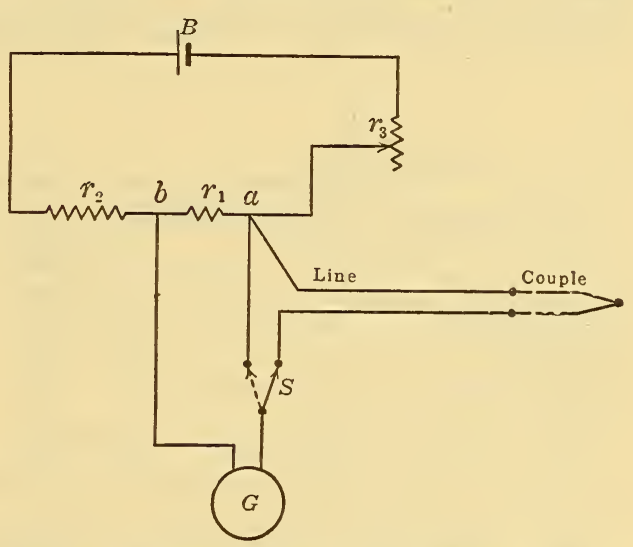

FIG. 28.-Wiring diagram of a deflection potentiometer

and $b$ within the potentiometer, and when the instrument is to operate in the lower range the battery circuit is opened. Thus the potentiometer setting $e^{\prime}$ is made zero without changing the value of $r_{1}$. In this range the instrument operates as an ordinary galvanometric indicator. If the temperature of the thermocouple is above 450 or $500^{\circ} \mathrm{C}$, a current of such magnitude is made to flow through $r_{1}$ that the potential drop $e^{\prime}$ across $r_{1}$ balances the emf developed by the couple when at $45^{\circ} \mathrm{C}$. The temperature will then be indicated on the high range scale.

The total resistance of the galvanometer circuit is almost exactly equal when operating in either range, since when operating in the upper range the shunting effect of $r_{2}+r_{3}$ on $r_{1}$ is negligible. Since a separate galvanometer scale is provided for each setting of the potentiometer (o and $e^{\prime}$ ) it is not really necessary that the sensitivity be equal in the two cases. 
For the high range, the current from the battery is adjusted by connecting switch $S$, as shown by the dotted line, and setting $r_{3}$ so that the galvanometer deflects to a marked position. Provision must be made for reversing the direction of the current from the battery through the galvanometer after this adjustment has been made. The figure does not show this or the switch for opening the circuit when the instrument is to be used for the low range.

Fig. 29 is the wiring diagram of the Leeds \& Northrup deflection instrument for thermocouples. When a range suitable for thermocouples is used and the condition of a balanced Wheatstone bridge with arms of equal resistance is never far departed from, the resistance of that part of the galvanometer circuit which is constituted by the potentiometer usually remains con-

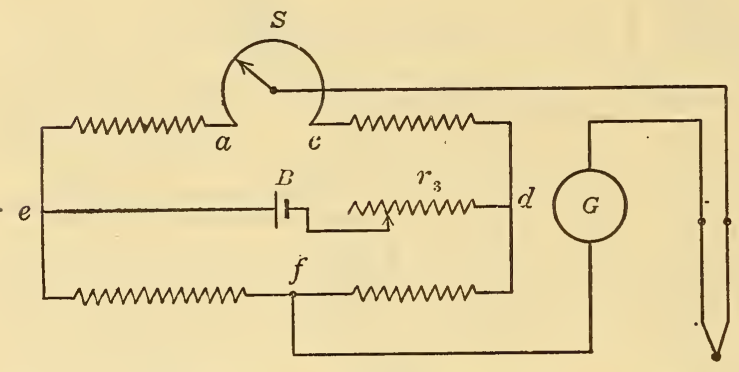

FIC. 29.-Wiring diagram of a deflection potentiometer stant within a few tenths of I per cent.

The value of this resistance for instruments of the same range and using the same battery current is considerably higher than that of the designs discussed above.

The following table shows suitable values of the different resistances which will give to a satisfactory degree, conditions of a nearly balanced Wheatstone bridge with equal arms:

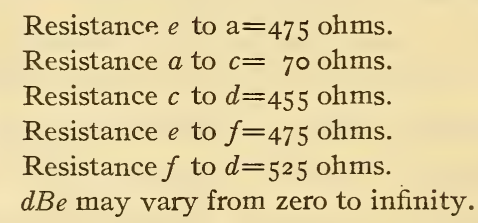

These values may be divided or multiplied by any number in order to obtain any desired range of currents and a proper critical damping resistance for the galvanometer. The above design allows a range of 70 millivolts over the slide wire. The curves in Fig. 30 show the manner in which the resistance $r_{\mathrm{p}}$ of that part of the galvanometer circuit consisting of the parallel paths within the potentiometer varies with different settings of the slide wire, ranging from zero to 70 millivolts, and with resistance $r_{3}$ in the outside battery circuit $d B e$, varying from zero to infinity. 
Actually $r_{3}$ would not be likely to vary beyond the limits Ioo and 500 ohms. An average value for $r_{\hat{\mathrm{p}}}$ may be obtained by setting the slide wire to read about 7 millivolts, and the variation of $r_{p}$ from this value will be, in general, less than o.I per cent, whatever the setting of the slide wire on the battery resistance $r_{3}$. Therefore, if the galvanometer is calibrated when the slide is set to read 7 , it will be more nearly accurate with varied settings and adjustments of the instrument. Since $r_{\mathrm{p}}$ constitutes only part of the galvanometer circuit, the galvanometer sensitivity will remain constant within proportionally less than o.I per cent.

This instrument is designed primarily so that the slide wire may be set to read the exact temperature required. The galvanometer $G$ accordingly indicates the departure of the actual temperature from the temperature desired. It thus serves as a very convenient guide to the operator of a furnace, who can see at a glance by how many degrees the temperature at any time differs from the temperature at which the furnace should be operated.

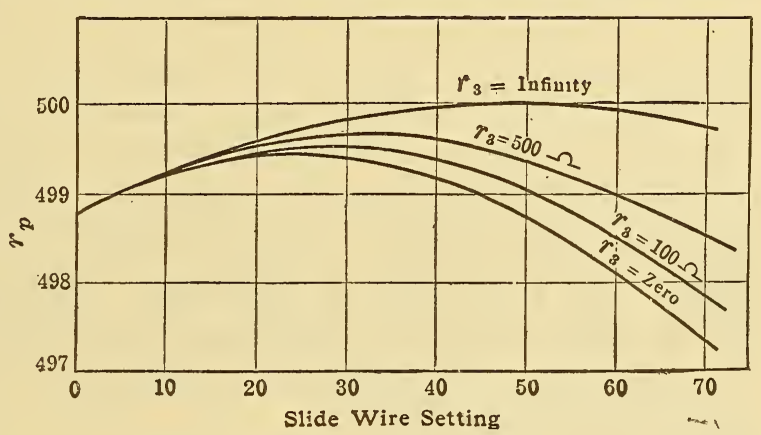

The Cleveland Instrument Co. deflection potentiometer is a modification of their Wheatstone bridge cold-junction compensating instrument. By altering the ratio of the coils, Fig. 37, in suitable steps, the emf of the couple is opposed by potential drops of different values, the indicator showing the unbalanced portion of the thermocouple emf, as in the other instruments described.

(I) Deflection Potentiometer Graduated to Read Temperature Directly.-The above discussion has assumed that the scale of the galvanometer may be graduated to read either emf or temperature. If the thermocouple has a linear relation between emf and temperature, the theory outlined is just as applicable to a scale and dial graduated in terms of temperature as in terms of emf. For example, the dial may consist of 13 points, representing o, Ioo, $200, \ldots \mathrm{I} 200^{\circ} \mathrm{C}$, and the galvanometer scale may be graduated 
from $\mathrm{o}$ to $100^{\circ} \mathrm{C}$ for the intermediate temperatures. Thus a temperature of $\mathrm{II} 24^{\circ} \mathrm{C}$ would be indicated by a dial setting of I I and a scale reading of 24 . If the temperature-emf relation of the couple is not linear, a given temperature change corresponds to different changes in emf, depending upon the temperature of the couple. Hence, in order that the scale of the galvanometer may be graduated in degrees, it is necessary to modify its sensitivity in the various temperature ranges by means of series resistance. Thus, instead of maintaining the total resistance, $R+r_{\mathrm{p}}$, of the galvanometer circuit constant, as in the case of the deflection potentiometer graduated in terms of emf, this resistance must be so adjusted that the change in sensitivity of the galvanometer compensates for the change in thermoelectric power of the couple with temperature. The change in thermoelectric power of the couple over the temperature range represented by the scale of the galvanometer is usually small, and hence causes only slight errors.

In the "range control board" the above-mentioned difficulty is avoided by the provision of a separate scale for each range; but it would not be practical to apply this method to the ordinary deflection potentiometer, which may have ro to 20 different scale ranges. In the Leeds \& Northrup deflection potentiometer, Fig. 29, the ratios of the resistances may be so modified as to produce very closely the proper compensation in $r_{p}$ for any type of couple used industrially.

(e) Temperature of the Cold Junctions of ThermoCOUPLES. - The emf developed by a thermocouple depends upon the temperature of the cold junctions, as well as upon the temperature of the hot junction. For certain base-metal couples having a nearly linear relation between temperature and emf, the emf is approximately proportional to the difference in temperatures of the hot and cold junctions. With such couples a change of $50^{\circ} \mathrm{C}$ in the temperature of the cold junctions, unless corrected for, would result in an error of $50{ }^{\circ} \mathrm{C}$ in the final temperature measurement.

If a couple is calibrated with a cold-junction temperature of $t_{0}{ }^{\circ} \mathrm{C}$ and used with a cold-junction temperature of $t^{\prime}{ }^{\circ} \mathrm{C}$, the true temperature of the hot junction is obtained by adding to the observed temperature the value $\left(t^{\prime}{ }_{0}-t_{\mathrm{o}}\right) K$ where $K$ is a factor depending upon the particular couple employed and upon the temperatures of the hot and cold junctions. The factor $K$ varies from I.5 to 0.3 , but for rough work may be assumed to be 1.0 for base-metal couples and 0.5 for rare-metal couples. 
The following table gives the cold-junction factors for several different types of couple:

TABLE 9.-Cold-Junction Correction Factors

\begin{tabular}{|c|c|c|c|c|c|c|c|c|c|}
\hline \multicolumn{2}{|c|}{$\begin{array}{l}\text { Engelhard } \\
\text { "Le Chatelier", }\end{array}$} & \multicolumn{2}{|c|}{$\begin{array}{l}\text { Jobnson-Matthey } \\
\text { "Le Chatelier" }\end{array}$} & \multicolumn{2}{|c|}{ Copper-constantan } & \multicolumn{2}{|c|}{ Iron-constantan } & \multicolumn{2}{|c|}{ Chromel-alurmel } \\
\hline Temperature & $\mathbf{K}^{a}$ & Temperature & $\mathbf{K}^{a}$ & Temperature & $\mathbf{K} a$ & Temperature & $\mathbf{K}^{a}$ & Temperature & $\mathbf{K}^{a}$ \\
\hline${ }^{\circ} \mathrm{C}$ & & ${ }^{\circ} \mathrm{C}$ & & ${ }^{\circ} \mathrm{C}$ & & ${ }^{\circ} \mathrm{C}$ & & ${ }^{\circ} \mathrm{C}$ & \\
\hline $265-450 \ldots \ldots$ & 0.65 & $250-400 \ldots .$. & 0.60 & $0-50 \ldots$ & 1.00 & $0-100 \ldots \ldots$ & 1.00 & $0-800 \ldots . .$. & 1.00 \\
\hline $450-650 \ldots \ldots$ & .60 & $400-550 \ldots .$. & .55 & $50-80 \ldots$ & .95 & $100-600 \ldots .$. & .95 & $800-1100 \ldots$ & 1.05 \\
\hline $650-1000 \ldots .$. & .55 & $550-900 \ldots \ldots$ & .50 & $80-110 \ldots$ & .90 & $600-1000$ & .85 & & $\ldots \ldots$ \\
\hline \multirow[t]{4}{*}{$1000-1450 \ldots .}$. & .50 & $900-1450 \ldots$ & .45 & $110-150 \ldots .$. & .85 & & & & ....... \\
\hline & & & & $150-200 \ldots .$. & .80 & & & & \\
\hline & & & & $200-270 \ldots \ldots$ & .75 & & & & \\
\hline & & & & $270-350 \ldots$ & .70 & & & & \\
\hline
\end{tabular}

$a$ Based on calibration with $t_{0}=0^{\circ} \mathrm{C}$,

The corrections may be applied directly, without computing, by setting the pointer of the galvanometer to read the coldjunction temperature on open circuit. The setting is made by turning the zero-adjustment screw of the indicator when the couple is disconnected. This method of correcting for the cold-junction temperature is accurate, but requires, of course, new settings whenever the temperature of the cold junction is altered. The Bristol compensator performs this adjustment automatically by means of a bimetallic spring connected to one of the control springs of the moving coil. The cold junctions of the couple must be accordingly located at the instrument either by direct connection or by means of extension leads, described later. Indicators of the potentiometric type frequently have a movable slide on the temperature scale or an auxiliary dial (see discussion below) which when set to the temperature of the cold junction gives perfect compensation at all temperatures of the hot junction. These two compensation methods also require new settings whenever the temperature of the cold junction is altered. With large and permanent installations the applying of corrections for the temperature of the cold junction by any of the above methods is frequently troublesome, since the temperature may vary considerably in practice over a few hours. There are several methods for obviating the necessity of applying such corrections and of making manual adjustments. The head of the couple may be fitted with a water jacket through which water flows at practically constant temperature from the water main. Copper wires lead from the ter- 
minals of the couple inside the water jacket to the indicator, and the pointer of the indicator on open circuit is set to read the mean temperature of the water, usually ro to $20^{\circ} \mathrm{C}$. If a potentiometer indicator is used, the zero adjustment slide or the cold-junction dial is set at this temperature.

(I) Compensating Leads.-The use of compensating lead wires from the couple to the indicator is, in general, the most satisfactory method of minimizing the cold-junction errors in industrial installations. For base-metal couples these lead wires are of nearly the same materials as those employed in the couple. For exam-

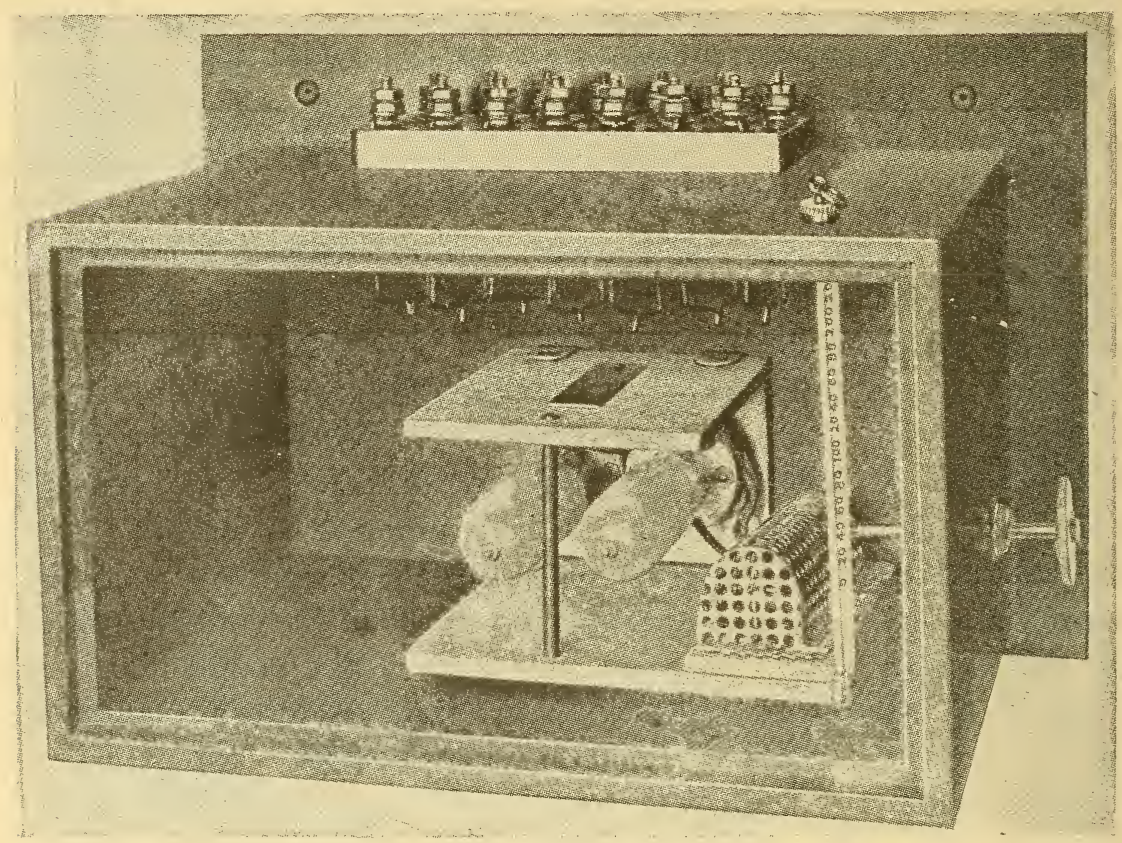

FIG. 3I.-Thermostated cold-junction box

ple, with a chromel-alumel couple one lead wire is chromel, which is connected to the chromel wire of the couple. The other lead wire of alumel is connected to the alumel wire of the couple. Small stranded wires are used for flexibility. Thus, the cold junction is carried away from the head of the couple, where the temperature varies, to a point at some distance from the furnace, where the temperature is reasonably constant, and from this point copper wires lead to the indicator. The compensating wires may terminate in a thermostated cold-junction box, as illustrated by Fig. 31, or may be buried under ground. At a depth of ro feet beneath the floor of a large building the tempera- 
ture remains constant to within $2^{\circ} \mathrm{C}$ throughout the year. Usually this mean temperature is about $\mathrm{I}^{\circ} \mathrm{C}$ for temperate climates, but may differ from this value somewhat if the location is in the immediate vicinity of a large furnace. To use this method for controlling the temperature of the cold junctions, an iron pipe of the proper length, closed at the bottom, is driven into the ground, and the two cold junctions-for example, copper-alumel and copperchromel, well soldered and carefully insulated-are threaded to the bottom of the pipe in such a manner as to be conveniently removable when necessary. The top of the pipe may be plugged with asbestos or waste and covered with pitch to keep water away from the insulation. The scale of the indicator is set to read the mean temperature of the bottom of the tube. It is convenient to have an extra pair of compensating leads or an extra thermocouple with its junction at the bottom of the pipe to measure this temperature occasionally. Usually the compensating leads of a basemetal couple are marked or are equipped with one-way terminals, so that they are easily connected properly to the head of the couple. If reversed at the couple, the leads will cause an error double the amount of the compensation. When compensating leads of a base-metal couple are properly connected to the couple, no deflection of the indicator is obtained by heating the head of the couple.

The high cost of platinum prevents the use of compensating leads of the same metal in the case of a rare-metal couple, but inexpensive lead wires of copper and an alloy of nickel-copper are now available for use with the platinum, platinum ( 90 per cent)rhodium (Io per cent) couple (Bristol compensating leads). These lead wires do not compensate individually, but taken together they compensate to within $5^{\circ} \mathrm{C}$ for a variation of $200^{\circ} \mathrm{C}$ in the couple-lead wire junctions. Since the compensating lead wires for the rare-metal couple do not compensate individually, both terminals on the head of the couple should be always as nearly as possible at the same temperature. The copper wire of the compensating leads is connected to the platinum-rhodium wire of the couple, and the copper-nickel wire is connected to the platinum wire of the couple-that is, alloy wire to pure metal-in each case. The cold junction is then located at the indicator end of the compensating leads. The temperature of this junction may be controlled, if necessary, by one of the methods described above. Copper wires are carried from this point to the indicator. 
(2) Potentiometric Compensation Methods.-The wiring diagram for the Leeds \& Northrup portable potentiometer equipped with a hand-adjusted, cold-junction compensator is given in Fig. 32 . The emf of the thermocouple $H$ is balanced against the potential drop across $D G$, a condition obtained when the galvanometer reads zero, as discussed previously in the section on potentio-

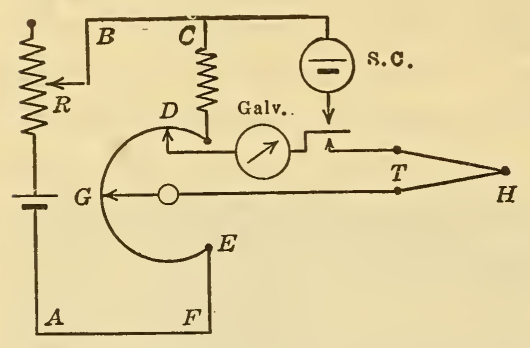

FIG. 32.-Hand-adjusted cold-junction compensator

metric indicators. The dial $G$ is calibrated to read the temperature of the hot junction of the couple. If this temperature remains constant and the temperature of the cold junction $T$ increases, the emf of the couple decreases and the point $G$ would have to be moved nearer $D$ to obtain a balance. If this were done, however, the temperature indicated on the scale would be too low. Hence, instead of moving $G$, the contact $D$ is turned nearer $G$ by an amount depending upon the temperature of the cold junction. A portion of the slide wire $D G E$, containing the contact $D$, is mounted as a separate dial, empirically graduated, for any particular type of couple, to read the temperature of the cold junction. The pointer on this dial is set at the cold-junction temperature, by which setting the contact $D$ is moved the proper amount for exact compensation. The balance is then made in the usual manner by adjusting the contact $G$. The temperature now indicated on the main dial is the correct temperature of the hot junction of the couple. The arrangement shown in Fig. 32, while illustrating the principle of this method of compensation, does not provide for emf measurements to zero emf. The temperature scale on the main dial must start at the highest temperature on the cold-

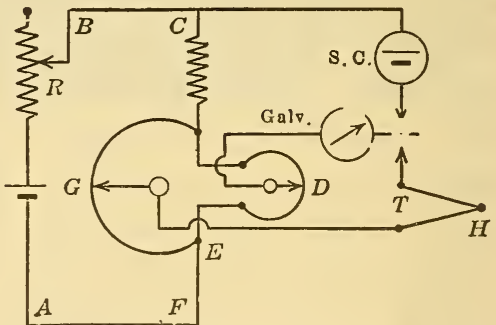

Fig. 33.-Hand-adjusted cold-junction compensator junction dial. Fig. 33 illustrates the wiring diagram more usually employed. The cold-junction dial is in parallel instead of in series with the main dial. This method permits settings on the main dial to zero emf. The theory of the compensation is similar to the theory of the automatic compensator described in the following paragraph: 
The above principle has been applied in an automatic compensator which has been used satisfactorily with the Leeds \& Northrup recorders, Fig. 34. The present circuit and the one just described are similar, with the exception that the contact $D$ in Fig. 33 is mechanically fixed between the two resistances, $M$ having a zero temperature coefficient of resistance and $N$ of nickel having a hightemperature coefficient. The resistance $N$ is located near the cold junction of the couple, so that its temperature and that of the cold junction are identical. If the temperature of the cold junction and of $N$ increases, the emf ceveloped by the couple decreases; but the accompanying increase in the resistance $N$ automatically produces the same

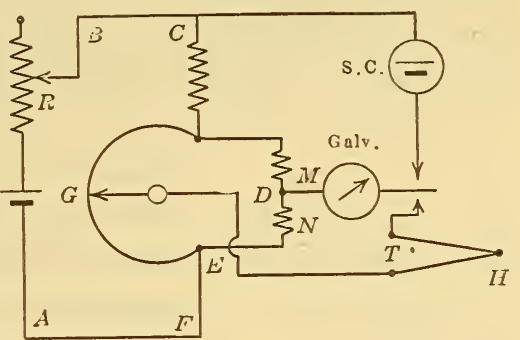

Fig. 34.-Automatic cold-junction compensator

effect as moving the contact $D$ toward $G$ in Fig. 32. The circuit is more simply represented by Fig. 35. Let $e=$ emf developed by the couple when the hot junction is at a temperature $t^{\circ} \mathrm{C}$ and

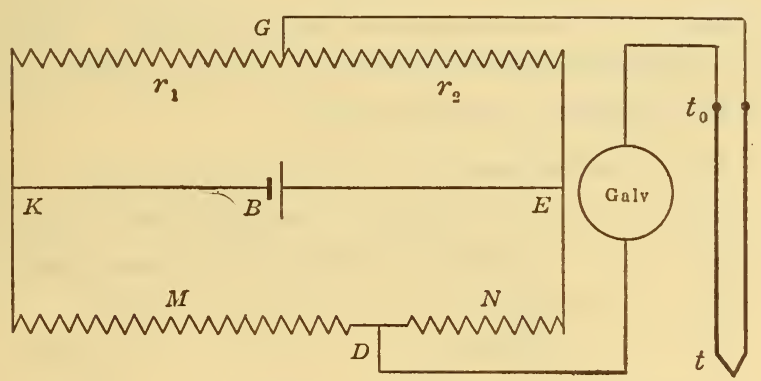

Fig. 35.-Automatic cold-junction compensator the cold junction $\mathrm{O}^{\circ} \mathrm{C}$, and $e_{\mathrm{o}}=$ the emf developed for a hot-junction temperature $t_{\mathrm{o}}{ }^{\circ} \mathrm{C}$ and a cold-junction temperature $\mathrm{O}^{\circ} \mathrm{C}$. Then the emf developed by the couple when

its hot junction is $t^{\circ} \mathrm{C}$ and cold junction is $t_{0}^{\circ} \mathrm{C}$ is $e-e_{0}$. Neglecting the slight effect of the variation of the resistance $N$ with the temperature, the potential drop from $K$ to $E$ due to the battery $B$ is constant $=e^{\prime}$. Whence the potential difference between $G$ and $D$ when the galvanometer in the thermocouple circuit indicates zero is simply derived as follows and is equal to the emf, of the couple:

P. D. between $G$ and $D=e^{\prime}\left(\frac{r_{2}}{r_{1}+r_{2}}-\frac{N}{M+N}\right)=e-e_{0}$.

For exact compensation the position $G$ must be independent of the cold-junction temperature $t_{\mathrm{o}}$; hence on differentiating the above 
expression we obtain the following as a condition which must be satisfied.

$$
\frac{d e_{\mathrm{o}}}{d t_{\mathrm{o}}}=e^{\prime} \frac{M}{(M+N)^{2}} \frac{d N}{d t_{\mathrm{o}}}
$$

By properly proportioning $N$ and $M$ this condition is well satisfied for either base-metal or rare-metal couples.

(3) Compensation by Use of a Shunt.-The use of a resistance having a high temperature coefficient shunted across the terminals of the couple at the cold junction was suggested by Foote ${ }^{11}$ in I9I 3 as a possible method of partially correcting for the cold-junction errors. A modification of this method has

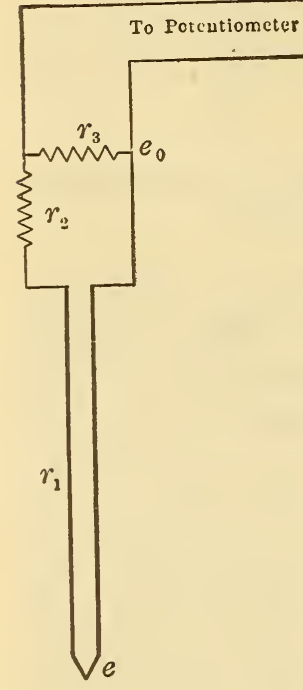

FIG. 36.-Cold-junction compensation by shunt and series resistances been since patented by Mertelmeyer ${ }^{12}$, and is used by the Bristol Co. When the temperature of the cold junction of a couple increases the emf developed by the couple decreases. If, however, the resistance of the coil shunted to the couple increases with the temperature, the potential drop measured across the coil tends to increase, and by properly proportioning the various constants of the circuit a fair degree of compensation is obtained. Fig. 36 illustrates the method of compensation for potentiometric measurements.

Let $r_{1}=$ resistance of the thermocouple.

Let $r_{2}=$ series resistance having a zero temperature coefficient (manganin).

Let $r_{3}=$ resistance at $o^{\circ} \mathrm{C}$ of the shunt.

Let $r_{3}^{\prime}=$ resistance of shunt at temperature $t^{\circ} \mathrm{C}$.

Let $\alpha=$ temperature coefficient of resistance of the shunt reckoned from $\mathrm{O}^{\circ} \mathrm{C}$.

Let $\beta=$ thermoelectric power of the couple (assumed constant).

Let $t=$ any temperature of the hot junction.

Let $t_{\mathrm{o}}=$ any temperature of the cold junction and of $r^{\prime}{ }_{3}$.

Let $t^{\prime}=$ temperature of hot junction for which perfect compensation is desired.

Let $t_{\mathrm{o}}{ }^{\prime}$ and $\mathrm{o}^{\circ} \mathrm{C}=$ temperatures of cold junction for which perfect compensation is desired.

11 Foote, B. S. Sci. Papers, No. 202, D. I2.

12 Mertelmeyer, assignor to Bristol Co. U. S. Patent No. I 228803 ; I9I 7. 
Let $e^{\prime}=$ potential drop across $r_{3}^{\prime}$, hot junction $=t^{\circ} \mathrm{C}$, cold junction $=t_{\mathrm{o}}{ }^{\circ} \mathrm{C}$.

Let $e-e_{0}=\beta\left(t-t_{0}\right)=$ emf developed by couple.

In order to reduce the effect of the variation in the resistance $r_{1}$ of the couple to a minimum, a relatively high resistance $r_{2}$ of manganin is mounted in series with the couple as illustrated. If $r_{2}$ is sufficiently high in comparison with $r_{1}$, we may neglect consideration of the latter in the present discussion. The potential drop $e^{\prime \prime \prime}$ across $r_{3}^{\prime}$ for a cold-junction temperature $t^{\prime}{ }_{0}^{\circ}{ }^{\circ} \mathrm{C}$ and a hot-junction temperature $t^{\prime}{ }^{\circ} \mathrm{C}$ is as follows:

$$
e^{\prime \prime \prime}=\frac{\beta\left(t^{\prime}-t^{\prime}{ }_{\mathrm{o}}\right)\left(\mathrm{I}+\alpha t^{\prime}{ }_{\mathrm{o}}\right) r_{3}}{r_{2}+r_{3}\left(\mathrm{I}+\alpha t^{\prime}{ }_{\mathrm{o}}\right)}
$$

For a cold-junction temperature $t_{0}=0^{\circ} \mathrm{C}$ and a hot-junction temperature $=t^{\prime}{ }^{\circ} \mathrm{C}$ the potential drop $e^{\prime \prime}$ is given by equation (2):

$$
e^{\prime \prime}=\frac{\beta t^{\prime} r_{j}}{r_{2}+r_{3}}
$$

For exact compensation $e^{\prime \prime}$ must equal $e^{\prime \prime \prime}$. Hence on equating (I) and (2) we obtain (3) as a conditional equation.

$$
\frac{r_{3}}{r_{2}}=\frac{\alpha\left(t^{\prime}-t_{0}^{\prime}\right)-\mathrm{I}}{\mathrm{I}+\alpha t^{\prime}{ }_{0}} \text {. }
$$

For a cold-junction temperature $=t_{o}{ }^{\circ} \mathrm{C}$ and a hot-junction temperature $=t^{\circ} \mathrm{C}$ the potential drop across $r_{3}^{\prime}$ is as follows:

$$
e^{\prime}=\frac{\beta\left(t-t_{\mathrm{o}}\right)\left(\mathrm{I}+\alpha t_{0}\right) r_{3}}{r_{2}+r_{3}\left(\mathrm{I}+\alpha t_{0}\right)}
$$

For a cold-junction temperature $=0^{\circ} \mathrm{C}$ and a hot-junction temperature $=t^{\circ} \mathrm{C}$ the potential drop across $r^{\prime}{ }_{3}$ is given by equation (5).

$$
e^{\prime \prime \prime \prime}=\frac{\beta t r_{3}}{r_{2}+r_{3}} .
$$

The error in the compensation, expressed in degrees, at any hotjunction temperature $t$ and cold-junction temperature $t_{o}$ is given by equation (6).

$$
\text { Error, in degrees }=\frac{e^{\prime \prime \prime \prime}-e^{\prime}}{e^{\prime \prime \prime \prime}} t=t-\frac{\left(\mathrm{I}+\frac{r_{3}}{r_{2}}\right)\left(t-t_{0}\right)\left(\mathrm{I}+\alpha t_{0}\right)}{\mathrm{I}+\frac{r_{3}}{r_{2}}\left(\mathrm{I}+\alpha t_{0}\right)} \text {. }
$$

On substituting the value of $\frac{r_{3}}{r_{2}}$ given by equation (3) one obtains:

$$
\text { Error, in degrees }=t_{0}\left(\frac{t^{\prime}-K t}{t^{\prime}-K t_{0}}\right)
$$

where

$$
K=\frac{\mathrm{I}+\alpha t_{0}^{\prime}}{\mathrm{I}+\alpha t_{n}}
$$


Suppose an iron-constantan couple is employed, and perfect compensation is desired for a hot-junction temperature of $800^{\circ} \mathrm{C}$ when the cold-junction temperature is $0^{\circ} \mathrm{C}$ or $50^{\circ} \mathrm{C}$. The potentiometer indicator is graduated to read correctly all temperatures of the hot junction when the temperature of the cold junction is $0^{\circ} \mathrm{C}$.

If the shunt is constructed of nickel wire we have the following data:

$$
\begin{aligned}
\alpha & =0.006 \\
\beta & =0.05 \text { millivolts per degree } \\
t^{\prime} & =800^{\circ} \mathrm{C} \\
t^{\prime}{ }_{0} & =50^{\circ} \mathrm{C}
\end{aligned}
$$

On substituting these values in equations (3) and (7) we obtain:

$$
\begin{gathered}
\frac{r_{3}}{r_{2}}=2.7 \\
\text { Error, in degrees }=t_{0}\left(\frac{800+4.8 t_{0}-\mathrm{I} \cdot 3 t}{800+3.5 t_{0}}\right) .
\end{gathered}
$$

Equation (9) states that the ratio of the values of the resistance of the nickel shunt at $\mathrm{o}^{\circ} \mathrm{C}$ and that of the manganin should be 2.7. Equation (IO) gives the error in degrees for any temperature of the hot junction $t$ and of the cold junction $t_{0}$. The following table shows the magnitude of these errors:

TABLE 10.-Error in Compensation by Shunt Method

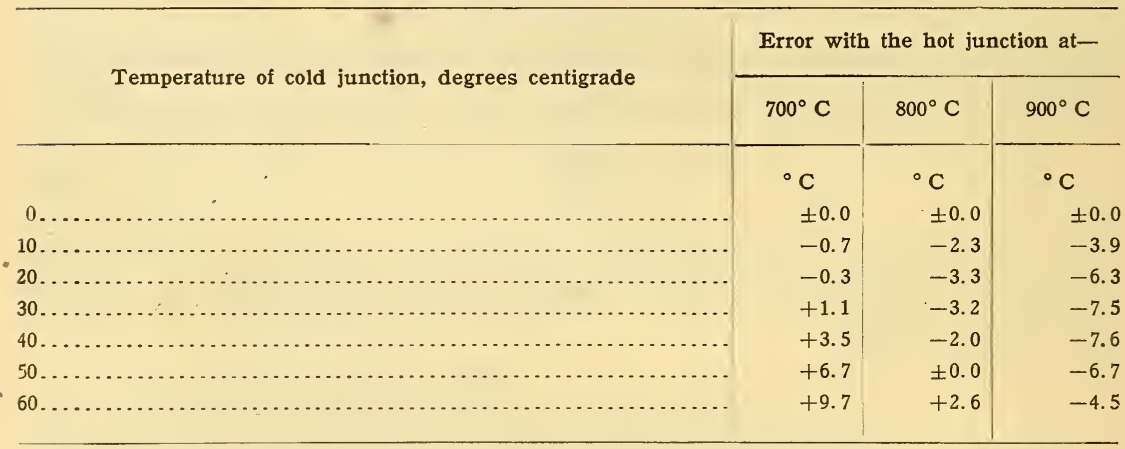

Thus, over a variation of $200^{\circ} \mathrm{C}$ in the temperature of the hot junction and of $60^{\circ} \mathrm{C}$ in the temperature of the cold junction the method compensates to within $10^{\circ} \mathrm{C}$.

The above method may be applied even more satisfactorily when a galvanometer is used instead of a potentiometer. By carrying through a series of computations for the more complicated circuit with a galvanometer of resistance $R$, a relation may 
be obtained between $R, \mathrm{r}_{2}, r_{3}, \alpha$, and $\beta$. The following values give slightly better compensation around $800^{\circ} \mathrm{C}$ than that illustrated in the table above:

TABLE 11.-Shunt Compensation with Galvanometric Indicator

\begin{tabular}{|c|c|c|}
\begin{tabular}{c|c} 
Galvanometer \\
resistance
\end{tabular} & $\begin{array}{c}\text { Series } \\
\text { zesistance } \\
\text { (manganin) }\end{array}$ & $\begin{array}{c}\text { Shunt } \\
\text { resistance } \\
\text { (nickel) }\end{array}$ \\
\cline { 1 - 2 } Ohus & Ohms & Ohms \\
100 & 126 & 150 \\
100 & 59 & 100 \\
100 & 23 & 50 \\
\hline
\end{tabular}

It is evident that this method of compensation has certain advantages in the control of the temperature of a furnace and in maintaining this temperature at an approximately constant value. It should not be employed when the variation in temperature of the furnace is much greater than $100^{\circ} \mathrm{C}$. There are few processes in which the use of compensated leads and a cold-junction box or a buried cold junction, as described above, is not to be preferred. The shunt method has been described at some length, however, because a discussion of it has never appeared elsewhere, and in certain restricted applications the method offers desirable features.

(4) Wheatstone-Bridge Compensation.-This method of compensation for the temperature of the Frg. 37.-Cold-junction compensation by cold junction as applied by the

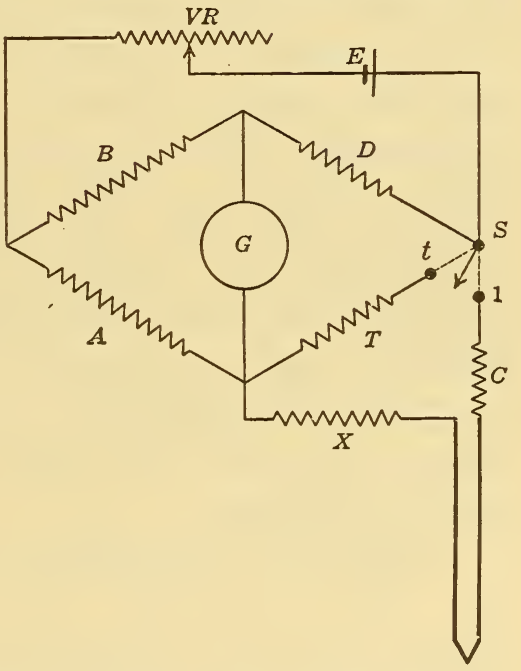

Wheatstone bridge method Cleveland Instrument Co. is illustrated in Fig. 37. The switch $S$ is first thrown to position $t$. The fixed resistances $A$ and $B$ are equal, so that if $T=D$ the bridge would be balanced and the galvanometer would show no deflection. The resistance of $T$ is, however, made considerably greater than that of $D$, so that the bridge is out of balance. By varying the resistance $V R$, the pointer of the galvanometer may be adjusted to some definite mark on the scale. This preliminary setting fixes the value of the current flowing in the main circuit due to the battery $E$. 
For a temperature measurement the switch $S$ is thrown to position I. The couple and the resistances $X+C$ now constitute an arm of the bridge. The resistance $C$ has a high temperature coefficient and is located at the cold junction of the couple. Suppose the apparatus is standardized for a cold-junction temperature of $\mathrm{o}^{\circ} \mathrm{C}$. Then, at this temperature $X+C=D$, and the galvanometer shows no deflection when the temperature of the hot junction is $0^{\circ} \mathrm{C}$. As the temperature of the hot junction increases the emf developed by the couple increases and the bridge is thrown out of balance, causing a deflection of the galvanometer. The scale of the instrument is accordingly empirically graduated to read the temperature of the hot junction when the cold junction is at $\mathrm{o}^{\circ} \mathrm{C}$, and when the proper current flows through the main battery circuit as determined by the preliminary adjustment. When the temperature of the cold junction increases, the emf of the couple decreases, but the resistance $C$ increases. An increase in the resistance $C$ tends to increase the deflection of the galvanometer, while a decrease in the emf of the couple tends to decrease the deflection. On account of the fact that the thermoelectric power of a couple and the temperature coefficient of resistance of the coil $C$ are nearly constant or vary similarly with temperature over a small range of temperature, by properly proportioning the various resistances of the circuit the increase in deflection due to an increase in the resistance of $C$ compensates to all practical purposes for the decrease in deflection due to the decrease in emf of the couple as the temperature of the cold junction increases.

(f) Methods Employed in CORRECTING FOR VARIATIONS IN THE TEMPERATURE-EMF RELATION OF DIFFERENT COUPLES OF THE SAME TYPE.-Platinum and platinum-rhodium suitable for thermocouples are refined by Engelhard in this country and by Johnson-Matthey in England. The temperature-emf relations for the couples obtained from these two sources differ from each other somewhat, but as stated elsewhere, the reproducibility of either of these general types of Le Chatelier couple is highly satisfactory. Base-metal couples of any given type, however, may produce under the same temperature conditions emfs differing by o to 5 per cent or more. Expressed in temperature, these differences may amount to $50^{\circ} \mathrm{C}$ at $1000^{\circ} \mathrm{C}$. If the manufacturer of the couples exercises special care in the choice of the wire, these differences may be considerably reduced. For example, a certain 
length of chromel wire and a length of alumel wire may be selected as representative of the standard couple for which the scale of the pyrometer indicator is graduated. The various stock coils of alumel are tested thermoelectrically against the standard alumel wire, and the stock coils of chromel against the standard chromel wire. If no emf is developed by heating the junction of the standard wire and the wire under test, it indicates that the two are similar. Suppose, however, that an emf of 0.5 millivolt is observed, the standard alumel wire being positive. This coil of alumel wire should be used accordingly with a coil of chromel wire to which the standard chromel wire tested 0.5 millivolt positive. By carrying through a series of such tests on many coils of wire, always heating to the same temperature, various pairs of chromel and alumel coils may be selected so that their temperature-emf relations are nearly the same as that of the standard couple. When these pairs of coils are made into couples, any differences in calibrations from that of the standard couple will be due mainly to heterogeneity of the wire itself. Usually a coil of wire is drawn from a single ingot, and the variations in the thermoelectric properties through a single ingot are likely to be far less than the differences in two ingots which may come from different melts.

After taking these precautions in regard to the selection of the wire, small variations in the emf-temperature relation of different couples still exist. Usually, they are of little practical importance, and most manufacturers consider couples made from wire thus selected to be sufficiently reproducible for industrial purposes. The difficulty in further correcting for the variations of individual couples from the manufacturing standpoint will be readily apparent.

(I) Compensation by Series Resistance.-It is entirely impractical to graduate the scale of the indicator for an individual couple because every time the couple is renewed a new scale would be required, and frequently many couples are used at the same time with a single indicator. If a couple shows higher emf than the standard or normal couple for which the indicator is graduated, an amount of resistance necessary to reduce the deflection to the proper value may be placed in series with the galvanometer. Usually, if a base-metal couple shows an emf, say 2 per cent high at $1000^{\circ} \mathrm{C}$, it will be 2 per cent high at all other temperatures; so that this method of correction is satisfactory for all temperature ranges. The series resistance must be located at the couple and $8513^{\circ}-21-5$ 
not inside the galvanometer, for in the latter case compensation would apply for one couple only. If a couple shows emf 2 per cent high, the extra series resistance must be 2 per cent of the total resistance of the circuit. Thus, with a 300-ohm galvanometer and negligible line resistance, 6 ohms of magnanin is placed in series with the couple. This is mounted on a spool inside the terminal head of the couple. The use of a comparatively high constant line resistance is no serious disadvantage when an instrument is calibrated to read correctly for this resistance. The same galvanometer may be used with different couples; for example, one showing emf 2 per cent high, another I per cent high, and another 0.5 per cent high. When the resistances mounted in the heads of these couples have the values 6 ohms, 3 ohms, and I.5 ohms, respectively, the temperature scale of the galvanometer is correct for all three. If the emf of a couple is low, resistance must be taken out of the circuit. The scale of the instrument is accordingly designed for the normal couple and for a certain normal series resistance, the latter being sufficient to permit adjustment for couples showing low emf. In the above example 6 ohms could be removed from the indicator and mounted in the head of the normal couple. This is the normal series resistance. For couples showing emf 2 per cent, I per cent, and 0.5 per cent high, the series resistances are I2, 9, and $7.5 \mathrm{ohms}$, respectively. For couples showing emf 2 per cent, I per cent, and 0.5 per cent low the series resistances are 0,3 , and $4.5 \mathrm{ohms}$, respectively.

This method of compensation is open to objection, however, especially from the standpoint of the pyrometer manufacturer, in that the resistance of the series coil required for exact compensation depends upon the resistance of the indicator. The following table shows the values of the series resistances required for indicators of various resistances in order to take care of a maximum variation of \pm 2 per cent in the emf of different couples. The resistance of the line proper-that is, lead wires and couple without series coil--is assumed negligible.

Now, the development of the past five years in pyrometry has tended toward the production of indicators of higher resistance with the object of minimizing the errors arising from variations in the line resistance. The result of this development has placed on the market during this interval, in the case of a single manufacturer, instruments having resistances anywhere from 5 to 600 ohms and even $\mathrm{r} 200 \mathrm{ohms}$. Hence, in ordering couples for replace- 
ment, the customer must state the resistance of the indicator and the manufacturer must carry in stock an almost endless assortment of couples compensated and calibrated to fit all the instruments he has manufactured possibly in the past ro years. A large plant which has purchased instruments during an interval of five years may have an assortment of say 100 indicators with resistances from 50 to $600 \mathrm{ohms}$, all calibrated to read correctly for the normal couple. Since the compensated renewing couples are no longer interchangeable, this plant would be required to carry a stock of couples for every indicator. The chances for confusion of records and mixing of couples and the extra cost of such a complete stock are serious items.

TABLE 12.-Compensation by Series Resistance

\begin{tabular}{|c|c|c|c|c|c|c|}
\hline Resistance of indicator & $500 \mathrm{ohms}$ & $250 \mathrm{ohms}$ & 125 ohms & 50 ohms & 25 ohms & $10 \mathrm{ohms}$ \\
\hline Emf of couple & \multicolumn{6}{|c|}{ Resistance of series coil for compensation } \\
\hline 2 per cent low.. & $\begin{array}{r}\text { Ohms } \\
0\end{array}$ & $\begin{array}{r}\text { Ohms } \\
0\end{array}$ & $\begin{array}{r}\text { Ohms } \\
0\end{array}$ & $\begin{array}{r}\text { Ohms } \\
0\end{array}$ & $\begin{array}{r}\text { Ohms } \\
0\end{array}$ & $\begin{array}{l}\text { Ohms } \\
0\end{array}$ \\
\hline 1 per cent low.... & 5.0 & 2.4 & 1.2 & 0.50 & 0.24 & 0.10 \\
\hline 0.5 per cent low.. & 7.5 & 3.7 & 1.9 & .75 & .37 & .15 \\
\hline Normal. ......... & 10.1 & 5.0 & 2.5 & 1.01 & .50 & .20 \\
\hline 0.5 per cent high.... & 12.7 & 6.3 & 3.1 & 1.27 & .63 & .25 \\
\hline 1 per cent high.... & 15.2 & 7.6 & 3.8 & 1.52 & .76 & .30 \\
\hline 2 per cent high...... & 20.3 & 106.1 & 5.0 & 2.03 & 1.01 & .40 \\
\hline
\end{tabular}

The error which is possible when a compensated couple is used with the wrong indicating instrument is illustrated by the following example: Suppose the couple originally read 2 per cent high and that it is compensated to read correctly with a $500-0 h m$ indicator. In the plant the couple, by mistake, is connected to a $50-0 h m$ instrument. The series resistance for the $500-0 h m$ instrument is $20.3 \mathrm{ohms}$ (see Table I 2 ), while that for the $50-\mathrm{ohm}$ indicator is only $2.03 \mathrm{ohms}$. The introduction of $20.3 \mathrm{ohms}$ in series with a $50-\mathrm{ohm}$ galvanometer when only $2.03 \mathrm{ohms}$ in series should be used makes the instrument read 26 per cent low. Hence if the temperature of the furnace were $1000^{\circ} \mathrm{C}$, the $5 \mathrm{O}-\mathrm{ohm}$ indicator would read about $740^{\circ} \mathrm{C}$, or in error by $260^{\circ} \mathrm{C}$. If no compensation whatever had been employed, and if the instruments were graduated for the normal couple with no resistance in series, each instrument when connected to the couple showing emf 2 per cent high would read 2 per cent high, or in error by about $20^{\circ} \mathrm{C}$ at $1000^{\circ} \mathrm{C}$. Thus the error developed by an interchanging of instruments with the 
compensated couple, a mistake which will occur frequently in any large plant, may cause an error many times as great as the error arising from the worst case of irreproducibility of couples when no compensating device at all is employed. A remedy for the prevention of such mistakes is to use instruments of one resistance only. Pyrometer indicators having a practically fixed preassigned resistance are made by certain manufacturers. All parts of the galvanometers are constructed according to specifications rigidly adhered to. The swamping resistance is finally adjusted until the total resistance of the instrument has the preassigned value found by experiment to be satisfactory. Slight differences will then exist in the calibration of different instruments. These can be corrected for by any of several methods such as illustrated below: (a) Calibrate the instrument by direct experiment and make a handdrawn scale. Different instruments will have slightly different temperature ranges. (b) Use stock printed scales and adjust the sensitivity of the galvanometer by means of a magnetic shunt. (c) Use stock printed scales.in several different temperature ranges and select the one which best fits the instrument. (d) Shunt the moving coil to give a specified deflection on a specified current. Then adjust the external resistance until the total resistance has the proper value. Use stock printed scales.

The adoption of a fixed standard instrument is, however, likely to thwart development work on the part of the manufacturers. Furthermore, instruments of all resistances and scale ranges are in daily use in the industries, and they are giving satisfactory service. It is impossible to consider the discarding of all indicators except those having a certain definite resistance in order that a convenient method of compensation by series resistance may be adopted. The objection to compensation by series resistance is further emphasized when indicators or an indicator and a recorder are operated in parallel on the same couple. Suppose a couple reading normally 2 per cent high is installed with a 500-ohm indicator. The series resistance in the head of the couple, as seen from Table I 2 , is $20.3 \mathrm{ohms}$. It is desired to operate another similar indicator in parallel, making two indicators of the same type on the same couple. The resistance of two 500-ohm indicators in parallel is $250 \mathrm{ohms}$. The two indicators accordingly act as a single indicator having a resistance of $250 \mathrm{ohms}$. Hence the series resistance must be IO.I ohms instead of $20.3 \mathrm{ohms}$. If the couple is to be used with both indicators, it can never be used with the indicators 
separately. If a 500-ohm indicator and a $\mathrm{I} 25$-ohm recorder are operated in parallel, the two instruments act as a single indicator, having a resistance of 100 ohms. For the above couple reading 2 per cent high the series resistance must be $20.3 \mathrm{ohms}$ when the $500-\mathrm{ohm}$ indicator is used, $5 \mathrm{ohms}$ when the $125-\mathrm{ohm}$ recorder is used, and about 4 ohms when both are used in parallel. In purchasing such compensated couples it is accordingly necessary to specify the resistances of the indicators and recorders, and to state the method of use - that is, to give a wiring diagram of the installation - and then the couple must be used always in the manner specified. When instruments of very low and different resistances are operated in parallel, the problem of choosing the proper compensation for the couple becomes very complicated. In fact, it is frequently necessary to use cut-out switches, so that the recorder is thrown out of the circuit when the indicator is read, and vice versa. A final objection to the method of compensation by series resistance is that it has no effect whatever when a potentiometric or semipotentiometric indicator or recorder is employed. A couple showing emf 2 per cent high and compensated to read correctly on a $500-0 h m$ indicator will still read 2 per cent high when the measurements are made with a potentiometer. Since the use of potentiometric instruments is becoming more extensive every year, especially for laboratory and checking work, and for recorders, this additional objection to the method of compensation by series resistance is worthy of consideration.

(2) Compensation by Shunt Resistance.-In this method of compensation the thermocouple is shunted usually by a small resistance. If the couple normally reads high, the resistance of the shunt is decreased; if low, the resistance is increased. An ordinary base-metal couple for industrial purposes has a resistance of the order of 0.1 to $0.3 \mathrm{ohm}$ at room temperature. Suppose this couple is shunted by a resistance of about the same magnitude. Such a shunted couple may be used.with a potentiometer or with a galvanometer having almost any resistance from, say, Io ohms up.

Various couples are thus perfectly interchangeable and may be used with instruments in parallel when desired. Hence none of the objections to the method of compensation by series resistance apply to the shunted couple. A very serious objection, however, may be raised against the method of shunted resistance in that the resistance of the shunt for proper compensation depends upon the resistance of the couple. The latter is subject to change with 
temperature of the furnace and depth of immersion, depending upon the temperature coefficient of resistance of the two alloys forming the couple, and the resistance is, of course, altered by deterioration of the couple with use. The following example illustrates the error which may be expected from a slight change in resistance of the couple when the resistance of the shunt is low.

Suppose the couple having a resistance at room temperature of $0.2 \mathrm{ohm}$ is shunted by $0.2 \mathrm{ohm}$ of manganin. If $e$ is the emf developed by the couple, the potential drop over the shunt is e/2 The scale of the galvanometer is arbitrarily graduated to take account of this reduction of emf. Let the resistance of the couple change from 0.2 to $0.25 \mathrm{ohm}$. This small change may be due to oxidation or may be accounted for by immersing the couple to half its length in a furnace at about $600^{\circ} \mathrm{C}$. The potential drop across the shunt of $0.2 \mathrm{ohm}$ on a couple of $0.25 \mathrm{ohm}$ is $0.44 e$ instead of $0.5 e$, for which the galvanometer is graduated. The galvanometer accordingly reads $\mathrm{I} 2$ per cent low or in error by about $\mathrm{I} 20^{\circ} \mathrm{C}$ at $1000^{\circ} \mathrm{C}$. This error is many times greater than any error which would be introduced on account of irreproducibility of the couples if no compensation device whatever were employed. Hence, in attempting to correct for a small error of, say, 15 or $30^{\circ} \mathrm{C}$, we have employed a method which is very likely to develop errors amounting to $100^{\circ} \mathrm{C}$ or more. The use of a shunt of low resistance has been discontinued. The method of shunted resistance is satisfactory, however, when the shunt has a resistance many times that of the couple. As the resistance of the shunt is increased the range for adjustment of different couples is decreased. However, it is not difficult to secure matched wire which is thermoelectrically reproducible to \pm 2 per cent, and this variation can be taken care of by means of shunts of fairly high resistance. For generality the method is discussed under the following heading:

(3) Compensation by Shunt and Series Resistance ${ }^{13}$. - Let $r_{1}=$ resistance of the couple + a small resistance (if necessary) in series with the couple; $\mathrm{r}_{2}=$ resistance of the shunt; and $R=$ resistance of the galvanometer. The potential drop $E^{\prime}$ across the shunt is given by the following equation where $e$ is the emf developed by the couple at any given temperature; for example, $900^{\circ} \mathrm{C}$ :

$$
E^{\prime}=\frac{r_{2} e}{r_{1}+r_{2}+r_{1} r_{2} / R}
$$

${ }^{13}$ Zimmerschied, U. S. L.etters Patent Nc. 776252; 1915. 
In case the potential drop is measured by a potentiometer we have:

$$
E=\frac{r_{2} e}{r_{1}+r_{2}} .
$$

The shunt resistance $r_{2}$ is adjusted to compensate for the variation in emf of the different couples. On account of the term $\frac{r_{1} r_{2}}{R}$ in equation (I) galvanometers having different resistances in general will be differently affected by variations in $r_{2}$ from couple to couple. If, however, the term $\frac{r_{1} r_{2}}{R}$ is small enough compared with $\left(r_{1}+r_{2}\right)$ this effect is negligible, and the potential drop across $r_{2}$ will be practically the same for all values of $R$ and for a potentiometer, regardless of the values of $r_{2}$ for different couples. Let us impose the condition that the value of $r_{1}, r_{2}$, and $R$ must be such that the reading with a potentiometer can never differ from that with a galvanometer by more than 0.5 per cent (that is, $5^{\circ} \mathrm{C}$ at $1000^{\circ} \mathrm{C}$ ). In general, this difference will be found to be very much less. This condition is expressed by equation (3):

$$
\frac{r_{1} r_{2}}{R} \rightleftharpoons 0.005\left(r_{1}+r_{2}\right)
$$

We desire to compensate for couples showing emfs differing from the normal couple by $< \pm 2$ per cent. For convenience in making the adjustments on the shunt it is better to allow a little more variation for couples showing low emf. We will make the computations so that a couple reading 3 per cent low could be compensated for by using a shunt of infinite resistance-that is, with no shunt at all. If $e$ is the emf of any couple, at some fixed temperature, and $e^{\prime}$ is the emf of a couple 3 per cent below normal, we have from equation (2) for compensation:

$$
\frac{r_{2} e}{r_{1}+r_{2}}=\frac{r_{2}^{\prime} e^{\prime}}{r_{1}+r_{2}^{\prime}}=e^{\prime} \text {, since } r_{2}^{\prime}=\infty \text {. }
$$

Hence, substituting in (3) the value of $r_{2}$ found from (4),

$$
r_{1}=\frac{R e}{200 e^{\prime}}=\frac{R}{200} \cdot \frac{\text { emf of any couple }}{\text { emf of couple } 3 \text { per cent low }} \text {. }
$$

It is of advantage to make $r_{1}$ as large as possible. This can be done by making $R$ large, but the value of $R$ must be small enough to take care of all galvanometers likely to be employed. If we denote by $R_{\mathrm{o}}$ the lowest galvanometer resistance for which com- 
pensation is required, we have equation (6) for the maximum desirable value for the resistance of the couple.

$$
r_{1}=\frac{R_{\mathrm{o}}}{2 \mathrm{OO}} \cdot \frac{e}{e^{\prime}}=\text { total resistance of couple. }
$$

Substituting this value of $r_{1}$ in equation (4) we obtain:

$$
r_{2}=\frac{r_{1}}{\left(\frac{e}{e^{\prime}}-\mathrm{I}\right)}=\frac{R_{\mathrm{o}}}{200\left(\mathrm{I}-\frac{e^{\prime}}{e}\right)}=\text { shunt resistance. }
$$

Table I 3 shows the values of the shunt resistances and couple resistances for the minimum galvanometer resistances $100,80,60$, and $40 \mathrm{ohms}$. The compensation is better the more the resistance of the galvanometer exceeds these minimum values, and in no case does the error in compensation amount to more than 0.5 per cent (that is, $3^{\circ}$ at $600^{\circ} \mathrm{C}$ or $5^{\circ}$ at $1000^{\circ} \mathrm{C}$ ).

\begin{tabular}{|c|c|c|c|c|}
\hline Series resistance & $r_{1}=0.5 \mathrm{ohm}$ & $r_{1}=0.4 \mathrm{ohm}$ & $r_{1}=0.3 \mathrm{ohm}$ & $r_{1}=0.2 \mathrm{ohm}$ \\
\hline Couple & Shunt & Shunt & Shunt & Shunt \\
\hline & Ohms & Ohms & Ohms & Ohms \\
\hline 3 per cent low... & $\infty$ & $\infty$ & $\infty$ & $\infty$ \\
\hline 2 per cent low............. & 48.50 & 38.80 & 29.10 & 19.40 \\
\hline 1 per cent low... & 24.25 & 19.40 & 14.55 & 9.70 \\
\hline Normal................ & 16.17 & 12.94 & 9.70 & 6.47 \\
\hline 1 per cent high.................. & 12.12 & 9.70 & 7.27 & 4.85 \\
\hline 2 per cent high................. & 9. 70 & 7.76 & 5.82 & 3.88 \\
\hline Minimum galvanometer resistance..... & 100 & 80 & $60 \cdot$ & 40 \\
\hline
\end{tabular}

TABLE 13.-Compensation by Shunt and Series Resistance

In using this method of compensation the standard galvanometer scale is graduated in the usual manner for the couple which reads 3 per cent low. Thus, if we have a table of emf versus temperature for the normal couple, we decrease all the values of the emfs in the table by 3 per cent. If $800^{\circ} \mathrm{C}$ corresponds to an emf of 33.2 millivolts for the normal couple, the $800^{\circ} \mathrm{C}$ point on the galvanometer scale must be equivalent to (0.97)(33.2), or 32.2 millivolts, and, similarly, for all the other graduations.

It was noted in the case of shunting a couple of 0.2 by $0.2 \mathrm{ohm}$, that if the resistance of the couple increased from 0.20 to $0.25 \mathrm{ohm}$ due to change in depth of immersion, the galvanometer would be in error by $120^{\circ}$ at $1000^{\circ} \mathrm{C}$. On referring to Table $\mathrm{r} 3$ the greatest error which a change in the couple resistance from 0.20 to 0.25 ohm can produce when properly shunted occurs with the couple 
reading normally 2 per cent high, in which case the shunt has a resistance of $3.88 \mathrm{ohms}$. The error due to this change in resistance amounts to I.2 per cent, or about $\mathrm{I} 2^{\circ}$ at $1000^{\circ} \mathrm{C}$ instead of $\mathrm{I} 2 \mathrm{O}^{\circ} \mathrm{C}$. For the normal couple the error is only $7^{\circ} \mathrm{C}$ and for the couple reading 2 per cent low only $3^{\circ} \mathrm{C}$. The error thus decreases as the resistance of the shunt increases. This shows that it is more desirable to use a couple having a resistance of $0.5 \mathrm{ohm}$. If the resistance of the couple alone is only $0.2 \mathrm{ohm}, 0.3 \mathrm{ohm}$ of manganin may be used in series, and the shunt is connected over the total of $0.5 \mathrm{ohm}$. If the resistance of the couple is now altered by $0.05 \mathrm{ohm}$ from 0.50 to $0.55 \mathrm{ohm}$ the couple reading 2 per cent high, normal, and 2 per cent low will be in error by only 5,3 , and $\mathrm{I}^{\circ} \mathrm{C}$ at $1000^{\circ} \mathrm{C}$, respectively. It is evident that this method of compensation is far superior to the use of a shunt of low resistance.

(4) Summary Statement on Reproducibility of Couples.-The object of this section has been primarily to call the attention of the pyrometer engineers in the industrial plants to the difficulties encountered by the pyrometer manufacturer, when he attempts to correct for small variations in the calibration of different couples. The Bureau of Standards has calibrated chromel-alumel couples submitted for test which deviated from the normal couple by $20^{\circ} \mathrm{C}$ at $1000^{\circ} \mathrm{C}$. On the other hand, the Bureau has purchased chromel-alumel wire at different times for which the maximum deviation from the specified temperature-emf relation was only $4^{\circ} \mathrm{C}$. It is certainly possible, if necessary, to hold the manufacturer to within $\pm 5^{\circ} \mathrm{C}$ of the specifications for an uncompensated couple. There are few industrial processes, however, using base-metal couples which require an accuracy of even $20^{\circ} \mathrm{C}$ at $1000^{\circ} \mathrm{C}$, and there are still fewer processes in which temperatures are measured to this accuracy or would be likely to be measured to this accuracy even if a perfectly compensated couple were secured. The users of pyrometers have forced these compensation methods upon the manufacturer by insisting upon greater precision than is really necessary. In so doing the user obtains a couple which when installed in a certain precise manner and frequently checked may give satisfactory results; but usually the compensation device is a source of more serious error than would be occasioned by the slight irreproducibility of the uncompensated couples. In the past few years remarkable advances have been made in the precision required and attained by certain few industries in temperature regulation, notably in the heat treatment of high-grade steel, such as dies, etc. Here an accuracy 
of $5^{\circ} \mathrm{C}$ is frequently necessary, and some manufacturers claim even greater precision than this in the reproducibility of the furnace conditions. Such precision, however, can not be expected of compensated couples. A solution of the problem from the manufacturing point of view is to secure as well matched wire as possible, do away with compensation devices, and sell at different prices two grades of couples, one which is guaranteed to $\pm 5^{\circ} \mathrm{C}$ and the other to $\pm 10^{\circ} \mathrm{C}$. Possibly at a later time, closer specifications could be adopted. If any industrial process requires greater precision than this, the couples should be individually calibrated and correction curves prepared similar to those furnished with high-grade voltmeters and electrical instruments. If exact reproducibility and higher accuracy are both required, the rare-metal couple should be employed. In objecting to compensating methods, we refer only to those devices which are supposed to correct for variations in the thermoelectric characteristics of the couple wire. Compensation methods for eliminating cold-junction errors, ordinary "compensating lead wires," etc., are, of course, necessary, and these methods must not be confused with the methods discussed in the above section, which operate for an entirely different purpose.

(g) Thermocouple Installations-(i) Special Precautions.The installation of a large thermocouple equipment requires the services of competent electricians. Just as much attention, if not more, should be given to the wiring, switches, switchboards, etc., as is given in the case of ordinary power installations. Proper fixtures should be used to mount the couple in the furnace. Lead wires should have a weatherproof covering and should be run in a metal conduit except for a short length of flexible cable at the ends of the conduit. The conduit should be grounded to prevent leakage from power installations or lighting circuits. All joints in the lead wires should be soldered and taped. When indicators or recorders of low resistance are employed, it is of the greatest importance to have a well-constructed electrical installation to insure a constant line resistance. Since instruments of low resistance are usually calibrated for a low line resistance of definite value, the size of copper wire required for a long line may be as large as No. I 2 or No. Io. Special attention must be given to contact resistances at switches. Frequently switches rated at IOo amperes are required, although the actual thermoelectric current is only a few milliamperes. If the indicator is of high resistance, or is equipped with a compensating device, or operates 
upon the potentiometric or semipotentiometric principle, the factor of very low line resistance is not of great importance, but the wiring should be well installed for the psychological effect, if nothing else. Stationary indicating and recording instruments usually should be mounted upon switchboards, with suitable selective or commutating switches when several couples are used with one indicator. When the head of the couple is exposed to severe conditions, rain, etc., as in outside kilns, a weatherproof terminal head should be used. This consists of an outside casing which fits over both binding posts. The cover may be tapped for conduit wiring or provided with a packing gland or stuffing box if a length of flexible cable is used between the couple and the conduit. The lead wires should be carried from the couple to the indicator through as cool rooms as conveniently possible. Copper has a high temperature coefficient of resistance, and the frequent practice of running wires over the top of a long row of furnaces may cause large variations in line resistance.

The indicator or recorder should be conveniently located. If the instrument is desired especially for the use of the operator of a furnace, it should be placed where it is readily available. It should be mounted where vibration and excessive dirt and dust will not injure the delicate part of the mechanism. In almost all industrial installations outside protecting cases are required to prevent dust from filtering through the case of the indicator. A suitable protecting case is illustrated by Fig. 38. The various screws and gears of a recorder require occasional attention. The tools for this work, screw drivers, oil can, etc., may be mounted inside the outer protecting case, where they are available for immediate use. Special devices are employed to damp out vibration when this is serious, as in the neighborhood of a trip hammer or rolling mill. Frequently the instruments are suspended on spiral springs. One convenient method especially suitable for heavy instruments, such as a recorder, is to mount the instrument on a shelf which is supported on a pier by four ordinary tennis balls, one at each corner of the shelf. The tennis balls damp out vibration very satisfactorily.

The proper location of couples in the furnace depends upon the particular process and use to which the furnace is put. The primary consideration must be to locate the hot junction at the point the temperature of which is required. A secondary consideration, however, is to locate the couple where the lead wires may 
be conveniently taken care of. The space between the protecting tube of the couple and the furnace wall should be tightly plugged with refractory cement, so that hot air can not strike through the hole onto the head of the couple, nor cold air be drawn in, thus cooling the hot junction of the couple.

The use of extension or compensating lead wires, cold-junction boxes, etc., has been discussed elsewhere. The cold-junction box should be so located as to reduce the amount of compensating lead wire required to a minimum. Compensating lead wires, except ordinary iron-constantan leads, are somewhat costly and should not be employed extravagantly. Also the use of long

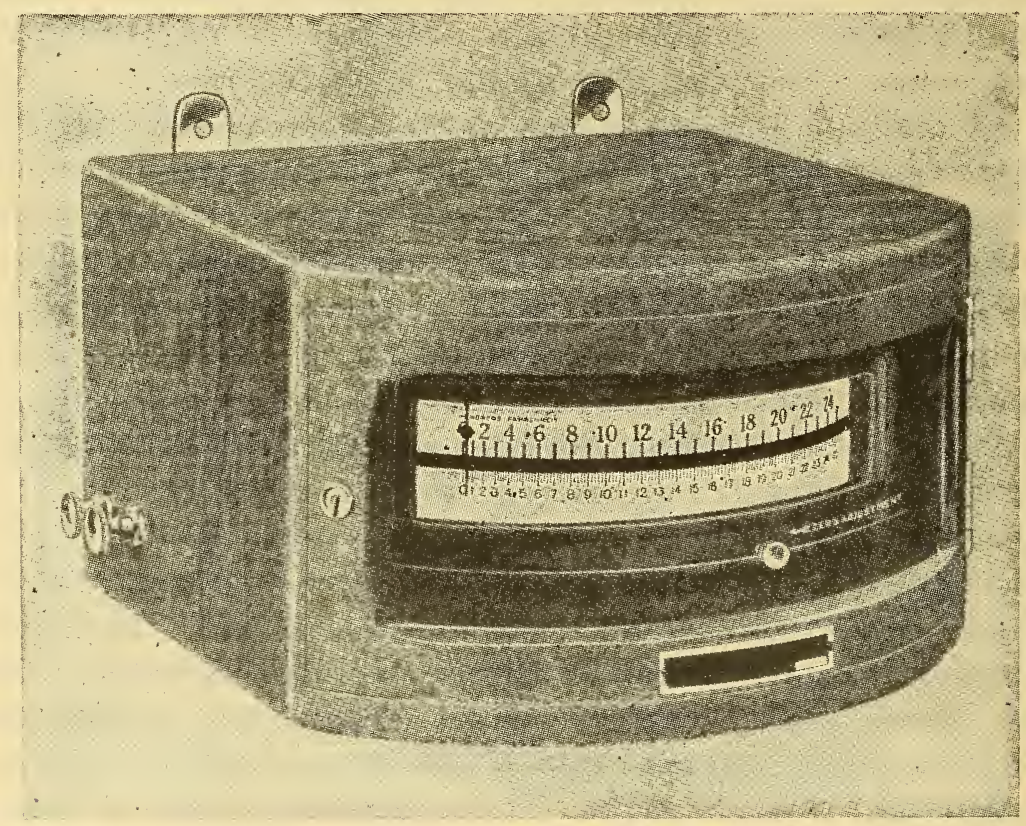

Fig. 38.-Indicator in hardwood protecting case

lengths of compensating wire increases the line resistance, since the resistivity of these leads is much higher than that of copper.

In case the cold junction is buried under ground, it must not be located too near a large furnace. Either the distance from the furnace or the depth at which the junction is buried must be increased. A depth of ro feet at at least ro feet from a large furnace is usually satisfactory.

(2) Common Return.-The use of a common-return wire for a multiple installation is in general unsatisfactory. With such installations, short circuits through the metal protecting tube of 
the couple to the furnace and other couples are likely to occur. The trouble which the short circuits can produce is sufficient to warrant the extra cost of copper required to prevent them. With the common return, leakage from a power installation affects the reading of every couple connected to the return and a leakage through a high resistance may alter the readings of every couple by the same amount so that the presence of such leaks is not always readily detected. It is also possible by leakage from different couples to the ground to obtain very erratic and erroneous readings when the common return is employed. Base-metal couples are frequently constructed with the hot junction welded to the end of the iron protecting tube in order to reduce thermal lag. Even when this welded junction is not made, the hot junction usually touches the protecting tube and is in good electrical contact with it, especially since at high temperatures insulation resistance becomes very low. Suppose that the iron tubes of two chromel-alumel couples are grounded to the iron casing of a furnace. The two hot junctions are thus connected to each other by a circuit of iron. The electrical circuit is represented by Fig. 39. On the indicator we have a chromel-alumel couple, one leg of which is shunted by an alumel-ironalumel differential couple. If the temperatures of the hot junctions of the couples are the same, this differential couple produces no effect. It will, how-

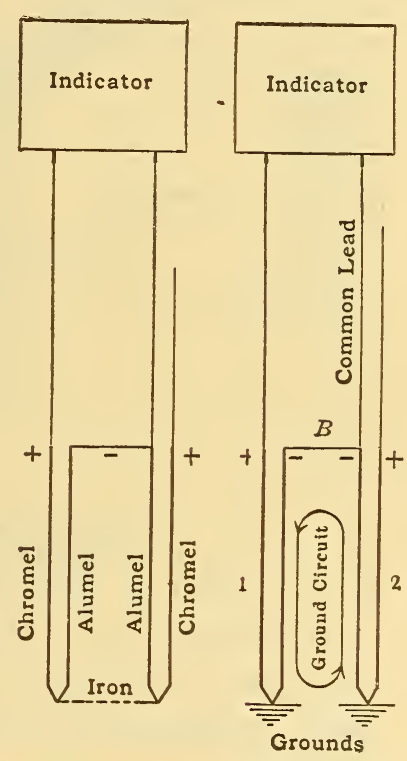

FIG. 39.-Illustrating objection to common return ever, alter the reading of the indicator whenever the two temperatures differ. Both the chromel-alumel couples will accordingly give erroneous results. If individual returns are used, the iron circuit produces no effect. When grounds occur farther back from the hot junction-for example, between the common return and the other lead wire of a single couple-all couples on the common return have, in addition to their own emf, an impressed potential drop due to the current flowing in the shorted couple. This may cause a large error in the reading of every couple on the line. Installations employing a common return are extensively used in the 
industries. It is an unwise practice, and one which should be avoided as far as possible.

(3) Wiring Diagrams of Thermocouple Installations.-Fig. 40 illustrates a simple thermoelectric installation for a rare-metal couple. The couple is properly protected by a porcelain or quartz tube and, if necessary, by an outer tube of iron, chromel, fire clay, etc. From the head of the couple compensating lead wires are carried to the bottom of a pipe driven ro feet under ground. From the bottom of the pipe copper lead wires are carried to the indicator.

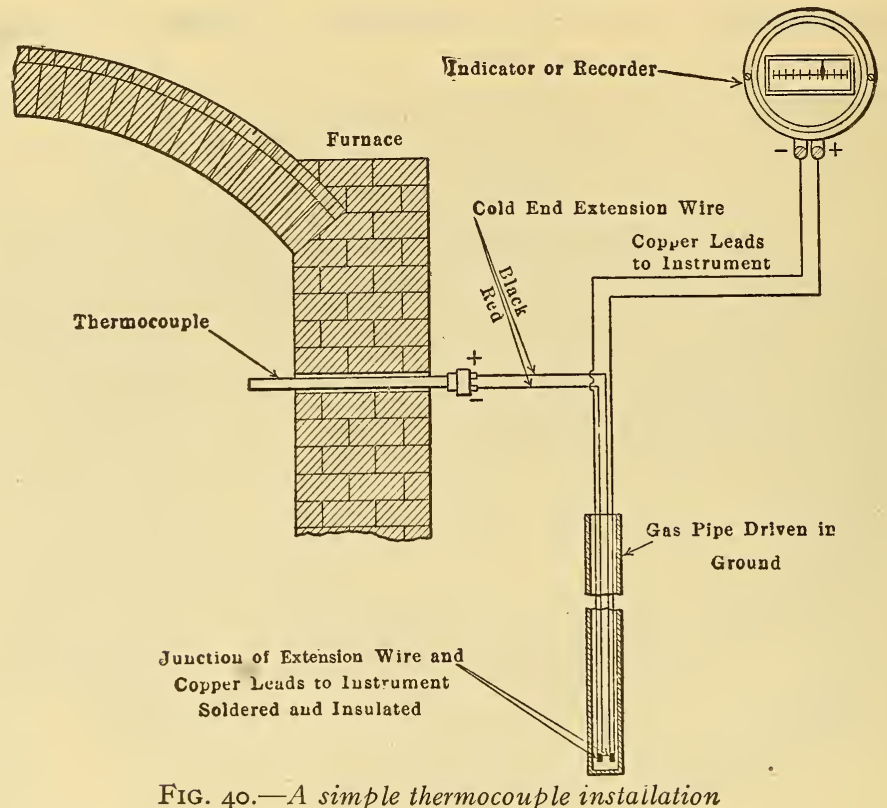

Fig. 4I illustrates a multiple installation for five thermo- couples. In this case a common return is employed, although, as stated above, the use of an individual return is preferred. By use of the common return for this installation four lengths of copper wire (or compensating leads) from the couples to the recorder have been saved, and the commutating switch is simpler. The indicator for the operator of the furnaces and the recorder for the superintendent's office are mounted in parallel. The indicator or recorder may be connected to any couple desired by setting the commutating switch illustrated in the lower halves of the cases of the instruments. Such an installation can be employed only when the instruments have a high resistance. The recorder and indicator, when connected to the same couple at the same time, act as 
a shunt on each other, and this tends to make both instruments read low, whereas, if the two instruments are calibrated to read correctly in parallel, they will both read high when connected to different couples. An example illustrates this point. Suppose the line and couple resistance for each circuit is $3 \mathrm{ohms}$ and the resistances of the recorder and indicator are 500 ohms each. Assume that both instruments are calibrated to read correctly when connected separately to any couple. The potential drop $E$ across the terminals of either instrument bears the following relation to $e$, the emf of the couple:

$$
E=\frac{500}{503} e
$$

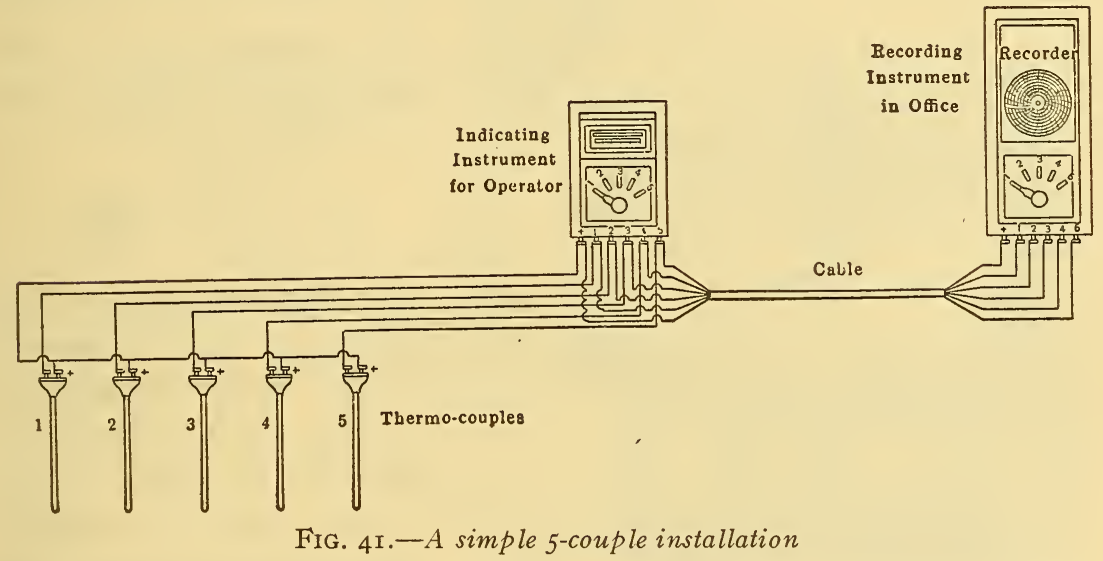

The scale of the instrument is graduated to take account of this reduction in emf. When the two instruments are connected in parallel, the potential drop across the indicator and recorder is given by the following equation, where $R$ equals resistance of indicator or recorder and $r$ equals line resistance:

$$
E^{\prime}=\frac{R e}{R+2 r}=\frac{500}{506} e .
$$

There is, accordingly, a reduction in emf by the factor $\frac{500}{506}$, when the instruments are connected in parallel to the same couple. Each instrument is calibrated for a reduction in emf of $\frac{500}{503}$. The error resulting from the parallel connection is thus 0.6 per cent, or about $6^{\circ} \mathrm{C}$ at $1000^{\circ} \mathrm{C}$. Hence, if the operator of the furnace switches couple number 5 , for example, on the indicator when this couple also is connected to the recorder, both instru- 
ments will read about $6^{\circ} \mathrm{C}$ low at $1000^{\circ} \mathrm{C}$. This error is usually insignificant.

A similar example will be considered for an indicator and recorder of low resistance. Let the line resistance $r$ equal 3 ohms as before, and the resistances $R$ of the indicator and of the recorder equal io ohms each. The potential drop $E$ across the terminals of either instrument bears the following relation to $e$, the emf of the couple:

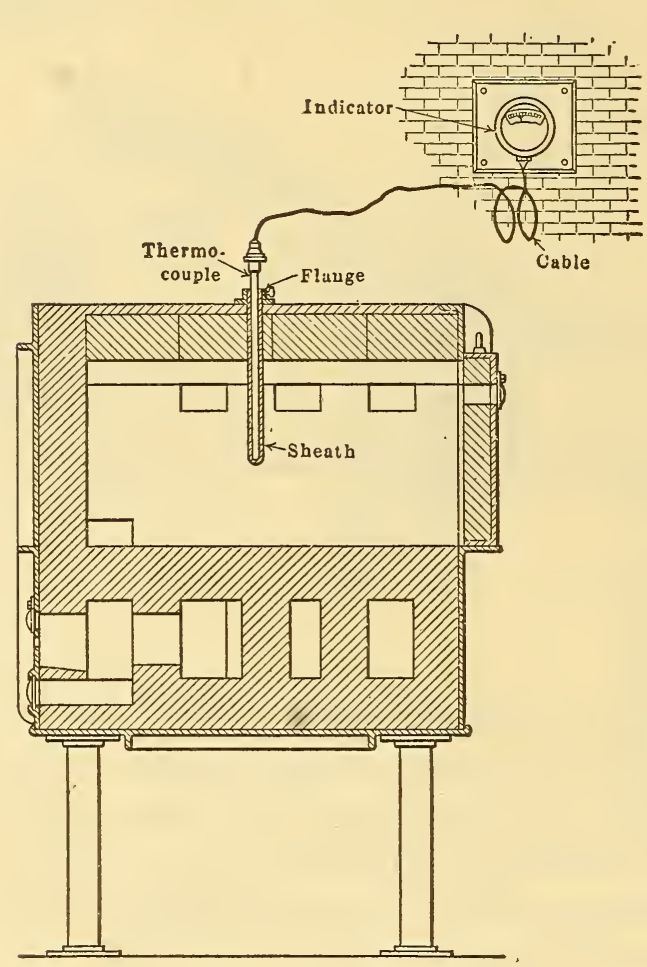

FIG. 42.-Thermocouple in oil-fired furnace

$$
E=\frac{R e}{R+r}=\frac{10}{\mathrm{I} 3} e .
$$

When the two instruments are connected in parallel, the potential drop across the indicator and recorder is as follows:

$$
E^{\prime}=\frac{R}{R+2 r} e=\frac{10}{\mathrm{I} 6} e .
$$

The instruments are calibrated to read correctly when used separately; that is, for a reduction in emf by the factor Io/I3. When used in parallel there is a reduction of emf by the factor Io/ı6. The error resulting from a parallel connection is thus I9 per cent or about $190^{\circ} \mathrm{C}$ at $1000^{\circ} \mathrm{C}$. Hence, if the operator of the furnace switches couple number 5, for example, on the indicator when this couple is connected to the recorder, both instruments will read low by $190^{\circ} \mathrm{C}$ at $1000^{\circ} \mathrm{C}$. This is a very serious error. Accordingly, instruments of low resistance can not be operated alternately separately and in parallel on the same couple. They must be used always either separately or in parallel. In either case the scales of the instruments are graduated for the proper reduction in emf due to line drop. The paralleling of simple galvanometric instruments having a resistance of 300 ohms and more, when the line resistance is less than 3 ohms, or of potentiometric instruments, is a safe practice. The paralleling of instruments of 
lower resistance requires specially graduated scales or the use of special wiring circuits. Instruments of low resistance designed for parallel operation should not be used separately. Instruments of low resistance operated with cut-out switches may be used separately, since the switches are designed so, that while a recorder and indicator are operated on the same line, they are never connected to the same couple at the same time.

Fig. 42 illustrates a simple thermocouple installation in an oil-fired furnace. The thermocouple is mounted in an additional metal sheath to protect it from mechanical shocks and breakage. Compensating lead wire is carried to the indicator. The cold junction is located at the indicator and is not thermostatically

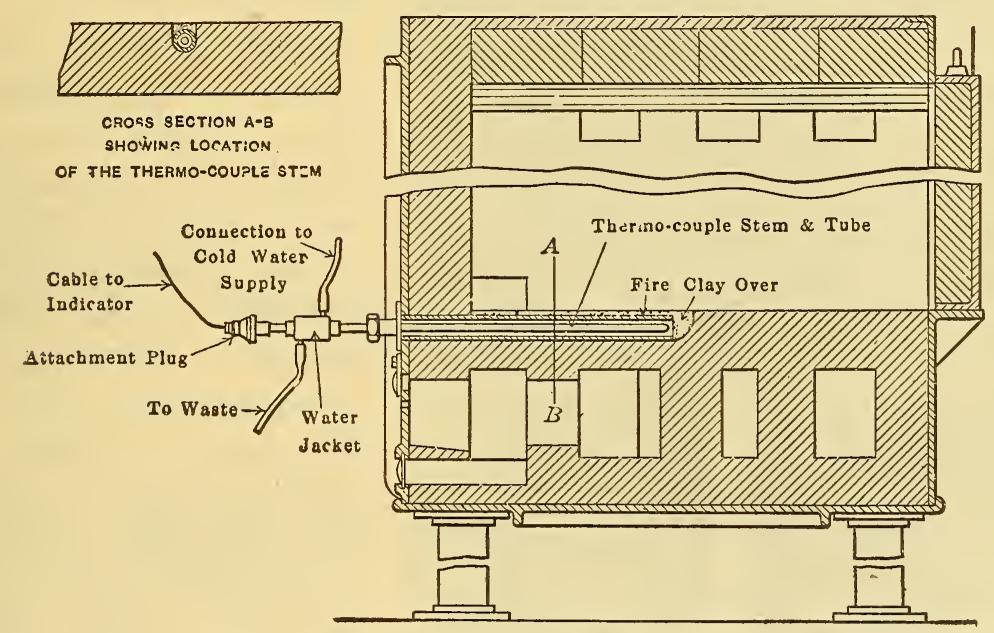

FIG. 43.-Thermocouple in oil-fired furnace

controlled. The indicator is set to read the room or cold-junction temperature on open circuit. Otherwise correction for "coldjunction error" must be applied.

Fig. 43 shows a thermocouple embedded in the floor of an oil-fired furnace. The couple thus requires no space in the heating chamber. The cold junction is water jacketed.

Fig. 44 shows a method of installing a couple in the wall of a large furnace.

Fig. 45 shows a method of installing a couple in a galvanizing tank or a pot of stereotype metal, babbitt, or tin.

Fig. 46 illustrates a multiple thermocouple installation connected to a single indicator. Compensating lead wires are carried $8513^{\circ}-21-6$ 
from the couples to a conveniently located cold-junction box. The temperature of this box is thermostatically controlled. From the cold-junction box copper wires are carried to the terminal block and selective switch illustrated. A common return has been employed between the cold-junction box and the switchboard. In general, it is preferable to use individual return wires for each couple. The switchboard illustrated is designed for six couples. By pressing one of the buttons shown any desired couple is connected directly to the indicator.

(4) Commutating Switches.-Fig. 47 shows a multiple rotary switch. By turning the dial to the proper posi-

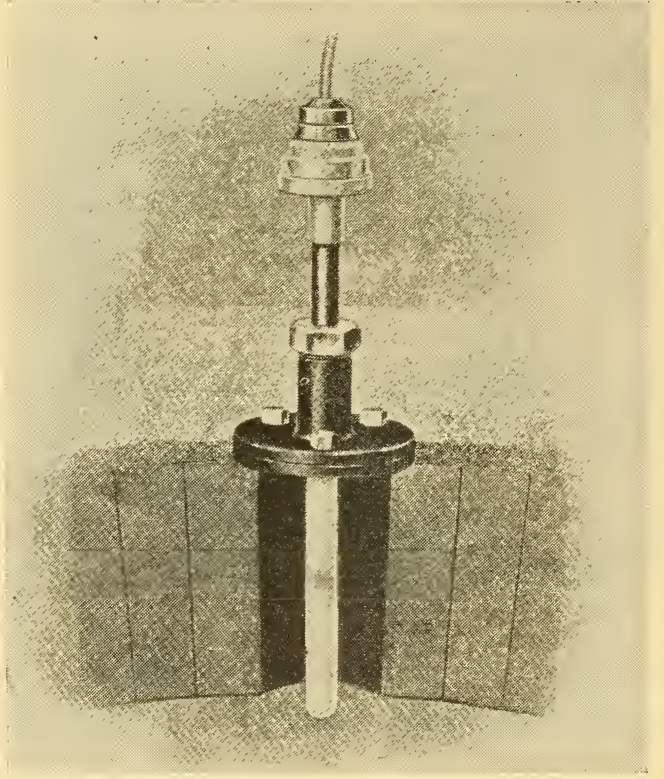

FIG. 44.-Method of mounting couple in furnace tion any one of 12 couples may be connected to the indicator. The commutating brushes in this switch are laminated phosphorbronze, diagonal wiping, and have a long spring action to follow up all possible wear. Fig. 48 illustrates a switch of different design, but similar in principle. Double points of contact are required when individual return wires are employed. The positive wires of each couple are connected to the outer ring of contacts and the negative wires to the inner ring. The galvanometer is connected across the two solid rings. Commutating switches are designed so that variable contact resistance is reduced to a minimum.

For large installations several hundred couples may be connected to a switchboard, and the operator of the board connects the couples successively to the indicator. The switchboard is frequently designed somewhat similar to an ordinary telephone switchboard. Often in these large installations communication between the operator of the switchboard and the operator of the 
furnace is maintained by a system of colored electric lamps. This method of temperature control is meeting with great success in the industries. A description of its applications is presented elsewhere.

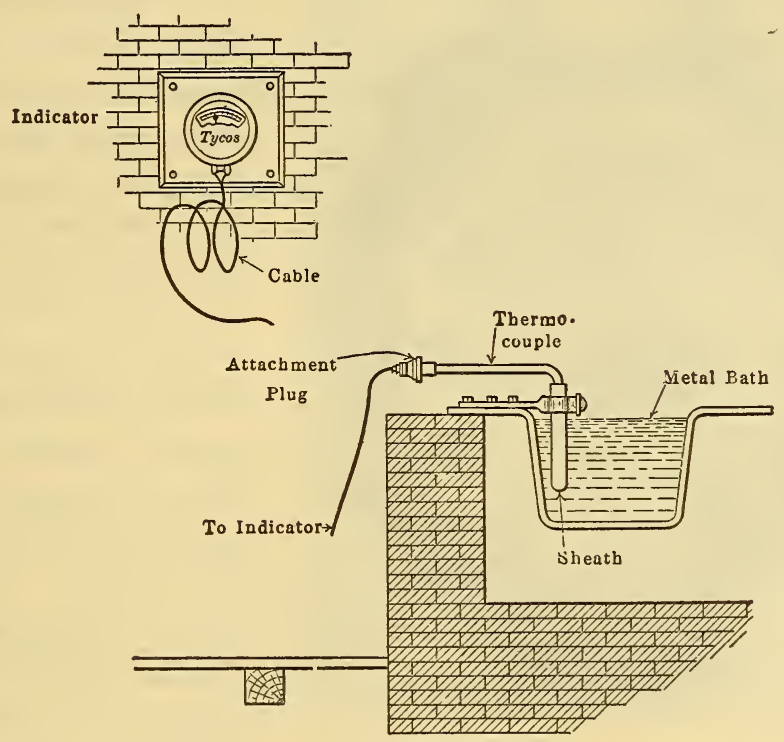

FIG. 45.-Thermocouple in lead bath

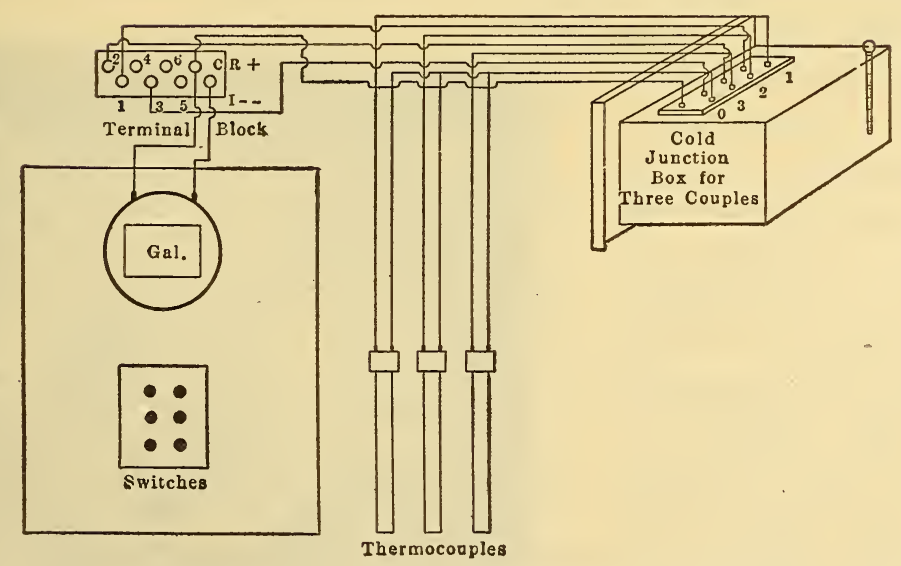

FIG. 46.-Multiple-couple installation with thermostated cold-junction box and compensating leads

(5) Use of Junction Box.-Fig. 49 illustrates a wiring diagram for a multiple-couple installation which is very useful in saving compensating lead wire and in thus reducing the cost and the resistance of the line. The junction box is a cast-iron box such as 
those used for underground telephone wiring. The box is not thermostated, since a constant and measured temperature is not

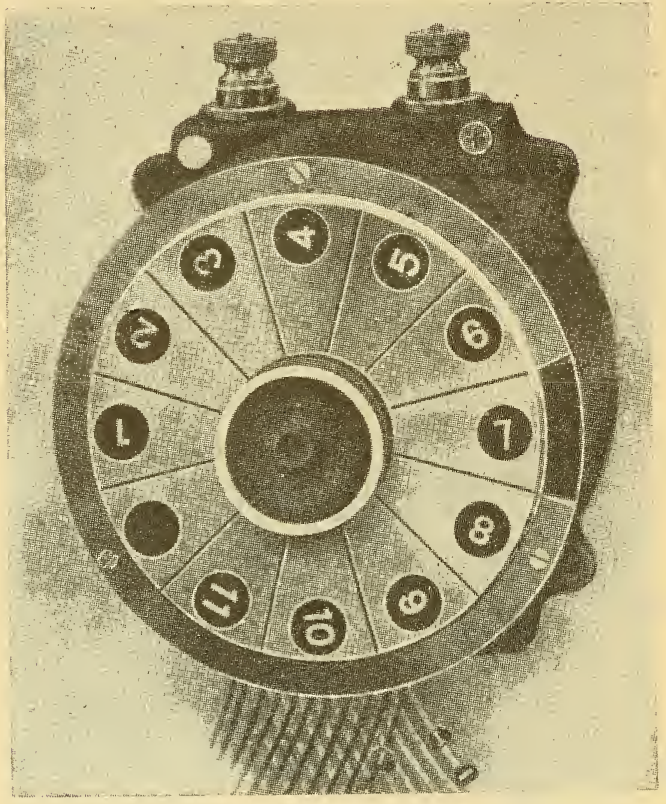

FIG. 47.-Type of commutating switch required. It is merely necessary to have a uniform temperature in the box. This is secured when the box is constructed of heavy metal. The emf developed at the junctions of compensating leads and copper leads is compensated for by a common junction in the opposite direction inserted between the selective switch and the indicator or recorder. A common cold junction is also placed here. In the illustration this is located in a pipe buried Io feet under ground.

The selective switch and recorder or indicator are usually mounted in a single case. The common cold junction and the junction-box compensating couple are connected at the recorder between the switch and the bindingpost terminals of the instrument, as illustrated. The cold junction is placed near the junction box and the recorder or indicator (with switch, if desired) may be any distance away, since only copper leads are used from this point to the junc-

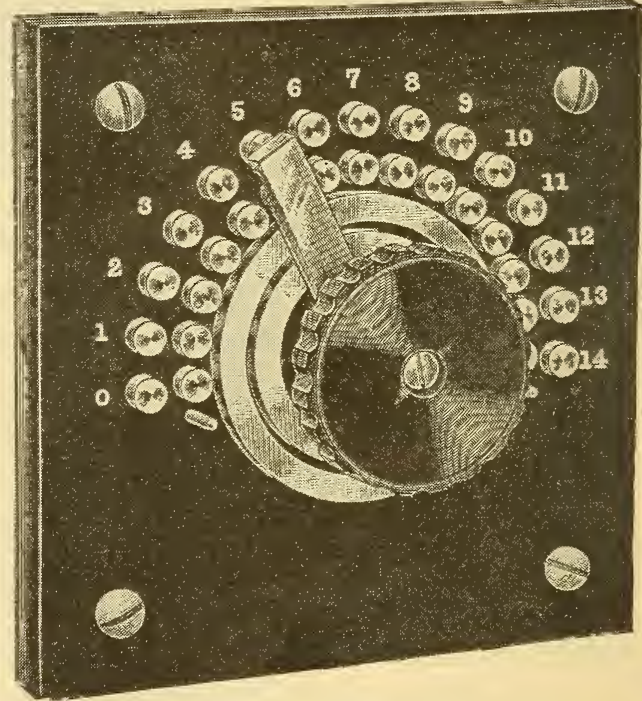

FIG. 48.-Type of commutating switch tion box. The method is especially useful where separate cold 
junctions require too long compensating leads. The following example illustrates a case where such an installation is desirable. Suppose the temperature of a coke oven 20 by 40 by I 50 feet is measured by nine couples inserted in the top. The indicator is located at the ground level 40 feet from the furnace. The buried cold junctions are 20 feet in front of the oven. The following illustrates the amount of compensated lead wire required to reach the buried cold junctions when the junction box is not employed: Three couples at rear of furnace 3 ( $15 \mathrm{O}+2 \mathrm{O}+2 \mathrm{O}+\mathrm{IO}$ ) feet, three couples at center of furnace $3(75+20+20+10)$, three couples at front of furnace $3(20+20+\mathrm{IO})$. Total compensating cable, I, I 25 feet.

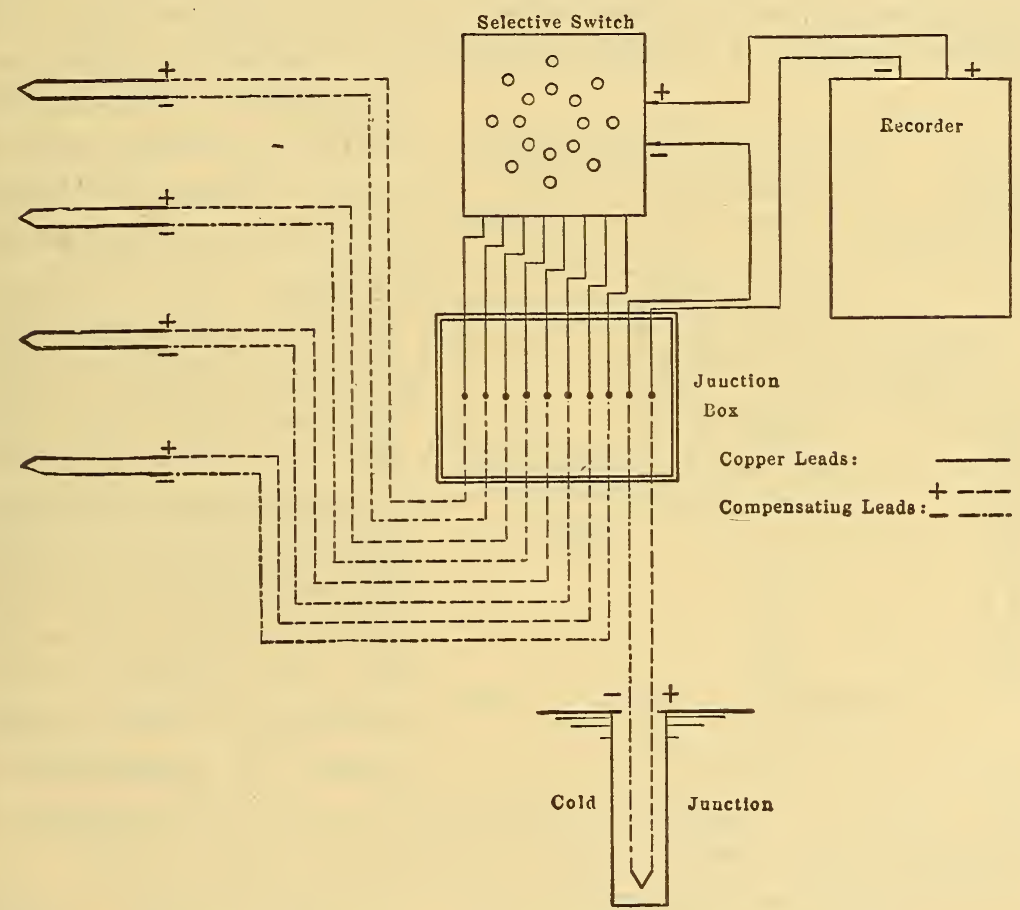

FIG. 49.-Illustrating use of junction box with controlled cold-junction temperature

Consider the same installation when a junction box is located on top of the furnace at the center: Three couples at rear of furnace 3 (75) feet, three couples at center of furnace 2 (IO), three couples at front of furnace $3(75)$, from box to cold junction ( $75+$ $20+20+\mathrm{IO}$ ). Total compensating cable, 595 feet.

By means of the junction box we effect a saving of some 500 feet in the compensating cable, and need to bury only one pair of junctions, and just as satisfactory an installation is obtained. In installing a large multiple-couple equipment with a junction box, 
it is very important to insure that the common cold-junction couple is connected with the correct polarity as illustrated. Although we have used a common cold junction for all couples, we have not employed the objectionable common return. Individual returns are used with every couple shown in the diagram.

In case the recorder is placed where the temperature is quite uniform from day to day, the use of a buried cold junction or thermostated cold-junction box is not absolutely essential. The emf generated at the junction box in Fig. 49 is then compensated for by running one pair of compensating leads from the recorder to the junction box, taking care to connect the negative lead to the negative terminal of the recorder and the positive lead to the selective switch. A simple installation of this kind is illustrated

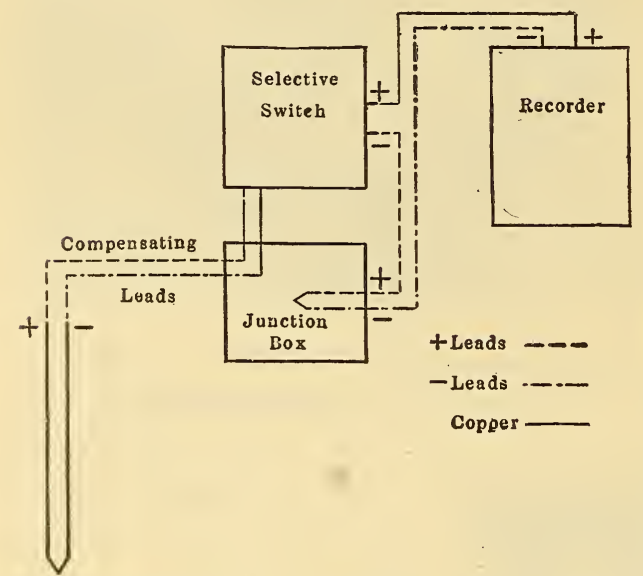

FIG. 50.-Illustrating use of junction box without cold-junction control

by Fig. 5o. Here only one couple is shown, but as many couples as desired may be connected to the multiple pole sclective switch. The compensating lead wires are soldered together inside the junction box. The auxiliary couple formed by the compensating leads is in series with the couple connected in by the selective switch. The cold junction is accordingly at the recorder where the temperature is fairly constant. Changes in temperature of the distributing or junction box thus will not affect the reading of any couple.

The principle of the junction box is somewhat similar to that of the Wilson-Maeulen zone box, except that no thermocouple in the junction-box system is connected directly to the compensating couple, all connections to the auxiliary couple being made through the selective switch. The couple $y z$ is connected directly to the zone box, Fig. $5^{\mathrm{I}}$. Two pairs of wires lead from the zone box, one pair of copper being connected to the main line and indicator. The other pair $Y Z$ are carried to the cold-junction box or are buried underground. For base-metal couples, $Y$ and $y$ are of the same material and also $Z$ and $z$. For rare-metal couples $Z$ and $Y$ are copper and an alloy of copper-nickel, respec- 
tively, the compensating leads already described for use with the Le Chatelier couple. The zone box thus saves running an extra pair of copper lead wires to the bottom of the cold-junction well. If this principle is used for a multiple installation with the object of saving compensating leads, a selective switch may be mounted between the zone box and the different couples. However, this requires that the selective switch be mounted near the furnace, unless a complicated interlacing electrical circuit is employed, whereas it is always desirable to have the switch at the indicator. It appears, therefore, that for large installations the junction box described above is preferable as far as economy in compensating lead wire is concerned. If a single-couple installation be tused, or separate cold-junction wells for different couples in multiple installations, the zone box accomplishes practically the same results as the junction box, namely, a small saving in copper and frequently a more desirable wiring system. The main advantage, however, of the junction box is the saving of compensating lead wire in a multiple-couple installation. The main advantage of the zone box is the simple method by which the cold junction is extended to some

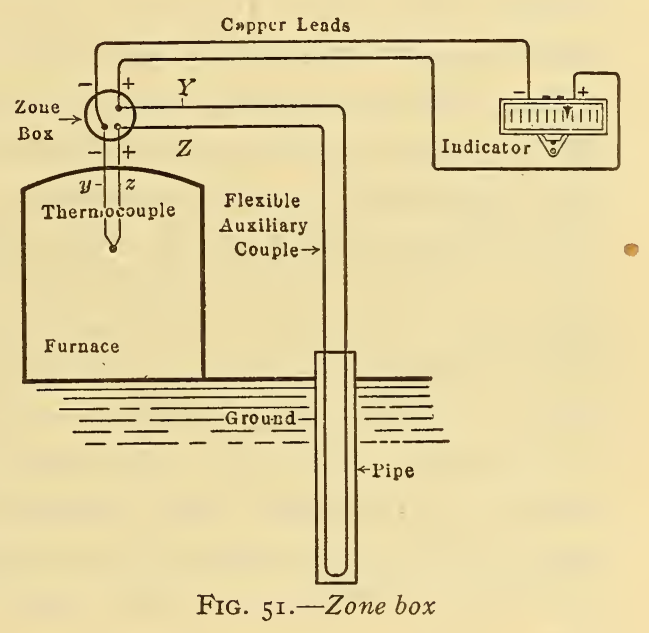
point at which the temperature can be controlled or is constant; for example, the bottom of the cold-junction well.

(6) Determination of Temperature of Buried Cold Junction.Several methods are available for obtaining the temperature at the bottom of the junction well. The simplest is to use a thermocouple consisting of the compensating leads. Insert this in the well, connect it to a portable indicator, and measure the secondary cold-junction temperature at the indicator with a thermometer. With a well ro feet or more deep and properly located it is necessary to measure the temperature possibly only once a month. Another method is to lower a thermometer into the well. The thermometer is wrapped with a few layers of cloth, leaving the stem exposed near the expected reading. It is left in the well for thirty minutes and read quickly after raising. The recorder or indicator is 
adjusted to correct for this temperature or the records may be marked "cold junction - ${ }^{\circ}$ " and corrections applied later.

All buried leads to the cold junction should be rubber insulated, and the junction well should be made water-tight. The compensating leads, particularly those for base-metal couples, will generate a large voltaic emf if they are wet and not insulated with water-proof covering.

(7) Depth of Immersion of Couples. - Thermocouples immersed in furnaces of the various industrial types must be carefully protected. Heavy iron tubes and frequently larger auxiliary protecting tubes of various materials are employed. The thermocouple calibrated in the laboratory will give the temperature of the hot junction when installed in the protecting tubes, but there is no certainty that this temperature is that of the furnace. Conduction along the protecting tubes may be so great that the temperature of the couple is far below that of the furnace. Conduction losses affecting the temperature of the hot junction may be reduced and even eliminated by using a sufficient depth of immersion, but it is not always possible to do this, and it is difficult to determine when the depth of immersion is sufficient. For example, an immersion of $5 \mathrm{~cm}$ might be satisfactory for one type of furnace or for a molten bath, and $30 \mathrm{~cm}$ insufficient for a certain muffle furnace. Each particular case requires special consideration. The general practice is to use as deep immersion as is conveniently possible and trust that this is satisfactory. Two methods may be suggested for investigating the question, but neither one is very conclusive. First, remove the couple alone from the fixed installation, leaving all protecting tubes in place. If it is impossible to remove the couple from the iron tube, use a similar tube in the fixed installation without the couple. Explore the temperature inside the protecting tube with an unprotected couple. If the temperature for several centimeters along the axis of the tube at the inner end is practically uniform, the depth of immersion is satisfactory. If, however, the temperature falls rapidly in this region, the immersion is not deep enough. Second, the couple previously standardized is mounted complete in the fixed installation and compared with another checking couple mounted along the side of the permanently installed couple. The checking couple must have a small cross section and must be used either unprotected or protected by an extremely thin tube in order to minimize the loss of heat by conduction. The hot junctions of this couple and of 
the couple under test are brought closely together, but not in contact. The checking couple should indicate a temperature more nearly that of the furnace than the temperature observed with the fixed couple. If the temperature difference between the two couples is large, a greater depth of immersion should be employed. These methods are complicated by local variations in the temperature of the furnace, but checks of this nature, although somewhat unsatisfactory, are better than no checks at all.

(8) Couples Purposely Insufficiently Immersed.-It is frequently desirable to purposely immerse the couple to an insufficient depth. In many processes the furnace is operated at such a high temperature that a thermocouple or protecting tube can not withstand the severe conditions to which it may be subjected. In this case the couple may be immersed only part way through the furnace wall, or to a distance flush with the inner wall of the furnace. The temperatures indicated by couples installed in this manner are always lower than those of the furnace interior, but they bear a fairly definite relation to the temperature of the furnace, and hence the method is satisfactory for temperature control and reproduction of furnace conditions from day to day.

(9) Protecting Tubes for Thermocouples. - The choice of a proper protection tube for a thermocouple is nearly as important as the selection of the material for the couple. Among others, the following properties of a protection tube should be considered in reference to the particular process for which the tube is required:

(a) Low Porosity to Gases.-Many tubes become very porous to furnace gases at high temperatures. Furnace gases usually attack the couple.

(b) Low Volatility.-Certain metal tubes are undesirable at high temperatures because the metal distills upon the couple, thus altering its calibration.

(c) Ability to withstand high temperature.

(d) Ability to withstand sudden changes in temperature.

(e) Ability to withstand mechanical shocks and strains.

(f) High Rigidity or Viscosity.-Protecting tubes frequently deform and exhibit the phenomenon of plastic flow at high temperatures.

(g) Thermal Conductivity.- - High thermal conductivity is frequently desirable when rapidly changing temperatures are measured. Usually, however, low thermal conductivity is desired so that the flow of heat along the tube is as small as possible. 
(h) Ability to resist corrosion from molten metals or furnace gases.

Excellent protecting tubes are obtainable for many different industrial processes. However, for certain processes satisfactory tubes have not as yet been developed. The problem is one for the ceramist or metallurgical engineer. This is particularly true in obtaining suitable tubes for molten metals, especially iron and brass. The following describes the more important protecting tubes now in use industrially:

Fused Quartz (Fig. 52, Nos. I, 2, and 3).-No. I or smaller tubes are used as insulators through which the wires of a rare-metal couple are threaded before inserting into the protecting tubes 2 or 3 . Tube 2 is of fused-quartz powder, which is afterwards

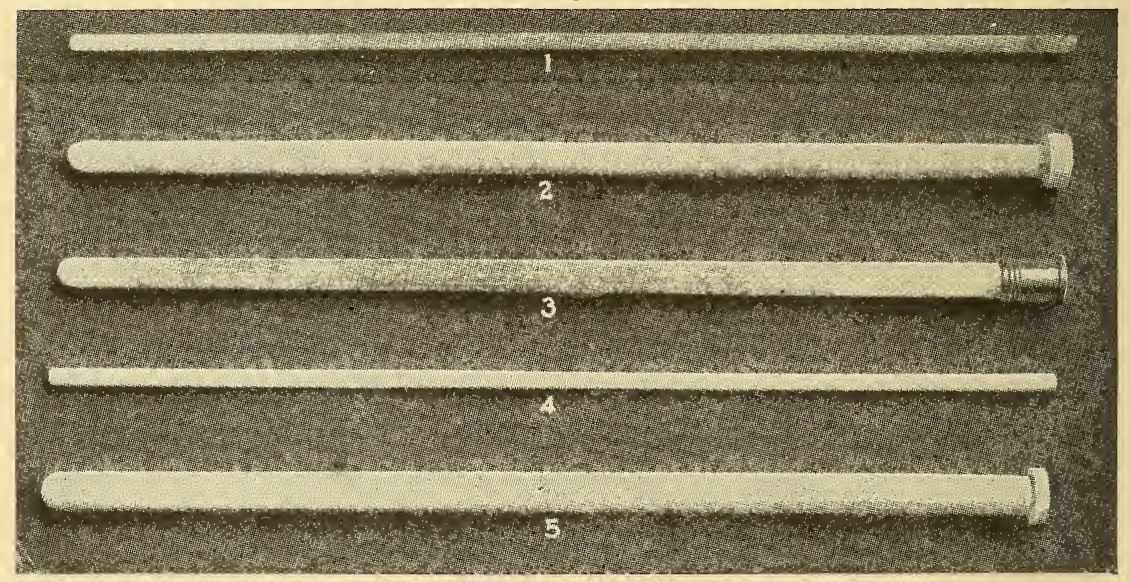

FIG. 52.-Protecting tubes for couples

fired. The preparation of such tubes is similar to that of porcelain tubes. Tubes $\mathrm{I}$ and 3 are drawn from molten quartz in much the same manner as in the manufacture of glass tubing. These tubes are translucent, like china. Transparent quartzglass tubing is manufactured, but on account of its cost is not used industrially for thermocouple protection. Fused quartz affords good protection up to $\mathrm{r}_{5} \mathrm{O}^{\circ} \mathrm{C}$ in an oxidizing atmosphere free from alkalies. The material is somewhat pervious to hydrogen and probably to other reducing gases, but is not pervious at this temperature to oxygen or carbon dioxide. Any reducing gas within the protecting tube of a rare-metal couple is disastrous, particularly when the tube contains silica. The silica is reduced to silicon which is readily absorbed by platinum. Above $1050^{\circ} \mathrm{C}$ 
and even at lower temperatures after prolonged heating, quartz devitrifies and crumbles away. Quartz tubes withstand sudden changes of temperature without breaking. Heavy molded quartz tubes are sometimes used for extra protection; for example, against acid fumes.

Porcelain (Fig. 52, Nos. 4 and 5).--Porcelain is used primarily for protection of rare-metal couples. Previous to 1914 a highly refractory porcelain known as Marquardt was imported from Germany. A better grade of this material was developed through the research work of the Bureau of Standards and is now manufactured in this country under the trade names "Usalite" and "Impervite." These two porcelains have a melting point above that of platinum. However, they are impervious to gases only when glazed. The softening point of the glaze used is about $1300^{\circ} \mathrm{C}$. If the tubes are glazed on the outside only, they are serviceable as pyrometer-protection tubes up to $\mathrm{I} 500^{\circ} \mathrm{C}$. The glaze on the German tubes softens at $1200^{\circ} \mathrm{C}$. The insulating tubes are not glazed. Pyrometer porcelain for use at lower temperatures is made by a number of American and foreign companies. The glaze on the Japanese porcelain softens at about $I I 00^{\circ} \mathrm{C}$ and its maximum serviceable temperature is about I $250^{\circ} \mathrm{C}$. An unprotected porcelain tube suddently thrust into a furnace at $1000^{\circ} \mathrm{C}$ will usually break. If it is inserted very slowly, however, there is little danger of breakage.

In permanent installation quartz and porcelain tubes and also the iron or chromel tubes of base-metal couples are frequently further protected by heavy outer tubes of fire clay, carborundum, graphite, etc. The outer tube is usually cemented in place in the furnace wall, forming a well into which the couple is inserted. In case the outer tube introduces too large a temperature lag or where there is danger of lowering the temperature of the hot junction of the couple by conduction of heat through the heavy tube, the latter is made open at both ends The couple and its smaller protecting tube are so mounted that the hot junction projects a few centimeters beyond the end of the outer protecting tube.

Carborundum (Fig. 53, No. I).-Carborundum or silicon carbide, $\mathrm{SiC}$, is used for outer protecting tubes. It has a very high thermal conductivity, low coefficient: of expansion, and a high mechanical strength. When heated in an oxidizing atmosphere, slow oxidation occurs, which does not become disadvantageously 
rapid at $1500^{\circ} \mathrm{C}$. Carborundum without clay binder is infusible and, if heated sufficiently, decomposes. Within the carborundum tube the atmosphere is made reducing by the products of slow decomposition of the tube, and porcelain is acted upon so that the life of the porcelain tube is materially reduced. This action may be minimized by permitting ready access of air into the open end of the carborundum tube. At the higher temperature ranges carborundum is unsuitable as a protection tube, particularly for glazed porcelain not vitrified. Ordinary furnace gases do not act upon carborundum. Fused alkali or alkali carbonates, basic slags, and most metals and metallic oxides

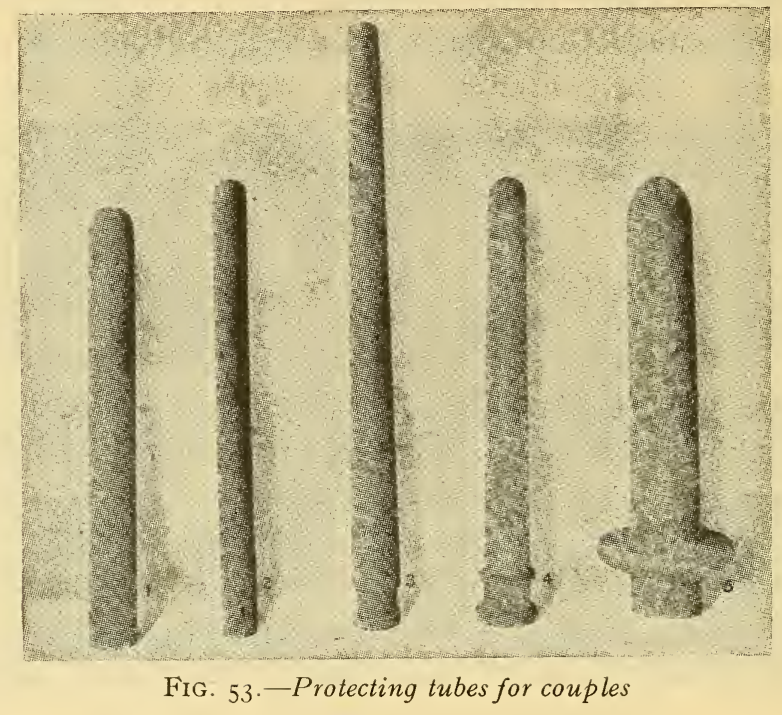

react with it at high temperatures. Carborundum tubes made with a refractory clay binder have slightly different characteristics, a lower thermal conductivity, higher coefficient of expansion, and are somewhat less refractory, although still possessing a softening point much above the temperature range suitable for couples.

Carborundum tubes have been used with some success in molten glass and open-hearth slag.

Nichrome or Chromel (Fig. 53, No. 2).-Cast nichrome or chromel tubes are used very extensively for protection of both basemetal and rare-metal couples. These tubes resist oxidation remarkably well, and, although much more costly than iron tubes, in many processes their longer life warrants and requires their use. Chromel "A" may be used continuously up to $\mathrm{I}_{200^{\circ}} \mathrm{C}$. The tubes are heavy enough to withstand considerable mechanical 
strain. It has not been possible as yet to draw these alloys into tubes. The material can be machined, but with difficulty, and threads may be cut for pipe fittings. In order to economize on the use of these materials, iron tubes are frequently employed having short tubes of nichrome or chromel welded to them. Only the part of the protecting tube exposed to the furnace need be of the expensive alloy. In processes carried out at low temperatures, where either iron tubes or chromel and nichrome tubes may be employed, experiments should be performed to determine the relative values of these tubes in life in hours per dollar of cost. There are practically no data available in this regard. In heat-treating furnaces and carbonizing furnaces chromel " $\mathrm{A}$ " is often used. Chromel "C" and nichrome last many months in lead baths. Chromel and nichrome do not volatilize as readily as iron. Basemetal couples are thus better protected by these tubes than when iron or steel is employed. Chromel "A" contains practically no iron. Chromel " $\mathrm{C}$ " and nichrome have a rather high iron content.

Graphite (Fig. 53, No. 3).-Graphite tubes afford an excellent protection to quartz or porcelain tubes on rare-metal couples and are frequently used with base-metal couples for molten metals. Porcelain incased in a sheath of graphite can be used in molten aluminum. Platinum couples must be thoroughly protected against the vapors distilled from graphite or carbon and from the reducing atmosphere present near heated graphite.

Clay (Fig. 53, No. 4).-Fire-clay outer protecting tubes are used for protection in kilns, glass and steel furnaces, annealing ovens, etc. Usually they are mounted vertically in the top of the furnace and may be cemented in place. Small fire-clay insulating tubes are used on base-metal couples.

Corundite (Fig. 53, No. 5).-Corundite consists of emery with a plastic clay binder. It is used in ceramic and glass industries for outer protecting tubes.

Alundum.-Natural corundum usually contans a large amount of iron. The artificial product, fused $\mathrm{Al}_{2} \mathrm{O}_{3}$, known under the trade name of "Alundum," is practically free from iron. This material is very desirable for protecting rare-metal couples and for use also as outer protecting tubes. The tubes are made from ground alumina mixed with a clay binder. The more refractory the tubes, the less impervious to gases they become. 'The inner protecting tubes are glazed in order to reduce the porosity, and 
the glaze is coated with an outer layer of alundum. This method of preparation permits the use of the tubes at temperatures above the softening point of the glaze. Such tubes are serviceable up to $\mathrm{I} 400^{\circ} \mathrm{C}$. Outer protecting tubes without glazing are made to withstand temperatures up to $1550^{\circ} \mathrm{C}$, and even higher. Alundum is mechanically strong and resists temperature changes much better than porcelain.

Nickel.-In an oxidizing atmosphere a thick tough coating of the oxide forms on nickel which does not readily scale. The oxide thus affords protection against further corrosion.

Pure nickel is frequently used for cyanide baths. It is very important, however, for this purpose, that the nickel be free from oxides and other impurities soluble in cyanide.

Steel and Iron.-Seamless steel and wrought-iron tubes are usually furnished with base-metal couples. They are satisfactory for many processes up to $800^{\circ}$ or $900^{\circ} \mathrm{C}$; for example, in a muffle furnace.

Calorized Iron.-Calorizing is a process by which the surface of a wrought-iron tube is impregnated with metallic aluminum. Calorized tubes resist oxidation better than the pure iron or steel tubes.

Duriron.-Duriron, a high silica iron alloy, is sometimes used at lower temperatures as a protection against acid fumes. When subjected to sudden temperature changes, the material may fracture.

Chromon.-An alloy of 75 per cent iron and 25 per cent chromium, useful for molten brass and bronze. A light protecting tube may be used for 100 to 200 determinations of less than one minute each.

\section{GENERAL THEORY OF OPTICAL AND RADIATION PYROMETRY}

The temperature of a material may be obtained from a measurement of the intensity of the radiant energy it emits. This measurement may refer to the radiation of all wave lengths emitted by the material, or, if the material is glowing, the measurement may refer to the visible light emitted or to the radiation in a very restricted portion of the visible spectrum. However, in general, the intensity of radiation depends not alone upon the temperature of the source, but also upon the particular material constituting the source. Thus glowing carbon appears to the eye about three times as bright as glowing platinum when both are at the same 
temperature. This is technically expressed by the statement that the emissive power or emissivity of carbon is about three times that of platinum. A material having the highest theoretically possible emissivity is known as a black body. In general, it is customary to assign a numerical value of $\mathrm{I}$ to the emissivity of a black body. Hence all other materials have an emissivity less than I. A black body is experimentally realized by uniformly heating a hollow inclosure and observing the radiation coming from a small opening in the wall. The intensity of radiation emitted from this opening depends only on the temperature of the walls. It does not depend upon the material of which the walls are constructed. If $E$ equals the emissivity of any nontransparent material and $R$ equals its reflection coefficient, it can be shown that $E+R=\mathrm{I}$. If a material having an emissivity of, say, 0.40 , and hence a reflection coefficient of 0.60 , is placed inside a black body it becomes indistinguishable from its surroundings. The total intensity of radiation leaving the material is the same as that emitted by the black body. Thus while the material actually emits only 40 per cent of the intensity of a black body at the same temperature, under the above conditions 60 per cent of the radiation falling upon it from the walls of the inclosure is reflected, with the net result that the object appears of the same intensity as its surroundings. However, if the material is removed from the black body and placed in the open air, the reflected intensity is no longer present and the object appears but 40 per cent as bright as a black body at the same temperature. Optical and radiation pyrometers are usually calibrated to read correctly when sighted upon a black body. Fortunately many technical processes are carried out under black-body conditions. Muffle furnaces, many annealing furnaces, etc., are sufficient approximations to "black bodies" to give practically correct temperature readings with the optical or radiation pyrometer. Some materials in the open are nearly "black;" for example, the oxide formed on iron and steel ingots, rails, etc. In general, however, corrections must be applied to the pyrometer readings to obtain the correct temperature of materials in the open. These corrections are very large in the case of clean molten metals. The presence of an oxide film on the molten metal surface greatly reduces the corrections. For temperature control it is not always necessary to correct the observed readings. As far as the factor of emissivity is concerned, the actual pyrometer readings, although known to be too low, will always be too low by the same amount from time to time for 
the same observed temperature, and hence will furnish as good information for temperature control and uniformity as could the true temperatures.

\section{OPTICAL PYROMETER TEMPERATURE SCALE}

The temperature scale for the optical pyrometer is based upon Wien's law for the distribution, in the spectrum, of the energy of a black body. This law may be stated by the following equation, where $\lambda$ denotes the wave length in microns, $c_{2}$ a constant $=14350$, $\vartheta$ the absolute temperature of the black body, $J_{\lambda}$ the intensity at the wave length $\lambda$ (that is, at a particular color such as red), and $c_{1}$ a constant, the value of which is of no moment in pyrometry since, as will be seen, it disappears from the actual working equation.

$$
J_{\lambda}=c_{1} \lambda^{-5} e^{-\frac{c^{2}}{\lambda \vartheta}} \text { for a back body. }
$$

The intensity of radiation $J^{\prime}{ }_{\lambda}$, of wave length $\lambda$, from a nonblack body of temperature $\vartheta$ and emissivity $E_{\lambda}$ is given by equation 2 .

$$
J^{\prime}{ }_{\lambda}=c_{1} E_{\lambda} \lambda^{-5} e^{-\frac{c_{2}}{\lambda \vartheta}}=c_{1} \gamma^{-5} e^{-\frac{c_{2}}{\lambda S \lambda}} \text { for a nonblack body. }
$$

In the third term of (2) we define $S_{\lambda}$ as the apparent temperature in degrees absolute of the nonblack body. This is the temperature measured by the optical pyrometer and is less than the true temperature $\vartheta$ for all materials except black bodies, when it becomes equivalent to $\vartheta$. From (2) we have:

$$
\frac{\mathrm{I}}{\vartheta}-\frac{\mathrm{I}}{S_{\lambda}}=\frac{\lambda \log E_{\lambda}}{0.4343 c_{2}}=\frac{\lambda \log E_{\lambda}}{623^{2}}
$$

Thus knowing $\lambda$ and $E_{\lambda}$, it is always possible to obtain the true temperature $\vartheta$ from the observed temperature $S_{\lambda}$.

An optical pyrometer is simply a photometer using monochromatic light (usually red) in which the intensity of radiation from either a standard or a constant source (electric lamp, oil flame, etc.) is compared with that from the object of which the temperature is desired. Frequently the two intensities are made equal by adjusting various types of absorbing devices (absorption glasses, iris diaphragms, etc.), interposed either on the furnace side or the standard lamp side of the pyrometer, depending upon which source is normally the brighter. In this process of comparison the term $c_{1} \lambda^{-5}$ of equation (I) is embodied as one of the calibration constants of the instrument. 


\section{RADIATION PYROMETER TEMPERATURE SCALE}

The temperature scale for the radiation pyrometer is based upon the Stefan-Boltzmann law expressing the relation between the total energy $J$ radiated per unit time per unit area by a black body and its absolute temperature, $\vartheta^{0}$ abs. as follows:

$$
J=\sigma\left(\vartheta^{4}-\vartheta_{0}^{4}\right),
$$

where $\vartheta_{0}$ denotes the absolute temperature of the surroundings or of the measuring instrument receiving the radiation, and $\sigma$ an empirical constant. In general $\vartheta_{0}^{4}$ is negligible in comparison with $\vartheta^{4}$, so the above relation becomes:

$$
J=\sigma \vartheta^{4} .
$$

For a nonblack body we have:

$$
J^{\prime}=\sigma E \vartheta^{4}=\sigma S^{4},
$$

where $E$ is the total emissivity and $S$ is the apparent absolute temperature of the object sighted upon as measured by the radiation pyrometer. From (6) one obtains:

$$
E=\frac{S^{4}}{\vartheta^{4}} \text { or } \log E=4(\log S-\log \vartheta) .
$$

Thus, knowing the total emissivity $E$ of any material, it is possible to obtain the true temperature $\vartheta$ from the apparent temperature $S$ as measured by a radiation pyrometer.

Summary statement of the two radiation laws: Equation (I) states that the intensity of radiation of a fixed wave length from a black body is proportional to $\frac{\frac{- \text { constant }}{\vartheta}}{\vartheta}$

Equation (5) states that the total radiation of all wave lengths emitted by a black body is proportional to $\vartheta^{4}$. These two laws, which form the basis of optical and radiation pyrometry respectively, are in agreement with the temperature scale defined by the gas thermometer up to $1550^{\circ} \mathrm{C}$, the upper limit at which a gas thermometer has been used satisfactorily. Above this range to $2500^{\circ} \mathrm{C}$ the scales defined by these two laws have been found, experimentally, to be in mutual agreement, and it is believed that they correctly represent the thermodynamic scale for all temperatures.

$8513^{\circ}-21-7$ 


\section{OPTICAL PYROMETRY}

\section{FERY OPTICAL PYROMETER}

Fig. 54 illustrates the principle of the Fery optical pyrometer. $G$ is a means for producing a divided photometric field. In the later instruments a Lummer-Brodhun or silver-strip cube is employed. Part of the field of view is illuminated by the source sighted upon and part by the gasoline lamp L, which burns at a constant brightness. By moving the wedges of black glass, $p p^{\prime}$,

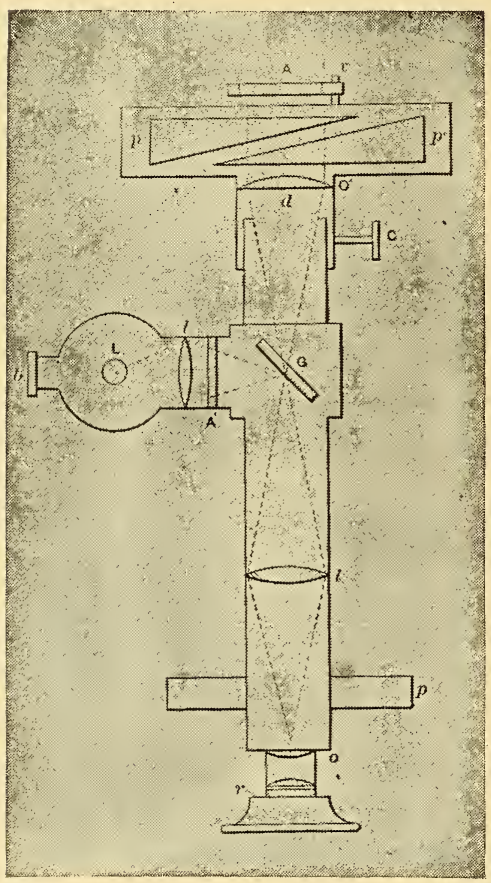

FIG. 54.-Types of optical pyrometers

In this pyrometer photographic match is obtained by the use of absorbing wedges the thickness of absorbing glass in the line of sight can be varied until the part of the field illuminated by the source has the same brightness as that illuminated by the lamp. A red glass screen is used in the ocular, so that fairly monochromatic light of this color $(0.65 \mu$ to $0.63 \mu$ ) is compared. The relation between the thickness of the wedges $x$ read on a scale and the absolute temperature $\vartheta$ is $x-P=-Q / \vartheta$ where $P$ and $Q$ are positive constants determinable by two calibration points. The instrument must be focused upon the radiating source, but no corrections for sighting distance need be applied. The Le Chatelier optical pyrometer designed earlier is similar in principle, but is not of constant aperture, and important corrections must be made with change of focus.

Brief directions for use of Fery optical pyrometer: Fill standard lamp about two-thirds full of gasoline (or amylacetate if originally calibrated for this material) and adjust flame until tip burns on a level with the top of the slit cut in the tube incasing the lamp. Focus eyepiece by drawing in or out until the field illuminated by the lamp is well defined. Focus the objective by thumbscrew $C$ until the source sighted upon is clearly outlined. Turn into the field the red glass screen in the eyecup and match the two photometric fields by adjusting the thumbscrew which moves 
the wedges. Observe the reading on the scale. This reading is converted into temperature by use of a table or plot of scale reading versus temperature furnished with the instrument. For varying and extending the range of the instrument two removable absorption glasses are used-one, $A^{\prime}$, on the standard lamp side and one, $A$, immediately in front of the adjustable wedges. In making observations care must be taken to note whether these glasses are in or out, and in converting scale readings to temperature, to observe that the correct table or plot corresponding to the particular combination employed is used.

\section{SHORE PYROSCOPE}

The Shore pyroscope, Fig. 55 , operates upon a principle very similar to that of the Fery optical pyrometer. The instrument has a scale graduated to read temperature directly, which is a material advantage. The design of optical parts is rather unnecessarily complicated, and it is difficult to match the two fields on account of color differences. The pyroscope is used extensively and with satisfactory results where high precision is not required.

(a) DIRECTIONS FOR USE OF SHORE Pyroscope.-Fill

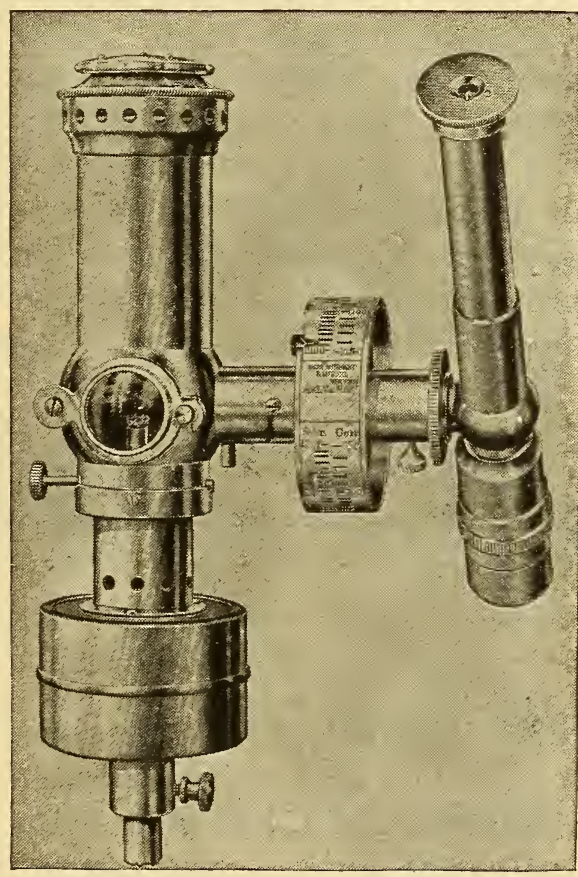

Fig. 55.-Types of optical pyrometers

This instrument employs a principle similar to that shown in Fig. 54

lamp two-thirds full of kerosene oil. Adjust the flame to burn at a height of about three-fourths of an inch. Focus on source by turning knurled ring on end of telescope tube. Turn knob by side of scale until the inner and outer fields match in brightness. Read temperature of source directly from scale setting. 


\section{WANNER PYROMETER}

Fig. 56 illustrates the arrangement of the optical parts in the Wanner pyrometer. The comparison light is a 6-volt incandescent lamp illuminating a glass matt surface in front of the slit $S_{2}$. The slit $S_{1}$, is illuminated by the source sighted upon. Light from each slit passes through the collimating lens $O_{1}$, the direct vision spectroscope $P$, a Wollaston prism $R$, a biprism $B$, the second collimating lens $O_{2}$, and is brought to a focus at $F$. The Wollaston prism produces two images of each slit which are polarized at right angles to each other. The biprism again doubles the number of images, so that there are finally four images of each slit at $F$. Six of these images are diaphragmed off by the screen $D$. The two remaining images, one of each slit, are superposed and are polarized at right angles to each other. From this point the light passes through the nicol prism $A$ and the ocular lens $E$. The direct-vision spectroscope is so adjusted that only red light of wave length about $\lambda=0.65 \mu$ reaches the eye, the other colors

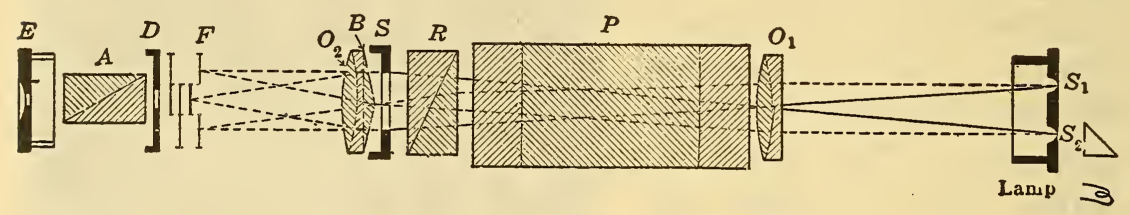

FIG. 56.-Types of optical pyrometers

In this instrument photometric match is obtained by the use of a polarizing system

being diaphragmed off by the screen $D$. The ocular is focused on the dividing edge of the biprism $B$. The eye perceives a circular photometric field, half of which is illuminated by the slit $S_{1}$ and half by the slit $S_{2}$. The light from the two fields is plane polarized and the plane of polarization in one field is at right angles to the plane of polarization in the other field. Consequently, on rotating the nicol prism $A$, one field increases and the other field decreases in intensity. A setting is obtained when the two fields match.

In order to determine the proper brightness at which to operate the electric lamp illuminating the slit $S_{2}$, the pyrometer is sighted on a source of standard brightness. This consists of an amylacetate lamp with a flame gage having a window of ground glass. The flame is adjusted to a specified height. The ground glass window illuminates the slit $S_{1}$. The analyzer nicol $A$ is set at specified normal point or angle marked on the instrument. The current through the electric lamp is then varied by means of a 
rheostat until the two fields are matched, and the current is read from the ammeter. This process should be repeated several times and a mean value of the current settings obtained. In using this instrument the current is adjusted to this mean value. The electric lamp burns at a high temperature and consequently deteriorates noticeably. Hence the above adjustment on the normal point requires frequent redetermination. For high precision the adjustment should be made both before and after a series of temperature readings. In the industrial plant once a day or once a week is sufficient, depending upon the amount of use.

The calibration of the instrument follows the law

$$
\log \tan \varphi=a-b / \vartheta,
$$

where $\varphi$ is the angular reading of the analyzer, $\vartheta$ the absolute temperature, and $a$ and $b$ positive empirical constants. The relation between $\log \tan \varphi$ and $\mathrm{r} / \vartheta$ is linear. Two calibration points serve to determine $a$ and $b$ and a table or plot may be made of $\varphi$ versus $t^{\circ} \mathrm{C}$. Usually such a table is furnished with the pyrometer, or the instrument may be sent to the Bureau of Standards for calibration.

The instrument described above is satisfactory for temperatures greater than $900^{\circ} \mathrm{C}$. In the temperature range 700 to $900^{\circ} \mathrm{C}$ the intensity of light from the furnace sighted upon is insufficient to permit accurate settings. Hence for temperatures from $700^{\circ}$ up the direct vision spectroscope $P$ is replaced by a red glass screen, or the objective lens $O_{1}$ is made of red glass, and the slits $S_{1}$ and $S_{2}$ are of much wider opening.

On account of stray light the Wanner pyrometer is not accurate at very small or very large angular readings. Moreover, the temperature increases so fast at large angles that the angles would have to be observed with extreme precision in order to obtain any accuracy when expressed in degrees of temperature. . The range of the instrument is thus confined to from about to to 80 angular degrees.

Table I $_{4}$ illustrates the relation between angular reading and temperature for a particular instrument. Different instruments may have entirely different or practically identical calibrations, as desired, depending upon the adjustment of the normal point. 
TABLE 14. - Relation Between Angular Readings and Temperature for a Particular Instrument

\begin{tabular}{|c|c|c|c|c|c|}
\hline \multirow[b]{2}{*}{ Angle } & \multicolumn{2}{|c|}{ Temperature } & \multirow[b]{2}{*}{ Angle } & \multicolumn{2}{|c|}{ Temperature } \\
\hline & $\begin{array}{l}\text { Without } \\
\text { absorption } \\
\text { glass }\end{array}$ & $\begin{array}{l}\text { With } \\
\text { absorption } \\
\text { glass }\end{array}$ & & $\begin{array}{l}\text { Without } \\
\text { absorption } \\
\text { glass }\end{array}$ & $\begin{array}{l}\text { With } \\
\text { absorption } \\
\text { glass }\end{array}$ \\
\hline $10 \ldots$ & ${ }^{\circ}{ }^{\circ}{ }_{900}$ & ${ }^{\circ}{ }_{1390}$ & $50 \ldots$ & ${ }^{\circ}{ }^{\circ} \mathrm{C}$ & ${ }^{\circ}{ }_{2020}$ \\
\hline $20 \ldots$. & 990 & 1575 & $60 .$. & 1255 & 2200 \\
\hline $30 \ldots$ & 1060 & 1725 & $70 .$. & 1360 & 2460 \\
\hline $40 .$. & 1120 & 1865 & $80 .$. & 1535 & 3030 \\
\hline
\end{tabular}

Thus the above instrument is satisfactory for temperatures up to $1500^{\circ}$ C. For higher temperatures an absorption glass is mounted in front of the slit $S_{1}$, which decreases the light from the furnace in a known ratio. The third column shows the temperatures which may be measured when an absorption glass of suitable density is employed. If still higher temperatures are desired a denser absorption glass must be used.

With the Wanner pyrometer the tip of the flame of the amyl acetate lamp should burn level with the top of the flame gage. The setting on the normal point is tedious work, as the flame flickers over the field. A screen of black paper placed around the lamp helps to reduce the flicker. The observations must be made in a closed room free from drafts. Any error in the setting of the normal current is carried over to the final temperature measurements, so that it is exceedingly important to exercise all possible care in these preliminary adjustments. Examine the screen of the flame gage to assure that no smoke has deposited upon it. A slight film of smoke from the lamp may cause an error of $100^{\circ}$ or more. The amyl acetate used in the lamp need not be of high purity. Any good grade of amyl acetate is satisfactory.

From experience with several hundred instruments in use in the technical industries it is evident that these pyrometers are subjected to great abuse. The instrument is made up of delicate optical parts and should not be allowed to become heated. Many of the parts are set in wax and the various optical surfaces are cemented by Canada balsam. The Wollaston prism and the nicol prism in the rotating eyepiece are made of calcite. This material is very soft, and, although it has the appearance of glass, may be scratched with the finger nail. In practically half the instruments examined these parts have been deeply cut by knives 
or pointed steel tools. All persons using this pyrometer should be cautioned not to touch any optical part except the lens in the eyecup, which requires occasional cleaning. Do not change the setting of any screw. The moving of a single screw may throw out the adjustrinent of the pyrometer and cause errors of $500^{\circ}$. If the position of any screw on the body of the instrument is altered, do not attempt to readjust the instrument, but return it to the maker. The replacement of the electric lamp will not alter the calibration of the pyrometer.

\section{SCIMATCO PYROMETER}

Fig. 57 illustrates the Scimatco pyrometer, an improved form of the Wanner pyrometer. All but one of the screws, the tampering with which affects the calibration of the instrument, are inclosed in a metal sheath, and hence are not readily accessible. The instrument has both an angular scale and a scale graduated directly in degrees of temperature. The box at the left contains a 6-volt storage battery, an ammeter, and an adjustable rheostat. For obtaining the proper setting of the current the pyrometer is clamped in its carrying case. The amyl acetate lamp and flame gage are so mounted that the ground glass of the gage is directly in contact with the glass

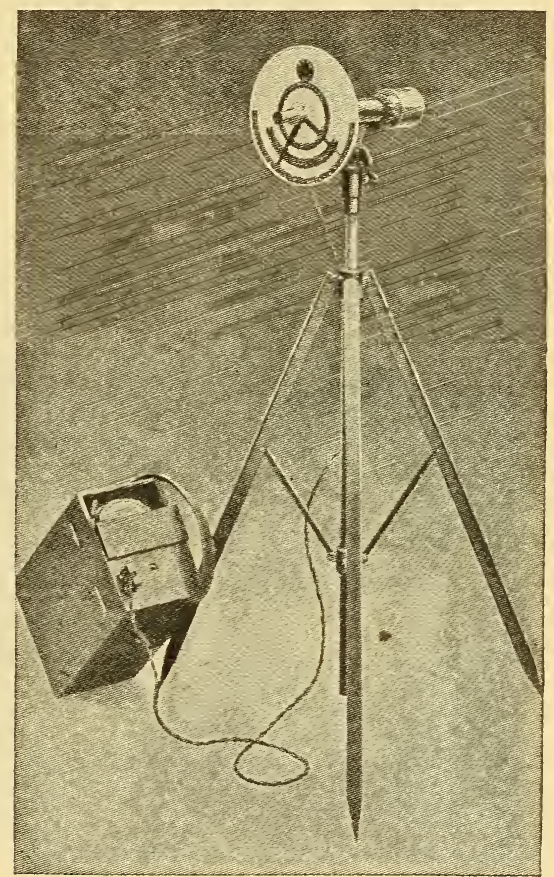

FIG. 57.-Types of optical pyrometers

This instrument employs a principle similar to that shown in Fig. 56

window of the pyrometer, opening to the slit $S_{1}$ of Fig. 56. The tip of the flame is adjusted until it is just visible on looking through the bottom of the red glass window in the dial of the instrument. With the Wanner or Scimatco pyrometer the observer can not see through the instrument the object sighted upon. This may cause inconvenience if it is desired to measure the temperature of a small crucible in a furnace. 


\section{F AND F PYROMETER}

Fig. 58 illustrates the arrangement of optical parts in the $F$ and $\mathrm{F}$ pyrometerr. Light from the furnace is focused at the center of the silver strip cube $C$. This cube produces a circular field divided through the middle. One half of the field receives light from the furnace and the other half from the ground-glass

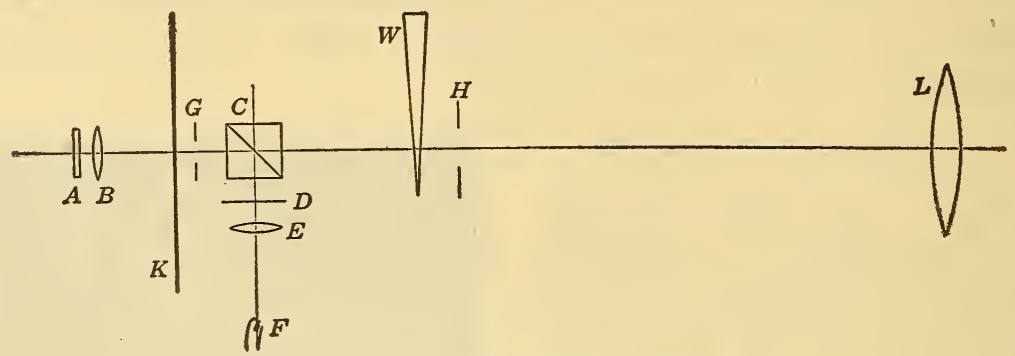

Fig. 58.-Types of optical pyrometers

In this instrument photographic match is obtained by the use of an absorbing wedge

screen $D$, which is illuminated by the electric lamp $F$ through the condenser lens $E$. The ocular containing the red glass screen $A$ and lens $B$ is focused on the dividing edge of this photometric field. $G$ and $H$ are diaphragms which limit the cone of rays employed. The two fields are matched by turning a thumbscrew,

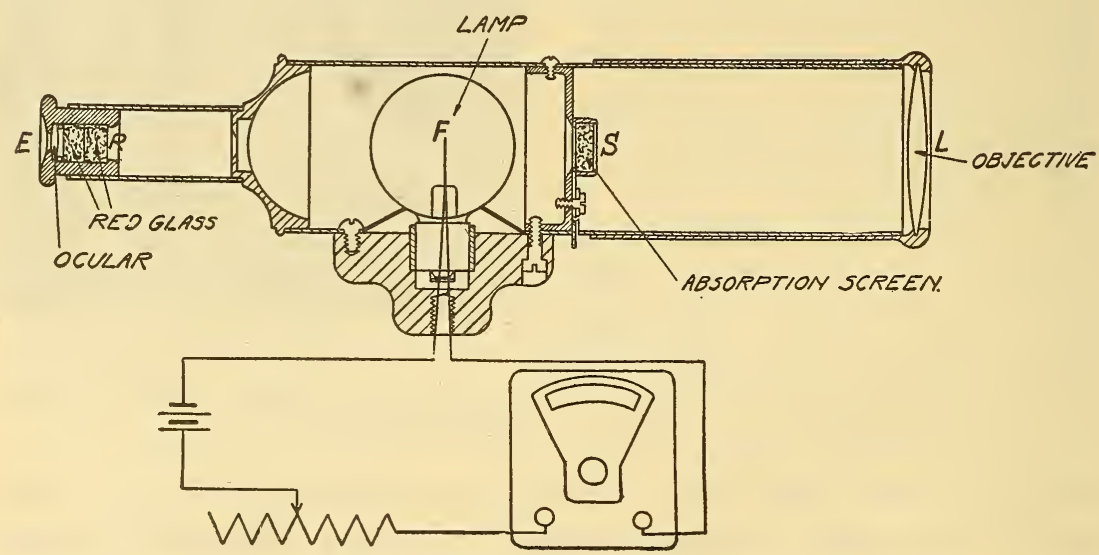

FIG. 59.-Types of optical pyrometers

Optical system and electric circuits of disappearing filament type of optical pyrometer.

which moves the black glass wedge $W$ across the path of the light from the furnace. By a system of gears this movement is transferred to a circular scale on the dial $K$ of the instrument. In appearance the pyrometer resembles the Scimatco, and is used in the same manner. For a normal-point setting the pointer is adjusted 
to read the normal angle and after removing the tube carrying the lens $L$ the instrument is clamped in its case. The flame gage of the amyl acetate lamp is so mounted that its ground-glass window is adjacent to the diaphragm $H$. A table is furnished with the instrument giving the relation between the scale reading in angular degrees and degrees of temperature, or a temperature scale is engraved upon the dial. This instrument is so designed that the object sighted upon is clearly imaged, a distinct advantage over the Wanner pyrometer. The relation between the scale reading $\alpha$ and the absolute temperature $\vartheta$ is $\alpha-P=-Q_{\prime}^{\prime} \vartheta$ where $P$ and $Q$ are positive constants determinable by two calibration points.

A more recent improvement of this pyrometer consists in the use of a semicircular instead of linear wedge. A semicircular disk is so ground that the thickness decreases linearly along the straight

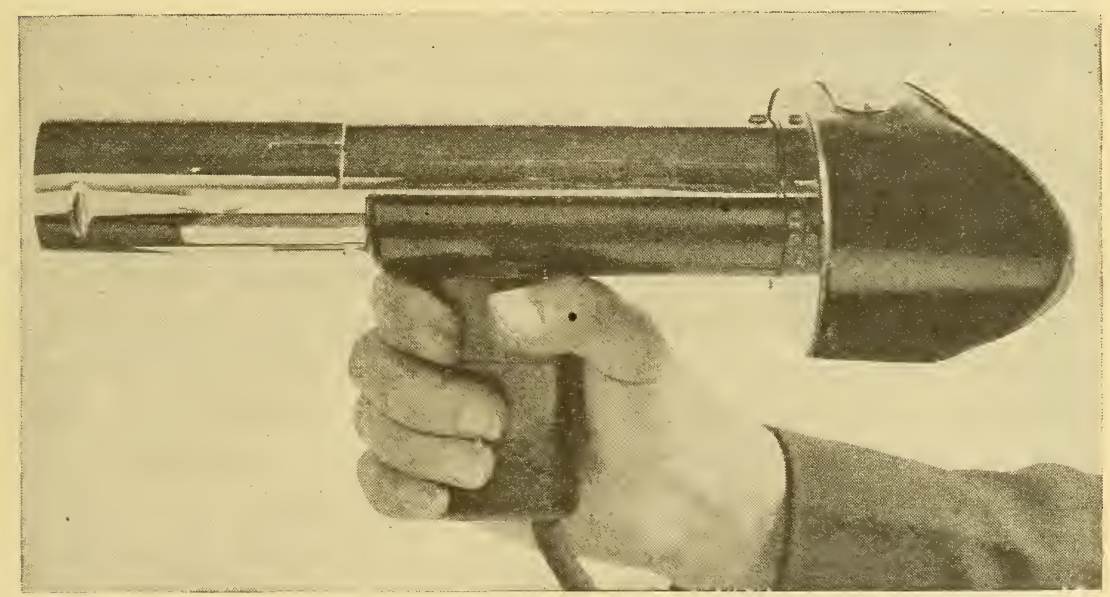

FIG. 60.-Types of optical pyrometers

Disappearing filament type

edge. A compensating wedge of clear glass is cemented to the absorbing glass to correct for refraction. The comparison lamp is mounted in the line of sight, replacing the cube $C$, Fig. 58, and the brightness of the filament, burning at a constant specified current, is matched against the source sighted upon by rotating the semicircular wedge. The relation between angular scale reading and temperature has the form: $\operatorname{Cos} \alpha-P=\mathrm{I} / \vartheta$.

\section{DISAPPEARING FILAMENT OPTICAL PYROMETER}

The disappearing filament optical pyrometer was first devised by E. F. Morse (U. S. Letters Patent Nos. 696878; 6969I6). Figs. 59 and 60 illustrate the Leeds \& Northrup form of this instrument. 
The filament of a small electric lamp F, Fig. 59, is placed at the focal point of an objective $L$ and ocular forming an ordinary telescope, which superposes upon the lamp the image of the source viewed. Red glass, such as Corning "high-transmission red," is mounted at the ocular to produce approximately monochromatic light. In making a setting the current through the lamp is adjusted by means of a rheostat until the tip or some definite part of the filament is of the same brightness as the source viewed. The outline or detail of this section of the filament is then indistinguishable from the surrounding field. This is illustrated by Fig. 61. In the third setting the central portion of the filament vanishes against the background. The current is read on an ammeter and the corresponding temperature is obtained from a plot or table of current versus temperature. The empirical relation between the current $i$ through the lamp and the temperature $t^{\circ} \mathrm{C}$ is of the form:

$$
i=a+b t+c t^{2}
$$

where $a, b, c$, are constants requiring for their determination at least three standardization points. ${ }^{14}$

The lamps should not be operated at temperatures higher than I $500^{\circ} \mathrm{C}$ on account of deterioration of the tungsten filament. If this temperature is not exceeded, the calibration of the lamp is good for hundreds of hours of ordinary use. For higher temperatures absorption glasses, S, Fig. 59, are used between the lamp and the objective, or in front of the objective, to diminish the observed intensity of the source. The relation between the temperature of the source $\vartheta^{\circ}$ abs. and the observed temperature $\vartheta_{0}^{\circ}$ abs., measured with the absorption glass interposed, is as follows:

$$
\frac{\mathrm{I}}{\vartheta}-\frac{\mathrm{I}}{\vartheta_{0}}=A
$$

where $A$ is for most practical purposes a constant.

Usually the instrument is furnished with a table showing the relation between the current through the lamp and the temperature both with and without the absorption glass. If, however, this relation is not given when the absorption glass is used, it may be readily determined by measuring the constant $A$ in the above formula. To do this, sight without the absorption glass on a muffle furnace or any uniformly heated furnace at $\mathrm{I} 200^{\circ}$ to $\mathrm{I} 500^{\circ} \mathrm{C}$ 
and observe the temperature $\vartheta$ in degrees absolute. Then, with the absorption glass in place, match the filament again and observe to what temperature $\vartheta_{0}$ in degrees absolute the current through the lamp corresponds. The difference in the reciprocals of these two temperatures is the constant $A$, which is usually of the order of magnitude -0.00015 . This determination should be repeated several times and at several different temperatures of the furnace. The separate values of $A$ should not differ by more than I per cent, and the mean value is used for computing the relation between the observed absolute temperature with the absorption glass and the true temperature of the source. In making these computations care must be exercised that all temperatures are converted to degrees absolute. Table I 5 illustrates the calibration of a certain pyrometer, both with and without the absorption glass. The constant of this particular absorption glass has the value $A=-0.000280$. By use of this glass temperatures as high as $2730^{\circ} \mathrm{C}$ can be measured, although the temperature of the lamp does not exceed $1360^{\circ} \mathrm{C}$.

It will be noted that the range of currents required is small, from, in general, about 0.3 to $0.6 \mathrm{am}$ pere. Thus, if the ammeter is designed to give full scale deflection on 0.6 ampere, nearly half

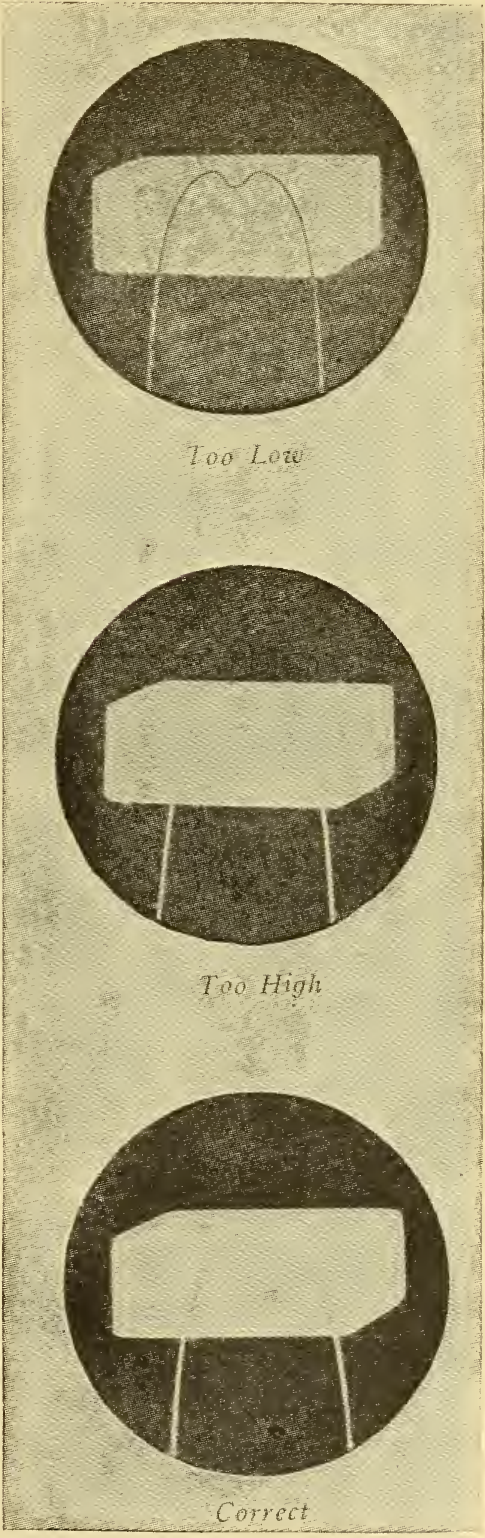

FIG. 6r.-Appearance of field of view when adjusting the current through the lamp of the disappearing filament pyrometer

of the scale, from o to 0.26 ampere, is never used. The depressed zero ammeter now available meets this objection. The 
moving-coil system, including the supports, pivots, and pointer, may be adjusted relative to the magnet by turning a lever on the case of the instrument to one of two positions. In one position the pointer is adjusted on open circuit, so that it falls over the first graduation on the scale. This adjustment is similar to the ordinary zero adjustment on any ammeter. In the second and working position the zero is depressed off the scale an amount equivalent to 0.26 ampere. The lowest scale reading is, accordingly, 0.26 , and the entire scale from 0.26 to 0.60 ampere is thus utilized for the range of the pyrometer lamp.

TABLE 15.-Typical Calibration of Lamp

\begin{tabular}{|c|c|c|c|c|c|}
\hline \multirow[b]{2}{*}{$\begin{array}{l}\text { Current, in } \\
\text { amperes }\end{array}$} & \multicolumn{2}{|c|}{ Temperature } & \multirow[b]{2}{*}{$\begin{array}{l}\text { Current, in } \\
\text { amperes }\end{array}$} & \multicolumn{2}{|c|}{ Temperature } \\
\hline & $\begin{array}{l}\text { Without } \\
\text { absorption } \\
\text { glass }\end{array}$ & $\begin{array}{l}\text { With absorp- } \\
\text { tion glass }\end{array}$ & & $\begin{array}{l}\text { Without } \\
\text { absorption } \\
\text { glass }\end{array}$ & $\begin{array}{l}\text { With absorp- } \\
\text { tion glass }\end{array}$ \\
\hline & ${ }^{\circ} \mathrm{C}$ & ${ }^{\circ} \mathrm{C}$. & & ${ }^{\circ} \mathrm{C}$ & ${ }^{\circ} \mathrm{C}$ \\
\hline $0.26 \ldots$ & 634 & 943 & $0.36 \ldots$ & 1060 & 1854 \\
\hline $.28 \ldots$ & 765 & 1190 & $.38 \ldots$ & 1113 & 1992 \\
\hline $.30 \ldots \ldots \ldots \ldots$ & 860 & 1386 & $.42 \ldots \ldots \ldots \ldots$ & 1201 & 2237 \\
\hline $.32 \ldots \ldots \ldots$ & 936 & 1555 & $.46 \ldots \ldots \ldots \ldots$ & 1281 & 2478 \\
\hline $.34 \ldots \ldots \ldots$ & 1002 & 1710 & $.50 \ldots \ldots \ldots$ & 1359 & 2733 \\
\hline
\end{tabular}

Fig. 62 shows the depressed zero ammeter. The batteries and the rheostat for controlling the current through the lamp are in the same case with the ammeter. This may be carried by the observer by means of the shoulder strap illustrated.

\section{GENERAL USE OF OPTICAL PYROMETERS}

Optical pyrometers and radiation pyrometers, described later, afford the only means yet developed for measuring temperature above $1500^{\circ} \mathrm{C}$. The high-temperature scale above $1500^{\circ} \mathrm{C}$ is based on the extrapolation of Wien's radiation law by means of a pyrometer of the disappearing-filament type. When the instrument is especially designed for precision work, it is possible to measure a temperature difference of $0.2^{\circ} \mathrm{C}$ at $\mathrm{I} 500^{\circ} \mathrm{C}$. The commercial form of the instrument when properly calibrated can be relied upon to $5^{\circ} \mathrm{C}$. With a well-designed optical pyrometer there is a perfect color match of the two fields at all times. Hence, contrary to the general impression, color is not matched at all, but simply brightness of uniform color. A color-blind observer will obtain the same settings as a normal observer. Forsythe ${ }^{15}$ 
has compiled data obtained with an optical pyrometer of the disappearing-filament type by six observers, none of whom had ever used an optical pyrometer before. The average variation from the mean for the six inexperienced observers was $3^{\circ} \mathrm{C}$ and the maximum variation, $5^{\circ} \mathrm{C}$.

Although the optical pyrometer is essential for the measurement of temperatures above $1500^{\circ} \mathrm{C}$, its usefulness is by no means confined to the high-temperature range. The thermocouple can not be adapted to many processes at low temperatures; for example,

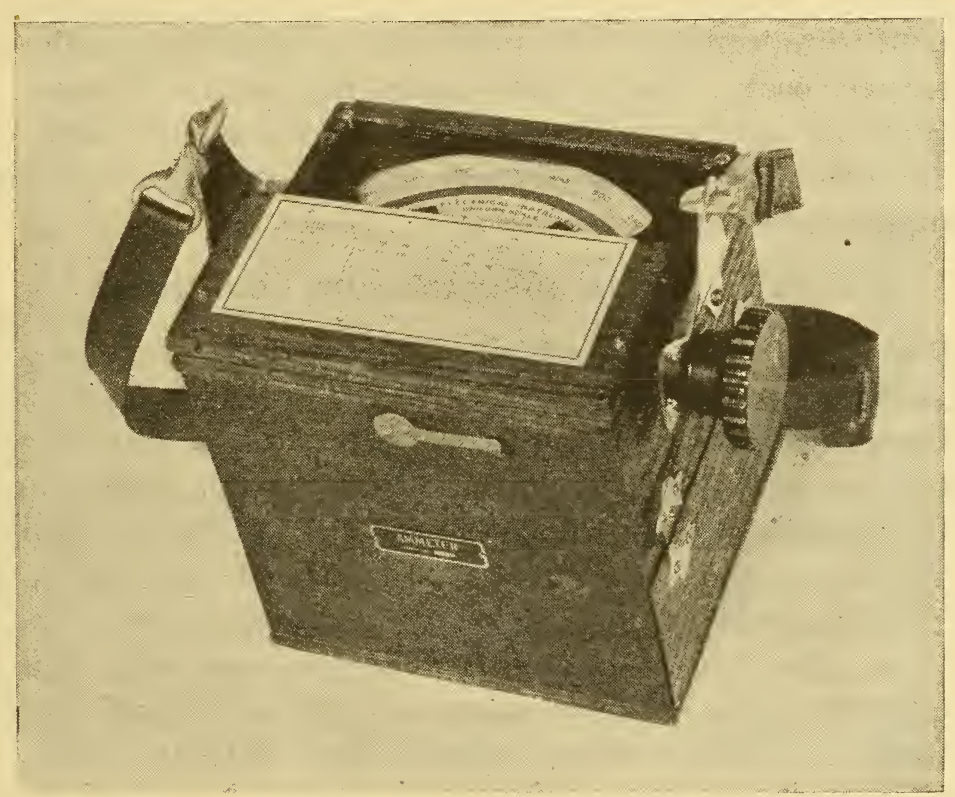

FIG. 62.-Depressed zero ammeter used with optical pyrometer shown in Fig. 60

the measurement of the temperature of steel rails as they pass through the rolls, ingots and forgings in the open, and small sources such as a heated wire or lamp filament. The temperatures used in the above processes may be accurately measured by the optical pyrometer. The temperature of a microscopic sample of any material can be measured by a modified form of the disappearing-filament pyrometer ${ }^{16}$. Also in many processes a thermocouple is not so convenient to use as an optical pyrometer, especially when the temperature is not required often enough to warrant a permanent installation of thermocouples.

${ }^{16}$ Burgess, B. S., Sci. Papers, No. 198. (See Fig. I4I of this paper.) 
One serious objection to the optical pyrometer from the industrial point of view is the fact that it has not been made automatically recording. Since a photometric match is required for every setting, the instrument necessitates the attention of an observer, although possibly a satisfactory automatic device will be developed eventually. Another objection is the introduction of the human element into the readings, thus affording an opportunity for dishonest or prejudiced settings. The observer, if he is the operator of the furnace, should be taught that the instrument is for his own assistance and that it is not to be considered as a policial measure; otherwise the measurements should be made by a disinterested party. In a plant operating several furnaces an intelligent boy can be profitably employed whose sole work is to make the rounds of the various furnaces and measure and record the temperatures.

\section{BLACK-BODY AND NONBLACK-BODY CONDITIONS}

Optical pyrometers are usually calibrated to "read correctly when sighted on a black body. Many furnaces approximate black-body conditions very satisfactorily. In a perfect black body the details of the inside of the furnace vanish, and a piece of steel, for example, which is being heated can not be distinguished from the background. If the objects in the furnace can be distinguished but only on close observation, and if much of the detail is lost, after they have been in the furnace some time, it is not likely that the temperature measurement will be seriously in error. If in error at all, the observed temperature will be too high when the furnace walls are brighter than the material being heat treated and too low when the walls are less bright. This latter condition is possible if the heat supply is variable or if it is shut off and the furnace allowed to cool.

That a steel ingot placed in a heated furnace may appear much hotter than it really is is a fact not always appreciated. The surface of the ingot appears hot because it reflects the bright light from the walls of the furnace. Of course in comparison to the much greater brightness of the walls, the cold ingot appears black, but this is due to the intense contrast. If the ingot is viewed alone when the direct radiation from the furnace is screened from the eye, it also is bright. Thus when an optical pyrometer is sighted on an ingot in the furnace, part of the light reaching the instrument comes from the side walls and is reflected by the surface of the ingot. Iron oxide reflects about the least amount of 
any material met with in metallurgical practice. Its emissivity is approximately 0.95; hence its reflection coefficient is 0.05 . Suppose an iron ingot at room temperature were suddenly placed in a furnace at $1200^{\circ} \mathrm{C}$. Although the surface of the ingot is cold, it reflects 5 per cent of the light falling upon it from the hot side walls. This 5 per cent of reflected radiation gives the ingot the appearance of an object at $950^{\circ} \mathrm{C}$. The measurement by the optical pyrometer accordingly would be $950^{\circ} \mathrm{C}$, although actually the ingot is at room temperature. The higher the reflecting power of the material the greater the observed temperature under the above conditions. Thus cold platinum would appear to be at about $1160^{\circ}$ or at almost the same temperature as that of the furnace. One method for reducing the error due to reflected radiation is to view the object through a large open door on a surface parallel to the opening. If the door is large enough, the surface of the ingot thus loses a great portion of the furnace wall contributing the reflected light. The ingot reflects the image of the opening left by the door, and this opening is not radiating. It must be pointed out that the reflection from any material being heat treated is diffuse, like that from a matt surface, so that an opening in a furnace wall is not truly imaged, as would be the case with a polished-mirror surface. Stray reflection is, however, greatly minimized even with matt surfaces by sighting through a large door on a surface parallel to the opening. When the material has attained the temperature of the side walls, it is, of course, not desirable to open a large door, since then the opening affects the black-body conditions. The pyrometer should be sighted through a small peephole as soon as approximate temperature uniformity is obtained. A more satisfactory method for reducing the stray reflections than by opening a large door is to sight into a deep, wedge-shaped cavity or hole made in the metal being heat treated. If this cavity is deep enough, very little radiation from the side walls can be reflected from it. If such a hole can not be made conveniently, a length of iron pipe closed at one end or a porcelain tube may be placed in good thermal contact ${ }^{*}$ with the material and so aligned that the pyrometer may be sighted through a peephole directly into the bottom of the tube.

The effect of reflected light is very noticeable in an empty coke oven. The reflection coefficient of the brick walls is comparatively high. If a perfect mirror were placed parallel to a glowing surface, the mirror would appear as hot as the surface. This 
effect of reflection takes place in a coke oven so that both walls appear of approximately equal brightness, even though they may differ considerably in temperature. Frequently a patch of the wall on one side becomes coated with a layer of coke. Since the coke has a higher emissive power than brick, this patch appears much hotter. Actually it is at about the same temperature as the less-bright surrounding wall. On account of reflection a corresponding bright patch appears on the opposite wall, although this wall may be free from coke. It is evident that the measurement of temperature of a portion of a nonuniformly heated furnace by means of an optical pyrometer is difficult unless the precautions suggested above are taken. As soon as the furnace attains temperature uniformity and equilibrium the optical pyrometer gives the true temperature very easily and readily.

When an optical pyrometer is sighted on a glowing material in the open, it reads too low. Certain materials, important industrially, have a very high emissivity, so that the corrections necessary to add to the observed temperatures to convert them into true temperatures are small. Thus with iron oxide the correction is only $10^{\circ}$ at $1200^{\circ} \mathrm{C}$. The corrections are very large for clear, molten metals, but are smaller for the oxides which soon form on the molten surface when exposed to the air. Table 16 shows the true temperatures corresponding to the temperatures observed when sighting on certain materials in the open. For temperature control it is unnecessary to apply these corrections. The observed temperatures, although known to be low, will be low by the same amount from time to time, and hence will serve just as satisfactorily for reproducing temperature conditions in any process as the corrected temperatures. The above statement must be modified if factors other than emissivity of the material require consideration. For example, reproducible results can not be expected if heavy clouds of smoke are in the line of sight one day and not on the next day. If the pyrometer is sighted on a stream of molten iron during pouring or tapping, the surface of the metal is usually clear and free from oxide. If the stream should at any time contain much slag, the surface will show bright patches on account of the higher emissivity of the slag. To make the readings conform with those taken on the clear stream, one must sight on the darker spaces between the slag or sight upon the slag and correct both sets of data according to Table 16 . 
TABLE 16.-True Temperature Versus Apparent Temperature Measured by Optical Pyrometers Using Red Light $(\lambda=0.65 \mu)$ When Sighted Upon the Following Materials in the Open

\begin{tabular}{|c|c|c|c|c|c|c|c|}
\hline \multirow{2}{*}{$\begin{array}{c}\text { Observed } \\
\text { temperature, } \\
\text { degrees } \\
\text { centigrade }\end{array}$} & \multicolumn{7}{|c|}{ True temperature, degrees centigrade } \\
\hline & $\begin{array}{l}\text { Molten } \\
\text { copper }\end{array}$ & $\begin{array}{l}\text { Molten } \\
\text { iron } a\end{array}$ & $\begin{array}{l}\text { Solid iron } \\
\text { oxide }\end{array}$ & $\begin{array}{c}\text { Solid nickel } \\
\text { oxide }\end{array}$ & $\begin{array}{l}\text { Nichrome } \\
\text { or chromel }\end{array}$ & $\begin{array}{l}\text { Molten } \\
\text { slag } b\end{array}$ & $\begin{array}{l}\text { Bright } \\
\text { platinum }\end{array}$ \\
\hline $700 \ldots \ldots$ & .... & ..... & 700 & 701 & 702 & & 750 \\
\hline $800 \ldots \ldots \ldots$ & $\ldots$. & $\ldots \ldots$ & 801 & 802 & 804 & .. & 861 \\
\hline $900 \ldots \ldots \ldots$ & ....... & ….. & 902 & 904 & 906 & ..... & 973 \\
\hline $950 \ldots \ldots . .$. & 1088 & ..... & 953 & 955 & 958 & $\ldots .$. & 1030 \\
\hline $1000 \ldots \ldots \ldots$ & 1150 & ...... & 1004 & 1007 & 1010 & $\ldots .$. & 1087 \\
\hline $1050 \ldots \ldots \ldots$ & 1213 & ....... & 1055 & 1058 & 1063 & ...... & 1144 \\
\hline $1100 \ldots \ldots \ldots$ & 1277 & 1183 & 1106 & 1110 & 1116 & ...... & 1202 \\
\hline $1150 \ldots \ldots \ldots$ & 1341 & 1239 & 1158 & 1162 & 1170 & ........... & 1260 \\
\hline $1200 \ldots \ldots .$. & 1405 & 1296 & 1210 & 1215 & 1224 & - $\cdots$ & 1320 \\
\hline $1250 \ldots \ldots \ldots$ & 1470 & 1353 & . & 1267 & $\ldots$. & ...... & 1375 \\
\hline $1300 \ldots \ldots$. & 1536 & 1410 & 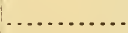 & 1320 & 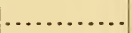 & ...... & 1435 \\
\hline $1400 \ldots$. & ..... & 1525 & & & . & 1455 & 1555 \\
\hline $1500 \ldots$. & & 1641 & & & & 1565 & 1675 \\
\hline $1600 \ldots \ldots \ldots \ldots$ & & 1758 & & & & 1670 & ..... \\
\hline $1700 \ldots \ldots \ldots$. & & 1876 & & & & 1780 & \\
\hline $1750 \ldots \ldots \ldots$. & (n.......... & 1935 & ............ & ....... & $\cdots$ & 1830 & ........... \\
\hline
\end{tabular}

$a$ Computed for $E_{\lambda}=0.40$, this being the best value for ordinary steel practice.

$b$ Computed for $E_{\lambda}=0.65$, an average value for liquid slags.

The above table was computed from the following equation where $\vartheta$ is the true absolute temperature, $S$ the observed absolute temperature, and $E_{\lambda}$ the emissivity for the wave length $\lambda$. This wave length has been selected as $\lambda=0.65 \mu$, the approximate value for optical pyrometers.

$$
\frac{\mathrm{I}}{\vartheta}-\frac{\mathrm{I}}{S}=\frac{\lambda \log E_{\lambda}}{623^{2}}=\frac{\log E_{\lambda}}{95^{88}}
$$

Table 17 gives the emissivity of various materials for this wave length. The change of emissivity with temperature is usually small for metals.

$$
8513^{\circ}-21-8
$$


TABLE 17. -Monochromatic Emissivity for Red Light ( $\lambda=$ about $0.65 \mu)$

\begin{tabular}{|c|c|c|c|c|c|}
\hline & Material & $E(0.65 \mu)$ & Material & \multicolumn{2}{|c|}{$E(0.65 \mu)$} \\
\hline \multicolumn{2}{|l|}{ Silver. . } & 0.07 & Cuprous oxide. . & & 0.70 \\
\hline \multirow{2}{*}{ Gold. . } & solid... & .13 & $800^{\circ} \mathrm{C} \ldots$. & & .98 \\
\hline & liquid. . & .22 & Iron oxide $\ldots .\left\{1000^{\circ} \mathrm{C} \ldots\right.$ & & .95 \\
\hline \multirow{2}{*}{ Platinum... } & \{solid... & .33 & $1200^{\circ} \mathrm{C} \ldots$ & & .92 \\
\hline & \{liquid. . & .38 & $\int 800^{\circ} \mathrm{C} \ldots$ & & .96 \\
\hline \multirow{3}{*}{ Palladium. } & ssolid... & .33 & Nickel oxide... $\left\{1300^{\circ} \mathrm{C} \ldots \ldots \ldots\right.$. & & .85 \\
\hline & $\{$ liquid. . & .37 & Iron, solid and liquid.......... & & .37 \\
\hline & \{solid... & .11 & Nickel, solid and liquid....... & & .36 \\
\hline \multirow[t]{2}{*}{ Copper. } & liquid.. & .15 & Iridium............. & & .30 \\
\hline & $\int 1100^{\circ} \mathrm{C}$. & .60 & Rhodium.............. & & .30 \\
\hline \multirow[t]{2}{*}{ Tantalum... } & $2600^{\circ} \mathrm{C}$. & .48 & Graphite powder (estimated)........ & & .95 \\
\hline & $\left(1000^{\circ} \mathrm{C}\right.$. & .46 & Carbon............................. & & .85 \\
\hline \multirow[t]{3}{*}{ Tungsten. . } & $\left\{2000^{\circ} \mathrm{C}\right.$ & .43 & Porcelain ( ? ? )..................... & .25 to & .50 \\
\hline & $3000^{\circ} \mathrm{C}$. & .41 & & & \\
\hline & $600^{\circ} \mathrm{C}$. & .95 & & & \\
\hline \multirow[t]{2}{*}{ Nichrome.. } & $900^{\circ} \mathrm{C}$ & .90 & & & \\
\hline & $1200^{\circ} \mathrm{C}$. & .80 & & & \\
\hline
\end{tabular}

Table i 8 shows the corrections which must be added to the readings obtained with an optical pyrometer using light of wave length $\lambda=0.65 \mu$, for various emissivities, in order to obtain the true temperatures. These data are especially useful when carefully plotted on a large scale, similar to Fig. 63 .

TABLE 18.-Correction to Observed Temperature for Pyrometer Using Red Light $\left(\lambda=0.65 \mu, c_{2}=14350\right)$

\begin{tabular}{|c|c|c|c|c|c|c|c|c|c|c|c|}
\hline \multirow{2}{*}{ Emissivity } & \multicolumn{11}{|c|}{ Add corrections below for the following observed temperatures, degrees centigrade } \\
\hline & 700 & 800 & 900 & 1000 & 1100 & 1200 & 1300 & 1400 & 1600 & 1800 & 2000 \\
\hline 0.30 . & 55 & 67 & 80 & 95 & 111 & 129 & 148 & 168 & 213 & 264 & 322 \\
\hline $.40 \ldots$ & 41 & 50 & 60 & 71 & 83 & 96 & 110 & 125 & 158 & 195 & 237 \\
\hline $.50 \ldots$ & 31 & 37 & 45 & 53 & 62 & 71 & 82 & 93 & 117 & 144 & 175 \\
\hline $.60 \ldots$ & 22 & 27 & 33 & 39 & 45 & 52 & 59 & 67 & 85 & 104 & 126 \\
\hline $.70 \ldots$ & 16 & 19 & 23 & 27 & 31 & 36 & 41 & 47 & 59 & 72 & 87 \\
\hline $.80 \ldots$ & 10 & 12 & 14 & 17 & 19 & 22 & 25 & 29 & 36 & 44 & 54 \\
\hline $.90 \ldots \ldots \ldots$ & 5 & 6 & 7 & 8 & 9 & 10 & 12 & 14 & 17 & 21 & 25 \\
\hline $1.00 \ldots \ldots$. & 0 & 0 & 0 & 0 & 0 & 0 & 0 & 0 & 0 & 0 & 0 \\
\hline
\end{tabular}

\section{TEMPERATURE OF GLOWING GAUZE}

An interesting application of the optical pyrometer is for the measurement of the temperature of gauze electrically or otherwise heated. In certain chemical processes platinum gauze electrically heated is used as a catalyzing agent, and must be maintained at a constant temperature. This is readily done by sighting normally on the surface of the gauze with an optical pyro- 
meter. The observed temperatures may be thus exactly reproduced from day to day. If it is required to convert the observed temperatures into exact true temperatures of the wire forming the gauze the problem is difficult. An approximate solution satisfactory for all industrial work is, however, easily obtained.

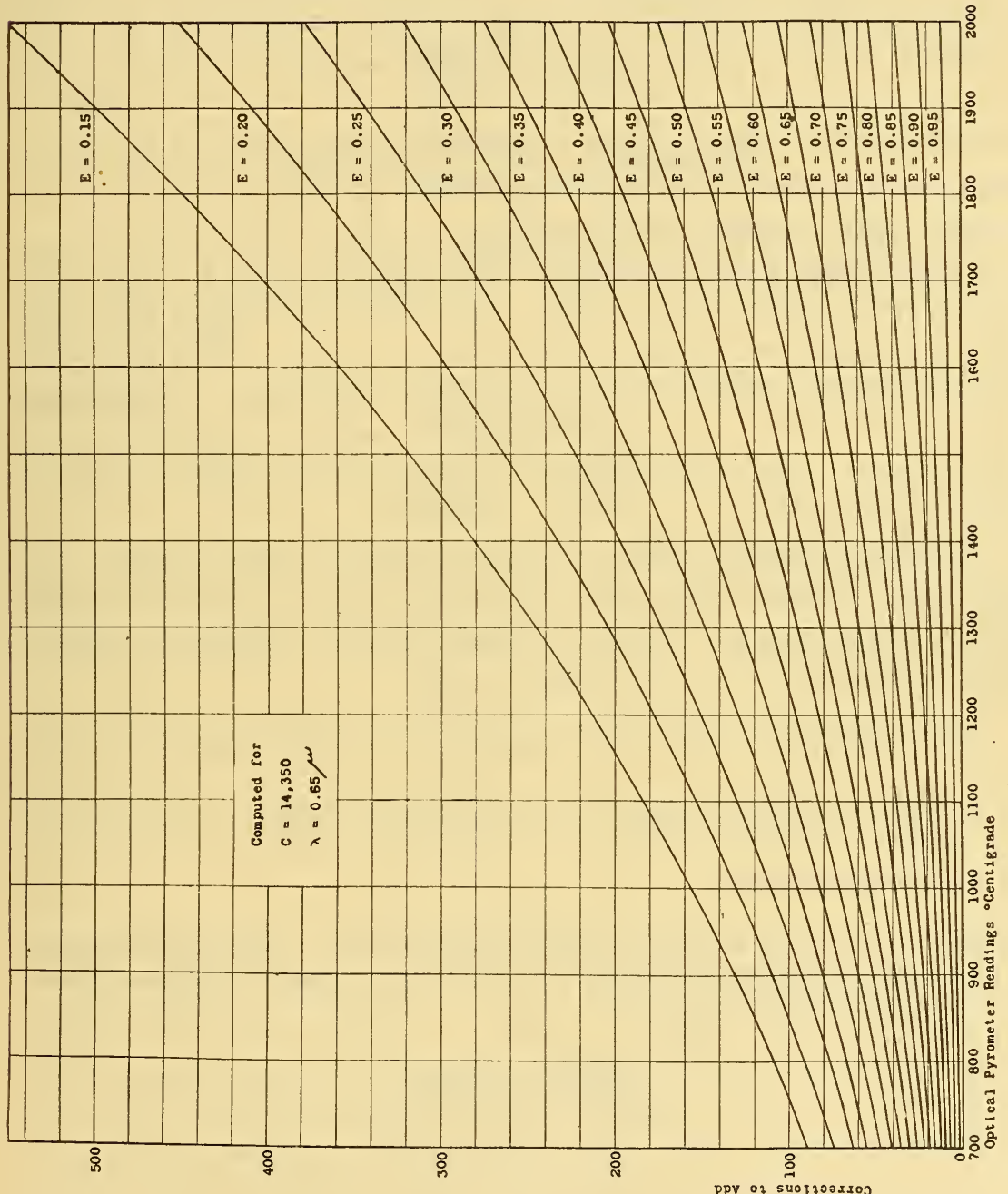

We will assume that the mesh of the gauze is sufficiently coarse that multiple reflection between the separate wires is negligible. Let $A_{1}$ equal the fractional part of the total area of the gauze comprised by the wire, and $A_{2}$ equal the fractional part of the total area representing the space between the wires. Let $E_{\lambda}$ equal the emissivity of the metal employed, and $E_{\lambda}^{\prime}$ equal the effective 
emissivity of the gauze as a whole; that is, taking into consideration the spaces between the wires which, of course, are not radiating surfaces. The following equations are readily apparent:

$$
\begin{gathered}
E_{\lambda}^{\prime}=\frac{A_{1}}{A_{1}+A_{2}} E_{\lambda}=A_{1} E_{\lambda} \text {, since } A_{1}+A_{2}=\mathrm{I} . \\
\frac{\mathrm{I}}{\vartheta}-\frac{\mathrm{I}}{S}=\frac{\log A_{1} E_{\lambda}}{9.588}
\end{gathered}
$$

where $\vartheta$ is the true absolute temperature of the wire of the gauze and $S$ is the absolute temperature observed with an optical pyrometer sighted normal to the surface.

A platinum gauze commonly employed is No. 80 mesh $(80$ wires to the inch) of 0.003 -inch wire. For this gauze $\mathrm{A}_{1}=0.42$. The emissivity of bright clean platinum is 0.33 . The platinun of this gauze soon becomes somewhat corroded. Possibly an emissivity of, say, 0.4 is more nearly the correct value under these conditions. Hence the effective emissivity of the gauze $=A_{1} E_{\lambda}=$ $(0.42)(0.4)=0.17$.

Usually the gauze must be viewed through a glass window. A thin glass window (see below) transmits about 90 per cent of the light falling upon it. Hence the final effective emissivity using a glass window $=E_{\lambda}^{\prime}=(0.17)(0.90)=0.15$.

The following table was computed by the formula $\frac{\mathrm{I}}{\vartheta}-\frac{\mathrm{I}}{S}=$ $\frac{\log \text { O.I5. }}{9588}$ A similar table for other gauzes may be computed in the manner outlined.

TABLE 19.-Platinum Gauze No. 80 Mesh, 0.003-inch Wire. Temperatures Observed Through One Window by Optical Pyrometer Sighted Normal to Surface of Gauze, versus True Temperature of Gauze.

\begin{tabular}{|c|c|}
\hline $\begin{array}{c}\text { Observed } \\
\text { temperature }\end{array}$ & $\begin{array}{c}\text { True } \\
\text { temperature }\end{array}$ \\
\hline${ }^{\circ} \mathrm{C}$ & ${ }^{\circ} \mathrm{C}$ \\
600 & 675 \\
650 & 730 \\
700 & 790 \\
750 & 850 \\
800 & 910 \\
850 & 975 \\
900 & 1035 \\
950 & 1095 \\
1000 & 1160 \\
1050 & 1220 \\
& \\
\hline
\end{tabular}




\section{USE OF A WINDOW}

It is frequently necessary, especially in the laboratory, to sight an optical pyrometer into a furnace through a window. What correction must be applied to the observed temperatures to take account of the loss of light at the window? Kanolt has measured the transmission coefficient for a number of ordinary glass windows at $\lambda=0.65 \mu$ and obtained a mean value of 0.904 . Hence we have

$$
\frac{\mathrm{I}}{\vartheta}-\frac{\mathrm{I}}{\mathrm{S}}=\frac{\log 0.904}{9588}=-0.0000046
$$

where $\theta$ is the true absolute temperature of the source and $S$ is the observed absolute temperature. Table 20 is computed from the above formula.

TABLE 20.-Correction to Observed Temperatures for Absorption of Light by a Single Clean Window

\begin{tabular}{|c|c|}
\hline $\begin{array}{c}\text { Observed } \\
\text { temperature }\end{array}$ & $\begin{array}{c}\text { Correction } \\
\text { to add }\end{array}$ \\
\cline { 1 - 2 }${ }^{\circ} \mathrm{C}$ & ${ }^{\circ} \mathrm{C}$ \\
600 & 3.5 \\
800 & 5.4 \\
1000 & 8 \\
1200 & 10 \\
1400 & 13 \\
1600 & 16 \\
1800 & 20 \\
$200 \mathrm{C}$ & 24 \\
2500 & 36 \\
3000 & 50 \\
\hline
\end{tabular}

\section{FLAMES AND SMOKE}

The optical pyrometer can not be used satisfactorily when sighted through flames or smoke. Usually the presence of dense flames increases the temperature reading and the presence of smoke clouds absorbs so much radiation that the pyrometer may read several hundred degrees low. The optical pyrometer can be used to measure the temperature of the slag in an openhearth furnace, but the flames prove a serious hindrance except during reversals, when observations may be taken to advantage. In a cement kiln the dust, smoke, and flames all combine to make the observations rery untrustworthy. Carbon dioxide, water vapor, and other invisible gases produce no effect. 


\section{MÉTHOD OF SIGHTING INTO A CLOSED TUBE}

In many processes where smoke can not be eliminated or where black-body conditions are not satisfactory, a porcelain or other refractory tube with a closed end is inserted into the furnace. The pyrometer is sighted into this tube, which, if fairly uniformly heated over a sufficient area, affords an excellent black body. This method has been employed also for obtaining the true temperature of molten metals, but suitable refractory tubes for many molten metals have yet to be developed.

\section{RADIATION PYROMETRY}

An optical pyrometer measures the intensity of a narrow spectral band of radiation emitted by a glowing object. The radiation pyrometer measures the intensity of all wave lengths, the light rays and the heat rays combined. Usually the energy of all wave lengths radiated by the source is focused in some manner upon the hot junction of a small thermocouple. The temperature to which this junction rises is approximately proportional to the rate at which energy falls upon it, which in turn, by the StefanBoltzmann law, is proportional to the fourth power of the absolute temperature of the source. The rise in temperature of the hot junction of the couple generates a thermoelectric emf. Hence the calibration of a radiation pyrometer consists in determining the relation between the emf developed and the temperature of the source sighted upon. This relation follows the law $e=a \vartheta^{\mathrm{b}}$ where $\vartheta$ is the absolute temperature of the source, $e$ is the emf developed by the instrument, and $a$ and $b$ are empirical constants determinable by two standardization points. The emf may be measured by a potentiometer or galvanometer or by any of the methods discussed under thermoelectric pyrometry. The galvanometer should have as high a resistance as is consistent with the requirement of robustness. The same type of instrument is used with the radiation pyrometer as with the ordinary thermocouple. In fact, the entire discussion on galvanometric methods of measuring emf of thermocouples is equally applicable to radiation pyrometry. The temperature of the cold junction of the couple in the radiation pyrometer is not controlled. The hot and cold junctions are in fairly close proximity and hence are equally affected by changes in room temperature. The cold junction is always shaded from the heat radiated by the source sighted upon. 


\section{THWING RADIATION PYROMETER}

Fig. 64 illustrates the principle of the Thwing radiation pyrometer. Radiation from the furnace enters the diaphragm $A$ and falls upon the hollow conical mirror $K$. The hot junction $C$ of

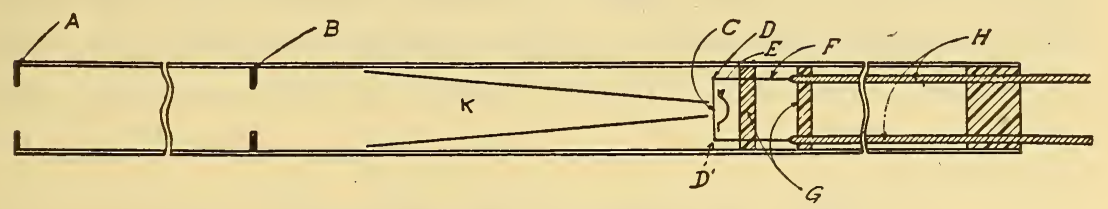

FIG. 64.-Types of radiation pyrometers

Receiving system of instrument employing a conical mirror

a minute thermocouple is located at the apex of the cone, and the cold junctions are at $D$ and $D^{\prime}$. By multiple reflection along the sides of the conical mirror the radiation is finally concentrated upon the hot junction of the couple. The emf is measured by a

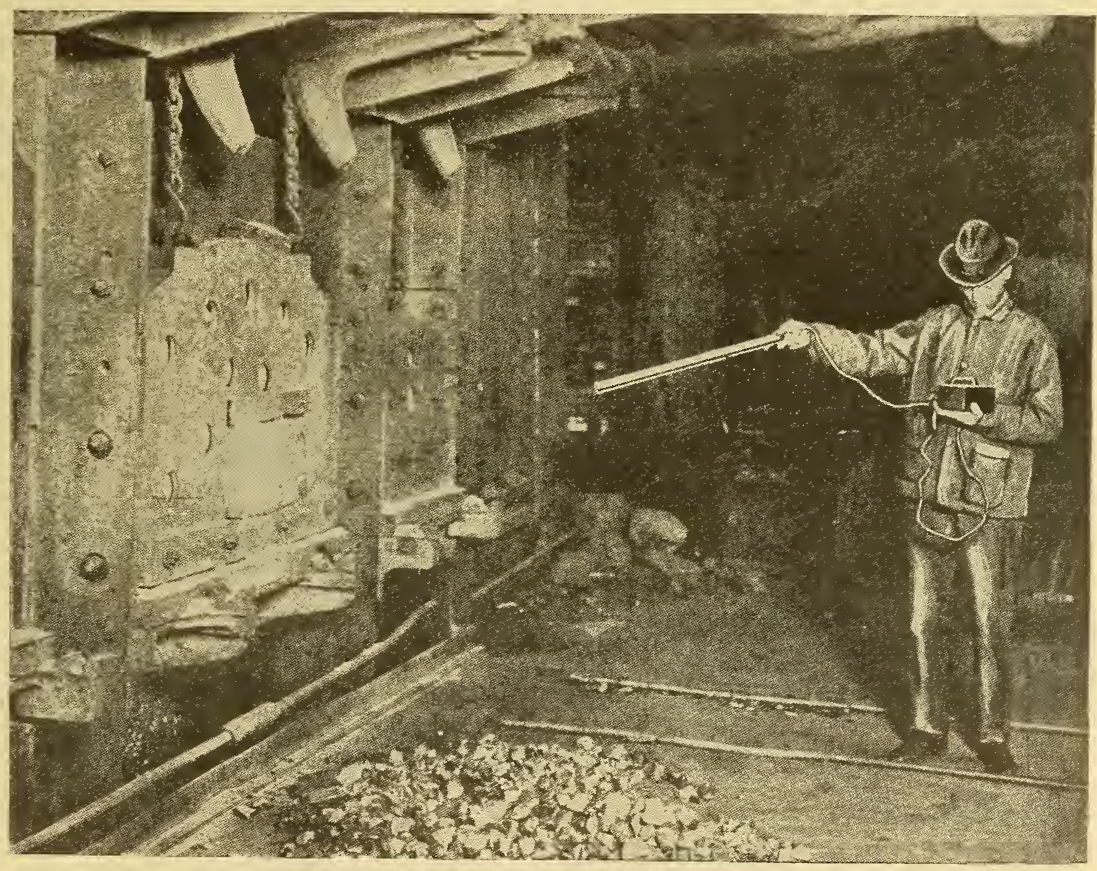

FIG. 65.-Observing the temperature of a furnace with the pyrometer illustrated in Fig. 64

galvanometer graduated to read temperature directly. Fig. 65 shows the method of using this instrument for measuring the temperature of a large furnace. Except for incidental errors, which will be briefly considered later, the reading of the instru- 
ment is independent of the sighting distance, provided the diameter of the source is sufficient to fill the cone of rays defined by the geometrical construction of the receiving tube. Fig. 66 illustrates this fact. The amount of radiation falling upon an element, $D$, of the conical mirror is proportional to the solid angle $H D A^{\prime}$ which is independent of the distance from the point $D$ to the source. This is true of every point on the base of the cone

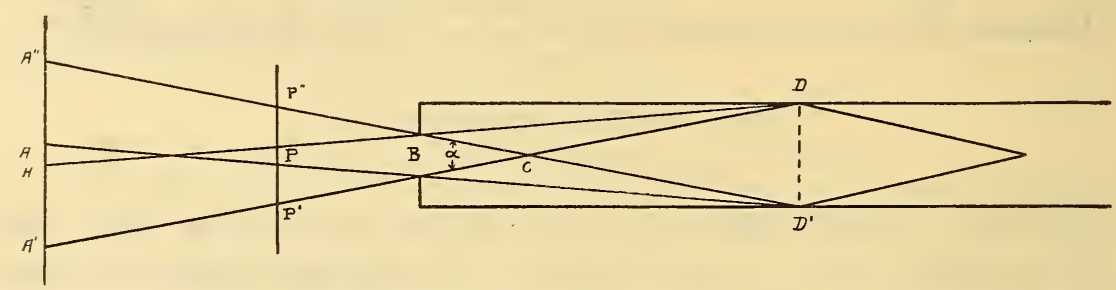

FIG. 66.-Cone type of fixed-focus radiation pyrometer

$D D^{\prime}$. Hence the total quantity of energy entering the cone is independent of the distance from the pyrometer to the source, provided the source is of sufficient size. The minimum size of source for any distance is determined by the lines $A^{\prime \prime} D^{\prime}$ and $A^{\prime} D$. Thus for the distance $B A$ the diameter of the source must be at least $A^{\prime} A^{\prime \prime}$ and for the distance $B P$ the diameter of the

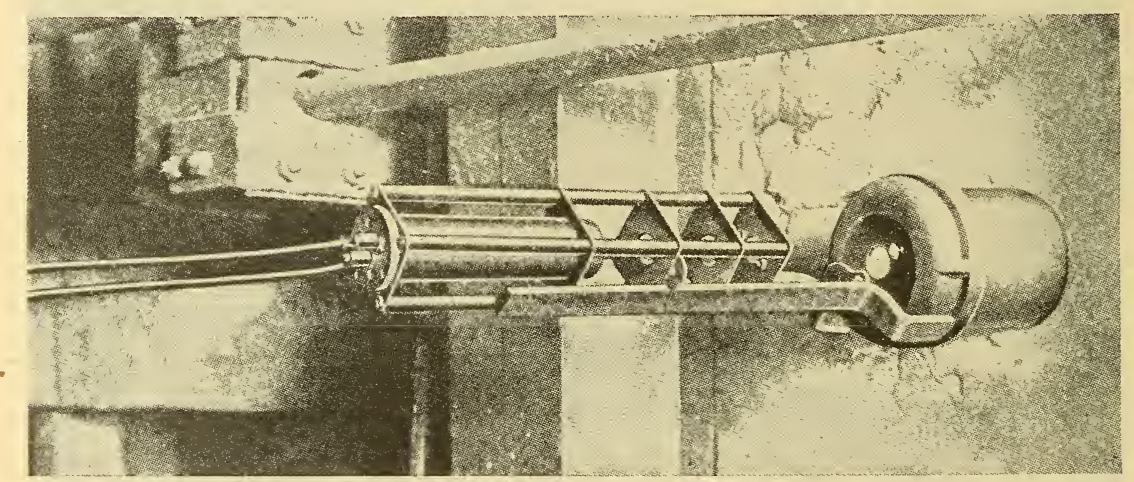

FIG. 67.-Radiation pyrometer for fixed installation on furnace wall

source must be at least $P^{\prime} P^{\prime \prime}$. The Thwing instrument is so constructed that the source must have a diameter at least oneeighth of the distance from the source to the receiving tube. Thus at 8 feet from a furnace the opening into the furnace must be $\mathrm{I}$ foot in diameter.

For permanent installations the tuibe is ventilated and has several extra diaphragms to prevent local heating of the instrument and reradiation to the couple. Fig. 67 shows a perma- 
nently installed Thwing radiation pyrometer sighted into the bottom of a closed metal tube which projects into the furnace.

\section{FOSTER RADIATION PYROMETER}

Fig. 68 illustrates the principle of the Foster radiation pyrometer. The thermocouple, $b$, and a front diaphragm, $B$, are

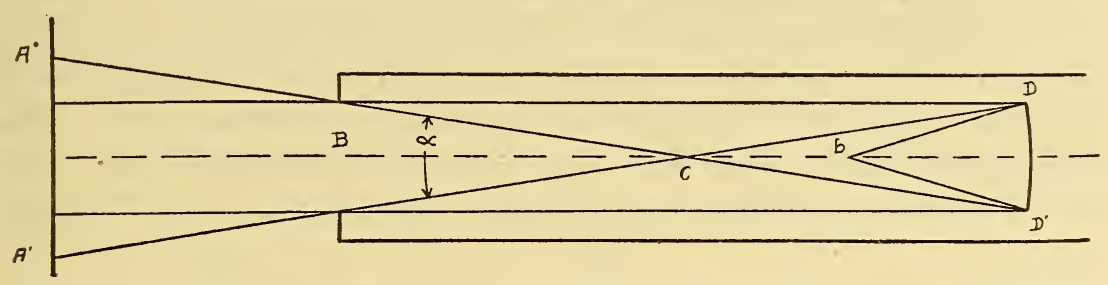

FIG. 68.-Mirror type of fixed focus radiation pyrometer

located at the conjugate foci of a concave mirror, $D D^{\prime}$. As in the case of the Thwing pyrometer, the source must be large enough to fill the cone of rays defined by the angle $\alpha$ or the lines $A^{\prime} C A^{\prime \prime}$. The position of the point $C$ is marked by a wing nut on the tele-

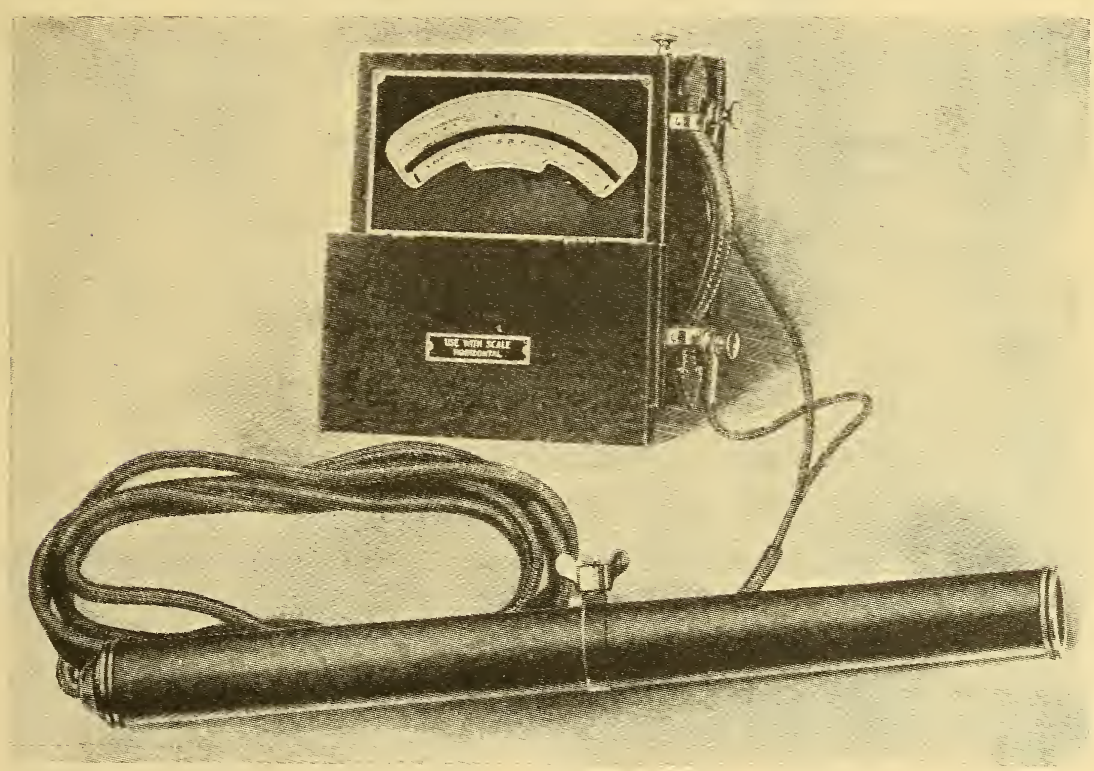

FIG. 69.-Types of radiation pyrometers

The receiving system of this pyrometer is shown in Fig. 68

scope tube. The angle $\alpha$ is made such that the diameter of the source sighted upon must be at least one-tenth the distance from the source to the wing nut. Thus for a distance of ro feet the diameter of the opening into the furnace must be at least I foot. Fig. 69 illustrates the complete instrument with an indicator 
graduated to read temperature directly. The Brown radiation pyrometer, which is similar in principle to the Foster pyrometer, is made with the receiving tube collapsible for convenience in carrying.

\section{FERY RADIATION PYROMETER}

Fig. 70 is a cross-section drawing of the Fery pyrometer made by the Taylor Instrument Cos. Radiation from the source sighted upon is concentrated by the concave mirror of speculum metal or gold, upon the hot junction of a minute thermocouple. This instrument is not fixed focus, as is the case with the other types of radiation pyrometer, but requires focusing for each sighting

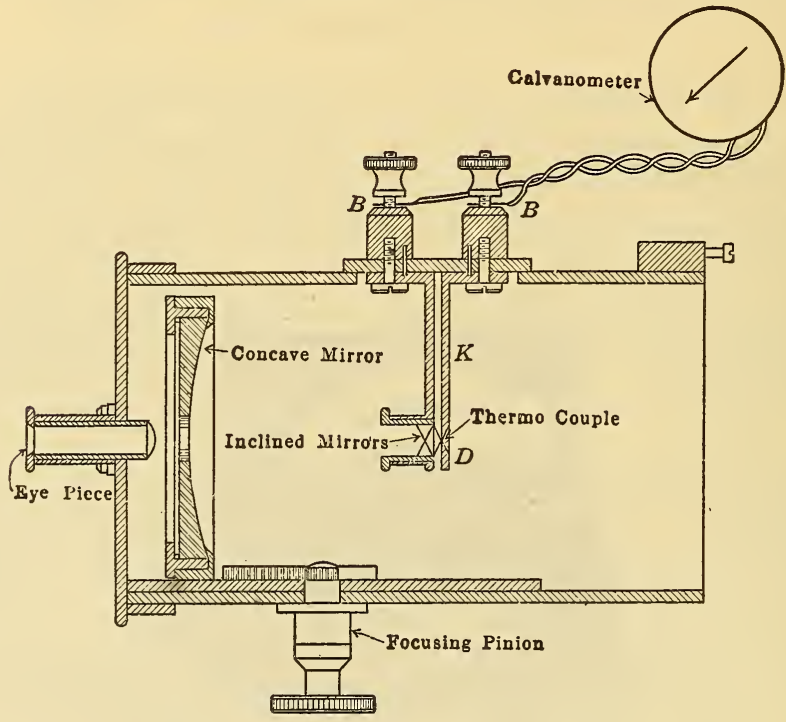

Fig. 70.-Types of radiation pyrometer

Mirror type of variable-focus radiation pyrometer

distance. The focusing is accomplished simply by means of an ingenious device ascribed to Fery. Two semicircular mirrors, Fig. $7 \mathrm{I}(a)$, inclined to one another at an angle of $5^{\circ}$ to $10^{\circ}$, are mounted in the thermocouple box, an opening of about $1.5 \mathrm{~mm}$ at the center of the mirrors forming the limiting diaphragm immediately in front of the couple. The observer views by means of the telescope $V$ the image of the furnace formed by the large concave mirror $M: M^{\prime}$ and reflected by the inclined mirrors $x x^{\prime}$ and $y y^{\prime}$ through a hole in the large mirror. If the image is not correctly focused at $O$, the intersection of the two small mirrors, the image appears broken in half as shown by $(b)$. Correct focus is obtained when the two halves of the image are in alignment $(c)$. 
Thus until correct focus is obtained certain straight lines of the source appear broken in the image. This breaking of a line is illustrated on a magnified and distorted scale by $(d)$. Suppose that the pyrometer were incorrectly focused upon a line source, an arrow, the image falling at position $A B$ instead of at $O$. The image of the arrow reflected from the mirror $y y^{\prime}$ lies at $A^{\prime \prime} B^{\prime \prime}$, and that reflected from the mirror $x x^{\prime}$ at $A^{\prime} B^{\prime}$. To the observer at $D$ the projections of these images appear as two distinct arrows, thus $\rightarrow \rightarrow$. As the pyrometer is brought nearer into the correct focus. by turning the pinion screw, and thus moving the large concave mirror in the direction $O D$, the points $P^{\prime}$ and $P^{\prime \prime}$ of the reflected images move along the lines $P^{\prime} O$ and $P^{\prime \prime} O$, coinciding at $O$ when the correct focus is obtained. The two arrows are then superposed, forming a single image.

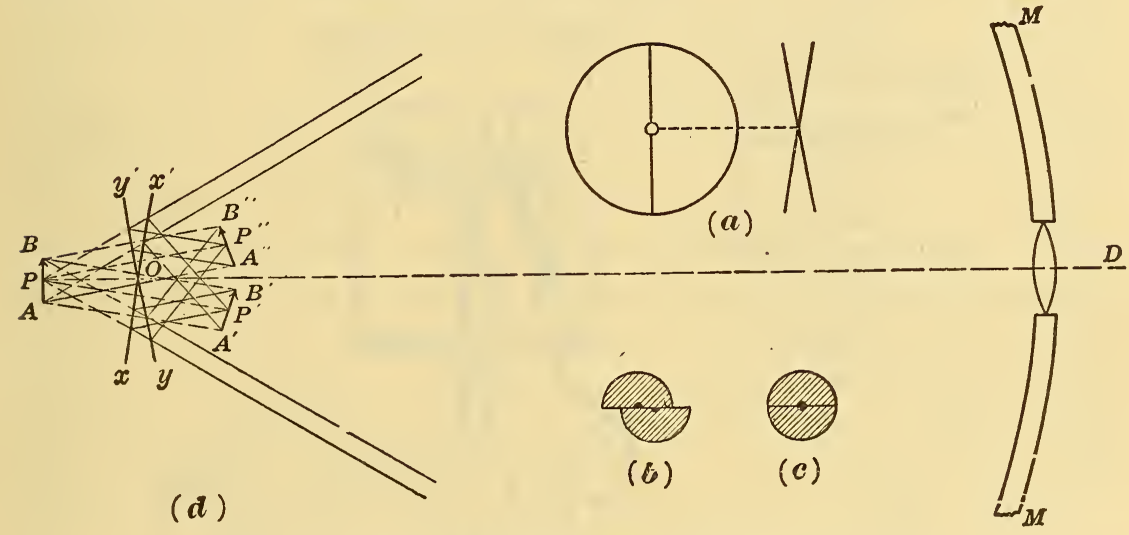

FIG. 7I.-Optical system of pyrometer shown in Fig. 70

For the measurements of very high temperatures, usually above $I_{5} 50^{\circ} \mathrm{C}$ the cover to the front of the telescope is provided with a sectored opening, which may be adjusted to reduce the radiation falling upon the receiver by any definite amount, and in this manner the upper temperature range of the instrument is practically unlimited. This adjustment is made by the manufacturer and should not be altered. For the lower scale range the cover is open, as shown in Fig. 72.

The readings with a Fery pyrometer, when properly focused, neglecting secondary errors discussed later, are independent of the sighting distance, as is the case with the fixed-focus radiation pyrometer. ${ }^{17}$ The image of the source, as viewed through the small

\footnotetext{
${ }_{17}$ See B. S. Sci. Paper No. 250, p. 97 , for the geometrical demonstration of this fact.
} 
telescope, must cover completely the limiting diaphragm to the thermocouple. This diaphragm appears as a black, circular area shown at the center of the field in Fig. $7 \mathrm{I},(b)$ and $(c)$. An excellent rule to follow is to sight at such a distance that the area of the image overlaps this hole and extends half way to the edge of the focusing mirrors. The Fery pyrometer requires a smaller source than the fixed focus instruments. The following table illustrates the size of source required for various sighting distances in order that the image cover the limiting diaphragm and extend about half way to the edge of the focusing mirrors, i. e. diameter of image $=4 \mathrm{~mm}$.
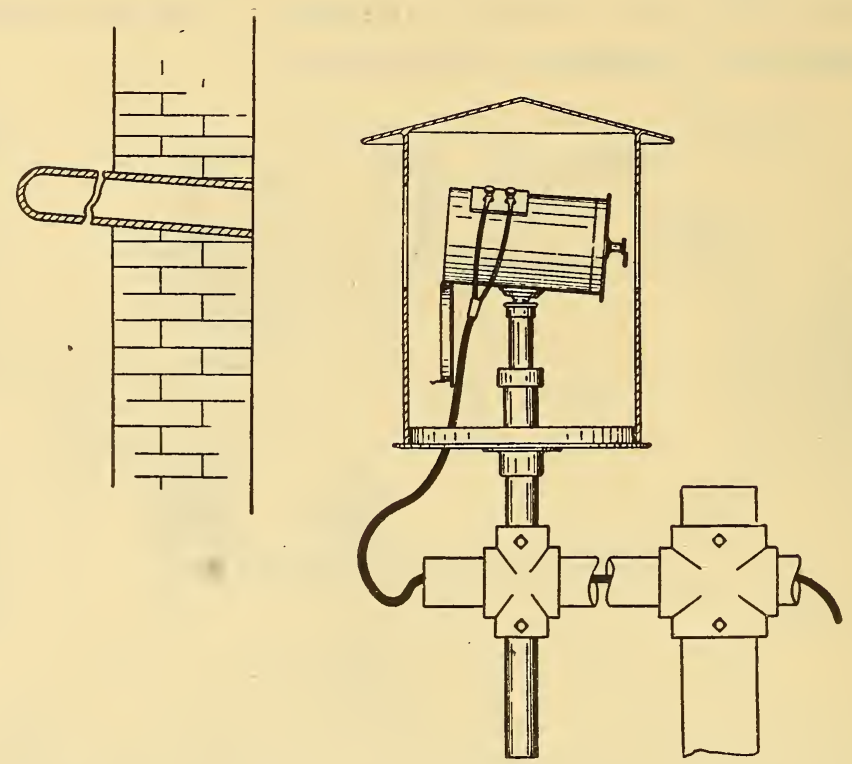

FIG. 72.-Radiation pyrometer in weather hood, sighted into a fire-clay tube

TABLE 21.-Size of Source versus Sighting Distance

\begin{tabular}{|r|r|}
\hline $\begin{array}{c}\text { Sighting } \\
\text { distance }\end{array}$ & $\begin{array}{c}\text { Diameter } \\
\text { of source }\end{array}$ \\
\hline $\begin{array}{r}\text { Centimeters } \\
70\end{array}$ & $\begin{array}{r}\text { Centimeters } \\
3.2 \\
80\end{array}$ \\
100 & 3.7 \\
150 & 4.8 \\
200 & 8.3 \\
300 & 11.2 \\
500 & 16.8 \\
& 28.5 \\
\hline
\end{tabular}




\section{ERRORS TO WHICH RADIATION PYROMETERS ARE SUBJECT}

Dust and dirt allowed to accumulate upon the concave reflecting mirror may so decrease its reflection coefficient as to develop errors amounting to $100^{\circ}$ or even $200^{\circ} \mathrm{C}$. Frequently the dust can be removed from the mirror by carefully brushing with a camel's-hair brush. The mirror may be removed from the instrument and washed, but this must be done with great care to insure that the delicate thermocouple or its mounting is not disturbed. The safest practice is to take all possible precautions to prevent dust from entering the instrument. Keep the case closed or the front diaphragm of the fixed-focus instruments plugged with a cork when not in use.

As shown above, the simple theory of the radiation pyrometer states that the readings are independent of the sighting distances or size of source, provided the source is larger than the minimum size of source demanded by the geometry of the instrument. Actually this ideal condition is not always realized. Some stray radiation is reflected down the walls of the telescope case. The walls become heated by the furnace and reradiate to the couple. Limiting diaphragms are similarly heated and reradiate to the couple. For these reasons a radiation pyrometer tends to read lower the greater the sighting distance or the smaller the size of source. It is thus in general desirable to use a radiation pyrometer as nearly as possible in the same manner from day to day and to have it specially calibrated for such use. For example, it a 6-inch source be employed, decide upon a convenient sighting distance, say 4 feet, and always use this distance, and not sometimes I foot and sometimes 5 feet. In the Fery pyrometer both the proper size of source and the proper focusing distances are secured by following the rule suggested above, viz, focus at such a distance that the image extends halfway between the black hole and the outside edge of the focusing mirrors.

\section{ADVANTAGES AND DISADVANTAGES OF RADIATION PYROMETERS}

For temperatures above 1400 or $1500^{\circ} \mathrm{C}$ either a radiation pyrometer or an optical pyrometer must be employed. The optical pyrometer is capable of higher accuracy and is less susceptible to errors than the radiation pyrometer. Smoke and dust affect the readings of both instruments, but the radiation pyrometer is seriously affected by the presence of cooler strata of carbon dioxide and other gaseous combustion products in the furnace. Carbon 
dioxide and water vapor absorb the heat rays, and hence the radiation pyrometer will read too low when sighted through such gases. The main advantage of the radiation pyrometer is the fact that it can be made automatically recording. The recording mechanism is the same as that employed for ordinary thermocouples, discussed in the section on recording pyrometry. The radiation pyrometer is desirable for many processes of lower temperature where thermocouples can not be conveniently installed. It is also useful in measuring the surface temperature of large ingots.

\section{BLACK-BODY AND NONBLACK-BODY CONDITIONS}

Radiation pyrometers are calibrated to read correctly when sighted upon a black body. Most furnaces approximate blackbody conditions sufficiently well. When sighted on materials in the open certain corrections must be applied to the observed temperature to convert them into true temperatures. For temperature control or reproducibility the apparent temperatures

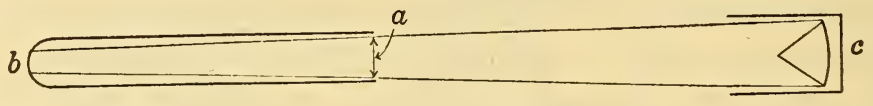

FIG. 73.-Method for determining proper diameter of sighting tube

may be used uncorrected, if desired, since, although known to be low, they will be low by the same amount from day to day. In case the temperature of one section of an unequally heated furnace is required, or if the furnace contains much smoke or dust, it is frequently desirable to sight the radiation pyrometer into the bottom of a fire-clay or porcelain tube, as illustrated by Fig. 72 . The tube should be uniformly heated at the end for a length at least three times its diameter. The tube must have a diameter such that the cone of rays entering the telescope is not intercepted by the front of the tube. Fig. 73 illustrates this condition. Suppose the distance from the bottom of the tube to the mirror of the Fery pyrometer is $100 \mathrm{~cm}$. Referring to the above table, the diameter of the source required is $4.8 \mathrm{~cm}$. Lay off the distance $b=4.8 \mathrm{~cm}$ and draw straight lines from the bottom of $b$ to the bottom of the mirror $c$, and from the top of $b$ to the top oi the mirror. The tube must have such a diameter at its front end $a$ that it does not cut in on the cone of rays represented by these two straight lines. This can be determined only by actually making the above drawing to scale or by computing. It can not 
be decided by looking through the telescope of the pyrometer, since the front end of the tube would not necessarily show even if it did cut in on the cone of rays. In the case of the fixed-focus instruments the diameter of the tube must be such that the cone of rays represented by the diameter $A^{\prime} A^{\prime \prime}$, Fig. 66, cuts the tube in the region which is uniformly heated.

Table 22 shows the true temperatures corresponding to the apparent or measured temperatures observed with a radiation pyrometer when sighted upon various materials in the open. This table must not be confused with Table i 6 for the optical pyrometer.

It will be noticed that the corrections are entirely different for the two types of pyrometer.

TABLE 22.-True Temperature versus Apparent Temperature Measured by Radiation Pyrometers when Sighted upon the Following Materials in the Open

\begin{tabular}{|c|c|c|c|c|c|}
\hline \multirow{2}{*}{ Observed temperature, degrees centigrade } & \multicolumn{5}{|c|}{ True temperature, degrees centigrade } \\
\hline & $\begin{array}{c}\text { Molten } \\
\text { iron }\end{array}$ & $\begin{array}{l}\text { Molten } \\
\text { copper }\end{array}$ & $\begin{array}{l}\text { Copper } \\
\text { oxide }\end{array}$ & $\begin{array}{c}\text { Iron } \\
\text { oxide }\end{array}$ & $\begin{array}{l}\text { Nickel } \\
\text { oxide }\end{array}$ \\
\hline $600 \ldots$ & & 1130 & 720 & 630 & 710 \\
\hline $650 \ldots$ & …. & 1210 & 775 & & 755 \\
\hline $700 \ldots$ & .... & 1290 & 830 & 735 & 800 \\
\hline $750 \ldots$ & & & 890 & & 845 \\
\hline $800 \ldots$ & 1200 & & 945 & 840 & 895 \\
\hline $850 \ldots \ldots \ldots \ldots$ & 1270 & & 1000 & 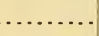 & 940 \\
\hline 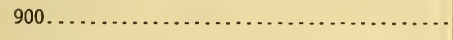 & 1340 & & 1060 & 945 & 985 \\
\hline $950 \ldots \ldots \ldots \ldots \ldots \ldots \ldots \ldots$ & 1410 & & 1115 & …. & 1030 \\
\hline $1000 \ldots$. & 1475 & & 1170 & 1050 & 1075 \\
\hline $1050 \ldots \ldots \ldots \ldots \ldots \ldots$ & 1550 & & & & 1120 \\
\hline 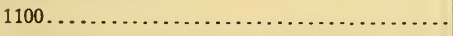 & 1610 & & & 1155 & 1165 \\
\hline 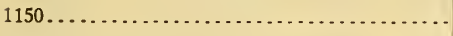 & 1680 & & & $\cdots$ & 1210 \\
\hline $1200 \ldots \ldots \ldots \ldots \ldots$ & 1750 & & & 1260 & 1255 \\
\hline
\end{tabular}

\section{RESISTANCE THERMOMETRY}

The operation of a resistance thermometer depends upon the variation with temperature of the resistance (mass resistivity) of an electrical conductor. A high temperature coefficient of resistivity might accordingly appear of primary importance. Certain oxides possess this property, having a resistance at room temperature from a hundred thousand to a million times that at $1000^{\circ} \mathrm{C}$. However, the resistance of a suitably mounted material may be easily and accurately determined so that the consideration of high temperature coefficient is rarely, if ever, the deciding factor in the choice of the material. The fundamentally important property 
is reproducibility and constancy of resistance. A further desirable feature is a simple and convenient relation between temperature and resistance. Platinum of the highest obtainable purity meets these requirements up to about $1100^{\circ} \mathrm{C}$. On the basis of the reproducible temperature scale defined by the platinum resistance thermometer, when calibrated according to certain specifications, temperatures may be measured to possibly o. ${ }^{\circ} \mathrm{C}$. at $1000^{\circ} \mathrm{C}$. To obtain such precision, however, requires accurately constructed apparatus and very careful experimental manipulation.

For industrial purposes, resistance thermometers are made usually either of platinum or of nickel, the latter material being fairly satisfactory for temperatures below $300^{\circ} \mathrm{C}$. In a common form of platinum thermometer about $40 \mathrm{~cm}$ of fine wire is wound upon a mica frame into a spiral coil about $4 \mathrm{~cm}$ in length and $0.7 \mathrm{~cm}$ in diameter. The length of the wire is so adjusted that the resistance of the coil is about $8.3 \mathrm{ohms}$ at $\mathrm{o}^{\circ} \mathrm{C}$. The frame is made of crossed strips of thin mica notched at the edges to hold the wire in place. From the ends of this coil lead wires of platinum or gold are carried to the terminal head of the thermometer. The lead wires are insulated and held apart by mica disks through which the wires are threaded. The thermometer is protected by a porcelain tube glazed on the outside, or by a quartz tube which fits into the terminal head. For industrial use the refractory tube is itself protected by an outer metal tube. The metal tube is usually fitted to an extra terminal head, through which the lead wires to the line are carried. On account of the deterioration of the platinum, thermometers constructed of fine wire are not very satisfactory for industrial use above $900^{\circ} \mathrm{C}$. Heavy platinum wire, $0.6 \mathrm{~mm}$ in diameter, may be employed up to $1 \mathrm{I}^{\circ} 0^{\circ} \mathrm{C}$, but the resistance of the thermometer is so low that it is necessary to use methods of measurement which do not depend upon constancy in the resistance of the lead wires-e. g., Thomson bridge or potentiometer.

In another form of resistance thermometer a coil of fine platinum wire having a resistance of 50 ohms at $0^{\circ} \mathrm{C}$ is wound upon a quartz rod $3 \mathrm{~mm}$ in diameter and $6 \mathrm{~cm}$ in length and fused inside a quartz tube, so that the platinum is entirely embedded in quartz. Gold leads are carried from the coil to the terminal head. The quartz insures high electrical insulation, while still preserving excellent heat conduction. The thermometer is very compact, 
and the platinum is thoroughly protected against deterioration. For work of the highest precision, in which measurements to o. ${ }^{\circ}$ or $0 . \mathrm{OI}^{\circ} \mathrm{C}$ are required, this type of thermometer is inferior to that in which the platinum is loosely mounted on a mica frame, since its calibration is slightly altered with use on account of the strain introduced by the differential expansion of quartz and platinum. For most purposes this objection is of small consequence.

The relation between the temperature $t^{\circ} \mathrm{C}$ and the resistance $r_{t}$ of the platinum resistance thermometer is of the following form where $a, b$, and $c$ are constants determinable by three standardization points:

$$
r_{\mathrm{t}}=a+b t+c t^{2} .
$$

If only two lead wires are carried from the thermometer coil to the indicator, the resistance measured is the sum of the resistance of the coil, the platinum or gold lead wires to the head of the thermometer, and the copper lead wires from this point to the indicator. The resistance of the platinum or gold lead wires will depend upon the form of temperature gradient along the thermometer from the bulb to the head and upon the depth of immersion. Hence this variable resistance is introduced into the temperature measurement, and changes in the resistance of the lead wires will be interpreted as changes in the temperature of the thermometer coil.

There are three general methods for minimizing or eliminating the effect of variation in lead resistance.

(I) Use of a Coil of High Resistance.-The resistance of the lead wires from the coil to the thermometer head and the variation in resistance of the copper lead wires are small compared to a 50-ohm platinum coil. Hence such variations in resistance as are likely to occur do not introduce serious error. Thus, only two lead wires are necessary, from the thermometer to the indicating instrument.

(2) Compensating Lead Wires of the Callendar or Siemens Form.-Both of these methods of compensation are applicable to measurements made with the Wheatstone bridge. The Callendar form of compensation requires four lead wires. Two of the lead wires joined to the ends of the coil are connected in one arm of the bridge, and the other two lead wires, which are "dummy" leads formed of a single loop of wire extending to the top of the $8513^{\circ}-21 \longrightarrow 9$ 
coil, are connected in the corresponding arm of the bridge. The two sets of lead wires are alike, so that variations in temperature affect each set similarly. The Siemens method of compensation described below requires three leads and is more often employed industrially in this country.

(3) Potential Terminals.-The use of potential terminals is necessary when the resistance of the thermometer is made very low or when the precision required is such as to make variations in difference of lead resistances inadmissible.

The general methods for measuring resistance include the Wheatstone bridge, the Thomson bridge, potentiometer, and differential galvanometer. The Wheatstone bridge is used the most extensively for industrial installations.

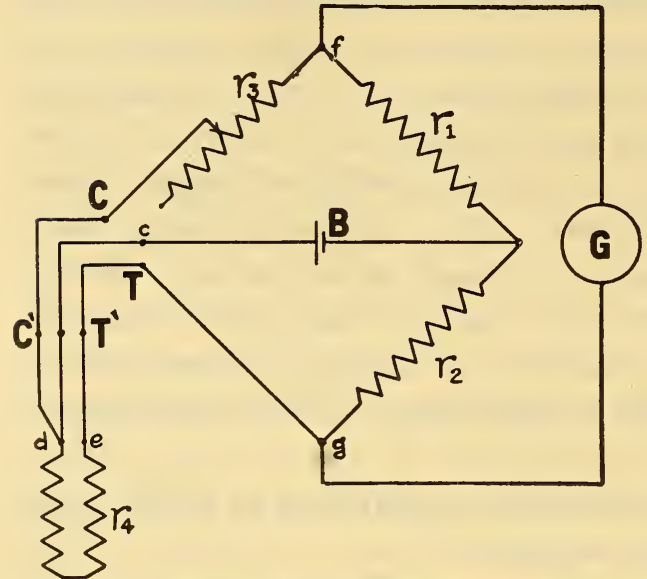

FIG. 74.-Wheatstone bridge and thermometer with Siemens 3-lead compensation

\section{THREE-LEAD WHEATSTONE} BRIDGE METHOD

Fig. 74 illustrates the wiring diagram for a simple Wheatstone bridge and thermometer with the Siemens three-lead compensation.

The platinum or gold lead wires $C^{\prime} d$ and $T^{\prime} e$ in the thermometer are constructed of as nearly the same resistance as possible, and the copper lead wires $C C^{\prime}$ and $T T^{\prime}$ must also be of

equal resistance. The battery $B$ is connected between the ratio arms $r_{1}$ and $r_{2}$ of the bridge and to the compensating lead wire $c d$. A sensitive galvanometer $G$ is connected to the points $f$ and $g$, as illustrated. By changing $r_{3}$ the bridge is balanced until the galvanometer shows zero deflection. Whence from the principle of the balanced Wheatstone bridge we obtain the following relation:

$$
\frac{r_{3}+C d}{r_{1}}=\frac{r_{4}+T e}{r_{2}} .
$$

If $r_{1}$ is constructed equal to $r_{2}$ then since $C d=T e, r_{3}=r_{4}$. Hence the measured resistance is independent of the resistance of the lead wires. 


\section{FOUR-LEAD WHEATSTONE BRIDGE METHOD}

Fig. 75 illustrates the wiring diagram for a simple Wheatstone bridge and thermometer of the four-lead potential terminal type. This method is used mainly for precision measurements in the laboratory. The battery $B$ is first connected to $c$. As before, when the bridge is balanced we obtain:

$$
r_{3}+C d=r_{4}+T e .
$$

The battery is then connected to the terminal $t$ and the terminals $T$ and $C$ are interchanged by means of a commutating switch. We accordingly have for a balance: $r_{3}^{\prime}+T e=r_{4}+$ $C d$. Whence upon combining these two equations:

$$
r_{4}=\frac{r_{3}+r^{\prime}}{2}
$$

Thus the measured resistance of the coil is independent of the resistance of the lead wires, and any error which would result from inequality of the lead resistances $C d$ and $T e$ in the ordinary three-lead thermometer is eliminated.

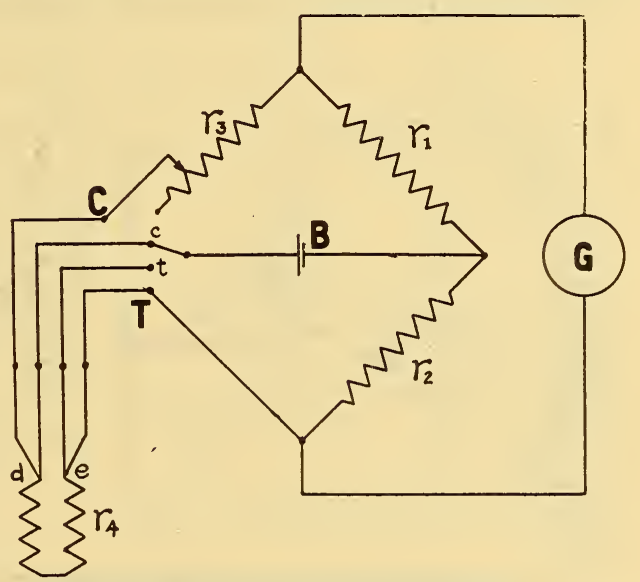

FIG. 75.-Wheatstone bridge and thermnmeter with 4-lead compensation

The diagrams of Figs. 74 and 75 are schematic and show a moving contact in the bridge arm $r_{3}$. Since resistance thermometers are nearly always made of comparatively low resistance, the variations of such a contact resistance would usually introduce serious errors. In practice the simple circuits shown in Figs. 74 and 75 are rarely used, but various modifications of the circuits are made for the purpose of avoiding the introduction of a contact resistance into the arm $r_{3}$. (See references quoted elsewhere.)

In the unbalanced bridge shown in Fig. 77 , or in a bridge arranged to be connected to any of a number of resistance bulbs, a superior type of switch should be employed. If a mercury switch is not used, the switch contacts should be kept clean and tight. 


\section{FOUR-LEAD POTENTIOMETRIC METHOD}

Fig. 76 illustrates the wiring diagram for a four-lead thermometer used with a potentiometer. The current, adjusted to a suitable value by the rheostat $r_{1}$, flows through a resistance standard $R$ and the thermometer coil $r_{4}$. The potential drops across $R$ and $r_{4}$ are measured by the potentiometer. Whence from Ohm's law, $\frac{e}{R}=\frac{e^{\prime}}{r_{4}}$ where $e$ and $e^{\prime}$ are the potential drops across $R$ and $r_{4}$, respectively. Thus, knowing $e, e^{\prime}$, and $R$, the coil resistance $r_{4}$ is readily obtained. The resistance $R$ may be made adjustable, so that by varying $R$ until $e=e^{\prime}$ the dial setting gives directly the value of $r_{4}=R$. This method is useful in precise laboratory work.

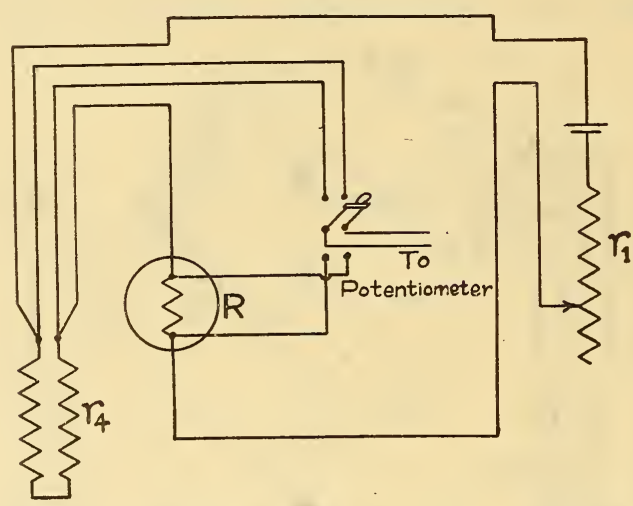

FIG. 76.-4-lead thermometer used with potentiometer

\section{DEFLECTION-GALVANOM- ETER METHOD}

The bridge methods so far described require the adjustment of the bridge until the galvanometer shows zero deflection. The temperature is then read from the setting of the dial or slide wire. It is possible, however, to use an unbalanced bridge with all resistances fixed, except that of the thermometer, which depends upon its temperature, and the temperature is obtained from the scale reading of the galvanometer. Fig. 77 shows the wiring diagram for an unbalanced Wheatstone bridge. With the switch $S$ thrown to the right we have the simple Wheatstone bridge, except that the resistances $r_{1}, r_{2}$, and $r_{3}$ are fixed. At a certain temperature of the thermometer-for example, $10^{\circ} \mathrm{C}$-its resistance is such that the bridge is balanced and $\frac{r_{3}}{r_{1}}=\frac{r_{4}}{r_{2}}$. If the temperature of the thermometer increases, its resistance increases and the bridge is no longer balanced. Thus a current flows through the galvanometer, producing a deflection. In general, the deflection of the galvanometer depends upon two factors-the temperature of the thermometer and the current flowing from the battery $B$. This current is adjusted to a definite value in the following manner. The switch $S$ is thrown to the left, thus replacing the thermometer by a fixed 
resistance of such magnitude that a suitable value of the current from $B$ deflects the galvanometer to its full scale deflection or to a normal point marked on the scale. The resistance $R$ is adjusted until the galvanometer deflects the proper amount. The switch is then thrown to the right, and, since the factor of variable battery emf or current is eliminated by this preliminary setting, the scale of the galvanometer may be empirically graduated to read directly the temperature of the thermometer. Thus no further adjustments are necessary as long as this current remains at the proper value, and any temperature is readily observed without the necessity for balancing required in the ordinary bridge. It is possible to have two or more temperature scales on the galvanometer. When two scales are providedas, for example, $0^{\circ}$ to $300^{\circ} \mathrm{C}$ and $300^{\circ} \mathrm{C}$ to $700^{\circ} \mathrm{C}$-one of the arms of the bridge is made adjustable to either of two resistances, one giving zero deflection for a thermometer temperature of $\mathrm{o}^{\circ} \mathrm{C}$ and the other zero deflection for a temperature of $300^{\circ} \mathrm{C}$.

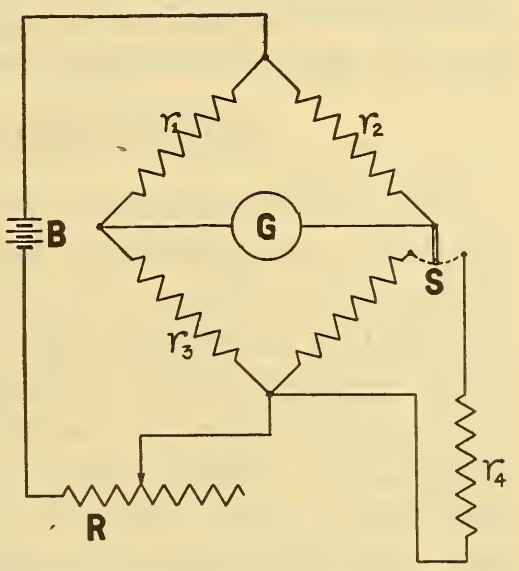

FIG. 77.-Unbalanced Wheatstone bridge

\section{SUMMARY STATEMENT ON RESISTANCE THERMOMETRY}

Although the resistance thermometer is of the greatest importance in the laboratory for work of extremely high precision, its importance for industrial processes at high temperatures is waning. To obtain the precision of which the instrument is capable requires many precautions easily overlooked. Bad contacts in the line, a broken strand in a flexible cable, etc., may cause large errors. The thermometer is very fragile, and its calibration may be altered by severe handling or by the slightest contamination or by volatilization of the platinum wire forming the coil. The range is confined usually below about $800^{\circ} \mathrm{C}$. In the early development of pyrometry, before satisfactory galvanometers were devised for thermocouples, the resistance thermometer afforded about the only accurate means of temperature measurement, but with the highly sensitive and reliable thermocouple indicators and portable potentiometers, and with the reproducible Le Chatelier couple now obtainable, there are few industrial processes carried out above 
$300^{\circ} \mathrm{C}$ where a thermoelectric installation is not to be preferred. When very small temperature changes or intervals must be measured with high precision, the resistance thermometer may be.employed effectually. Also, in certain processes where an integrated or average temperature over the entire bulb is required, the resistance thermometer is highly desirable. A homogeneous thermocouple indicates the temperature of its hot junction, while a resistance thermometer indicates the average temperature of the entire coil or bulb, which may be constructed as long as desired. There are no cold-junction corrections for the resistance thermometer-a decided advantage over the thermocouple, but one which is offset by the disadvantages mentioned.

\section{RECORDING PYROMETRY}

One of the fundamental principles of efficiency is the use of adequate and permanent records. The rapid increase in the manufacture and use of recording pyrometers is a proof of the appreciation of efficiency principles on the part of the manufacturers engaged in the various technical industries. Where recording pyrometers are not employed in an industry in which temperature measurements are necessary, one will generally find that a printed form is used upon which is written, periodically, the temperatures measured by an indicating instrument. It is no serious condemnation of the workman to state that such records are often "doctored;" but it is rather a reflection upon the executive who places such temptation in the way of a workman. One of the well-known "tricks of the trade" is to "force" a furnace so that it will be at the proper temperature during the periodic trips of the foreman or other official.

Indicating instruments and recorders may be used together to great advantage. The recorder furnishes a printed record and a check upon the operator of the furnace, and a record of value in correlating properties of the finished product with the heat treatment. The indicator should be of assistance to the operator in controlling the furnace or oven.

Modern practice requires a temperature-recording instrument that is as simple as possible, rugged, reliable, and sufficiently accurate. Of these qualifications reliability is paramount, particularly in cases where the recorder is used for controlling the temperature automatically. By a reliable instrument is meant one that will run continuously with little attention and with a con- 
sistent degree of accuracy; that is, if it is in error by 5 per cent one day it must be in error by a like amount on any other day, and not by 2 per cent or io per cent. The earlier forms of recorder were complex and delicate, or mechanically unsatisfactory and inaccurate, and required considerable attention to keep them in operation. Within the past few years, however, the development in the manufacture of temperature recording devices has been highly satisfactory, and many excellent instruments are now available.

The pyrometers that can be made to record automatically fall under the following classifications: (I) Gas, saturated vapor, and liquid thermometers; (2) resistance thermometers; (3) thermoelectric pyrometers; (4) radiation pyrometers.

Of these four types, the thermoelectric pyrometer recorder has the greatest applicability, especially for the higher temperatures at which the first two named are not suitable. The constantvolume industrial gas thermometer is successful up to about $400^{\circ} \mathrm{C}$. The resistance thermometer is capable of very high accuracy up to $1000^{\circ} \mathrm{C}$. At such high temperatures, however, thermocouples are more serviceable, since deterioration of the wire from continual heating does not so seriously alter the emf developed by a couple as it does the resistance of a resistance thermometer. Base-metal couples serve satisfactorily up to I $100^{\circ} \mathrm{C}$, and platinum platinum-rhodium couples up to I $500^{\circ}$ $\mathrm{C}$, although above $\mathrm{I} 400^{\circ} \mathrm{C}$ it becomes very difficult to protect the couple from contamination by the furnace gases and vapors. Radiation pyrometers are useful at the highest attainable temperatures, but processes in which temperatures greater than $1600^{\circ} \mathrm{C}$ are used are not, in general, subjected to very precise temperature control.

\section{FORMS OF TEMPERATURE RECORDS}

The most usual form of temperature record is that in which temperature appears as one coordinate and time as the other coordinate. The temperature-time curve drawn on such a record has been called an autographic record. This type of curve is the most easily obtained mechanically and is valuable as a continuous record of the temperature of a furnace over a prolonged run. It is also occasionally used to detect transformation points in steel, which appear as flexures or indentations on the plot when the furnace containing the sample is uniformly heated or cooled. 
For this latter work, however, the " differential-temperature " curve recorder is especially adapted and will be described later. Other types of curves obtained with special recorders, and used mainly for laboratory work, are the temperature-rate curve, the inverserate curve, and the derived-differential curve. The various special methods have been discussed by Burgess ${ }^{18}$ and will not be considered in the present paper.

\section{GENERAL TYPES OF THERMOCOUPLE RECORDERS FOR TEMPERATURE-TIME CURVES}

A recorder for obtaining a temperature-time curve consists essentially of an electrical measuring instrument with a mechanism for periodically recording its indications upon a chart that moves with a uniform speed. As in the case of simple indicators, there are two general types of recorder, one operating on the galvanometric principle and one operating on the potentiometric principle. Also, as in the case of indicating instruments, the potentiometric principle, while somewhat more complicated, has the especially desirable feature that its indications are independent of the thermocouple resistance. The readings of a recorder operating on the galvanometric principle depend on the variations in the resistance of the external electric circuit, although the effect of these variations can be reduced by using an instrument of high internal resistance or, with less satisfaction, by keeping the resistance of the external circuit very low. In many of the recorders now obtainable the resistance is sufficiently high that these effects become of little practical importance, or the instrument may be equipped with one of the compensation devices described elsewhere.

\section{RECORD CHARTS}

There are three types of record paper in general use-the roll charts and drum charts shown in Fig. 78 and the disk or circular charts shown in Fig. 79. The roll chart may contain enough paper to last a month or more, while usually the drum or circular charts are renewed every 24 hours. For single-point recorders all of these forms are employed; but, with one or two exceptions, multiple-point recorders use record paper in the roll form.

Upon circular charts, the lines of equal temperature (time coordinates) are represented by concentric circles, and lines of equal time (temperature coordinates) by arcs following the course

28 B. S. Sci. Papers, No. 99. 
of the galvanometer pointer. The distorted appearance of such a record is at first somewhat confusing, but, with practice, the record is easily read even when the complete curve is allowed to

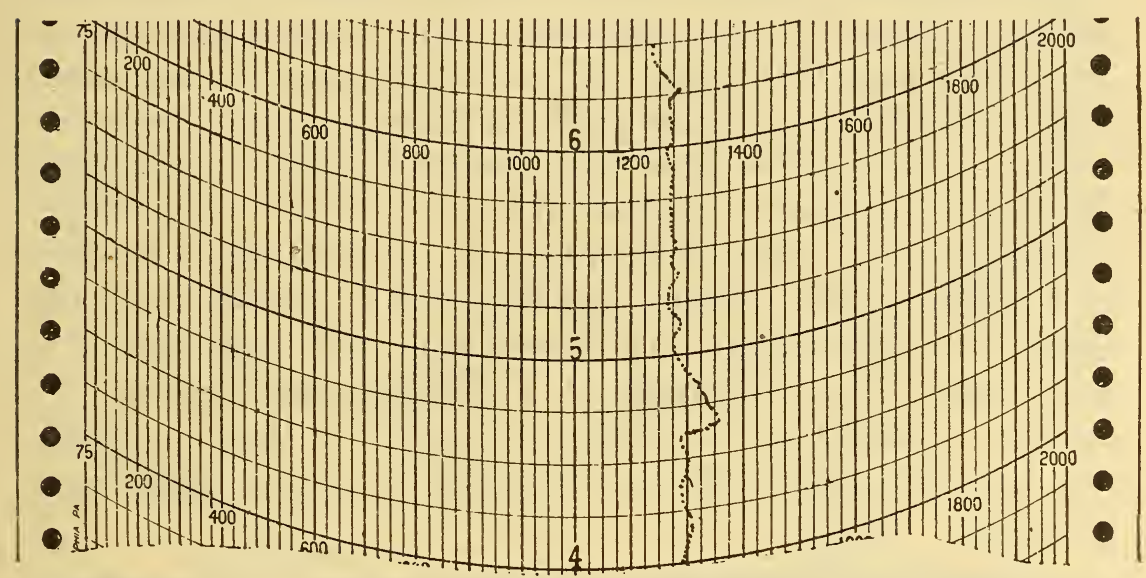

FIG. 78.-Roll or drum chart

extend around the chart for several revolutions. If an extended and open-temperature scale is required, the disk record becomes somewhat unsatisfactory on account of its size, since the diameter of the circular sheet must be more'than twice the width of the

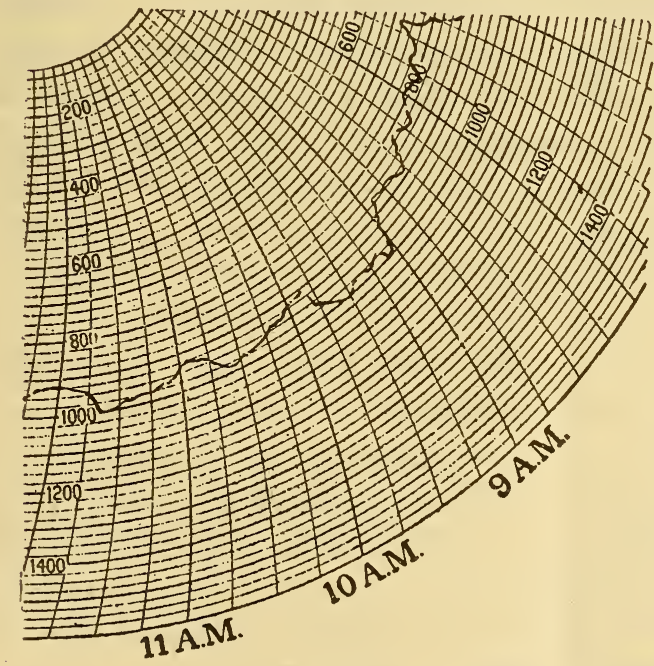

Fịg. 79.-Disk chart temperature scale. The necessity of opening the recorder case, usually every 24 hours, to mount a new record sheet means greater liability for an accumulation of dust.

In filing records for future reference the circular and drum type oneday records offer some advantage over the continuous-roll records, although the latter may be cut to any convenient length when removed from the recorder. With the drum and roll records the coordinates can be made rectangular. In some cases, however, the temperature coordinates are parts of a circle, as shown in Fig. 78, where the radius of the circle is the 
length of the galvanometer needle. The roll record is particularly adapted to multiple-point recording and for adjusting to very slow or very rapid record speeds.

The method by which the printing of the record is accomplished will be described in the discussion of the various instruments. The record may be obtained by pen and ink, stylus and inked ribbon, inked thread, carbon paper, or coated paper; by puncturing the record paper by means of an electric spark; or by stamping upon the record sheet some imprint from a stencil. The difficulty in making several distinct records upon the same chart; required by a multiple-point recorder, has been met in various ways, but there is still opportunity for development and improvement in this regard. The advantage of a multiple-point recorder for extensive installations is obvious in that for a small additional cost one instrument will do the work of several single-point recorders.

\section{THERMOCOUPLE RECORDERS OPERATING ON THE GALVANOMETRIC PRINCIPLE}

The recording millivoltmeter, or galvanometer, may employ the same galvanometer system used in the portable indicating instruments. A more rugged instrument is desired, however; this ruggedness is obtained by increasing the strength of the springs, boom, coil, and jewel bearings of a pivot instrument, etc. To compensate for the resulting decrease in sensitivity, the density of magnetic flux through the coil may be increased, the number of turns of wire on the moving coil may be increased, and the so-called swamping resistance in series with the moving coil may be decreased. Most indicators have a single magnet, while recorders have as many as four or six magnets. A portable instrument would become too heavy with so many magnets. In general, the resistance of a recorder is less than that of an indicator of the same type. On account of the very small emfs developed by thermocouples, and on account of the necessity of using a comparatively high resistance in series with the galvanometer coil to minimize the effect of a varying resistance, the torque that can be produced on the moving coil is small. Pivots, suspensions, etc., must be very carefully made, and the moving coil must be accurately balanced and so mounted that it swings perfectly free.

The galvanometer pointer can not be used to trace a legible record directly, since the friction between the paper and the pointer would entirely alter the readings. One common method 
for obtaining the record is illustrated by the Siemens \& Halske recorder, shown in Fig. 8o. The paper is unwound by clockwork at a uniform speed from a roll. An inked ribbon lies below the paper and above a metal plate. At periodic intervals the chopper bar $B$ falls; pressing a stylus on the end of the galvanometer boom $N$ into contact with the paper and against the ribbon-and metal plate underneath. This makes a small dot on the underside of the thin record paper, which shows through from the top, as illustrated. The paper is ruled with the proper time and temperature coordinates, and the row of dots obtained by continuous operation constitutes the required temperature-time curve. The maximum frequency with which the dots can be recorded depends on the natural period of vibration of the moving coil. In general practice, the dots appear at intervals of Io to 30 seconds.

This principle is employed in many instruments of American make. The chopper bar may be operated by an electric motor, clockwork, or electromagnet, and the design must be such that the galvanometer boom swings clear of the bar, between the intervals of depression, and such that the depression of the bar against the boom in no way damages the coil mounting. These conditions may be satisfied in pivot, suspension, and combination pivot-suspension systems.

Recorders may be classified according to the type of support

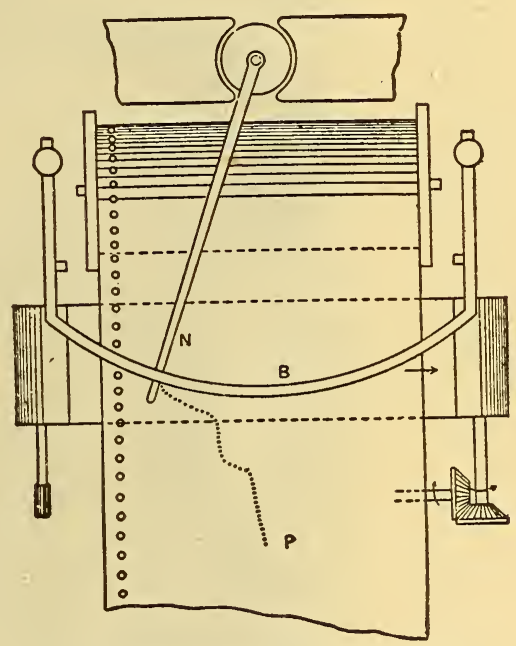

FIG. 80.-Principle of autographic recorder employed in mounting the moving coil. The double-pivot support is the most common and was developed for its greater ruggedness and the constancy of sensitivity obtained. The outstanding fault of the double-pivot instruments is the tendency to stick and the failure of the manufacturers to make them sufficiently dust proof. An indicator of the double-pivot type may have its moving coil so mounted that the pivots do not center in the jewel bearings, but roll around in a small arc over the cup-shaped surface of the bearings. This reduces the friction considerably, but the method is not applicable for recorders in which the pointer is periodically struck by a bar with sufficient force to move the pivots in their bearings. 
Unipivot-suspension instruments are not common and are not widely known. The suspension can be made very long, and hence comparatively heavy and strong. By crimping the suspension or by supporting it on a spring, a definite fraction of the weight of the moving coil may be taken from the pivot. The coil requires clamping during shipment and must be mounted in such a way that it will always seat properly if jarred out of the bearing. There is, of course, very little danger of the suspension breaking, and this type of construction can be followed in making an instrument of good constancy and sensitivity at relatively low cost.

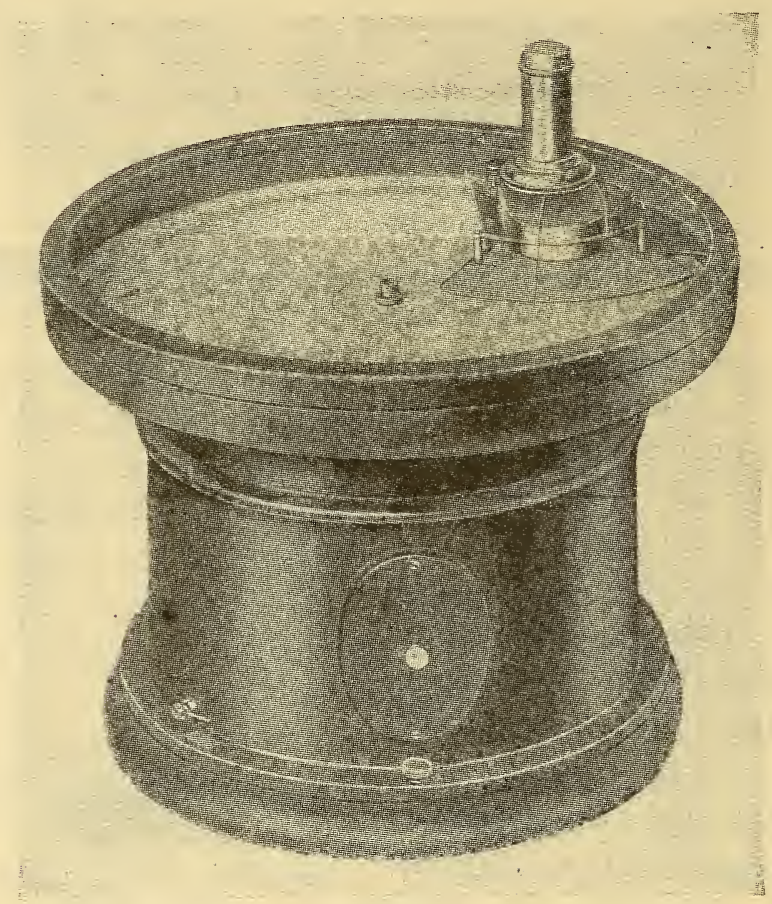

FIG. 8I.-Types of circular-chart galvanometric recorders

The instrument shown is made either for single or multiple records

All the advantages of the foregoing types of galvanometer construction are found in the double suspension, and with the latter a still greater sensitivity is possible. The coil is mounted so that jarring can move it a very small distance, and the spring supports of the suspensions are so made as to allow this amount of motion without undue tension. The coil is usually short-circuited when the instrument is not in use.

Recorders may also be classified according to their motive power. For most precise movement of the chart a clock must be used either alone or in conjunction with an electromagnet, which 
may be applied to do most of the work. The clock alone must be large, and have plenty of power, particularly for multiple-point recorders. The commutators of multiple-point recorders must operate with considerable friction; this is best overcome by means of an electromagnet operated by the clock. The magnet is also used in some instruments to operate the chopper bar. By having the magnet lift a weight with each stroke, it can be used to furnish power for driving the chart, as is done in one of the instru-

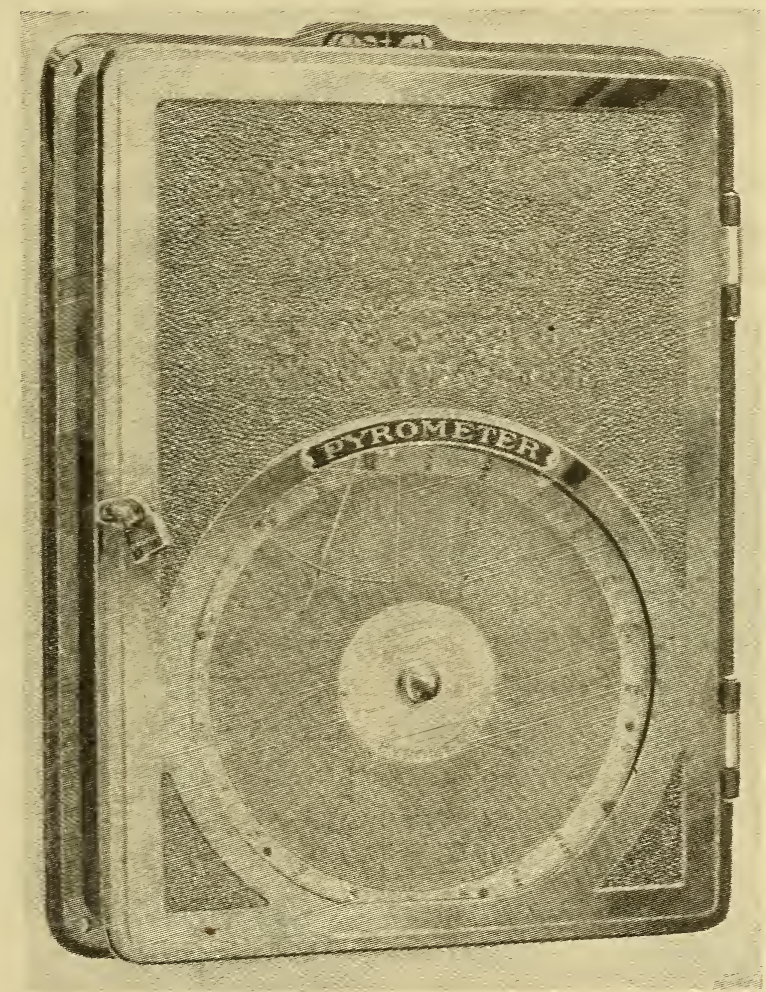

FIG. 82.-Types of circular-chart, single-point galvanometric recorders

ments described below. A motor may be used for the driving power, and by attaching a governor very satisfactory control of the chart motion is obtained. The motor requires somewhat more attention than a clock, but in some recorders it is indispensable.

(a) Industrial Types of Recordens.-Foreign designs will not be described, for the writers believe that no foreign-made recorders compare favorably with the American makes in simplicity of construction, ruggedness, and general applicability for 
industrial use. The American manufacturers first in the field supplied a need by making a fairly cheap form of single-point recorder simply made and sufficiently accurate to be well worth its use. This was done at a time when the imported instruments were expensive, complicated, and accurate only when cared for by an expert.

(I) The Circular-Chart Single-Point Recorder.-The circularchart single-point recorders are made by a number of American firms. Figs. 8I to 83 illustrate the various forms of this type of instrument. With the exception of the Cleveland recorder, Type c, Fig. 8I, this type is not made for multiple records. Attention might be called to the fact that the time coordinates of the circular-chart recorders are relatively short and thus these charts are unsuited to the measurement of rapidly changing temperatures. They are best suited for furnace or oven operation where a fixed temperature is maintained or desired or a slow rise or fall as met with in the ceramic industry and in annealing ovens.

Fig. 83 shows the construction of the Brown recorder. The millivoltmeter is mounted on the door so that charts may be renewed without danger of injuring the millivoltmeter pointer. The presser frame is also mounted on the door and is operated by the reciprocating arm shown at the upper left-hand side of the chart. This arm is slowly drawn back by the clock (hidden by chart) against the tension of a spring. Its release throws the presser frame against the pointer and a dot is registered on the chart. In Fig. 83 is seen a metal guide extending across the paper just below the point where the record is made. A heavy paper having a slot along this guide is placed underneath the thin paper chart and over the carbon paper so that in changing records the paper is not easily soiled. The small spool seen in the upper right-hand corner is a resistance in series with the moving coil of the meter. One of its purposes is for calibrating the instrument or changing the temperature range-e. g. to change a range $\mathrm{o}$ to $1000^{\circ}$ to o to $\mathrm{I}_{500^{\circ}}$ the total resistance of the instrument would be increased by approximately 50 per cent. This should not be done with low-resistance instruments without also correcting the lead resistance; that is, the correction for range should apply to the entire electric circuit.

The Hoskins millivoltmeter is unique in the fact that the meter is behind the record and the glass window closes flat against the chart, sustaining the pressure of the pointer when the latter is 
raised by the presser bar. This construction gives greater convenience in changing records.

The Bristol recorder is shown in Fig. 82. The pointer has a flattened part so that when depressed by the chopper bar there

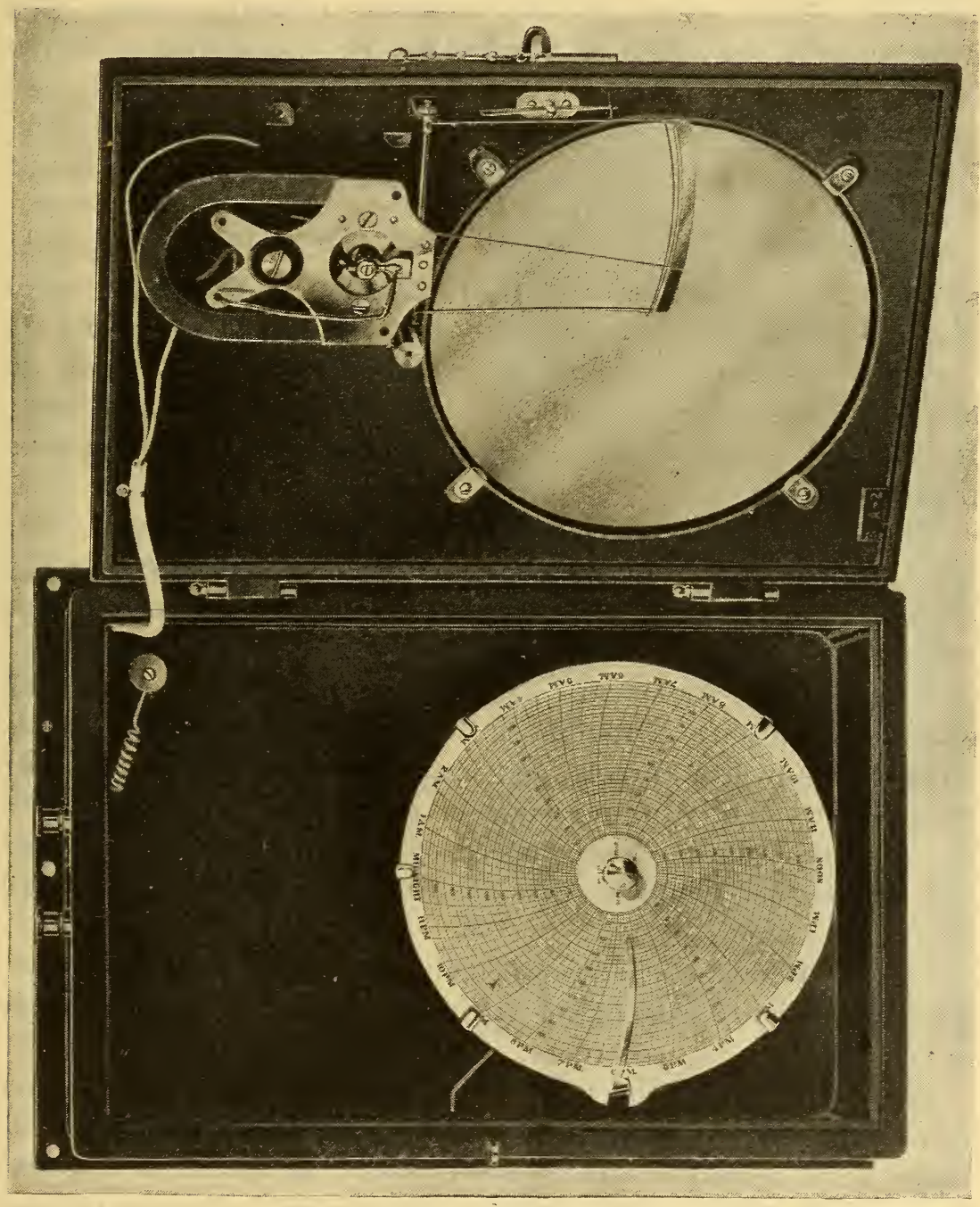

FIG. 83.-Types of circular-chart, single-point galvanometric recorders

is the slight rubbing action on the chart necessary with the use of smoked paper.

The Cleveland recorder shown in Fig 8I is much less simple in construction than the foregoing types. Its distinctive features are a somewhat more open scale (larger chart), a unipivot-suspension meter, and a unique method of making the record. The 
pointer is not periodically depressed, but swings free with its tip close to the record. Every 30 seconds an electric spark passes from the pointer to the chart plate, puncturing the paper. The record is a series of holes with seared edges which are easily seen. There is a tendency of the spark to jump the gap at an angle, causing a slight error, which, however, is not serious. This instrument is not designed particularly for installation near the furnace, but in an office or central pyrometer station. Its high resistance makes it possible to use very long leads of small wire. The clock is electrically wound and requires no attention except regulation. To accomplish multiple recording with this instrument, the chart is divided into sectors, and, if six records are to be made, the chart moves forward slightly more than a sixth of a revolution each one-half minute. The time coordinates are very short, and the instrument is adapted in this form only to large furnaces with constant or slowly changing temperatures. It should be used combined with an indicator for controlling temperatures.

The Stupakoff recorder is the only drum chart type. This instrument is of the double-pivot type with a high resistance and ordinary scale length. The drum is rotated once in a day or a week.

(2) Roll-Chart Recorders.-The general advantages of the roll chart are legibility (coordinates are parallel and may be made rectangular), width of scale, and adaptability to rapidly changing temperatures, and the recording of more than one record on a single chart. With a paper speed of $\mathrm{I}$ inch an hour a 20-yard roll will last I month. Rolls are usually furnished in 20-yard lengths and longer.

Fig. 84 illustrates the Brown continuous recording pyrometer. The fundamental principles of its operation are the same as those of the circular-chart recorders.

Fig. 85 illustrates the Brown multiple-recording pyrometer for recording I to io temperatures. Records are obtained in five colors from a multicolored ribbon placed as shown in Fig. 8o. For more than five records double dots are used. This is a method which has been found to give very easily distinguishable records. The record is made by pressing the galvanometer pointer down upon the chart which runs over a nearly sharp straight edge with the ribbon between. This results in rectangular coordinates. The length of scale is $61 / 4$ inches $(\mathrm{I} 6 \mathrm{~cm}$ ). The recording interval is 30 seconds, which gives a five-minute cycle for 10 records. The 
galvanometer is of the two-pivot type with a moving coil of 50 ohms of copper and a swamping resistance of 350 ohms of manganin.

The instrument is run by electric power obtained from I Io or 220 volts alternating-current or direct-current lighting circuits or from storage batteries or dry cells. The speed is controlled by a clock automatically wound. The clock closes an electric contact in series with a solenoid magnet, which operates the depressor, and the selective switch advances the chart paper and ribbon, and is also linked to the reroll cylinder. The entire mechanism including the galvanometer is mounted on the back of the recorder case and hinged to be swung outward. The case of aluminum is designed to be dust and fume proof without an outer extra cabinet.

The Engelhard recorder which has been recently developed is of the high-resistance double-suspension type. The chart has rectangular coordinates and a graduated width of $4 \frac{\mathrm{T}}{2}$ inches (I I.4 cm). The resistance is upward of 700 ohms for rare-metal thermocouplęs.

The Hoskins multiple recording pyrometer, which has been recently developed, is

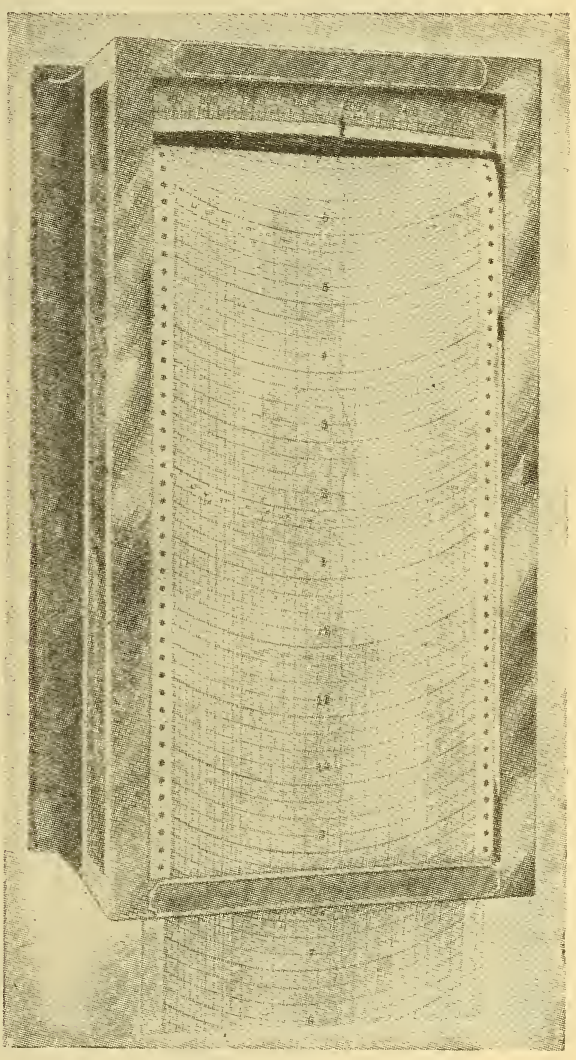

FIG. 84.-Types of roll-chart galvanometric recorders, single-point recorder shown with cover and chart removed in Fig. 86. It is made for Io records of base-metal thermocouples. The scale is over 7 inches $(17 \mathrm{~cm})$ wide, the highest range instrument reading to $2500^{\circ} \mathrm{F}\left(1370^{\circ} \mathrm{C}\right)$. The essential features are an electric drive ( $C$ is a solenoid arm) and a clock operating only the electric contact device $D$. The motion of the solenoid arm raises the frame $I$ (which is depressed in other instruments), changes the $8513^{\circ}-21-10$ 
commutator or rotary selective switch $H$, and moves the chart a step forward every third contact. Records are made every 20 seconds, the change from one couple to the next occurring every minute. Instead of using different colors or symbols for different couples the chart is divided by parallel lines, between any two of which only one couple records. The spaces are numbered con-

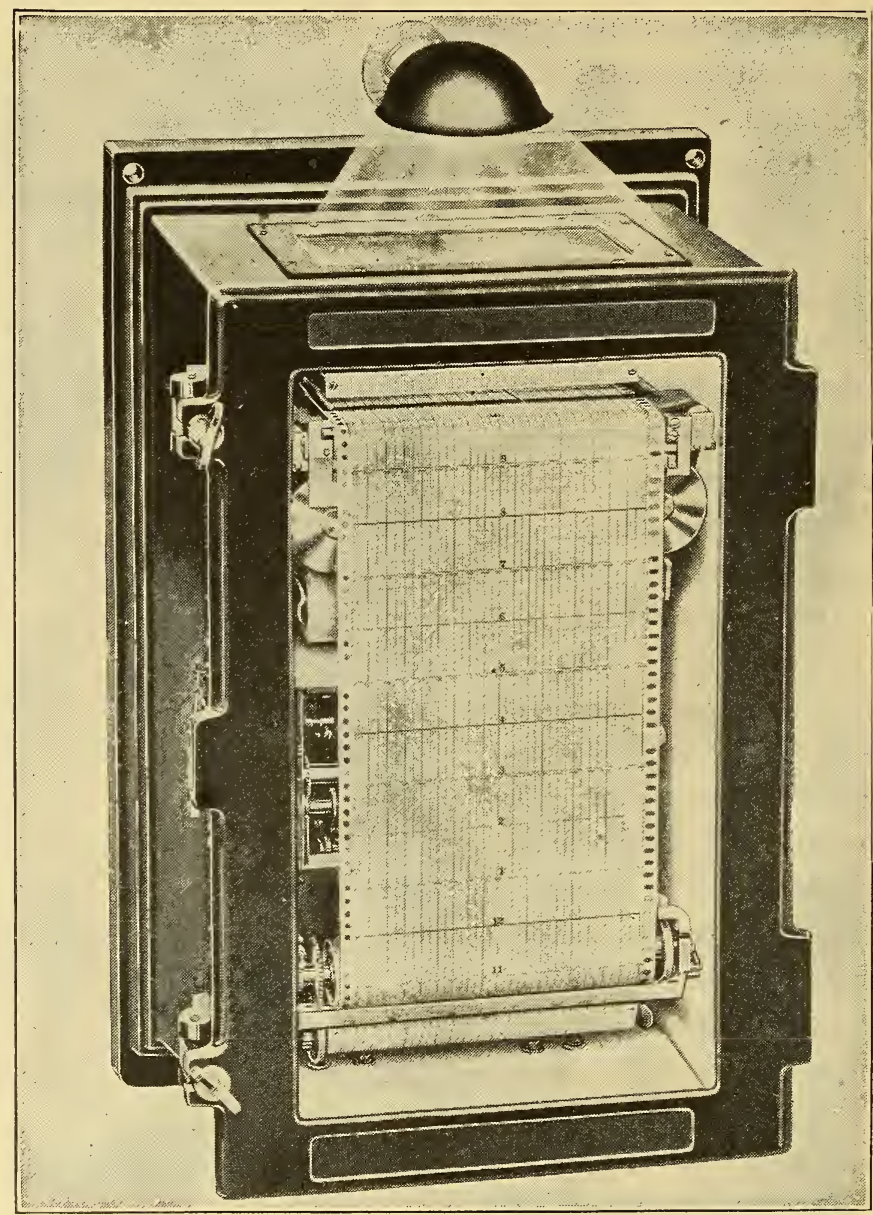

Fig. 85.-Types of roll-chart galvanometric recorders, multiple-point recorder

secutively and the records are distinguished by referring to the parts of the chart in which they fall. This is more clearly seen by pointing out that the selective switch is fastened directly on the shaft $G$, which holds the toothed wheels engaging perforations in the paper chart. A plug switchboard $L K$ mounted within the case is so made that it is possible to arrange the couples in any 
order or to put any couple on more than one number. The resistance of the millivoltmeter is 150 to $300 \mathrm{ohms}$ and is intended to operate with a fixed lead resistance of $50 \mathrm{ohms}$. The high external resistance is selected to take care of compensating leads the resistance of which is about $0.13 \mathrm{ohm}$ per foot. Calibration

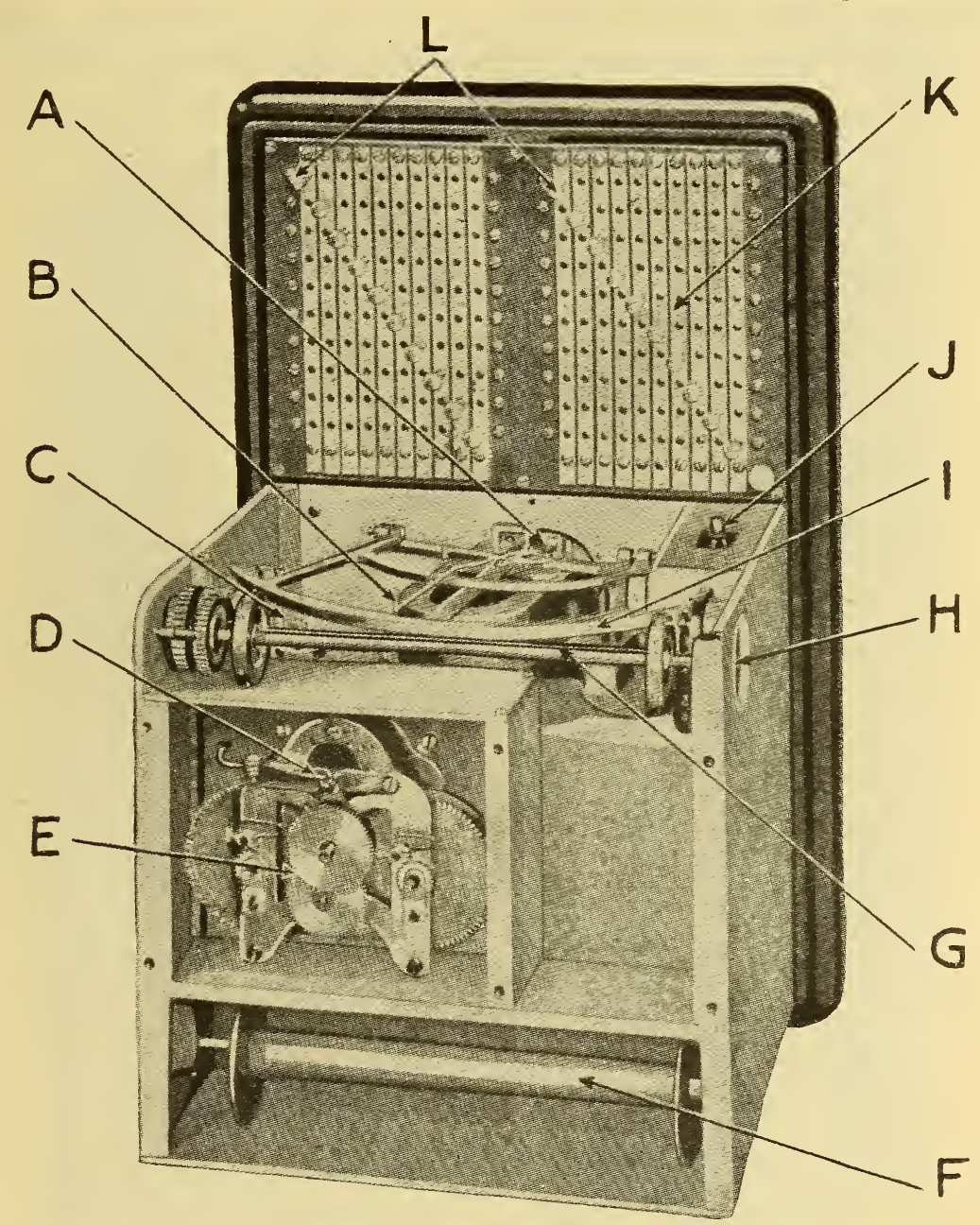

Fig. 86.-Types of roll-chart galvanometric recorders, multiple-point recorder

can of course be made for any external resistance met with in practice.

Fig. 87 illustrates the Taylor Instrument Co.'s thread recorder for single records. The instrument may be obtained with double scale range and the change from one range to the other is accomplished by means of a removable plug resistance located in the 
lower part of the case. Using an inked thread gives rectangular coordinates. The illustration shows the ease with which replacements are made over the five small pulleys, one of which keeps the thread taut. The large clock has two parts, one of which moves the chart, while the other lifts the depressor frame. The depressor frame or bar is lifted by the clock through a pawl, which engages a slowly turning rachet. The pawl is tripped out of en-

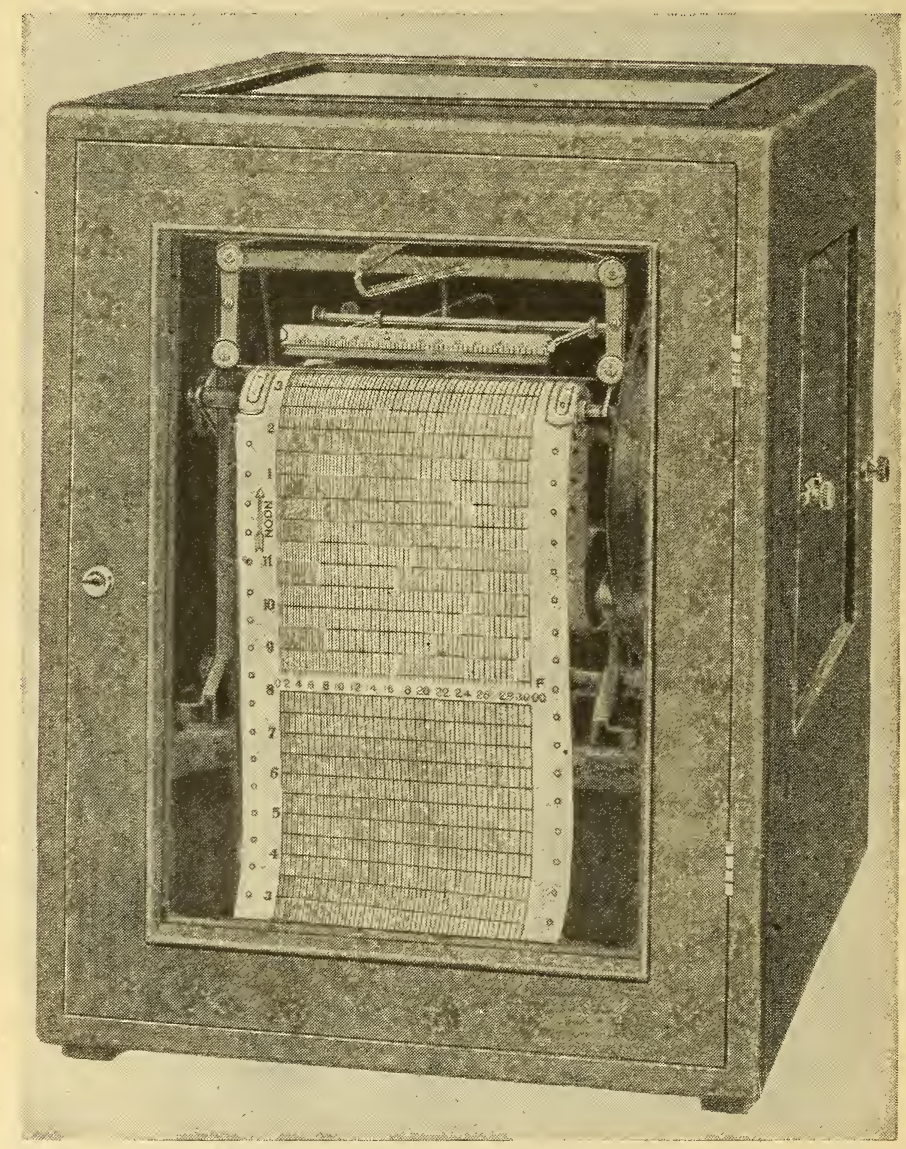

FIG. 87.-Types of roll-chart galvanometric recorders

The record is obtained by pressing an inked thread against the paper

gagement, allowing the bar to fall, pressing the pointer against the thread and record paper.

Figs. 88,89 , and 90 are three views of the multipyrograph, a thread recorder for multiple records. The mechanism is built on a heavy metal frame, which slides in grooves, so that the entire works may be pulled forward for examination and cleaning, etc. The principal additions to the thread recorder are an electro- 
magnet, commutating switch, and index dial showing numbers through the small opening in the left-hand box. The commutating switch is inclosed in the rectangular box below and is connected through the sprocket chain, which is turned in steps by

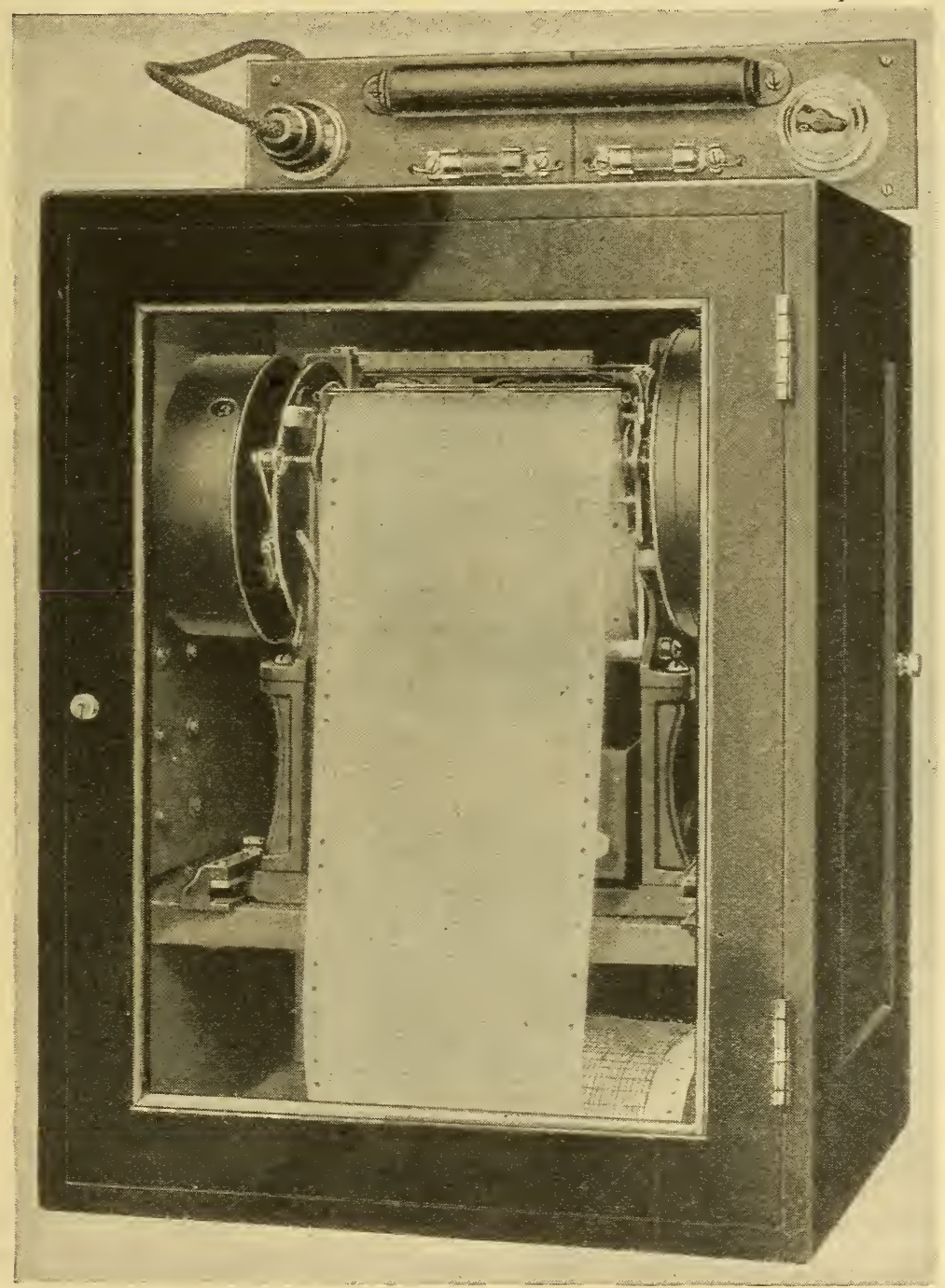

FIG. 88.-Types of roll-chart galvanometric recorders, multiple-point recorder The record is made with inked threads

the electromagnet. The switch consists of a row of long, flat, phosphor-bronze springs with platinum-rhodium contacts. One spring at a time is pressed by a cam, which is properly synchronized with the numbers and colors of the threads. A wiping action 
is given the contacts by an offset arrangement, assuring good contact. This principle of a wiping, or sliding, action of contacts is applied quite universally, though only in the better class of recorders are rare-metal contacts used. A high-resistance (500 ohms and more) galvanometer would render unnecessary the assured perfection of contact which is afforded by a wiping action and platinum points.

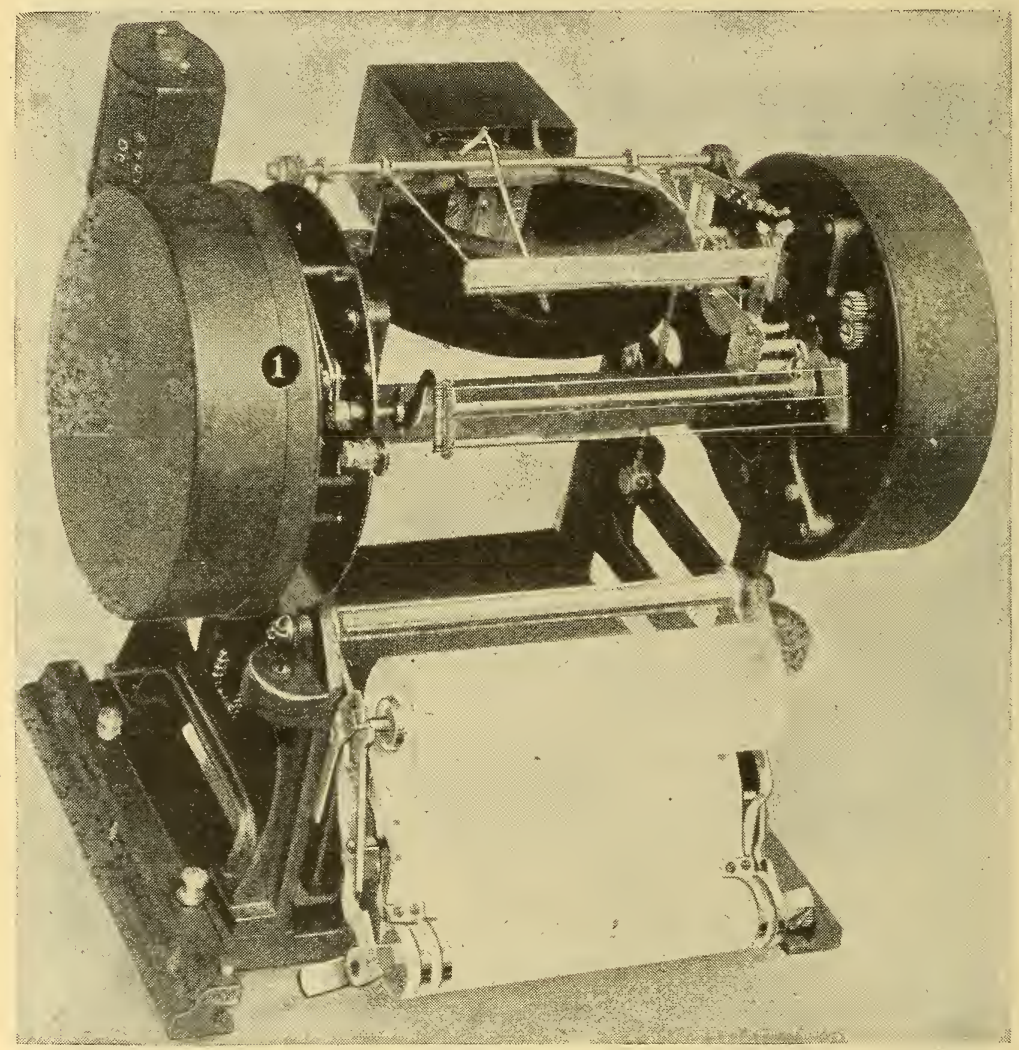

FIG. 89.-Detail of instrument shown in Fig. 88

The clock of this recorder does not require winding, but only provides an escapement for the gradual release of power supplied by the electromagnet. The solenoid coil raises a heavy bar, which is retained in the upper position by a pawl catching on a ratchet. The latter is allowed to turn at a uniform rate by the escapement. The motion of the magnet turns the switch cam shaft and operates the depressor arm. The chart is run through the power supplied to the ratchet by the fall of the lifted parts. Electric contact is made every one-half minute, the interval being 
governed by the position of the pawl; that is, the number of ratchet teeth passed during the rise of the marnet.

Three inked threads of different colors are mounted on a frame, as shown in Fig. 89, in which the chart rolls are shown lowered into the position for replacement. The threads are brought alternately into positions governed by the commutating mechanism. Six records are obtained by changing the order of records in such a way that three records appear as a line of uniformly spaced dots and three appear as lines of dots spaced in pairs. The proper selection of numbers will usually keep records of the

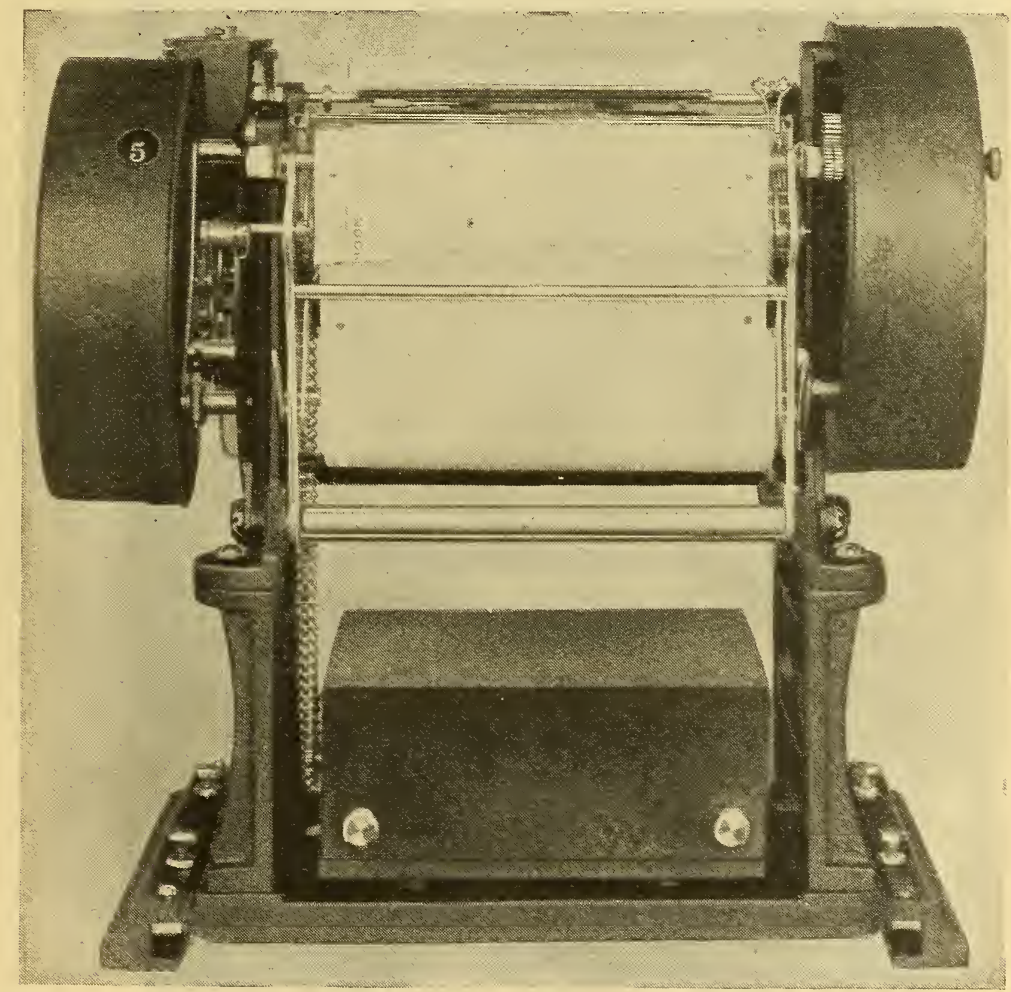

Fig. 90.-Detail of instrument shown in Fig. 88

same color well separated. The moving coil of the millivoltmeter is given additional protection from dust by the casing shown in Fig. 89.

To make this recorder operate on one thermocouple, the couple is connected to the six terminals in parallel. If a record of only one color is desired the threads may be replaced by three of like color. The scale of the chart is $4 \frac{\mathrm{I}}{2}$ inches (I $.4 \mathrm{~cm}$ ) wide and may 
be graduated in various ranges of degrees Fahrenheit and centigrade.

The Thwing recording pyrometer is illustrated in Fig. 9I, which shows a type making six records with two galvanometers. Records are distinguished on this instrument by-making one record of 3 -minute dashes, the second of $1 / 2$-minute dashes, and the third

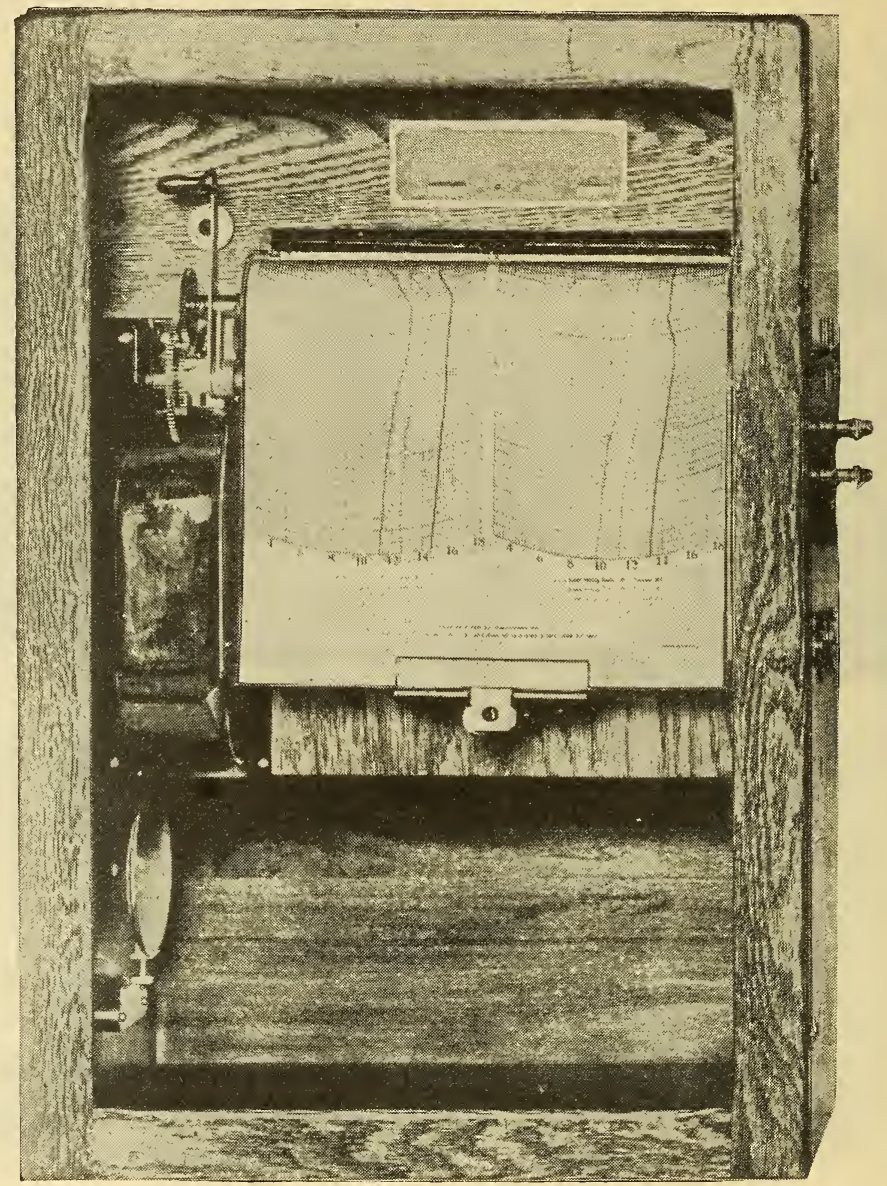

FIG. 91.-Types of roll-chart galvanometric recorders, six-point recorder with two galvanometers

with dots. The cycle requires 7 minutes, since both galvanometer pointers are depressed at once. After the third record the thermocouple circuit is opened, and the pointer returns to zero, where it is pressed upon an inking pad. This method of making easily distinguished records is simple and results in no confusion. The Thwing recorders are also made with three or four galvanometers 
giving 9 or 12 records. The width of scale decreases with the number of records, and for $\mathrm{I} 2$ records, is but $2 \mathrm{I} / 2$ inches $(6.3 \mathrm{~cm})$ wide. The mechanism of the instrument is very simple and can be made so, even with considerable friction in operation, because the clock is very powerful and is wound once daily. The clock does all the work, turning the paper roll, depressing the needle, and closing and opening the thermocouple circuits. The commutator contacts are of silver and should give good results with a slight

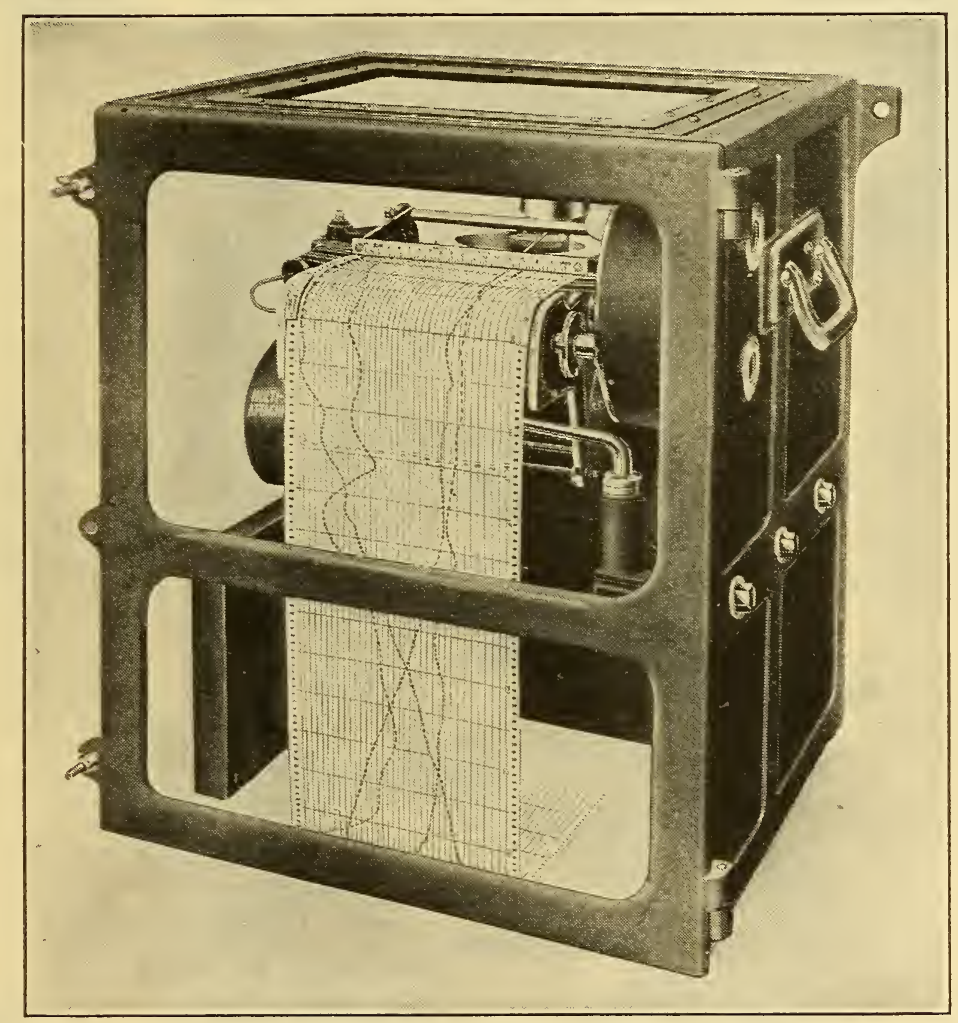

FIG. 92.-Types of roll-chart galvanometric recorders, multiple-point recorder

wiping action, which is obtained. This recorder is often furnished to operate on combinations of thermocouples and one or more radiation pyrometers, which is frequently very convenient. The galvanometers used are double-pivot instruments of a standard Thwing type, which has been described previously. The resistance can be made very high-in the more recent instruments as high as 20 ohms per millivolt.

The Wilson-Maeulen recorder, known as the Tapalog, is made for either single or multiple point records and is illustrated in 
Figs. 92 and 93. The figures show a four-record instrument, which is the most common form. The very heavy and strong construction of this instrument is easily recognized in the illustra. tions. The case is heavy enameled cast iron with one-fourth inch plate-glass windows. The works are mounted on a bronze casting supported on an iron shelf. There is no need of an extra dustproof cabinet, and this case is bolted directly to a wall.

Rectangular coordinates on the chart are obtained by pressing the galvanometer pointer against a nearly sharp, straight edge extending across and underneath the record paper. In Fig. 93

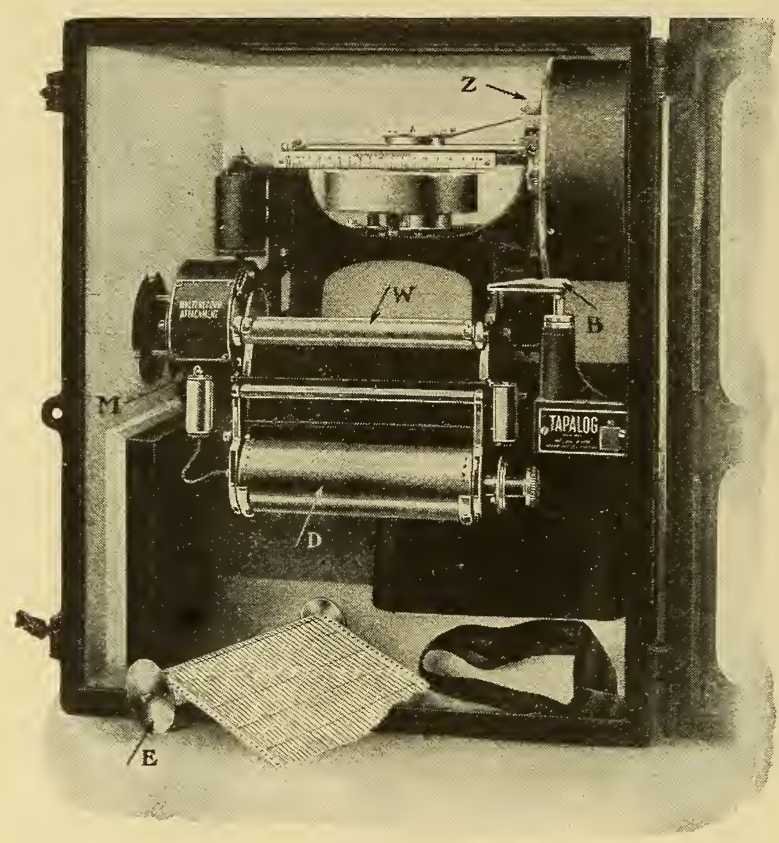

FIG. 93.-Detail of recorder shown in Fig. 92

the chart carriage is lowered into position for change of paper and inked ribbon, which here have been removed. The sharp, straight edge is visible midway between the sprocket roller $W$ and the sprocket drum $D$. The front of the depressor frame, sometimes called the chopper bar, is straight and in line with the sharp, straight edge. The pointer is thus caught between two edges, giving a dot under the paper, which is thin and translucent. The records are distinguished by different colors. Dots are made at ro-second intervals, 8 dots to a thermocouple, and a change of couples every 80 seconds. The recorder may be made to persist in indicating the temperature of one couple by moving the lever 
to "Single," which disconnects the commutator within the cylindrical box on the left.

Power is obtained from three dry cells placed within the case, and the speed of recording operations is regulated by the clock in the

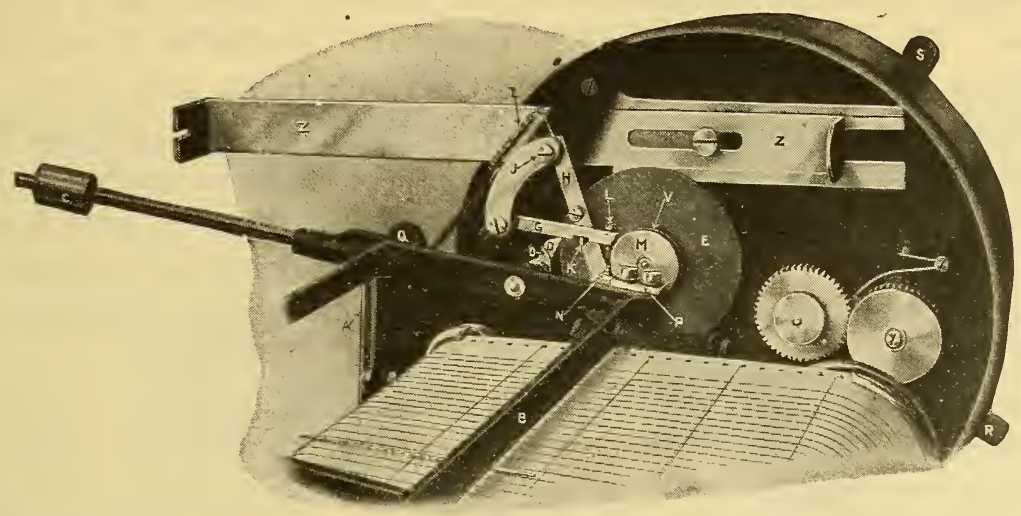

FIG. 94.-Contact mechanism of recorder shown in Fig. 92

box at the upper right-hand side. The clock case is the background in Figs. 94 and 95. It is a powerful eight-day clock with two springs. The escapement is mechanically linked with the recorder parts through the sprocket $E$, Fig. 94, which is engaged by the

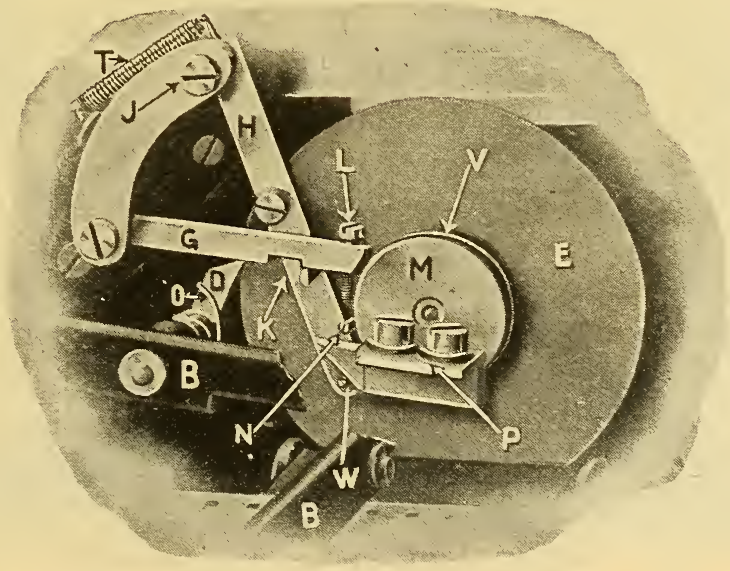

FIG. 95.-Contact mechanism of recorder shown in Fig. 92

pawl $D$. The chart drum is driven by the pair of gear wheels seen in the lower right-hand corner. The following description explains the operation of the contact mechanism and the method of taking the load off the clock. The depressor frame $B$ is held up by 
the overbalancing weight $C$ and is allowed to rise slowly by the slow motion of the escapement sprocket $E$, which is engaged by the pawl $D$ and its spring $O$. In Fig. 95 the corner of $B$ has been cut away to show parts $P$ and $W$. When electric contact (battery circuit) is made, the electromagnet forces the frame $B$ down with a sharp blow. This lifts the weight $C$. The part $P$, bolted to $B$, engages the lug $W$ on the arm $H$, forcing the latter to the left until it is caught by a lug entering the notch $K$ in the $\operatorname{arm} G$. As soon as the frame $B$ is depressed it starts rising slowly with the motion of $E$. When the frame $B$ reaches a certain point, it lifts the arm $G(P$ strikes $L$ ) disengaging it from the lug on $H$, allowing $H$ to be pulled into its original position by the spring $T$. The latter operation is sudden, so that the lower end of $H$ strikes the spirally wound wire $V$ on $M$, making electric contact in the battery circuit. Thus the cycle is complete. In this cycle, energy is intermittently stored up in the spring $T$ and the weight $C$. The contact on $H$ and the wire $V$ are platinum-rhodium and platinum, so that good contact is always made.

Eight strokes of an electromagnet store up sufficient energy to shift the commutator. This is not shown in detail, as the parts are so small and superposed as to make it difficult to show their operation photographically. The contacts are of rare metal and a combined blow-and-wipe contact is employed.

The galvanometer of this recorder has the unipivot system described in the section on indicating instruments and may be obtained with a resistance of 450 ohms for a 55 -millivolt range.

\section{THERMOCOUPLE RECORDER OPERATING ON THE POTENTIO- METRIC PRINCIPLE}

The principle of operation of the potentiometer circuit has been described elsewhere. The three steps in the operation-adjusting the current through $D E$, Fig. 24, connecting the thermocouple (the galvanometer is deflected), and moving the point $G$ until the galvanometer deflection is zero-are done automatically in the multiple-point potentiometer recorder.

The Leeds and Northrup curve-drawing recorder is a potentiometer recorder for one couple only. The ink pen remains in contact with the paper chart at all times, and a continuous terraced curve is drawn. In this single-point recorder, the current through the slide wire is set by hand, since a commutator would be required to do this automatically, and the addition of a commutator converts the instrument into a multiple-point recorder, as illustrated 
in Figs. 96 and 97. The potentiometer recorder consists essentially of a potentiometer with the mechanical device shown in Fig. 98 for automatically changing the slide-wire contact and moving the pen across the chart. It is the perfection of this simple device that has made possible the application of the potentiometric principle to the autographic recorder.

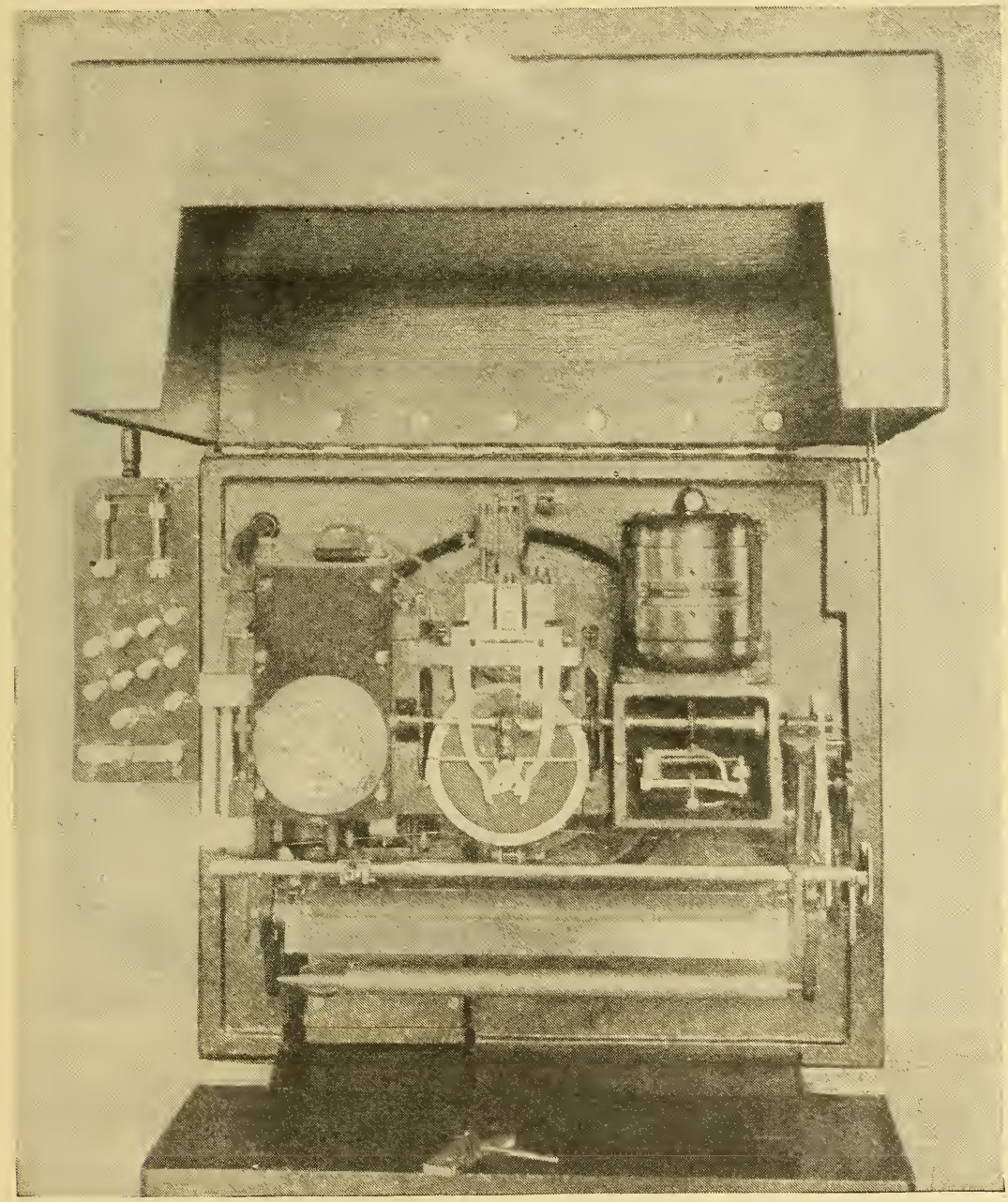

FIG. 96.-Potentiometric recorder

Figs. 96 and 97 show the construction of this instrument in some detail. The case consists of a cast-iron back with an oakand-glass cover, raised in the illustrations. On the left of the case are shown the main switch, the thermocouple, and battery binding posts, and, on this particular instrument, an automatic 
cold-junction compensator. On the left, within the case, is the double-pole commutator with five sectors, one of which is for the standard-cell connection. The motor is mounted above the box containing the governor, which is attached to the end of the motor shaft. The gears and cams on the right connect the main shaft with the commutator, print wheel (in place of pen), and paper chart. In Fig. 98, the moving coil of the galvanometer is marked

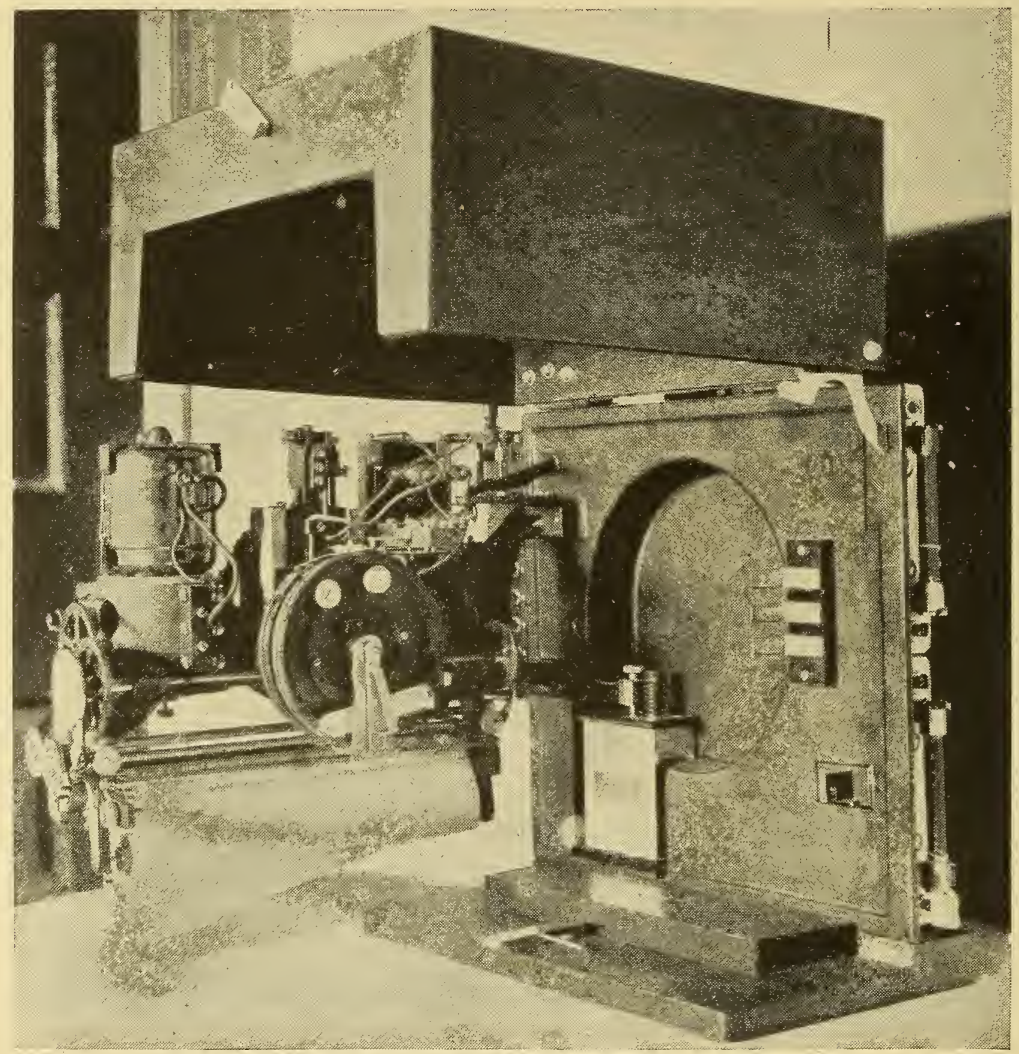

FIG. 97.-Detail view of instrument shown in Fig. 96

7 and this is seen in Fig. 96 between the pole pieces of the horseshoe magnet.

The chart of the Leeds and Northrup recorder is Io inches (25 $\mathrm{cm}$ ) wide and has rectangular coordinates. The paper has been removed in Fig. 96, showing, near the bottom of the case, the empty roll. The paper goes from this roll over the drum and out of the case through a slot in the bottom. The pen in single-point recorders, or the print wheel in the instrument illustrated, travels on a rod above the drum and is attached to a cord. In Fig. 96 
this rod is turned in synchronism with the commutator, so that the proper number on the print wheel is down. The rod is kept away from the paper, against the tension of two helical springs, by arms at each end, which are pressed against the peripheries of two cams on a shaft running in the rear of the frame seen in Fig. 97 , where the entire works is swung outward on a heavy cast-iron frame.

A short description of the device shown in Fig. 98 and its cycle of operations will explain how the deflection of the galvanometer results in a movement of the slide wire and pen without requiring that the galvanometer do any work. The disk I is mounted on

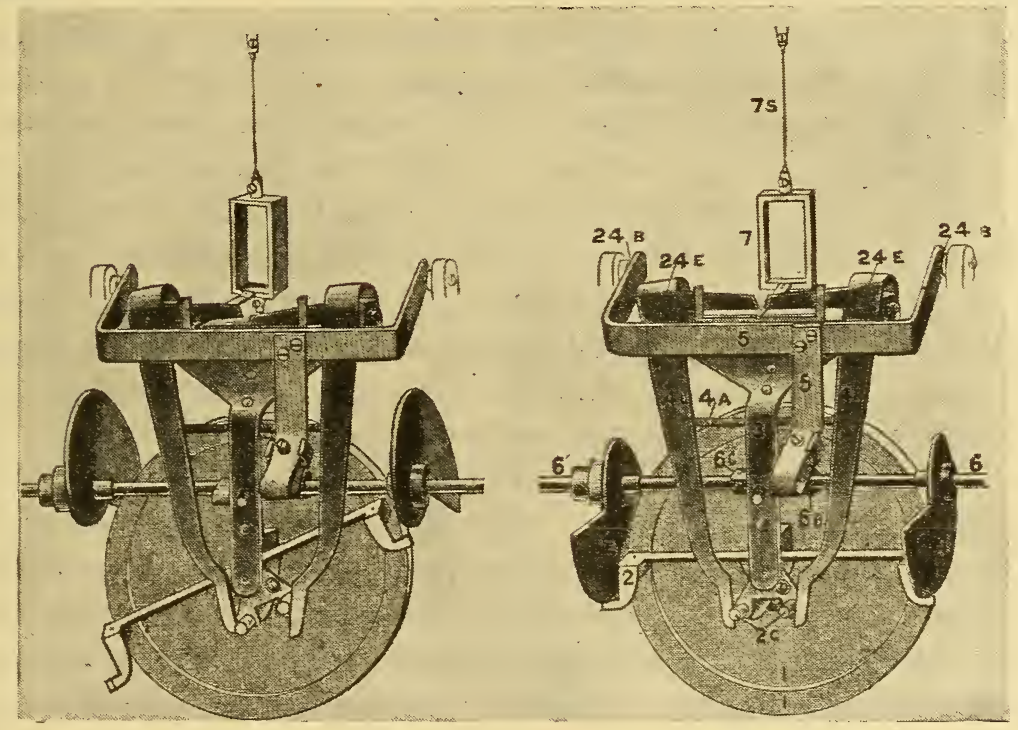

(a) mechanism uubalanced

(b) mechanism balanced

FIG. 98.-Balancing mechanism of potentiometric recorder

the same shaft as the slide resistances $R$ and $D E$, Fig. 24, which are wound on the circumferences of the disks visible in Fig. 97. The power supplied by the motor enters this mechanical system through the shaft 6 carrying the large cams $6 E$ and the small cams $6 B$ and $6 C$. At each revolution of the shaft 6 the cams $6 E$ straighten out the arm 2, which, perchance, has been tilted a moment before, and in doing this rotate the disk I, arm 2 being pressed at this time against the disk I by the spring 3. The arm 2 is pivoted on the spring 3 , which is fast to the frame of the instrument. When the cams $6 E$ have rotated until their longest radii are passing the extensions of arm 2, the cam $6 C$ begins to 
raise 3 , lifting 2 away from the disk. When 2 is free, the cam $6 B$ raises the rocker arm 5, which, in case the galvanometer is unbalanced, catches the pointer under one of the right-angle levers ${ }_{4} L$ and ${ }_{4} R$ pivoted at $24 E$. The lever ${ }_{4} L$ or ${ }_{4} R$ is thus made to swing the arm 2 by pressing against one of the eccentrically located lugs $2 C$. The rocker arm 5 is then immediately lowered to allow the galvanometer to swing freely. Cam $6 C$ is so shaped and fixed on the shaft 6 that it will recede from the spring 3 , allowing 3 to press 2 against the disk just before the cams $6 E$ begin once more to straighten 2 .

This mechanism, in its cycle of operations, moves the contact on the slide wire whenever the potentiometer is out of balance with the thermocouple and in so doing operates to obtain or restore the balance. The shaft 6 rotates once in about 2 seconds, which is slow enough to allow the galvanometer time to come to rest, or nearly so. This design is such that the amount of rotation of the arm 2 increases with the extent of the galvanometer deflection, since the pointer approaches the fulcrum of the lever ${ }_{4} L$ or ${ }_{4} R$ as the deflection increases. The motion of 5 is adjusted so that the rotation of 2 will correspond to a rebalancing step of the pen of three-fourths of an inch ( $19 \mathrm{~mm}$ ) when the deflection is a maximum, and decreases uniformly to about one-fiftieth of an inch when the deflection is just sufficient to catch the boom under one of the right-angle levers. This gives sufficient rapidity of the various actions to take the pen the width of the scale in somewhat less than I minute. A record is made once a minute on the multiple-point recorders of standard design. The position of the pen, when a balance has been obtained just before each record, corresponds to a definite point on the slide wire, for the pen is fixed to a cord fast to the slide-wire disk and is wound or unwound with the rotation of the disk.

Once during a revolution of the commutator, the thermocouple is disconnected and the standard-cell connection made. At the same time the potentiometer slide wire is let loose from its shaft, and the clutch engages a second resistance $R$, Fig. 24 . Movements of the disk then result in changing the resistance of the battery circuit, and the current is thus set to its proper value. The pen does not follow this adjustment, and no record is made of variations in the current. With batteries in fair condition, the current is easily maintained constant; but if there arises any doubt of this constancy, the recorder may be watched for a few 
minutes, and when the standard-cell connection is made the first deflection of the galvanometer is an indication of the change in the current since the last adjustment. A short-circuiting contact on the slide wire carries the pen to zero on the chart when the battery has run down, thus providing ample warning under most circumstances.

The scale of this recorder is uniform when graduated in millivolts, and departs from uniformity for a temperature graduation according to the temperature-electromotive force relation of the thermocouple. The standard galvanometer is sufficiently sensitive to work satisfactorily with a full-scale range of ro millivolts, which gives a very open scale, particularly for base-metal couples, when $0 . \mathrm{I}$ inch $(2.5 \mathrm{~mm})$ of scale corresponds to $2.5^{\circ} \mathrm{C}$. This recorder is used with resistance thermometers, in which case the electrical circuit takes the form of a Wheatstone bridge, and the scale can be opened until the entire range corresponds to as little as $2^{\circ} \mathrm{C}$. The zero of the scale can be adjusted to correspond to any fixed electromotive force, so that the scale may be put within any range of temperature desired. The great adaptability of the instrument is readily apparent. Some of the applications will be considered in the section on temperature control.

\section{TRANSFORMATION-POINT INDICATORS AND RECORDERS}

Instruments for obtaining transformation or critical points in steels have been in use for a number of years and are fairly well known in the steel industries. The simplest, being also the least accurate method of measuring the temperature at critical points, is to record or plot from indicator readings the temperature-time curve of the sample of steel when placed in a furnace and heated. This method requires a very steady rate of heating, and the sensitivity is ordinarily only sufficient for high-carbon steels. It is possible, by using a potentiometer and very sensitive galvanometer and forcing the furnace to rise in temperature at a fixed rate, to obtain a temperature-time curve that will indicate plainly all the transformations. There is great difficulty in keeping the rate of heating constant, and it is not necessary to control this rate better than to keep it from changing rapidly. However, in the research laboratory, good heating-rate control, sufficient sensitivity, and an accurate method of measuring time intervals, as with a chronograph or stop watch, will give all the necessary $8513^{\circ}-21-11$ 
data, sometimes requiring for a complete interpretation the replotting of the data ${ }^{19}$ as temperature versus inverse rate, $\frac{\Delta t}{\Delta \vartheta}$.

Burgess has described the use of a neutral body and the differential-couple arrangement (first devised by Sir Roberts-Austen) which tends to avoid to a large extent the destruction of useful data by variations in heating and cooling rates. The differential couple is mounted with one hot junction in the test piece and the other in a neutral body (one with no transformation points).

The data obtained are curves of temperature of test piece versus temperature difference between test piece and neutral body. These are mounted side by side in the furnace and must be of such size, specific heat, emissivity, etc., as to heat and cool at nearly the same rate.

The industrial laboratory requires a method of automatic, or at least semiautomatic, recording of cooling curves. Simplicity of the apparatus implies a limited scale range, incompatible with the direct $\vartheta$ versus $t$ method, and requiring the use of the differential thermocouple. To avoid errors due to improper measurement of time intervals, the records of temperature and temperature difference must be on one chart and are best obtained from a single galvanometer connected alternately to the thermocouple in the test piece and the differential couple. A curve of temperature versus temperature difference may be obtained simply in a semiautomatic recorder using two galvanometers.

(a) The Brown Transformation-Point Recorder.-Fig. 84 illustrates the type of recorder applied for this purpose. The single galvanometer of this instrument has two windings of low resistance, one of which has in series with it a high resistance and is employed to measure temperatures. This coil is connected permanently to the thermocouple in the test piece. At intervals a record is made of its temperature. At alternate intervals the other coil is connected to the differential couple, and a record made of its temperature difference. This difference shows on the chart as the distance apart of the two curves of $\vartheta$ and $\vartheta-\vartheta^{\prime}$. If the first coil were disconnected during the time the other is connected, $\vartheta-\vartheta \vartheta^{\prime}$ would be measured by the deflection from zero on the chart. But $\vartheta-\vartheta^{\prime}$ may be positive or negative, and this arrangement would necessitate a reversing switch and offer no advantage. To obtain sufficient sensitivity base-metal couples

${ }^{19}$ G. K. Burgess, On Methods of Obtaining Cooling Curves, B. S. Sci. Papers, No. 99. 
and rather large test pieces are used. The instrument will show a curve with a very marked jog at the eutectoid transformation point, and by close observation the allotropic transformation point $A_{3}$ may be detected in pure iron. There is not an excessive sensitivity, but practically the best that can be done with a doublepivot galvanometer.

(b) The LeEds \& Northrup Transformation-Point IndiCATOR.-This instrument is a semiautomatic recorder giving a

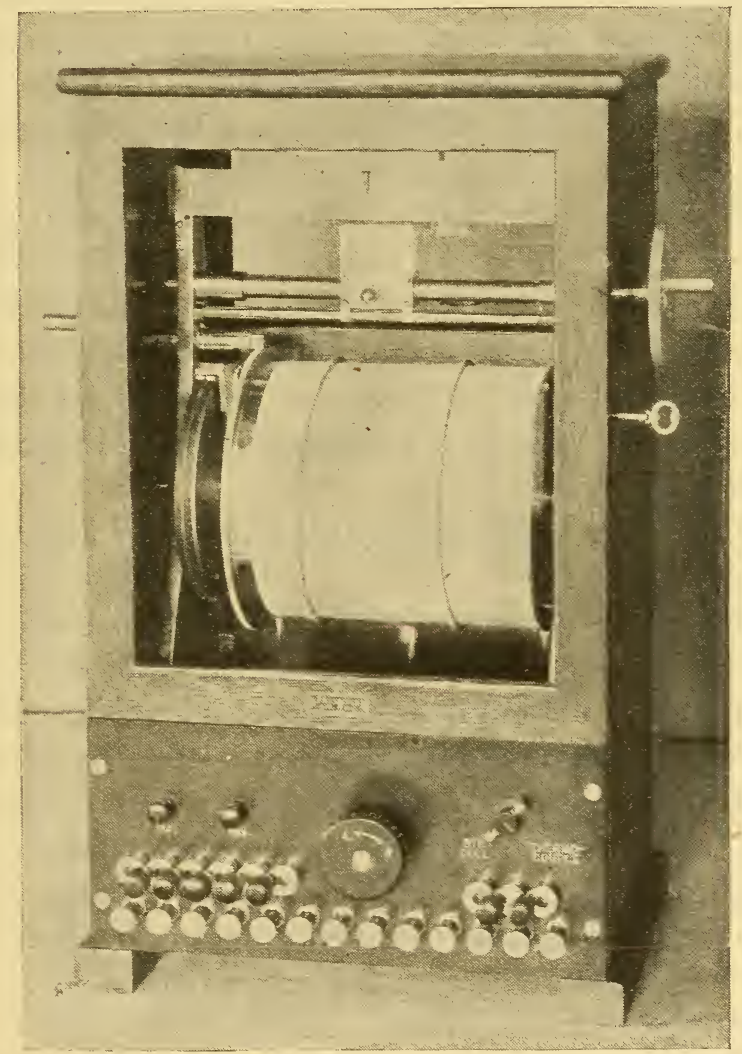

FIG. 99.-Transformation-point indicator

continuous curve of $\vartheta$ vs. $\vartheta-\vartheta^{\prime}$. One observer is required and no time measurements are made; it is illustrated, in part, by Fig. 99. The wall-type reflecting galvanometers (two) are not shown. The complete apparatus includes the two galvanometers, furnace, rheostat, and thermocouples.

The recorder consists, in the main, of a potentiometer with a slide wire and drum chart on the same shaft. In moving the slide wire to obtain a balance of the potentiometer galvanometer, 
the chart, with ordinates graduated in degrees (or millivolts), turns simultaneously and its position with reference to a pen held in contact indicates the temperature of the test piece. The pen is mounted above the chart on a carriage, which may be moved by turning a screw across the width of the chart. Upon the carriage is a ground-glass screen with a central vertical mark. A spot of light from the differential galvanometer is focused on this screen. The differential couple is connected directly to this galvanometer, and its deflections are followed by turning the pen-carriage screw, keeping the mark on the glass coincident with the light beam from the galvanometer. A separate glass scale is provided for balancing the other galvanometer. The observer has two motions to perform-viz, turning the slide wire (and drum) and turning the pen-carriage screw. The zero-temperature point is suppressed, and the end of the slide wire is usually made to correspond to 2 millivolts or the corresponding temperature. The other end of the slide wire corresponds to ro millivolts, giving 8 millivolts, or about $800^{\circ} \mathrm{C}$, over the whole chart length of 20 inches $(50.8 \mathrm{~cm})$. This is a sufficiently long temperature scale for all practical purposes.

In series with the differential galvanometer is a resistance used to cut down the sensitivity of the galvanometer, which will be usually deflected off the scale at a eutectoid transformation point. The sensitivity is ample for detecting all the transformation points ordinarily required in practice. The period of the galvanometer is short enough to allow the heating and cooling to be done in less than I hour. The curve obtained is a continuous line, slightly ragged due to manual operation, and is easily translated into metallurgical terms. It can be replotted into a curve of $\vartheta$ versus $\Delta\left(\vartheta-\vartheta^{\prime}\right) / \Delta \vartheta$, the so-called "derived-differential curve," due to Rosenhain ${ }^{20}$ especially if errors are suspected on account of considerable differences in the cooling curves of the test piece and the neutral. This curve also aids in the interpretation of results and corresponds to the inverse-rate curve when only measurements of temperature and time are made without the use of a neutral body and differential thermocouple.

20 Observations on Recalescence Curves, Proc. Phys. Soc. Lond., 21, p. I80; 1908. 


\section{HIGH-TEMPERATURE CONTROL}

The meaning of temperature control may be extended to cover not only the control of temperatures, but also the control of processes through a knowledge of the temperatures involved. In this sense it has a very wide interest. Temperature control is involved in nearly all industrial processes, but the present discussion is confined to high temperatures.

Some of the factors increasing the difficulty in the regulation of furnaces, ovens, kilns, tanks, etc., are inconstancy of heat supply, variation in internal absorption or generation of heat, variation in heat lost by radiation, etc., and unsteady supply or composition of material to be heat treated. Each of these items is intimately associated with temperature and temperature variation. Hence control is best accomplished when the relation between these factors and temperature and temperature variations is understood.

In many cases the accumulated experience of years enables an operator to control a process fairly successfully without the assistance of a pyrometric installation. Frequently the process is characterized by a definite chemical or physical change in itself sufficient for control. Occasionally the operating conditions may be maintained so constant-for example, in distillation processesthat a pyrometer merely indicates a fixed temperature within the accuracy of the instrument. The operators of open-hearth furnaces secure remarkably reproducible results by visual-observation of the slag and roof. In a pyrites dead-roast furnace the ore tends to become too hot and ball up, but before disastrous consequences result the operator has sufficient time to cool the furnace. Glass can be worked without a pyrometer because there is a considerable temperature range over which this is possible.

However, the operation of these and other processes may be improved by a suitable pyrometric equipment. Maximum efficiency is usually secured over a narrow range of temperature through which a process may be carried out. Thus in forcing the openhearth furnace to maximum production the rate of heating of the charge is limited by the refractoriness of the roof brick. If the brick melts at $1710^{\circ} \mathrm{C}$, the deterioration of the roof is very rapid at this temperature. But at say $1690^{\circ} \mathrm{C}$, at which temperature there is no physical change to aid the operator, the life of the roof is much greater. The process, therefore, can be carried out more efficiently if this temperature is maintained by the use of an optical pyrometer. With a thermocouple installed in the hottest 
bed of a pyrites furnace the temperature may be controlled below the danger point, and thus avoid the necessity of occasional drastic action to cool the furnace and lower production. Glass works best in a small range of temperature; hence, production and quality may be increased by installing pyrometers. In the lehrs, where visual estimates of temperature are impossible, the pyrometer is essential. Here it is necessary to know the highest temperature reached and also the rate of cooling of the ware. In numerous industrial processes the pyrometer is recognized as an indispensable aid to temperature control, especially in the metallurgical industries, where such close adherence to temperature specifications is required that frequently the pyrometer is taxed to furnish the required accuracy.

In order to control a furnace with a pyrometer it is often desirable to locate the instrument at the point where the largest temperature variations occur and where these variations take place the earliest. It is necessary not only to obtain correct temperatúre where this is desired, but also to obtain the temperature at some other point in the furnace, variations of which have a known significance and relation to proper operation. For example, a thermocouple may be so located that should a furnace tend to become too hot this tendency would be evidenced by the pyrometer indication and a cooling action could be initiated before any vital part of the furnace is overheated. The pyrometer should function in a like manner during cooling. Unfortunately in many processes the temperatures are too great for such an installation, and this fact is responsible for the slow development of automatic control at higher temperatures. Thus a thermocouple could not be employed for this purpose in steel and glass manufacture or in many ceramic processes, but possibly a radiation pyrometer could be used.

Often the position must be so chosen as to protect the couple even though the temperature indicated is not the one most desirable to know. One of the severest tests of permanently installed couples is in a pyrex glass tank. Rare-metal couples are mounted in the tank wall without extending to the inner face. Although the indicated temperatures of 1475 to $1500^{\circ} \mathrm{C}$ are possibly $100^{\circ}$ below the actual temperature of the glass, the method affords a fair degree of control and is about the only means suitable for recording instruments.

The methods of rendering temperature information usefulthat is, the method of indicating and recording temperatures and 
their variations-are very numerous and must be adapted to special needs in a plant. The simplest plan is to mount the pyrometer indicator, preferably of the wall type, in a conspicuous place, where the operator can read it with the least trouble; special means must often be employed for making the scale sufficiently legible. The scale must be as open as possible, so that small changes of temperature may be readily detected. Some indicators are provided with two pointers, one of which can be turned to the temperature desired. This second pointer may be made a single line or a double one, the latter indicating the limits of variation allowable. In the absence of a recorder the second pointer is nearly indispensable as a means of judging rates of change. In poorly lighted rooms illuminated scales may be used. If cheap labor is employed and the operator is unable to adequately interpret the indicator reading, signaling lights or alarms may be operated either manually or automatically. In larger plants the indicators may be situated at a central station, where the pyrometer man reads them and transmits the proper signal to the operator. Colored lights are best used for this purpose, white for correct temperature, green for too low, and red for too high, and white with red or green for small departures from the correct temperature. The lighting circuit is, of course, completely separated from the pyrometer circuit. If the plant electrician is allowed to install the wiring, he must be convinced that electrical insulation and leakage assume a new significance in electrical pyrometry. The use of these colored lights or a signal system is not restricted to control at a fixed temperature; they may be employed for governing the rate of heating up a kiln, for instance. In the ceramic industries, pyrometers are particularly useful as a means of avoiding loss of time and curtailment of production on account of a nonuniform rate of firing.

The operator, in controlling a furnace to reach a desired temperature, bases his action on experience and judgment. Possibly, he turns a gas or oil valve a certain part of one turn, according to the change just observed in the condition of the furnace. Obviously the adjustment can best be made with a knowledge of the changes which have occurred over a considerable interval of time rather than by watching the change occurring from moment to moment. This is one of the most prominent advantages of a recording pyrometer; and when it is possible and the class of labor employed warrants it, the operator should be allowed the advantages of inspecting the recorder chart. It is necessary that 
the record be made with a frequency greater than that of significant changes likely to occur in the furnace, particularly when a multiple recorder is used. In case a single-record recorder is used or a multiple-galvanometer recorder in which each galvanometer is always connected to one pyrometer, the position of the pointer or pen shows the change occurring. The recorder should be so constructed that the record is visible to the last minute.

The application of the multiple-point recorder affords further advantages in control. These arise from the paralleling of records on one chart, showing, for instance, the time-temperature curves of a battery of furnaces. It is also of advantage to parallel the records of two or more points in a single furnace which is being heated. Thus the rate of increase in temperature of a central portion of the furnace may be made as high as possible, while the record shows at the same time the temperature in dangerous portions of the furnace likely to be damaged by an excessive rate of firing.

When pyrometers are newly installed in a process where they have not been used before, the immediate result is usually confusion. Difficulty is encountered in correlating the indicated temperatures with other conditions, so that the operation may be at first even less efficient. Too much attention is given to temperature, and the attempt is made to operate without regard to other necessary factors, or the new knowledge is misinterpreted because of the errors of previous ideas. These usually exist and must be recognized. An operator after years of experience develops a temperature scale of his own, which, while useful to him, may differ considerably from the true temperature scale. The change in position of a damper may produce very peculiar results in the temperature of one portion of a furnace, quite contrary to the effect expected by the most experienced operator. The transition period following the adoption of a pyrometric equipment is bridged with difficulty and every effort should be made to correlate facts as they really exist, not as they have been thought to exist, with temperature.

The employment of pyrometers is generally least in those industries in which operating conditions are the worst because of the great difficulty met with in maintaining uniformity of conditions. It is particularly true of such an industry as the ceramic, in which the chemical constitution and physical condition of raw materials are of prime importance and difficult to control. The kiln operator often can not fire his kiln according to any certain 
time-temperature relations because he does not know how, not because it is impossible. He will never know how so long as pyrometers are not installed and the time-temperature relations are not associated with other variables. Pyrometers are now being used in kilns in order that the rate of heating may be maintained with greater certainty and a loss of time due to too slow firing be avoided, but the finishing time is determined with Seger cones. It is hoped that the Seger cones will eventually be supplanted by even more useful pyrometric methods.

\section{DEVICES USED FOR CONTROL}

Control, either automatic or manual, and automatic signaling are always accomplished by the employment of a method of forcing the furnace to heat or cool between limits, at a rate and through a range depending on the process and constancy required. Ordinarily there is little difficulty in obtaining enough sensitivity of the instrument for either automatic signaling or control, except in the research-laboratory furnaces, where at times the limit of sensitivity is employed. In control of a furnace through pyrometric measurement, it is not necessary that the pyrometer be accurately calibrated. It must, however, be particularly reliable and have a consistent error, as has been stated elsewhere.

(a) AUTOMATIC AlARM.-An ordinary pyrometer galvanometer of low resistance may be fitted with two contacts on pivoted arms, between which the meter pointer plays. No relay is necessary for the small current and voltage required to operate a bell. The automatic alarm is seldom used in this form, since the alarm operates for too large a part of the time unless the contacts are placed wide apart, when their usefulness is much lessened. The action may be made intermittent on more complicated instruments. The alarm should properly be employed only when the departure from a certain temperature range results in real danger to life or property.

(b) Manual Signaling.-The development of manual signaling has taken place, for the most part, in the larger plants having extensive pyrometric installations of such scope that a central pyrometer station is necessary. Fig. I oo illustrates a form of central station developed at the Ford Motor Co. by W. G. Vesey. With such an installation only the thermocouples and signal lights are in the furnace room and the indicators in the station are connected to different couples by means of push buttons on switchboards. The more sensitive types of double-suspension or uni- 
pivot-suspension millivoltmeters or thermocouple potentiometers are used and can indicate easily temperature variations of 0.2 per cent of the scale range. The substitution of manual operation for automatic is necessary to attain the closest correlation of temperature measurements and other physical conditions and the physical and chemical properties of the products.

Signaling is accomplished by colored lights, using three at each furnace. Temperatures are read at frequent intervals, determined by furnace operation and the operator's interpretation of signals. In some plants pneumatic tubes are installed between the furnace room and the station for transmitting notes concern-

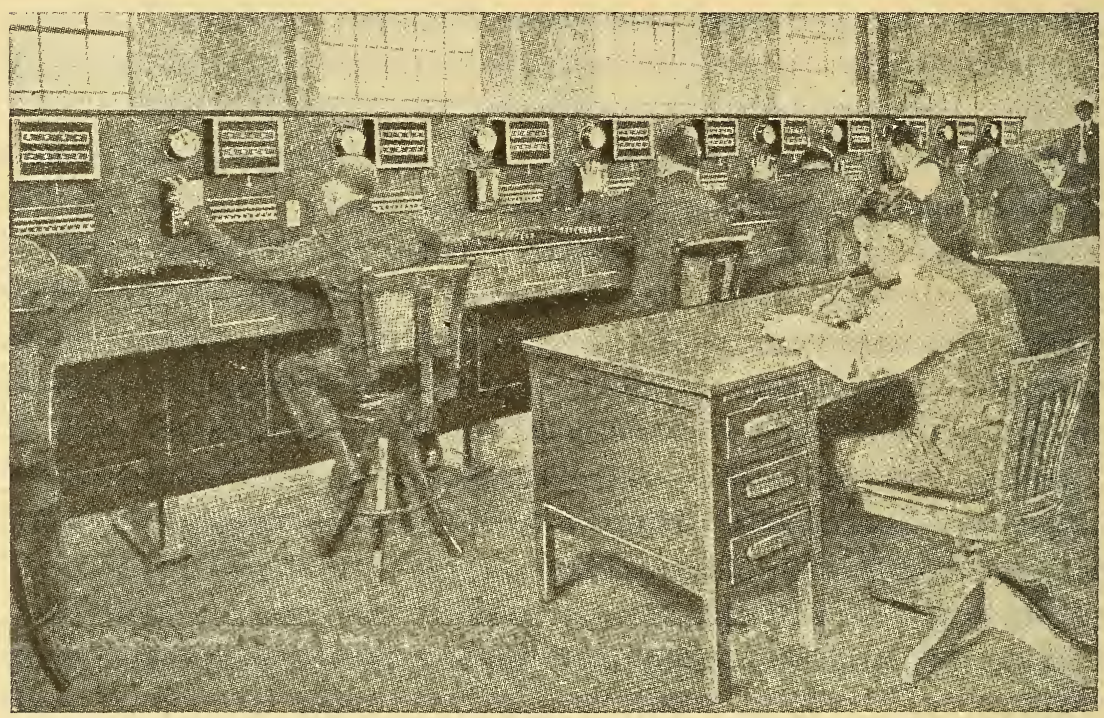

Fig. 100.-A central pyrometer station

ing the process or measurements. The furnace man can signal the station with the ordinary enunciator, as shown in Fig. Ioo, at the top of the switchboard. Such a signaling system of colored lights, enunciators, and pneumatic tubes is, of course, not peculiar to pyrometry.

(c) Automatic Signaling.-The descriptions of the various types of recorder make it clear how automatic signaling may be accomplished with pyrometer galvanometers. In a similar manner to that in which autographic records are made, a depressor bar or frame, in its rise and fall, closes either of two pairs of contacts depending on the position of the pointer. The device may be attached to an indicator, according to Fig. Ior, or a recorder may 
be made to do both recording and signaling. The latter arrangement has not been very successfully applied to the galvanometer type of recorder, but is easily adapted to the Leeds \& Northrup potentiometer recorder. In this case two contacts move with the slide wire on its shaft and the third is stationary.

The galvanometric instrument illustrated in Fig. Ior has two platinum contacts mounted on an arm which swings across the scale to adjust the signaling range. The signaling-circuit current does not flow to the galvanometer pointer, but each platinum contact is double, one above the other, and the two are brought together by the pressure of the depressor arm transmitted through the pointer. The pointer is, in this case, a selector. The arrangement of Bristol differs from this in that a tipping device

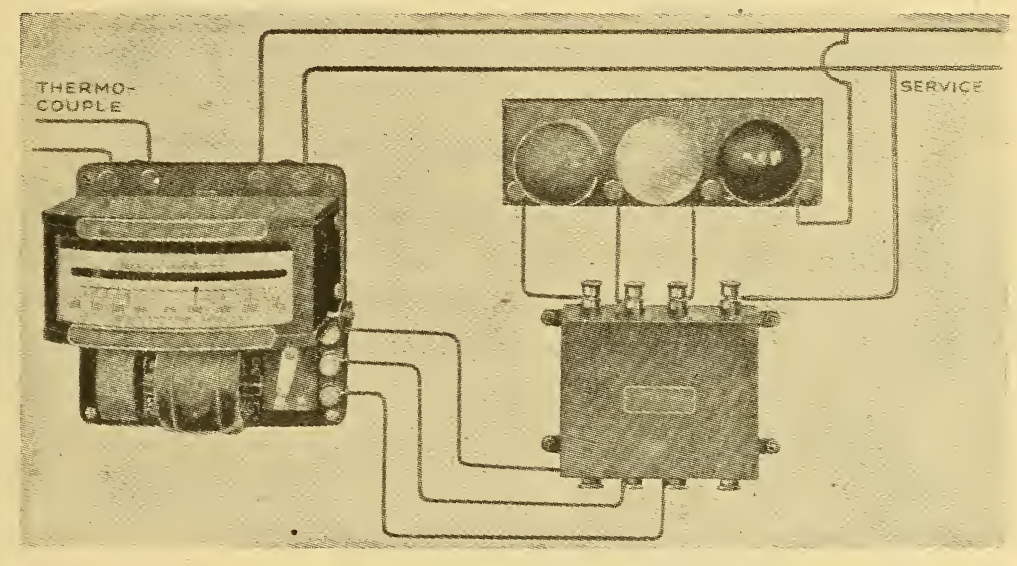

FIG. I0I.-A Atomatic signaling pyrometer

restrained by springs is tipped by the pointer when the depressor arm falls in a direction determined by the pointer. The springs are arranged to snap the contact when the pressure has reached a certain value. By this means the selection can be made to take place over a smaller interval than with two contacts placed side by side and close together. The electromagnetic switches operated by closing these contacts scarcely need description. They are made applicable to any range of voltage and require a small fraction of an ampere. The circuit may include any of the wellknown signaling devices, some of which have been mentioned.

Figs. I02, I03, and 104 illustrate, diagrammatically, the arrangement of the curve-drawing potentiometer recorder with signaling lamps and furnace indicator. The diagrams are selfexplanatory, but the new method of supplying power to the in- 
dicator may be mentioned. This is not taken from the thermocouple, but the indicator is shown in a Wheatstone bridge attached to the line, preferably i Io-volt alternating current. The movement of the disk carrying the slide wire $S$ and the three contacts

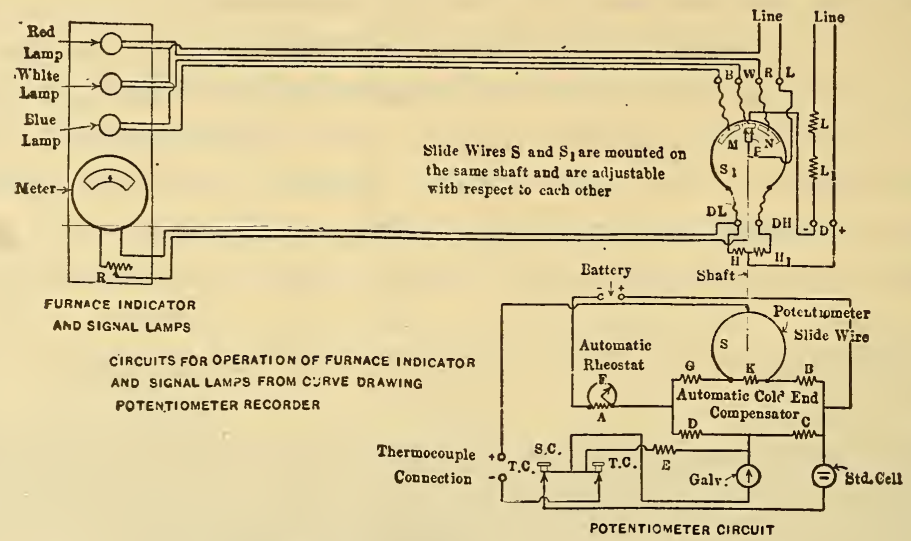

FIG. I02.-A Atomatic signaling recorder, potentiometer type

connected to the lamps results in connecting the proper lamp and in unbalancing the bridge circuit. The meter may be of high resistance and its indications are easily checked by the recorder indication and correction accomplished with the adjustable resistance in the circuit. It is only intended to show the magnitude

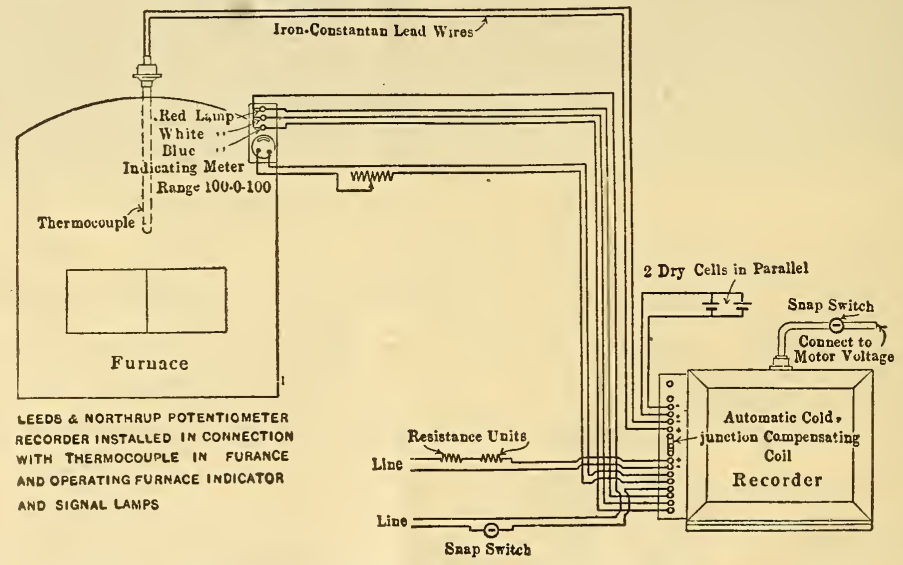

FIG. I03.-A Atomatic signaling recorder, potentiometer type

of departures from correct furnace temperatures and a large variation in line voltage would not be serious. Fig. IO4 shows the use of the double recorder, or curve-drawing recorder, with a commutator for two couples, which gives the form of record 
shown in Fig. I05. This instrument is also used in the automatic control arrangement shown in Fig. I08.

The advantages of automatic signaling are not always well defined, and the method has not been put to very extensive use.

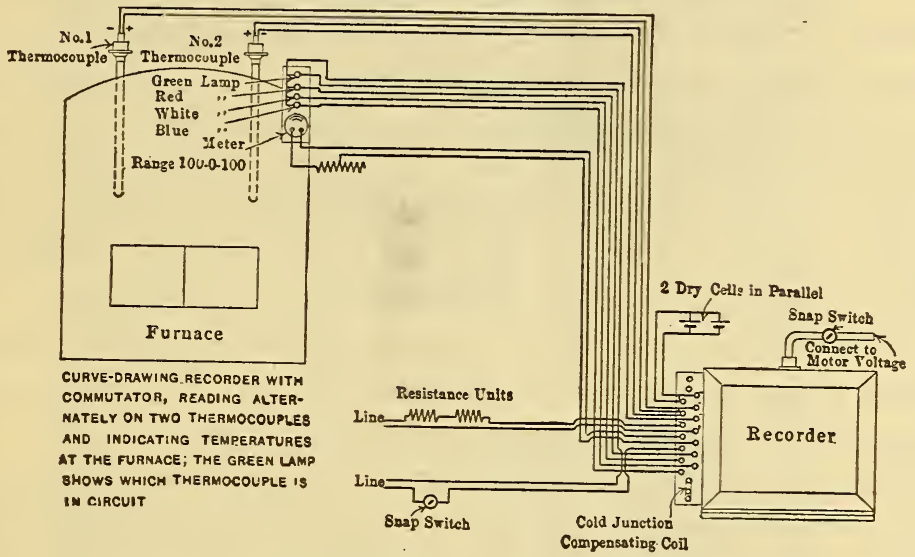

FIG. I04.-Automatic signaling recorder, potentiometer type

In cases where very exact temperature measurements and control are necessary and the sensitivity demanded results in an instrument which must be removed a distance from the furnace or oven, automatic signaling may be required; but in most cir-

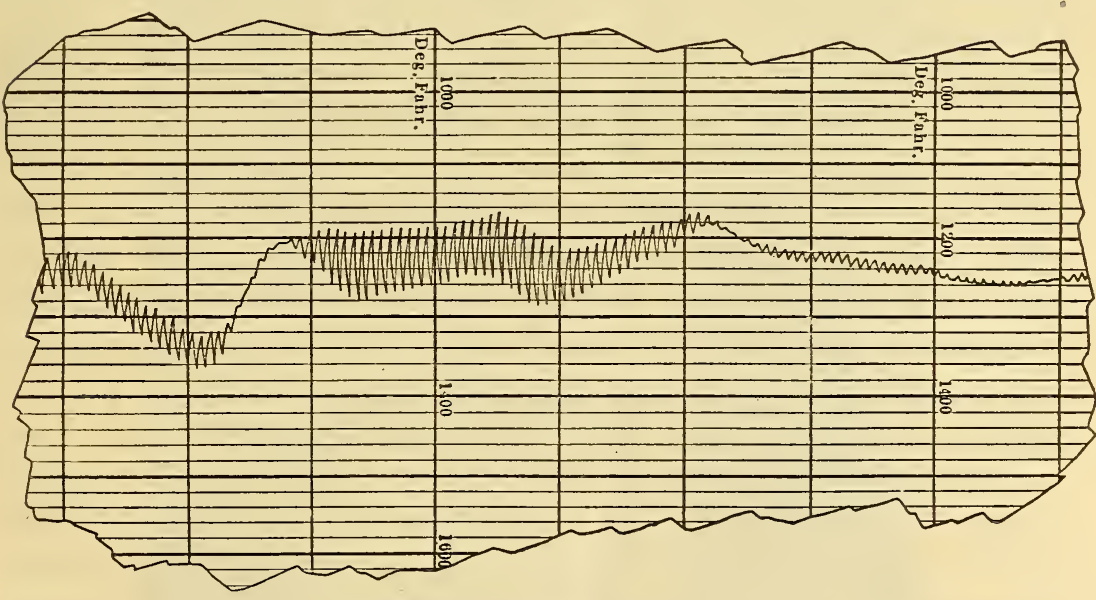

FIG. I05.-Record of double curve drawing recorder

cumstances the proper placing of the indicator or indicators will make automatic signaling unnecessary. To make one indicator operate many separate systems of signaling would require a complicated and cumbersome commutator. Such an arrangement 
might take the place of some of the observers in the central pyrometer station of a large plant, but sensitivity and flexibility would be sacrificed.

(d) Automatic Temperature Control.-Temperature control at low ranges is termed thermostating and is ordinarily obtained by means of the movement of bimetallic springs, or the thermal expansion of rods or fluid columns. At high temperatures these devices are utilized only with great difficulty, and thermoelectric instruments are substituted. The principle of operation is similar to that used in automatic signaling. Electromagnetic impulses arising in the pyrometer indicator or controller are utilized to operate oil or gas valves or electric switches. In case the valve or switch is large and the electromagnetic operation requires more than a few hundred watts, electric relays are incorporated between the controller and the valve.

Automatic temperature control is complicated by the interrelation of such factors as thermal lag and the magnitude of the corrective change in heat supply during each period of reversal. The sensitivity of the control instrument determines the range of temperature that must be covered by the thermocouple (or other pyrometer) during each reversal of the valve or switch. The range of temperature at the source of heat is greater than this, and the difference is determined by the type of furnace, the method of heating, and the locating of the thermocouple. For closest regulation, which is best accomplished electrically, the couple must be very near if not in contact with the heater. Considering the cycle of operations, if a furnace is cooling the current will be increased when the couple has dropped a certain amount, the heater being at a still lower temperature. The current may be increased by a single step upon reaching this point or a mechanical arrangement may be used, which will continue to increase the current so long as the couple has not reached the upper limit of temperature. The single step will ordinarily give the least overcorrection of heat supply, and the method is the most simple to apply. It will fail to give the desired result, however, if there exists a condition of wide variation in the amount of heat absorbed or lost in the furnace operation, for in this case the heating current change during a reversal must be large. A mechanism which is always increasing or decreasing the heat supply in small steps may be often advantageous and is practically indispensable in the arrangement mentioned below for automatic control of heating or cooling rates. 


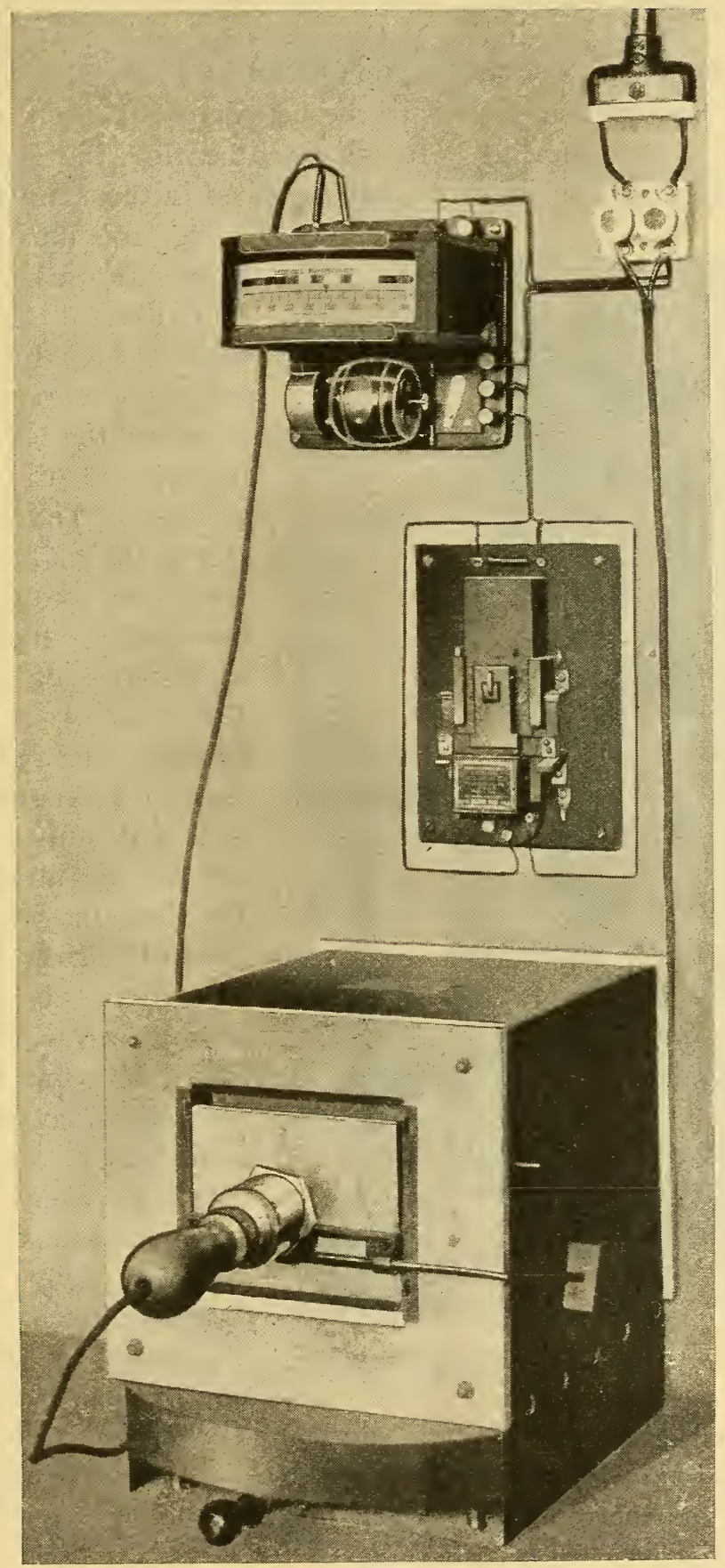

FIG. 106.-Automatic temperature control pyrometer 
In industrial equipment usually the single-step method has been applied. In electric heating, the heating current is changed by opening or closing a switch, which will shunt a rheostat, change the series or parallel connections of the heater, or change the secondary side of a transformer. In gas or oil heating, the supply pipe is by-passed and the control valve is placed in this shunting line.

Fig. I06 illustrates the

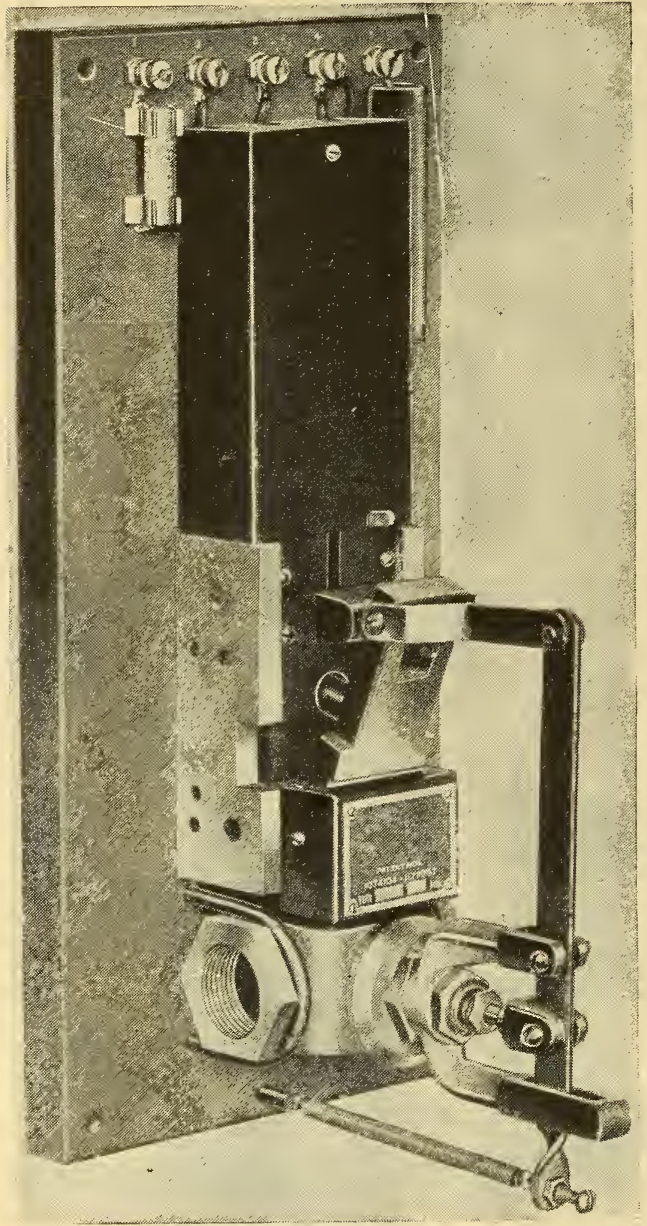

FIG. I07.-Electromagnetically operated gas or oil valve Brown automatic temperature-control pyrometer, showing the galvanometer, motor for raising and lowering the depressor frame, and the solenoid switches. The galvanometer is the high-resistance type with 300 ohms for a base-metal couple and a scale range of $1100^{\circ} \mathrm{C}$. The control is between limits I per cent of the scale range in extent; that is, it will control to about $10^{\circ}$ for the above range. The range may be lowered considerably for closer regulation, say to $3^{\circ} \mathrm{C}$, without serious disadvantage. By using a method for manual correction for resistance and lowresistance meter with a very open sale, the control may be easily perfected for less than a $\mathrm{I}^{\circ}$ variation. Obviously the cold junction of the thermocouple must be either buried deeply in the ground or placed in ice or a thermostated box. For temperatures below $425^{\circ} \mathrm{C}$ Brown substitutes a nitrogen-gas thermometer, which makes it possible to control to $0.2^{\circ}$ and better, with a scale range of $10^{\circ}$ or $15^{\circ} \mathrm{C}$. Fig. 107 illustrates the form of the electromagnetically operated valve developed. The Bristol company has also adapted the instrument described for 
signaling, so that it will control the furnace temperature automatically. This company has patented a valve somewhat different than the one illustrated, but designed to accomplish the same results.

An apparatus for automatically controlling the operation of electric furnaces for hardening steels, etc., has been recently developed.

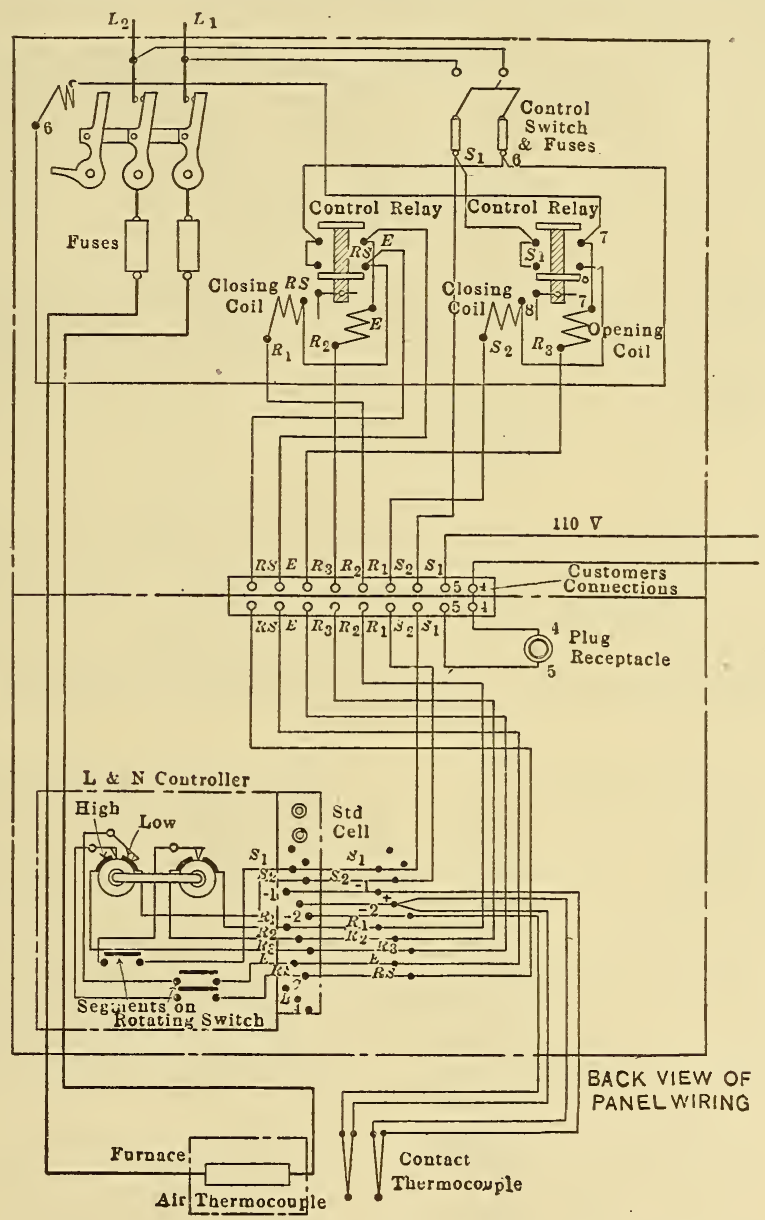

FIG. I08.-Wiring diagram of automatic control panel for electric furnaces

Fig. 108 is a wiring diagram of the control panel. This equipment automatically heats a hardening furnace up to a temperature, say $1000^{\circ} \mathrm{C}$, somewhat above that desired, say $900^{\circ} \mathrm{C}$, and holds it there until the piece being treated reaches $900^{\circ}$, when the furnace temperature is dropped to this value and held there. This unique operation is accomplished by placing one thermo$8.513^{\circ}-21-12$ 
couple, called the contact couple, in contact with the piece, and another, called the air couple, in the furnace near the wall. The air couple is kept hot until the contact couple reaches the proper temperature, when the air temperature is lowered to this value.

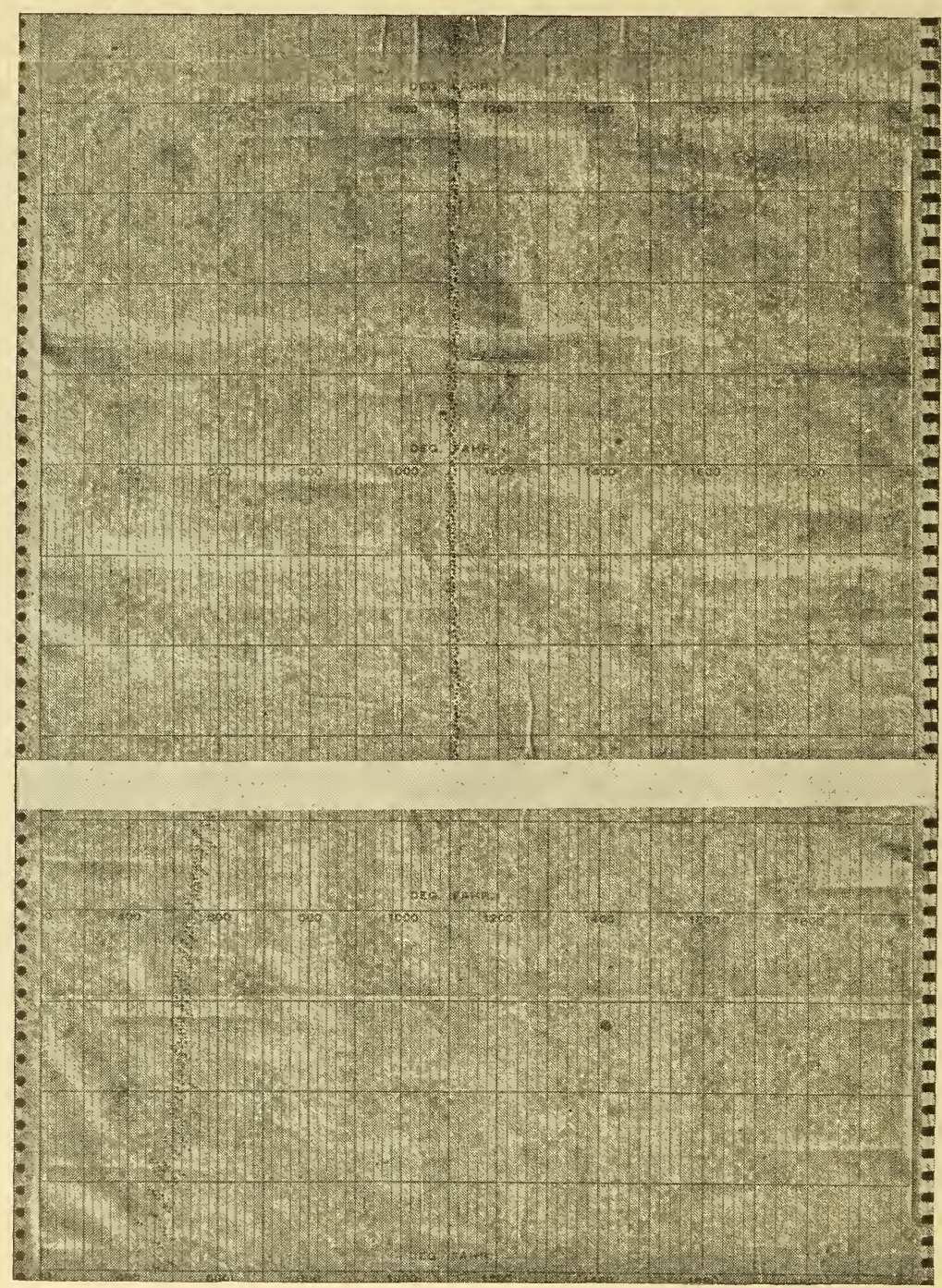

FIG. 109.-Sections from record of 24-hour annealing heat

The wiring diagram of Fig. 108 is clear upon close study of the connections and a discussion is scarcely necessary. ${ }^{21}$ The con-

${ }^{21}$ In this diagram the following must be noted: (I) Control relays are closed when $E$ is connected to 6 and $S_{1}$ is connected to 7 . (2) Connecting $S_{1}$ and 7 closes the heating circuit by the magnetic switch on the left. (3) Contact thermocouple operates through contacts marked $S_{1}, R_{1}$, and $R_{2}$, and air-couple contacts $S_{2}, R_{3}, R S$ and $E$. (4) $S_{1}$ and $R_{2}$ are connected and open a relay when contact couple reaches the desired temperature. 
tacts operating the relays are opened and closed by the automatic adjustment of the potentiometer slide wire. By disconnecting either of these thermocouples and one of the control relays the instrument may be used simply to hold a furnace at a fixed temperature. Another application of the potentiometer type of automatic controller maintains the temperature of the box ovens used in japanning at the proper point. It is made to control at one temperature for a certain interval and then automatically changes to another temperature and maintains this.

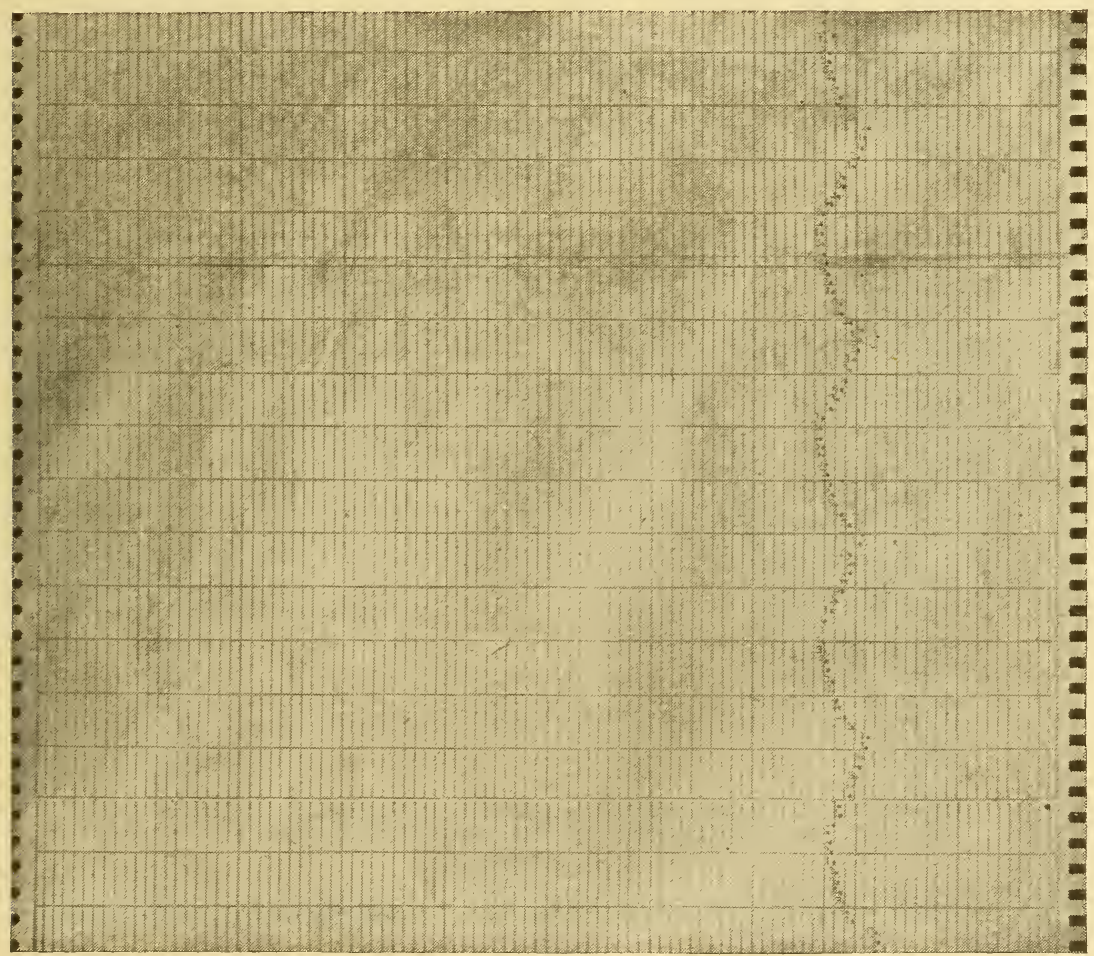

FIG. IIO.-Sections from record obtained of reversals in regenerative firing

Still another operation can be performed with this instrument. One of the writers has constructed an apparatus which wrill heat or cool a furnace at a predetermined rate, either constant or a function of the temperature. This apparatus has been used in studying the annealing of glass, especially the fine annealing of optical glass. This type of control is obtained by placing one contact on the potentiometer slide wire and the other two contacts upon a disk which is made to turn slowly at a chosen rate. The disk is turned by the controlling recorder itself, so that the 
whole apparatus is automatic. This principle of moving contacts is applicable to any of the controllers described, but at present such control has not been utilized industrially.

As a thermostat, the potentiometer-recorder controller can be made capable of operating upon o.or $^{\circ}$ changes in the tempera-

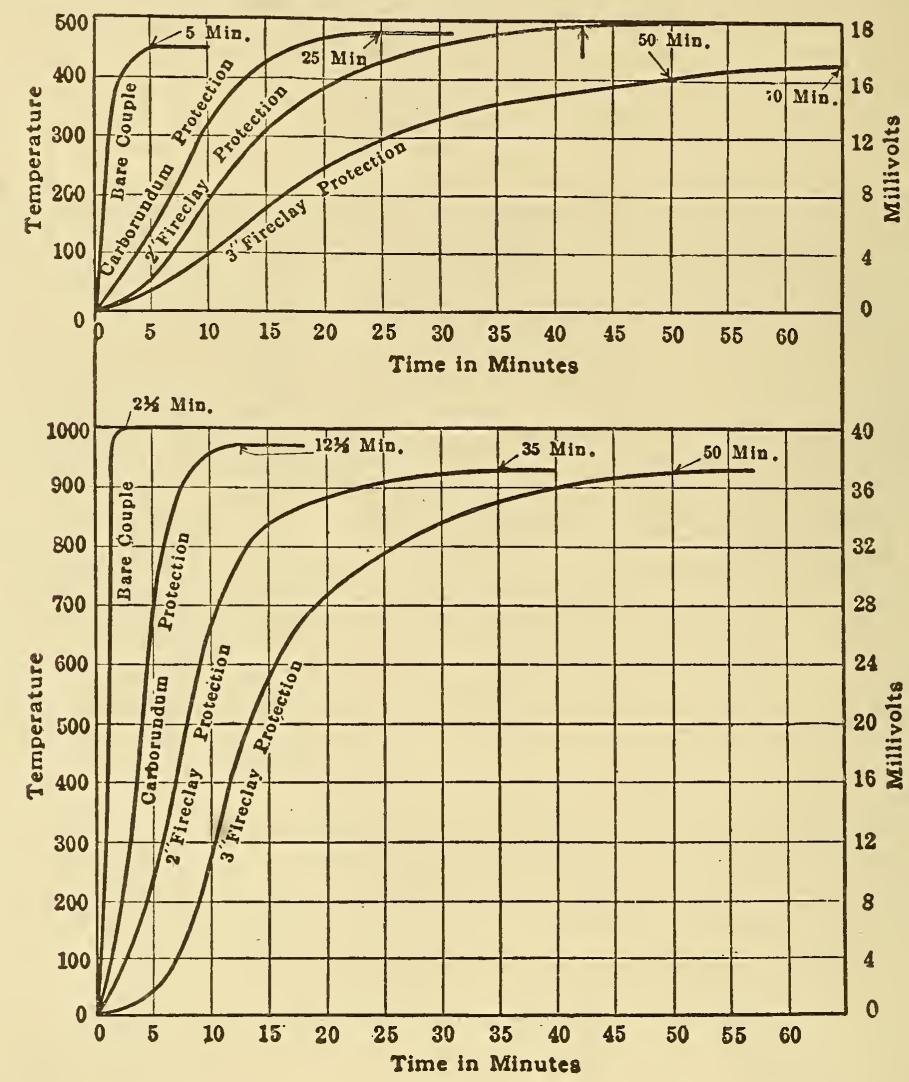

FIG. III.-Curves showing lag in pyrometer reading for thermocouples in various protection tubes

ture of a resistance thermometer and upon less than $0.5^{\circ}$ with base-metal thermocouples. Very rapid progresis is properly expected in the future development of instruments for automatic temperature control at higher temperatures. The field is a comparatively new one and undoubtedly offers possibilities not fully appreciated at the present time. 


\section{INTERESTING EXAMPLES OF THE UTILITY OF RECORDERS IN FUR- NACE CONTROL}

Fig. IO9 is a reproduction of a chart from a six-point recorcier showing the operation of a car annealer furnace $3 \mathrm{I}$ feet 4 inches long, 6 feet wide, and $44 \frac{1}{2}$ inches high from floor of car to top of rough arch. This furnace was fired from combustion chambers placed at the side and below the level of the car floor. The six points for which temperatures were recorded were in six different locations so chosen as to give the average condition of the entire heating chamber. The record shows how quickly after starting the operator was able to obtain a fair degree of temperature uniformity, and how at the last of the run the entire furnace was held for hours at a remarkably uniform temperature. This chart shows at a glance the condition desired, viz, uniformity.

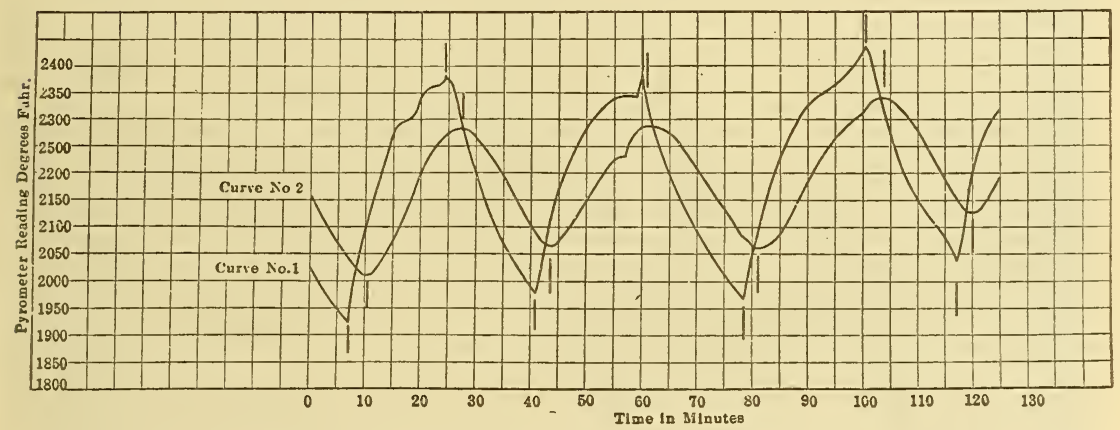

FIG. II2.-Curves showing temperature variations of slag pocket of open-hearth furnace

Curve No. $x$, couple protected by $x^{7} / 8$-inch carborundum tube. Curve No. 2, couple protected by 3 -inch fireclay tube (Hutchins)

Fig. I I is a simple example of the excellent record obtained of furnace temperatures during changes in regenerators. It illustrates the method of temporarily connecting one thermocouple to all the points of the recorder, thus securing a more complete record of the variations. If, for example, connections were made only to No. I point, the record obtained would be misleading. However, the precise shape of this curve has little meaning, for, as shown in Figs. II I and II 2, the record obtained depends considerably upon the characteristic lag of the thermocouple. ${ }^{22}$

Fig. II 3 shows waste-heat temperatures taken when a furnace was changed from nonregenerative to regenerative firing. Properly marked with day and hour, this becomes a permanent record of the operation.

${ }^{22}$ Otis Hutchins, Pyrometer Protection Tubes, Trans. A. I. M. M. E., 153, p. r8r r; Sept., rgrg. 
Fig. II 4 is a record of experimental work done in a by-product coke oven. Five points of a six-point potentiometer recorder are in use, the sixth point being short-circuited in order to bring its record to zero. (At the beginning of the record, No. 6 left on

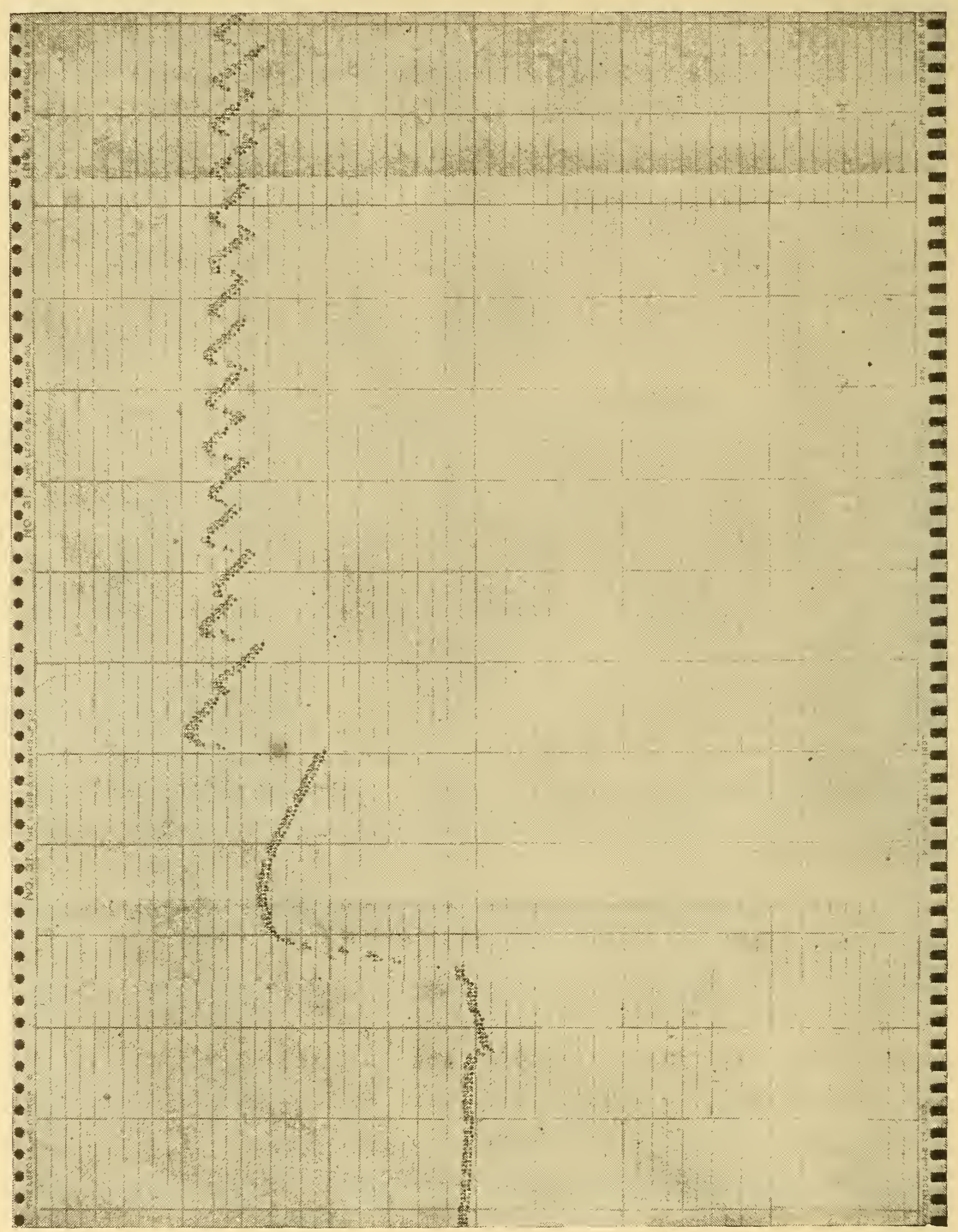

FIG. II3.-Record of changes in method of firing

open c1rcuit became confused with No. 5.) This is a recora of the last I 3 hours of a "charge." No. I is the record of temperature of the vapors above the coal in the oven. Nos. 2, 3, and 4 are from thermocouples in the center of the coal charge inserted a 
few hours before the temperature at this point rose above $100^{\circ} \mathrm{C}$. At the end of the coking period couples $\mathrm{I}, 2,3$, and 4 were withdrawn, and as the couples cooled the readings dropped to zero. No. 5 is the waste-heat record. This curve shows how incomplete

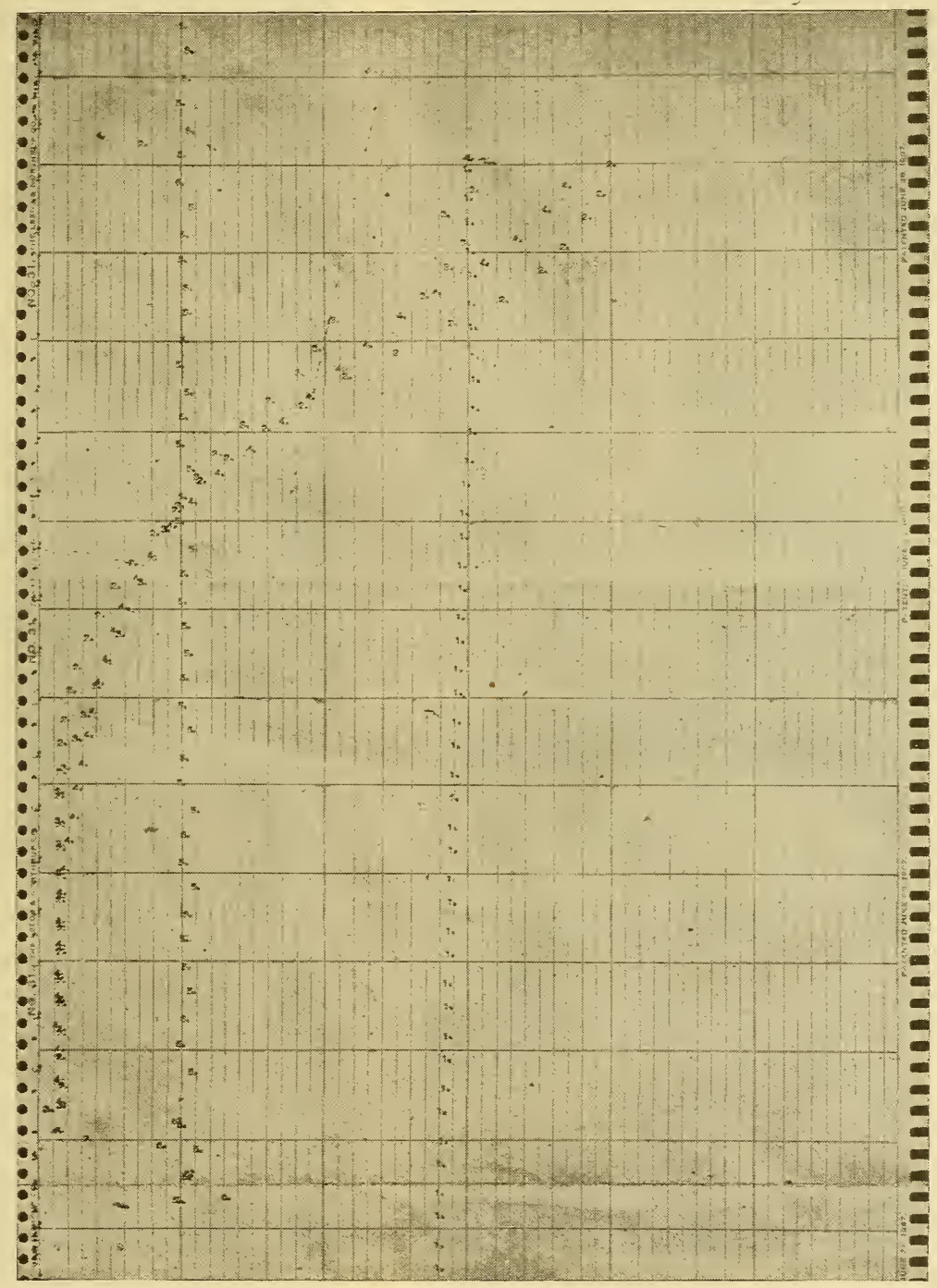

Fig. II4.-Record of experiments in a by-product coke oven

is a record obtained when the time interval between records is too long. The record of No. I is useful for a check on the operation of the recorder. Knowing that this record should show a gradual and uniform rise in temperature, any departure from a smooth 

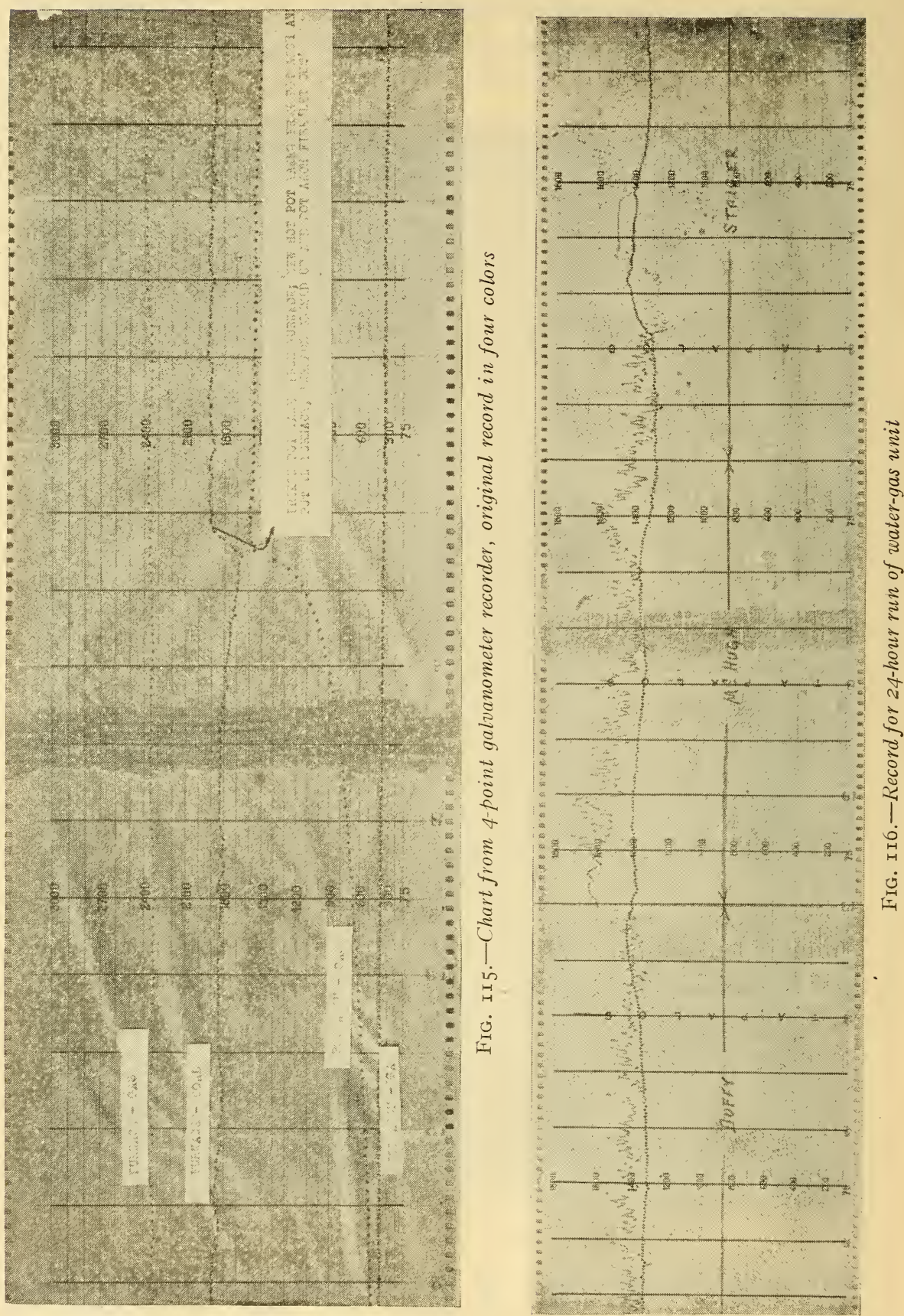
curve is an indication of instrument trouble or tampering. For experimental work these curves are best replotted with the time coordinate shortened and a continuous smooth curve drawn through the points.

Fig. I I 5 is the chart from a 4-point galvanometer recorder with the thermocouples placed as indicated on the chart. The four records are made in colors and with dots differently placed, which makes them very legible. Operations are here clearly shown upon a permanent record.

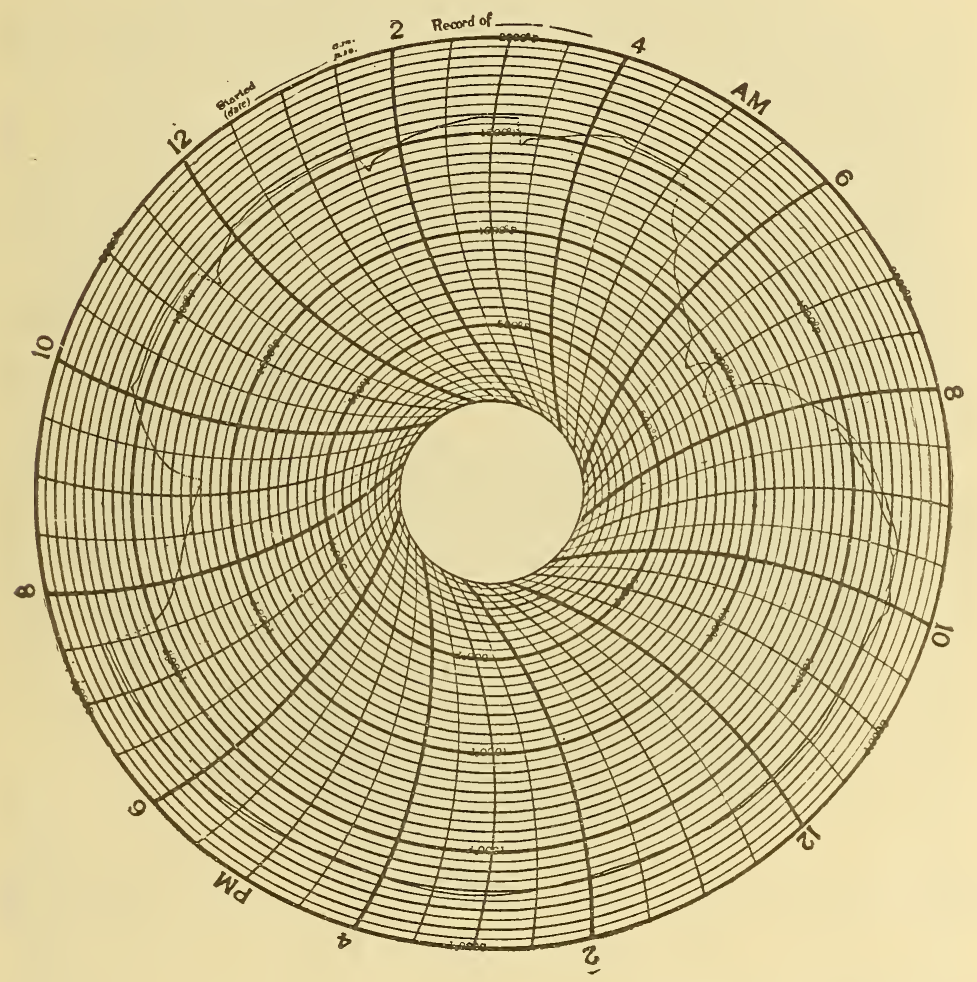

FIG. II 7.-Record of furnace used for case hardening during the day and annealing at night

Fig. I 6 is a record of temperatures in a water-gas unit. This record was selected to show how recorder charts may be used to indicate the comparative efficiency of operators. In this case the three shift operatora, Messrs. Duffy, McHugh, and Staiger, were stimulated to better work when this record was framed and hung in the gas house. The lower curve should have been maintained within $25^{\circ}$ of $1350^{\circ} \mathrm{C}$ and the upper curve between $1350^{\circ}$ and $155^{\circ} \mathrm{C}$. Mr. Duffy very evidently secured the most satisfactory results. 
Fig II7 is the record of the operation of a gas furnace used alternately for casehardening and for annealing. At $7.30 \mathrm{a} . \mathrm{m}$. the furnace was charged and the temperature raised to approximately $1750^{\circ} \mathrm{F}$ at 9.30, where it was maintained with good control until $7.30 \mathrm{p}$. m. Then a new charge was put in, and the temperature was raised to $\mathrm{I} 575^{\circ} \mathrm{F}$, this being repeated three times before $5 \mathrm{a} . \mathrm{m}$, when the fire was cut off. This record was used by the operator for obtaining proper temperatures and serves

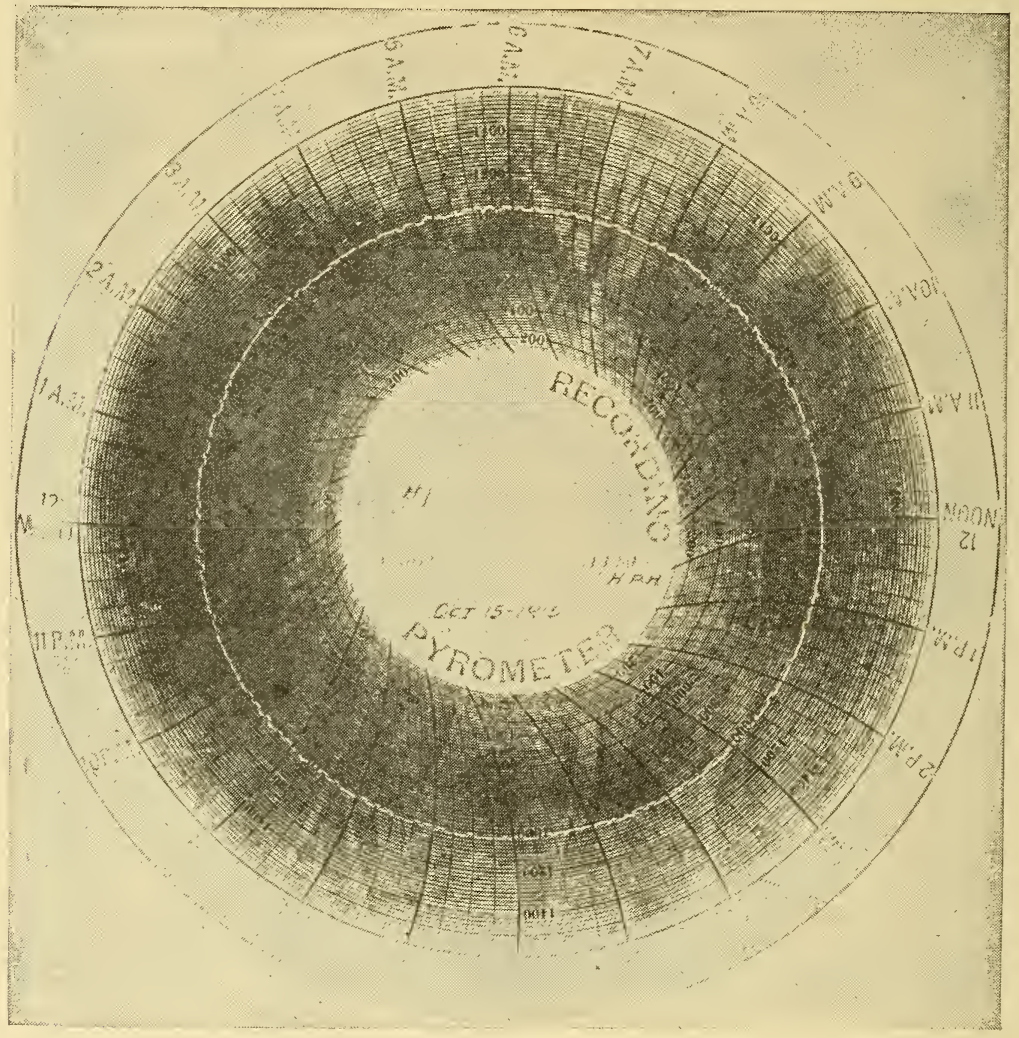

FIG. I 8.-Example of excellent control of annealing furnace (oil or gas)

also as a permanent record of the number of heats. Knowing the processes at this plant, it is a record of what types of heat treatment were accomplished.

Fig I I 8 is an example of excellent control of a gas or oil furnace for annealing brass. Any laxity on the part of the operator would be very apparent in this record. However, such good control is exceptional. Fig. II9 is the record of stack gas temperature in a billet-heating furnace, and in this case the control was considered good. 
Fig. I 20 is another illustration of the clarity with which the relative efficiency of shift operators is shown. In this case the work of the day shift was superior to that of the night shift.

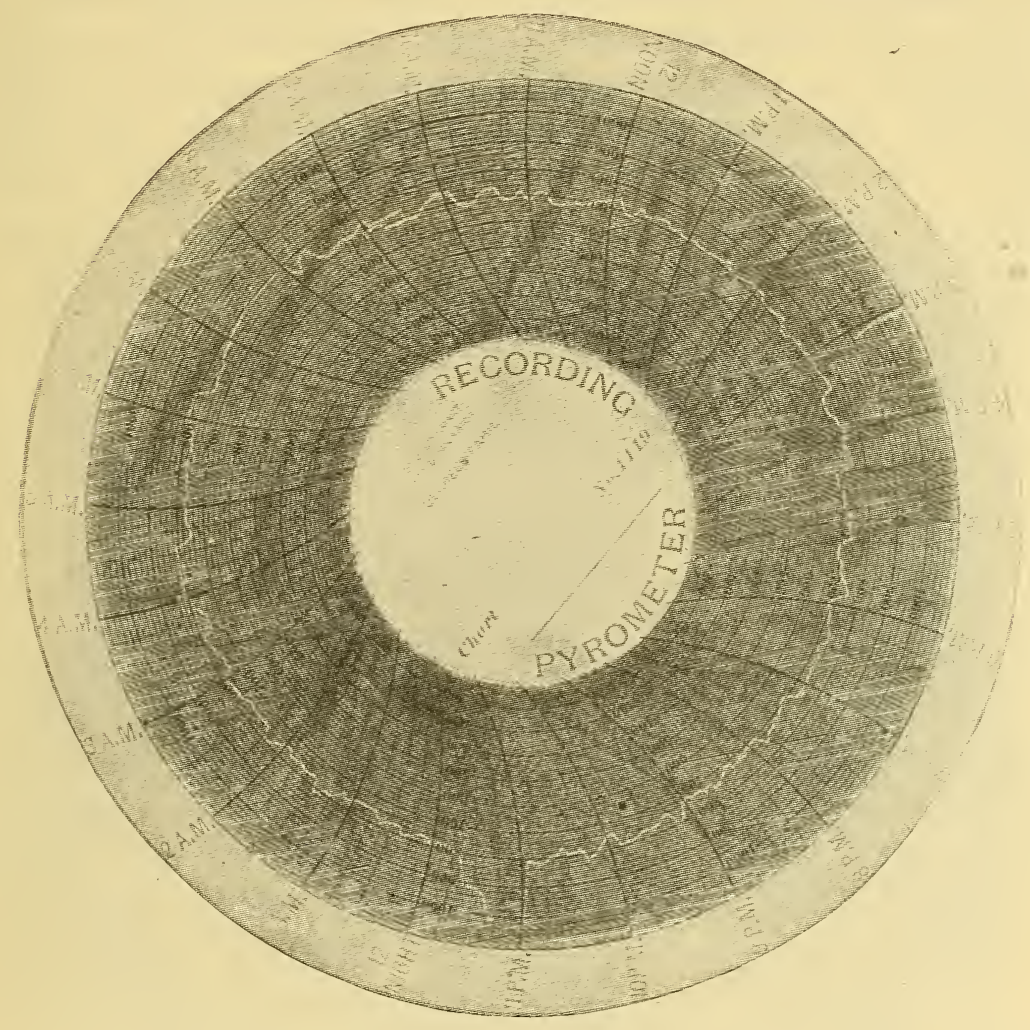

FIG. II9.-Example of good control

\section{MELTING-POINT METHODS AT HIGH TEMPERATUERS ${ }^{23}$}

\section{INTRODUCTION}

The determination of a melting or freezing point, whether for a physical or physical-chemical purpose, resolves itself into the measurement of a temperature under particular conditions and with appropriate technique. The accuracy and ease with which melting points at high temperatures may be determined is due to the comparatively recent developments in pyrometry, electricfurnace construction, and refractory materials.

The general classes of substances considered are metals, alloys, salts, and refractory materials. For the temperature measure-

\footnotetext{
${ }^{23}$ Dana and Foote, Chem. and Met. Eng., 22, p. 23 and D. 63; r920. Also see Foote, Harrison, and Fairchild, same, 18, p. 343 and p. 403; rgr8.
} 
ments, thermoelectric, resistance, and optical pyrometers are employed, depending upon the temperature range and other controlling conditions.

The melting or freezing point of a pure crystalline substance is defined as the temperature at which the solid phase can remain in equilibrium with the liquid phase at normal atmospheric pressure. The effect of pressure is ordinarily insignificant; it is only when the pressure reaches many atmospheres that any change in the

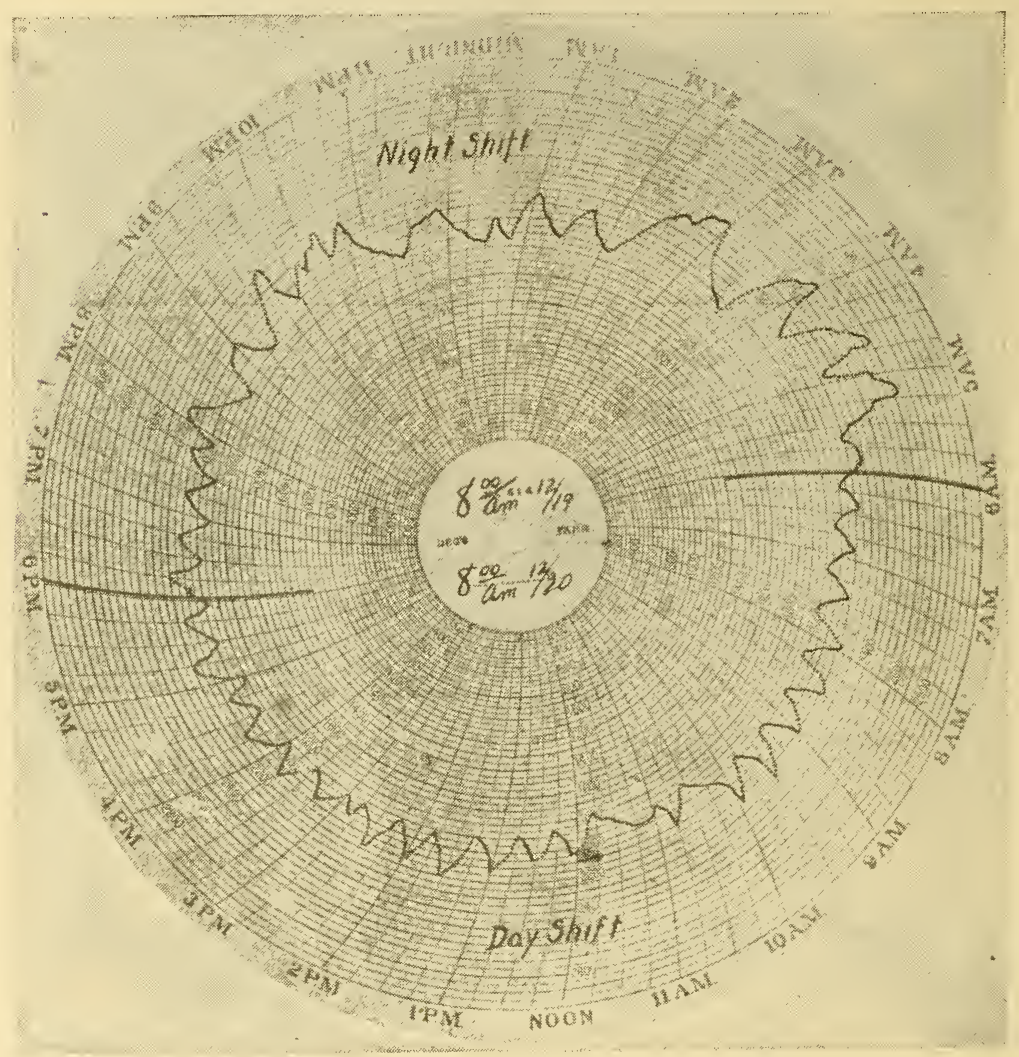

FIG. I20.-Illustrating relative efficiency of day and night shift

melting or freezing point can be readily detected. At the melting point there is a discontinuous change of a number of the physical properties of a material; e. g., thermal conductivity, electrical resistivity, specific volume, etc. In general, alloys, excepting eutectoids, mixtures of refractory oxides, and materials with impurities do not have a definite melting point, but become liquid gradually; that is, they melt over a temperature interval, and consequently are considered as having a "melting range." 


\section{USE OF THERMOELECTRIC PYROMETERS}

(a) Pure Materials-(i) General Discussion.-The experimental arrangement of this method consists of a crucible containing the substance in which a thermocouple either protected or unprotected is centrally inserted. The crucible is placed in a uniformly heated portion of an electric furnace. On account of cleanliness, clarity of the atmosphere, and preciseness of control, electric resistance furnaces are preferable to gas or fuel fired furnaces for accurate melting-point determination. When a melting or freezing point is to be observed, the temperature of the furnace is gradually raised or lowered, and the temperature as indicated by the thermocouple is noted at frequent, and preferably uniform, intervals of time. If all conditions were ideal, the temperature of the charge would remain constant during melting or freezing. Thus the temperature-time curve would be characterized by a straight line exactly parallel to the axis of time and by a discontinuous change of slope at either end of the line marking the beginning and ending of the period of melting or freezing. Actually, however, the change of state shown by the curve, even for pure materials, is not sudden, but more or less gradual. Only a part of the freezing curve will be flat, and the melting curve usually possesses greater obliquity than the freezing curve. For this reason freezing-point determinations are usually more reliable and are employed whenever possible, although the temperature thus obtained is frequently called the melting point, to which it is numerically equivalent.

The reasons for obliquity, and for its existence to a greater degree in the melting curves, have been discussed in detail by White. ${ }^{24}$ On heating, the furnace walls are hotter than the crucible, and hence heat flows from the furnace to the crucible. When a layer of the charge near the wall of the crucible reaches the melting point, the heat absorbed by the charge goes to supply that necessary for the latent heat of fusion, and the temperature of the outside layer tends to remain fairly constant. The thermocouple measures the temperature of the solid metal in the center of the charge and when thus surrounded by an isothermal layer the rate at which the inside temperature increases is greatly diminished, resulting in a rounding off of the temperature-time curve. As the isothermal layer progresses inward the temperature of the couple slowly rises until the material immediately surrounding it

${ }^{24}$ White, Melting-Point Determinations, Am. J. Sci., 28, p. 453; 1909. 
begins to melt, when a condition of stationary temperature is maintained for a short time during which the thermocouple reading gives the true melting point. While the center of the charge is melting, however, the temperature of the outer layer of material in the crucible rises very rapidly, and a large temperature gradient is established between the outside and center. The resulting rapid flow of heat to the center of the crucible accelerates the melting and, if the layer of remaining solid metal is unequally distributed about the couple, tends to increase the temperature readings and cause another rounding off of the temperature-time curve. The apparent degree of obliquity found near the beginning and end of the melting period depends considerably on the sensitivity of the temperature measurements and scale of plotting. Thus, highly accurate data obtained with a precision potentiometer may be plotted on so large a temperature scale that the curve may appear quite oblique, while the same data replotted on a less extended scale appear fairly flat. Aside from this, however, the degree of obliquity varies according to the factors controlling the differences in temperature within the charge, such as dimensions and thermal properties, rate of heat supply, etc. If the solid metal could be stirred during a melting-point determination the temperature gradients could be diminished, but even with powdered materials where stirring is possible it is not convenient and does not appear to be-very satisfactory. The larger the diameter of the charge, the faster the rate of heating, the smaller the diffusivity, and the lesser the latent heat of fusion, the greater will be the tendency to obliquity. The obliquity may be decreased by slow heating and by using a charge of fairly small diameter. The protection tubes of the thermocouple should be small, of thin wall, and should be deeply immersed in the charge. With metals the obliquity is not so great as with salts, on account of their higher heat diffusivity and latent heat of fusion; in fact, for most cases the obliquity is of negligible importance with pure metals. But with salts and certain refractory materials, unless special care is taken, the obliquity may be so great as to almost completely obscure the true melting phenomenon.

On cooling, the crucible is hotter than the furnace walls, and heat flows from the crucible to the furnace. The outer layer of the charge solidifies first and during freezing acts as an isothermal layer, thus decreasing the temperature differences between the center and outside of the charge. The rate at which the temperature 
of the couple falls decreases, causing a rounding off of the first part of the temperature-time curve. While the center of the charge is freezing, the outside is completely frozen and rapidly falls in temperature, increasing the temperature gradient and the outward flow of heat, and hence the latter part of the freezing is accelerated.

The first part of the approximately flat portion of the curve for freezing corresponds to the true freezing point, and the latter part of the approximately flat portion of the curve for melting corresponds to the true melting point. The same precautions of high narrow charges, narrow protecting tubes, sufficient depth of immersion, and small thermocouple wires apply to both cases.

In general, the freezing curves are more sharply defined than the melting curves. A liquid cooling and subject to convection currents is likely to possess a more uniform temperature distribution than a solid on heating. Furthermore, the freezing point is chosen at a time immediately following a period of small temperature gradients, while the melting point must be chosen near the end of the period of melting when there is possibility of temperature non-uniformity. A further factor of importance is the constancy of heat supply. On cooling with a furnace well insulated, the freeze may extend over a considerable time with all power supply shut off. The furnace thus acquires a uniform rate of cooling. In heating, however, the effect of slight variations in

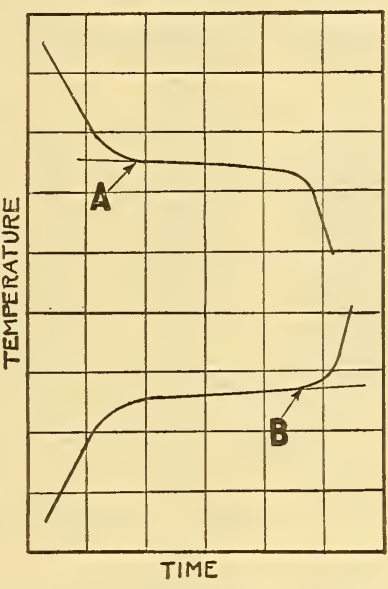

Firg. I2I.-Oblique freezing and melting curves the power supply may alter the true character of the melting curve. Fig. I I s shows a freezing and a melting curve in which considerable obliquity is present. The true temperature for freezing is obtained by extrapolating the straight portion of the freezing curve and noting at what temperature $A$ the continued straight line deviates from the observed curve. The melting point $B$ is similarly obtained from the melting curve. The first part of the freezing curve and the last part of the melting curve determine these two temperatures as stated above.

It is impossible to obtain satisfactory freezing curves with certain materials, such as silicates, on account of extremely slow crystallization, and the melting curves, even though frequently poorly defined, must be employed. The phenomenon of surfusion 
or supercooling is well illustrated by water which may be cooled to $-3 \mathrm{O}^{\circ} \mathrm{C}$ without freezing. Surfusion is due to conditions which do not favor the attainment of equilibrium between the solid and liquid phases at the true freezing point. The conditions tending to produce equilibrium and the elimination of surfusion are slow cooling, stirring of the liquid and the presence of nuclei which serve as centers of crystallization. When a supercooled liquid freezes, it crystallizes very rapidly and the temperature rises to the true freezing point provided the supercooling has not proceeded too far. The subsequent freezing takes place in a normal manner. Surfusion in most metals seldom exceeds $0 . \mathrm{I}$ or $0.2^{\circ} \mathrm{C}$, but in the case of antimony it is very marked and may amount to $30^{\circ} \mathrm{C}$, depending upon the rate of cooling, as shown by Fig. I22. Supercooling also occurs in alloys, especially in eutectics. While the contrary phenomenon of superheating is negligible with pure

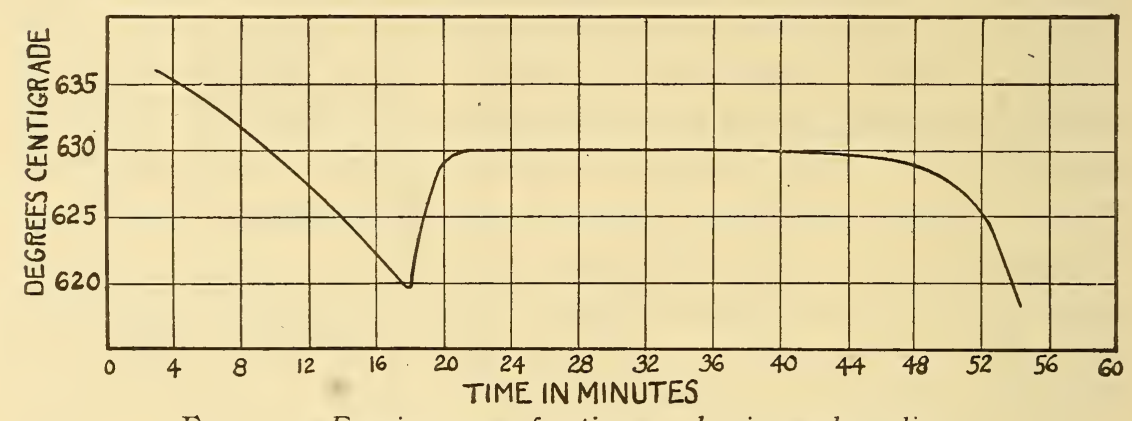

FIG. I22.-Freezing curve of antimony, showing undercooling

metals, it may become of importance with alloys, particularly in allotropic transformation and with salts and silicates.

(2) Precision Work with Pure Metals. - The primary method for the standardization of thermocouples consists in determining the emf developed by the couple at the freezing points of several very pure metals. The metals so employed are those the freezing points of which have been accepted as fixed points on the high temperature scale; e. g., tin, cadmium, zinc, antimony, aluminum, silver, gold, copper, etc. For this work and for the reverse problem of accurately determining the freezing points of metals and alloys the greatest precautions against contamination of the couple and the metal are requisite. Precision work at high temperatures requires the use of homogeneous platinum, 90 per cent platinumIo per cent rhodium couples. The diameter of the wire usually employed is 0.5 or $0.6 \mathrm{~mm}$, but for certain special purposes wires of o.I $\mathrm{mm}$ are useful. 
(3) Crucibles and Protecting Tubes.-Of all crucible materials Acheson graphite has the greatest utility. It is very pure, can be machined into any desired shape, and is not attacked by most of the common metals. At high temperatures the gases formed from its oxidation provide the reducing atmosphere necessary for the protection of the metal. The following table illustrates sizes of graphite crucibles, which have been used in various investigations. For resistance thermometry the largest size shown is necessary, but for general work the intermediate size is recommended. In this case a smaller protecting tube is desirable-for example, one with an outside diameter of $5 \mathrm{~mm}$.

Some metals, such as nickel and iron, combine with graphite, and certain other metals also should not be heated under the reducing conditions present with graphite. Crucibles of magnesia alumina, or mixtures of the two, are suitable for iron and nickel. Porcelain crucibles may be used for many of the metals, but there is always danger of these cracking when the metal melts or freezes.

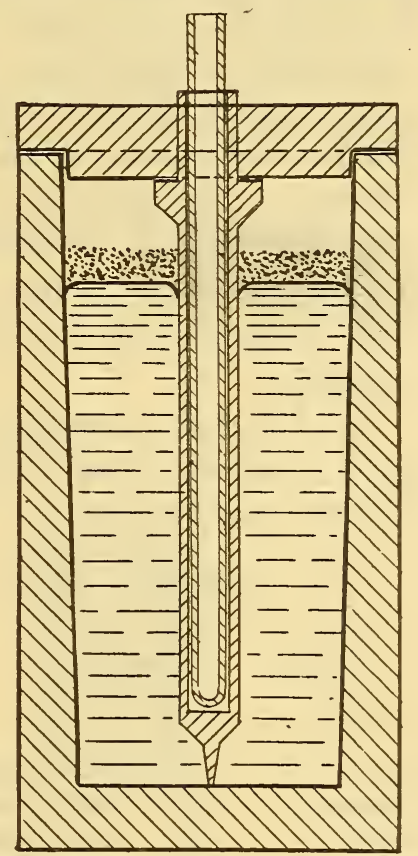

Fig. I23.-Graphite sheath for protection of porcelain tube in aluminum

TABLE 23.--Size of Crucibles

\begin{tabular}{|c|c|c|c|c|c|c|c|c|c|}
\hline \multirow{3}{*}{ Laboratory } & \multicolumn{4}{|c|}{$\begin{array}{l}\text { Crucible dimensions, all in } \\
\text { centimeters }\end{array}$} & \multicolumn{2}{|c|}{$\begin{array}{c}\text { Charge } \\
\text { dimensions }\end{array}$} & \multirow{2}{*}{\multicolumn{2}{|c|}{$\begin{array}{c}\begin{array}{c}\text { Protection } \\
\text { tube }\end{array} \\
\text { Diameter }\end{array}$}} & \multirow{3}{*}{$\begin{array}{l}\text { Dis- } \\
\text { tance } \\
\text { from } \\
\text { bottom } \\
\text { of } \\
\text { charge } \\
\text { to pro- } \\
\text { tection } \\
\text { tube }\end{array}$} \\
\hline & \multicolumn{2}{|c|}{ Inside } & \multicolumn{2}{|c|}{ Outside } & \multirow{2}{*}{$\begin{array}{l}\text { Diam- } \\
\text { eter }\end{array}$} & \multirow{2}{*}{ Height } & & & \\
\hline & $\begin{array}{l}\text { Diam- } \\
\text { eter }\end{array}$ & Height & $\begin{array}{l}\text { Diam- } \\
\text { eter }\end{array}$ & Height & & & $\begin{array}{l}\text { Out- } \\
\text { side }\end{array}$ & Inside & \\
\hline Bureau of Standards. & 7.0 & 15 & 8.5 & 16 & 7.0 & 11 & 1.0 & 0.8 & 1.0 \\
\hline Geophysical laboratory & 2.7 & 8 & 3.7 & 10 & 2.7 & 5 & .8 & .5 & .5 \\
\hline Bureau of Standards. & 1.2 & 3 & 2 & 3.5 & 1.2 & 2 & .15 & .1 & .3 \\
\hline
\end{tabular}

Up to $500^{\circ} \mathrm{C}$ protection tubes and insulating tubes for the couple, made of pyrex glass, are useful. From 500 to $\mathrm{I} \mathrm{I}^{\circ} \mathrm{O}^{\circ} \mathrm{C}$ lower grade porcelain, glazed on the outside only, or fused quartz, $8513^{\circ}-21-13$ 
are satisfactory. Above $1100^{\circ} \mathrm{C}$ tubes having a composition approximating that of sillimanite, $\mathrm{Al}_{2} \mathrm{O}_{3}, \mathrm{SiO}_{2}$, are recommended. ${ }^{25}$ Porcelain tubes, or crucibles, or any material containing silica, can not be used in contact with aluminum, as the silica is readily attacked. Aluminum may be melted in a graphite crucible, and the porcelain protecting tube may be itself protected by a very thin sheath of graphite. Fig. I 23 illustrates one convenient manner in which the sheath may be mounted in the crucible. The sheath is held down in the metal by the weight of the cover and is allowed to remain in the crucible after the aluminum is frozen. The thermocouple-protecting tube fits tightly inside the sheath. At present there is no satisfactory protecting tube for nickel. This material and its alloys may be best studied by optical methods.

(4) Table of Melting Points.-The following table lists the more common metals, the melting points of which can be used for the standardization of thermocouples by the crucible method:

TABLE 24.-Melting Points of Metals from. Tin to Nickel

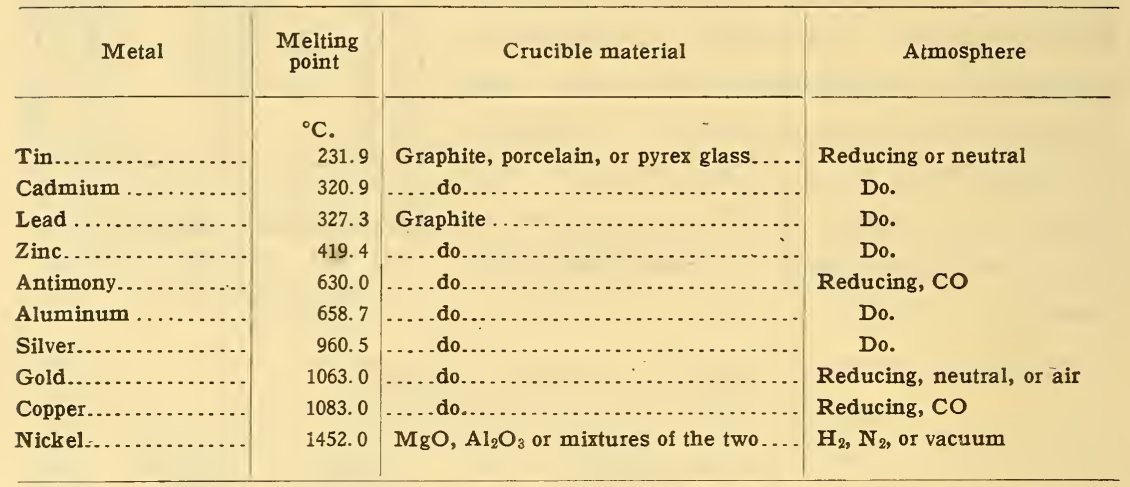

The surface of the metal, except nickel, should be covered with a layer of powdered or flaked graphite, and the crucible should be provided with a cover having a hole through which the thermocouple tube is inserted. Under no circumstances should graphite be exposed to an air current above $500^{\circ} \mathrm{C}$, as it readily oxidizes and crumbles away. The furnace should be tightly closed at the bottom and no larger openings exposed at the top than absolutely necessary. When fairly small graphite" crucibles are employed, it is frequently desirable to use a larger outside crucible of porcelain and fill the space between the two crucibles with

\footnotetext{
${ }^{25}$ Such porcelain is known as Usalite or Impervite.
} 
graphite powder, as shown in Fig. I24. When larger crucibles are used, the graphite powder may be poured directly into the furnace tube to a depth sufficient to entirely cover the crucible.

(5) Construction of Furnace.-Fig. I24 shows the construction of a furnace using crucibles of the intermediate size given in Table 23 for operation up to $1100^{\circ} \mathrm{C}$. The graphite crucible $H$, containing the metal $G$ and the pyrometer tube $A$, rests in a porcelain crucible $F$ and is completely covered by powdered graphite $E$. The porcelain crucible is supported by the alundum tube $I$. The heater tube is of alundum, R. A. 98; length, $25 \mathrm{~cm}$; inside diameter, $5 \mathrm{~cm}$; and wall thickness, $3 \mathrm{~mm}$. This is- preferably corrugated on the outside and is wound with 60 to 80 turns of No. I4 "chromel A" wire, $C$, to the ends of which are welded the lead-in terminals $K$ of No. Io chromel. The alundum tubes $B$ and $I$ rest on a good nonconducting fire brick about 5 by 8 by $8 \mathrm{~cm}$. The shell $M$, $32 \mathrm{~cm}$ high, is of sheet iron or monel metal painted with aluminum paint. The ends of the shell are closed by impregnated asbestos boards $N$, I $\mathrm{cm}$ thick and $\mathrm{I} 7 \mathrm{~cm}$ in diameter. The annular space $O$ is packed with silocel powder. The lead-in wires are insulated from the metal shell

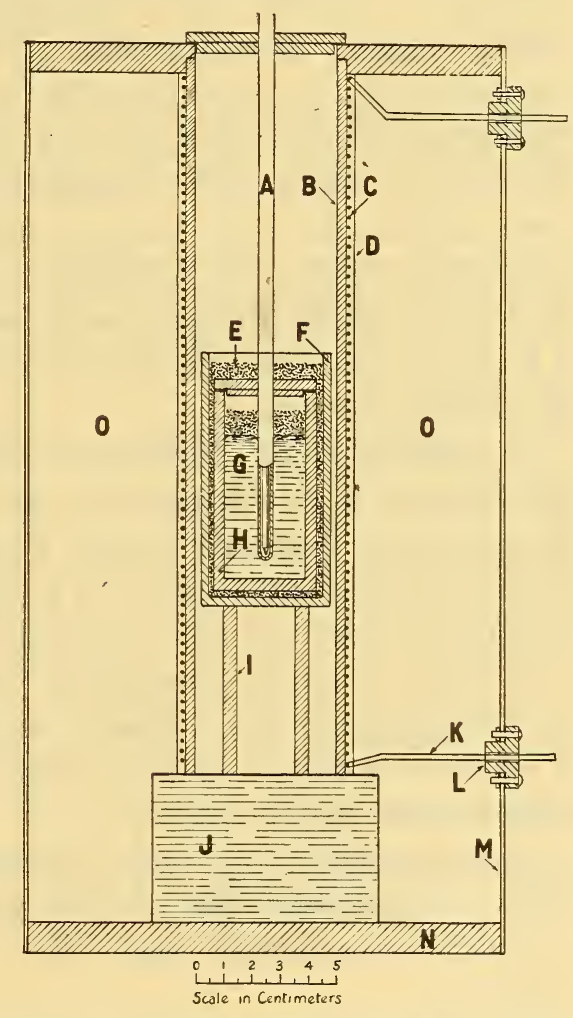

FIG. I24.-Melting point furnace for metals and alloys by asbestos board or steatite bushings. The furnace in series with a rheostat for control may be operated on IIo volts and consumes about I kilowatt at $1100^{\circ} \mathrm{C}$.

Larger furnaces are required for the large crucibles described in Table 23. Such a furnace may be constructed from the following stock material. Alundum tube, R. A. 98 ; length, $45 \mathrm{~cm}$ : inside diameter, $8 \mathrm{~cm}$; wall thickness, $6 \mathrm{~mm}$. Chromel A winding No. 5 wire; 60 turns separated by and covered over with 
alundum cement. Diameter of shell, $36 \mathrm{~cm}$; height of shell, $60 \mathrm{~cm}$; silocel powder insulation, general construction similar

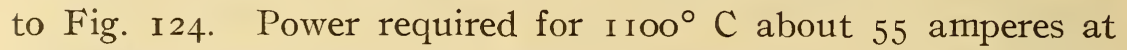
60 volts. Using the large crucibles a freeze of copper with all power off extends over a period of about 20 minutes.

(6) General Precautions.-In order to melt for the first time a metal which is in small pieces, the graphite crucible, filled with the material, fitted with a cover having a hole in which the thermocouple fits tightly and surrounded by powdered graphite, is slowly heated. If convenient, $C O$ or $N_{2}$ may be passed into the furnace, but $\mathrm{CO}_{2}$ should not be used. The surface of the metal should not be covered with graphite at first, as it may filter into the interior and become imprisoned there. When the metal has been melted, however, the graphite layer on the surface is quite desirable. With the exception of antimony, which should be stirred just as the freezing point is reached to reduce supercooling, it is not advisable to stir the molten metals, as pockets of graphite are thereby formed. .

In melting large crucibles of copper care must be taken that the furnace is not burned out before the copper is melted. It is frequently desirable to use a thermocouple connected to the heating element attached to a temperature control apparatus and

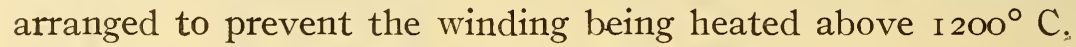

If there is doubt concerning the proper depth of immersion, several determinations should be made at different depths until a position is found where a slight variation does not affect the temperature measurement.

The pyrometer tube may be allowed to remain in the metal for a few degrees below the freezing point without breaking, and if it can not be removed then, the metal should be immediately remelted.

A uniform rate of cooling of about $2^{\circ} \mathrm{C}$ per minute from $10^{\circ} \mathrm{C}$ above the melting point is satisfactory for a freezing-point determination. The values obtained from melting and freezing curves should agree to from o.I to o.or $^{\circ}$ for pure metals, and the curves should have a flat portion extending over at least ro minutes. If the curve is not fairly flat the metal is likely impure, and hence possesses a melting range instead of a true melting point. Fig, I 25 illustrates an observed freezing and melting curve for zinc. The freezing curve is constant to $0.03^{\circ} \mathrm{C}$ for a period of i 5 minutes and the melting curve for ro minutes. The obliquity in the latter part of the melting curve amounts to O. $^{\circ} \mathrm{C}$ in six minutes. 
(b) Metals with Impurities, and Alloys.-An impurity in a metal usually lowers the melting point and produces obliquity in the melting and freezing curves, the magnitude of both of these effects increasing, within limits, as the amount of impurity is increased. Assuming that the amount of impurity is small and that an ideal solution with the metal in the liquid state is formed, the lowering of the freezing point $\Delta t$ may be expressed approximately by the Raoult-Van't Hoff equation:

$$
\Delta t=\frac{R \vartheta^{2}}{L} \frac{N_{1}}{N}
$$

in which $R=1.99, \vartheta=$ absolute temperature of the freezing point of the pure metal, $L=$ molar latent heat of fusion of the pure metal, $N_{1}=$ number of mols of impurity, and $N=$ number of mols of pure metal. In Fig. 126, $A$ represents the freezing curve of a pure substance and $B$ the curve obtained when an impurity is present. The lowering of the freezing point is $4^{\circ} \mathrm{C}$, an amount readily caused by a few tenths of a per cent of an impurity at about $800^{\circ} \mathrm{C}$. In general, the above equation for freezing-point lowering holds only for an impurity

which forms the simplest Fig. I25.-Freezing and melting curves of pure zinc type of eutectic alloy with

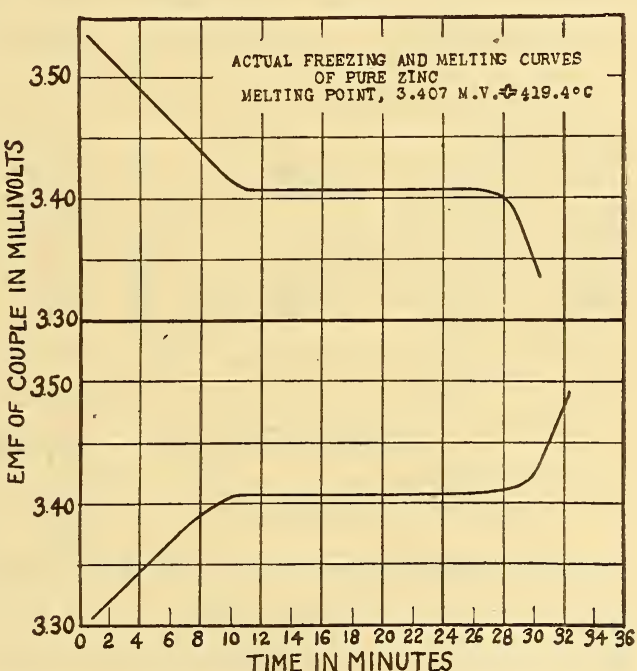
the pure metal. Evidently it can not be applicable to those impurities which are wholly or partially soluble in the metal in the solid state, that is, which form solid solutions, for in some of these cases the melting point is raised by the addition of the impurity, as, for example, nickel added to copper.

For most of the common metals the oxide is insoluble in the pure metal, but with copper this is not the case, and special care must be taken, preferably by the maintenance of a strongly reducing atmosphere, to avoid the formation of cuprous oxide $\mathrm{Cu}_{2} \mathrm{O}$. This oxide forms a eutectic with copper saturating it at 3.45 per cent of $\mathrm{Cu}_{2} \mathrm{O}$ or 0.395 per cent of oxygen by weight, and freezing 
at $1063^{\circ} \mathrm{C}$. As an example of the application of the above equation, the freezing point lowering of copper at the eutectic, considering the $\mathrm{Cu}_{2} \mathrm{O}$ as the dissolved impurity, will be calculated.

$$
\Delta t=\frac{R \vartheta^{2}}{L} \frac{N_{1}}{N}=\frac{2(1083+273)^{2}(2.19)}{(63.6)(43.3)(143)}=20.4^{\circ} \mathrm{C}
$$

The lowering, found experimentally, is $20.0^{\circ} \mathrm{C}$.

If the copper contains only a small amount of the oxide, the first solidification begins slightly below the melting point of pure copper and crystals of copper separate from the solution. The concentration of the dissolved impurity $\mathrm{Cu}_{2} \mathrm{O}$, accordingly, increases so that the freezing point of the remaining part of the solution is still lower. The pure copper will continue to separate from the liquid at a gradually decreasing temperature until the

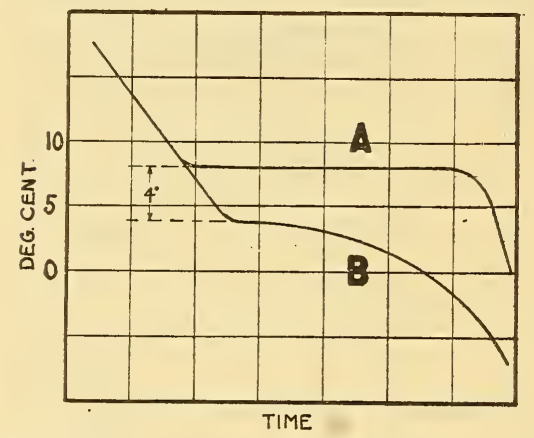

FIG. I26.-Freezing curves without and with impurity solution becomes saturated with $\mathrm{Cu}_{2} \mathrm{O}$. This results in rounding or obliquity of the cooling curve. When the remaining solution is saturated, both the $\mathrm{Cu}$ and the $\mathrm{Cu}_{2} \mathrm{O}$ solidify together at a constant temperature as a eutectic mixture. At this point a halt occurs in the cooling curve, the magnitude of which depends upon the amount of the eutectic present. The effect of a small amount of $\mathrm{Cu}_{2} \mathrm{O}$ will escape notice in an ordinary melting-point determination; but, if considerable oxide is present, the freezing curve shows the two retardations-one below $1083^{\circ}$ and the other at $1063^{\circ} \mathrm{C}$. Observation continued through the latter point may serve as a rough criterion of the purity of copper suspected of contamination by its oxide. The obliquity of the curve also may be an indication of impurity. If a copper wire is slowly heated in air the melting usually begins at the eutectic point, $1063^{\circ} \mathrm{C}$, instead of at $1083^{\circ} \mathrm{C}$.

Oxygen dissolves in silver and may lower the melting point as much as $5^{\circ} \mathrm{C}$ if the silver is heated in air.

An alloy may be considered as a metal containing large proportions of one or more impurities. Let us consider the simplest case of an alloy of two metals, $A$ and $B$, which are mutually soluble when liquid and insoluble when solid. If $A$ first begins to separate 
from the solution, the remaining liquid becomes richer in $B$, and, as shown above, this part of the freezing curve gradually changes in slope, as illustrated by Fig. 127 , curve $I, L$. As the temperature is lowered the solution finally becomes saturated with $B$, and $A$ and $B$ crystallize together as a eutectie, shown in Fig. I 27, by the lower flat portion of the cooling curve. The alloy accordingly has a melting or freezing range extending from the temperature at which separation of $A$ first begins to the temperature at which the eutectic mixture solidifies. If the original composition of the alloy is the eutectic mixture, there exists a true melting point as shown in Fig. I27, curve $I I$, and not a melting range.

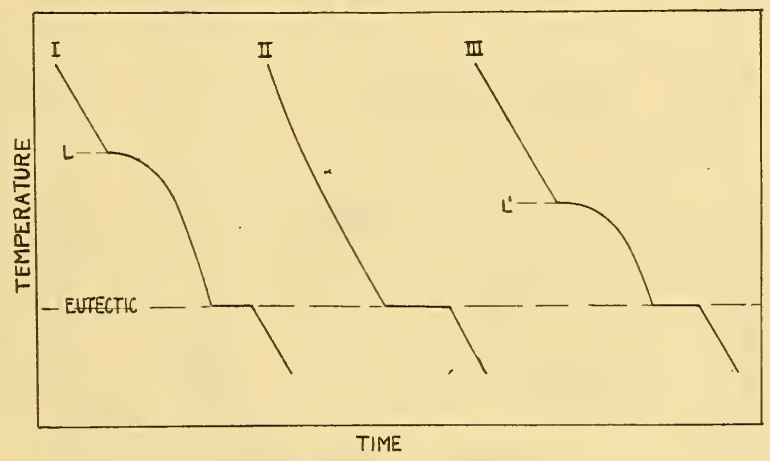

FIG. I27.-Freezing curves of simple eutectic alloys

The general form of the temperature-composition diagram of the simple binary alloy described above is illustrated by Fig. I 28 . The curves $I, I I$, and $I I I$ of Fig. I 27 show the form of the cooling curves obtained with the alloys of compositions $I, I I$, and $I I I$ of Fig. I 28.

Often a binary alloy exhibits two halts in the melting and freezing curves-one oblique and one flat or both oblique. Furthermore, other complications may arise, such as the formation of solid solutions, compounds, and allotropic transformations accompanied by heat changes which require more sensitive methods of detection than by a simple cooling curve. In general, the larger the melting range in a binary alloy the more difficult it becomes to locate the lower transformation point because of the small amount of crystallization per degree drop in temperature and the resulting small heat effect. The same general details for melting point of pure metals apply to alloys. The eutectic alloy of silver and copper, 7I.9 per cent $\mathrm{Ag}$, 28. I per cent $\mathrm{Cu}$, by weight, melts at 
$779^{\circ} \mathrm{C}$ and is useful as a standardization temperature for thermocouples.

For alloys having a large melting range accurate control of the rate of heating and cooling is essential. Automatic control of the rate may be obtained by a salt-water rheostat as applied by Burgess and Crowe, ${ }^{26}$ or an automatic magnetic control system may be installed. Scott and Freeman ${ }^{27}$ describe a modified Rosenhain furnace especially useful for critical point work. This consists of a vertical tube furnace, heated at the top and cooled at the bottom, through which the material under investigation is automatically moved at a definite rate.

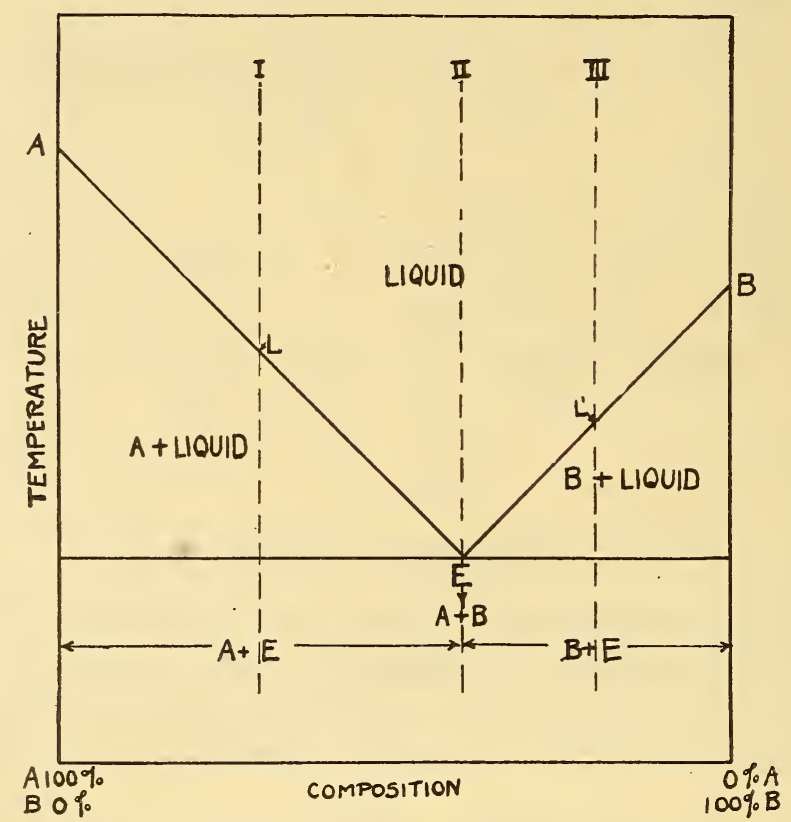

FIG. I28.-Temperature-composition diagram of simple eutectic alloy

If the ordinary temperature-time curve does not give evidence of a change of state at the beginning or end of a melting range or at a transformation point, the more sensitive means of detection, such as the inverse-rate method and the differential method, may be employed.

The inverse-rate method consists in determining the time required for the specimen to fall a definite temperature intervalfor example, $2^{\circ} \mathrm{C}$-and in plotting this time against the tempera-

26 B. S. Sci. Papers No. $21_{3}$.

${ }^{27}$ Bull. Am. Inst. Min. and Met. Eng.; Aug., 1919. 
ture. In effect, it amounts to differentiating the temperaturetime curve in respect to temperature. The experimental arrangements are the same as for the ordinary-time method, except that the time must be measured more accurately. Merica ${ }^{28}$ has described a simple and effectual device consisting of - two stop watches, one of which is stopped and one started at the end of

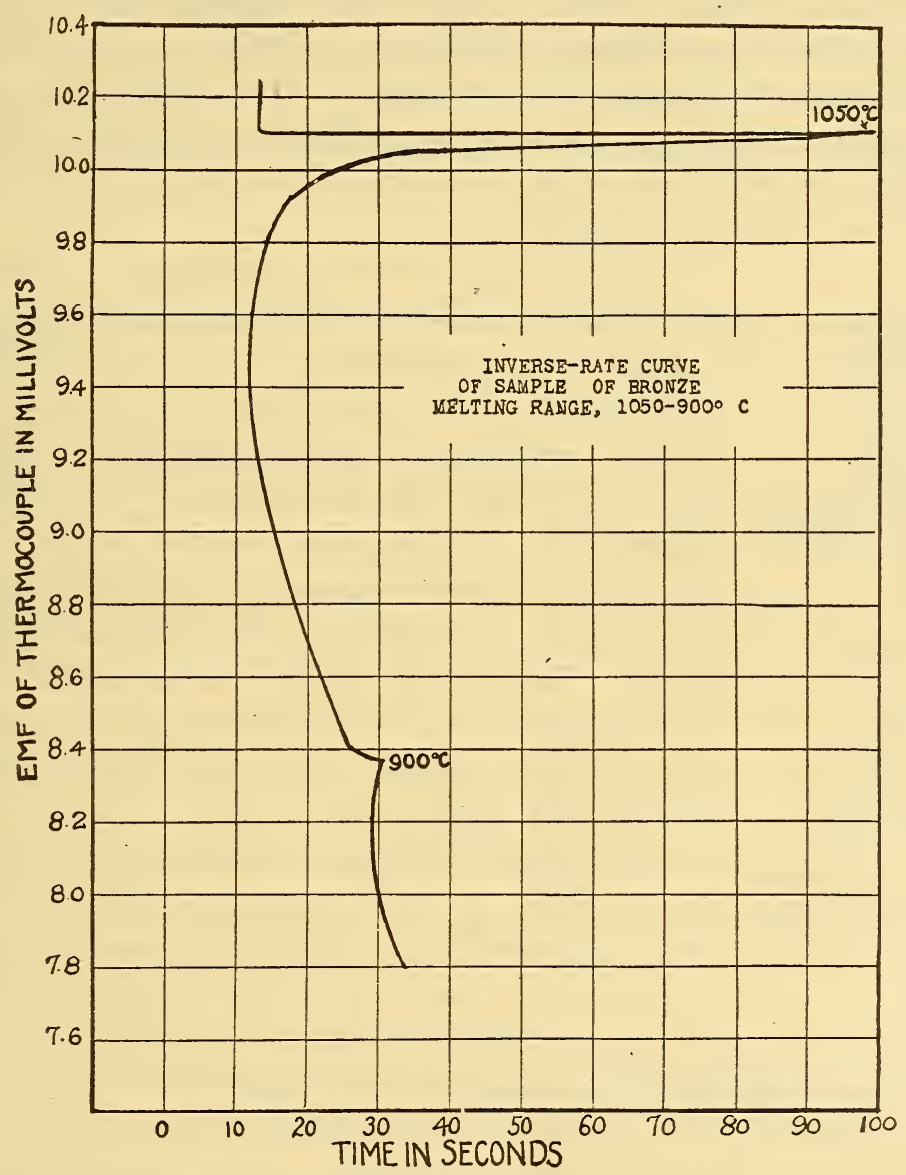

FIG. I29.-Inverse-rate curve of a bronze

each time interval. Fig. I 29 shows an inverse-rate curve through the melting range of a bronze. The lower transformation at $900^{\circ} \mathrm{C}$ could not be detected by the ordinary temperature-time method.

The differential method is still more sensitive to small thermal changes. This consists in measuring the temperature dif-

${ }^{28}$ Bull. Am. Inst. Min. and Met. Eng.; July, rgrg. 
ferences between the material in which the transformation takes place, and a neutral body having no transformation points in the same temperature interval, such as platinum or nickel, and plotting this difference against the temperature of the material. Two thermocouples are employed as illustrated by Fig. I30, one a differential couple and the other an ordinary couple which measures the temperature of the sample under investigation. By use of a sensitive galvanometer with the differential couple extremely minute thermal transformations may be easily detected. Since the material under test and the neutral body are placed in close proximity in the furnace, slight variations in the rate of heating produce no noticeable effect.

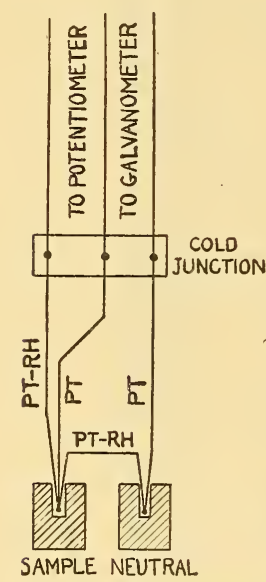

FIG. I30.-Diagrammati representation of differential method

Another method of interpreting the data is by means of the derived differential curve, first suggested by Rosenhain. ${ }^{29}$ This amounts to differentiating the differential curve in respect to the temperature and plotting the value so obtained against the temperature. The derived differential is accordingly related to the differential curve in the same manner that the inverse rate is related to the temperature-time curve. Thus, if $\vartheta$ is the temperature of the material being investigated and $\vartheta^{\prime}$ the temperature of the neutral body, the derived differential is the graph $d\left(\vartheta-\vartheta^{\prime}\right) / d \vartheta$ versus $\vartheta$.

The method consists in observing successive values of $\left(\vartheta-\vartheta^{\prime}\right)$ for equal increments of $\vartheta$, say $2^{\circ} \mathrm{C}$, and plotting successive differences in these values of $\left(\vartheta-\vartheta^{\prime}\right)$ against $\vartheta$. The experimental arrangement is the same as that required for the differential method. Fig. I3I shows both differential and derived differential curves for the same sample of pure iron at the transformation points $A_{2}$ and $A_{3}$.

(c) InORganic Salts and Silicates.-On account of the low heat diffusivity and latent heat of fusion of salts and silicates, considerable difficulty is encountered because of the obliquity of the melting or freezing curves. Consequently, it is necessary to use tall, narrow charges and slow rates of heating. Few salts can be heated in a reducing atmosphere and hence graphite crucibles are prohibited. Porcelain and fire clay are attacked by most salts, so that metal crucibles must be employed. Of these, plati-

${ }_{29}$ Burgess, B. S. Sci. Papers No. 99. 
num, piatinum alloys, and nickel have the widest utility. The thermocouple protecting tube of porcelain must be itself protected by a thin-metal sheath. In many cases salts do not attack platinum perceptibly, so that the thermocouple may be immersed directly in the molten salt. When this is done the crucible must be free from volatile metals, such as iridium, especially for work at high temperatures; otherwise the couple will become contaminated. If no protecting tube is employed, very small crucibles may be used, from $\mathrm{I}$ to $2 \mathrm{~cm}$ in diameter and 2 to $4 \mathrm{~cm}$ high. If

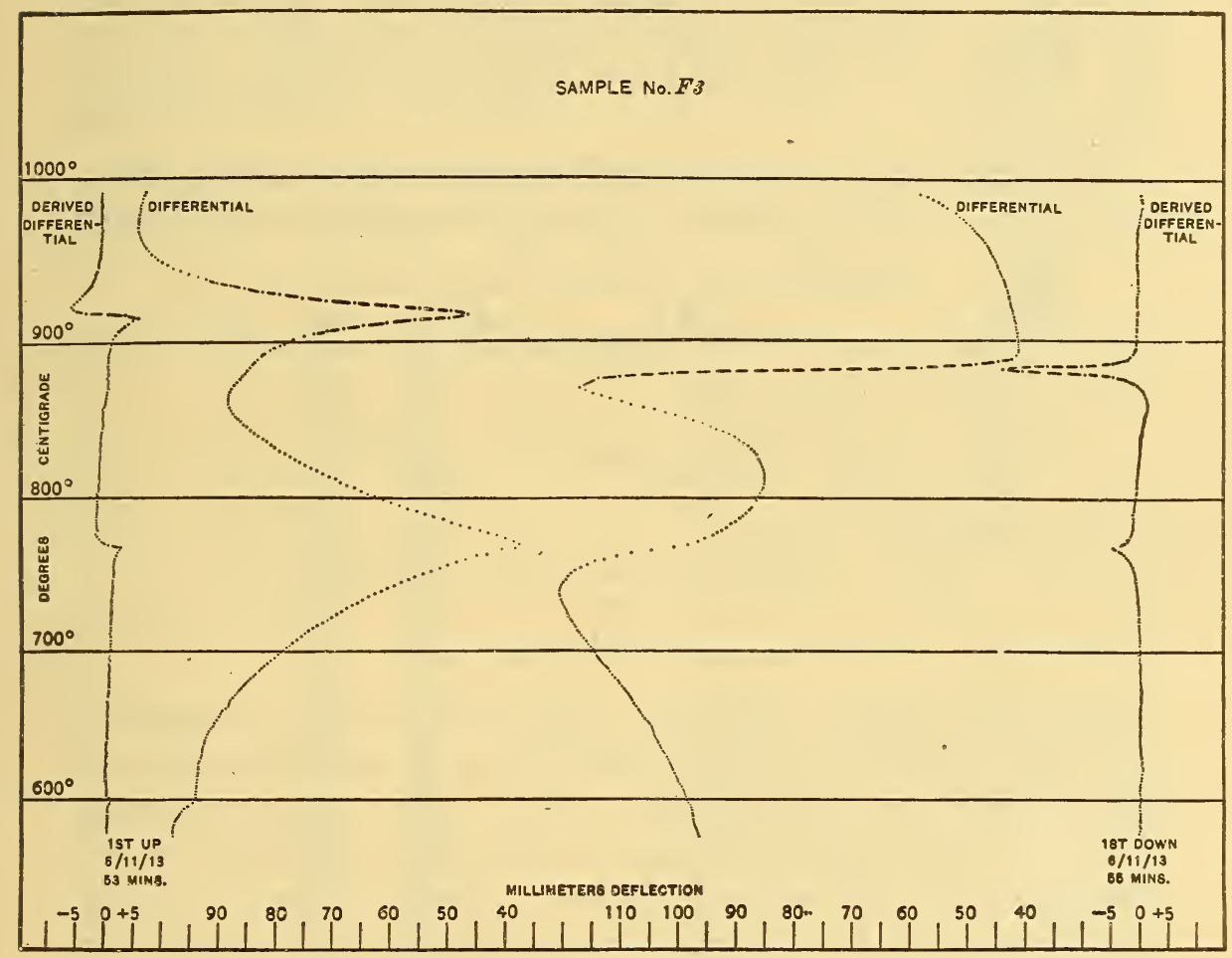

FIG. I3I.-Comparison of differential and derived differential methods

the salt is quite volatile and the vapors distill into the furnace an additional protection tube for the furnace heater is necessary. The melting or freezing points of the nitrates, chlorides, and sulphates of the alkali and alkali earth metals may be determined in the above-described manner.

The melting points of silicates and other compounds of metallic oxides are usually comparatively high and their great viscosity when liquid, slow melting, slow crystallization, and under-cooling introduce many difficulties in the melting-point determination. 
Many crystalline silicates which have a definite melting point do not show a definite freezing point on account of the high viscosity of the liquid, thus preventing the formation of crystal nuclei. The liquid supercools and the viscosity may so increase that a glass is formed; thus borax may be melted but once, and on cooling a clear glass results, which with reheating shows no marked heat transformation at the melting point. Other materials acting in this manner to a lesser degree are diopside, $\mathrm{MgSiO}_{3} \cdot \mathrm{CaSiO}_{3}$, melting at ${ }_{1} 391^{\circ} \mathrm{C}$, and lithium metasilicate, $\mathrm{Li}_{2} \mathrm{SiO}_{3}$, melting at $\mathrm{I} 2 \mathrm{O}^{\circ} \mathrm{C}$. Consequently, in silicate melting-point determinations the melting curves are almost exclusively considered. In Fig. 132, $A$ represents about as good a curve as is usually obtained with a silicate.

In another class of silicates tne meting requires a large time interval, probably on account of the high viscosity of the liquid,

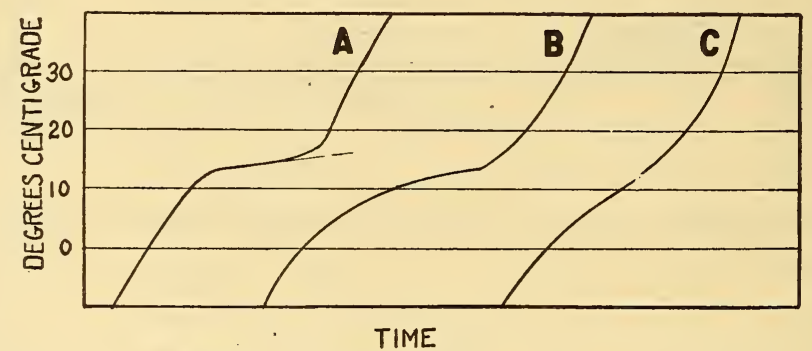

FIG. I32.-Silicate melting curves

so that the material will easily superheat as well as supercool. Both the melting and freezing curves may be so oblique that no halt in the curves is perceptible. Fig. I32, $C$, shows a heating curve for a slow-melting silicate, which gives practically no information as to the true melting point. Silicates of this class may be differentiated by studying the melting curves obtained for different rates of heating. If the faint trace of a halt in the melting curve shifts with rate of heating, it is fairly conclusive evidence that this method is not applicable. A method which has been employed for such materials by the Geophysical Laboratory is to heat the substance for a considerable time at various constant temperatures and, after quenching in mercury, investigate the crystalline properties under a petrographic microscope. The crystalline structure is destroyed by the melting. Materials of this type are albite $\mathrm{NaAlSi}_{3} \mathrm{O}_{8}$, melting below $\mathrm{I}_{200^{\circ}} \mathrm{C}$, ortho-

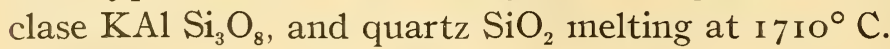


The apparatus used by the Geophysical Laboratory for silicate work is shown in Fig. I33. The marquardt or sillimanite porcelain tube $A$ is joined to a platinum sleeve $B, 5$ by $\mathrm{I} .2 \mathrm{~cm}$, into which tightly fits the platinum crucible $C$, containing the charge. The platinum thermocouple, carefully centered, is inserted with no protection. Great care must be taken to avoid the presence of impurities, such as chips of porcelain from the tube $A$, etc., as the lowering of the freezing point by impurities is proportional to the square of the absolute temperature and becomes very serious at high temperatures. Fig. I $32, B$, shows a heating curve of a silicate containing an impurity.

White has found that in general the electrical leakage through the molten salt is not of serious importance. However, this must be considered

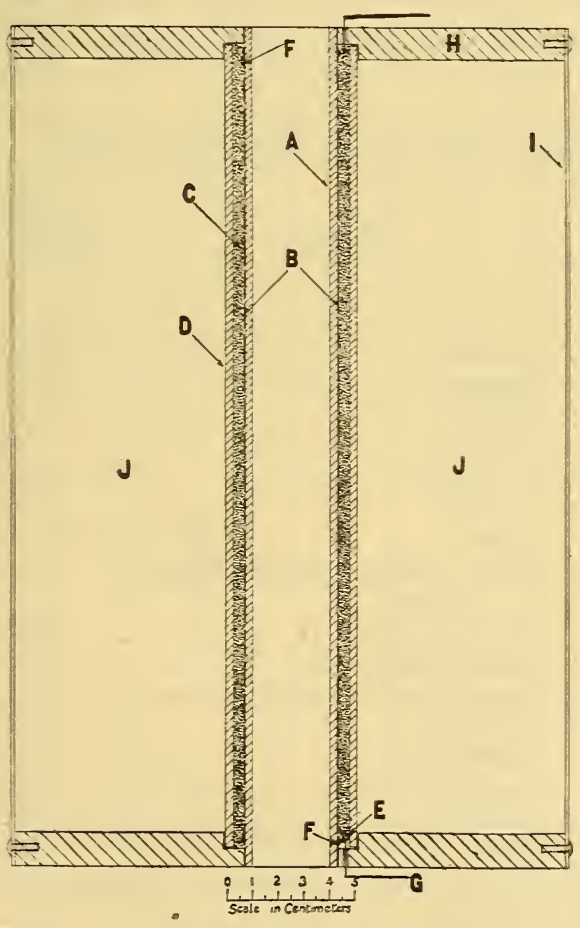

FIG. I34.-Platinum wound melting-point furnace for silicates and metals for precision work at high temperatures, Fig. I33.-Silicate as well as the leakage from

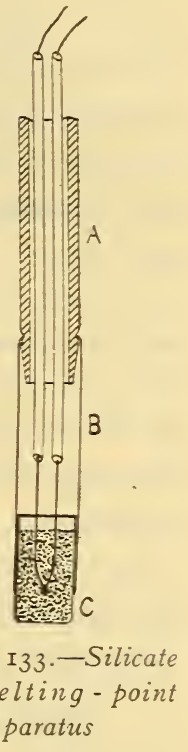
the furnace heating current to the couple. The latter may be diminished by prerenting any part of the heated couple or crucible from coming into contact with the furnace wall. Thermionic leakage to the couple may be minimized by electrostatic shielding. Leakage may be usually detected by moving the couple and by reversing or cutting off temporarily the heating current.

The rate of heating should be from $2^{\circ}$ to $8^{\circ} \mathrm{C}$ per minute. On account of the tendency of the melting curres to obliquity and on account of the small heat transformations involved, it is necessary to maintain a careful furnace control, so that irregularities in heating will not appear on the melting curve. Even then it is possible 
that more sensitive methods of thermal analysis may be desirable, such as the inverse rate or the differential methods described elsewhere. ${ }^{30}$.

Since an oxidizing atmosphere is necessary, the furnace employed for high melting-point work with salts and silicates must be wound with platinum. Fig. I 34 shows a furnace suitable for temperatures up to $155^{\circ} \mathrm{C}$. The tube $A$ of Norton alundum, R. A. $98,33 \mathrm{~cm}$ long, $3 \mathrm{~cm}$ bore, and $3 \mathrm{~mm}$ wall, is wound with platinum or platinum-rhodium ribbon $B$, I to $2 \mathrm{~cm}$ wide and 0.01 or 0.02 $\mathrm{mm}$ thick, with the turns about $3 \mathrm{~mm}$ apart. The ends of the foil are bound with a turn of platinum wire $F$, to which are fused the heavy-silver lead wires $G$; Around the tube $A$ is a second tube $D$, and the space between the two tubes is packed with Norton $\mathrm{R}$. R. alundum, I2O mesh, to prevent local heating of the ribbon. The space between the shell $I$ and the tube $D$ is filled with sintered magnesite.

(I) Table of Melting Points.-The following table gives the melting points of several salts and silicates determined at the Geophysical Laboratory:

TABLE 25.-Melting Points of Salts, Silicates, and Other Compounds

\begin{tabular}{|c|c|c|}
\hline Substance & Formula & $\underset{\text { point }}{\text { Melting }}$ \\
\hline 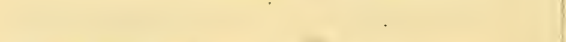 & & ${ }^{\circ} \mathrm{C}$ \\
\hline Sodium molybdate...... & $\mathrm{Na}_{2} \mathrm{MoO}_{4} \ldots$ & 687 \\
\hline Borax & $\mathrm{Na}_{2} \mathrm{~B}_{4} \mathrm{O}_{7} \ldots \ldots \ldots \ldots$ & 741 \\
\hline Sodium chloride $\ldots . . . \ldots \ldots \ldots$ & $\mathrm{NaCl} . \ldots \ldots \ldots \ldots . . . . . . .$. & 801 \\
\hline Sodium sulphate ..... & $\mathrm{Na}_{2} \mathrm{SO}_{4} \ldots$ & 884 \\
\hline Sodium metasilicate... . & $\mathrm{Na}_{2} \mathrm{SiO}_{3} \ldots \ldots \ldots \ldots \ldots$ & 1088 \\
\hline Lithium metasilicate ........... & $\mathrm{Li}_{2} \mathrm{SiO}_{3} \ldots \ldots \ldots \ldots \ldots$ & 1202 \\
\hline Lithium orthosilicate $\ldots \ldots \ldots \ldots \ldots \ldots$ & $\mathrm{Li}_{4} \mathrm{SiO}_{4} \ldots \ldots \ldots \ldots \ldots \ldots$ & 1255 \\
\hline Eutectic between diopside and calcium metasilicate.. & $\begin{array}{l}\mathrm{MgSiO}_{3} \cdot \mathrm{CaSiO}_{3} 60 \text { per cent, } \mathrm{CaSiO}_{3} \\
\quad 40 \text { per cent }\end{array}$ & 1357 \\
\hline Penta calcium trialuminate... & $5 \mathrm{CaO} .3 \mathrm{Al}_{2} \mathrm{O}_{3} \ldots \ldots \ldots . .$. & 1382 \\
\hline Diopside .......................... & $\mathrm{MgSiO}_{3} \cdot \mathrm{CaSiO}_{3} \ldots \ldots \ldots \ldots \ldots \ldots \ldots$ & 1391 \\
\hline Eutectic between calcium metasilicate and cristobalite & $\mathrm{CaSiO}_{3} 77$ per cent, $\mathrm{SiO}_{2} 23$ per cent... & 1426 \\
\hline Calcium metasilicate....... & $\mathrm{CaSiO}_{3} \ldots \ldots \ldots \ldots \ldots$ & 1540 \\
\hline Anorthite................. & $\mathrm{CaSiO}_{3} \cdot \mathrm{Al}_{2} \mathrm{SiO}_{3} \ldots \ldots \ldots \ldots \ldots \ldots \ldots$ & 1551 \\
\hline Magnesium metasilicate ....... & $\mathrm{MgSiO}_{3} \ldots \ldots \ldots$ & 1554 \\
\hline 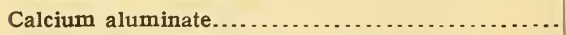 & $\mathrm{CaO} . \mathrm{Al}_{2} \mathrm{O}_{3} \ldots \ldots \ldots$ & 1592 \\
\hline Cristobalite ............................... & $\mathrm{SiO}_{2} \ldots \ldots$ & a 1710 \\
\hline
\end{tabular}

a Quenching method.

(d) Technical Melting-Point Determinations.-For ordinary technical testing, in which an accuracy of 5 .to $10^{\circ} \mathrm{C}$ is sufficient, the general procedure is the same as that discussed for pre-

\footnotetext{
${ }^{30}$ For further details on silicates see publications of the Geophysical Laboratory in the Am. J. Sci., and Jaeger, Physico-Chemical Measurements at High Temperatures, Walters, Groningin, Holland; 1913.
} 
cision work, except that less care is required in experimental details. When large couples are used it is necessary to procure much larger crucibles, approximately in the same proportion. illustrated by Table 23. It is not a wise practice to immerse a bare base-metal couple directly in a molten salt or metal. The couple should be protected by a metal or ceramic tube, preferably of thin wall, in order to reduce heat conduction. For metals, Dixon graphite crucibles, which are less susceptible to oxidation than the Acheson graphite, or pure fire clay (except for aluminum) are satisfactory; while iron, calorized iron, nickel, nickel-chromium alloys, or duriron are serviceable for salts. For the checking of thermocouples pots of commercial tin, lead, zinc, sodium chloride, and copper of a fair degree of purity are sufficient. The melting points of these materials will rarely differ from those of the pure substances by $10^{\circ} \mathrm{C}$, and, if close checks are required, the melting points may be determined by means of an accurately calibrated couple. A gas furnace may be employed for heating, but the flame should not be allowed to strike the crucible on one side only. If the burner is directed along a radius of the furnace instead of tangential to its surface a baffle may be interposed before the crucible, or the crucible may be mounted on a pedestal above the flame. Under these circumstances it is desirable to protect the crucible by an outer metal tube. An excellent form of gas furnace is the melter's furnace illustrated by Fig. I35. For ordinary laboratory work size No. 3 is suitable. The furnace is provided with three burners $D$, only one of which appears in the drawing, mounted tangentially to the circumference in order to produce a spiraling flame. Excellent temperature uniformity is thus obtained.

(e) WIRE METHOD.-The wire method of determining melting points of metals consists in inserting at the hot junction of the couple a small length of wire drawn from the material to be investigated. Usually a rare-metal couple is employed. The couple is cut apart at the hot junction and the sample wire, preferably of about the same diameter as the wire of the couple and about I $\mathrm{cm}$ or less in length, is fused (not soldered) in. The couple is then mounted in a narrow tube, which may be closed at the lower end, and inserted into a uniformly heated furnace, as illustrated by

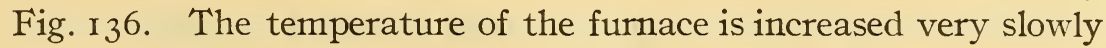
as the melting point is approached, and the emf of the couple is observed when it comes to a halt during melting. This occurs 
slightly in advance of the instant that the circuit is broken by the melting. The temperature corresponding to this emf on the calibration curve of the couple is the desired melting point. The introduction of the short piece of metal produces no effect on the thermoelectric circuit, provided it is at a uniform temperature. The two metals most satisfactorily employed with this method are gold and palladium, although many other metals could be used with the proper atmosphere. The experimental

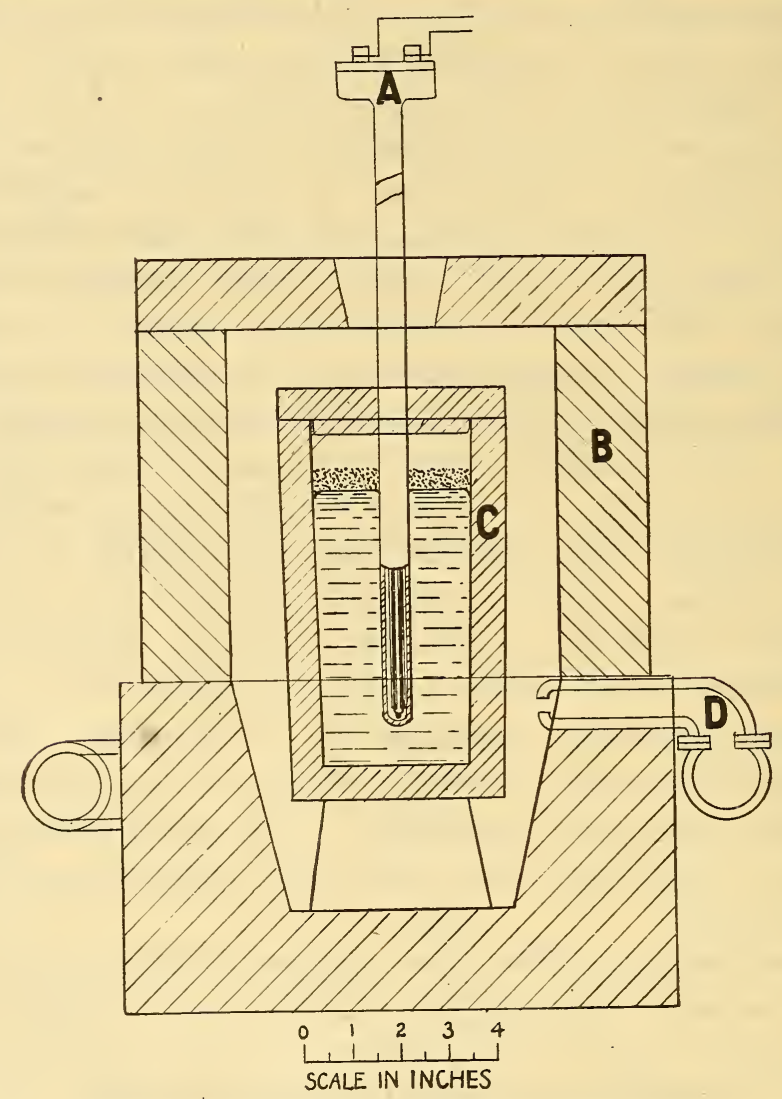

FIG. I35.-Gas furnace for technical melting points

manipulation for palladium is a little difficult. A platinumwound furnace similar to Fig. I 34 must be used, and the two insulating tubes of the couple must not come into contact with each other or with the furnace walls; otherwise electrical leakage occurs. A leak between the wires of the couple produces a peculiar effect at the instant of melting of the palladium. Instead of the galvanometer showing zero deflection, it will indicate the very large voltaic emf developed when the porcelain acts as an electrolyte, 
since the metallic shunt of low resistance across this emf is broken when the palladium melts. With proper care results by this method are reproducible to $\mathrm{I}^{\circ} \mathrm{C}$. It is frequently convenient to sight a microscope of low power on the loop of wire and observe the melting. - If the rate of heating is slow, sufficient time elapses from the incipient melting to secure several reliable potentiometric readings before the circuit is broken.

The wire method is occasionally employed for copper heated in air. The temperature so obtained however, is not reliable. It may be any value from 1063 to $1083^{\circ} \mathrm{C}$, depending upon the rate of heating, and hence upon the amount of oxide formed. With very slow heating the wire may be converted into $\mathrm{CuO}_{2}$, which melts at about I I $90^{\circ} \mathrm{C}$.

\section{USE OF RESISTANCE THERMOMETERS}

The standard temperature scale as used and distributed by the Bureau of Standards is defined in the interval -40 to $45^{\circ} \mathrm{C}$ by means of platinum resistance thermometers calibrated in ice, steam, and sulphur vapor $\left(444.6^{\circ} \mathrm{C}\right)$. Platinum of a high degree of purity is readily, obtainable, and its purity is assured if the mean temperature coefficient of resistance of the thermometer between $\mathrm{o}$ and $100^{\circ} \mathrm{C}$ is not less than 0.00388 , and if the constant $\delta$ of the Callendar equation used for expressing the relation between temperature and resistance is not greater than $\mathrm{I} .5^{2}$. The temperature scale from $45^{\circ}$ to $\mathrm{IIOO}{ }^{\circ} \mathrm{C}$ is FIg. I36.-Apparatus defined by the melting points of pure antimony,

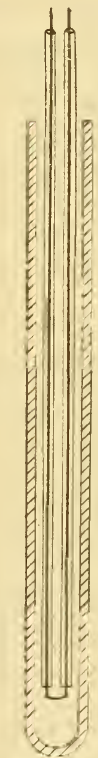
silver, gold, and copper, and interpolation between these points is based on the temperature scale defined by the rare metal couple (Pt, 9o per cent $\mathrm{Pt}$ - ro per cent $\mathrm{Rh}$ ) calibrated at two of these fixed points, antimony and copper, and a third point, zinc, fixed by the resistance thermometer.

It has been found that the scale defined by the resistance thermometer calibrated as above agrees with the thermocouple scale as closely as this may be reproduced, even up to $800^{\circ} \mathrm{C}$. Above this range the behavior of a resistance thermometer is likely to be erratic. The calibration is subject to changes on account of volatilization of the platinum and strains introduced by the warp$8513^{\circ}-21-14$ 
ing of the mica frame upon which the wire is wound. Reliable precise measurements can be obtained only by intercomparison of several thermometers. Below $800^{\circ} \mathrm{C}$, however, the temperature scale defined by the resistance thermometer is probably more closely reproduced by different resistance thermometers than is the thermocouple scale reproduced by different thermocouples. Hence when very high precision is required, $0.1^{\circ} \mathrm{C}$ or better, the resistance thermometer offers some advantages in the range $45^{\circ}$ to $800^{\circ} \mathrm{C}$. Below $450^{\circ} \mathrm{C}$ the resistance thermometer is the most accurate means of temperature measurements, since its temperature scale is accepted as standard. In melting-point work of the highest precision from 450 to $500^{\circ} \mathrm{C}$ there is some question as to whether the error resulting in a temperature gradient over the entire bulb of a resistance thermometer introduces as large an error as may arise with a thermocouple, which requires a much smaller region of temperature uniformity; for example, the error due to inhomogeneity of the wires of the couple.

The general technique to be observed in melting-point determinations with the resistance thermometer is the same as that required in thermocouple work of the highest precision. The large size of crucibles described in Table 23 is necessary, since the bulb of the thermometer is from 5 to $8 \mathrm{~cm}$ long. The most extensive paper on the application of resistance thermometers to melting-point determinations is by Waidner and Burgess. ${ }^{31}$ This paper contains a complete bibliography up to 1909. Later developments have been made in only the methods of measurement, such as bridges, etc., and are described by Waidner, Dickinson, Mueller, and Harper and by Mueller. ${ }^{32}$

\section{USE OF OPTICAL PYROMETERS}

(a) Metals and Alloys.-Above $1400^{\circ} \mathrm{C}$ the difficulties involved in the use of thermocouples for melting-point determinations are very great. Refractory tubes are attacked by the metals and are permeable to gases, which contaminate the couple. The calibration of the couple changes, inhomogeneity develops, and troubles arise from electrical leakage. For these reasons the use of optical pyrometers is desirable and, above $\mathrm{r} 55^{\circ} \mathrm{C}$, necessary. The disappearing-filament type of optical pyrometer is most satisfactory, since it is capable of high precision and requires a very

31 Waidner and Burgess, B. S. Sci. Papers, No. I 24.

:- Vaidner, Dickinson, Mueller, and Harper, A Wheatstone Bridge for Resistance Thermometry, B. S. Sci. Papers, No. 24I. Mueller, Wheatstone Bridges and Some Accessory Apparatus for Resistance Thermometry, B. S. Sci. Papers, No. 288. 
small source upon which to sight. Special care must be given in the use of optical pyrometers to insure that black-body conditions are closely approximated.

For metals and alloys melting below $\mathrm{I} 750^{\circ} \mathrm{C}$ the experimental device shown in Fig. $\mathrm{I} 37$ is suitable. The outer tube is made of sillimanite porcelain, melting at $\mathrm{I} \mathrm{IO}^{\circ} \mathrm{C}$, and constitutes the unit to be inserted into a furnace. The crucible inside this tube is about $\mathrm{I} 8 \mathrm{~mm}$ in diameter and $20 \mathrm{~mm}$ high, and is provided with a cover having two holes, 2 to $3 \mathrm{~mm}$ in diameter, in the positions illustrated. The pyrometer is focused on the outer hole and the central hole is for observing the progress of a freezing or melting. If the metal in the crucible is molten the central hole appears quite dark against a brighter background because the level metal surface reflects the image of the upper and cold part of the furnace. The surface under the side hole, however, is convex on account of the surface tension of the metal, and the $V$-shaped depression formed between the metal and the side wall affords a satisfactory black body upon which to sight the pyrometer. The outer tube should be uniformly heated for a distance at least twice its diameter, and the short crucible should be filled about half full with metal. Tall crucibles are not desirable, as they are less likely to be uniformly heated, and any nonuniformity disturbs the black-body conditions. Although it is realized that such a system is

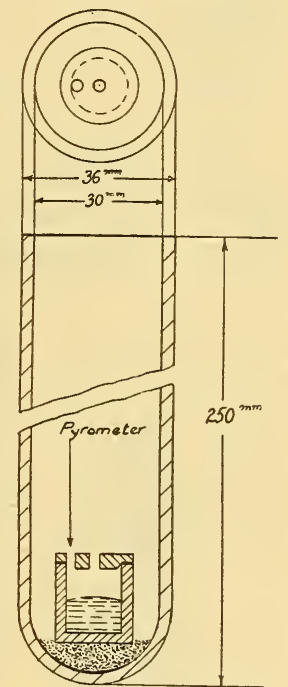

Fig. I37.-Apparatus for melting points of metals and alloys with optical pyrometer not ideal for producing black-body radiation, especially when the temperature is continually changing, as is necessary in meltingpoint determinations, still it may be concluded on the basis of many careful observations that the error in general does not amount to more than 2 or $3^{\circ} \mathrm{C}$. For more precise work it is possible to fix to the cover of the crucible a reentrant tube immersed directly in the metal, and into which the pyrometer is sighted. This method is frequently employed for the standardization of an optical pyrometer by direct melting point determinations. ${ }^{33}$

\footnotetext{
:3 Kanolt, B. S. Tech. Papers No. ro. Foote, Met. and Chem. Eng., 11, p. 97, 1913,
} 
(1) Table of Melting Points.-The following table gives the melting points of several pure metals, which with their alloys may be investigated by the above method:

TABLE 26.-Melting Points from 1400 to $1800^{\circ} \mathrm{C}$

\begin{tabular}{|c|c|c|c|}
\hline Metal & $\begin{array}{l}\text { Melting } \\
\text { point }\end{array}$ & Crucible material & Atmosphere \\
\hline Silicon...... & 1420 & $\mathrm{MgO}, \mathrm{Al}_{2} \mathrm{O}_{3}$, or mixtures. & Vacuum, $\mathrm{N}_{2}, \mathbf{H}_{2}$. \\
\hline Nickel..... & 1452 & 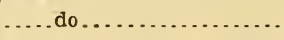 & Vacuum, $\mathbf{N}_{2}$ \\
\hline Cobalt...... & 1480 & ..... do.. & Vacuum. \\
\hline Iron . . . . . . . & 1530 & ..... do.. & Do. \\
\hline Palladium... & 1550 & ..... do.. & Air. \\
\hline Chromium... & 1615 & ..... do.. & Vacuum, $\mathbf{N}_{\mathbf{2}}$. \\
\hline Platinum... & 1755 & ..... do.. & Air. \\
\hline
\end{tabular}

(2) Crucibles.-Crucibles containing either graphite or silica should not be used for precision work with these metals and their alloys. Silica-free crucibles are not sold by the porcelain manu-

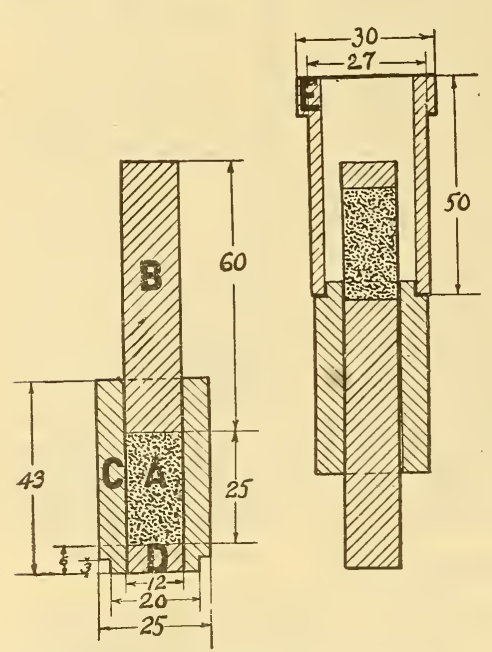

FIG. r38.-Briquetting mold for refractory materials (dimensions in $\mathrm{mm}$ ) facturers, but can be made in the laboratory without much difficulty from magnesia, alumina, or mixtures of the two. Pure magnesium aluminate does not begin to melt below $1925^{\circ} \mathrm{C}$, and hence is sufficiently refractory for the above metals. Pure fused alumina and pure fused magnesia are ground to I 50 to 200 mesh and mixed in the approximate proportion 75 per cent alumina and 25 per cent magnesia (the exact proportion is immaterial). The powder is slightly moistened and compressed in a briquetting mold, similar to that illustrated by Fig. I38, into a cylinder having the outside diameter of the crucible. This cylinder is fired in a graphite crucible at a temperature of $\mathrm{I} 700^{\circ} \mathrm{C}$ for a few moments. The Arsem furnace, described later, or any carbon-resistor furnace having a clean atmosphere and capable of maintaining this temperature, is satisfactory. The cylinder sinters to a hardness sufficiently strong to be handled, and with care it is possible to drill out a hole large enough for the metal with an ordinary twist drill. The crucible is then refired at a temperature between 1800 and $1900^{\circ} \mathrm{C}$. A cover is readily made in the same manner. 
For ordinary testing with metals other than palladium and platinum, the sillimanite crucible ("impervite" or "usalite") may be employed. These are, of course, attacked and must be discarded after each determination, but experiments indicate that the impurity introduced in the metal by a single heating affects the melting point less than $10^{\circ} \mathrm{C}$. Such crucibles are useful for ordinary work with steels and nickel-chromium alloys.

(3) Furnaces.-None of the metals or alloys of high melting point, except some of the rare metals, can be melted in air without serious oxidation. The use of a vacuum furnace, the residual gases being $\mathrm{CO}$ and $\mathrm{N}_{2}$, is satisfactory except for palladium and platinum. Fig. I 39 illustrates such a furnace designed by Arsem. ${ }^{34}$ It is readily and accurately controllable and a clear atmosphere is assured. The graphite spiral $C$ is mounted in water-cooled copper electrodes. $F$ is a radiation shield of graphite filled with magnesia. The pyrometer is sighted through the glass window $D$ into the sillimanite tube $B$ (see also Fig. 137), which is suspended from the top of the furnace. In the place of $A$ the crucible shown in Fig. 137 is mounted on a bed of alundum. The porcelain tube is fairly impermeable to the hot gases and carbon vapor. With the first heating in a vacuum furnace the liquid metal usually sputters on account of evolution of gases, and may throw off the cover of the crucible, but after the first melting satisfactory readings can be taken. Suitable rates of heating or cooling near the melting point are from ro to $20^{\circ} \mathrm{C}$ per minute. In this range temperature measurements should be made very frequentlyevery ro seconds, if possible. Fig. I 40 illustrates typical freezing curves for pure iron and chrome steel by this method.

For temperatures below $1550^{\circ} \mathrm{C}$ the sillimanite tube of Fig. I 37 may be heated in a platinum-wound furnace, if desired. The proper atmosphere for the metal can be obtained by fitting the sillimanite tube with a metal top containing a window and two side tubes through which $N_{2}$ is drawn. Another method is to insert a long porcelain tube through the furnace, in the center of which is mounted the crucible. Gas is led in at the top of this tube and out at the bottom.

Crucible melts of palladium and platinum are seldom made. Both of these metals must be melted in an oxidizing atmosphere, since in a reducing atmosphere the slightest trace of silica, which is present in nearly every type of furnace, is disastrous. Palla-

${ }^{34}$ Arsem, J. Am. Chem. Soc., 28, p. 921; 1906. Kanolt, B. S. Tech. Papers No. ro; B. S. Sci. Papers, No. 212 . 
2 I4 Technologic Papers of the Bureau of Standards

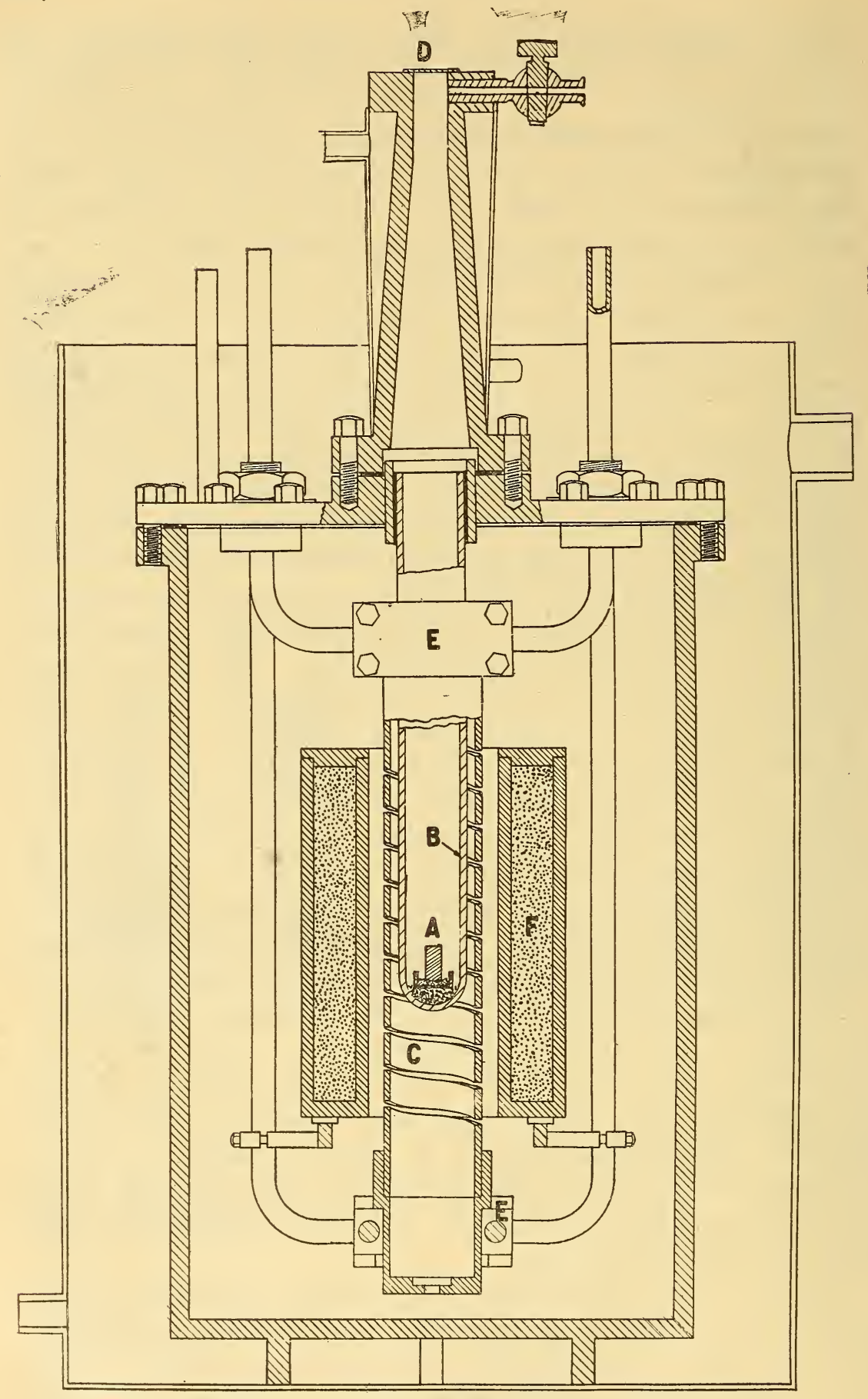

FIG. I39.-Graphite resistance vacuum furnace 
dium may be heated in a lime, alumina, magnesia, or magnesiumaluminate crucible in a platinum-wound furnace. Platinum could be melted in a furnace wound with a platinum-rhodium alloy, but the method has never been attempted. In several cases a furnace consisting of an iridium tube in which the tube serves as the resistor element has been used. The method is open to serious objections, however, on account of the rapid volatilization of

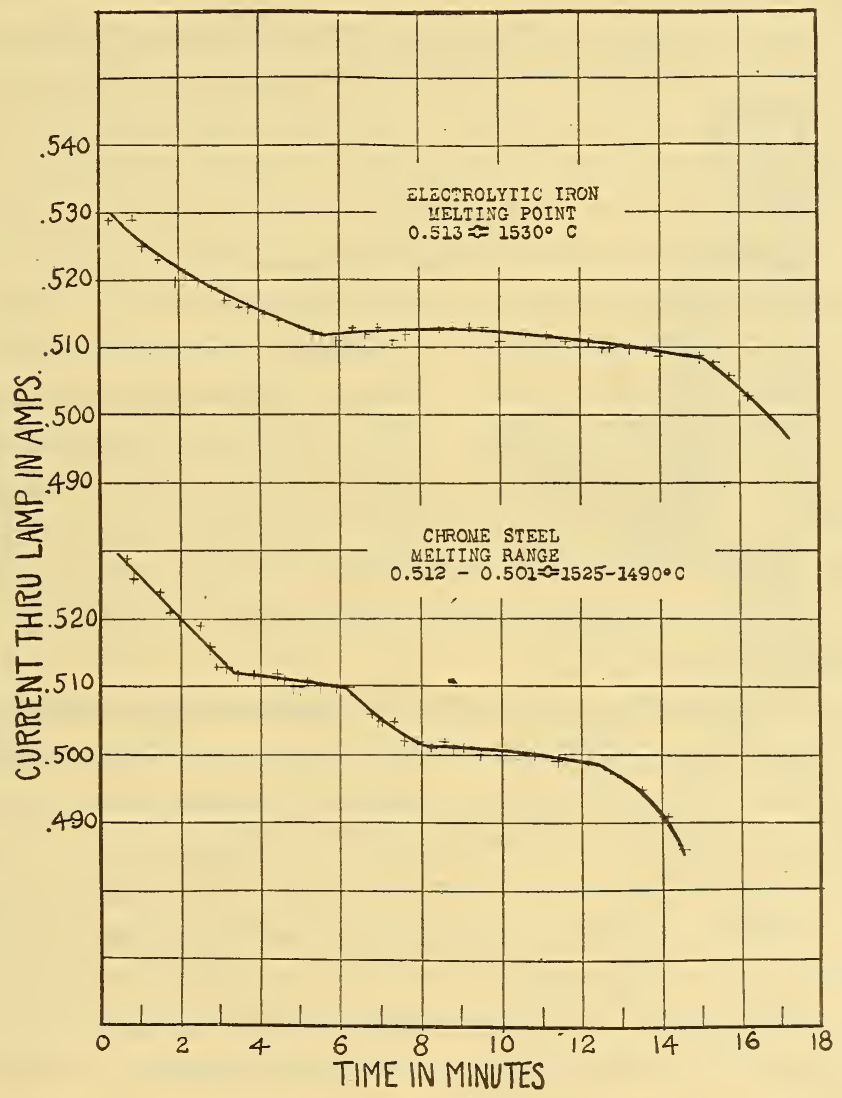

FIG. I40.-Freezing curves of pure iron and chrome steel

iridium even when the material is coated with a protective paint, such as a refractory earth mixture..$^{3 \overline{ }}$

A furnace recently developed by Northrup ${ }^{36}$ heats any electrically conducting material by inducing eddy currents in it. This method is admirably adapted to the melting of easily contaminated metals. The heating may be made with any desired

35 Waidner and Burgess, B. S. Sci. Papers, No. 55.

36 Northrup-Ajax High-Frequency Induction Furnace, Chem. Met. Eng.; Aug. r, r9r8. Principles of Induction Heating with High-Frequency Currents, Trans. Am. Electrochem. Soc.; IgIg. 
gas as an atmosphere or in vacuum. Limitations upon proper refractory containers and insulators are reduced to a minimum. It is even unnecessary to use silica-free crucibles for metals such as palladium and platinum. Temperature uniformity through the charge is secured, thus permitting satisfactory measurements with the optical pyrometer. The Northrup furnace operates on an entirely new principle and its many possible applications are still being developed.

(4) Other Methods.-For metals melting much above the range of platinum a modification of the wire method may be used. The material in the form of wire or ribbon is mounted as a filament of an electric lamp and is gradually heated electrically until it melts. The apparent or brightness temperature is measured by an optical pyrometer at the instant of melting. In order to correct the observed temperature to true temperature the value of the emissivity of the material must be known. This involves a separate and difficult experiment. In some cases the material in sheet form is folded into a wedge and electrically heated, and the pyrometer is sighted into the opening of the wedge, the radiation from which is approximately black. Various other methods are employed, such as sighting on a bead of the molten metal in an electric arc, extrapolation of the temperature-current curve for a lamp filament, etc. ${ }^{37}$

(5) Micropyrometer.-In order to determine the melting points of microscopic samples of materials, Joly devised the meldometer. This consists of an electrically heated platinum strip the linear expansion of which is a measure of its temperature. The relation between elongation and temperature is determined by placing samples of pure materials on the strip and observing the melting point through a microscope while the elongation is measured by a screw adjustment. A more satisfactory method is by use of the Burgess ${ }^{38}$ micropyrometer, which operates on the same principle as the disappearing-filament pyrometer, except that the lamp is mounted in a microscope instead of a telescope. (See Fig. I4I.) This is sighted on a platinum strip and on the strip in the field of view is placed a sample of the material to be melted, usually from o.I to o.OoI $\mathrm{mg}$ in weight. The strip is inclosed in a water-cooled container having a clear-glass window and a re-

${ }^{37}$ Waidner and Burgess, B. S. Sci. Papers Nos. 40 and 55; J. d. Phys. 6, p. 830; 1907. Forsythe, Astrophys. J., 12, p. 364; 19ז1. Mendenhall and Ingersoll, Phys. Rev., 25, p. I; I907. Pirani and Meyer, Verh. d. Phys. Ges., 14, p. 426 ; 1912. Wartenberg, Verh. d. Phys. Ges., 12, p. I20; 1909. Langmuir, Phys. Rev., 6, p. I52; 1915. Worthing, Phys. Rev. 10, p. 377; 1917.

${ }^{38}$ B. S. Sci. Papers Nos. 198, 205, and 242. 
ducing or neutral atmosphere is maintained. The temperature of the platinum strip is measured at the instant of melting, which is indicated with unmistakable evidence, since the material either gathers into a ball or spreads out on the strip. The pyrometer is calibrated by employing metals of known melting points. A natural question which arises is whether the sample actually takes up the temperature of the strip. If the sample were heated by radiation alone, since it receives radiation from a solid angle $2 \pi$ and radiates through a solid angle $4 \pi$, it can be shown on the basis of the Stefan-Boltzmann law. that the absolute temperature of the sample would be roughly only 0.8 that of the strip. On the other hand, the microscopic sam ple acts as a shunt on the platinum strip, and more heat may be developed at this point, tending to increase the temperature. Thermal conduction along the strip reduces this effect. The fact that samples of different sizes appear to give the same value for the melting point indicates that all these effects tending to produce temperature non-uniformity are small and that the sample actually assumes the temperature of the strip, the heat conduction and gas convection compensating for the deficiency in the direct radiation. The method must be used with

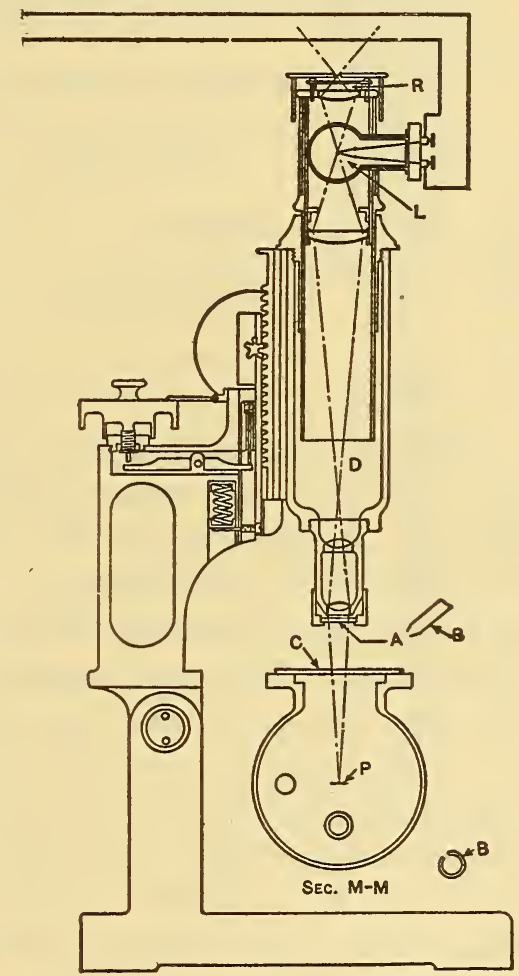

Fig. I4I.-Micropyrometer caution, however, when applied to other materials than metals, especially in a vacuum. It has been found that errors of $50^{\circ}$ or more resulted when gold was heated on a graphite strip in vacuum.

(b) REFRACTORY MATERIALS-(I) Refractory oxides.-Refractory oxides are the basic constituents of minerals and of ceramic refractory materials. Their melting points are very high, and it is necessary to observe special precautions to avoid contamination and to eliminate the smoke produced by the vaporization of the 
oxide. The Arsem furnace shown in Fig. I39 was used by Kanolt ${ }^{39}$ for determining the melting points of magnesia, lime, alumina, and chromium oxide. In each case heating curves by the temperature-time method were obtained with an optical pyrometer, and the results were confirmed by examination of the materials heated above and below the observed melting points. The rate of heating was about $100^{\circ} \mathrm{C}$ per minute near the melting point.

The following table of melting points is a summary of Kanolt's work:

TABLE 27.-Melting Points of Refractory Oxides

\begin{tabular}{|c|c|c|c|}
\hline Oxide & M. P. ${ }^{\circ} \mathrm{C}$ & Crucible material & Atmosphere \\
\hline $\mathrm{MgO}$. & 2800 & Graphite... & Stream of $\mathrm{CO}$ and $\mathrm{N}_{2}$ at atmospheric pressure \\
\hline $\mathrm{CaO} .$. & 2570 & Tungsten, $\mathrm{CaO}$ & Stream of $\mathrm{H}_{2}$ at atmospheric pressure \\
\hline $\mathrm{Al}_{2} \mathrm{O}_{3 \ldots} \ldots$ & 2050 & Tungsten, graphite.... & $\begin{array}{l}\mathrm{CO} \text { and } \mathrm{N}_{2} \text { vacuum; stream of } \mathrm{CO} \text { and } \mathrm{N}_{2} \text { at atmos- } \\
\text { pheric pressure }\end{array}$ \\
\hline $\mathrm{Cr}_{2} \mathrm{O}_{3 . .}$ & 1990 & do & $\mathrm{CO}$ and $\mathrm{N}_{2}$ vacuum \\
\hline
\end{tabular}

Magnesia was melted in a special form of graphite crucible, Fig. I 42, which was mounted in the Arsem furnace. The pyrometer was sighted through the tube $B$, on the bottom of the tube $A$, immersed in the magnesia. $C O$ and $N_{2}$ at atmospheric pressure were passed through $B C D$, thus clearing the field of view from smoke. Lime was melted in a similar container, except that the parts in contact with the lime were made of tungsten. Fig. I 43 shows another method employed for lime. The tube $O$, compressed from pure lime, was suspended in the furnace, and a stream of hydrogen was passed in at $P$ and out at $Q$, to avoid the presence of smoke. Alumina was melted in vacuo in the same form of crucible used for magnesia and also in a graphite crucible, shown by Fig. I44. This latter method with crucibles of both graphite and tungsten was employed for chromium oxide.

(2) Fire Bricks and Ceramic Materials. ${ }^{40}$ - Refractory materials such as fire bricks, fire clays, and minerals are composed of compounds of the refractory metallic oxides, solid solutions of the oxides, pure oxides, and mixtures of these classes, together with a variety of chemical compounds. .The oxides, silica, and alumina occur most commonly in refractory materials, and frequently in combination with these are the oxides of the alkaline-earth, the alkali, the iron group, and the rare-earth metals.

${ }^{39}$ Kanolt, B. S. Tech. Papers, No. זо; B. S. Sci. Papers No. 212.

40 For a more detailed discussion see L. I. Dana, Bull. Am. Inst. Min. and Met. Eng.; Sept., rgrg. 
(3) Meaning of the Melting Point.-In the case of refractory materials, which are either amorphous or heterogeneous mixtures of oxides and other substances, there is no definite melting point. The change from the solid state to one in which the material will flow is gradual over both a temperature and time interval. In addition, physical and chemical reactions which are not equilibrium reactions of ten take place during melting. These phenomena practically prohibit the use of melting curves and, for ordinary technical purposes, the criterion of marked flow, although indefinite from the pure scientific standpoint, is adopted. Accordingly, the practical definition of the melting point of a refractory material is that temperature at which under specified conditions a marked and distinct flow of the material begins. In terms of the deformation of a cone or cylinder the melting point

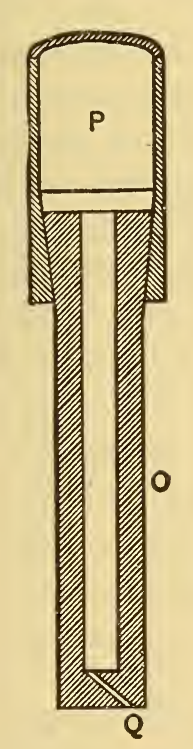

FIG. I43.-Arrangement for melting lime is midway between the temperature at which the deformation begins and the temperature at which the material fuses into a lump or ball, or is completely bent over. The softening or fusing point of a refractory should be defined in the same manner, making the three terms synonymous. If the refractory is a pure Fig. 142.-Arrangecrystalline compound, it possesses a true melting point, but often,

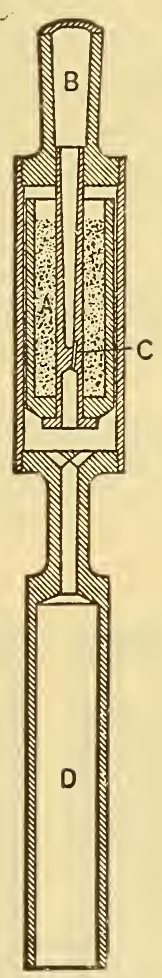

ment for melting magnesia

as, for example, with quartz and albite, the change of state takes place so slowly at the melting point that the substance may be superheated. Thus with quartz the viscosity is so high that a marked flow is not perceptible until the material is superheated by 40 or $50^{\circ} \mathrm{C} .{ }^{41}$ For technical testing the criterion of marked flow is usually taken to define the so-called melting point even for pure crystalline compounds, and thus, on the basis of such a definition, the melting point of quartz is about $1750^{\circ} \mathrm{C}$.

\footnotetext{
${ }_{41}$ Quartz begins to flow at $1750^{\circ} \mathrm{C}$. (Kanolt, B. S. Tech. Papers, No. Ic) while its true melting point is ${ }_{17} 70^{\circ} \mathrm{C}$ (Ferguson and Merwin, Am. J. Sci., 46, D. I: 1918.]
} 
(4) Conditions Affecting Observed Values of the Melting Point.The results obtained on the basis of the above definition of melting point are influenced by a number of experimental factors as follows: (a) Chemical composition; (b) size of the particles, and size, shape, and orientation of the sample; $(c)$ time and rate of heating; and $(d)$ pressure and chemical nature of atmosphere.

The relations between these factors are so complex that in most cases it is not possible to predict the magnitude or sign of the change in melting due to a variation in one or more of the above

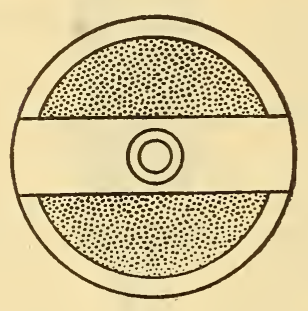
conditions. It must be noted that the melting point in actual use of the refractory material may differ considerably from that observed in the laboratory on account of the fact that the conditions of use are not reproduced in the laboratory. Thus contamination of the refractory by molten metals, slags; flue dust, etc., may alter the melting point because of the chemical reaction; aiso, the

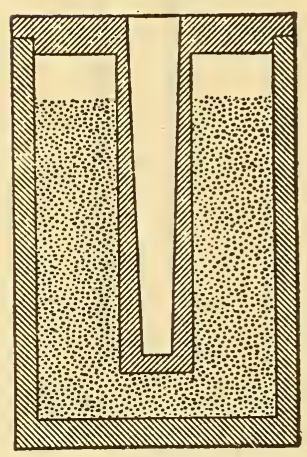
time and rate of heating may be different. Under load the softening or melting point of a refractory is lowered on account of plastic flow. For this and other reasons the value of the melting point determined in the laboratory is not the only factor, as far as failure is concerned, to be considered in the choice of a refractory for an industrial installation.

(5) Method of Melting-Point Determination-Sampling, Grinding, and Molding.FIG. I44.-Crucibleformelt- Fire brick and other refractories of non-
ing alumina and chromic uniform texture and composition or with

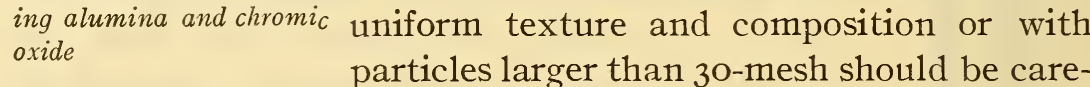
fully sampled. It is accordingly necessary to grind the material and mold the powder into the form of a cylinder or cone. If the refractory is of fine and uniform texture, however, a piece may be chipped off and shaped to the proper form. Fine refractory powders may be briquetted directly. Since the size of the particles may affect the melting point, the degree of fineness should be specified, at least approximately. For a large variety of substances, grinding to pass an 8o-mesh screen has proven satisfactory. The material is then slightly moistened and compressed into a cylinder about $2.5 \mathrm{~cm}$ high and $\mathrm{I} .2 \mathrm{~cm}$ in diameter, by means of the brass or polished steel mold shown in Fig. I38. In 
this figure $C$ is a cylinder, $B$ the piston, $D$ a plug, and $A$ the powder. In order to remove the compressed cylinder, the mold is inverted, and the cylinder $E$ is placed on top and the sample is slowly pushed out.

(6) Time and Rate of Heating.-The rate of heating from room temperature to about $1000^{\circ} \mathrm{C}$ for substances melting above

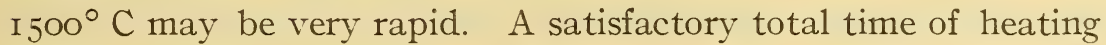
is from 30 minutes to 2 hours with a rate of from 5 to $10^{\circ} \mathrm{C}$ per minute from about $50^{\circ}$ below the melting point and during melting. Since the observed melting point depends to some extent upon the rate of heating, it is necessary to specify the temperaturetime curve in order that results be reproducible. The following table illustrates the manner in which this may be done with sufficient exactness:

Typical Time-Temperature Relation

\begin{tabular}{|c|c|}
\hline Temperature interval & Time \\
\hline $\begin{array}{l}\text { Room temperature to } 1000^{\circ} \mathrm{C} \ldots \ldots \ldots \\
1000 \text { to } 1650^{\circ} \ldots \ldots \\
1650 \text { to } 1700^{\circ} \mathrm{M} . \mathrm{P} . \ldots .\end{array}$ & $\begin{array}{l}\text { Minutes } \\
20 \\
25 \\
5\end{array}$ \\
\hline
\end{tabular}

(7) Type of Furnace and Conditions Existing Therein.-A furnace for melting refractories should be capable of easily maintaining a temperature of $1800^{\circ} \mathrm{C}$, and means must be provided for close temperature regulation. The atmosphere within the furnace must not react appreciably with the specimen; otherwise the material must be protected. Graphite or carbon-tube and spiralresistance furnaces operated in air oxidize rapidly, but may be somewhat protected by passing a neutral or reducing gas through them. The gas may also serve the purpose of carrying away smoke, so that accurate optical temperature measurements may be made. Crushed carbon, graphite, kryptol, or carbon-plate resistor furnaces do not permit of very precise regulation. Some forms of furnaces are so constructed that it is difficult to use an optical pyrometer. The vacuum furnace of the Arsem type, although requiring greater accessory apparatus, is more desirable on account of its freedom from smoke and because good control is possible at extreme temperatures, even at $2.500^{\circ} \mathrm{C}$. Furthermore, the atmosphere can be made much less reducing than that in an ordinary graphite furnace. Many refractories are seriously attacked in a carbon and carbon-monoxide reducing atmos- 
phere, and it is usually not possible to judge by simple inspection of the melted sample whether the reduction is appreciable. In many cases the surface of the specimen is attacked, and a shell of higher melting point is formed, so that, while the inner uncontaminated material may have been melted, there is no outward evidence of this fact. In general, the sample should be protected from the reducing atmosphere by a refractory tube of low porosity. In the Arsem furnace the sample $A$, Fig. I39, is protected by the sillimanite tube $B$. For temperatures above $1800^{\circ} \mathrm{C}$ no suitable protection tubes have been made. In certain special cases it may be desirable, if possible, to determine the melting point of a refractory under the atmospheric conditions to which it is subjected in actual use. Indirectly the pressure of the atmosphere may affect the melting point. For example, in a vacuum the more volatile and fusible substances, such as alkalies, may distill, and thus cause a rise of melting point.

There appear to be some possibilities in the development of gas furnaces for high melting-point determinations. With a suitable construction and air pressure of 10 pounds and more, temperatures of $1650^{\circ} \mathrm{C}$ may be maintained with illuminating gas and $\mathrm{I}^{8} 80^{\circ} \mathrm{C}$ with natural gas. By recuperating the waste heat or by preheating the gases the above temperature limits may be increased. It should be pointed out that for reliable melting-point work it is necessary to maintain a high temperature, not on a restricted small surface, but throughout a considerable volume.

(8) Temperature Measurements.-Pyrometric cones have been employed as a rough measurement of the melting temperature of refractories. The refractory is thus said to have a softening point corresponding to a certain cone number. Seger and similar cones serve a useful purpose under slow rates of heating in measuring heat effects during the firing of ceramic products, but in the laboratory where the rates of heating are comparatively rapid and may vary considerably the determination of the cone softening point is not of much significance. For it has been repeatedly shown by numerous investigators ${ }^{42}$ that the softening point of Seger cones depends upon the nature of the atmosphere and especially upon the time and rate of heating. Usually the more rapid the rate of heating the higher the softening temperature, variations of $50^{\circ} \mathrm{C}$ being easily obtained. In order that the indications by a Seger cone possess reliability, it is necessary to specify

42 Sosman, Trans. Am. Ceram. Soc., 15, p. 482 ; 1913. 
the atmosphere and the time-temperature curve in the manner illustrated above for refractories. Thus in the ordinary use of Seger cones a variable factor is introduced in the temperature scale defined by the cone numbers, which may have no relation to the properties and characteristics of the refractory being tested. Hence it is essential to use a strictly fixed and reproducible temperature scale. This is best accomplished by means of the disappearing-filament type of optical pyrometer.

A very slight departure from black-body conditions enables one to discern the specimen and to watch the progress of melting. It is important to keep the eye fixed on the sample in order that the melting may be observed with certainty. Sintering and vitrification take place on heating, sometimes producing shrinkage and bending; this must not be confused with the change in shape on melting. As in the case of metals, the pyrometer should be focused on a surface of the sample oblique to the line of sight, but it must be assured that this surface is not reflecting light from a hotter section of the furnace, thus causing too high a reading. After the determination the sample is removed from the furnace, broken apart, and inspected. The inside surface should appear homogeneous and amorphous or glassy. When the sample has been melted in a vacuum furnace, a cellular structure is usually apparent, due to gases evolved during melting.

(c) Softening Temperature of Coal Ash.-The clinkering of coal ash is closely related to its softening temperature. Consequently, the determination of this temperature is of considerable practical importance. The Bureau of Mines has made a very complete investigation of the factors and conditions affecting the fusibility of coal ash and have recommended a standard procedure. $^{43}$ The results of their investigations will be briefly described.

Coal ash is composed principally of silica, alumina, iron oxides, and lime, together with smail a $\pm_{4}$ : nts of other oxides. The compounds which exert the greatest nfluence on the melting point are the iron oxides, because they exist in several states of oxidation, depending on the chemical nature of the gases in which the ash is heated. When the ash is heated in air, the iron is oxidized to its highest states of oxidation, $\mathrm{Fe}_{2} \mathrm{O}_{3}$ and $\mathrm{Fe}_{3} \mathrm{O}_{4}$. When heated in a very strongly reducing atmosphere, such as exists in a carbon resistance furnace, all the oxides are reduced to metallic iron. The ash in both of these atmospheres has a higher softening point

${ }^{43}$ Fieldner, Hall, and Field, Bureau of Mines Bull. No. I29. 
than when it is heated in moderately reducing atmospheres, such as $\mathrm{H}_{2}$ and $\mathrm{H}_{2} \mathrm{O}$ or $\mathrm{CO}$ and $\mathrm{CO}_{2}$. Under these conditions the oxides are reduced to the ferrous state $\mathrm{FeO}$, forming low melting-point ferrocalcic silicates.

It has been found that the iron in the slag or clinker of an ordinary fuel bed exists in the ferrous state. Consequently, it is preferable to heat the ash in a furnace which will reproduce as nearly as possible the conditions in the fuel bed. A carbonresistance furnace or one with air as the atmosphere is not suitable. A furnace wound with molybdenum with a gas-tight case and equipped with a complicated arrangement for regulating the mix-

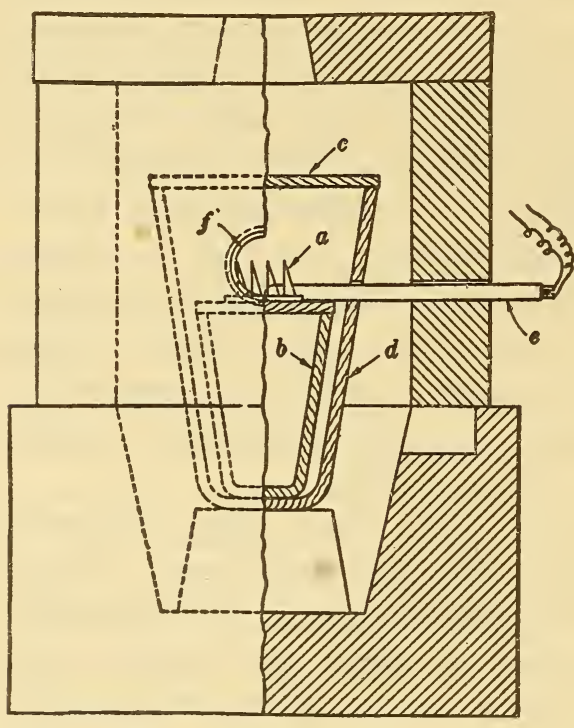

FIG. I45.-Arrangement for determining fusibility of coal ash ture of $\mathrm{H}_{2}$ and $\mathrm{H}_{2} \mathrm{O}$ has been used; but it is too complex for industrial use. The simplest and most satisfactory furnace has been found to be the gas furnace shown in Figs. I 35 and I45, operated with the maximum excess of gas over air, and containing an atmosphere approximately 50 per cent $\mathrm{CO}$ and 50 per cent $\mathrm{CO}_{2}$. Since some samples of ash may melt as high as $1500^{\circ}$ $\mathrm{C}$, it may be necessary to $\mathrm{mix}$ a small quantity of oxygen with the air to reach this temperature.

The following procedure is recommended: After thoroughly roasting the coal the ash is ground to 200 mesh and roasted again in a current of oxygen. Using a ro per cent dextrin solution as a binder, small triangular pyramids, $20 \mathrm{~mm}$ high and $6 \mathrm{~mm}$ wide at the base, are made from the powdered ash. These are placed vertically in a muffle or crucible as shown in Fig. I45. A yellow flame burning at least $15 \mathrm{~cm}$ above the cover is maintained, and the rate of heating before and during softening should be from 5 to $10^{\circ} \mathrm{C}$ per minute. The softening temperaure is the point at which the cone fuses down to a lump or ball, which, it will be noticed, is at a further stage of melting than is recommended by the Bureau of Standards for general ceramic material. For the 
temperature measurements a thermocouple or optical pyrometer is employed.

(d) Table of Melting Points of Refractory Materials.The melting points in the following table were determined at the Bureau of Standards, principally by Kanolt, under-conditions approximating those described in the section on refractory materials. With the exception of pure kaolin and silica, different. samples of the same general type of material may have melting points considerably different from the values tabulated.

TABLE 28.-Melting Points of Common Refractory Materials

\begin{tabular}{|c|c|c|c|}
\hline Material & $\begin{array}{c}\text { Tempera- } \\
\text { ture, } \\
\text { degrees } \\
\text { centigrade }\end{array}$ & Material & $\begin{array}{c}\text { Tempera- } \\
\text { ture, } \\
\text { degrees } \\
\text { centigrade }\end{array}$ \\
\hline Fire bricks: & & Clays and sands-Continued. & \\
\hline Fire clay $\ldots \ldots \ldots \ldots \ldots \ldots \ldots$ & $1500-1750$ & Bauxite clays......................... & 1800 \\
\hline Silica....................... & $1700-1750$ & Bauxite $\mathrm{Al}_{2} \mathrm{O}_{3}\left(2 \mathrm{H}_{2} \mathrm{O}\right) \ldots \ldots \ldots$ & 1820 \\
\hline Magnesia ....................... & 2150 & Chromite $\mathrm{FeO} . \mathrm{Cr}_{2} \mathrm{O}_{3} \ldots \ldots \ldots \ldots$. & 2180 \\
\hline 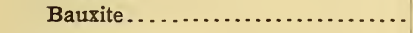 & $1600-1800$ & Silica......................... & 1750 \\
\hline Chromite........................... & 2050 & 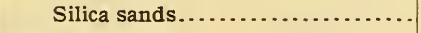 & $1700-1750$ \\
\hline Clays and sands: & & Miscellaneous: & \\
\hline Kaolin, $\mathrm{Al}_{2} \mathrm{O}_{3} 2 \mathrm{SiO}_{2}\left(2 \mathrm{H}_{2} \mathrm{O}\right) \ldots \ldots$ & 1740 & Coal ash...... & $1100-1500$ \\
\hline Fire clays. . . . . & $1400-1740$ & Alundum cements... . & $1750-200 J$ \\
\hline
\end{tabular}

\section{STANDARDIZATION OF PYROMETERS}

\section{STANDARDIZATION OF HIGH-TEMPERATURE THERMOMETERS}

High-temperature thermometers may be standardized by comparison with a standard instrument, or the thermometers may be calibrated by immersion in vapors of various boiling liquids. Thus the $100^{\circ}$ point may be checked by use of the hypsometer or steam-point apparatus. Other satisfactory temperatures are the boiling points of naphthalene, $218^{\circ} \mathrm{C}$, benzophenone $306^{\circ} \mathrm{C}$, anthracene $340^{\circ} \mathrm{C}$, and sulphur ${ }^{44} 444.6^{\circ} \mathrm{C}$. The boiling-point method, however, is less convenient and in general is more subject to error than the comparison method.

For the range $\mathrm{IOO}$ to $320^{\circ} \mathrm{C}$ the comparator consists of an electrically heated, stirred oil bath, into which the thermometers are immersed. For temperatures up to $55^{\circ} \mathrm{C}$ an electrically heated lead or tin bath is most satisfactory. The comparator is constructed of iron throughout and contains a stirrer for circulating the molten metal. The thermometers are inserted in thin steel tubes closed at the bottom and immersed in the liquid metal.

\footnotetext{
${ }^{44}$ See p. 278 for description of the apparatus required
} $8513^{\circ}-21-15$ 
It is not possible to read thermometers totally immersed in the above-described comparators, and hence special attention must be given to the stem corrections. If both the standard and the thermometer under test have the same dimensions and the same number of degrees of mercury column emergent, the stem corrections may be omitted. This is seldom the case, however, and it is usually necessary to compute stem corrections for both thermometers, as described on page I4. Thermometers graduated for short lengths of immersion (e. g., 3 to $5 \mathrm{~cm}$ ) should not be tested for such immersion on account of the uncertainty as to the temperature of the upper part of the bath. It is preferable to immerse to a greater depth and to correct the observed data for the desired depth of immersion.

The calibration of an improperly annealed thermometer changes with time and use, and even the best constructed thermometers are subject to some variation. If the thermometer is graduated for the ice or steam point, the amount of any change may be detected by check observations in ice or steam directly after a reading at a high temperature. Where no ice-point graduation is provided it is advisable to test the highest point on the scale first. If a preliminary test shows a considerable change in the readings with moderate heating, the thermometer should be annealed in an electrically heated oven as described by Dickinson..$^{45}$

The above brief description of thermometer standardization applies more especially to laboratory thermometers of the etched or inclosed-scale types. In many cases the comparators do not reproduce satisfactorily the conditions to which industrial thermometers are subjected in technical practice; as, for example, the measurement of the temperature of gases or superheated steam. Unfortunately, practically no information is available in regard to the best procedure for such tests. ${ }^{46}$

\section{STANDARDIZATION OF THERMOCOUPLES}

Thermocouple testing may be divided into two general classesprimary standardization and secondary standardization. In primary standardization the thermocouple is calibrated directly in terms of certain fixed temperatures, defined by the melting or freezing points of a series of chemically pure metals. In a secondary standardization the couple under test is compared with

45 Dickinson, B. S. Sci. Papers No. 32.

46 See Wilhelm, Chicago Pyrometry Symposium. 
another couple, which has been calibrated as above. Primary standardization is not recommended for most industrial laboratories, as it requires a considerable amount of extra apparatus and laboratory technique. This Bureau undertakes work of such character for a nominal fee and recommends that the industrial laboratory maintain at least two standard rare-metal couples, which may be submitted to the Bureau at intervals for a primary calibration. All the couples are then calibrated in the industriai laboratory by comparison with these standards. However, a number of companies prefer to do their own primary standardization, and for this reason the best methods for primary calibrations as well as secondary calibrations are discussed below.

(a) Standardization of RARE-Metal Couples. -The raremetal couple may be purchased completely mounted in a porcelain or quartz tube with the cold junction arranged to operate at.room temperature or at the temperature of melting ice. The latter method is preferable for the highest accuracy, but the former method is usually sufficient for most requirements. A suitable ice box is described later.

If desired, the thermocouple wire may be purchased from the platinum refiners and made into couples in the laboratory. One wire should be platinum and the other an alloy of io per cent rhodium with platinum, both of the highest possible purity. For general work wires $0.5 \mathrm{~mm}$ or $0.6 \mathrm{~mm}$ in diameter and 100 $\mathrm{cm}$ long serve satisfactorily. A shorter length may be ised if the cold junction is operated at room temperature or in connection with the compensating leads described elsewhere.

The hot junction is formed by fusing the two wires in an oxy-gas flame or small electric arc. The wires are threaded through twohole porcelain insulators and placed inside of a thin-walled protecting tube of quartz or of porcelain glazed on the outside only. The two free ends of the couple are mounted in the ice box and from this point copper leads are carried to the potentiometer.

(I) Annealing.-Before standardization the couple should be removed from the mounting and the entire length of wire elec-

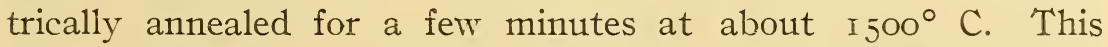
treatment eliminates the emfs which otherwise would be developed between the hard and soft portions of the wire. Often rare-metal couples contaminated by long-continued use may be restored to their original condition by annealing for an hour or more at this high temperature. Impurities, such as iron oxide, which have accumulated on the surface of the wires may be removed by 50 
per cent nitric or hydrochloric acid, or by melting borax on the heated wires and allowing the beads of borax to flow along the wire and drop off. The borax should drop off instead of vaporize in order that it may carry with it any absorbed matter. In case long-continued alternate heating and treatment with borax or acid fail to restore sections of the couple, these badly contaminated portions should be cut out and the couple fused together again with as small a junction as possible. The insulators should be cleaned by heating to incandescence in a gas flame, or, when badly corroded, should be replaced by new tubes.

For annealing electrically the couple is suspended between two binding posts and sufficient current passed through it to raise the temperature of the wire to about $5_{500^{\circ}} \mathrm{C}$. A couple made of wires $0.6 \mathrm{~mm}$ in diameter requires about $\mathrm{I} 4$ amperes, $0.5 \mathrm{~mm}$ about I 2.5 amperes, and $0.4 \mathrm{~mm}$ about io amperes.

In general, the contaminated portions do not glow as brightly as the purer sections of the wire. If the wire is not badly contaminated, the darker spots will gradually disappear. If more extreme local treatment is required, the contaminated portions may be wound into a small spiral and the couple heated as before. The coiled portion of the wire loses less heat per unit length by radiation, and hence can be maintained at the desired temperature while the rest of the wire is heated to a dull red. Usually foreign matter produces a gray or dark appearance of the wire when cold, and these contaminated sections are stiffer than the uncontaminated wire.

While couples may be often restored to their original condition by annealing, the disappearance of the gray spots on the wire does not prove absolutely that the couple is free from contamination. The absence of contamination is shown, however, by a homogeneity test. Where high accuracy is required a homogeneity test should be made.

(2) Homogeneity Test.-For work of precision each wire of the couple must be uniform in chemical composition and physical condition throughout its entire length. The magnitude of the emf developed by the couple depends upon the composition of the couple in the region of the temperature gradient. The emf developed by an inhomogeneous couple is not necessarily a measure of the temperature of the hot junction. In fact, it is a measure of the temperature of the hot junction only when the region of inhomogeneity lies entirely outside of the region of temperature gra- 
dient. Such a fortunate condition would be obtained very rarely in practice.

Suppose, for example, in a furnace $40 \mathrm{~cm}$ long, that the temperature is uniform and at $1000^{\circ} \mathrm{C}$ over the central $20 \mathrm{~cm}$ of the furnace and that the temperature drops, linearly with distance, to $0^{\circ} \mathrm{C}$ over the remaining $10 \mathrm{~cm}$ on each side. Omitting consideration of conduction losses, a homogeneous couple would show the true temperature of any desired portion of this furnace, and the temperature indicated would be that corresponding to the position of the hot junction of the couple.

Consider the use of an inhomogeneous couple in which the wires are badly contaminated for a length of $5 \mathrm{~cm}$ from the hot junction. This couple would give the correct temperature of the central portion of the furnace when immersed to any depth between $\mathrm{I} 5$ and $30 \mathrm{~cm}$, because for such depths of immersion the inhomogeneous portion of the couple lies wholly within the region of temperature uniformity. It would not be possible to measure with this couple the temperature of any portion of the furnace less than $\mathrm{r} 5 \mathrm{~cm}$ from the outside, because the region of inhomogeneity would lie in the region of temperature gradient. Under such conditions the couple acts as a compound or differential couple, and it is difficult to predict how much in error its indications may be, since the magnitude of the error depends upon the amount of contamination and the steepness and location of the temperature gradient.

The above example illustrates the fact that the reading of an inhomogeneous couple depends upon the depth of immersion and does not, except by accident, indicate the temperature of the hot junction. It would be impossible to measure, by means of an inhomogeneous couple, the temperature of any portion of a furnace which was nonuniformiy heated, unless the couple were calibrated in the position used. ${ }^{47}$

For a homogeneity test a short length of each thermocouple wire is selected as a standard reference specimen. These lengths may be cut from the uncontaminated cold-junction ends of the couple, and one set may be used repeatedly for various couples of the same type. One end of the standard wire-for example, the pure platinum wire-is placed in contact with a section of the corresponding wire of the thermocouple, and the emf is observed between the cold-junction end of the thermocouple wire and the

${ }^{48}$ See section b (4), Checking Thermocouples in Fixed Installations, p. 224. 
free end of the standard wire when the point of contact between the standard and the thermocouple is heated to some convenient high temperature, maintained constant.

The point of contact of the two wires is then moved to a different section of the thermocouple wire and the process continued until the platinum wire of the thermocouple is completely explored. The process is repeated for the alloy wire of the couple, except that in this case the specimen of the alloy wire is used as the standard.

If $e$ is the maximum difference in microvolts between two readings of emf made along one wire of the thermocouple, and $s$ the value of the thermoelectric power for the couple (micro-

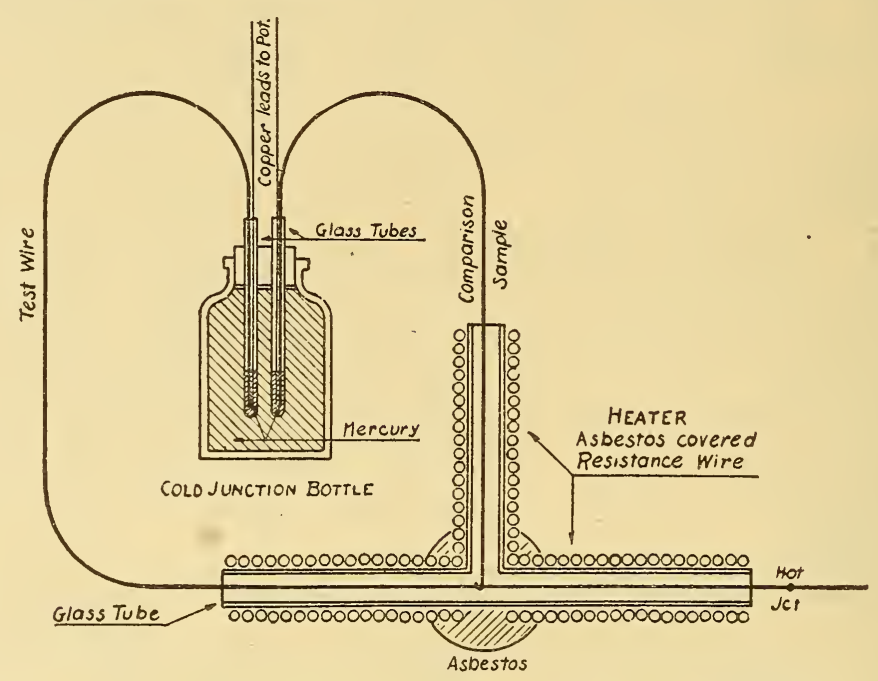

FIG. I46.-A pparatus for homogeneity tests of rare-metal couples

volts per degree of temperature), then the maximum error usually possible in the use of the couple due to inhomogeneity of this wire is $e / s$ in degrees, provided the temperature of the hot junction in general use does not exceed that employed in the homogeneity test.

If the temperature difference between the hot and cold junctions during the homogeneity test were $t_{1}-t_{2}$, and during the actual use of the couple $t^{\prime}{ }_{1}-t^{\prime}{ }_{2}$, the maximum error possible in the use of the couple, due to inhomogeneity of this wire, is usually approximately $\frac{t^{\prime}{ }_{1}-t^{\prime}{ }_{2}}{t_{1}-t_{2}} \cdot \frac{e}{s}$ degrees. Similar conditions hold for the other wire of the couple. 
The effects of inhomogeneity in both wires may be either subtractive or additive, so that it is evident that to quantitatively correct temperature readings for errors due to inhomogeneity is practically impossible. Merely qualitative results can be obtained from tests of this nature. If the variations in emf observed during the homogeneity test are less than 5 microvolts for $t_{1}-t_{2}=$ $500^{\circ} \mathrm{C}$, the couple can be relied upon for precision work. If certain sections of the couple show larger emf, these sections

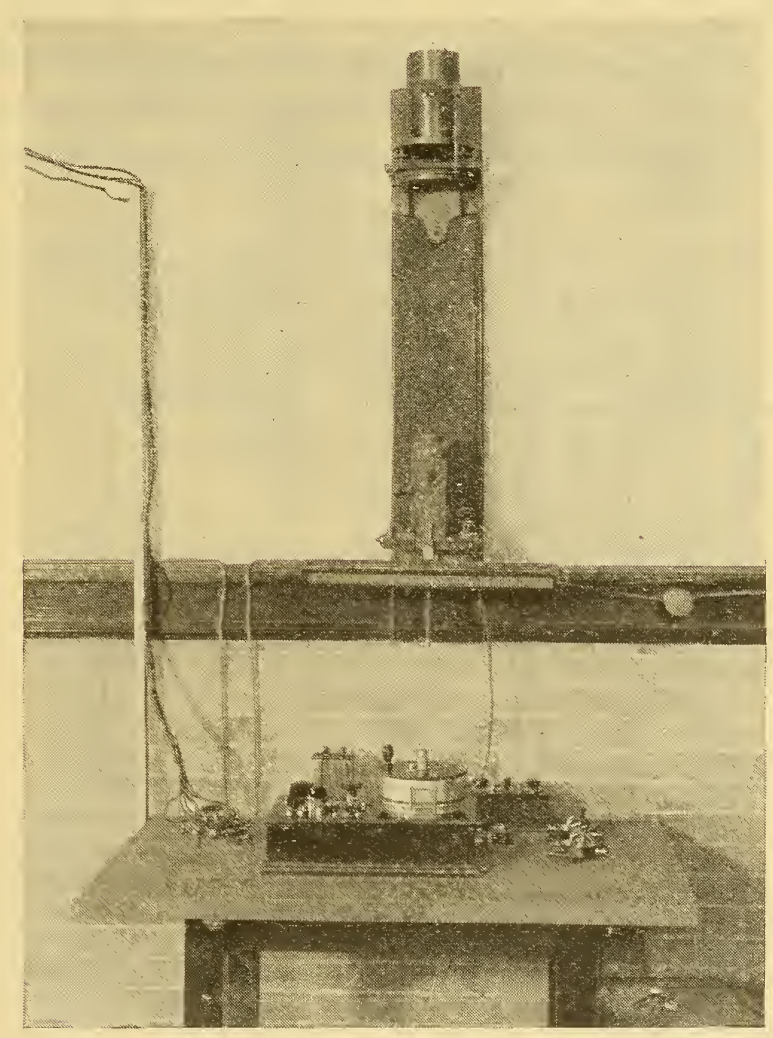

FIG. I47.-Laboratory potentiometer and accessories

should be treated by annealing until their condition is satisfactory or should be cut away in case they can not be brought to a proper condition.

The point of contact between the couple wire and the standard wire may be heated by a Bunsen flame, but a more satisfactory method is as follows: The heater, Fig. I 46 , consists of a pyrexglass or porcelain $T$ tube having the top and leg of the $T$ wound with resistance wire, through which sufficient current is passed to heat this tube to at least $400^{\circ} \mathrm{C}$. One of the wires of the thermo- 
couple is threaded through the top of the $T$ and the standard wire is brought into contact with the couple by threading it through the lower part of the $T$. The end of the standard wire is bent into a small hook, through which the couple passes, sufficient tension being given to the standard wire to secure good electrical contact with the couple. The free ends of the couple wire and the standard are maintained at the same temperature by use of a cold-junction box.

The cold-junction box may consist of a small bottle of mercury, into which dip two small glass tubes closed at the lower end. A few drops of mercury are placed in each tube, and the free end of the couple wire is inserted into one tube and the free end of the standard into the other tube. Copper wires making contact with

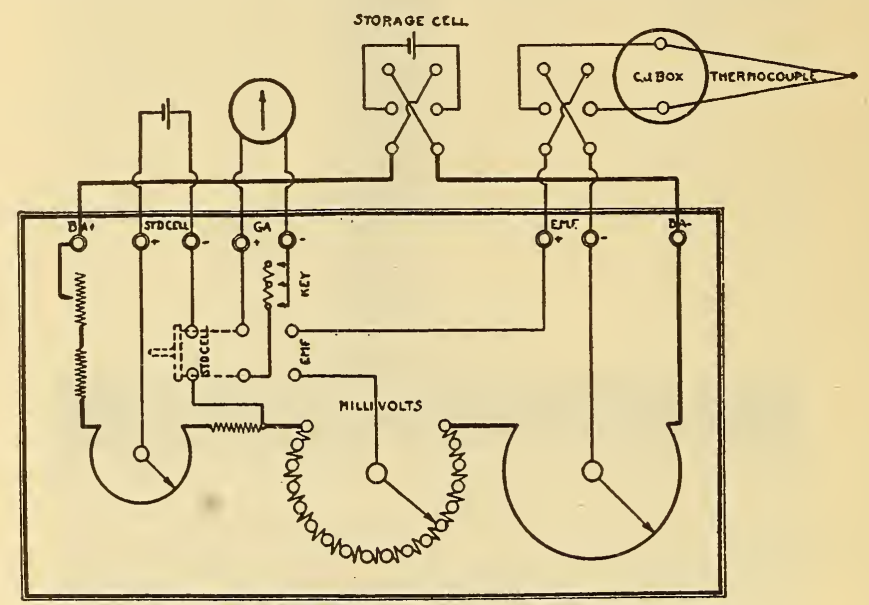

FIG. I48.-Wiring diagrams of potentiometer installation

the platinum wires by means of the mercury inside the tubes lead to the millivoltmeter or potentiometer. The copper and platinum must not come in electrical contact except in the mercury. Readings of emf are made for various points of contact along the couple by pulling the couple through the $T$ tube and allowing a minute or so for temperature equilibrium at each new position.

(3) Laboratory Potentiometer.-In precision thermocouple calibration a potentiometer especially designed for this type of work should be used. Fig. I 47 illustrates a complete potentiometer installation, including galvanometer and reading scale. The wiring diagram is given in Fig. 148.

For work of the highest precision objection has been raised to the use of a potentiometer employing an adjustment of a slide 
wire for obtaining either part or all of the emf reading. Instruments are obtainable in which all adjustments are performed by dial settings, the circuits being so arranged that parasitic emfs introduced at moving contacts produce insignificant effects on the measured emf. Examples of this type of instrument are the White and the Diesselhorst potentiometers. Such refinements are, however, seldom essential, except in special research work.

In order to eliminate the effect of accidental thermal emfs developed in the instrument itself, two double-pole, double-throw switches are used, one in the main battery circuit and one in the emf circuit. Readings are made with the two switches thrown first in one direction and then in the other. In one case the parasitic emfs arising in the potentiometer and the galvanometer are additive to that of the couple, and in the other case they are subtractive, whence a mean of the two readings gives the true emf of the thermocouple circuit. In comparing the emfs of two or more couples readings should be taken in the following order and the final means obtained should be used as the calibration data:

Desirable Order for Readings

\begin{tabular}{|c|c|c|c|}
\hline Position of switches & Couple I & Couple II & Cuuple III \\
\hline Normal. . & 1 & 2 & 3 \\
\hline Reversed......... & 6 & 5 & 4 \\
\hline Normal........................ & 7 & 8 & 9 \\
\hline \multirow[t]{2}{*}{ Reversed... } & 12 & 11 & 10 \\
\hline & Mean emf & Mean emf & Mean emf \\
\hline
\end{tabular}

Some observers prefer to take all readings with the switches in the normal position. One reversed reading is made and half the difference between a normal and reversed reading is added to or subtracted from the mean of all normal readings. This method is allowable if the parasitic emfs do not vary dering the observations.

Care should be taken that the battery switch is in the normal position when attempting to balance the instrument against the standard cell. The main battery of the potentiometer should be a 2-volt storage cell of about 50 ampere-hours capacity and should be charged every two months. Details in regard to the use and care of a potentiometer are given in the instruction manual furnished with the instrument.

(4) Cold-Junction Ice Box.-A satisfactory ice box is obtained by fitting a small battery jar with a fiber or hard-rubber cover. Through the cover are inserted several small glass tubes closed at 
the lower end, two tubes for each couple used. A few drops of mercury are placed in each tube, and the battery jar is kept filled with clean ice shaved or ground into a wet snow.

The cold-junction ends of the couples are inserted, one in each tube, and copper wires lead from the mercury to the potentiometer. The copper wires should be insulated from the couple wires throughout the length of the glass tubes, except where they dip into the mercury. This precaution is very important, for, if the copper and platinum (or alloy wire) are in electrical contact at the top of the glass tubes, the whole value of the ice box is lost.

The glass tubes should be kept clean and dry inside. Moisture

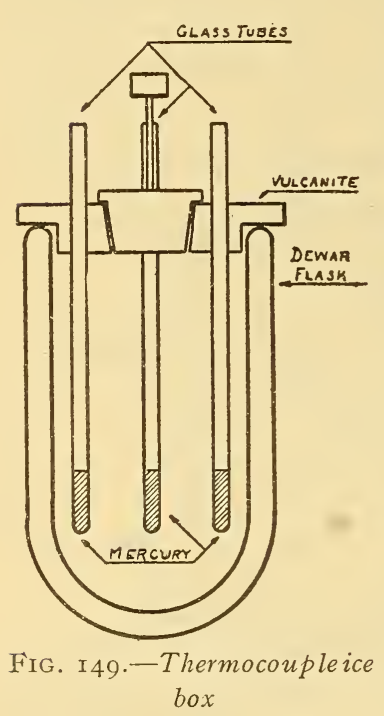
is likely to condense in the tube from the atmosphere and should not be allowed to accumulate. A little moisture and dirt at the bottom of the tube will form a galvanic cell giving a large emf. It is desirable, but not necessary, to replace the battery jar by a Dewar flask or thermos bottle having a large mouth, since the ice melts more slowly in this form of container. Fig. I49 shows an ice box suitable for thermocouple work.

(5) Calibration of Primary Standards.The theory and technique of melting-point determinations have been discussed in an earlier chapter. Only the practical application to thermocouple standardization is considered in the following: When a crucible of pure molten metal is allowed to cool, the cooling curve obtained by plotting temperature of the metal versus time is continuous until the freezing point (or transformation point) is reached. During freezing the metal gives out enough internal latent heat to supply the losses due to radiation and convection, and the temperature of the metal remains constant until all the metal has frozen. In this region of the cooling curve the curve is parallel to the time axis. After the metal has frozen it cools in a somewhat similar manner as when completely molten. Fig. I 50 shows cooling and heating curves for several metals.

The freezing point of copper $\left(1083^{\circ} \mathrm{C}\right.$ ) is clearly indicated by the flat portion of the second curve. On heating a metal the temperature gradually increases with time until the melting 
point is reached. The temperature of the metal then remains constant, as shown in Fig. I50, until all the metal is melted, after which the temperature again increases with time.

The temperatures at which melting and freezing occur are identical for pure metals. When molten antimony is allowed to cool, the temperature usually falls below the freezing point before the metal starts to freeze, a phenomenon known as undercooling. When any of the metal starts to solidify, however, the temperature immediately rises to the freezing point, as shown in Fig. I50, unless the undercooling has proceeded too far. Undercooling may be greatly reduced by stirring the metal or tapping the crucible for an instant just as the freezing point is approached.

Aluminum does not appear to have as welldefined a melting or freezing point as do many other metals. The material is "pasty" over a small temperature range. This may be clue to the presence of impurities and is probably not a characteristic of the pure metal.

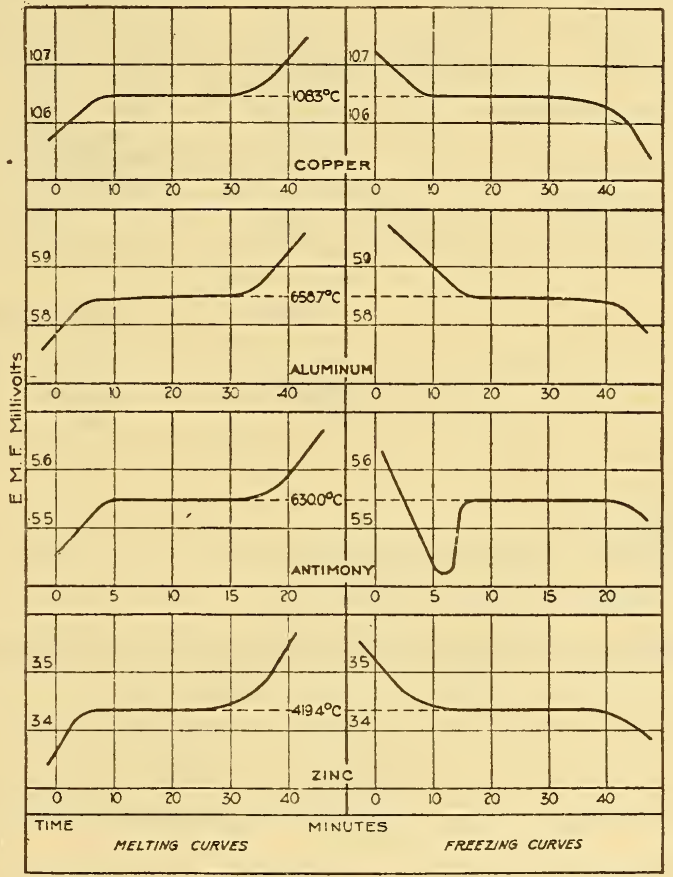

FIG. I50.-Typical heating and cooling curves

In the primary calibration of rare-metal couples use is made of the type of cooling and heating curves above described. The couple, properly protected, is immersed in the molten metal, which is then allowed to cool slowly. The emf of the couple is observed as frequently as possible and the time noted for each observation. These values are plotted, and the emf corresponding to the flat portion of the cooling curve is the emf at the freezing point of the metal. A similar process may be carried through by melting the metal. 
The temperature versus emf relation for the platinum, platinumrhodium couple has the following form in the temperature range

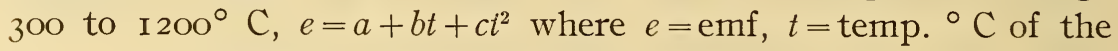
hot junction of the couple (cold junction $=0^{\circ} \mathrm{C}$ ), and $a, b$, and $c$ are constants. Three standard points are required to determine $a, b$, and $c$ for any particular couple. These fixed temperatures may be the melting or freezing points of zinc $419.4^{\circ} \mathrm{C}$, antimony $630^{\circ} \mathrm{C}$, or aluminum $658.7^{\circ} \mathrm{C}$, and copper $1083^{\circ} \mathrm{C}$. The metals must be of the highest possible purity. This is especially the case with antimony, which is very difficult to obtain in sufficient degree of purity. The Bureau of Standards furnishes standard samples of zinc, aluminum, and copper (also tin and lead; see Circular No. 66) with certified melting points. Aluminum of the highest obtainable purity is recommended in preference to antimony for the industrial laboratory. The following shows the method of obtaining the constants $a, b$, and $c$ :

Observed Calibration Points

\begin{tabular}{|c|c|c|}
\hline Metal & Temperature & emf \\
\hline & ${ }^{\circ} \mathrm{C}$ & Millivolts \\
\hline Zinc.. & 419.4 & 3. 436 \\
\hline 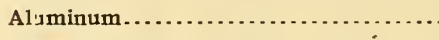 & 658.7 & 5. 839 \\
\hline 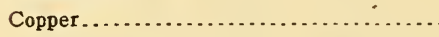 & 1083 & 10.561 \\
\hline
\end{tabular}

Whence, from the above equation, it follows that:

$$
\begin{gathered}
3.436=a+(419.4) b+(419.4)^{2} c \\
5.839=a+(658.7) b+(658.7)^{2} c \\
\text { I0.56I }=a+(\text { I } 083) b+(\operatorname{IO8} 3)^{2} c
\end{gathered}
$$

These three equations are solved for $a, b, c$, giving $a=-0.323$, $b=+0.008276, c=+0.000001638$. The final equation for this couple thus becomes:

$$
e=-0.323+0.008276 t+0.000001638 t^{2},
$$

where $e$ is the emf in millivolts corresponding to a hot-junction temperature of $t^{\circ} \mathrm{C}$, and a cold-junction temperature of $\mathrm{o}^{\circ} \mathrm{C}$. From this equation $e$ may be computed for various values of $t^{\circ} \mathrm{C}$ (for example, $300^{\circ} \mathrm{C}=2.306$ millivolts; $35^{\circ} \mathrm{C}=2.774 ; 400^{\circ} \mathrm{C}=$ 3.249 ; $1200^{\circ} \mathrm{C}=\mathrm{I} 1.967$, etc.), and a curve or table of $e$ versus $t^{\circ} \mathrm{C}$ may be constructed. Using this curve or table one may readily 
find the temperature corresponding to any emf observed in the future use of the couple.

The above equation serves to determine the temperature scale in the interval from 300 to $1200^{\circ} \mathrm{C}$. An entirely different equation must be used for points below $300^{\circ} \mathrm{C}$. For discussion of this see Burgess and Le Chatelier, "Measurement of High Temperatures." At temperatures higher than $1200^{\circ} \mathrm{C}$ the following corrections must be added to the temperatures given by the above equation. These corrections apply for all platinum, 9o per cent platinumIo per cent rhodium thermocouples.

Corrections to Extrapolated Thermoc
\begin{tabular}{|c|c|}
\hline $\begin{array}{c}\text { Temperature } \\
\text { computed from } \\
\text { parabolic } \\
\text { equation }\end{array}$ & $\begin{array}{c}\text { Correction } \\
\text { to add }\end{array}$ \\
\hline${ }^{\circ}$ C & ${ }^{\circ}$ C \\
1200 & 0 \\
1300 & 2 \\
1400 & 6 \\
1500 & 14 \\
1600 & 25 \\
1700 & 39 \\
\hline
\end{tabular}

The standard metal may be used in an Acheson graphite crucible (Dixon graphite is also satisfactory for copper) the inside diameter of which is slightly less at the bottom than at the top. Convenient inside dimensions are about $5 \mathrm{~cm}$ diameter and $15 \mathrm{~cm}$ deep. For use with the $50 \mathrm{~cm}^{3}$ metal samples furnished by the Bureau of Standards a crucible $3.5 \mathrm{~cm}$ inside diameter and $8 \mathrm{~cm}$ deep is recommended. Crucibles should be fitted with a cover having a small hole in the center through which the couple is inserted. The crucible may be heated either in an electric or gas furnace. Fig. I24 shows a convenient form of electric furnace. The crucible containing the metal is shown within a larger crucible and surrounded by graphite. If the furnace is too small for the extra crucible, the graphite may be poured directly into the furnace tube until the crucible is entirely covered. This prevents the oxidation and consequent crumbling away of the crucible.

If a gas furnace is used, the crucible should be placed inside a length of iron pipe, capped at the bottom, and the space between the crucible and the pipe should be filled with powdered or flaked graphite. Instead of an iron pipe a larger crucible of Dixon graphite or fire clay may be used. Fig. I35 shows a suitable gas furnace, 
but does not show the iron pipe. In all cases the top of the crucible and the surface of the metal should be covered with 2 or $3 \mathrm{~cm}$ of powdered or flaked graphite. This is especially important with copper, as the oxide of copper formed on exposure of the hot metal to the air is soluble in the metal, and thus lowers the melting point to an appreciable extent.

Protecting Tube for Rare-Metal Couple.-The thermocouple must not come in direct contact with the molten metal or metallic vapors. A suitable protecting tube is of porcelain glazed on the outside and closed at the lower end. The diameter of the tube may be I cm or less, and the walls should be thin. The couple wires are again protected and insulated from each other by small porcelain insulating tubes or by two-hole porcelain tubing.

It is good practice to remove the couple and insulators from the outside protecting tube when immersing the latter in the molten metal, as frequently the tubes are broken in this operation. This precaution saves the couple in case such an accident takes place. The procedure is, therefore, as follows: Hold the pyrometer tube above the furnace in the heated air until it takes up this temperature. Gradually lower it into the furnace until the top of the crucible is reached. When thoroughly heated, gently immerse it in the metal. Observe that the tube is not broken. If in doubt, insert a small porcelain tube inside the pyrometer tube. If the outer tube is broken, the small tube will be discolored or contaminated with the metal. Another method of detecting a broken tube is to pull it out of the metal, and, if intact, replace it before it has had time to cool. After having determined that the pyrometer tube is good insert the thermocouple. This should be done very slowly, as the rapid insertion of the cold thermocouple may break the protecting tube. After the metal has frozen the protecting tube should be withdrawn, or the furnace should be immediately heated for a melting-point determination. An immersed tube which is allowed to cool with the metal far below the melting point is usually broken. For a small temperature range below the melting point the tube can be readily withdrawn.

Either glazed porcelain or fused quartz (silica) protecting tubes may be used in this work. With aluminum, however, the protecting tube must be itself protected from the metal. This is easily done by making a tubular sheath of Acheson graphite, with walls about I mm thick, which slips over the quartz or porcelain tube, as shown in Fig. I23. The porcelain tube need not be removed from the metal before cooling when thus protected. It 
is better to have one set of tubes for each metal; but when necessary to use the same tube in various metals this can be done if care is taken to thoroughly clean the tube with. nitric and hydrochloric acid. The greatest care should be exercised to see that no dirt of any kind gets inside the pyrometer tubes. Dust or carbon inside the tubes, when heated, forms a reducing atmosphere which

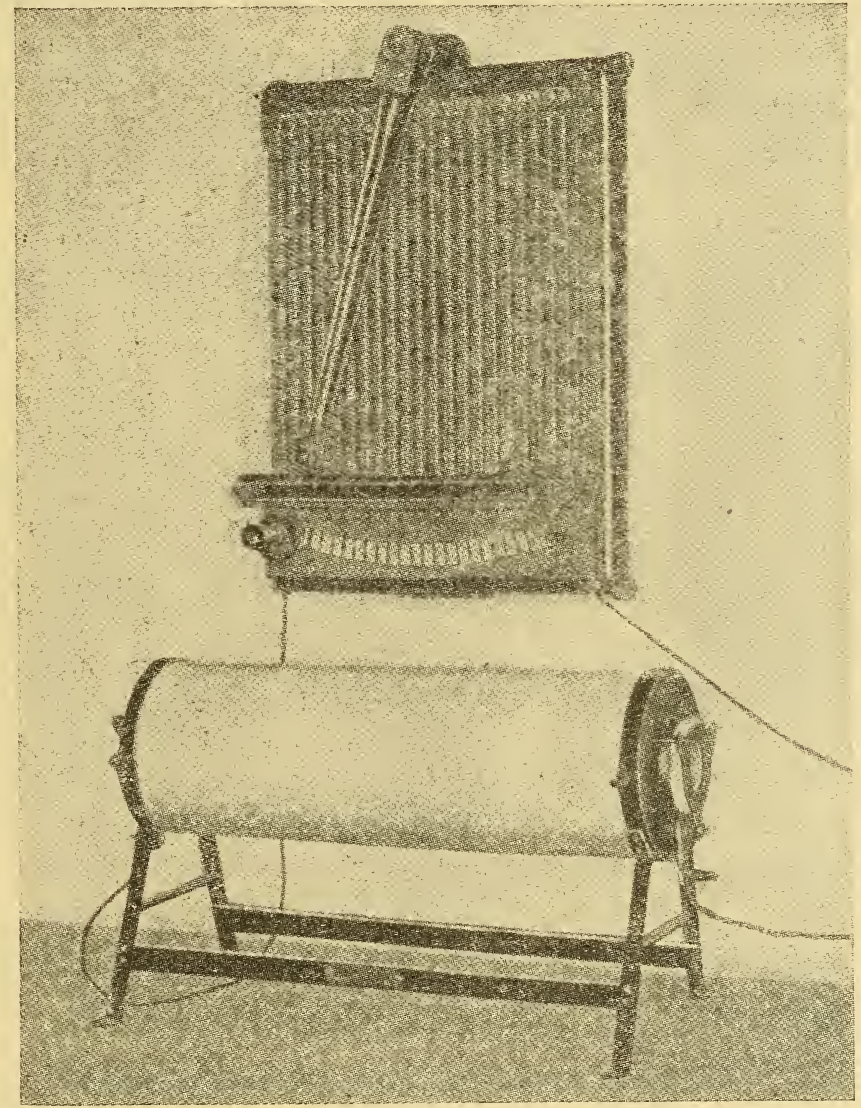

FIG. I5I.-Convenient form of electric furnace for intercomparing rare-metal couples

acts upon the silica, freeing silicon, which rapidly attacks the platinum.

(6) Secondary or Comparison Calibration of Rare-Metal Couples.The secondary method, as follows, is recommended for industrial laboratories: One, or preferably three, rare-metal couples of wire about $\mathrm{I}$ meter in length and $0.6 \mathrm{~mm}$ in diameter are maintained as primary standards. These couples should be submitted to the Bureau of Standards for test and certification at intervals, depend- 
ing upon the amount of use to which they are put, or whenever contamination is suspected. The couples to be tested are compared with these standards. Suitable electric furnaces for such comparison tests are available, designed to operate on either I Io or 220 volts, and may be obtained complete with adjustable rheostat for regulating the current, as shown in Fig. $5_{5} \mathrm{x}$. The furnace consists of a porcelain tube wound with platinum foil, surrounded by a larger porcelain tube, around which is packed thermal insulating material. The annular space between the two tubes should be filled with No. I 20 mesh Norton R. R. alundum to prevent local heating of the platinum. A convenient size of heating tube is $\mathrm{x}$ by $\mathrm{x} 8$ inches, but larger sizes can be secured if desired. The furnace should be kept clean and should not be used for testing base-metal couples, annealing iron rods, or other processes likely to cause contamination.

The rare-metal couples to be calibrated are removed from the protecting tubes and insulating tubes and annealed, as described previously. The insulating tubes are cleaned, when necessary, by the method described above and then replaced and the couples are tested without the protecting tubes, if the furnace is clean. The hot junctions of all the couples, the standard couple, and the couples under test are connected together with as good thermal contact as possible. The junctions may be fastened together with a small platinum wire and then wrapped with platinum foil. Since the couple wires are thus in electrical contact at the hot junction they must not be allowed to touch one another at any other point. The use of platinum foil around the hot junction reduces temperature gradients to a minimum. If the couples are tested in the protecting tubes this precaution becomes still more important.

The cold junctions of all the couples are maintained, preferably, at $\mathrm{O}^{\circ} \mathrm{C}$. If three couples are being compared with one standard the readings should be taken in the following order:

Desirable Order for Readings

\begin{tabular}{|c|c|c|c|c|}
\hline Reversing switch & Standard & No. 1 & No. 2 & No. 3 \\
\hline Normal........ & 1 & 2 & 3 & 4 \\
\hline Reversed....... & 8 & 7 & 6 & \\
\hline Normal............. & 9 & 10 & 11 & 12 \\
\hline Reversed............................. & 16 & 15 & 14 & 13 \\
\hline 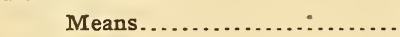 & & & ... & ... \\
\hline
\end{tabular}


The means of the observations on each couple are taken and the temperature is obtained from the reading of the standard couple. Several calibration points are made in this manner in the range 300 to $1200^{\circ} \mathrm{C}$. These are plotted, temp. vs. emf, and a smooth curve is drawn through the experimental points for each couple. If it is inconvenient or undesirable to use a cold-junction temperature of $0^{\circ} \mathrm{C}$ for the couples under test, any other constant temperature may be employed. This can be measured by means of a mercury thermometer. It is nearly as essential to know the temperature of the cold junction as that of the hot junction, since the magnitude of the emf observed depends upon both temperatures.

When a large number of couples must be accurately calibrated, the following method, requiring more elaborate apparatus, is satisfactory. In order to insure equality of temperature between the standard couple and the couple being calibrated without waiting for the furnace to acquire a constant temperature, the hot junctions are fused together. Two separate potentiometers are employed, one connected to each couple. Each potentiometer is provided with a reflecting galvanometer. The two spots of light are reflected onto a single scale, the galvanometers and lamps being so adjusted that the spots coincide with the zero point of the scale on open circuit or when the potentiometers are balanced.

The potentiometer connected to the couple under test is set at an integral number of millivolts, and the temperature of the furnace is increased or decreased until the galvanometer indicates a balance. At this instant the emf, and hence the temperature, of the standard is observed with the other potentiometer. This may be done easily if the spot moves slowly across the scale. By making observation first with a rising and then with a falling temperature, minor errors, such as time lag, are eliminated. Observations are taken at nearly every millivolt for the couple under test, from its lowest to its highest range; the furnace is then cooled and the readings thus repeated in the reverse order. The mean of the observed temperatures corresponding to the various millivoltages gives the final calibration of the couple. For best results about two hours are required, the couple being tested at ro to i 5 points. It is necessary to use a furnace which is not well insulated thermally in order that it may cool sufficiently rapidly.

A form of furnace especially adapted to the rapid testing of a large number of couples consists of a tube of nickel-chromium $853^{\circ}-21-16$ 
alloy (chromel) clamped between two water-cooled terminals. A heavy current from a suitable transformer is passed through the tube, the tube itself serving as the heating element or resistor. For use with base-metal couples a porcelain insulating tube may be placed inside the metal tube, or the base-metal couple may be wound with asbestos cord to electrically insulate it from the walls of the tube. In this case the standard couple, insulated in a nonporous porcelain tube, is placed with its hot junction as near as possible to the hot junction of the base-metal couple. Rare-metal couples may be standardized by taking proper precautions to prevent contamination by the metal vapors from the heating unit. The heating tube of this furnace is exposed to the air, and no thermal insulating material is employed. A large concentric tube may be mounted around the heating tube to reduce the radiation loss, although this is not necessary. A furnace of this type may be heated very rapidly to any temperature up to $\mathrm{I} 100^{\circ} \mathrm{C}$, and it is possible to completely calibrate a couple at five or six different temperatures in a half hour.

(b) Standardization of Base-Metal, Couples. - It is difficult to secure equality of temperature between the hot junctions of two base-metal couples or between a base-metal and a rare-metal couple. One arrangement for precision calibration is to place the junction of the rare-metal couple in a small saw cut made in the hot junction of the base-metal couple and pinch the jaws of the saw cut until good thermal contact is secured. Small basemetal couples may be fused directly to the rare-metal couple. The rare-metal couple is protected by porcelain tubes to within a few millimeters of the hot junction, and the end of the porcelain tube is sealed to the couple by a small amount of kaolin and waterglass cement or by pyrex glass. This prevents contamination of the rare-metal couple, with the exception of the small length of 2 or $3 \mathrm{~mm}$, which is necessarily exposed to the metal vapors. If the furnace is uniformly heated in this region a small amount of contamination will not cause any error. The method, however, is not feasible for work on a large scale. With fused or squeezed junctions the double potentiometer may be used in calibrating base-metal couples.

(I) Use of a Muffle Furnace.- Several base-metal couples and the standard rare-metal couples mounted in nonporous porcelain tubes are packed together as closely as possible and tied into a bundle with asbestos cord. The hot junctions are inserted into a length of very heavy iron pipe capped at one end and the open 
end from which the couples protrude is tightly packed with asbestos wool. The iron tube and couples are mounted in the furnace and the emf measurements are made as soon as temperature equilibrium is reached. If the muffle furnace is large enough the iron tubes protecting the base-metal couples need not be removed. A satisfactory size of furnace measures 8 by 8 by 30 inches deep. Precision work is impossible in this method of calibration. Even when every precaution is taken to reduce temperature gradients, differences in temperature of ro or $20^{\circ} \mathrm{C}$ frequently will be found to exist between the different couples mounted side by side. However, this accuracy is sufficient for many industrial processes.

This method may be greatly improved by employing a large copper block drilled with holes into which the hot junctions of the couples are inserted. The heavy protecting tube is removed from each couple during the test.

(2) Use of Molten-Metal Bath.-A satisfactory method for calibrating base-metal couples on a large scale is by use of a bath of molten metal. An iron pot about I 2 inches in diameter, or larger, and 15 inches deep is filled with tin or lead covered with powdered graphite. This may be heated in a gas furnace or an electric fur. nace wound with nickel alloy, such as nichrome or chromel. Into the tin bath dip about six iron tubes closed at the lower end and immersed to a depth of $\mathrm{I} 2$ inches. The tubes are just large enough to permit easy insertion of the base-metal couple with its iron protecting tube. The standard couple is mounted in one of the six tubes and the base-metal couples are placed in the others. As soon as temperature equilibrium is reached the emfs are measured, that of the standard couple giving the temperature of the bath. A large number of base-metal couples may be tested one after the other at this temperature, and the process is then repeated at another temperature. The tin bath may be used satisfactorily in the range 300 to $1000^{\circ} \mathrm{C}$, since tin is a metal of low volatility. Data obtained by the above method are plotted, temperature versus electromotive force, for each couple and a smooth curve drawn representing the calibration. For some types of basemetal couple the curve is slightly S-shaped, showing small inflections near the critical points of the metals of which the couple is constructed. In precision work the calibration must be made at short-temperature intervals over these critical regions to determine the exact form of the temperature-emf curve. 
(3) Single-Point Calibration or Check Point.-It is frequently desirable to check a base-metal couple at a single point. If the couple is reading emfs, say 2 per cent higher at this temperature than the usual emf of that shown by the original calibration data, it is customary to assume that it reads 2 per cent high at all temperatures. This method of calibration at best is only approximate, and experimentally it is frequently found that a couple reading 2 per cent high at $800^{\circ} \mathrm{C}$ indicates correctly at $1000^{\circ} \mathrm{C}$. However, single-point calibrations are of value, especially as a check upon the behavior of a couple in any particular temperature range.

A convenient standard temperature for this work is the melting or freezing point of sodium chloride (common salt), chemically pure. This material melts or freezes at 8 or $^{\circ} \mathrm{C}$. The salt may be used in a duriron crucible heated in a gas or electric furnace. The base-metal couples are immersed without protecting tubes from four to six inches in the bath. Melting or freezing point curves may be observed as already described. These curves are rather oblique on account of the low thermal conductivity of the salt, as discussed in the section on melting-point methods. The exact portion of the curve representing the true freezing point is reached when crystals begin to form along the entire length of the immersed end of the couple. The couple may be used as a stirring rod during the freezing. If the salt has not been previously fused, it must be melted the first time with a cover on the crucible as the imprisoned water in the salt crystals will cause the salt to sputter out badly. After the first melt, however, there will be no trouble in the use of this material. Molten salt reacts somewhat with most base-metal couples, but the reaction is so slow that the couple will not be injured. We have not found any serious change in melting point resulting from heating the salt in contact with iron for several hours.

(4) Checking Thermocouples in Fixed Installations.-It is frequently desirable to check the readings of a thermocouple without removing it from the furnace in which it is installed. Tests of this nature are of extreme importance in the technical industries. They are also difficult to perform with precision, and the precision which is obtainable in a laboratory test can not be expected in tests of this nature. However, an ordinary calibration in the laboratory of a base-metal couple which has been in use in a fixed installation for some time, while furnishing results apparently of high precision, does not necessarily hold when the couple is returned to its original installation. This is on account of the heterogeneity developed in a base-metal couple after prolonged heating. 
The presence of heterogeneity could be detected by a homogeneity test, but such tests on a large scale are riot worth while for base-metal couples except for investigational work and, moreover, do not show how to correct the readings satisfactorily. Such a test would simply indicate that the couple should be thrown away, whereas in its permanent installation it still has a useful life. The throwing away of base-metal couples in a fixed installation as soon as heterogeneity sets up would be a very costly and unnecessary procedure, except in the comparatively rare cases where the highest possible accuracy is required. The degree of heterogeneity developed depends upon the type of installation, depth of immersion, location and character of temperature gradient, etc. These quantities are fairly definite and fixed for any particular installation. If, however, the couple is removed from the installation and calibrated in the laboratory, these quantities are altered and the conditions under which the couple is actually operating can not be reproduced. The calibration of the laboratory may give results quite different from the calibration which would be obtained if it were possible to duplicate exactly all the physical characteristics of the permanent installation. Hence it is evident that if inhomogeneous couples are ever to be used, it is important that they be calibrated or checked in their fixed installation.

The exact method of procedure depends upon the type of installation. A standard checking couple is employed complete with a high-resistance galvanometer, "heat-meter," or portable potentiometer. The hot junction of the checking couple is placed as closely as possible to the hot junction of the couple under test, and the readings of the two couples compared. The main objection to the method is the difficulty in getting the two hot junctions together. One method is to drill a hole in the furnace at the side of each couple permanently installed large enough to permit insertion of the checking couple. The hole is kept plugged, except when comparison tests are being made. The checking couple is immersed in the furnace through this hole to the same depth as the couple under test. The hole should be located as close to the permanently installed couple as possible.

In many installations the base-metal couple and protecting tube are mounted inside another protecting tube of iron, fire clay, carborundum, or some other refractory which is permanently cemented or fastened into the furnace wall. Frequently there is room to insert a small test couple in this outer tube alongside of 
the fixed couple. A third method, much less satisfactory, is to wait until the furnace has reached a fairly constant temperature and make observations with the couple under test, then remove this couple from the furnace, and insert to the same depth the checking couple.

If desired, comparisons can be made preferably by either the first or second method at several temperatures, and a curve may be obtained for each permanently installed couple showing the corrections necessary to apply to its readings.

It may be thought that this method of checking couples is unsatisfactory, because in most furnaces used in industrial processes large temperature gradients exist and there is no certainty that the standard couple is at the same temperature as the couple under test. This objection, however, is not serious, because if temperature gradients do exist, in the furnace, of such a magnitude as to cause much difference in temperature between two similarly mounted thermocouples located closely together, the accuracy required by the measuring instruments need not be great, and the reading of the standard couple can be accepted for the standardization of the fixed couple just as satisfactorily as if the true temperature of the fixed couple were-known. If temperature gradients exist of such a magnitude that two correctly calibrated couples similarly mounted closely together read, say, $20^{\circ} \mathrm{C}$ apart, it may be certain that neither couple is registering the temperature of material within the furnace to this degree of accuracy.

(c) Cold-Junction Correction.-If a couple is calibrated for $a^{3}$ cold-junction temperature of $t_{\circ}^{\circ} \mathrm{C}$ and is used with a coldjunction temperature of $t_{0}{ }^{\circ} \mathrm{C}$, the true temperature of the hot junction is that on the calibration plot corresponding to the observed emf $e$ plus the emf $e^{\prime}$ where $e^{\prime}$ is the emf developed when the hot junction is at $t_{0}{ }^{\circ} \mathrm{C}$ and the cold junction is at $t_{\circ}{ }^{\circ} \mathrm{C}$. The value of $e^{\prime}$ is positive when $t_{0}{ }^{\prime}>t_{0}$ and negative when $t_{0}{ }^{\prime}<t_{0}$. Frequently the instruments used are graduated in temperature only, in which case to the observed reading is added the quantity $\left(t_{\circ}{ }^{\prime}-t_{0}\right) K$, where $K$ is a factor depending upon the couple and the temperatures employed. Values of the factor $K$ for several types of couple are given in Table 9. The factor is obtained from the following equation. This states that

$$
K=\frac{\left(\frac{\Delta e}{\Delta t}\right)_{\Delta t_{0}}}{\left(\frac{d e}{d t}\right)_{t}}
$$


is equal to the mean slope of the calibration curve over an interval $\Delta t_{\circ}=$ about $5 \mathrm{O}^{\circ} \mathrm{C}$, in the neighborhood of the cold-junction temperature, divided by the slope at the observed temperature $t$. The interval $\Delta t_{\circ}$ should embrace any likely cold-junction temperature. Thus, if the calibration were made for a cold-junction temperature of $30^{\circ} \mathrm{C}$, the mean slope from 5 to $55^{\circ} \mathrm{C}$ is the numerator of the above expression. This is obtained by dividing the difference in emfs at $5^{\circ} \mathrm{C}$ and $55^{\circ} \mathrm{C}$, by the difference in temperature, that is, $50^{\circ} \mathrm{C}$. If the calibration is made for a cold-junction temperature of $0^{\circ} \mathrm{C}$, the mean slope is determined from o to $50^{\circ} \mathrm{C}$ and not from -25 to $+25^{\circ} \mathrm{C}$, since in practice the cold-junction temperature seldom falls below $0^{\circ} \mathrm{C}$. However, since this method is only approximate and since the corrections are always small, the precise manner of determining the slope at the lower limit (that is, the numerator of the correction term) is immaterial.

(d) Method of Making Thermocouple Junctions. - The hot junction of a thermocouple should be made by soldering or fusing the two metals. Silver solder is useful at temperatures below $900^{\circ} \mathrm{C}$, or gold below $1000^{\circ} \mathrm{C}$, but, in general, the best type of junction is made by fusion. The junction of a rare-metal couple may be made by use of a small oxygen-illuminating-gas flame. Acetylene is not desirable. Special blast lamps suitable for this work are obtainable. The flame should be small, possibly $4 \mathrm{~cm}$ long, the tip of the lamp having an opening of from 0.5 to $\mathrm{I} \mathrm{mm}$. The junction should be fused into a ball about $\mathrm{I} \mathrm{mm}$ in diameter.

Another convenient method of fusing the junction is by use of an electric arc. The couple wires are connected to the positive terminal of the i Io-volt direct-current main and the negative terminal is connected through a resistance to a graphite pencil having an insulated handle. The graphite should be about 5 or $6 \mathrm{~mm}$ in diameter and should be sharpened to a point. For a couple of 0.6 $\mathrm{mm}$ wire a resistance of 30 to $40 \mathrm{ohms}$ is used. Touch together the ends of the two wires of the couple and then bring the point of the graphite pencil into contact with the junction. As soon as an arc is formed draw the pencil away. With a little practice excellent joints can be made in this manner.

This method is also good for repairing broken couples. In this case the junction should be made as nearly as possible of the same size as the wire itself.

Large base-metal couples may be fused either by an electric arc or oxy-gas torch. It is frequently the practice to twist the two wires, as shown in Fig. 6, for a few turns, as this gives greater me- 
chanical strength. Before fusing, the junction of the couple is coated thoroughly with borax. This is done by heating the ends of the two wires to a dull red heat and then dipping into the powdered borax. The borax adhering to the wire is then melted and the process continued until a heavy coating of the fused transparent borax is obtained. A stick of borax glass may be used instead of the powder. The couple may be mounted horizontally or suspended with the junction down. The arc or oxy-gas flame is allowed to play upon the junction uutil the metal fuses into a neat bead. If sufficient borax is used, the weld will show no signs of oxidation. The arc employed for this work is of the ordinary type, consisting of two carbons, although for small couples the form of arc described above may be used.

No flux should be employed when fusing rare-metal couples.

Do not allow borax to come in contact with the porcelain lining of a furnace or with porcelain tubes. What borax remains on a base-metal couple may be removed, if desired, by dipping the junction while hot into water. The borax glass will then crack away. In making thermocouple junctions dark glasses should be worn in order that the work may be watched closely. It is also essential that the glasses absorb the ultra-violet light, which may cause severe burns and injure the eyes. Special goggles for this type of work are advertised in the trade journals.

\section{USE OF STANDARDIZED COUPLES WITH VARIOUS TYPES OF MEASUR- ING INSTRUMENTS}

The temperature-emf relation of a couple may be known and it may be desirable to use the couple with different forms of indicator. Many of the instruments employed are complicated by different compensation devices, the exact operation of which must be understood in order to prevent the introduction of serious error in the observations. In the following we shall assume that the instruments themselves read correctly or that the correctionsto-scale readings are known. If the instruments are graduated in temperature only, it is essential to have a calibration of potential difference at the instrument terminals versus temperature scale. The readings of all galvanometric instruments, except the heat meter (Harrison-Foote method) and the pyrovolter, are affected by changes in line resistance. Hence the resistance of the couple must be equal to that for which the scale of the indicator is designed, or corrections as already described must be applied to the observed deflections. These corrections are assumed 
to have been applied when appreciable in the following special cases treated:

(a) Galvanometer Adjusted to Read Zero on Open CirCuit or Potentiometer without Cold-Junction CompensaTOR.-If the cold junctions of the couple are at a -temperature different from that for which the couple was standardized, a cold-junction correction as discussed above must be added to the observed reading. This also applies to the use of the pyrovolter.

(b) Galvanometer Adjusted to Read Cold-Junction TemPERATURE OR EQUivalent EMF ON OPEN Circuit.-This adjustment of the open-circuit reading may be either manually or automatically operated, in the latter case usually by a bimetallic spring. The standardization of the instrument itself should be made with the pointer adjusted to the cold-junction temperature employed in the standardization of the couple. If, howerer, the instrument has been calibrated with a different adjustment, the two calibrations may be coordinated by adding to the emf reading of the instrument calibration or by subtracting from the emfs of the couple calibration the emf on the couple calibration corresponding to the open-circuit temperature reading of the instrument. The latter operation changes the couple calibration to a new basic cold-junction temperature, shown by the opencircuit reading of the indicator.

If the instrument is graduated in emf, it should be adjusted on open circuit to read the emf shown by the couple calibration corresponding to the temperature of the cold junctions at the time of operation. When the instrument is used with several couples, the calibration of all the couples must be referred to the same basic cold-junction temperature.

(c) Potentiometer with Cold-Junction Compensator.The potentiometer compensator adds to the emf developed by the couple an amount equal to the reduction in emf of the couple occasioned by a rise in cold-junction temperature. The magnitude of the compensation may be measured by potentiometers graduated in emf by short-circuiting the emf terminals with a copper wire and then balancing the instrument in the usual manner. The main scale reading gives the amount of the compensation. When the main scale is calibrated with a manually adjusted compensator set to read a given value, the compensator scale is thereby made correct at this reading, though it may be in error at other points. Likewise, an automatic compensator is correct 
at the temperature at which it is maintained during a calibration of the instrument. The compensator, if manually operated, as well as the main scale, may be graduated either in emf or temperature. The possible combinations require separate consideration.

(1) Main Scale and Manually Operated Compensator Graduated in $E m f$.-During operation the compensator (corrected for scale error, if necessary) should be set at the emf corresponding to the temperature of the cold junctions at the time of operation, as indicated by the couple calibration regardless of the basic cold-junction temperature employed during the standardization of the couple. If, however, the cold-junction temperature during operation is lower than the basic cold-junction temperature used in the calibration of the couple, the latter should be changed to a lower value, unless the compensator can be set for negative compensation, an adjustment not furnished in the present designs.

(2) Main Scale and Manually Operated Compensator Graduated in Temperature.-The instrument should have a temperature-scalereading-versus-emf calibration, for which the compensator is set to read the temperature of the cold junctions of the couple during its standardization. This makes the compensator reading correct at this setting, although it may have scale errors at other settings. In further use the compensator (corrected for scale error) is set to read the cold-junction temperature. The hot-junction temperature is then obtained by use of the temperature-scale-readingversus-emf calibration of the instrument and the temperature-emf calibration of the couple.

(3) Main Scale Graduated in Temperature, Manually Operated Compensator Graduated in Emf. - The temperature-scale-readingversus-emf calibration of the instrument is made with the compensator set to read zero. The calibration of the couple is adjusted to the basic cold-junction temperature corresponding to the mainscale reading when the instrument is balanced with the emf terminals short-circuited and the compensator set to read zero. In use the compensator is set at the emf corresponding, on the revised thermocouple calibration, to the operating temperature of the cold junctions, and the hot-junction temperature is obtained by use of the revised temperature-emf calibration of the couple and the temperature-scale-reading-versus-emf calibration of the instrument.

With depressed zero potentiometers - that is, instruments having the lowest main scale reading greater than zero; for example, starting at $5 \mathrm{OO}^{\circ} \mathrm{C}$ or 5 millivolts - it is impossible to obtain a balance 
with the emf terminals short-circuited. In this case a temperature-scale-reading-versus-emf calibration of the instrument is made with the compensator set at zero. Select a temperature for which it is most desirable to have a correct scale reading-for example, $1000^{\circ} \mathrm{C}$-and note the corresponding emf. Then the calibration of the couple is revised by adding or subtracting from all emf values an amount sufficient to make the above emf value correspond to the chosen temperature. Plot the revised thermocouple calibration and determine the temperature corresponding to zero emf. This is the new basic cold-junction temperature. In the further use of the instrument the compensator is set to read the emf on the revised thermocouple plot corresponding to the operating cold-junction temperature, and the hot-junction temperature is obtained directly from the main temperature scale reading corrected for scaie errors. The scale correction is determined by comparing at equivalent emfs the temperature-scaleversus-emf calibration of the instrument with the revised temperature-emf calibration of the couple. Occasionally this method of calibration is desirable for ordinary potentiometers, since it makes a chosen scale reading correct.

(4) Main Scale Graduated in Emf and Manually Operated Compensator Graduated in Temperature.-The emf terminals are short-circuited, and with the main scale set to read zero the compensator is adjusted until a balance is obtained. The compensator reading gives the proper basic cold-junction temperature to which the thermocouple calibration is adjusted. Accordingly, for this coldjunction temperature the compensator scale is correct. In further use the compensator dial (corrected for scale error) is set at the operating cold-junction temperature, and the hot-junction temperature is determined from the main scale reading (corrected for scale error) and the revised temperature-emf relation of the thermocouple. Instruments having a depressed zero are used as described in the preceding paragraph.

(5) Scale Graduated in Emf and Automatic Compensator.-The compensating emf added to that developed by the couple depends upon the temperature of a nickel resistance which is so located as to acquire the cold-junction temperature of the couple. The calibration of the couple must have a basic cold-junction temperature determined by the temperature of the nickel resistance when the compensating emf is zero, that is, when some chosen scale reading corresponds to the emf at the terminals of the instru- 
ment. If inconvenient to determine this by actually varying the temperature of the compensating coil, it may be obtained as follows: Suppose that the chosen scale reading be zero millivolts, this must correspond to zero emf across the instrument terminals. Hence the emf terminals are short-circuited, and a balance is secured, as in an ordinary temperature measurement. The reading $e_{1}$ thus obtained shows the amount of compensation for the temperature $t_{1}$ of the compensating coil. The calibration data of the thermocouple must be adjusted to a basic cold-junction temperature such that for a hot-junction temperature $t_{1}$ the couple develops the emf $e_{1}$. This is done by subtracting from all emf values in the original couple calibration the quantity $e_{0}-e_{1}$, where $e_{0}$ is the emf corresponding to the temperature $t_{1}$ in the original calibration.

A given compensator can be correct for but one type of couple. To determine the accuracy of its construction, the emf terminals of the instrument are short-circuited and the scale readings for a balance observed with various temperatures of the compensating coil. The relation, scale reading versus temperature of the compensating coil, should be identical to the revised temperature-emf calibration of the couple.

If the instrument is of the depressed-zero type a calibrated potentiometer is connected across the emf terminals and the instrument balanced. The difference between the emfs indicated by the two potentiometers is the same $e_{1}$ appearing in the discussion above, and the further procedure is identical. The sign of $e_{1}$ is positive if the calibrated potentiometer reads lower than the instrument equipped with the compensator, and negative if higher.

(6) Scale Graduated in Temperature, and Automatuc Compensator.-A scale-reading-versus-emf calibration must be made. The thermocouple calibration should be adjusted to a basis cold-junc tion temperature equal to that of the compensating coil during the instrument calibration. In further use the temperature of the hot junction is obtained directly from the corrected temperature scale of the instrument. This is determined by comparing the temperature-scale-versus-emf calibration of the instrument at equivalent millivolts with the revised temperature-emf calibration of the couple. 


\section{STANDARDIZATION OF THERMOELECTRIC INSTRUMENTS}

(a) Laboratory Standard Potentiometer.-The potentiometer should be calibrated about three months after its manufacture and at intervals of two or three years. Two, or preferably three, standard cells should be available for intercomparison. In case one shows changes relative to the other two, it should be restandardized, and one or more of the cells should be occasionally calibrated, whether or not such variations are detected. The calibration of standard cells and potentiometers should be done either by the Bureau of Standards or the manufacturers. A simple method of intercomparison of cells is as follows: With cell number I and the standard cell dial set to the corresponding certified emf, the instrument is balanced against the standard cell in the ordinary manner. Cell number I is then replaced by cell number 2, and the standard cell dial adjusted until a balance is again obtained. If this reading is not the certified emf of cell number 2, one of the two cells has changed. The cell in error may be detected by an intercomparison with a third cell, unless the three cells have changed similarly, which condition is detected by the periodic standardization of one of the cells. The cells should not be subjected to air currents or other causes of temperature change immediately previous to or during use or intercomparison. They should not be allowed to freeze.

(b) Adjustment of Instruments for Calibration.-Instruments having a variable zero or open-circuit adjustment should be set to satisfy the conditions of use, as already described.

(c) Galvanometers. - Galvanometers may be calibrated in terms of the potential drop across the instrument terminals, or allowance may be made for a definite external resistance. Fig. I 52 shows a convenient wiring diagram. Adjustable resistances $R_{1}$ and $R_{2}$ are connected in series with a battery $B$. The galvanometer terminals are connected, with the proper polarity, across $R_{1}$. Either one of the resistances is adjusted until the desired scale reading is obtained, and the potential drop across the galvanometer terminals is measured by the standard potentiometer. If the galvanometer is to be calibrated for a definite external resistance, this amount $x$ may be placed in the circuit, as illustrated. If $x=o$ the calibration is in terms of the potential drop across the galvanometer terminals. Observations of instrument reading versus true emf should be made at least at 5 points over the scale, and any range showing irregularities should be further checked. 
The resistance $R_{1}$ may be varied from o to Ioo ohms and $R_{2}$ from Ioo to Io ooo ohms. In general, $R_{2}$ should be kept rather large to avoid drawing too much current and to prevent rapid decrease of current due to polarization, if old dry cells are employed. In making the first set up, $R_{1}$ should be small (or even zero) and $R_{2}$ large. $R_{1}$ should never be disconnected from the circuit without first disconnecting the galvanometer or opening the battery circuit at $R_{2}$ or $B$. It should be noted whether the instrument returns to the same open-circuit reading after the calibration, also after the full scale deflection is registered for some time. It is advisable to tap a deflection instrument before each reading during standardization in order to avoid sticking of the coil in its

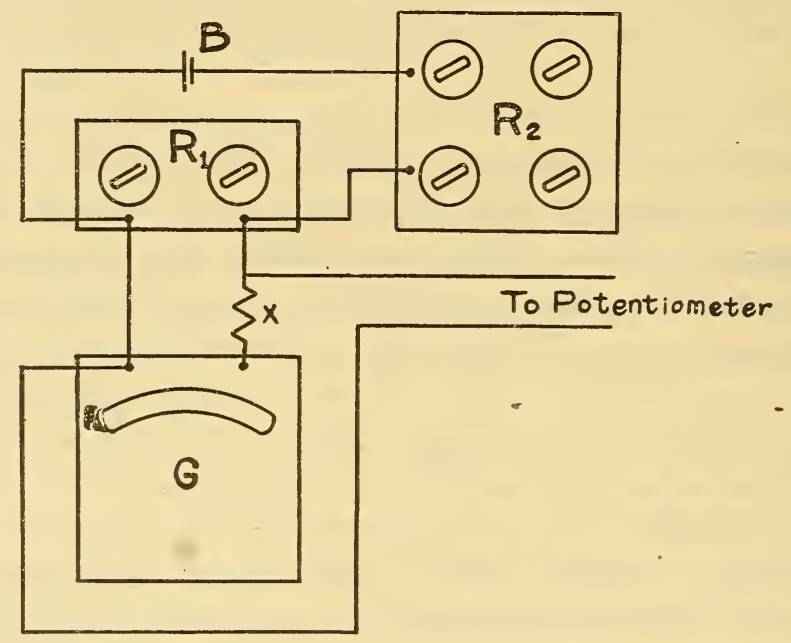

FiG. 152.-Wiring diagram for standardization of galvanometric indicators

mounting. Instruments equipped with a bimetallic spring zero adjuster should be calibrated at constant room temperature.

The temperature coefficient of a galvanometer may be observed by calibrating twice at two widely different room temperatures. Sufficient time-several hours - must be allowed for the instrument to reach a uniform temperature.

A bimetallic spring cold-junction compensator may be tested by varying the temperature of the instrument and observing the corresponding scale readings on open circuit. The relation, emf scale reading versus temperature, so obtained, must be identical to the temperature-emf relation of the couple based on the coldjunction temperature $t_{\mathrm{o}}$ where $t_{\mathrm{o}}$ is the temperature of the galvanometer for a zero-emf scale reading. If the scale is graduated 
in degrees, the pointer, on open circuit, should indicate the temperature of the galvanometer.

(d) Portable and Recording Potentiometers.-The emf or thermocouple terminals of the instrument are connected to the emf terminals of the laboratory standard potentiometer, + to + and - to - . Both instruments are balanced as usual against their individual standard cells, and with various settings of the scale of the instrument under test the true emf is obtained from the reading of the standard potentiometer. Different portable potentiometers may be similarly intercompared, in which case it is desirable to short-circuit one of the galvanometers after balancing against the standard cell in order to increase the sensitivity.

(e) Potentiometer with Compensator Graduated in TemPERATURE.-The compensator is set at the desired basic coldjunction temperature of the couple, and the main scale is tested as above. This makes the compensator scale correct at this particular setting. To calibrate the compensator scale, the main scale is set at any definite position, preferably greater than the highest setting on the compensator scale, and several measurements are made of the emf for different compensator settings, one setting being the same as that used for the main scale calibration. Each reading is subtracted from the value determined by this latter setting. The compensator readings versus the emf differences should be equivalent to the temperature-emf relation of the couple. If these do not agree sufficiently well, the compensator scale should be standardized in terms of scale reading versus true cold-junction temperature, and the necessary correction applied in subsequent use each time the compensator is set.

(f) Potentiometer with Compensator Graduated in emf.The main scale is calibrated with the compensator set at zero. This makes the zero setting of the compensator correct. To calibrate the compensator scale, the main scale is set at any definite position, preferably greater than the highest setting on the compensator scale, and measurements are made of the emf for various compensator settings. Each reading is subtracted from the value observed with the compensator set at zero. This gives the calibration of the scale reading versus true enf for the compensator.

(g) Potentiometer with Automatic Compensator.-These are calibrated in the same manner described for simple potentiometers, the temperature of the compensating coil being noted and 
maintained constant during the observations. ${ }^{48}$ If the scale is graduated in emf and accurately made, true emf across the potentiometer terminals is indicated for some definite temperature of the compensator, but at other temperatures this is not the case. Hence it is desirable to know the basic temperature of the compensator for which the scale graduation was made. This may be determined for an instrument which does not have a depressed zero by short-circuiting the emf terminals and varying the temperature of the compensator coil until a balance is obtained with an emf scale reading of zero. The temperature-emf relation of the couple must be adjusted for this basic cold-junction temperature.

It is not always convenient to vary the temperature of the compensator. The instrument may be tested with the temperature of the compensator that of the room, preferably at the approximate mean cold-junction temperature to be employed in subsequent use. A calibration of emf scale readings versus true emf is made in the manner described above, and the temperature-emf relation of the couple is adjusted to the basic cold-junction temperature equivalent to that of the room during the standardization of the potentiometer. By comparing the temperature-emf relation of the couple to the scale-reading-versus-true-emf relation for the instrument, a final table may be prepared of scale reading versus temperature. The general procedure is identical when the potentiometer scale is graduated in temperature.

(h) Instruments Employing the Harrison-Foote Method.If the laboratory potentiometer is of the low-resistance type, such as the Leeds \& Northrup precision thermocouple potentiometer, the "heat meter" is most easily and accurately standardized by connecting the emf terminals of the two instruments together, + to + and - to - . After balancing the potentiometer against the standard cell its galvanometer terminals are short-circuited and the potentiometer key is closed. The two instruments will then read approximately the same. The potentiometer is set to give the desired deflection of the heat meter, and before each reading the heat meter must be adjusted by the usual method to compensate for external resistance. The potentiometer setting gives the true emf corresponding to the scale reaaing of the heat meter. Heat meters having an adjustable resistance of $\mathrm{I}_{5} \mathrm{ohms}$ may be calibrated in the above manner by means of the Leeds $\&$

48 The accuracy of the compensator may be determined by the method given on page 240 . 
Northrup type $\mathrm{K}$ potentiometer, provided the ratio coils are so set that the fourth dial step is not exceeded.

If a potentiometer of high resistance must be used, the wiring diagram shown in Fig. ${ }^{2} 2$ may be employed, in which $x$ is zero and $R_{1}$ is less than $\mathrm{I}_{5} \mathrm{ohms}$ (the value of $r_{5}$ in Fig. $2 \mathrm{I} a$ ). The resistances $R_{1}$ and $R_{2}$ are adjusted for the desired deflection, the heat meter being set in the usual manner to compensate for external resistance. The true emf corresponding to the observed scale deflection is obtained by measuring the potential drop across $R_{1}$ with the potentiometer, while the heat meter is disconnected from the circuit. A good dry battery or storage cell is necessary for these determinations. If $R_{1}$ is less than o.I ohm and the potentiometer is connected directly across $R_{1}$, it is unnecessary to disconnect the heat meter.

A less. precise method of standardization is to set the adjustable resistance of the heat meter to the marked position and follow the procedure described for calibrating a galvanometer in terms of the potential drop across its terminals.

\section{CALIBRATION OF COUPLE AND INDICATOR AS A UNIT}

Often it is desirable to calibrate a couple and indicator as a unit, the calibration giving the relation between scale reading and true temperature.

(a) Galvanometric Instruments. - The calibration automatically takes account of line resistance. In future use, however, it is important that the resistance of the circuit does not change, especially if the instrument is of low resistance. If the galvanometer is always adjusted to read zero on open circuit, the cold-junction temperature of the couple during calibration is the basic cold-junction temperature to which subsequent cold-junction temperatures must be corrected, as described on page 246 . If the galvanometer is graduated in temperature in the low range, the pointer may be adjusted to read the cold-junction temperature both during calibration and subsequent use. This avoids the necessity for applying cold-junction corrections. Galvanometers equipped with a bimetallic spring compensation operate the zero shift automatically, and should be so adjusted that on open circuit the instruments indicate the cold-junction temperature.

(b) POTENTIOMETERS.-When a simple potentiometer without compensator is calibrated with a couple, as a unit, the cold-junction temperature during calibration is the basic cold-junction tem$8513^{\circ}-21-17$ 
perature of the couple to which subsequent cold-junction temperatures must be corrected, as described on page 246 . When the potentiometer has a compensator graduated in temperature, the compensator is set to read the cold-junction temperature of the couple both during calibration and subsequent use. When the main scale of the potentiometer and the compensator are both graduated in emf, a setting of the compensator should be so chosen during calibration that in subsequent use any likely cold-junction temperature may be compensated for. Usually the cold-junction temperature is not less than $\mathrm{o}^{\circ} \mathrm{C}$. Hence one method is to set the compensator at zero and calibrate with a cold-junction temperature of $0^{\circ} \mathrm{C}$. If the calibration must be made with the coldjunction temperature that of the room, it is not desirable to set the compensator at zero, because in subsequent use the room temperature may decrease, and no compensation could be afforded, since negative compensation is not provided for. Hence during calibration the compensator should be adjusted to such a position that it will read zero when the cold-junction temperature is $\mathrm{o}^{\circ} \mathrm{C}$ or the lowest temperature, $t_{0}$, likely to be encountered. This position may be estimated from a knowledge of the temperatureemf relation of the general type of couple employed. In future use of the pyrometer the compensation for cold-junction temperature must be referred to the basic cold-junction temperature for which the compensator reads zero; that is, $0^{\circ} \mathrm{C}$ or $t_{0}$ in the above example. If the main scale is graduated in temperature, shortcircuit the emf terminals, set the main scale to read the coldjunction temperature to be used in the calibration, and balance by adjusting the compensator. This gives the proper compensator setting if the main temperature scale is approximately correct for the type of couple employed. In subsequent use the compensator may be set by the same method, or the compensation may be estimated from a knowledge of the temperature emf relation of the general type of couple used. This relation must be referred to the basic cold-junction temperature determined by the reading of the main scale when the instrument is balanced with the emf terminals short-circuited and the compensator set at zero.

\section{STANDARDIZATION OF OPTICAL PYROMETERS}

An optical pyrometer may be standardized by either a primary or a secondary method. A precise and satisfactory primary calibration requires considerable technique, but the secondary 
method is sufficient except for special research work in the laboratory, when the highest possible precision is desired. In the secondary method the pyrometer under test is compared with an instrument which has been accurately standardized. The primary standard optical pyrometer of this Bureau, shown in Fig. I53, operates on the disappearing-filament principle, since this method is capable of the highest precision. In the following sections are considered methods of secondary standardization, after which a brief statement of the method employed for primary standardization is presented.

(a) Secondary Standardization.--(r) Comparison Source.A constant and uniform source of light is required upon which the two pyrometers are sighted. It is not essential that this

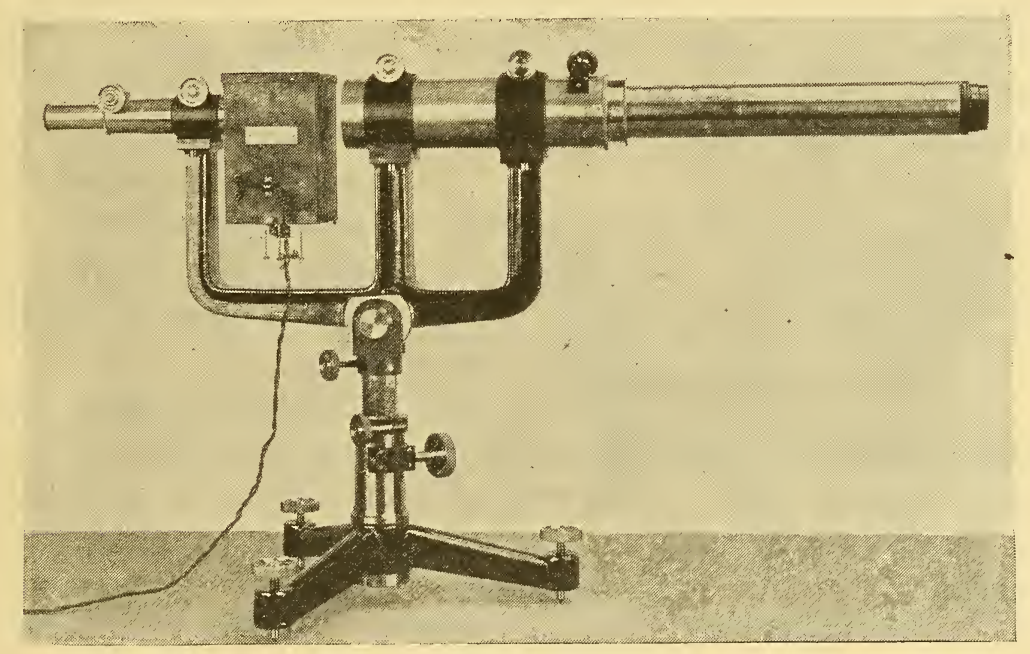

FIG. 153.-Bureau of Standards precision optical pyrometer

source be a black body, but if the radiation is very selective only pyrometers of the same type or instruments using the same wave length should be compared. Fig. I 54 illustrates a black-body furnace suitable for temperatures up to $1550^{\circ} \mathrm{C}$. Its construction is similar to that described under Fig. I 34 . A series of diaphragms of successively smaller openings lead into the interior inclosure, from which the radiation is emitted. The pyrometer is focused on the diaphragm $A$. The angle $b A c$ is properly chosen somewhat greater than the cone of rays required by the pyrometer; otherwise one of the front openings may act as a diaphragn to the optical system and cut down the aperture, thus giving an erroneous calibration. For temperatures up to $2500^{\circ} \mathrm{C}$ the Arsem furnace, 
already described, is useful. Thus, in Fig. I 39 the sample $A$ may be removed or both $A$ and $B$ may be replaced by a graphite crucible. A small furnace for operation up to $\mathrm{I} 60^{\circ} \mathrm{C}$ may be made by winding the inside walls of an alundum crucible (front, back, and side walls) 3.5 by $3.5 \mathrm{~cm}$, with $0.6 \mathrm{~mm}$ platinum or platinum-rhodium wire. The turns of wire are held in place by a thin layer of alundum cement, and the radiation is emitted from a front opening about I $\mathrm{cm}$ in diameter. If no insulation is employed, the temperature of the furnace may be readily adjusted to any desired value, and by using battery current can be maintained constant.

A carbon-strip lamp, consisting of a thin carbon ribbon about $8 \mathrm{~mm}$ wide, mounted in an evacuated glass bulb, may be operated up to $\mathrm{i} 800^{\circ} \mathrm{C}$, and the radiation is sufficiently black for ordinary calibration work, even with pyrometers using different quality of red glass. The General Electric Co. has made lamps of this character, requiring from io to I 5 amperes for $\mathrm{I}^{\circ} 80^{\circ} \mathrm{C}$. The temperature distribution across the strip is never very good, and

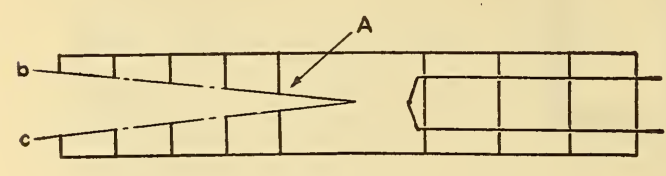

FIG. I54.-Black-body furnace great care is required that both pyrometers are sighted on exactly the same portion of the ribbon.

A strip of pure sheet nickel electrically heated in air forms a tough coating of oxide which does not scale. This type of source possesses the advantage that it may be made as large and uniform as required, but it

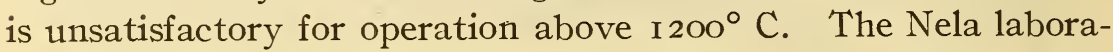
tory has developed the tungsten-strip lamp, which may be operated up to $2500^{\circ} \mathrm{C}$. A ribbon of tungsten about $2 \mathrm{~mm}$ wide and 4 $\mathrm{cm}$ long is mounted in a gas-filled bulb. While the source is not black, its emissivity at various wave lengths is well known and may be corrected for when required. The width of the source, however, is so small that its use is practically confined to the disappearing-filament pyrometer, and if the instruments compared have the same type of red glass, as is usually the case, the departure of the source from black-body conditions is of no importance.

(2) Disappearing-Filament Pyrometer.-Fig. I 55 illustrates the arrangement for comparing the pyrometer on the left with a standard instrument on the right. The comparison source is the small furnace described above, or a carbon or tungsten strip lamp. Since the radiation intensity from such a strip varies with the angle 
$\vartheta$ at which the pyrometer is sighted, it is desirable to make both $\vartheta$ and $\vartheta^{\prime}$ as small as convenient and as nearly equal as possible. It is also necessary to focus both pyrometers on the same portion of the strip. This may be done by locating some peculiarity of the strip visible in each pyrometer, or the pyrometer lamps may be heated to about I 300 or $\mathrm{I} 400^{\circ} \mathrm{C}$ and the images of the filaments superposed on the strip at the point desired. If the furnace shown in Fig. I 54 is employed, it is inconvenient to sight both pyrometers at the same time. A convenient means may be devised for shifting either the furnace or the pyrometers by the proper

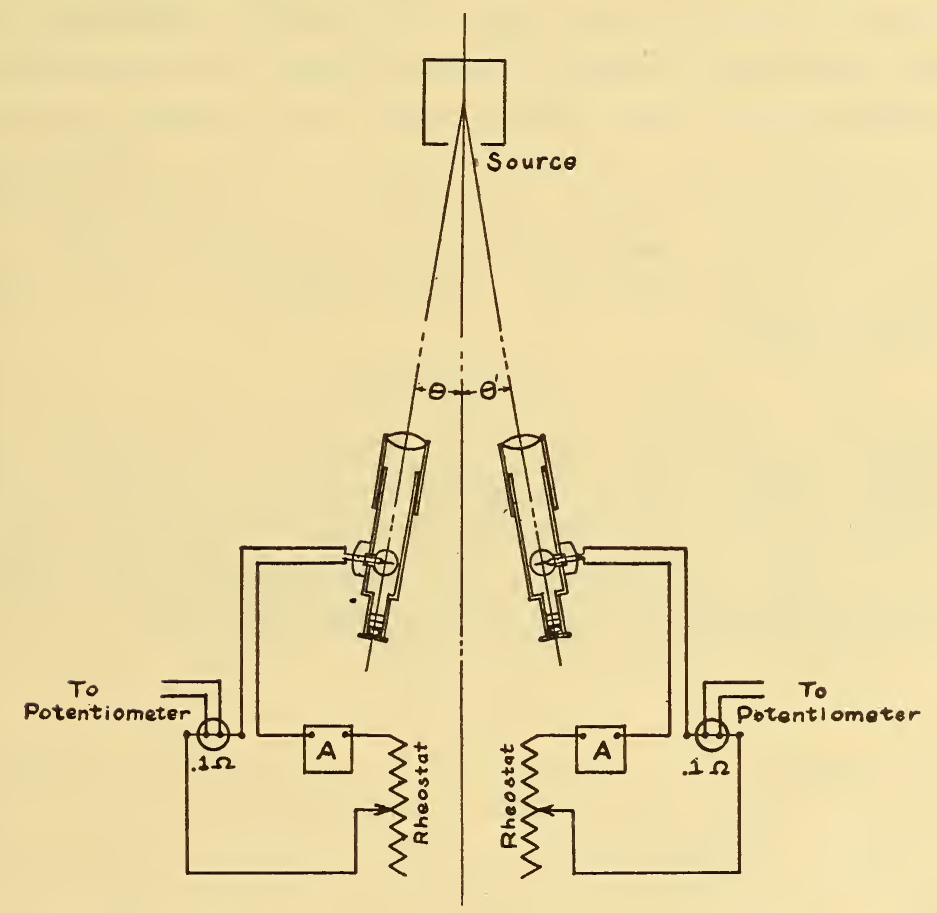

FIG. 155.-Comparison method of standardizing optical pyrometers

amount, so that readings may be made first with one instrument and then with the other. With the arrangement illustrated two observers may take readings simultaneously, thus eliminating any effect due to change in temperature of the source. If a new pyrometer lamp is employed, it should be first annealed at not less than $2000^{\circ} \mathrm{C}$ for 20 hours for a tungsten filament and $1800^{\circ} \mathrm{C}$ for a carbon filament. Tungsten filaments with welded terminals are now almost exclusively used.

For an approximate calibration the current may be measured by means of an ammeter, but for a precision calibration it is 
desirable to use a potentiometer. Resistance standards of O.I ohm are connected in series with the lamp, and the potential leads are carried to a potentioneter. The currents usually required are from 0.2 to 0.6 ampere, which, over a O.I ohm, give a potential drop of from 20 to 60 millivolts, a convenient range for the laboratory thermocouple potentiometer. The use of a deflection potentiometer for the current measurements is very satisfactory, this instrument furnishing sufficient accuracy (o.I per cent) and possessing nearly the convenience of an ammeter.

The current measurements with the standard instrument are converted to temperature from the primary calibration chart, whence a plot may be made of current versus temperature for the instrument under test. The plot may be prepared by drawing the best smooth curve through the observed data. It is, however, desirable in precise work to use a definite form of curve for which the constants are computed from the experimental data. The following equation holds very satisfactorily from 700 to $5^{00}{ }^{\circ} \mathrm{C}$ for carbon lamps:

$$
i=a+b t+c t^{2}
$$

where the constants $a, b$, and $c$ are determinable by three standardization points. This equation applied to tungsten lamps should not be extrapolated below the lowest observed point, nor for more than $\mathrm{ICO}^{\circ} \mathrm{C}$ above the highest point. An equation slightly more satisfactory for tungsten has the form:

$$
i=a t+b e^{\mathrm{ct}} \text {. }
$$

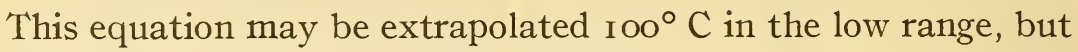
errors of $5^{\circ} \mathrm{C}$ may result from an extrapolation of $100^{\circ} \mathrm{C}$ in the high range. Accordingly, for precision work it is desirable to employ the more difficult cubic equation with four constants, viz.:

$$
i=a+b t+c t^{2}+d t^{3} .
$$

The constants are determinable by four standardization points, but it is usually desirable to obtain at least ro comparison points from which the best curve may be computed by least squares in the following manner: The values of $i, t, t^{2}, t^{3}, t^{4}, t^{5}, t^{6}, i t, i t^{2}, i t^{3}$, are tabulated for each calibration point and the sums of all the $n$ values of $i$ are denoted by $\Sigma i$, of all the $n$ values of $t$ by $\Sigma t$, etc. These sums are substituted in the following normal equations, which are solved for $a, b, c$, and $d$ : Normal equations: 


$$
\begin{aligned}
& a n+b \Sigma t+c \Sigma t^{2}+d \Sigma t^{3}=\Sigma i \\
& a \Sigma t+b \Sigma t^{2}+c \Sigma t^{3}+d \Sigma t^{4}=\Sigma i t \\
& a \Sigma t^{2}+b \Sigma t^{3}+c \Sigma t^{4}+d \Sigma t^{5}=\Sigma i t^{2} \\
& a \Sigma t^{3}+b \Sigma t^{4}+c \Sigma t^{5}+d \Sigma t^{6}=\Sigma i t^{3}
\end{aligned}
$$

Thus, knowing $a, b, c$, and $d$, the equation of the lamp may be computed for various temperatures, and a curve may be drawn through these points, or a table of current versus temperature may be calculated.

For extending the range of the pyrometer above $1400^{\circ} \mathrm{C}$ absorption glasses calibrated as described on page 106 are employed. In ordinary work the value of $A$ so obtained is a constant, but in precision testing it is found that $A$ usually varies somewhat with the temperature of the source sighted upon, as will be briefly considered later.

If the pyrometers are calibrated separate from the ammeters, the ammeters should be also tested at several points. This is readily done by connecting a $\mathrm{O} . \mathrm{I}-\mathrm{ohm}$ resistance standard in series with the ammeter and measuring the potential drop across this resistance for various settings of the ammeter, whence a table of corrections may be prepared for the instrument. This method is more desirable than to calibrate the ammeter and pyrometer as a unit, since if changes in the calibration occur it is then readily apparent whether the lamp or the ammeter is at fault.

In the comparison method of calibration of optical pyrometers no corrections need be applied for absorption of light by glass windows, glass bulbs of strip lamps, etc., since both instruments are affected similarly.

It is occasionally desirable to check an instrument at a single point. Any convenient source of light is satisfactory for this purpose. For example, a stone block in a neighboring building, illuminated by sunlight, or a concrete sidewalk, etc., furnishes a source of apparent temperature from 700 to $\mathrm{r} 200^{\circ} \mathrm{C}$. Such sources however, are far from black, and it is very important that the same type of red glass be used in each pyrometer so compared.

(3) Wanner Pyrometer. - This instrument requires a much larger source than the disappearing-filament pyrometer, and hence neither a carbon nor tungsten strip lamp is satisfactory. A wide nickel strip may be used, but for a sufficient temperature range either the black-body furnace or the Arsem furnace is necessary. Metal strips are also objectionable for use with the Wanner, since the emitted light is polarized and readings will be affected by the 
orientation of the instrument. The source sighted upon must be uniformly heated, and the instrument should be carefully aligned, so that the standard pyrometer may be focused on the center of the field used by the Wanner.

The relation between the log tan of the angular reading and the reciprocal of the absolute temperature is linear. The brightness of the black-body source sighted upon is matched against that of the electric lamp which burns at a constant brightness $X$. Since the photometric fields illuminated by the lamp and furnace are polarized at right angles, we obtain the following equation for the condition of a match, where $J$ is the intensity of the source at the absolute temperature $\vartheta$, and $\varphi$ is the angle through which the analyzer or eyepiece is rotated.

$$
J=X \tan ^{2} \varphi
$$

At the normal point scme definite angle $\varphi_{0}$ corresponds to an intensity $J_{0}$ thus:

Hence

$$
J_{0}=X \tan ^{2} \varphi_{0}
$$

or

$$
\begin{gathered}
J=J o \frac{\tan ^{2} \varphi}{\tan ^{2} \varphi_{0}}, \\
c_{1} \lambda^{-5} e^{\frac{-c_{2}}{\lambda \vartheta}}=c_{1} \lambda^{-5} e^{\frac{-c_{2}}{\lambda \vartheta_{0}}} \frac{\tan ^{2} \varphi}{\tan ^{2} \varphi_{0}} .
\end{gathered}
$$

Accordingly $\log \tan \varphi=\left[\frac{c_{2} \log e}{2 \lambda \vartheta_{o}}+\log \tan \varphi_{0}\right]-\frac{c_{2} \log e}{2 \lambda \vartheta}$,

or $\log \tan \varphi=a-\frac{b}{\vartheta}$ where $a$ and $b$ are positive constants deter-

minable by two standardization points, although more points are always desirable.

The adjustment for the normal point is made as described on page roo, and the instrument is then compared with the standard at about ro different temperatures well distributed over the angular scale of the pyrometer. After this comparison the normal setting is again determined to assure that no change has taken place. The reciprocal of the absolute temperature $\frac{\mathrm{I}}{\vartheta}$ is plotted against the logarithm of the tangent of the angle, $\log \tan \varphi$, as shown in Fig. I 56, and the best straight line is drawn through the observed points. The temperatures corresponding to the various angles are then read from this plot, and a table of temperature versus angle from $\varphi=10^{\circ}$ to $\varphi=80^{\circ}$ is prepared for subsequent use. 
If the instrument has a temperature scale engraved on the dial, it is unnecessary (although still preferable) to make the above computations. The correct temperature may be plotted against the temperatures indicated by the Wanner and a correction chart prepared similar to those furnished with precision voltmeters. However, since the curve $\varphi$ vs. $\vartheta$ is not linear, but

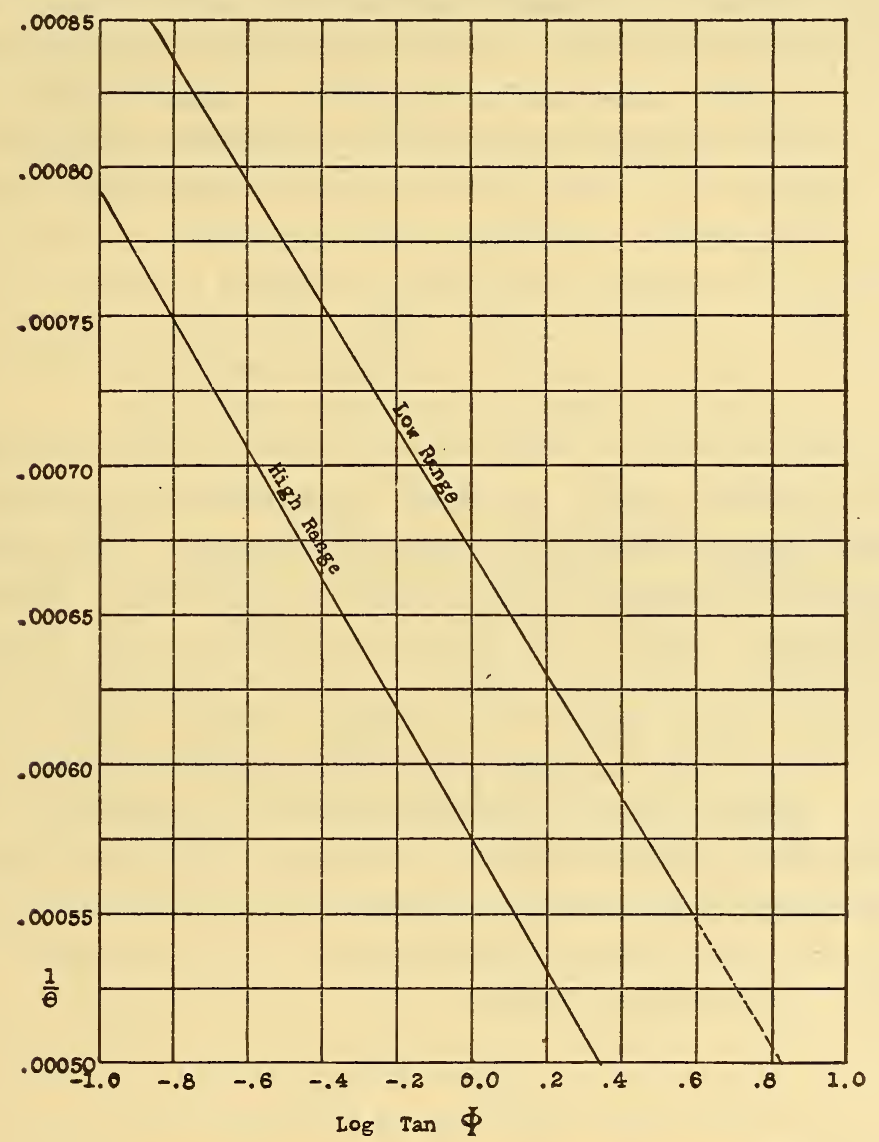

FIG. I56.-Calibration plot for Wanner pyrometer

$\mathrm{S}$-shaped, it is difficult to interpolate corrections on the temperature scale directly unless a large number of standardization temperatures are selected.

The high-range Wanner is equipped with an absorption glass which may be standardized in the manner described for the absorption glass of the disappearing-filament pyrometer, page Io6, or the instrument may be standardized directly with the absorption glass. 
The Wanner pyrometer contains several set screws, the movement of any one of which may throw the instrument out of adjustment. In general, it is inadvisable to attempt to readjust the optical parts, as the process is difficult and complicated. If the dividing line in the field is poor or if it is impossible to obtain a uniformly illuminated field, it is likely that the Wollaston prism has been moved or requires recementing with Canada balsam. Occasionally the set screw on the analyzer loosens, but this adjustment is easily made in the laboratory. Sight on any constant source and match the two fields by setting the analyzer in the first quadrant, as is done in an ordinary temperature measurement. Then make a setting with the pointer in the second quadrant. This can be done after removing a stop screw either on the dial or on the bearing upon which the eyepiece turns. The sum of the two angular readings should be $180^{\circ}$, and the analyzer set screw is adjusted until this is the case.

(4) Fery Optical and F and F Pyrometer..-These instruments employ a linear wedge of black glass which is moved across the photometric field until a match is secured. With the Fery instrument the motion of the wedge is read from a linear scale, and with the $F$ and $F$ this motion is transferred by a gear to a circular scale on the dial plate. In either case, if the scale is divided into equal spaces-for example, circular degrees for the $F$ and $F$-the scale reading plotted against the reciprocal of the absolute temperature is approximately a straight line. Thus, referring to Fig. 58 , the source $F$ is properly adjusted in the manner described to a constant brightness $X$. If the instrument is sighted on a black body of temperature $\vartheta$, we obtain for the condition of a photometric match:

$$
T c_{1} \lambda^{-5} e^{-\frac{c_{2}}{\partial \lambda}}=X
$$

where $T$ is the transmission coefficient of the wedge. If strictly monochromatic light were employed (the condition for the narrow band of radiation actually used is not seriously different as far as practical considerations are concerned), the transmission coefficient of the wedge bears the following relation to the thickness $x$ and to the angle $\alpha$, which is proportional to this thickness, $K$ and $K^{\prime}$ being constants:

$$
T=e^{-K x}=e^{-K^{\prime} \alpha} .
$$


On substituting this value of $T$ in the above equation and taking the logarithm of each member we obtain:

$$
\alpha+\left[\frac{\log _{e} X}{K^{\prime}}-\frac{\log _{e} c_{1} \lambda^{-5}}{K^{\prime}}\right]=-\frac{c_{2}}{K^{\prime} \lambda} \frac{\mathrm{I}}{\vartheta}
$$

or $\alpha-P=-Q / \vartheta$ where $P$ and $Q$ are positive constants. The constant $X$ is fixed by the normal setting of the pyrometer. Thus some particular angle $\alpha_{\circ}$ is made to correspond to a temperature $\vartheta_{\circ}$; whence

or

$$
e^{-K^{\prime} \alpha_{o}} c_{1} \lambda^{-5} e^{-\frac{c_{2}}{\lambda \vartheta_{o}}}=X=e^{-K^{\prime} \alpha} c_{1} \lambda^{-5} e^{-\frac{c_{2}}{\lambda \vartheta}}
$$

$$
\alpha-\left[\alpha_{\circ}+\frac{c_{2}}{K^{\prime} \lambda \vartheta_{\circ}}\right]=-\frac{c_{2}}{K^{\prime} \lambda} \frac{\mathrm{I}}{\vartheta}
$$

thus, $\alpha-P=-Q / \vartheta$, as before.

Hence the relation between the angle $\alpha$ and the reciprocal of the absolute temperature $\mathrm{I} / \vartheta$ is linear. The adjustment on the normal point is made in the manner already described, and the instrument is compared with the standard pyrometer at a series of temperatures. The angle $\alpha$ is plotted against I $/ \vartheta$, and the best straight line drawn, as shown in Fig. I 57; whence a table or plot of $\alpha$ versus $t^{\circ} \mathrm{C}$ may be prepared. If the instrument is graduated in temperature a simple table of corrections may be determined without plotting the data in terms of angular readings.

(b) Primary Standardization.-A secondary calibration of a properly designed disappearing-filament pyrometer may be easily made with an accuracy of $5^{\circ} \mathrm{C}$ and, if necessary, $2^{\circ} \mathrm{C}$, or even better. Hence there is no reason for making a primary calibration unless the highest possible precision for research work is required.

A primary calibration requires the use of a black body, and every precaution must be taken to assure that the radiation is as nearly black as possible. Two conditions should be fulfilled; the opening into the hollow inclosure, in general, should be small compared to the size of the inclosure, and the walls of the inclosure must possess a uniform temperature. The black body shown in Fig. I 58 satisfies these conditions. The crucible and radiator are constructed of graphite, and the radiation from the inclosure $B$ is emitted through the opening $C$. The space $D$ is filled with metal, such as copper. The bath of molten metal of high thermal conductivity insures tcmperature uniformity over the entire radiator. The radiation is so black that all detail of contour 
vanishes, and, in order to distinguish the opening $C$ for focusing and adjusting the pyrometer, it is necessary to allow a drop of water to fall into $B$, when momentarily $C$ appears in the field as a dark spot. This form of crucible has been made in smaller sizes for use in the Arsem furnace, but it has been found that no appreciable sacrifice in blackness is offered by the much-easier constructed crucible shown in Fig. I44. Using this type of crucible a primary calibration is made by observing, as described on page $2 \mathrm{I}$ I,

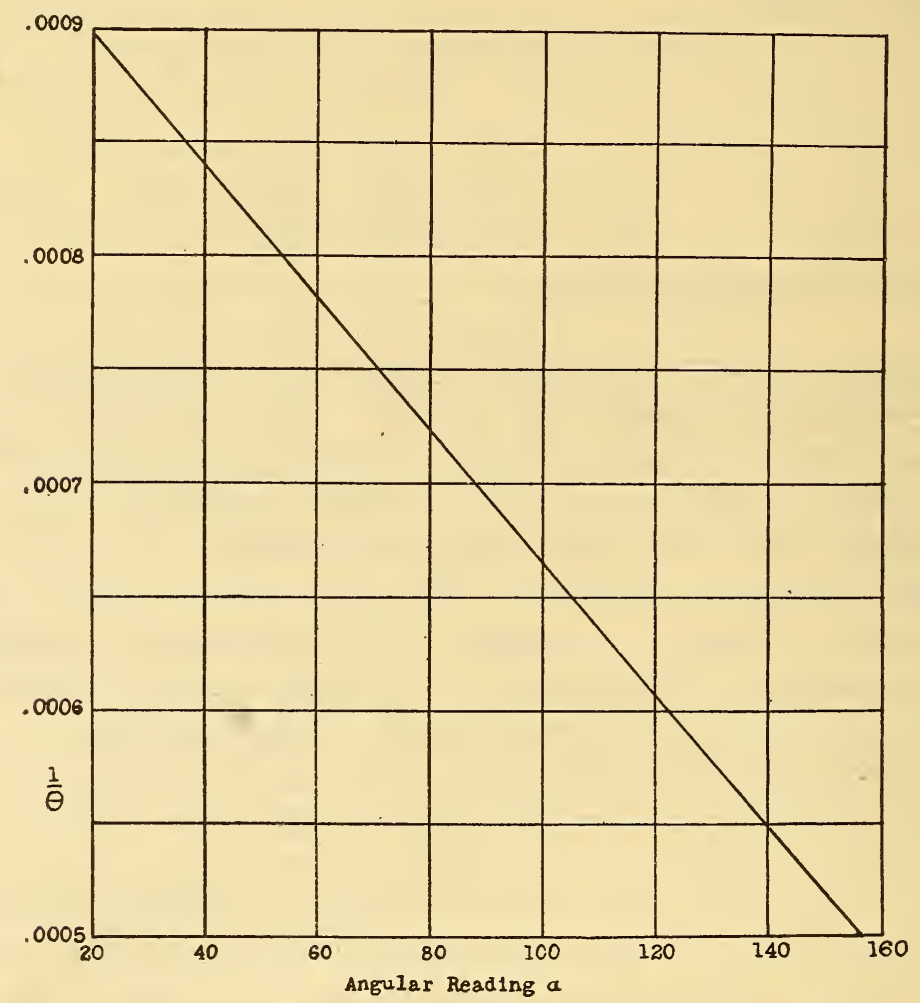

FIG. 157.-Calibration plot for $F$ and $F$ or Fery optical pyrometer employing a linear wedge

the freezing or melting curves of several metals the melting points of which are accurately known. The metals chosen are aluminum ${ }^{49}$ (99.66 per cent pure) $658.7^{\circ} \mathrm{C}$; copper-silver eutectic, $779^{\circ} \mathrm{C}$; silver, $960.5^{\circ} \mathrm{C}$; and copper, $1083^{\circ} \mathrm{C}$, or gold, $1063^{\circ} \mathrm{C}$. The time of melting or freezing is adjusted to about 20 minutes, and at least 30 or 40 observations are made during the change in phase. If a glass window is used on the furnace it is necessary to apply small corrections to the above melting points to allow for 
the absorption of the glass, as described on page II 7 . The experiments are repeated several times, and the mean values of the currents determined potentiometrically are substituted in the cubic equation $i=a+b t+c t^{2}+d t^{3}$, which is solved for the constants $a, b, c$, and $d$. This calibration curve is interpolated and extrapolated from 650 to $40^{\circ} \mathrm{C}$. The data so obtained are carefully

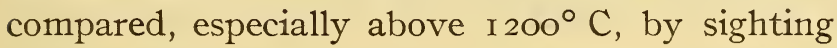
into the Lummer Kurlbaum black body shown in Fig. I 54, the temperature of which is measured by two accurately standardized rare-metal thermocouples. If any differences exist, the latter observations are given weight and a new calibration curve is recomputed. However, if the diaphragms have progressively smaller openings into the interior of the furnace, so that good black-body conditions obtain, the two methods of standardization are in excellent agreement.

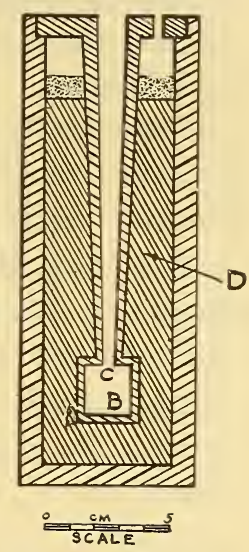

FIG. I58.-Black body immersed in molten metal

The precision calibration of the absorption device for extend-

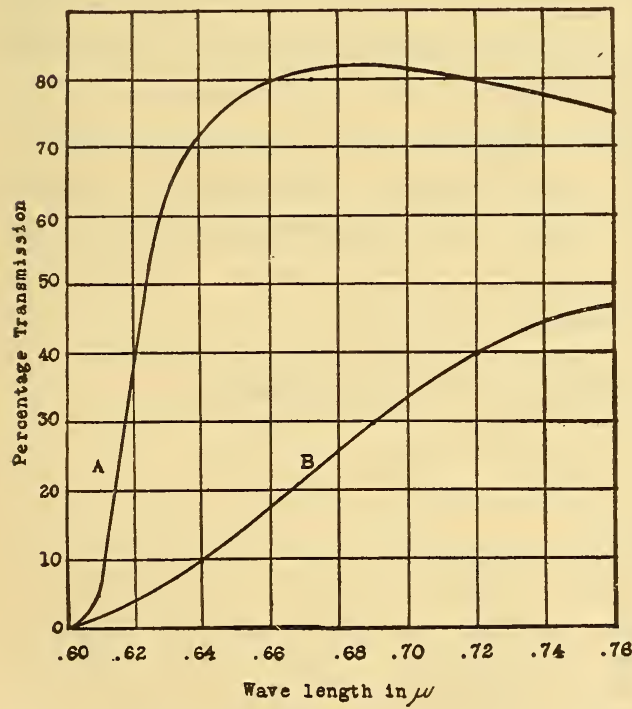

FIG. I59.-Spectral transmission of A- "Corning High-Transmission Red" marked I5o per cent, thickness $5 \mathrm{~mm} ; \mathrm{B}$ - Jena red glass No.2745, thickness $3.2 \mathrm{~mm}$ through such a glass increases, the color of the glass changes slightly from a deep red to a bright red, and this change in effective wave rometer above $1400^{\circ} \mathrm{C}$ is a complicated and laborious process. The quantity $A=\mathrm{I} / \vartheta-\mathrm{I} / S$, discussed on page 106 , is not, in general, a constant, but, with increasing temperature of the source, numerically increases for the usual type of absorption glass and decreases for a sectored disk. This is due to the fact that strictly monochromatic light is not employed. The red glass ocular transmits a band of light, as illustrated by Fig. I 59 .

As the temperature of the ing the range of the py- 
length of transmission must be considered. It is necessary to introduce several definitions before the calibration of absorption devices can be discussed. Visibility, $V=f(\lambda)$, is the relative sensitivity of the normal eye to equal intensities of radiation of different wave lengths. Luminosity, $L=f(\vartheta)$, is the luminous intensity of a heterochromatic source, and is proportional to the area under the curve obtained by plotting the product of visibility and energy intensity against the wave length. ${ }^{50}$ The effective wave length $\lambda_{L}$ of a color filter or screen is that wave length for which a small change in temperature of the black-body source sighted upon produces the same fractional change in luminosity and in the energy intensity at this wave length, as viewed and measured through the screen. This definition is expressed mathematically as follows:

$$
\text { Effective wave length }=\lambda_{\mathrm{L}}=\frac{\int_{0}^{\infty} T_{\lambda} V_{\lambda} J_{\lambda} d \lambda}{\int_{0}^{\infty} \frac{T_{\lambda} V_{\lambda} J_{\lambda}}{\lambda} d \lambda}
$$

where $T_{\lambda}$ is the transmission coefficient of the color screen and $J_{\lambda}$ is the energy intensity of the black-body source at the wave length $\lambda$. Thus $\lambda_{\mathrm{L}}$ is the $\lambda$-coordinate of the center of gravity of the area under the curve obtained by plotting $T_{\lambda} V_{\lambda} J_{\lambda} / \lambda$ versus $\lambda$. This may be computed for a series of temperatures, for any particular red glass, by graphical means, as described elsewhere. ${ }^{51}$ As an example of the variation of $\lambda_{\mathrm{L}}$ with $\vartheta$, the following table for Jena red glass No. 2745, thickness $3.2 \mathrm{~mm}$, is illustrative. The variation is much less with the Corning red glass.

Effective Wave Length of a Red Glass Screen

\begin{tabular}{c|c|}
\hline$\vartheta$ & $\lambda_{\mathbf{L}}$ \\
\cline { 1 - 2 } ○ absolute & $\mu$ \\
1000 & 0.6607 \\
1200 & .6560 \\
1400 & .6532 \\
1600 & .6513 \\
1800 & .6498 \\
2000 & .6487 \\
& \\
\end{tabular}

\footnotetext{
${ }^{50}$ If luminosity in watts $/ \mathrm{cm}^{2}$ is divided by the mechanical equivalent of light, the brightness of the source in candles $/ \mathrm{cm}^{2}$ is obtained.

${ }^{51}$ Foote, B. S. Sci. Papers No. 260.
} 
Analogous to the simple Wien equation strictly applicable only for monochromatic light, the following equation may be written as a definition of the mean effective wave length $\lambda^{\prime}{ }_{\mathrm{L}}$ when an absorption device is employed with the pyrometer using heterochromatic radiation:

$$
\frac{\mathrm{I}}{\vartheta}-\frac{\mathrm{I}}{S}=\frac{\lambda_{\mathrm{L}}^{\prime} \log _{10} T r}{c_{2} \log e}=A(\vartheta)
$$

In this equation $\vartheta$ is the true absolute temperature of the blackbody source, and $S$ is the absolute temperature measured through the absorption device of effective transmission $T r$. The effective transmission of the absorption device having a transmission coefficient $T_{\lambda}{ }^{\prime}=f(\lambda)$ is defined as the ratio of the luminosity of the source viewed through the red glass to the luminosity of the source viewed through the absorption device and the red glass, as follows:

$$
T r=\frac{\int_{0}^{\infty} T T^{1} V J(\lambda, \vartheta) d \lambda}{\int_{0}^{\infty} T V J(\lambda, \vartheta) d \lambda}=\frac{\int_{0}^{\infty} T V J(\lambda, S) d \lambda}{\int_{0}^{\infty} T V J(\lambda, \vartheta) d \lambda}
$$

A definite relation holds between $\lambda_{L}^{\prime}$ and $\lambda_{\mathrm{L}}$ expressed by equation (4)

$$
\frac{\mathrm{I}}{\lambda_{\mathrm{L}}^{\prime}}=\frac{\int_{1 / \mathrm{s}}^{1 / \vartheta^{\prime}} \frac{d\left(\frac{\mathrm{I}}{\vartheta}\right)}{\lambda_{\mathrm{L}}}}{\frac{\mathrm{I}}{\vartheta}-\frac{\mathrm{I}}{\mathrm{S}}}
$$

Whence the reciprocal of the mean effective wave length is equal to the average value of the reciprocal of the effective wave length for the system (red glass + black body) between the limits I/S and $I / \vartheta$. For all red glasses actually used this mean is equivalent within I part in 6000 to Io ooo to the simple mean of the effective wave lengths at two temperatures $\vartheta$ and $S$.

The method of applying the above equations to the use of a sectored disk is briefly as follows: Suppose the transmission coefficient of the disk as determined by a dividing engine is o.OI. Hence from equation (3) $T r=T^{\prime}=$ O.OI. We shall compute the value of $A$ corresponding to the true temperature $2000^{\circ}$ abs. The effective wave length of the special Jena red glass described, for $\vartheta=2000^{\circ}$, is $0.6487 \mu$. This is not the value of $\lambda^{\prime}{ }_{L}$, which is a mean of $\lambda_{\mathrm{L}}$ at $S$ and $\lambda_{\mathrm{L}}$ at $\vartheta$. However, as a first approximation 
let $\lambda_{L}^{\prime}=0.6487$. On substituting equivalent values in equation (2) we obtain:

$$
\begin{gathered}
A=\frac{\mathrm{I}}{2000}-\frac{\mathrm{I}}{S}=\frac{0.6487 \log \mathrm{O} . \mathrm{OI}}{(\mathrm{I} 435 \mathrm{O})(.43429)} \\
\text { or } S=\mathrm{I} 4 \mathrm{I} 2^{\circ} \text { abs. for a first approximation. }
\end{gathered}
$$

By interpolation of the data in the above table we find that for a temperature of $\mathrm{I}_{4} \mathrm{I} 2^{\circ}$ the effective wave length is $\lambda_{\mathrm{L}}=0.653 \mathrm{I} \mu$. The mean of $\lambda_{\mathrm{L}}$ for $\mathrm{I}_{4} \mathrm{I}_{2}{ }^{\circ}$ and $\lambda_{\mathrm{L}}$ for $2000^{\circ}$ is $\lambda_{\mathrm{L}}^{\prime}=0.6509 \mu$. Substituting this value in equation (2):

$$
\begin{aligned}
& A=\frac{\mathrm{I}}{2000}-\frac{\mathrm{I}}{S}=\frac{0.6509 \log 0.0 \mathrm{I}}{(\mathrm{I} 435 \mathrm{O})(.43429)}=-0.0002089 \\
& \text { or } S=\mathrm{I}_{4} \mathrm{II}^{\circ}{ }^{\circ} \text { abs. for a second approximation. }
\end{aligned}
$$

On carrying through higher approximations the value of $S$ (hence $\lambda^{\prime}{ }_{\mathrm{L}}$ ) remains unchanged; thus $A_{2000}=-0.0002089$ as above. The quantity $A$ may be computed in this manner for various temperatures of the source, giving the following illustrative table:

Variation of $A$ with Temperature of Source

\begin{tabular}{|c|c|}
\hline$\vartheta$ & $A$ \\
\hline 0 abs. & \\
1400 & -0.0002105 \\
1600 & -.0002098 \\
1800 & -.0002093 \\
2000 & -.0002089 \\
\hline
\end{tabular}

The calibration of an absorption glass is more difficult, because not only does the mean effective wave length change with the temperature but also the effective transmission $T r$ of equations (2) and (3). The value of $T r$ as a function of $\vartheta$ may be determined photometrically, or the integrals in equation (3) may be evaluated graphically and a curve drawn of $\log T r$ versus $\vartheta$ or $S$. However, the accuracy of such determinations is not sufficient. It is more desirable to measure directly $\vartheta$ and $S$ up to as high a value of $\vartheta$ as is possible without danger of altering the lamp calibration-that is, $\vartheta=1700$ or $1800^{\circ}$ abs. - and to extrapolate the observed curve of $A=\mathrm{I} / \vartheta-\mathrm{I} / S$ versus $\vartheta$, using the same form of curve as that obtained by the graphical computation of $\lambda_{\mathrm{L}}^{\prime} \log T r$. Check observation $\mathrm{s}$ at the higher extrapolated temperatures may be made by comparisons with a calibrated sectored disk.

Foote, Mohler, and Fairchild ${ }^{52}$ have showed that it is possible to obtain an absorption glass for which $A$ is a real constant. Such 
a glass must have a transmission coefficient of the form $T_{\lambda}^{\prime}{ }_{\lambda}=e^{K \lambda}$ where $K / c_{2}=A$.

Absorption glasses are very convenient for precision determinations, but their calibration must be made with exceedingly great care. Moreover, the transmission coefficient of an absorption glass depends to some extent upon the room temperature, thus necessitating further corrections not here considered. Many of these difficulties are avoided by using the more inconvenient sectored disk, but this method is also subject to uncertainties, as, for example, the proper location of the sector in the line of sight when a small source is sighted upon. It is accordingly desirable in precision work, such as the establishing of the high-temperature scale, to employ both methods.

There are other means of standardizing an optical pyrometer. For example, a black body may be maintained at the melting point of palladium and a calibration effected by the use of several sectored disks of measured aperture. A more detailed discussion, however, of this field of pyrometry and of the effective wave length entering into precision determinations of emissivity of nonblack radiators is beyond the scope of the present treatment. ${ }^{53}$

\section{STANDARDIZATION OF RADIATION PYROMETERS}

For precision work the radiation pyrometer must be standardized and used with extreme care. It has been found ${ }^{5 t}$ that many of these instruments are subject to peculiar errors requiring for their elimination rigid specifications in the method of calibration and use.

When a radiation pyrometer is exposed to the radiation from a source at a constant temperature, the instrument does not indicate immediately the temperature of the source, but exhibits a certain time lag during which the receiving system is heating up. Instruments are constructed in which to all practical purposes the equilibrium position is attained in a few seconds, but occasionally one finds receivers for which this is not the case. The readings may continue to increase for ro to 30 minutes, and sometimes increase for a few moments and then decrease appreciably. These effects should be investigated, and, if important,

\footnotetext{
${ }^{63}$ Worthing, Trans. A. I. M. M. E.; 19I9. Forsythe, Trans. A. I. M. M. E.; I9I9. Foote, Fairchild, and Mohler, Absorption Glasses, B. S. Sci. Papers (in preparation).

${ }^{64}$ Burgess and Foote, B. S. Sci. Papers No. 250. $8513^{\circ}-21-18$
} 
the instrument must be calibrated and used with a definite time of exposure.

The most serious error to which radiation pyrometers are subject is that arising from stray radiation. Some of the heat rays from the source are reflected down the walls of the telescope tube, eventually reaching the receiver. The walls near the receiver become warm and reradiate to the couple. The result of such stray radiation is to increase the readings as the size of the source is increased or the sighting distance decreased. Thus Burgess and Foote found for a certain fixed focus pyrometer that the reading at $100 \mathrm{~cm}$ from a large source was but 70 per cent of that obtained when the instrument was mounted close to the source. For a certain focusable pyrometer the reading at $250 \mathrm{~cm}$ distance was $50^{\circ} \mathrm{C}$ different from the reading at $\mathrm{I} O \mathrm{~cm}$ distance. These differences do not arise from the use of a source of insufficient size. In all cases the size of source was greater than that demanded by the geometry of the instrument, as described in the section on radiation pyrometry. In later instruments the construction has been modified to reduce these errors as much as possible. It is, however, advisable to employ approximately the same size of source and sighting distance in standardization and use of radiation pyrometers, or, if this can not be done, the same ratio of diameter of source to sighting distance.

(a) Primary Standardization.-In a primary standardization the radiation pyrometer is sighted into a black body, the temperature of which may be measured by rare-metal thermocouples. Since radiation pyrometers require a much larger source than optical pyrometers, the forms of black body previously described are not suitable. Fig. I6o illustrates one type of black body. The radiator $D$ is a hollow graphite cylinder I 6 by $8 \mathrm{~cm}$, containing three diaphragms. This is mounted in an electrically-heated, platinum-wound, tube furnace 60 by $8 \mathrm{~cm}$, with the back wall of the radiator $40 \mathrm{~cm}$ from the front of the furnace. The thermocouples $E$ and $F$ are protected by highly refractory porcelain tubes glazed on the outside. The furnace is inclined at an angle of about $30^{\circ}$ to minimize air circulation. At $B$ is located a water-cooled diaphragm of the proper opening. With the Fery pyrometer it is advisable to focus on the inmost opening $D$ of the radiator. This requires special attention to insure that the front openings do not cut in on the cone of rays subtended by the pyrometer. The fixed-focus instruments 
require so rarge a source that the front of the instrument must be within a few $\mathrm{cm}$ of the furnace. The diaphragms are accordingly cut with openings increasing in size toward the interior of the furnace, to fit the cone of rays illustrated in Figs. 66 and 68 . Graphite oxidizes rapidly at high temperatures and also contaminates the furnace, so that it is sometimes desirable to have the black body made of refractory porcelain.

(b) Secondary Standardization.-The use of an electric furnace and black body of the type described has certain disadvantages. Several hours are required to obtain satisfactory temperature equilibrium, and the source is too small for many instruments. Once having obtained a primary calibration with a standard radiation pyrometer, instruments of the same type

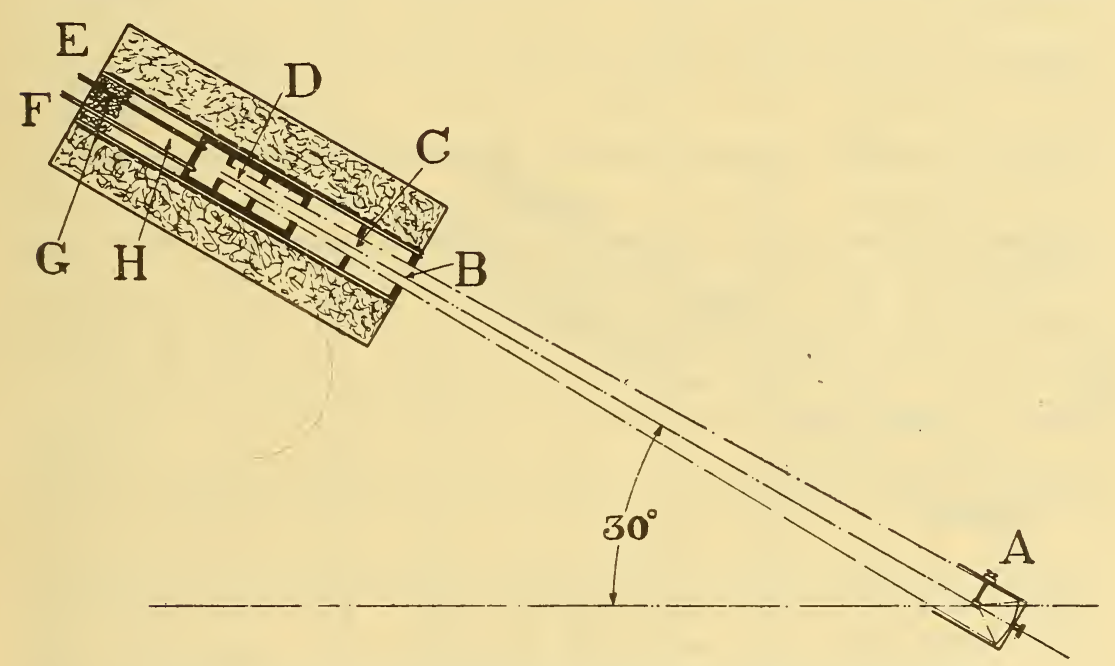

Fig. I60.-Graphite black body and furnace for radiation pyrometers

may be compared by sighting upon a uniformly heated source which is not emitting black-body radiation. A suitable source is obtained by electrically heating sheet nickel in air. A tough coat of nickel oxide forms which does not readily scale, provided the cooling from $500^{\circ} \mathrm{C}$ to room temperature is done slowly. A strip $17 \mathrm{~cm}$ long (exposed section), I $3 \mathrm{~cm}$ wide, and $0.015 \mathrm{~cm}$ thick furnishes a circular area of $\mathrm{I} 2 \mathrm{~cm}$ in diameter which is uniform in temperature to within $2^{\circ}$ at $1200^{\circ} \mathrm{C}$. A blackened water-cooled diaphragm having an opening of the required size is mounted directly in front of the glowing strip, and the pyrometer is sighted upon this opening. Readings are made alternately with the standard instrument and the pyrometer under test. 
(I) Computation of Standardization Data.-The method of computation of observed data is the same with either the primary or secondary standardization. The relation between emf $e$ and the absolute temperature $\vartheta$ is of the form $e=a \vartheta^{\mathrm{b}}$, where $b$ is approximately 4. This must be considered as a purely empirical equation. The fact that its form was suggested by the StefanBoltzman law is incidental, because there are many reasons why the latter law is not strictly applicable. The emf of the couple is not exactly proportional to the difference in temperature of the hot and cold junctions, and the temperature of the hot junction

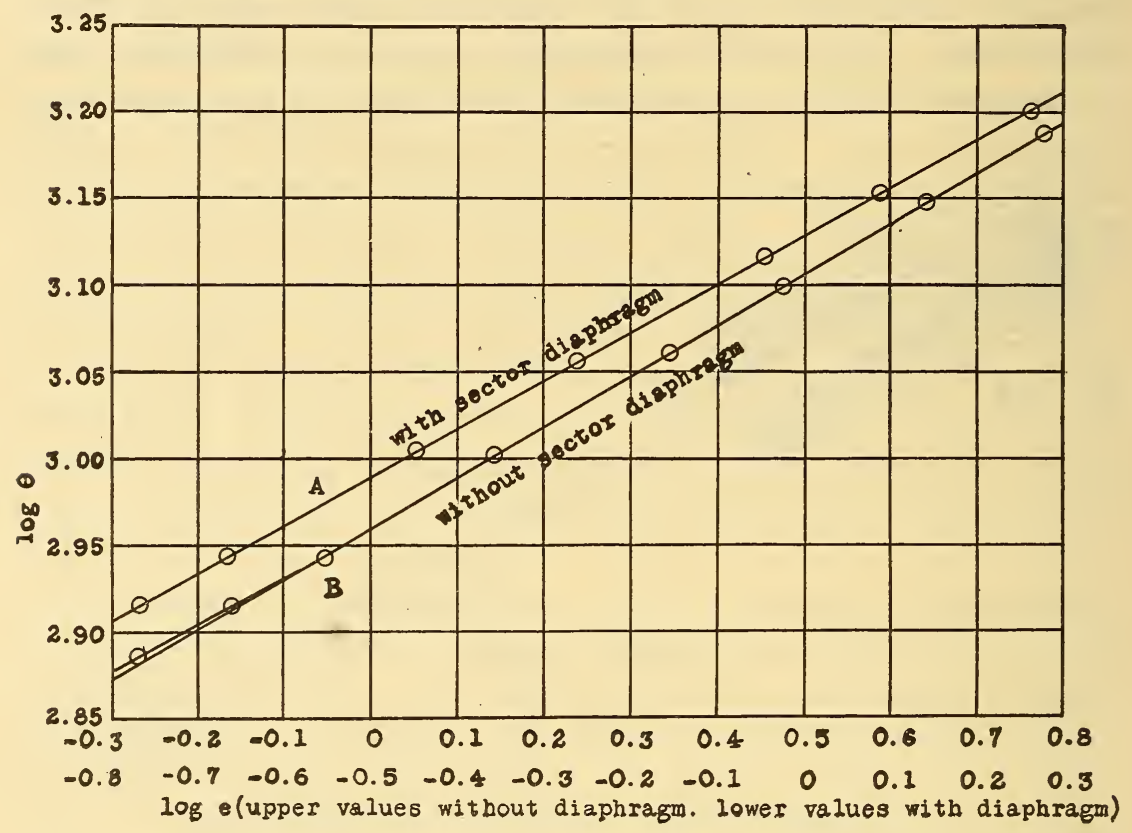

FIG. I6I.-Calibration plot for radiation pyrometer

is not proportional to the rate at which radiation is absorbed. With increasing temperature of the source the temperature of the receiver increases considerably in some of the Fery pyrometers, rising to $100^{\circ} \mathrm{C}$ above that of the room, thus introducing a complicated variation in conduction and convection losses. Furthermore, the total reflection coefficient of the mirrors depends upon the temperature of the source. Hence, the justification of the above equation lies in the fact that it appears to satisfy the observations over a considerable temperature range. The empirical relation has been extended to apply to lower temperatures by the addition of a $\vartheta_{0}$ term, as follows: $e=a\left(\vartheta^{\mathrm{b}}-\vartheta_{\mathrm{o}}^{\mathrm{b}}\right)$; but, in 
general, the simpler form is sufficient for the temperature ranges ordinarily employed. Accordingly, expressed in the logarithmic form, the equation for the radiation pyrometer becomes:

$$
\log e=\log a+b \log \vartheta .
$$

The observed data are plotted $\log e$ versus $\log \vartheta$ and the best straight line is drawn through the points, as shown by Fig. I6r. At the lowest temperatures there may be a slight tendency for the observed points to lie above this line, in which case the data may be given weight by allowing the line to curve a little in this interval.

The sectored diaphragm used for extending the temperature range of the Fery pyrometer should reduce the amount of radiation falling upon the receiver by a constant factor, and hence it might be expected that if $e=a \vartheta^{\mathrm{b}}$ represents the calibration without the diaphragm, $e=K a \vartheta^{\mathrm{b}}$, where $K$ is a constant, would represent the calibration with the diaphragm. Thus, on the log plot the two straight lines would be parallel. Frequently, however, the lines best satisfying the data are not exactly parallel, as shown by Fig. I6r. A table or curve of emf versus temperature is prepared from the values obtained in the above manner.

In work of high precision the emfs are measured with a potentiometer. If a galvanometer graduated in temperature is employed, the following method may be observed in determining the corrections to the scale at the higher range of temperature. Suppose the galvanometer is graduated from 600 to $1300^{\circ} \mathrm{C}$, using the pyrometer without the sector and from 1300 to $2000^{\circ} \mathrm{C}$ with the sector. The highest calibration point is $1400^{\circ} \mathrm{C}$, which is not sufficient to determine directly the scale error in the high range.

Accordingly, the instrument with the sectored diaphragm is calibrated from 600 to $1400^{\circ} \mathrm{C}$, the emf being measured by a potentiometer. The data are plotted $\log e$ versus $\log \vartheta$ and the curve linearly extrapolated to $2000^{\circ} \mathrm{C}$. The galvanometer highrange scale versus emf calibration is made as discussed under Fig. 152, the resistance $x$ being equal to that of the lead wires and couple of the radiation pyrometer. A table is made of true temperature versus emf of the instrument and of temperature scale of the galvanometer versus emf. These are compared at equal emfs, giving directly a table of temperature scale versus true temperature throughout the high range. 


\section{STANDARDIZATION OF RESISTANCE THERMOMETERS}

If an accurately calibrated resistance-measuring instrument is employed, the most precise method of standardizing a pure platinum resistance thermometer is by observations of the resistance at the temperatures of melting ice, steam, and sulphur vapor.

(a) ICE PoInT.-A jar or double-walled vacuum flask about 30 $\mathrm{cm}$ deep is filled with finely divided ice thoroughly wet with distilled water. The ice should reach to the bottom of the flask. The resistance $R_{\circ}$ of the thermometer immersed in this bath is determined.

(b) STEAm PoinT.-The Regnault hypsometer affords a satisfactory steam bath. With slow boiling, except for work of the highest precision, the pressure of the steam may be assumed the same as that of the outside air. The temperature is obtained from the following formula where $p$ denotes the barometric pressure in mm of $\mathrm{Hg}$ :

$$
t^{\circ} \mathrm{C}=100+0.03670(p-760)-0.00002046(p-760)^{2} .
$$

(c) Sulphur BoIling Point.-The following specifications have been prepared by Mueller and Burgess: ${ }^{55}$

All experimental work published has been drawn upon in preparation of these specifications. The specifications relate to apparatus and procedure suitable for the standardization primarily of resistance thermometers and thermocouples, and it was therefore considered permissible to limit the type of apparatus somewhat closely. The aim has been to impose conditions which are sufficient to insure that the thermometer shall assume a definite temperature, dependent only upon the pressure, when placed in the apparatus, and the question as to whether these conditions are really necessary has received only secondary consideration. Thus the question as to the temperature attained in tubes larger or smaller than those specified need not be considered. It may further be supposed that the thermometer or couple will be mounted in a protecting tube or sheath, and that the diameter of such a tube or width of the sheath will be less than $2 \mathrm{~cm}$.

The specifications relate to the following matters: (I) Boiling apparatus, (2) purity of sulphur, (3) radiation shield, (4) procedure, and (5) computations.

(I) Boiling Apparatus.- The boiling tube is of glass, fused silica or similar material, and has an internal diameter of not less than 4 or more than $6 \mathrm{~cm}$. The length must be such that the length of the vapor column measured from the surface of the liquid sulphur to the level of the top of the insulating material surrounding the tube shall exceed the length of the thermometer coil by at least $20 \mathrm{~cm} .{ }^{56}$

Heating is by any suitable heater at the bottom of the tube, and the arrangement must be such that the heating element, and all conducting material in contact with it, terminate at least $4 \mathrm{~cm}$ below the level of the liquid sulphur. If a flame is allowed

55 Mueller and H. A. Burgess, B. S. Sci. Papers No. 339.

56 This length was arrived at as follows: The minimum distance from the liquid surface to the bottom of the shield was taken as $6 \mathrm{~cm}$, excess of length of shield over length of thermometer coil $6 \mathrm{~cm}$, distance available for displacing thermometer $6 \mathrm{~cm}$, and minimum distance from top of shield to level of top of insulation $2 \mathrm{~cm}$. 
to impinge directly on the tube, the heat insulation must extend at least $4 \mathrm{~cm}$ below the level of the liquid sulphur. There should be a ring of insulating material above the heater, fitting the tube closely, to prevent superheating of the vapor by convection currents outside the tube. Above the heater the tube is surrounded with insulating material, not necessarily in contact with it, and of such character as to provide heat insulation equivalent to a thickness of not less than I cm of asbestos. The length of this insulation has already been specified. Any device used to close the top of the boiling tube must allow a free opening for equalization of pressure.

(2) Purity of Sulphur.-The sulphur should contain not over 0.02 per cent of impurities. It should be tested to determine whether selenium is present.

(3) Radiation Shield.-The radiation shield consists of a cylinder open at both ends and provided with a conical umbrella above. The cylindrical part is to be I. 5 to $2.5 \mathrm{~cm}$ larger in diameter than the protecting tube of the thermometer, and at least I $\mathrm{cm}$ smaller than the inside diameter of the boiling tube. The cylinder should extend I. $5 \mathrm{~cm}$ or more beyond the coil at each end. The umbrella should fit the thermometer tube closely, should overhang the cylinder, and be separated from the latter by a space 0.5 to I cm high. The inner surface of the cylinder must be a poor reflector, such as sheet iron, blackened aluminum, asbestos, or a deeply corrugated surface.

(4) Procedure.-The sulphur is brought to boiling ${ }^{57}$ and the heating is so regulated that the condensation line is sharply defined and is I $\mathrm{cm}$ or more above the level of the top of the insulating material. The thermometer, inclosed in its shield, is inserted into the vapor, taking care to have the thermometer coil properly located with respect to the shield, and the thermometer and shield centered in the boiling tube. After putting the thermometer into the vapor time must be allowed for the line of condensation again to reach its proper level. Simultaneous readings of the temperature and barometric pressure are then made. In all cases care should be taken to prove that the temperature is not affected by displacing the thermometer 2 or $3 \mathrm{~cm}$ either up or down from its usual position.

(5) Computations.-Temperatures are calculated from the pressure by use of the formula,

$$
t^{\circ} \mathrm{C}=444 .{ }^{\circ} 60+0.0910(p-760)-0.000049(p-760)^{2} .
$$

If necessary, account should be taken of any difference in pressure between the levels at which the thermometer bulb and the open end of the barometer, respectively, are located. Pressures are to be expressed in the equivalent millimeters of mercury at $\circ^{\circ}$ and under standard gravity $(g=980.665)$.

Fig. 162 shows a gas-heated sulphur boiling-point apparatus and radiation shield. Electrically heated baths, properly constructed, are more satisfactory. ${ }^{58}$

\footnotetext{
${ }^{57}$ If the sulphur has been allowed to solidify in the bottom of the tube, it must be melted from the top downward to avoid breaking the tube. A better procedure is that recommended by Rothe ( $Z$. S. für Instrk., 23, p. 366 ; 1903 ), viz, on completing work with the apparatus it is turned so that the tube makes an angle of $30^{\circ}$ or less with the horizontal, so that the sulphur on soiidifying extends along the sides of the tube, in which position it may be melted down with less danger of breaking the tube. Even when the procedure recommended is followed, breakage of tubes may be reduced by carefully melting the sulphur from the top downward over a bunsen burner before applying heat to it in the apparatus.

58 Mueller and H. A. Burgess, B. S. Sci. Papers No. 339.
} 

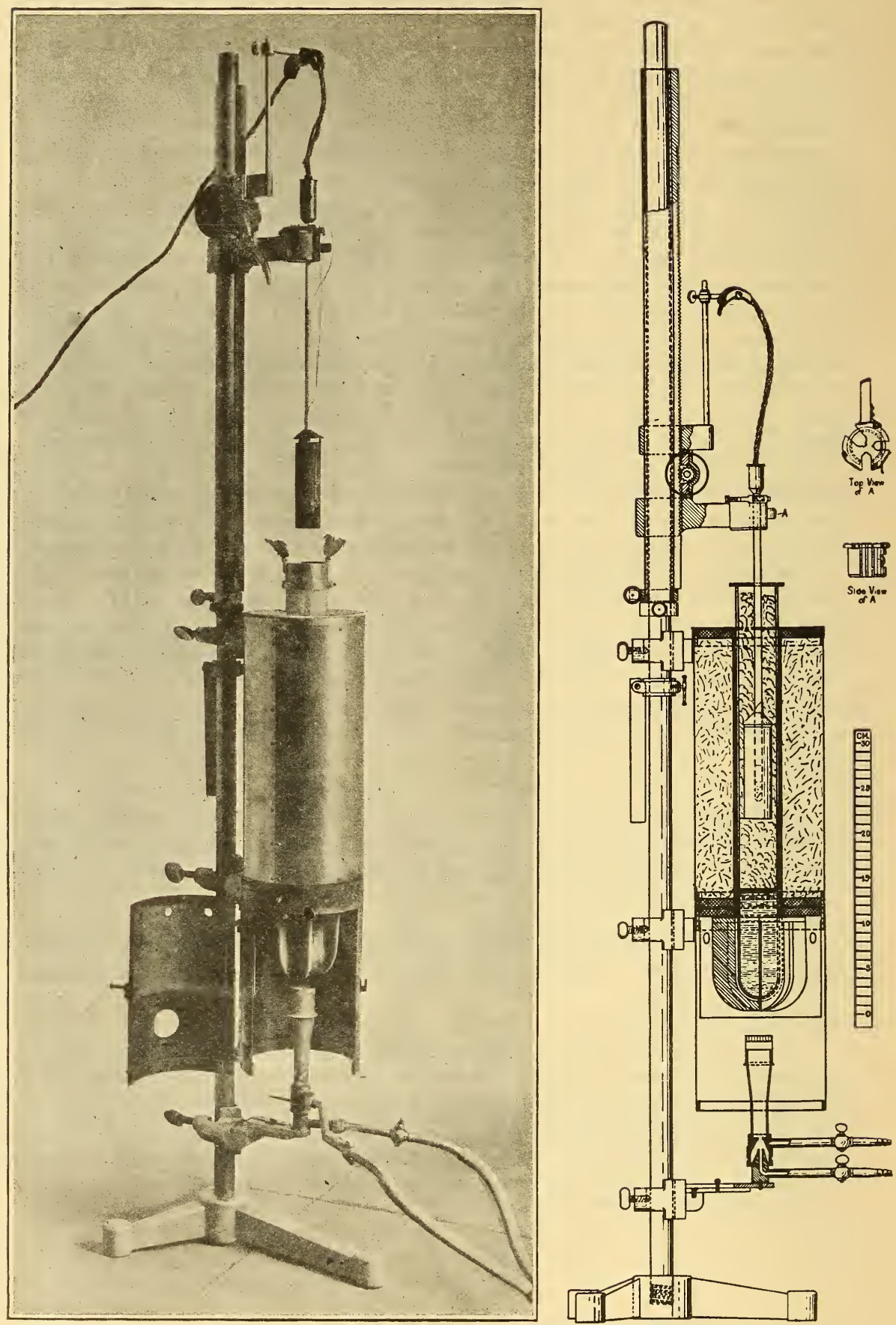

FIG. I62.-Sulphur boiling-point apparatus 
(d) Computation of Data.-The relation between temperature $t$ and resistance $R$ is expressed by the following equations where $p t$ is known as the "platinum temperature" and $\delta$ is a constant of value I.48 to I.52.

$$
\begin{gathered}
t-p t=\delta\left[\left(\frac{t}{\mathrm{IOO}}\right)^{2}-\frac{t}{\mathrm{IOO}}\right] \\
p t=\frac{R-R_{\mathrm{o}}}{R_{100}-R_{\mathrm{o}}} \text { IOO }
\end{gathered}
$$

From the resistance $R_{o}$ at $0^{\circ} \mathrm{C}$ and the observed resistance at the steam point, $R_{100}$ is calculated as follows: From the accompanying table of $t$ versus $p t$, the value of $p t$, corresponding to the actual steam temperature, is obtained. This and the observed resistance $R$ is substituted in the second equation above, which is solved for $R_{100}$.

\begin{tabular}{|r|r|}
\multicolumn{2}{|c|}{ Values of $t$ and $p t$} \\
\hline$t^{\circ} \mathrm{C}$ & \multicolumn{1}{|c|}{$p t$} \\
\hline 99.000 & 99.015 \\
99.400 & 99.409 \\
99.800 & 99.803 \\
100.000 & 100.000 \\
100.200 & 100.197 \\
100.600 & 100.591 \\
101.000 & 100.985 \\
\end{tabular}

The quantity $\delta$ is determined by substituting in the first formula for $t$ and $R$ their respective values at the sulphur-boiling point. The equations may be then computed and a table of $R$ as a function of $t$ prepared. This represents the standard temperature scale by definition, from -40 to $450^{\circ} \mathrm{C}$, and is in agreement with the standard temperature scale up to $1100^{\circ} \mathrm{C}$ as closely as can be determined at the present time.

(e) NICKEL CoILS.-Nickel is occasionally employed for lise below $300^{\circ} \mathrm{C}$. The equation $\log R=a+m t$ holds approximately from o to $300^{\circ} \mathrm{C}$. Two standardization points, not too close together, are required for the determination of the constants $a$ and $m$.

(f) Thermometers with Indicating Instruments.-It is frequently desirable, especially for technical work in the industries, to standardize a thermometer and indicator as a unit. The precision of the ordinary indicator is usually not sufficient to require 
primary standardization of the form described. A secondary method of standardization by comparing with other instruments, thermocouples, etc., in a uniformly heated electric furnace may be employed, the general procedure being that described in the section on standardization of couples. If, however, the laboratory is equipped for primary determinations, the following method is easier and more satisfactory. The relation $R=f(t)$ for the thermometer alone is determined from the ice, steam, and sulphurpoint measurements. The indicator alone is calibrated at several points by connecting, in the place of the thermometer, known resistances and making the usual adjustments as for temperature measurements. These measurements may be accordingly so computed that a table of corrections to the reading of the indicator, when used with the thermometer, is obtained.

Instruments of the type shown in Fig. 77 should be standardized with leads of the same resistance as that employed in the use of the thermometer. Indicators of the type shown in Fig. 74 are calibrated by connecting the resistance standards between the points $d$ and $e$.

Some of the strands of flexible leads may become broken, thus altering the resistance of the line and introducing an error of serious importance in precision measurements. Test for such a fault may be made by interchanging leads $C C^{\prime}$ and $T T^{\prime}$, Fig. 74, or replacing the leads by another set in Fig. 77. Bad contacts and corroded lead wires and soldered junctions, etc., occasion considerable difficulty in industrial installations and must be always guarded against.

Further details on precision standardization of resistance thermometers are given in the papers referred to below. ${ }^{59}$

\section{INDUSTRIAL APPLICATIONS OF PYROMETRY}

In the following paragraphs are considered several specific applications of pyrometry in the technical industries. This treatment is by no means intended to comprehensively review all compiled data in so extended a field, nor are the most important applications necessarily considered. The more or less obvious applications to temperature control of furnaces for annealing, ordinary heat treating, etc., are omitted entirely.

\footnotetext{
${ }_{59}$ Waidner and Burgess, B. S. Sci. Papers No. 124. Mueller and H. A. Burgess, B. S. Sci. Papers No. 339. Mueller, B. S. Sci. Papers No. 288.
} 


\section{GLASS MANUFACTURE}

(a) Temperature Measurements in Glass Melting.-In the manufacture of glasses of lower melting point, such as bottle glasses, it has been customary to estimate the proper working conditions and indirectly the temperature by observing the physical and chemical properties of the mix in different parts of the furnace. Since the complete removal of bubbles, striæ, and chemical impurities in ordinary glassware is not always esserıtial, accurate temperature control has not been considered necessary. There is no question, however, but that the application of pyrometry to the melting of ordinary glass is desirable. For the melting of optical glasses accurate control is one of the most important

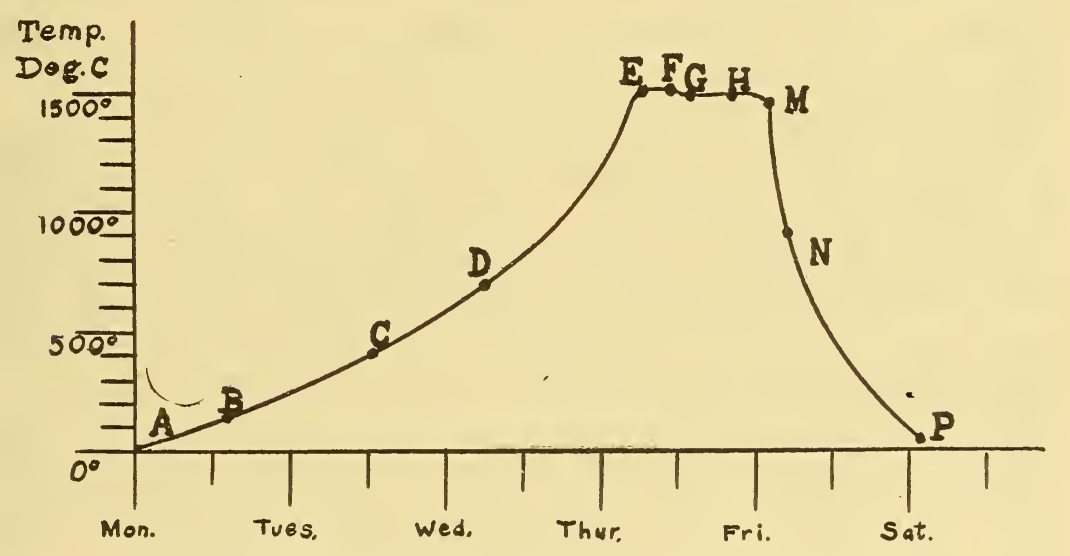

FIG. I63.-Heating and cooling curve of a pot of optical glass

factors affecting the quality of the glass. For example, there is a rather narrow range of temperature above which the increased solution of the pot material results in coloring of the glass and in leakage from the pot, and below which the glass does not fine successfully, but becomes milky and full of bubbles. If the temperatures are not reproduced from time to time the volatilization and solution are not constant, thus resulting in changes of composition and optical properties of the various batches. The proper heat treatment must be determined for each type of glass

Fig. I63 shows a complete heating and cooling of a particular pot of optical glass. ${ }^{60}$ After the clay pot is molded and dried it is fired very slowly to prevent cracking and to a high temperature so that the sintering increases the strength. The firing takes place from $A$ to $F$. From $G$ to $H$ the batch is introduced in several

$60 \mathrm{Keuffel}$, Chicago Pyrometry Symposium. 
charges. From $H$ to $M$ the glass is thoroughly mixed by a clay stirring rod. It is then cooled and the stirring continued to $N$, during which time the bubbles and striæ are removed. At $N$ the glass is so viscous that it can no longer be stirred. From $N$ to $P$ the glass is cooled sufficiently rapidly to break into chunks of the proper size. During the part of the curve represented by GH MN accurate control of the temperature is most essential.

On account of the flame in a tank furnace, a thermocouple placed at any point above the glass indicates the temperature of the hot gases surrounding it and not that of the glass. In such positions, pyrometers may often serve for general furnace control. The large differences in temperature in the molten glass and the crude process of tank-melting make it questionable whether a measure of the temperature of a particular portion of the glass affords any serviceable indication of temperature conditions. A thermocouple placed at the working end, especially in one of the arrangements used for removing glass at a definite rate, is valuable in regulating the temperature, and hence the viscosity of this portion of the glass. But the outstanding difficulty at present is the corrosion and short life of the thermocouple-protecting tubes when placed in contact with glass or even the furnace atmosphere. The destruction of the pyrometer may be avoided by sighting an optical pyrometer into a closed-end tube inserted into the glass through a diagonal hole cut in the wall just above the glass surface. The life of a tube used with an optical pyrometer is much longer than when employed as a protection to a couple.

The readings of an optical pyrometer sighted directly on the glass surface in a tank furnace are unreliable on account of the reflection of light from the flames. However, fairly satisfactory black-body conditions are obtained by sighting on the intersection of the glass surface and the opposite wall. Another method is to float a refractory block on the glass close to the wall and sight into the angle formed by the wall and the block. These methods may be checked by sighting into a closed tube.

The temperatures in pot furnaces are more uniform, and reliable measurements may be made by sighting the optical pyrometer into the pot or in some cases on the back or side walls of the furnace. Covered or hooded pots afford almost ideal black-body conditions. In general, in order to obtain the best temperature reproducibility the optical pyrometer should be used in exactly the same manner from time to time; that is, it should be sighted on the same portion of the furnace or pot. 
In some glass pot and tank furnaces rare-metal couples inserted in the wall, and often not projecting entirely through into the interior, have been employed. The advantage of such an installation lies in the fact that recorders may be used and a record of the entire temperature cycle is obtained. Even though the temperature indicated is not that of the glass, sufficient control is possible, and, if necessary, the departure of the thermocouple temperature from that of the glass may be determined by the optical pyrometer. However, the continued use of rare-metal couples in a glass furnace is very costly on account of deterioration.

(b) General Principles of Annealing.-The object of annealing is to remove the strain in glass, thus minimizing the danger of breakage, and in addition, for optical glass, to make the material optically homogeneous. Annealing is accomplished by heating the glass to a uniform temperature at which it is sufficiently soft to allow the strains to relax in a short time, and then cooling uniformly to room temperature and slowly enough so that no appreciable strains are introduced. The factors which should be known are the maximum allowable rate of heating, the annealing temperature, the time the glass is to be maintained at this temperature, and the temperature-time curve of cooling.

For each type of glass there exists a short temperature range, at the lower limit of which a considerable time is required for relieving stresses and at the upper limit the stresses vanish very rapidly. The upper limit is characterized by several phenomena, such as the rapid deformation of strips or rods of the glass under load, the rapid disappearance of the strain figures and colors revealed by polarized light, a thermal transformation marked on heating by an absorption of heat, and by a sudden increase in thermal expansion. While the deformation of the glass at the upper limit is rapid under load, the glass is by no means soft enough to produce a rounding of corners or a deformation of shape under its own weight in the time required for annealing.

Table 29 shows the temperatures of several optical glasses, made by the Bureau of Standards, at which the heat absorption and rapid change in thermal expansion take place on heating. For rapid removal of stresses temperatures 20 or $30^{\circ}$ higher may be used, but in general it is preferable to anneal at a temperature about $20^{\circ}$ lower, so that the cooling may be more rapid without introducing new strains. 
TABLE 29.-Annealing Temperature of Optical Glasses Made by the Bureau of Standards

\begin{tabular}{|c|c|c|c|}
\hline Glass & Temperature & Glass & Temperature \\
\hline Dense flint.. & $\begin{array}{l}{ }^{\circ} \mathrm{C} \\
460\end{array}$ & Light crown. & $\begin{array}{l}{ }^{\circ} \mathrm{C} \\
495\end{array}$ \\
\hline Medium flint.. & 455 & Borosilicate crown... & 515 \\
\hline Light flint.. & 485 & Light barium crown. & 575 \\
\hline Barium flint.. & 520 & & \\
\hline
\end{tabular}

The glass is held at some definite temperature within the annealing range until the strain has been sufficiently reduced and is then cooled at a rate depending upon the kind of glass, nature of the ware, dimensions, etc. Although the lower the annealing temperature the shorter the time required to cool, this gain is offset by the longer time necessary to reduce the strain at the annealing temperature. The optimum annealing temperature and rate of cooling may be determined empirically by examining for strain samples of glass cooled according to definite procedures. Without seriously increasing the strain in the finished glass the rate of cooling may be doubled for every $10^{\circ} \mathrm{C}$ decrease in temperature until it reaches a value consistent with a factor of safety from breakage, when it should be constant. The rate should be approximately inversely proportional to the square of the thickness of the glass. During annealing the surface of the glass must have a uniform temperature; otherwise new strains are introduced. ${ }^{61}$

(c) Temperature Measurements in Annealing Ovens.-The measurement of temperature in annealing ovens should serve the purpose of determining the exact temperature of the glass and the uniformity of heating. A decrease of about $10^{\circ} \mathrm{C}$ in the annealing temperature requires about twice the time for the same degree of annealing. Hence comparatively greater precision and sensitiveness of temperature measurement, especially with optical glass, is required in glass annealing than in ordinary annealing processes. The temperature should be known to better than $5^{\circ} \mathrm{C}$, and the sensitivity should be $\mathrm{I}$ or $2^{\circ} \mathrm{C}$. A portable potentiometer or a potentiometer recorder and a sensitive thermocouple afford the best equipment. As the temperature usually remains below $650^{\circ} \mathrm{C}$, a base-metal couple such as iron-constantan or preferably chromel-alumel in a calorized iron protecting tube is satisfactory.

The couples should be so located that the true temperature of the glass is being measured. Flames or cold blasts of air should

${ }^{61}$ For a detailed account of methods of annealing see Tool and Valasek, B. S. Sci. Papers No. 358. 
not come in contact with the couple or the glass. In order to ascertain the uniformity of heating a number of couples, depending on the size of the oven or kiln, should be located at various positions. In a lehr a minimum of two couples should be installed, one near the hot end and the other near the cold end, mounted down through the arch. A recorder is especially desirable in glass annealing, since improper temperature regulation at any time during the cooling may introduce serious strains, and the record chart shows whether or not the entire process has been carried out according to specification.

\section{ROTARY PORTLAND-CEMENT KILNS}

The high heat loss in cement kilns may be considerably reduced by close adherence to temperature specifications; experimentally determined under best working conditions, and the uniformity of the product may be improved by a suitable pyrometric equipment.

For controlling kiln operation a pyrometer installed in the rear end is most useful. It will indicate (or record) heat losses and kiln conditions such as draft, feed, fuel supply, rings, shutdowns, and under normal conditions the slow rise or fall of average temperature in the kiln.

The pyrometer best suited for this installation consists of a chromel-alumel thermocouple protected in a chromel or nichrome tube and connected to the ordinary indicator or recorder, which must be carefully protected from dust. The couple is inserted through the rear housing with its hot junction near the axis of the kiln and I or 2 feet within the kiln. A 6 or 7 foot couple will usually suffice. It should be kept away from the feed pipe in order to avoid the cooling action and the bridge of caked dust which forms. This cake should be removed at frequent intervals and at a regular hour, for its removal will change the indicated temperature.

In dry-process plants temperatures of $600^{\circ}$ to occasionally as high as $\mathrm{I} 100^{\circ} \mathrm{C}$ are obtained. In wet-process plants any thermocouple has a long life and requires little attention because of the low temperature existing in the rear end.

A thermocouple installed in the kiln stack will serve, but with somewhat less satisfaction, particularly when the leakage of cold air into the housing is unsteady. However with the shorter kilns the increase in life of the couple obtained by removing it to the stack may be an economic advantage. 
For obtaining data on the temperature of the clinkering zone, which are of greatest value during radical changes of operation such as a new fuel or raw mix, the optical pyrometer alone is suitable. A thermocouple inserted through the kiln wall with its hot junction flush with the inner face is a possibility, but the method is attended with many difficulties and uncertainties; for example, the connection to the recorder must be made through two collector rings.

Temperatures in the clinkering zone range from i 200 to $1500^{\circ} \mathrm{C}$, depending upon the composition of the raw material, length of kiln, and length of hot zone. The clinkering zone is heated uniformly over a few feet only, and measurements should be taken at the hottest portion.

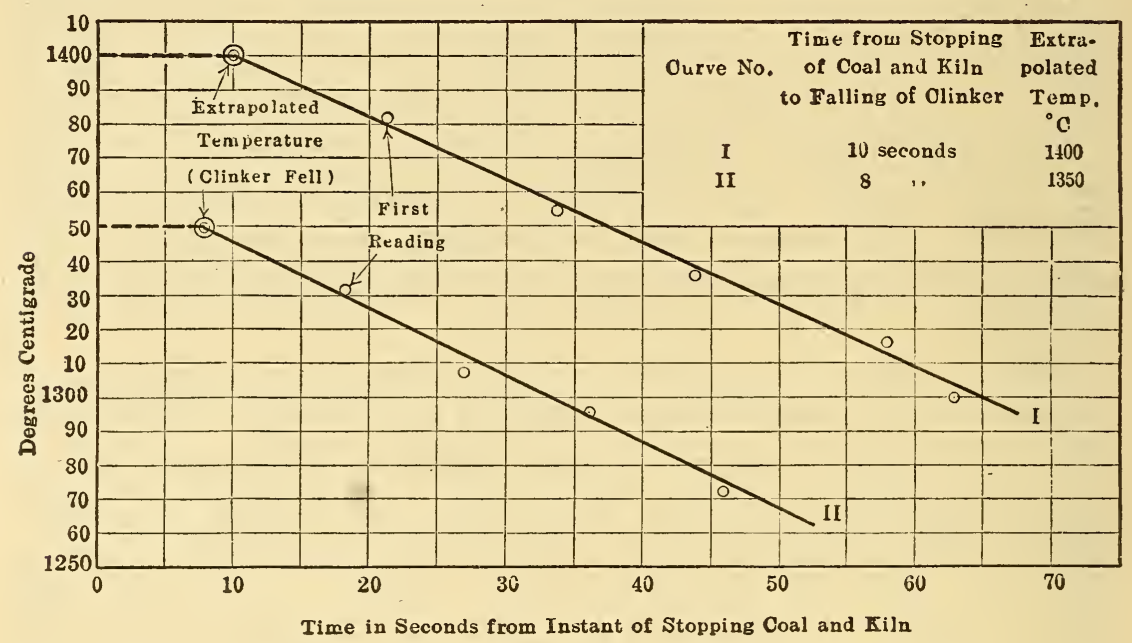

FIG. I64.-Typical extrapolation plot of temperature measurements on a rotary cement kiln

These measurements are made with difficulty, and only a long series (taken, for example, every 3 o minutes for 8 hours) is of value, as a single observation may be in gross error. The difficulties encountered are due to the presence of dust during operation and the high intrinsic brilliancy of the flame. Hence, in order to obtain useful measurements, it is necessary to stop the kiln and shut off the fuel and air. The optical pyrometer should be mounted in front of the kiln and sighted through an observation hole upon a spot on the inner wall which is uncovered as a clinker falls away. This spot is in the lower right-hand quadrant of the kiln, rotation being counterclockwise, facing the kiln. Upon stopping the kiln the clinker will soon fall, exposing the inner wall. It is not feasible 
to sight on the mottled surface of the clinker, which cools with great rapidity as soon as the flame is cut off. The inner wall, however, does not cool materially until the clinker falls, and it presents a fairly uniform surface upon which to sight. On account of the great roughness of this surface and the surrounding hot walls, the apparent emissivity is high enough to make it unnecessary to apply a correction. A series of observations may be obtained within $I^{1} / 2$ minutes, after waiting 5 to $\mathrm{I}_{5}$ seconds for the atmosphere to clear. Four or five readings should be made as rapidly as possible (every Io seconds), the time of each reading being determined by a stop watch. These readings, plotted as in Fig. I64, show the temperature at the moment the clinker fell. A reading taken within 5 seconds from the time the clinker fell is not more than $30^{\circ}$ lower than the temperature at zero time. The extrapolation, however, increases the accuracy if the observations are properly timed. ${ }^{62}$

\section{CERAMIC INDUSTRIES}

A brief historical review of the progress of pyrometry in the ceramic industries is of interest because at present there are in use both the most modern forms of temperature-measuring devices and the very old forms. Among the oldest of these is Wedgewood's contraction pyroscope. The principle of this method is still used in the present-day "Veritas firing system." "63 An improvement over Wedgewood's original pyroscope was announced in I 886 by Seger, who had constructed a series of fusible cones intended to indicate temperature intervals of $25^{\circ}$ between 600 and $1800^{\circ} \mathrm{C}$. Such cones are used in large quantities at present. They are known in this country as Seger cones, standard pyrometric cones, or Orton cones. They consist of small tetrahedra molded from various mixtures of kaolin, feldspar, lime, magnesia, quartz, iron oxide, lead oxide, and boric acid. Cones of higher number in the series have been developed to extend the range to $2000^{\circ}$ (pure $\mathrm{Al}_{2} \mathrm{O}_{3}$ melts at $2050^{\circ}$ ). These are made by adding progressively greater amounts of alumina to kaolin. It is well known at present that the melting points of these cones do not correspond to definite temperatures. ${ }^{64}$ However, the cone numbers have always been associated with temperature, the temper-

62 Dana and Fairchild, Chicago Pyrometry Symposium.

63 Cf. B. S. Tech. Papers No. 40: r9r 4.

64 B. S. Tech. Papers Nos. I 7 and 40. Sosman, Trans. Am. Ceram. Soc. 15, p. 482: rgr3. Bureau of Mines Bull. No. 129; 1918.

$8513^{\circ}-21-19$ 
atures given being in general higher than the final maturing temperature attained in a kiln heated to the corresponding cone number. The effect of time on the softening points of some cones is shown in Table 30, and the effects of oxidizing and reducing atmospheres is indicated in Table $3 \mathrm{I}$. These effects are greater and more irregular for cones melting in the lower ranges because of the admixtures of reducible and of volatile fluxes. The fact that the effects of time and of kiln gases on the softening of cones are somewhat similar to the effects of these variables on the progress of firing the ware is assigned as the reason for the successful and continued use of Seger cones. They do not give, however, a true measure of either of these effects, for they are never of the same material as the ware being fired. There is an advantage in favor of Veritas firing rings in this regard, since the latter may be made of the same mixture as that of the ware. Even in this case, however, there are differences readily apparent to the ceramist.

Neither Seger cones nor the firing rings are satisfactory gages of the efficiency obtained in firing, nor can they be used during drying, water smoking, or cooling. These faults have aided in the development and use of indicating and recording pyrometers. Nevertheless, the employment of cones will probably continue for many years, since they are inexpensive and can be placed at inaccessible points in a kiln as a check on the variation of heat conditions. Also. their use requires a minimum of technical knowledge and training of the burner.

The application of pyrometers to processes in the ceramic industry has been retarded by the great complexity of the phenomena accompanying the vitrification of ceramic materials and the resultant difficulty in correlating temperature measurements with other variables, some of which are difficult to control or are unknown. It is just this condition which we believe will finally compel ceramists to depend entirely on pyrometers, especially recorders, to determine proper rates of firing and the best finishing temperatures. The pyrometer gives at least a true indication of temperature and temperature variation with time.

The types of pyrometers available for employment by the ceramic engineer are (I) thermocouple (preferably platinum, platinum-rhodium), (2) optical pyrometer, and (3) radiation pyrometer. There is, of course, a great variety of pyrometers and thermometers which can be used for the drying operation and 
the low temperatures employed in enameling and decorating. The instruments are all described in detail in other sections.

(a) Installations.-In kilns fired between the temperatures 600 and about $1450^{\circ} \mathrm{C}$ the platinum, platinum-rhodium thermocouple is the most useful for recording the temperatures. This thermocouple protected with a porcelain tube and an extra fireclay or other outer tube is found to give satisfactory indications when the tip of the protection tube is extended into the kiln 3 to 4 inches beyond the inner face of the wall. Below $\mathrm{r} 300^{\circ} \mathrm{C}$ it is better to immerse the couple at least 6 inches, provided a sufficiently refractory tube is used. Above $145^{\circ} \mathrm{C}$ adequate protection is very difficult and the general practice is to withdraw the couple and tubes until the tip is just flush with the inner face of the wall or even within the wall. In such a position obviously the lag is very great, and also the indicated temperature will be considerably below the correct value. Base-metal thermocouples, which can not be used above $1200^{\circ} \mathrm{C}$, are more easily protected and should be inserted 6 inches or more beyond the inner wall. The best position to install the couple depends upon the type of kiln and its peculiar characteristics. Generally the couple is inserted through the roof, both in intermittent kilns and in continuous kilns of the tunnel and chamber týpes. In large kilns proportionately more couples are required. For example, in the tunnel kiln a couple installed every 20 feet of kiln length is advantageous. One of these should be in the hottest zone. Others should be opposite combustion chambers or grates, so that separate firing units may be controlled. In more complicated kilns, arranged to preheat air, to furnish heat for drying, etc., other couples are required. At present many manufacturers must do their own experimental work to determine the best points for installations. Portable outfits for this purpose are furnished by all pyrometer manufacturers.

The recording instrument is indispensable, and its great advantage over indicators is especially well recognized by ceramists. The most obvious advantage lies in the ability of the recorder to indicate plainly the occurrence of "setbacks" or otherwise the steady rise in temperature of intermittent kilns. This use alone is sufficient cause for the employment of pyrometers rather than cones. Multiple-point recorders are best for tunnel kilns and such intermittent kilns as require more than one couple. This recorder may confuse the records of a number of small kilns, making it desirable to use a simple recorder for each kiln. 
Radiation pyrometers are of value when thermocouples can not be used for recording temperatures. Their use is discussed in detail in the section on radiation pyrometers.

The optical pyrometer, particularly the portable form, is useful in experimental work and may be employed to great advantage for routine adjustment of kilns to a uniform temperature. It logically replaces cones used for this purpose. "True" temperatures are easily obtained, and an exploration is made more quickly and accurately with this instrument than by observing the condition of cones. A fire-clay tube of $\mathrm{I}$ inch inside diameter inserted through the kiln wall and extending 6 to 12 inches within forms a very convenient means of measuring temperatures optically; otherwise readings may be obtained by sighting through a small opening onto the ware.

TABLE 30.-Softening Points of Cones, ${ }^{\circ} \mathrm{C} a$

\begin{tabular}{|c|c|c|c|c|c|c|c|c|c|}
\hline \multirow{2}{*}{ Cone } & \multicolumn{4}{|c|}{ Rate of heating ${ }^{\circ} \mathrm{C} /$ hour } & \multirow{2}{*}{ Cone } & \multicolumn{4}{|c|}{ Rate of heating ${ }^{\circ} \mathrm{C} /$ hour } \\
\hline & 42.5 & 27.5 & 20.0 & .12 .5 & & 42.5 & 27.5 & 20.0 & 12.5 \\
\hline $010 .$. & 885 & 885 & 880 & 910 & $1 \ldots$ & 1100 & 1085 & 1090 & 1070 \\
\hline $09 \ldots$ & 920 & 930 & 950 & 890 & 2. & 1110 & 1090 & 1100 & 1075 \\
\hline $08 \ldots \ldots$ & 950 & 970 & 970 & 965 & $3 \ldots \ldots$ & 1120 & 1110 & 1110 & 1080 \\
\hline $07 \ldots \ldots$. & 960 & 975 & 950 & 970 & $4 \ldots \ldots$ & 1130 & 1125 & 1115 & 1090 \\
\hline $06 \ldots$ & 990 & 1000 & 990 & 995 & $5 \ldots$ & 1140 & 1135 & 1125 & 1110 \\
\hline $05 \ldots$ & 1015 & 1035 & 1025 & 1030 & $6 \ldots$ & 1170 & 1140 & 1135 & ..... \\
\hline $04 \ldots \ldots$. & 1040 & 1055 & 1040 & 1045 & $7 \ldots .$. & 1185 & 1155 & 1140 & 1125 \\
\hline $03 \ldots . .$. & 1055 & 1065 & 1050 & 1050 & $8 \ldots \ldots$ & 1200 & 1170 & 1160 & 1150 \\
\hline $02 \ldots \ldots$ & 1065 & 1070 & 1065 & 1060 & $9 \ldots$ & 1230 & 1190 & 1180 & 1190 \\
\hline $01 \ldots$ & 1080 & 1080 & 1080 & 1070 & & & & & \\
\hline
\end{tabular}

$a$ Brown and Murray, B. S. Tech. Papers No. I7.

TABLE 31.-Softening Temperatures, ${ }^{\circ} \mathrm{C}$, of Cones in Various Atmospheres $a$

\begin{tabular}{|c|c|c|c|c|c|c|}
\hline \multirow[b]{2}{*}{ Cone No. } & \multirow{2}{*}{$\begin{array}{l}\text { Per cent of } \\
\mathrm{Fe}_{2} \mathrm{O}_{3} \text { in } \\
\text { cone formula }\end{array}$} & \multicolumn{3}{|c|}{ Softening temperatures in- } & \multirow{2}{*}{$\begin{array}{c}\text { Differences } \\
\mathrm{H}_{2}-\mathrm{H}_{2} \mathrm{O} \\
\text { and } \\
\mathrm{CO}-\mathrm{CO}_{2} \\
\text { atmospheres }\end{array}$} & \multirow[b]{2}{*}{$\begin{array}{c}\mathrm{H}_{2}-\mathrm{H}_{2} \mathrm{O} \\
\text { and air }\end{array}$} \\
\hline & & $\begin{array}{c}\text { Atmosphere } \\
\text { of equal } \\
\text { parts of } \mathrm{H}_{2} \\
\text { and } \mathrm{H}_{2} \mathrm{O}\end{array}$ & $\begin{array}{c}\text { Atmosphere } \\
\text { of equal } \\
\text { parts of } \mathrm{CO} \\
\text { and } \mathrm{CO}_{2}\end{array}$ & Air & & \\
\hline $06 \ldots \ldots \ldots$. & 8.52 & 1018 & 1026 & 1040 & +8 & +22 \\
\hline $04 \ldots \ldots \ldots . . .$. & 8.54 & 1089 & 1062 & 1085 & -27 & -4 \\
\hline $02 \ldots \ldots \ldots$ & 8.57 & 1119 & 1119 & 1178 & 0 & +59 \\
\hline $1 \ldots \ldots \ldots \ldots$ & 8. 58 & 1146 & 1164 & 1182 & -18 & +36 \\
\hline $2 \ldots \ldots \ldots \ldots$ & 4. 38 & 1165 & 1170 & 1181 & +5 & +16 \\
\hline $4 \ldots \ldots \ldots \ldots$ & None & 1221 & 1240 & 1250 & +19 & +29 \\
\hline $6 \ldots \ldots \ldots$ & None & 1281 & 1292 & 1286 & +11 & +5 \\
\hline $8 \ldots \ldots \ldots \ldots$ & None & 1332 & 1330 & 1366 & -2 & +34 \\
\hline $10 \ldots \ldots \ldots$. & None & 1358 & 1340 & 1334 & -18 & -24 \\
\hline $12 \ldots \ldots \ldots \ldots$ & None & 1358 & 1370 & 1367 & +12 & $\dot{+} \bar{y}$ \\
\hline
\end{tabular}

a Fieldner, Hall, and Field, Bur. Mines Bull. No. I29: I9r8. 


\section{BY-PRODUCT COKE INDUSTRY}

The measurement of the temperatures in and about a byproduct coke oven is attended with special difficulties ar ising from inaccessibility of parts of the battery. Measurements may be taken at some points with comparative ease. If the construction engineer is informed regarding the desirability of making temperature measurements, he can readily provide the battery with openings for the insertion of thermocouples and for the use of the optical pyrometer:

(a) LOCATIONS FOR INSTALLING THERMOCOUPLES.-Measurements may be profitably made at the following points, which are usually accessible:

I. Waste-heat flue or tunnel at the end of the battery, and possibly on each side of the battery.

2. Heating walls (and recuperator walls) in the combustion flues or passages.

3. Regenerators, either individual or otherwise. The hot ends are usually inaccessible, but can very well be made accessible at some points.

4. Ascension or offtake pipes from the ovens.

5. In vapors and in coal in the ovens.

An inaccessible point, and one where probably more useful information can be obtained than at any other, is near the surface of the oven wall next to the coal. It is possible and wholly practicable to provide one or two ovens in a battery with holes in the walls about $3 \mathrm{~cm}$ in diameter and extending in about 2 meters from either the pusher side or coke side. Thermocouple protecting tubes are available which will not flux with silica brick below $1300^{\circ} \mathrm{C}$, a temperature never intentionally reached at this point.

Thermocouples are commonly used only at point No. I above, but other installations have occasionally been made during experimental work. For properly controlling a battery the wasteheat thermoelectric pyrometer, and an optical pyrometer for measuring heating-wall temperatures are indispensable.

The thermocouples best suited for coke-oven work are the platinum, platinum-rhodium couples, and the chromel-alumel couples. For use in the vapors above the coal in the oven in the offtake pipe, and also in the waste-heat tunnel (under regenerative firing), any of the common types of base-metal couples excepting copper constantan are suitable. For permanent installation the 
platinum couples should be of wire 0.5 or $0.6 \mathrm{~mm}$ in diameter, protected in glazed porcelain tubes of "Usalite" or "Impervite." The inner insulating tubes should be of the same material unglazed. This porcelain is easily cracked by sudden heating, and the successful insertion of the couple into a hot furnace is difficult to accomplish. There is no certainty of success without the use of a watch and a protection tube marked each centimeter of its length, so that the insertion may be made slowly and steadily. Ordinary tubes (walls 2 to $3 \mathrm{~mm}$ thick) may be thrust into a flue

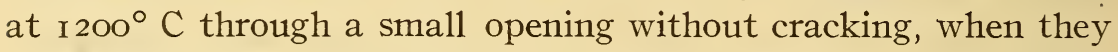
are inserted at a rate of about $5 \mathrm{~cm}$ per minute. Withdrawal should be made at a rate not greatly exceeding this, and a hot tube should not be allowed to touch cold objects other than poor conductors. of heat, such as asbestos.

The waste-heat tunnel pyrometer should be installed as near the battery as possible and connected to a single-point recorder placed conveniently for the burner and foreman. Special attention should be given the cold junction of the couple. This should be buried underground or placed well above the tunnel roof where the temperature is not greatly influenced by the heat from the flue. Under regenerative firing. waste gases are in the temperature range 200 to $350^{\circ} \mathrm{C}$ and vary $100^{\circ}$ or less during reversal. The recorder should have a total scale range of o to $500^{\circ} \mathrm{C}$. Records need not be made more frequently than once in two minutes; but, if it is desirable to connect this couple to a multiple-point recorder, more than one point on the recorder should be used, so that records are made at least every five minutes. A good thermocouple properly installed at this location will probably last for a number of years without serious deterioration.

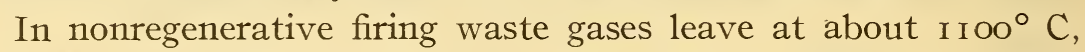
and a well-protected platinum couple must be used. It is best to employ the tubes mentioned above with an additional outer tube of fire clay, unglazed porcelain, etc., to protect the glazed porcelain from corrosion by the dust-laden gases. Fused quartz can not be used here.

(b) Measurements with the Optical Pyrometer.-An instrument with a scale range from 700 to $1400^{\circ} \mathrm{C}$ is required. A more extended range will seldom be used, since a temperature of $\mathrm{I} 400^{\circ} \mathrm{C}$ in the battery is dangerous. Heating wall temperatures can be easily measured in batteries having observation ports above each wall. The temperatures obtained by sighting on the nozzle 
brick at the base of heating wall flues have a very remote connection with oven temperatures, but such measurements are of great utility for controlling the battery. Observations should be made in a flue in which there is no flame and when the nozzle opening is plainly visible. Attempts to obtain useful information by observations made on other points in the flue will generally be attended with failure because of the lack of black-body conditions, particularly in the upper part of the flue. However, temperature gradients across the battery may be adjusted by such observations.

Measurements with an optical pyrometer may be made at various other points in a battery where the temperature is over $7 \mathrm{OO}^{\circ} \mathrm{C}$. It is difficult, but sometimes possible, to obtain a fairly good value for the temperature of the coke in the oven just before pushing the charge. This can be done only in ovens in which the top layer of coal is coked well enough to make it possible to clear the oven of smoke by removing a lid and opening the offtake pipe. Observations upon the coke during pushing as it emerges from the oven are practically impossible because of the very rapid cooling of the coke and the large clouds of smoke and dust which arise.

Observations made upon the oven walls after pushing are subject to very large errors on account of lack of black-body conditions, refraction of light by strong convection currents of air, and especially by the variation in emissivity of the oven face with changing layers of adhering coke.

(c) Experimental Measurements in Oven Proper.-There remains to be mentioned briefly the methods of employing couples within the coal charge during coking. One of the writers has successfully made a large number of such measurements, employing chromel-alumel thermocouples insulated with fire-clay nipples and protected in ordinary wrought-iron pipes. The latter were carefully inspected to see that there were no open seams, and before using they were thoroughly cleaned inside with dilute acid and dried. Such couples quickly deteriorate and the protection tubes must be removed after each charge. The maximum temperature attained is about $\mathrm{I} \mathrm{IOO}^{\circ} \mathrm{C}$, and carbonization is rapid. The couples can be used for two charges. These thermocouples are inserted through holes in the oven lids, preferably in the center of the charge (midway between walls). Insertion can be made easily for a number of hours after charging, since the center of the charge remains at $100^{\circ} \mathrm{C}$ for half the coking period or longer. The heads of the couples must be very strong, as con- 
siderable force is required in withdrawing the couples just before pushing. They can not be turned because the soft iron at $1100^{\circ}$ will collapse.

Measurements in the coal are difficult, expensive, and have seldom been attempted. A discussion of the time-temperature relation obtained is beyond the scope of this paper. These measurements are more properly made in small laboratory furnaces and ovens, the opportunity afforded for research of practical utility being very promising.

\section{STEEL MANUFACTURE}

(a) BLAST FURNACE.-Temperature control of blast furnaces by pyrometric methods is in the experimental stage. Measurements with the optical pyrometer obtained by sighting through a glass window into a tuyère show temperatures from $1600^{\circ}$ to above $1850^{\circ} \mathrm{C}$. During tapping the temperature of the metal in the ordinary iron furnace varies from $\mathrm{I} 43 \mathrm{O}$ to $1475^{\circ} \mathrm{C}$ and that of the slag from $\mathrm{I} 43 \mathrm{O}$ to $\mathrm{I} 55^{\circ} \mathrm{C}$. The method of observing these latter temperatures is described below.

(b) Bessemer Converter.- - It is questionable whether the use of pyrometers will ever prove fruitful in the control of this process. Observations with the optical pyrometer on the apparent temperature of the flame are of little value. The temperature of the steel after blowing may be measured with the optical pyrometer by sighting on the liquid metal surface when the converter is tilted, and no corrections for emissivity are necessary, as approximate black-body conditions exist. The temperature during pouring may be obtained as described later.

(c) Open-Hearth Furnace-(I) Dome.-The temperature of the dome may be measured with the optical pyrometer by sighting on the inner surface of the brick, care being taken to avoid flames and fume. This is best done during reversals or at times when the flames are the least visible. The roof temperature varies from 1550 to $170^{\circ} \mathrm{C}$; but by means of pyrometric measurements the temperature may be maintained as close as possible to I $710^{\circ} \mathrm{C}$, the melting point of the best silica brick.

(2) Slag Pocket.-Base-metal couples installed in the slag pocket assist in maintaining a proper supply of heat.

(3) Slag Surface. - The temperature of the slag surface in the furnace may be measured with the optical pyrometer, no correction for emissivity being necessary if the furnace temperature is fairly uniform. Care must be taken to avoid sighting through 
brilliant flames. Slag temperatures are as much as $150^{\circ} \mathrm{C}$ above the temperature of the metal during heating. When the fire is shut off, the slag cools rapidly and may be $75^{\circ} \mathrm{C}$ below that of the metal just before tapping.

(4) Metal Bath.-Measurements of the temperature of the metal within the furnace have not been as yet reduced to routine practice, although much investigational work has been done. ${ }^{65}$ One method is to immerse an Acheson graphite rod, block, or tube through the slag into the metal until it has attained temperature equilibrium and then raise the graphite above the slag surface and immediately observe its temperature with the optical pyrometer. The slag does not adhere, and no correction for emissivity is necessary. The method may be modified by removing the graphite quickly and observing the cooling curve in air and extrapolating the curve to the time at which the graphite was withdrawn from the bath. A similar method may be employed in spooning a sample of the metal, the optical pyrometer being sighted on the clear liquid surface. In this case the corrections for emissivity given in Table 16 should be made.

A few successful observations have been obtained by immersing a special refractory tube into the metal and sighting into the inside of the tube with the optical pyrometer. The tube was made of steel tipped with an Acheson graphite tube and protected by a fire-clay sleeve. Precautions must be taken to remove the smoke from the line of sight. All of these methods are inconvenient, but afford about the only means so far devised for obtaining the temperature of the metal before tapping.

(d) Tapping and TeEming.- The temperature of the clear metal in tapping and teeming may be easily and accurately measured by the optical pyrometer. The observer should stand from to to 20 feet from the stream and avoid sighting upon the slag, which appears as bright patches on the clear metal surface. Corrections for emissivity are applied as shown in Table 16. This table gives also the emissivity corrections for slag, temperature measurements of which are occasionally desirable.

(e) Rolling, ETC.-The oxide formed on the surface of iron or steel heated in air readily scales and does not adhere well to the metal. This results in a film of air between the two surfaces and a considerable lowering of the outside temperature. It is accordingly necessary to remove the loosely adhering oxide before making a temperature measurement. In many processes, such as

${ }^{65}$ Cf. Chicago Pyrometry Symposium. 
rolling, this is done automatically, and the optical pyrometer may be sighted directly upon the outside surface. This method has proved especially advantageous for steel rails while passing through the rolls. ${ }^{66}$ Thus in four rail mills it was found that the ingot temperatures vary from 1075 to $\mathrm{II}_{5} \mathrm{O}^{\circ} \mathrm{C}$, and the finishing temperatures of the rails from 880 to $1050^{\circ} \mathrm{C}$. The measurements are capable of an accuracy of $10^{\circ} \mathrm{C}$, so that close temperature specifications may be employed and greater uniformity of the product secured. The corrections for emissivity are usually negligible, but are given in Table 16 .

The temperatures measured by the optical pyrometer are for the outside surface of the thin coat of oxide adhering after the flaky oxide is chipped off. There still exists a considerable temperature gradient through the remaining film. The relation between the temperatures of outside and inside surfaces of the oxide is apparently fairly well given by the following table. ${ }^{67}$

Temperatures, Degrees Ce
\begin{tabular}{|c|c|}
\hline $\begin{array}{c}\text { Outside } \\
\text { of oxide } \\
\text { layer }\end{array}$ & $\begin{array}{c}\text { Inside } \\
\text { of oxide } \\
\text { layer }\end{array}$ \\
\hline 600 & 610 \\
700 & 715 \\
800 & 815 \\
900 & 930 \\
1000 & 1080 \\
\hline
\end{tabular}

\section{TEMPERATURES OF METAL SURFACES}

The measurement of temperatures of metal surfaces is of importance in many investigational problems. If the surface is accessible and above a red heat, the optical pyrometer may be employed, corrections for emissivity being applied to the readings. At lower temperatures the radiation pyrometer might be employed, but, in general, it is unsatisfactory below $600^{\circ} \mathrm{C}$. The most suitable method, especially for steam pipes, surfaces of stoves, etc., is by use of thermocouples. A special form of mounting must be made to reduce to a minimum the effect of heat conduction along the couple. Two small holes are drilled into the surface to a convenient depth and the bare end of each wire of the couple is peened into the separate holes. The ordinary welded junction is accordingly not employed, the circuit being closed through the metal surface. If the holes are drilled sufficiently close together that no serious temperature difference exists at the two points,

${ }^{66}$ Burgess, Rawdon, and Waltenberg, B. S. Tech. Papers, No. ${ }^{8}$.

${ }^{67}$ Burgess and Foote, B. S. S 2i. Papers No. 249. 
the introduction of a short length of iron, as a steam pipe, produces no effect. The temperature measured is that of the metal surface surrounding the point where the couple wires are peened, and if the wires are sufficiently small compared to the size of the surface the temperature of which is required, the conduction loss is negligible. This method has been employed in the measurement of heat transmission through boiler tubes, ${ }^{68}$ and by this Bureau successfully for temperature measurements of steel girders embedded in concrete and subjected to fire. For problems of the latter type it affords about the only means for assuring that the temperature of the metal itself is being observed.

\section{CONCLUSION}

The rapid development in the application of pyrometry to the technical industries has been responsible in no small measure for the remarkable advancements characterizing all industrial processes requiring heat control.

The history of the application of pyrometric methods to technical processes dates from several centuries, but the major development has been effected in the past 20 years, and the rate of development is increasing.

A decade ago the word pyrometry required definition for the average being. Few, if any, technical schools considered the subject in their curricula. To-day pyrometric engineering is a profession, and one which promises unlimited opportunity.

With this'recent growth of the industrial applications of pyrometry it is not surprising that the subject of pyrometry itself should have made rapid strides. New instruments are devised, new methods perfected, until at present a large pyrometric installation may be as extensive and intricate as a central telephone station.

This treatise has contained nothing of the historical, and the purely scientific aspects of pyrometry have been intentionally subordinated. It purports to describe practical methods in use in the industries for the year 1920. No doubt much will be antiquated in another half decade.

In conclusion, the writers desire to express their appreciation for the many valuable criticisms offered by their colleagues, especially Messrs. Waidner and Mueller, during the preparation of the manuscript.

Washington, February i I, I 920.

${ }^{68}$ Kreisinger and Barkley, Bur. Mines Tech. Paper No. II4. 


\section{APPENDIX}

\section{CONTENTS}

TABLE I.-Conversion table for degrees centigrade to degrees Fahrenheit (L. Page.

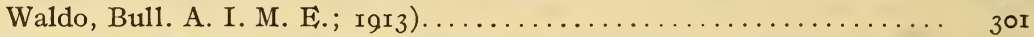

TABLE II.-Conversion table for degrees Fahrenheit to degrees centigrade ( $\mathrm{L}_{\text {. }}$. Waldo, Bull. A. I. M. E.; I913)....................... 302

TABLE III.-Logarithms of tangents to tenth degree for use with Wanner pyrometers..................................... 304

TABLE IV.-Calibration data of representative couples............... 306

Fig. 165.-Chart showing various thermoelectric data................ 307

TABLE V. -Data of Geophysical Laboratory for Pt-9o Pt-1o Rh Thermocouple. 308

TABLE VI.-Data of Geophysical Laboratory for Copper-Constantan Thermocouple........................................ 309

Fig. I66 to Fig. 185.-Modern Pyrometric Installations................ 310 
TABLE I.-Conversion Table Degrees Centigrade to Degrees Fahrenheit

\begin{tabular}{|c|c|c|c|c|c|c|c|c|c|c|c|c|}
\hline$c^{\circ}$ & 0 & 10 & 20 & 30 & 40 & 60 & 60 & 70 & 80 & 90 & & \\
\hline $\begin{array}{r}-200 \\
-100 \\
-0 \\
\end{array}$ & $\begin{array}{c}F \\
-328 \\
-148 \\
+32 \\
\end{array}$ & $\begin{array}{c}F \\
-346 \\
-166 \\
+14 \\
\end{array}$ & $\begin{array}{c}F \\
-364 \\
-184 \\
-4 \\
\end{array}$ & $\begin{array}{c}F \\
-382 \\
-202 \\
-22 \\
\end{array}$ & $\begin{array}{c}\mathbf{F} \\
-400 \\
-220 \\
-40 \\
\end{array}$ & $\begin{array}{c}F \\
-418 \\
-238 \\
-58 \\
\end{array}$ & $\begin{array}{c}F \\
-436 \\
-256 \\
-76\end{array}$ & $\begin{array}{c}F \\
-454 \\
-274 \\
-94\end{array}$ & $\begin{array}{c}F \\
\cdots \ldots \\
-292 \\
-112\end{array}$ & $\begin{array}{c}F \\
\ldots \ldots \\
-310 \\
-130\end{array}$ & & \\
\hline 0 & 32 & 50 & 68 & 86 & 104 & 122 & 140 & 158 & 176 & 194 & & \\
\hline $\begin{array}{l}100 \\
200\end{array}$ & $\begin{array}{l}212 \\
392\end{array}$ & $\begin{array}{l}230 \\
410\end{array}$ & $\begin{array}{l}248 \\
428\end{array}$ & $\begin{array}{l}266 \\
446\end{array}$ & $\begin{array}{l}284 \\
464\end{array}$ & $\begin{array}{l}302 \\
482\end{array}$ & $\begin{array}{l}320 \\
500\end{array}$ & $\begin{array}{l}338 \\
518\end{array}$ & $\begin{array}{l}356 \\
536\end{array}$ & $\begin{array}{l}374 \\
554\end{array}$ & $c^{\circ}$ & $F^{\circ}$ \\
\hline 300 & 572 & 590 & 608 & 626 & 644 & 662 & 680 & 698 & 716 & 734 & $\begin{array}{l}1 \\
2\end{array}$ & $\begin{array}{l}1.8 \\
3.6\end{array}$ \\
\hline $\begin{array}{l}400 \\
500\end{array}$ & $\begin{array}{l}752 \\
932\end{array}$ & $\begin{array}{l}770 \\
950\end{array}$ & $\begin{array}{l}788 \\
968\end{array}$ & $\begin{array}{l}806 \\
986\end{array}$ & $\begin{array}{r}824 \\
1004\end{array}$ & $\begin{array}{r}842 \\
1022\end{array}$ & $\begin{array}{r}860 \\
1040\end{array}$ & $\begin{array}{r}878 \\
1058\end{array}$ & $\begin{array}{r}896 \\
1076\end{array}$ & $\begin{array}{r}914 \\
1094\end{array}$ & 3 & 5.4 \\
\hline 600 & 1112 & 1130 & 1148 & 1166 & 1184 & 1202 & 1220 & 1238 & 1256 & 1274 & $\begin{array}{l}4 \\
5\end{array}$ & $\begin{array}{l}7.2 \\
9.0\end{array}$ \\
\hline $\begin{array}{l}700 \\
800\end{array}$ & $\begin{array}{l}1292 \\
1472\end{array}$ & $\begin{array}{l}1310 \\
1490\end{array}$ & $\begin{array}{l}1328 \\
1508\end{array}$ & 1346 & $\begin{array}{l}1364 \\
1544\end{array}$ & $\begin{array}{l}1382 \\
1562\end{array}$ & $\begin{array}{l}1400 \\
1580\end{array}$ & $\begin{array}{l}1418 \\
1598\end{array}$ & $\begin{array}{l}1436 \\
1616\end{array}$ & $\begin{array}{l}1454 \\
1634\end{array}$ & 6 & 10.8 \\
\hline $\begin{array}{l}800 \\
900\end{array}$ & $\begin{array}{l}1472 \\
1652\end{array}$ & $\begin{array}{l}1490 \\
1670\end{array}$ & $\begin{array}{l}1508 \\
1688\end{array}$ & $\begin{array}{l}1526 \\
1706\end{array}$ & $\begin{array}{l}1544 \\
1724\end{array}$ & $\begin{array}{l}1562 \\
1742\end{array}$ & $\begin{array}{l}1580 \\
1760\end{array}$ & $\begin{array}{l}1598 \\
1778\end{array}$ & $\begin{array}{l}1616 \\
1796\end{array}$ & $\begin{array}{l}1634 \\
1814\end{array}$ & 7 & 12.6 \\
\hline 1000 & 1832 & 1850 & 1868 & 1886 & 1904 & 1922 & 1940 & 1958 & 1976 & 1994 & 9 & 16.2 \\
\hline 1100 & 2012 & 2030 & 2048 & 2066 & 2084 & 2102 & 2120 & 2138 & 2156 & 2174 & 10 & 18.0 \\
\hline 1200 & 2192 & 2210 & 2228 & 2246 & 2264 & 2282 & 2300 & 2318 & 2336 & 2354 & & \\
\hline 1300 & 2372 & 2390 & 2408 & 2426 & 2444 & 2462 & 2480 & 2498 & 2516 & 2534 & & \\
\hline 1400 & 2552 & 2570 & 2588 & 2606 & 2624 & 2642 & 2660 & $26 \div 8$ & 2696 & 2714 & & \\
\hline 1600 & 2732 & 2750 & 2768 & 2786 & 2804 & 2822 & 2840 & 28.58 & 2876 & 2894 & & \\
\hline 1600 & 2912 & 2930 & 2948 & 2966 & 2984 & 3002 & 3020 & 3038 & 3056 & 3074 & $E^{\circ}$ & $\mathrm{c}^{\circ}$ \\
\hline 1700 & 3092 & 3110 & 3128 & 3146 & 3164 & 3182 & 3200 & 3218 & 3236 & 3254 & 1 & .56 \\
\hline 1800 & 3272 & 3290 & 3308 & 3326 & 3344 & 3362 & 3380 & 3398 & 3416 & 3434 & 2 & 1.11 \\
\hline 1900 & 3452 & 3470 & 3488 & 3506 & 3524 & 3542 & 3560 & 3578 & 3596 & 3614 & 3 & 1.67 \\
\hline 2000 & 3632 & 3650 & 3668 & 3686 & $37 \cup 4$ & 3722 & 3740 & 3758 & 3776 & 3794 & 4 & 2.22 \\
\hline 2100 & 3812 & 3830 & 3848 & 3866 & 3884 & 3902 & 3920 & 3938 & 3956 & 3974 & 6 & 3.33 \\
\hline 2200 & 3992 & 4010 & 4028 & 4046 & 4064 & 4082 & 4100 & 4118 & 4136 & 4154 & & \\
\hline 2300 & 4172 & 4190 & 4208 & 4226 & 4244 & 4262 & 4280 & 4298 & 4316 & 4334 & $\begin{array}{l}7 \\
8\end{array}$ & $\begin{array}{l}3.89 \\
4.44\end{array}$ \\
\hline 2400 & 4352 & 4370 & 4388 & 4406 & 4424 & 4442 & 4460 & 4478 & 4496 & 4514 & 9 & 5.00 \\
\hline 2500 & 4532 & 4550 & 4568 & 4586 & 4604 & 4622 & 4640 & 4658 & 4676 & 4694 & & \\
\hline 2600 & 4712 & 4730 & 4748 & 4766 & 4784 & 4802 & 4820 & 4838 & 4856 & 4874 & $\begin{array}{l}10 \\
11\end{array}$ & $\begin{array}{l}5.56 \\
6.11\end{array}$ \\
\hline 2700 & 4892 & 4910 & 4928 & 4946 & 4964 & 4982 & 5000 & 5018 & 5036 & 5054 & 12 & 6.67 \\
\hline 2800 & 5072 & 5090 & 5108 & 5126 & 5144 & 5162 & 5180 & 5198 & 5216 & 5234 & & \\
\hline 2900 & 5252 & 5270 & 5288 & 5306 & 5324 & 5342 & 5360 & 5378 & 5396 & 5414 & $\begin{array}{l}13 \\
14\end{array}$ & $\begin{array}{l}7.22 \\
7.78\end{array}$ \\
\hline 3000 & 5432 & 5450 & 5468 & 5486 & 5504 & 5522 & 5540 & 5558 & 5576 & 5594 & 15 & 8.33 \\
\hline $\mathbf{3 1 0 0}$ & 561 & 56 & 56 & 5 & 56 & 5 & 57 & 5733 & 756 & 5774 & 16 & 8.89 \\
\hline 3200 & 5792 & 5810 & 5828 & 5846 & 5864 & 5882 & 5900 & 5918 & 5936 & 5954 & 17 & 9.44 \\
\hline 8300 & 5972 & 5990 & 6008 & 6026 & 6044 & 6062 & 6080 & 6098 & 6116 & 6134 & 18 & 10.00 \\
\hline 8400 & 6152 & 6170 & 6188 & 6206 & 6224 & 6242 & 6260 & 6278 & 6296 & 6314 & & \\
\hline 3500 & 6332 & 6350 & $62: 68$ & 6386 & 6404 & $\begin{array}{l}6422 \\
6602\end{array}$ & $\begin{array}{l}6440 \\
6620\end{array}$ & $\begin{array}{l}6458 \\
6638\end{array}$ & $\begin{array}{l}6476 \\
6656\end{array}$ & $\begin{array}{l}6494 \\
66744\end{array}$ & & \\
\hline 8600 & 6512 & 6530 & 6548 & 6566 & 6584 & 6602 & 0020 & 6638 & 6656 & 6674 & & \\
\hline $\begin{array}{l}3700 \\
\mathbf{3 8 0 0}\end{array}$ & 6692 & 6710 & 67 & 6746 & 6764 & 678 & 6800 & 6818 & 6836 & 6854 & & \\
\hline $\begin{array}{l}8800 \\
8900\end{array}$ & $\begin{array}{l}6872 \\
7052\end{array}$ & $\begin{array}{l}6890 \\
7070\end{array}$ & $\begin{array}{l}6908 \\
7088\end{array}$ & $\begin{array}{l}6926 \\
7106\end{array}$ & $\begin{array}{l}6944 \\
7124\end{array}$ & $\begin{array}{l}6962 \\
7142\end{array}$ & $\begin{array}{l}6980 \\
7160\end{array}$ & $\begin{array}{l}6998 \\
7178\end{array}$ & $\begin{array}{l}7016 \\
7196\end{array}$ & $\begin{array}{l}7034 \\
7214\end{array}$ & & \\
\hline $\mathrm{c}^{\circ}$ & 0 & 10 & 20 & 30 & 40 & 50 & 60 & 70 & 80 & 90 & & \\
\hline
\end{tabular}

ExumpLes: $1347^{\circ} \mathrm{C} .=2444^{\circ} \mathrm{F} .+12.6^{\circ} \mathrm{F} .=2456.6^{\circ} \mathrm{F} . \quad 3367^{\circ} \mathrm{F} .=1850^{\circ} \mathrm{C} .+2.78^{\circ} \mathrm{C} .=1852.78^{\circ} \mathrm{C}$ 
TABLE II.-Conversion Table Degrees Fahrenheit to Degrees Centigrade

(Dots denote recurring decimals)

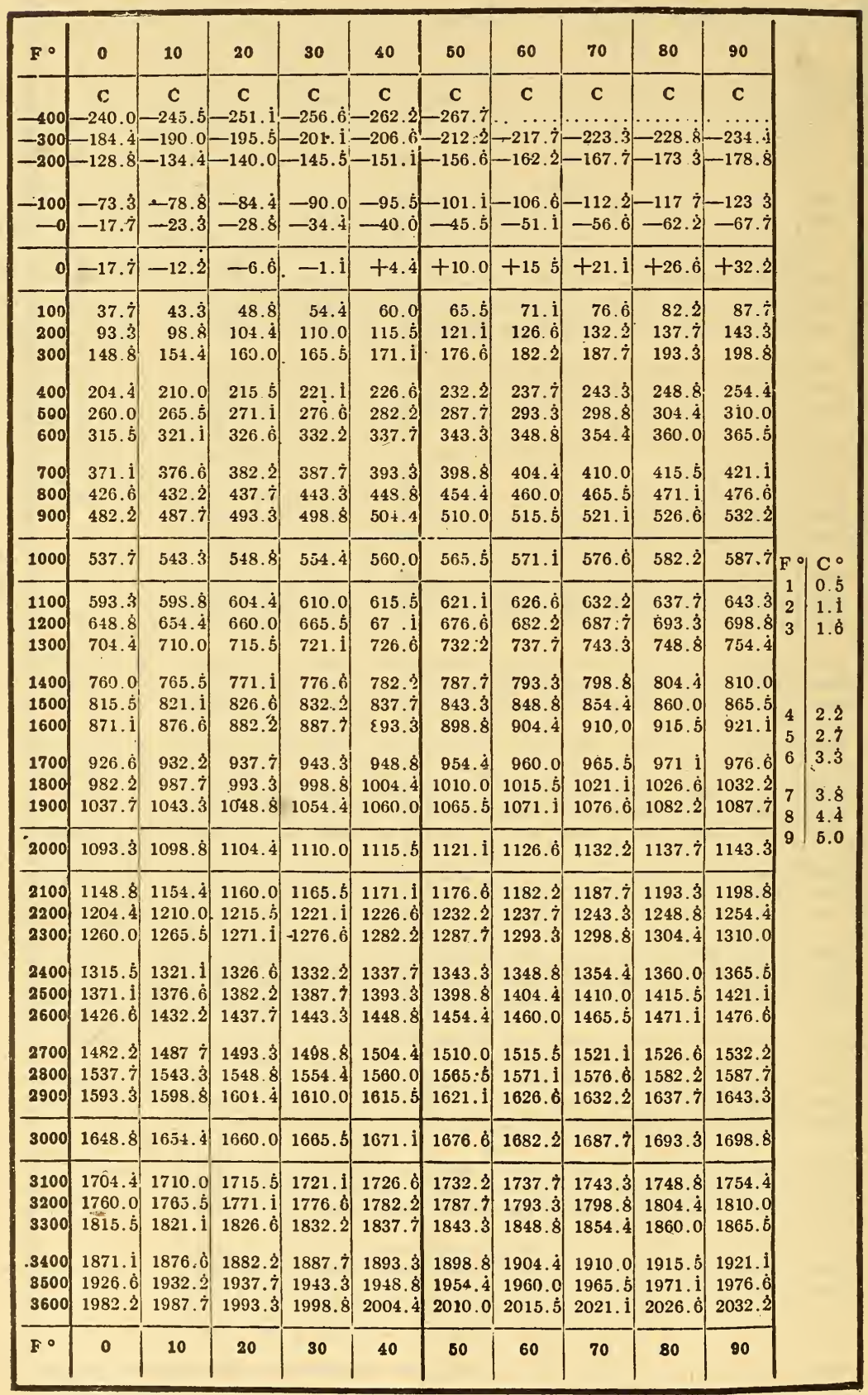

Examples: $-246.0^{\circ} \mathrm{F} .=-151.11^{\circ} \mathrm{C} .-3.33^{\circ} \mathrm{C} .=-154.44^{\circ} \mathrm{C}$.

$3762^{\circ} \mathrm{F} .=2071.11^{\circ} \mathrm{C} .+1.11^{\circ} \mathrm{C} .=2072.22^{\circ} \mathrm{C}$.

$2423.5^{\circ} \mathrm{F} .=1326.666^{\circ} \mathrm{C} .+1.666^{\circ} \mathrm{C} .+0.277^{\circ} \mathrm{C} .=1328.609^{\circ} \mathrm{C}$. 
TABLE II-Continued

${ }^{\circ} \mathrm{F}$ to ${ }^{\circ} \mathrm{C}$

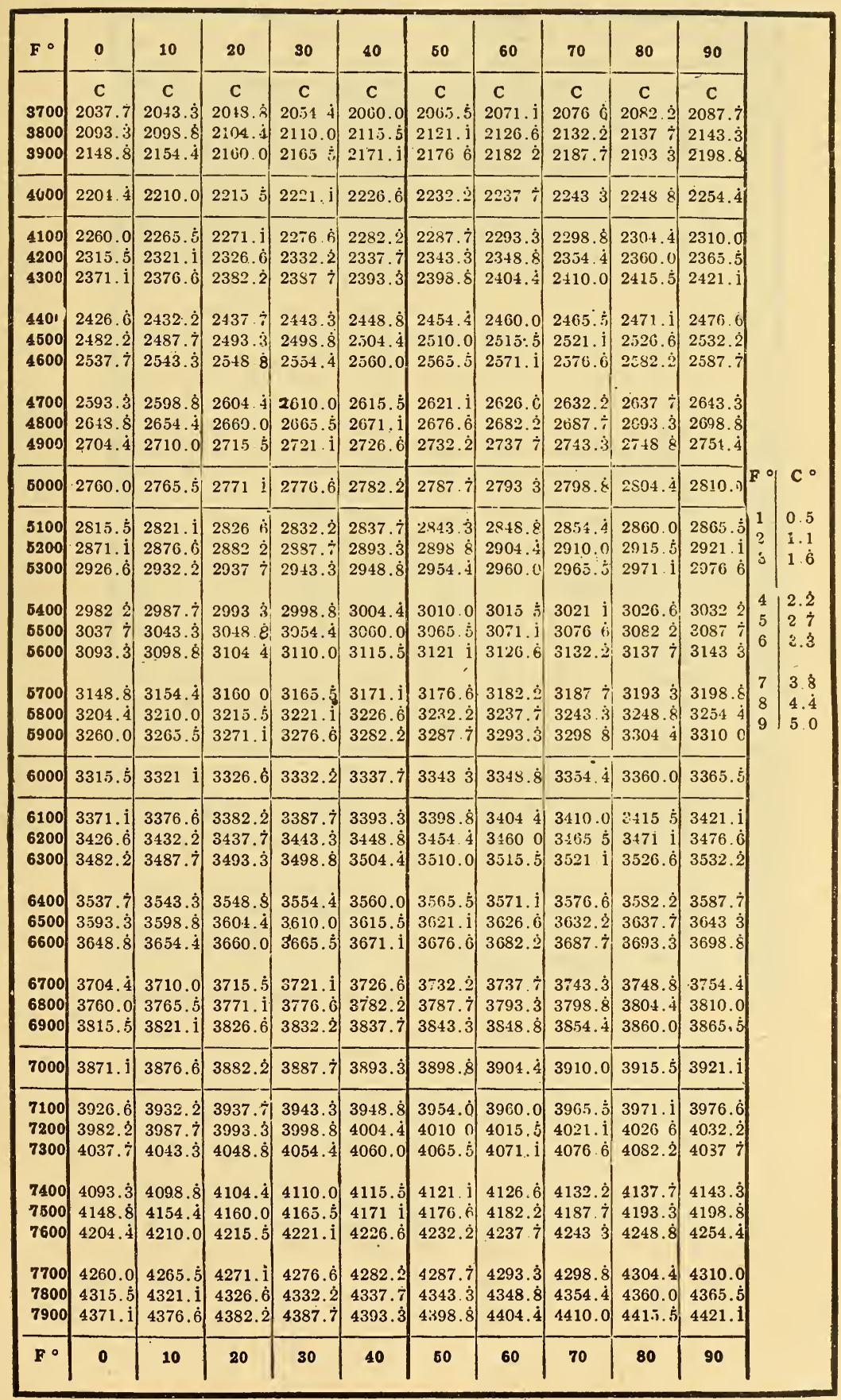


TABLE III.-Logarithms of Tangents

(For use with Wanner Pyrometers)

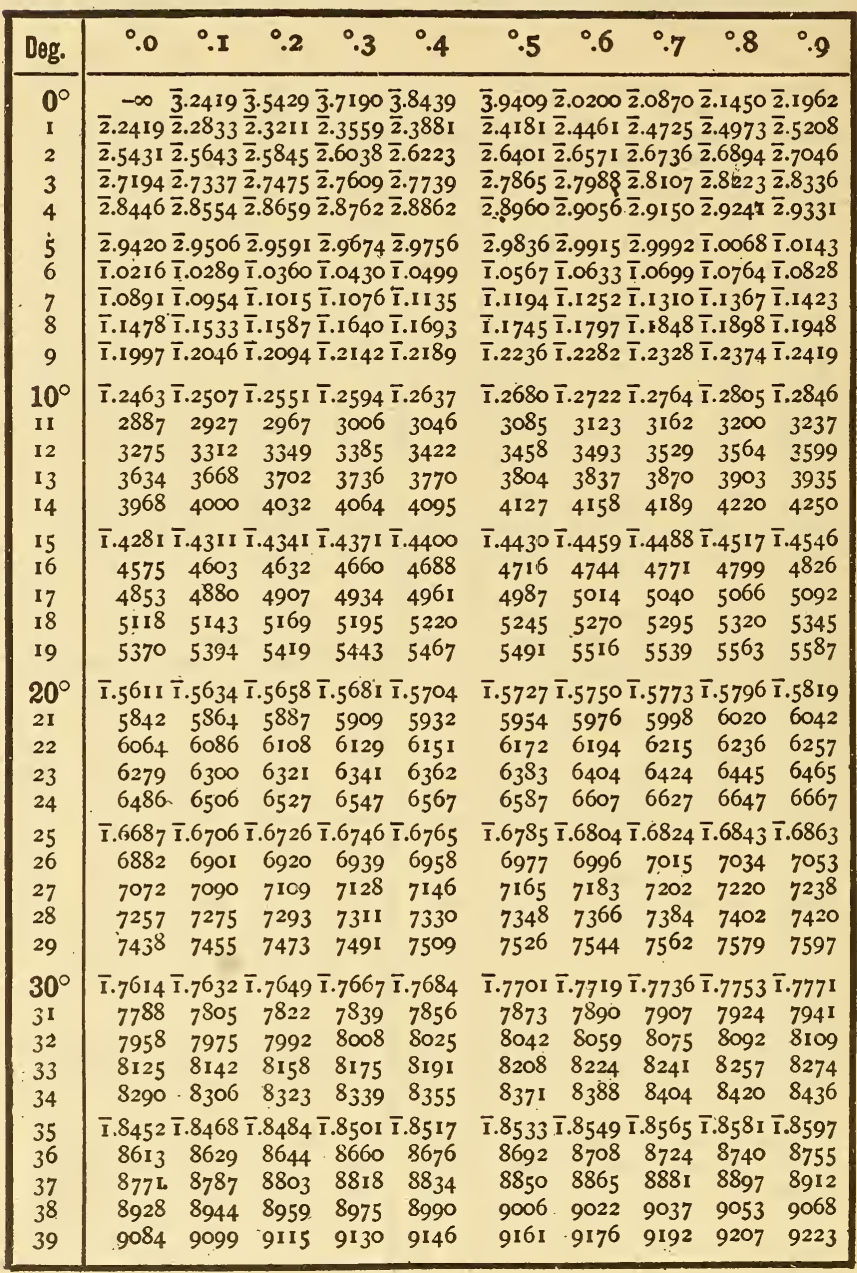


TABLE III.-Continued

Logarithms of Tangents

\begin{tabular}{|c|c|c|c|c|c|c|c|c|c|c|}
\hline Deg. & $\circ .0$ &.$I$ & .2 & $\cdot 3$ & .4 & ${ }^{\circ} \cdot 5$ & $\circ 6$ & .7 & .8 & 9 \\
\hline 3 & \multicolumn{5}{|c|}{ 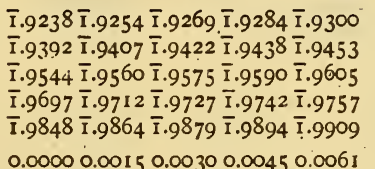 } & $\begin{array}{l}\overline{\mathrm{I}} .9315 \overline{\mathrm{I}} \\
\overline{\mathrm{I}} .9468 \overline{\mathrm{I}} \\
\overline{\mathrm{I}} .962 \mathrm{I} \\
\overline{\mathrm{I}} .9772 \overline{\mathrm{I}} \\
\overline{\mathrm{I}} .9924 \overline{\mathrm{I}}\end{array}$ & $.9330 \overline{\mathrm{I}}$ & $.9346 \overline{\mathrm{I}}$ & $.936 \div \bar{I}$ & $\begin{array}{r}.9376 \\
.9529 \\
.9681 \\
7.9833 \\
.9985\end{array}$ \\
\hline $\begin{array}{l}45 \\
46\end{array}$ & $\begin{array}{r}0.00000 \\
0152\end{array}$ & & $\begin{array}{l}0.00300 \\
0.182\end{array}$ & $\begin{array}{l}0.0045^{\circ} \\
0197\end{array}$ & 0.0061 & $\begin{array}{r}0.0076 \\
0.228\end{array}$ & $\begin{array}{l}0.0091 \\
0243\end{array}$ & 258 & & $\begin{array}{l}0136 \\
0288\end{array}$ \\
\hline 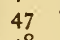 & 030 & & & 0349 & 0364 & 0379 & 0395 & 0410 & 425 & 0440 \\
\hline $\begin{array}{l}48 \\
49\end{array}$ & 8 & 04 & 0639 & $\begin{array}{l}0501 \\
0654\end{array}$ & $\begin{array}{l}0517 \\
0670\end{array}$ & $\begin{array}{l}0532 \\
0685\end{array}$ & $\begin{array}{l}0547 \\
0700\end{array}$ & $\begin{array}{l}0562 \\
0716\end{array}$ & $\begin{array}{l}0578 \\
0731\end{array}$ & $\begin{array}{l}0593 \\
0746\end{array}$ \\
\hline $\begin{array}{l}50^{\circ} \\
5^{\mathrm{I}}\end{array}$ & \multicolumn{5}{|c|}{0.07620 .07770 .07930 .08080 .0824} & \multicolumn{5}{|c|}{$0.08390 .08540 .08700 .08850 .090 \mathrm{r}$} \\
\hline 52 & 107 & & & IIIg & 1135 & IIร०. & 1166 & $1182^{\circ}$ & 1197 & 1213 \\
\hline 53 & 12 & & 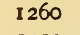 & 1276 & 1292 & 1308 & 1324 & 1340 & $135^{6}$ & 1371 \\
\hline 54 & 13 & & 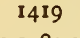 & 1435 & $145^{I}$ & 1467 & 1403 & 499 & 1510 & $15,5^{2}$ \\
\hline 55 & \multicolumn{5}{|c|}{0.15480 .15640 .15800 .15960 .1612} & \multicolumn{5}{|c|}{0.16290 .16450 .16610 .16770 .1694} \\
\hline 56 & 1710 & & 1743 & I 759 & 1776 & 1792 & 1809 & I 825 & 1842 & 1858 \\
\hline 5 & 政 & 18 & - & 1925 & 1941 & $195^{8}$ & 1975 & 1992 & 2000 & 2025 \\
\hline & 20 & & 207 & 2093 & 2110 & 2127. & 2144 & 2161 & $217^{8}$ & 2195 \\
\hline 59 & 2212 & 22 & 2247 & 2264 & 228I & 2299 & 2316 & 2333 & 2351 & 368 \\
\hline $60^{\circ}$ & \multicolumn{5}{|c|}{0.23860 .24030 .24210 .24380 .2456} & \multicolumn{5}{|c|}{$0.24740 .249 \mathrm{I} \cup .25090 .25270 .2545$} \\
\hline $6_{I}$ & 2562 & & 2598 & 2616 & 2634 & 2652 & 2670 & 2689 & 2707 & 2725 \\
\hline 62 & 274 & & $278 c$ & 2798 & 2817 & 2835 & 2854 & 2872 & 2891 & 2910 \\
\hline 63 & & & 29 & 2985 & 3004 & 23 & 30 & II & 3080 & 3099 \\
\hline 04 & & & & 3176 & 3196. & 5 & 3230 & 3234 & 3 & 294 \\
\hline & \multicolumn{5}{|c|}{0.33130 .33330 .33530 .33730 .3393} & \multicolumn{5}{|c|}{$0.34: 30.34330 .3453 \quad 0.34730 .3494$} \\
\hline & & & 355 & $357^{\circ}$ & 3590 & 7 & 3038 & 3059 & 3079 & 3700 \\
\hline & & & 3980 & 4002 & 4024 & 4046 & 4068 & 4091 & 4113 & $\begin{array}{l}-1 \\
4136\end{array}$ \\
\hline & 4158 & & 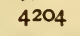 & 4227 & 4250 & 4273 & 4296 & 4319 & 4342 & 4366 \\
\hline $70^{\circ}$ & \multicolumn{5}{|c|}{0.43890 .44130 .44370 .44610 .4484} & \multicolumn{5}{|c|}{$0.45090 .45330 .45570 .45^{81} 0.4606$} \\
\hline 7I & 4630 & & & 4705 & & & $-S$ & & 4831 & 4857 \\
\hline $7^{2}-1$ & & & & & & & 503 & 5066 & & $5^{12}$ \\
\hline 73 & & & & 522 & 5256 & 284 & $53 \mathrm{I}$ & 534 & 5368 & 53 \\
\hline 74 & 5423 & 5454 & 3403 & $55^{12}$ & 5541 & 5570 & 5000 & $5^{629}$ & 5059 & 5089 \\
\hline $\begin{array}{l}75 \\
76\end{array}$ & \multicolumn{5}{|c|}{$\begin{array}{c}0.57190 .57500 .57800 .58110 .5842 \\
6032 \quad 6065 \quad 6007 \quad 6130 \quad 6163\end{array}$} & \multicolumn{5}{|c|}{0.58730 .59050 .59360 .59680 .6000} \\
\hline & 63 & & 04. & 6471 & 6507 & 6542 & $6_{578}$ & 6615 & 6651 & 6688 \\
\hline 7 & 672 & 676 & 6800 & $683^{8}$ & 6877 & 6915 & 6954 & 6994 & 7033 & 7073 \\
\hline 75 & 7113 & 7154 & 7195 & 7236 & 7278 & 7320 & 7363 & 7406 & 7449 & 7493 \\
\hline $\begin{array}{l}80^{\circ} \\
81 \\
82\end{array}$ & \multicolumn{5}{|c|}{$\begin{array}{l}0.75370 .7581 \quad 0.76260 .76720 .7718 \\
0.80030 .80520 .81020 .81520 .8203 \\
0.85220 .85770 .86330 .86900 .8748\end{array}$} & $\begin{array}{r}0.77640 \\
0.8255 \circ \\
.0 .88060\end{array}$ & $\begin{array}{l}0.78110 \\
0.8307 \\
0.88650\end{array}$ & $\begin{array}{l}.78580 \\
.83600 \\
.899240\end{array}$ & $\begin{array}{l}0.79060 \\
0.84130 \\
0.89850\end{array}$ & $\begin{array}{l}0.7954 \\
0.8467 \\
0.9046\end{array}$ \\
\hline 83 & \multirow{2}{*}{\multicolumn{5}{|c|}{ 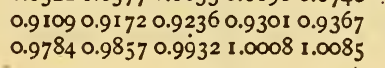 }} & \multicolumn{5}{|c|}{ 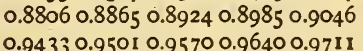 } \\
\hline 8 & & & & & & \multicolumn{5}{|c|}{ 1.0164 I.0244 1.0326 1.0409 1.0494 } \\
\hline 85 & \multicolumn{2}{|c|}{1.05801 .0669} & & & & \multicolumn{5}{|c|}{ I.1040 I.II38 I.I 238 I.I 34 I I.14 } \\
\hline & \multirow{2}{*}{\multicolumn{2}{|c|}{$\begin{array}{l}1.1554 \\
1.2306\end{array}$}} & & & & $1.2135 \mathrm{I}$ & & & & \\
\hline 87 & & & & & & & & & \\
\hline $88 \quad$ & \multicolumn{2}{|l|}{1.4569} & & & & 1. 5819 & & & & \\
\hline & I. & & 3. & & & 2.059 & & b & 2 & $2.75^{8}$ \\
\hline
\end{tabular}

$8513^{\circ}-21-20$ 
TABLE IV.-Calibration Data of Representative Couples

\begin{tabular}{|c|c|c|c|c|c|c|c|c|c|c|}
\hline \multicolumn{2}{|c|}{$\begin{array}{l}\text { Englehard } \\
\text { "Le Chatelier" }\end{array}$} & \multicolumn{2}{|c|}{$\begin{array}{l}\text { Johnson-Matthey } \\
\text { "Le Chatelier" }\end{array}$} & \multicolumn{2}{|c|}{$\begin{array}{l}\text { Copper- } \\
\text { constantan }\end{array}$} & \multicolumn{3}{|c|}{ Iron-constantan } & \multicolumn{2}{|c|}{$\begin{array}{l}\text { Chromel- } \\
\text { alumel }\end{array}$} \\
\hline \multirow{2}{*}{ Emf } & \multirow{2}{*}{$\begin{array}{l}\text { Temp., } \\
\text { degrees } \\
\text { C }\end{array}$} & \multirow{2}{*}{ Emf } & \multirow{2}{*}{$\begin{array}{l}\text { Temp., } \\
\underset{C}{\text { degrees }}\end{array}$} & \multirow{2}{*}{ Emf } & \multirow{2}{*}{$\begin{array}{l}\text { Temp., } \\
\text { degrees } \\
\text { C }\end{array}$} & \multirow{2}{*}{ Emf } & \multicolumn{2}{|c|}{$\begin{array}{l}a \text { Temp., } \\
\text { degrees C }\end{array}$} & \multirow{2}{*}{ Emf } & \multirow{2}{*}{$\begin{array}{l}\text { Temp., } \\
\text { degrees } \\
\text { C }\end{array}$} \\
\hline & & & & & & & $B$ & $L$ & & \\
\hline 0 & 0 & 0 & 0 & 0 & 0 & 0 & 0 & 0 & 0 & 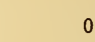 \\
\hline 1 & 147 & 1 & 146 & 1 & 25 & 5 & 105 & 95 & 5 & 122 \\
\hline 2 & 265 & 2 & 260 & 2 & 49 & 10 & 204 & 186 & 10 & 243 \\
\hline 3 & 374 & 3 & 364 & 3 & 72 & 15 & 299 & 277 & 15 & 363 \\
\hline 4 & 478 & 4 & 461 & 4 & 94 & 20 & 392 & 367 & 20 & 482 \\
\hline 5 & 578 & 5 & 553 & 5 & 115 & 25 & 483 & 457 & 25 & 601 \\
\hline 6 & 675 & 6 & 641 & 6 & 136 & 30 & 574 & 546 & 30 & 721 \\
\hline 7 . & 770 & 7 & 725 & - 7 & 156 & 35 & 662 & 632 & 35 & 844 \\
\hline 8 & 861 & 8 & 806 & 8 & 175 & 40 & 749 & 713 & 40 & 970 \\
\hline 9 & 950 & 9 & 884 & 9 & 194 & 45 & 836 & 792 & 45 & 1100 \\
\hline 10 & 1037 & 10 & 959 & 10 & 213 & 50 & 924 & 871 & & \\
\hline 11 & 1122 & 11 & 1032 & 11 & 232 & 55 & 1011 & 950 & & \\
\hline 12 & 1206 & 12 & 1103 & 12 & 250 & 60 & & 1030 & & \\
\hline 13 & 1290 & 13 & 1173 & 13 & 268 & & & & & \\
\hline 14 & 1373 & 14 & 1242 & 14 & 285 & & & & & \\
\hline 15 & 1455 & 15 & 1311 & 15 & 302 & $\ldots \ldots$ & & & & $\ldots$ \\
\hline & & 16 & 1379 & 16 & 319 & & & & & \\
\hline & & 17 & 1447 & $\begin{array}{l}-17 \\
\end{array}$ & 336 & & & & & \\
\hline & & & & 18 & 353 & & & & & \\
\hline
\end{tabular}

$a B$ represents mean calibration by U. S. Bureau of Standards of iron-constantan couples from all sources. $L$ represents mean calibration of Leeds \& Northrup's iron-constantan couple.

Emfs of various materials versus platinum, at different temperatures, the cold junctions being at zero ${ }^{\circ} \mathrm{C}$. From this chart the emf of any combination of metals or alloys is found by measuring the vertical distance between the curves for the chosen materials, the upper curve indicating the positive element. Thus at $500^{\circ} \mathrm{C}$ the emf of a copel-platinum thermocouple would be 23.4 millivolts, copel being the negative element, while at the same temperature a platinum-chromel couple would develop i6 millivolts, chromel being positive to platinum and hence also positive to copel. The emf of a copel-chromel would be $16+23.4$ millivolts at $500^{\circ} \mathrm{C}$.

Where two curves are parallel in a given temperature range the emf of a couple made of the materials indicated would remain constant throughout that range. Where two curves cross, the emf of the couple indicated would decrease, become zero, and then increase in the reverse direction as the temperature is varied uniformly through this range, provided the cold junctions are at zero ${ }^{\circ} \mathrm{C}$. 


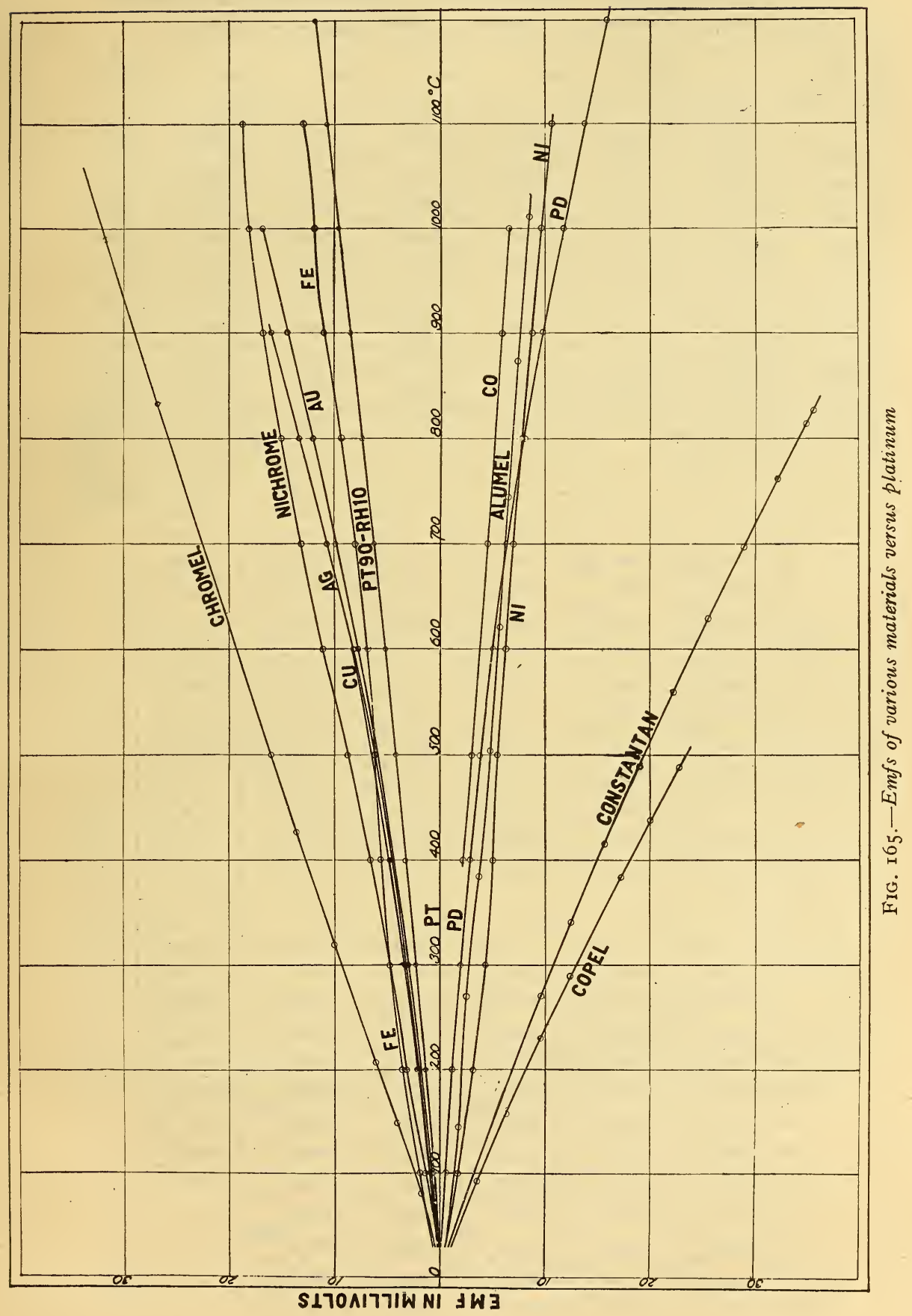


TABLE V.-Emf-temperature data for Pt-90 Pt $10 \mathrm{Rh}$ Thermocouple as determined by the Geophysical Laboratory. (Adams, Bull. A. I. M. M. E., 159, p. 2111, 1919)

[Emfs are expressed in microvolts; temperatures in degrees centigrade; cold junction $=0^{\circ} \mathrm{C}$ ]

\begin{tabular}{|c|c|c|c|c|c|c|c|}
\hline $\begin{array}{c}E \\
\mu v\end{array}$ & 0 & 1000 & 2000 & 3000 & 4000 & 5000 & 6000 \\
\hline 0 & $0 \quad{ }^{\circ} \mathrm{C}$ & $147.1^{\circ} \mathrm{C}$ & $265.4^{\circ} \mathrm{C}$ & $374.3^{\circ} \mathrm{C}$ & $478.1^{\circ} \mathrm{C}$ & $578.3^{\circ} \mathrm{C}$ & $675.3^{\circ} \mathrm{C}$ \\
\hline 100 & $\begin{array}{r}17.8_{16.7} \\
16.0\end{array}$ & 159. $7_{12.4}^{17.0}$ & 276.6 & $384.9^{10.0}$ & $488.3^{10.1}$ & $588.1^{Y .0}$ & $684.8^{3.0}$ \\
\hline 200 & 34. $5_{15.8}$ & 172. 1 & $287.7_{11.0}$ & $\begin{array}{r}395.4_{10.5} \\
10.0\end{array}$ & 498. $4_{10.1}$ & $\begin{array}{r}597.9 \\
9.8\end{array}$ & $\begin{array}{r}694.3^{5.5} \\
9.5\end{array}$ \\
\hline 300 & 50. 3 15.1 & 184.3 & 298. $7_{11.0}$ & $405.9^{10.4}$ & 508. $5_{10.1}$ & $\begin{array}{r}607.7 \\
9.7\end{array}$ & $\begin{array}{r}703.8 \\
9.5\end{array}$ \\
\hline 400 & $\begin{array}{r}65.4 \\
14.6\end{array}$ & 196. ${ }^{11.8}$ & 309. $7^{10.9}$ & 416. ${ }^{10.4}$ & $518.6_{10.0}$ & $\begin{array}{r}617.4 \\
\mathbf{9 . 7}\end{array}$ & $\begin{array}{r}713.3^{9.4} \\
\end{array}$ \\
\hline 500 & $80.0_{14.1}$ & 208.111 .6 & $\begin{array}{r}320.6 \\
10.9\end{array}$ & $\begin{array}{r}\text { 426. } 7 \\
10.4\end{array}$ & $528.6_{10.0}$ & $\begin{array}{r}627.1 \\
9.7\end{array}$ & $\begin{array}{r}722.7 \\
9.4\end{array}$ \\
\hline 600 & $94.1_{13.7}$ & 219. $7^{11.5}$ & $\begin{array}{r}331.5_{10.8} \\
10.0\end{array}$ & 437. $1_{10.3}$ & $538.6_{10.0}$ & $\begin{array}{r}636.8 \\
9.7\end{array}$ & $\begin{array}{r}732.1^{9.4} \\
\end{array}$ \\
\hline 700 & $107.8_{13.4}$ & $231.2_{11.5}$ & $\begin{array}{r}342.3_{10.7} \\
10.7\end{array}$ & $447.4_{10.3}$ & $548.6 \quad 9.9$ & $\begin{array}{r}646.5 \\
9.6\end{array}$ & $\begin{array}{r}741.5 \\
9.4\end{array}$ \\
\hline 800 & 121. 2 & $242.7^{11.4}$ & $\begin{array}{l}353.0_{10.7} \\
\text { (10. }\end{array}$ & 457. $7_{10.2}$ & $\begin{array}{ll}558.5 & 9.9\end{array}$ & 656. 1 & $\begin{array}{r}750.9 \\
9.3\end{array}$ \\
\hline 900 & 134. $3_{12.8}$ & 254. $1_{11.3}$ & 363. ${ }^{10.6}$ & $\begin{array}{r}467.9 \\
10.2\end{array}$ & 568.4 & 665.79 & $760.2^{9.3}$ \\
\hline 1000 & 147.1 & 265.4 & 374.3 & 478. 1 & 578.3 & 675.3 & 769.5 \\
\hline
\end{tabular}

\begin{tabular}{|c|c|c|c|c|c|c|}
\hline$\underset{\mu v}{E}$ & 7000 & 8000 & 9000 & 10000 & 11000 & 12000 \\
\hline 0 & $\begin{array}{r}769.5^{\circ} \mathrm{C} \\
9.3\end{array}$ & $\begin{array}{r}861.1^{\circ} \mathrm{C} \\
9.0\end{array}$ & $\begin{array}{r}950.4^{\circ} \mathrm{C} \\
8.8\end{array}$ & $\begin{array}{r}\text { 1037. } 3^{\circ} \mathrm{C} \\
\mathbf{8 . 6}\end{array}$ & $\begin{array}{r}1122.2^{\circ} \mathrm{C} \\
\mathbf{8 . 4}\end{array}$ & $\begin{array}{r}1205.9^{\circ} \mathrm{C} \\
8.3\end{array}$ \\
\hline 100 & 778.8 & ${ }^{870.1} 9.0$ & 959.28 .8 & ${ }^{1045.9} 8.5$ & 1130.68 .4 & 1214. ${ }^{2} 8.4$ \\
\hline 200 & 788. 0 & 879. 1 9.0 & $\begin{array}{l}968.0 \\
8.7\end{array}$ & 1054.48 .5 & $1139.0^{0}$ & 1222. $6 \mathrm{~S} .3$ \\
\hline 300 & 797. 2 9.2 & 888.1 & $\begin{array}{l}976.7 \\
8.7\end{array}$ & $\begin{array}{r}1062.9 \\
8.5\end{array}$ & $\begin{array}{r}1147.4 \\
8.4\end{array}$ & $\begin{array}{r}1230.9^{8.4} \\
\end{array}$ \\
\hline 400 & 806. 4 9.2 & 897.19 .0 & ${ }^{985.4} 8.7$ & $1071 .{ }^{4} 8.5$ & 1155.8 & $1239 .{ }_{8.3}$ \\
\hline 500 & 815.6 & 906. ${ }^{1} 8.9$ & $994.1_{8.7}$ & 1079.98 .5 & 1164.28 .3 & 1247.68 .3 \\
\hline 600 & 824. 79.1 & $915 .{ }^{0} 8.9$ & $\begin{array}{r}1002.8 \\
8.7\end{array}$ & $\begin{array}{r}1088.4 \\
8.5\end{array}$ & $\begin{array}{r}1172.5 \\
8.4\end{array}$ & $\begin{array}{r}1255.9 \\
8.4\end{array}$ \\
\hline 700 & 833. 8 & ${ }^{923.9} 8.9$ & ${ }^{1011.5}{ }^{8.6}$ & ${ }^{1096.9} 8.5$ & ${ }^{1180.9} 8.3$ & ${ }^{1264.3} 8.3$ \\
\hline 800 & 842. 9 & $\begin{array}{l}932.8 \\
8.8\end{array}$ & 1020. 18.6 & 1105.48 .4 & 1189. 2 & 1272. 68.4 \\
\hline 900 & 852.0 & 941.6 & 1028. 7 & 1113.8 & 1197.6 & $1281.0_{8}$ \\
\hline 1000 & 861.1 & 950.4 & 1037.3 & 1122.2 & 1205.9 & 1289.3 \\
\hline
\end{tabular}

\begin{tabular}{|c|c|c|c|c|c|c|}
\hline$\underset{\mu v}{E}$ & 13000 & 14000 & 15000 & 16000 & 17000 & 18000 \\
\hline $\begin{array}{r}0 \\
100 \\
200 \\
300 \\
400 \\
500 \\
600 \\
700 \\
800 \\
900 \\
1000\end{array}$ & 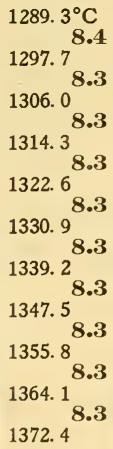 & $\begin{array}{c}1372.4^{\circ} \mathbf{C} \\
8.3 \\
1380.7{ }^{8.3} \\
1389.0 \\
1397.3^{8.3} \\
{ }^{8.3} \\
1405.6 \\
1413.8 \\
14.2 \\
1422.0 \\
1430.2 \\
8.2 \\
1438.4 \\
1446.6^{8.2} \\
1454.8\end{array}$ & 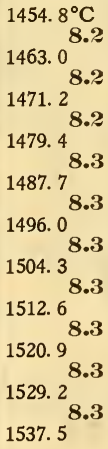 & 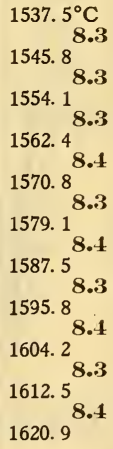 & 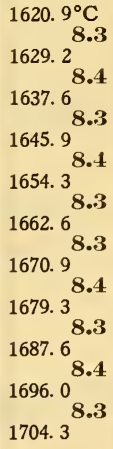 & 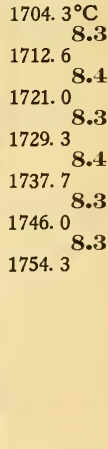 \\
\hline
\end{tabular}


TABLE VI.-Emf-temperature data for Copper-Constantan Thermocouple as determined by the Geophysical Laboratory. (Adams, Bull. A. I. M. M. E., 159, p. 2111, 1919)

[Emfs are expressed in microvolts; temperature in degrees centigrade; cold junction $=0^{\circ} \mathrm{C}$ ]

\begin{tabular}{|c|c|c|c|c|c|c|c|}
\hline $\begin{array}{c}E \\
\mu v\end{array}$ & 0 & 1000 & 2000 & 3000 & 4000 & 5000 & 6000 \\
\hline 0 & & 25.27 & 49.20 & 72.08 & 94.07 & 115. 31 & 135. 91 \\
\hline 100 & 2. $59_{2}$ & 27. 72 2.43 & $\begin{array}{l}51.53 \\
2.32\end{array}$ & ${ }^{74 .}{ }^{3} 2.23$ & 96. 23 2.15 & 117. 40 & 137. 94 \\
\hline 200 & $\begin{array}{r}\text { 5. } 16 \\
2.56\end{array}$ & 30. 15 & 53. 85 2.31 & 76. 54 2.22 & 98. 38 & ${ }^{119.48} 2.08$ & $\begin{array}{r}139.96 \\
2.02\end{array}$ \\
\hline 300 & $\begin{array}{r}7.72 \\
2.55\end{array}$ & $\begin{array}{r}32.57 \\
2.41\end{array}$ & 56. 16 2.30 & $\begin{array}{r}78.76 \\
2.21\end{array}$ & 100.52 & $\begin{array}{r}121.56 \\
2.07\end{array}$ & $\begin{array}{r}141.98 \\
2.01\end{array}$ \\
\hline 400 & ${ }^{10.27} 2.53$ & 34. 98 2.40 & ${ }^{58.46} 2.3$ & ${ }^{80.97} 2.2$ & ${ }_{\mathbf{2 . 1 3}}^{102.6^{6}}$ & ${ }_{2.0}^{123.63}$ & $\begin{array}{r}143.99 \\
2.01\end{array}$ \\
\hline 500 & $\begin{array}{l}\text { 12. } 80 \\
\mathbf{2 . 5 2}\end{array}$ & $\begin{array}{r}37.38 \\
2.39\end{array}$ & 60.76 & 83. 17 & $\begin{array}{r}104.79 \\
\mathbf{2 . 1 2}\end{array}$ & 125.69 & 146.00 \\
\hline 600 & $\begin{array}{r}15.32 \\
2.51\end{array}$ & 39. 77 2.38 & $\begin{array}{r}63.04 \\
2.27\end{array}$ & $\begin{array}{r}85.37 \\
2.19\end{array}$ & ${ }^{106.91^{2.11}}$ & $\begin{array}{r}127.75 \\
2.05\end{array}$ & $\begin{array}{l}148.00 \\
2.00\end{array}$ \\
\hline 700 & 17. 83 2.49 & ${ }^{42.15} 2.36$ & ${ }^{65.31} 2.27$ & ${ }^{87.56} 2.18$ & 109.02 .10 & ${ }^{129.80^{2.04}}$ & $\begin{array}{l}150.00 \\
1.99\end{array}$ \\
\hline 800 & 20. 32 2.48 & 44. 51 2.35 & $\begin{array}{c}67.58 \\
2 .\end{array}$ & $\begin{array}{r}89.74 \\
2.1\end{array}$ & $\begin{array}{ll}111 & 12 \\
& 2.10\end{array}$ & 131. ${ }^{84}$ & $\begin{array}{r}151.99 \\
1.99\end{array}$ \\
\hline 900 & 22.80 & 46.86 & 69.83 & 91.91 & 113. 22 & 133.88 & 153. 97 \\
\hline 1000 & 25.27 & 49.20 & $72.08^{7 \cdot 60}$ & 94.07 & 115.31 & 135. $91^{\circ}$ & 155.95 \\
\hline
\end{tabular}

\begin{tabular}{|c|c|c|c|c|c|c|c|}
\hline $\begin{array}{c}E \\
\mu v\end{array}$ & 7000 & 8000 & 9000 & 10000 & 11000 & 12000 & 13000 \\
\hline 0 & 155.95 & 175.50 & 194.62 & 213. 36 & 231.74 & 249.82 & 267.60 \\
\hline 100 & $157.92^{1.98}$ & $177.43^{1.50}$ & $196.51^{1.09}$ & $215.21^{1.00}$ & $233.56^{1.0 \%}$ & $251.61^{1.09}$ & $269.36^{1.76}$ \\
\hline & 1.97 & 1.93 & 1.89 & 1.85 & 1.82 & 1.79 & 1.76 \\
\hline 200 & $\begin{array}{r}159.89 \\
\mathbf{1 . 9 7}\end{array}$ & ${ }^{179.36} \mathbf{1 . 9 2}$ & ${ }_{198.40} 1.88$ & $217.06 \quad 1.85$ & ${ }^{235 .}{ }^{38} 1.82$ & ${ }_{1.78}^{253.40}$ & 271.12 \\
\hline 300 & 161.86 1.96 & 181.28 & 200. 28 & 218.91 1.84 & 237.20 & 255.18 1.78 & 272.88 \\
\hline 400 & $163.82 \mathbf{1 . 9 6}$ & 183. 20 & $202.16_{1.88}$ & 220.75 & 239.01 & $256.96{ }_{1.78}$ & 274.64 1.76 \\
\hline 500 & 165.78 & 185.11 & $204.04 \mathrm{sz}$ & 222.59 & 240.82 & 258.74 & 276.40 \\
\hline 600 & 167.73 & 187.02 & 205.91 & $224.43^{1.07}$ & 242.63 & 260.52 & 278.15 \\
\hline 700 & $169.68_{1.94}^{1.30}$ & 188.93 & 207.78 & $226.26{ }_{1.83}^{1.83}$ & $244.43^{1.80}$ & 262.29 & 279. $90_{1}$ \\
\hline 800 & 171.62 & $190.83 \quad 1.90$ & $209.64 \quad 1.86$ & 228.09 & 246. 23 1.80 & 264.06 1.7\% & 281.65 1.74 \\
\hline 900 & 173. 56 & 192. 73 & 211.50 & 229. 92 & 248.03 & $265.83^{x}$ & 283. 39 \\
\hline 1000 & $175.50^{1.94}$ & $194.62^{1.8}$ & $213.36^{\mathbf{1 . 8 6}}$ & $231.74^{1 .}$ & $249.82^{1}$ & $267.60^{1 .}$ & 285.13 \\
\hline
\end{tabular}

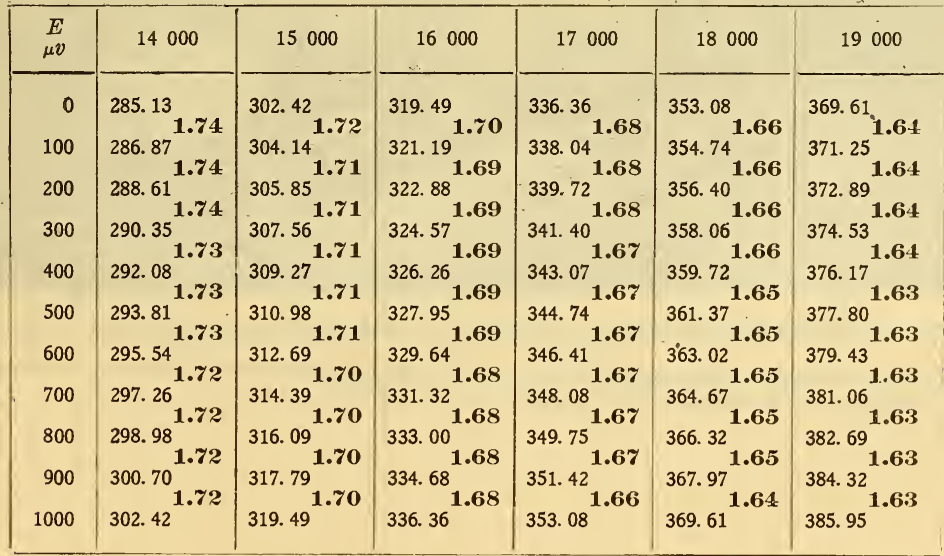




\section{PYROMETRIC INSTALLATIONS}

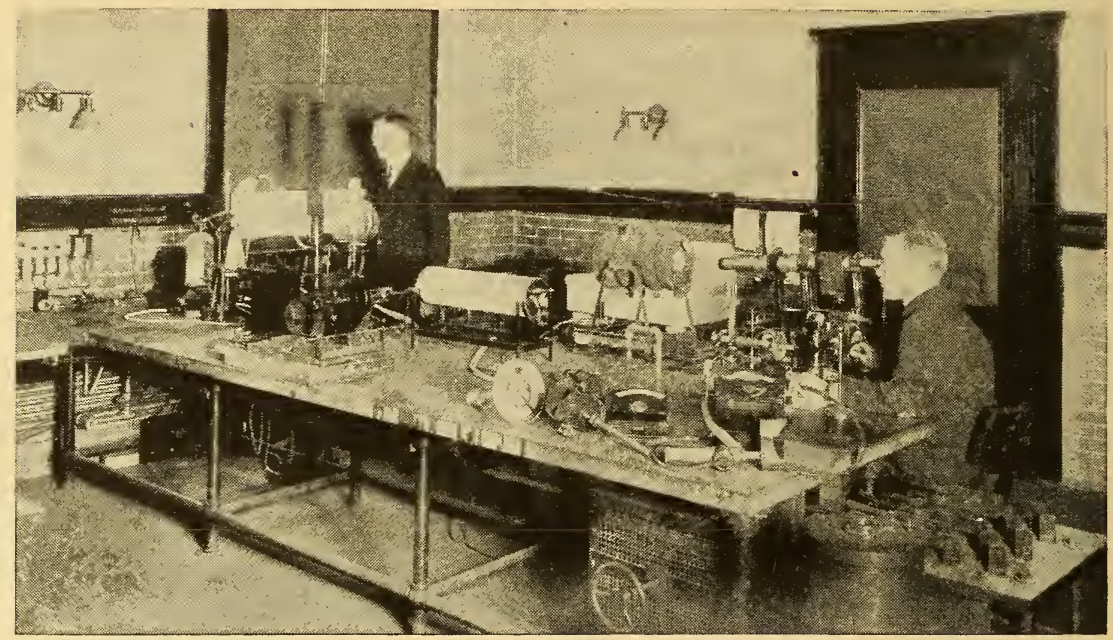

FIG. r66.-One of the high-temperaturemeasurement laboratories at the Bureau of Standards

In the foreground are placed some industrial types of optical and radiation pyrometers. The observer on the right is sighting into a "black body" furnace with the primary standard optical pyrometer

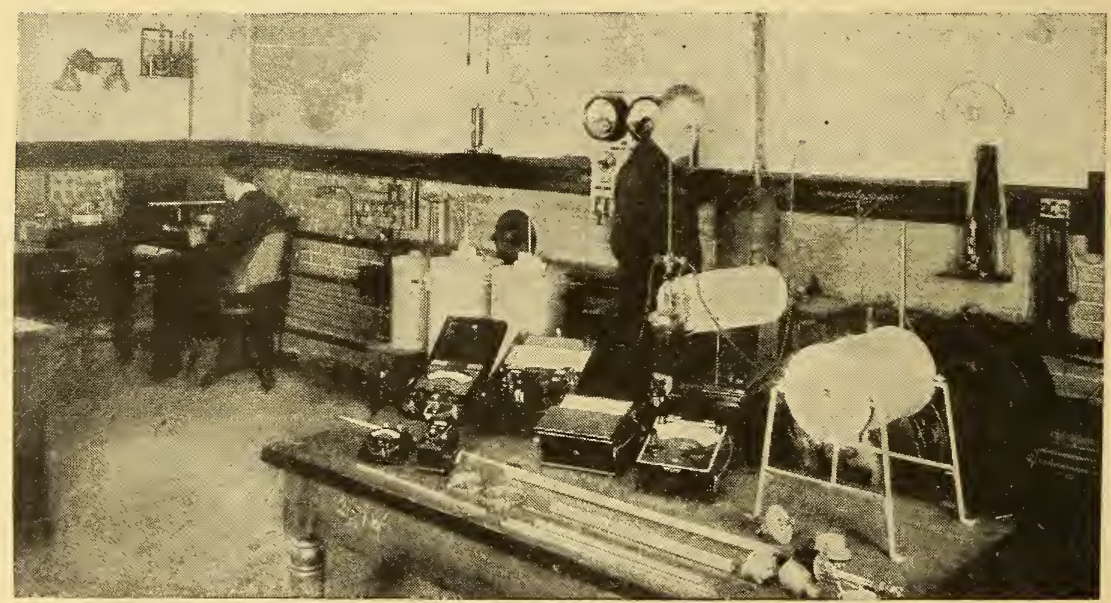

FIG. 167.-One of the high-temperaturemeasurement laboratories of the Bureau of Standards

In the foreground are shown some industrial types of thermocouples and millivoltmeters. Furnaces for melting standard melting-point metals are shown in the background 


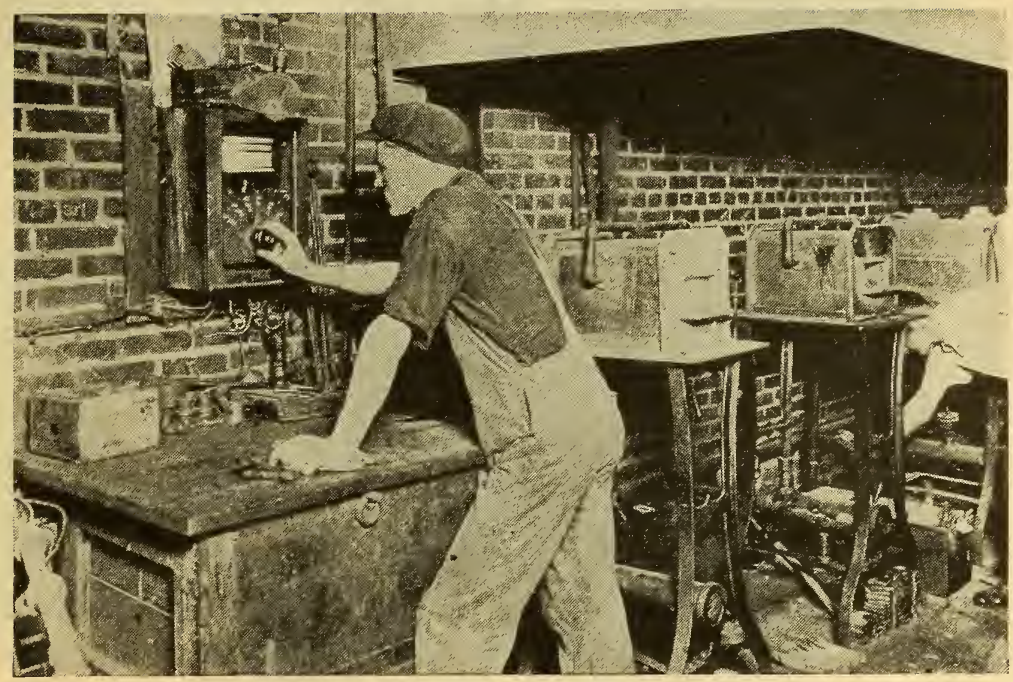

FIG. 168.-Six-point indicator with two temperature scales-one for rare-metal couples in high-speed steel furnaces and one for base-metal couples in carbon-steel furnaces

Plant of a large Philadelphia tool company

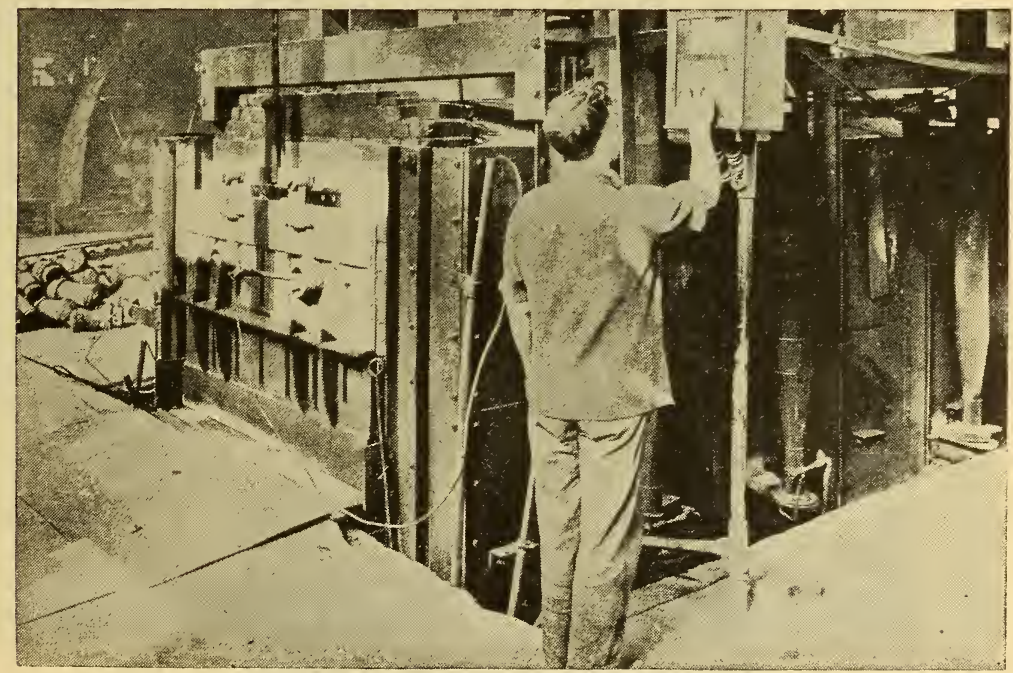

FIG. I69.-Two-point indicator in a furnace for heat treating airplane engine cylinders 


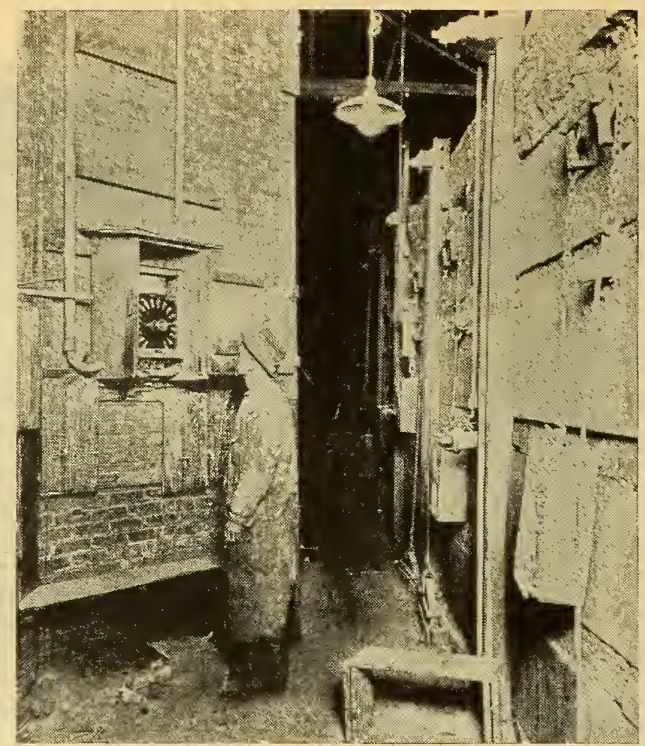

FIG. I70.-Eighteen-point indicating pyromeier in rear of furnace of a malleable steel company

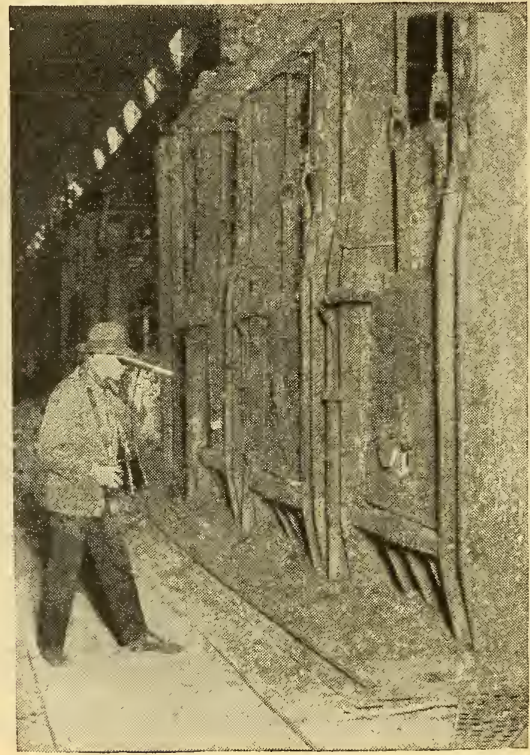

FIG. I 7. - Measurements of furnace temperature with the optical pyrometer

The operator is sighting with the disappearing-filament pyrometer into a furnace through a small observation hole in the door. True temperatures are obtained, since fairly good black-body conditions are assured (if the temperature distribution in the furnace is sufficiently uniform) 


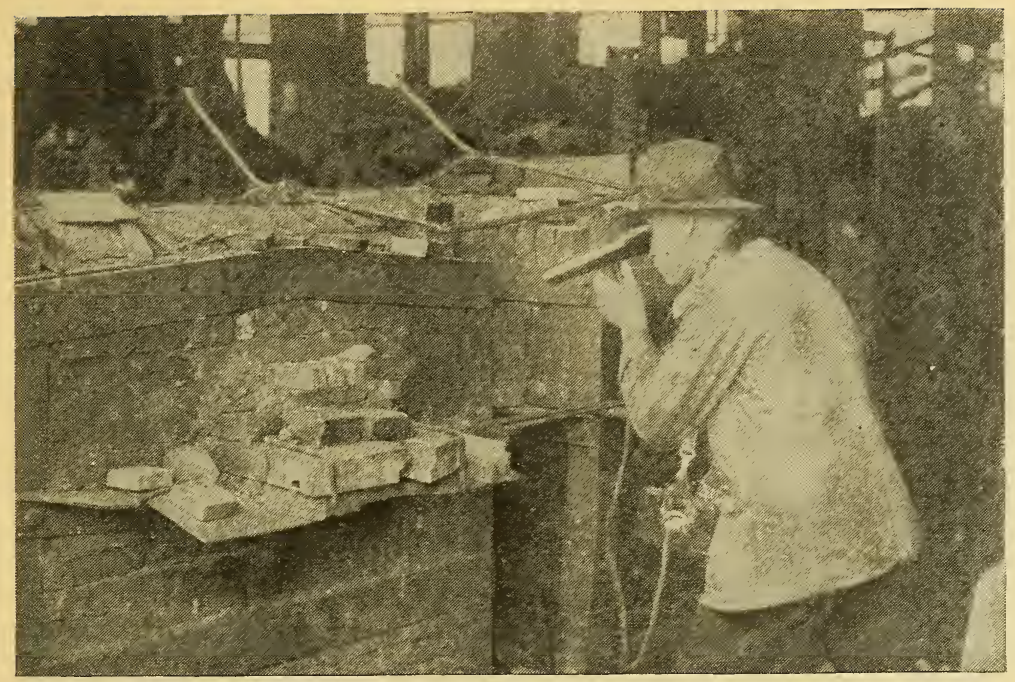

FIG. I72.-Measurements of temperature of a tool with the optical pyrometer

The operator is sighting with the disappearing-filament pyroneter at a small tool undergoing heat treatment. Since the temperature may be estimated to within $5^{\circ} \mathrm{C}$, the most exacting specifications for heat treatment can be followed

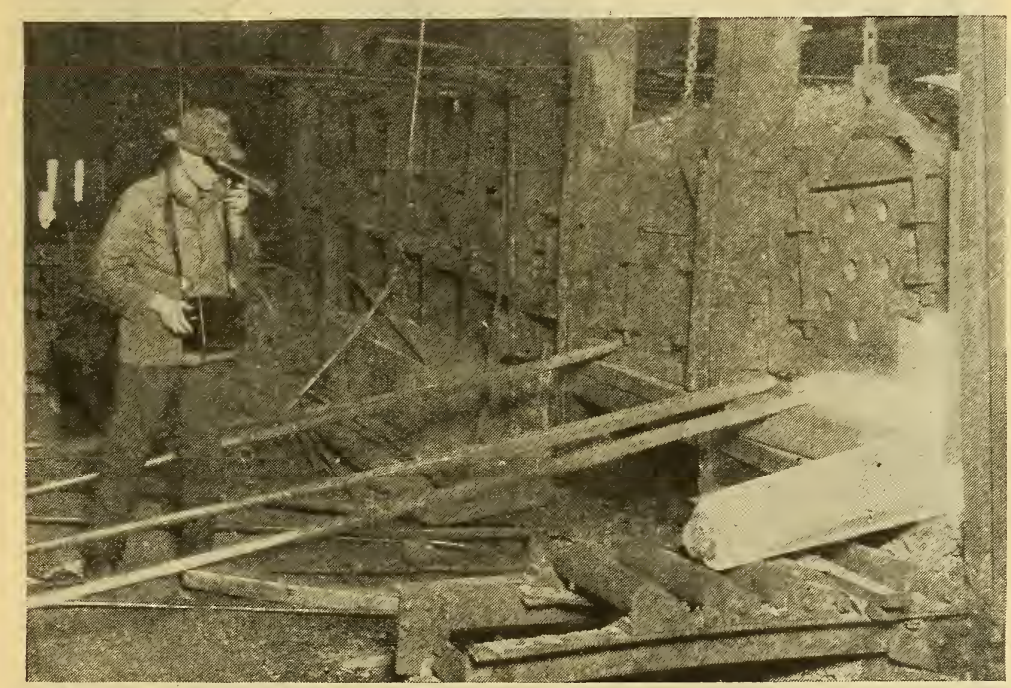

FIG. I73.-Measurements of ingot temperatures with the optical pyrometer

The operator is sighting with the disappearing-filament pyrometer on an ingot which is passing from the furnace to the rolls. Although the observation is being made in the open, practically true temperature of the oxide surface is obtained on account of the very high emissivity of iron oxide. Small corrections for emissivity amounting to less than $10^{\circ} \mathrm{C}$ may be applied when necessary, as shown in Table 16 of the text 


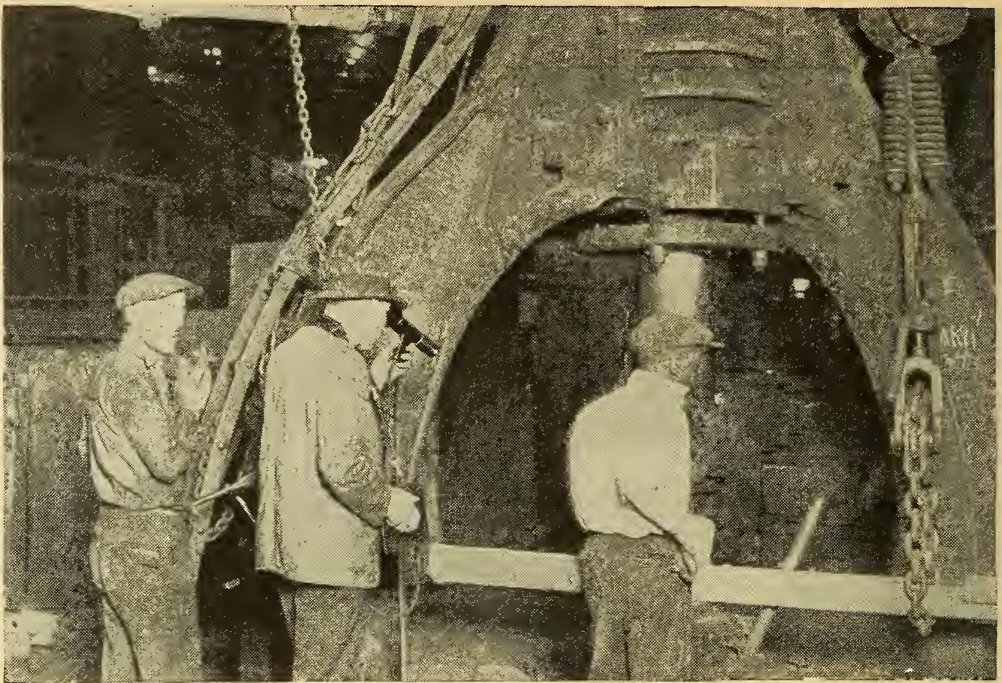

FIG. I74.-Measurements of forging temperature with the optical pyrometer

The operator is sighting upon an ingot during the forging process. In this manner the specifications for forging temperature of high-grade steel may be closely followed

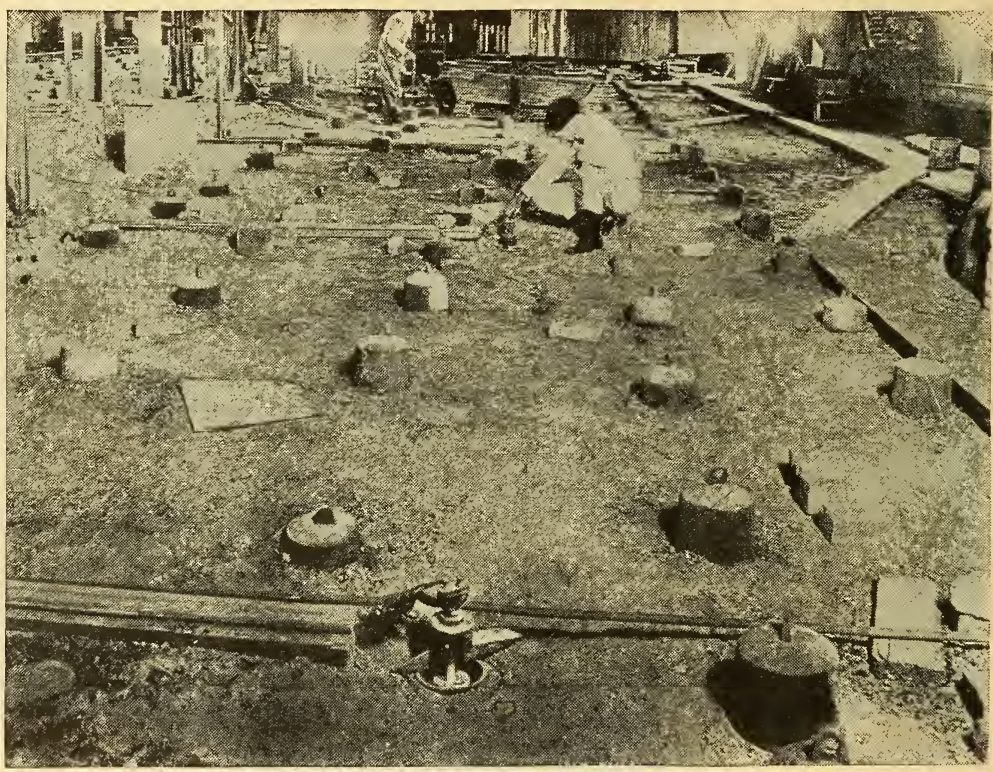

FIG. I75.-Use of pyrometry in the ceramic industry

Rare-metal thermocouples are shown installed at intervals of ${ }_{25}$ feet through the top of a tunnel kiln. The wiring is in conduit with outlets for connection to the thermocouple heads. Asbestos-covered compensated extension wire removes the cold junction to a point some distance from the kiln 


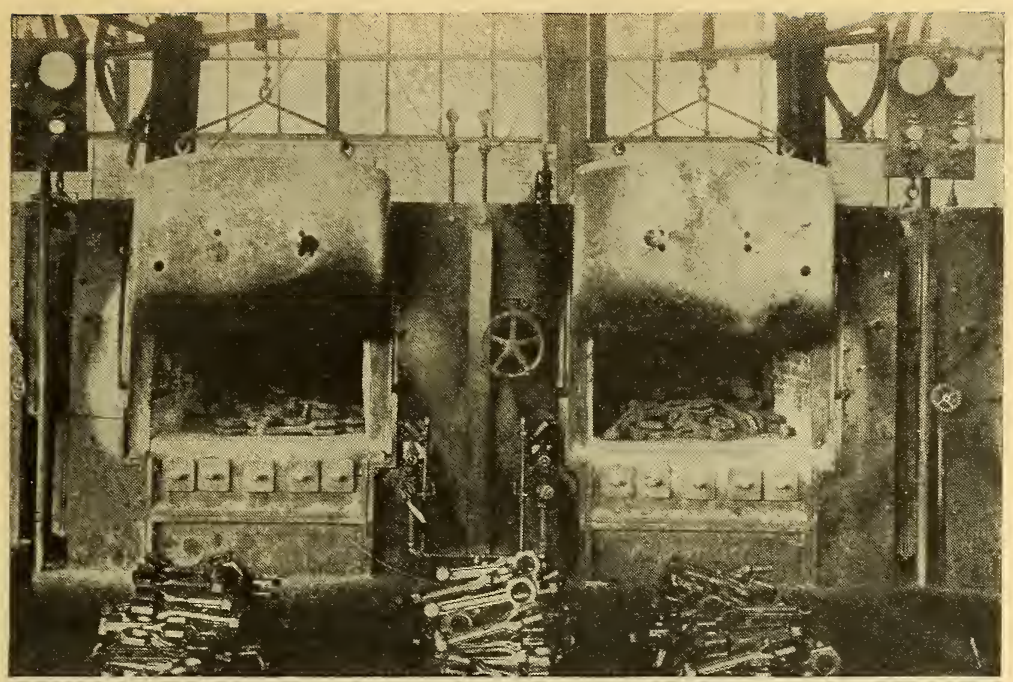

FIG. I76.-A utomatic signaling control

In the upper corners of the illustration are shown indicators and colored electric lights mounted on panel boards. The signals, made by the red, white, and green lights, and by the auxiliary indicators are controlled by a recorder equipped for automatic signaling. The recorder is connected to thermocouples installed in the furnaces

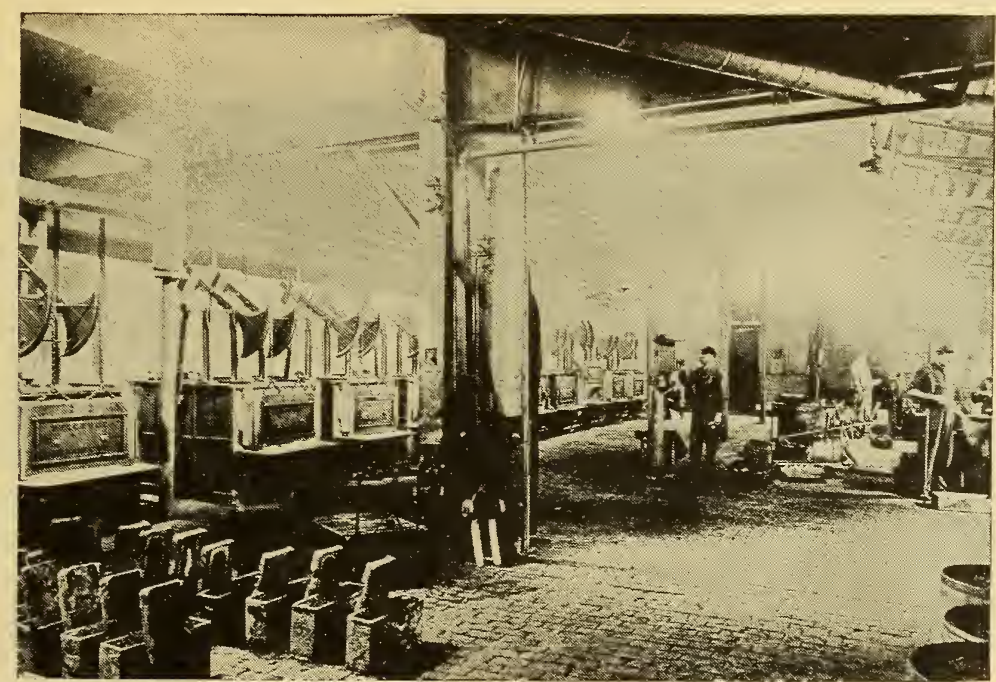

FIG. I77.-Furnace room of a steel plant where high grade automobile parts are heat treated

Thermocouples are installed in each furnace, and a multiple-point indicator is shown mounted on a column in the center of the room 


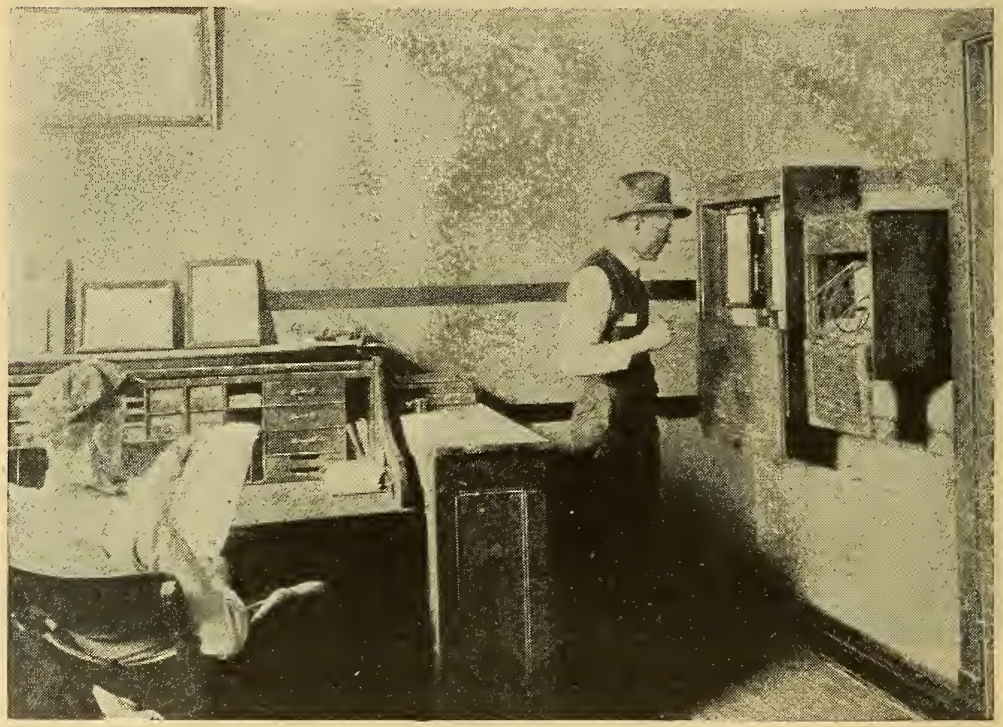

FIG. I78.-Pyrometric recorders in a superintendent's office

The thermocouples installed in the furnaces shown in Fig. 177 are connected to recorders in the superintendent's office. A continuous record is accordingly obtained of the operation of each furnace

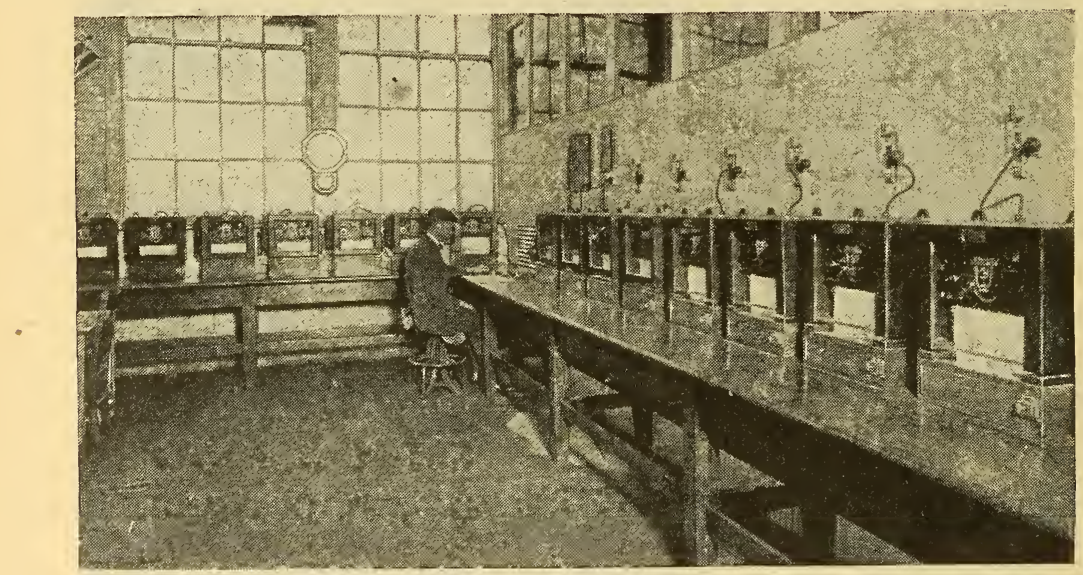

FIG. 179.-Central pyrometer station

In a large plant it is frequently desirable to install all recorders in a single room. An attendant has charge of the installation and the maintenance of the records 


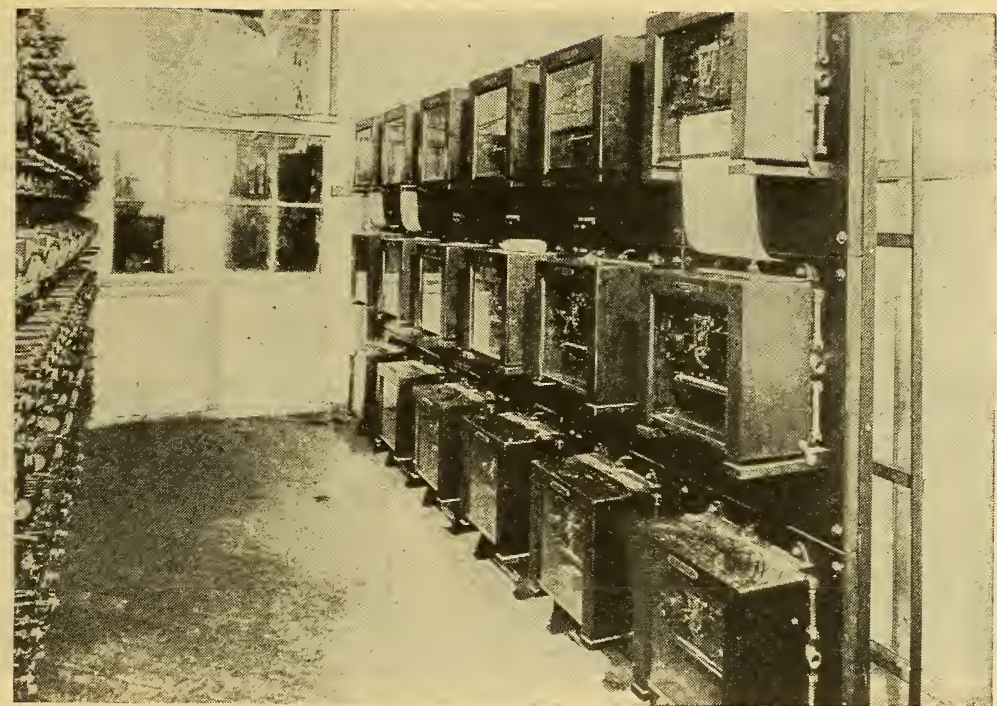

FIG. I80.-Central pyrometer station

The recorders are equipped with devices for operating the relay switches at the laft. By this means electric furnaces are maintained automatically at specified temperatures

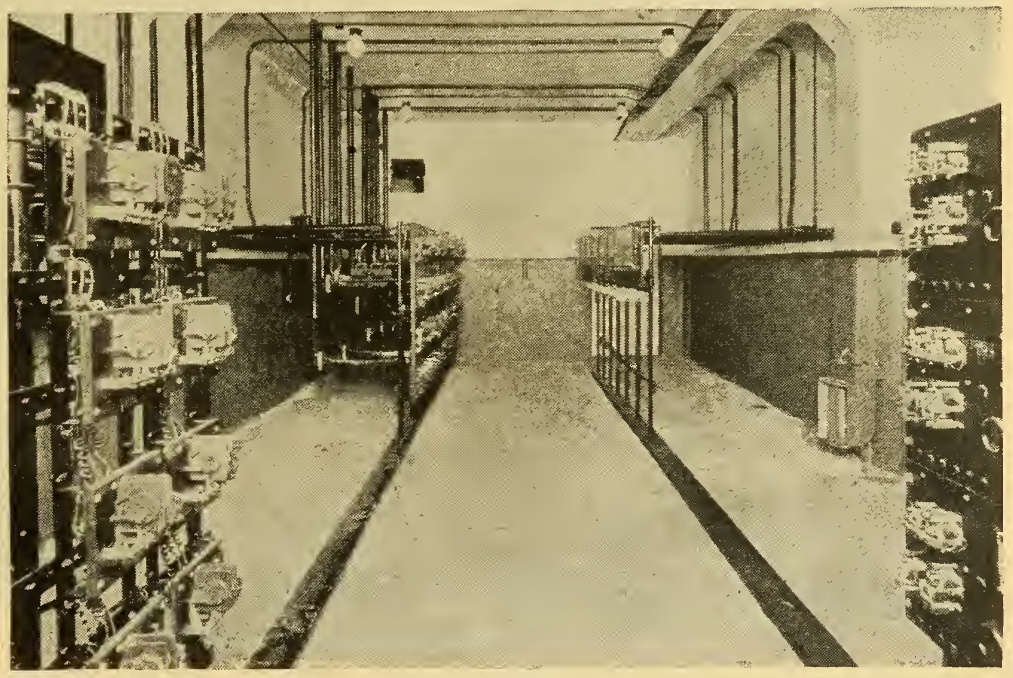

FIG. I8r.-A utomatic temperature control

View of another installation of automatic control devices 


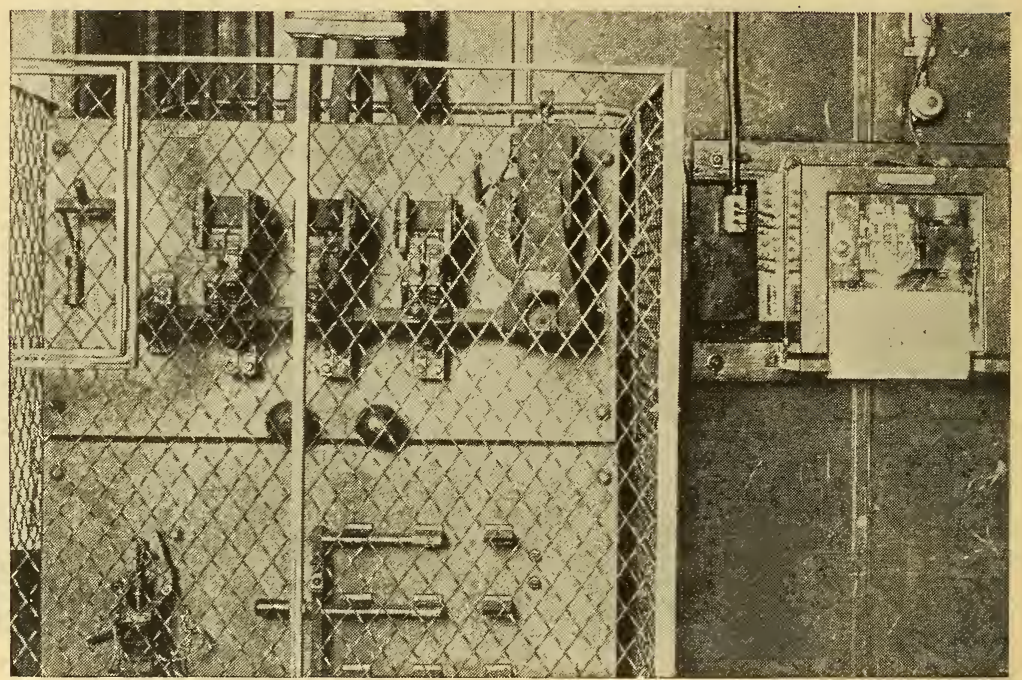

FIG. I82.-A Atomatic temperature control

The electromagnetic switch, operated by the relay, which in turn is operated by the recorder, is shown in greater detail

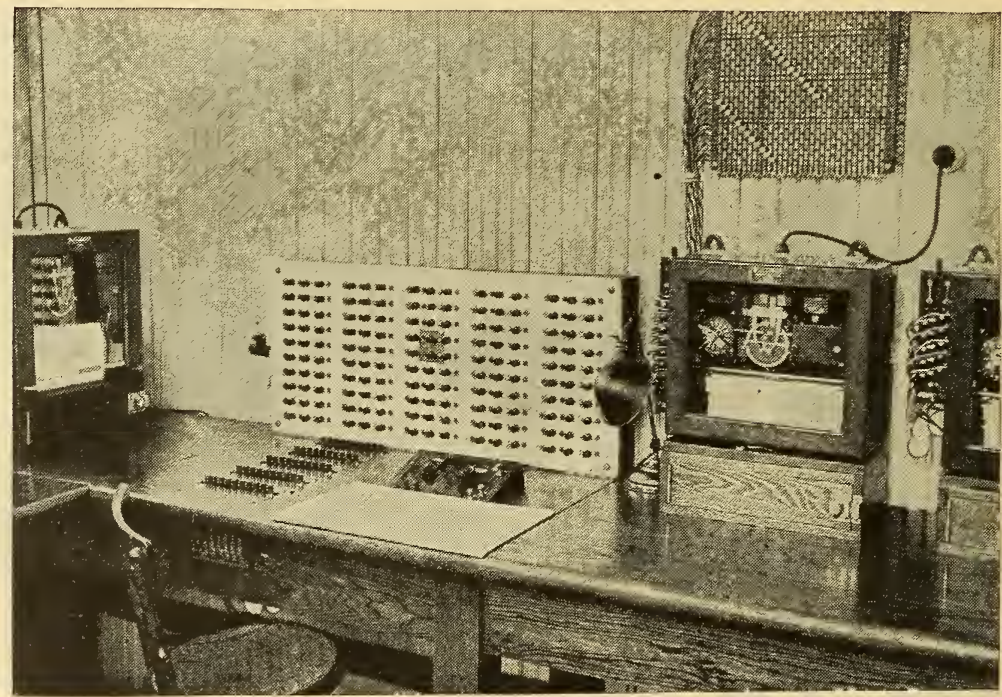

FIG. 183.-Central pyrometer station

The photograph shows one corner of a large central station. The plug board above is used for connecting the multiple-point recorders to any combination of furnaces. The switchboard is used for connecting couples to the potentiometer indicator and for transmitting signals to the furnace room 


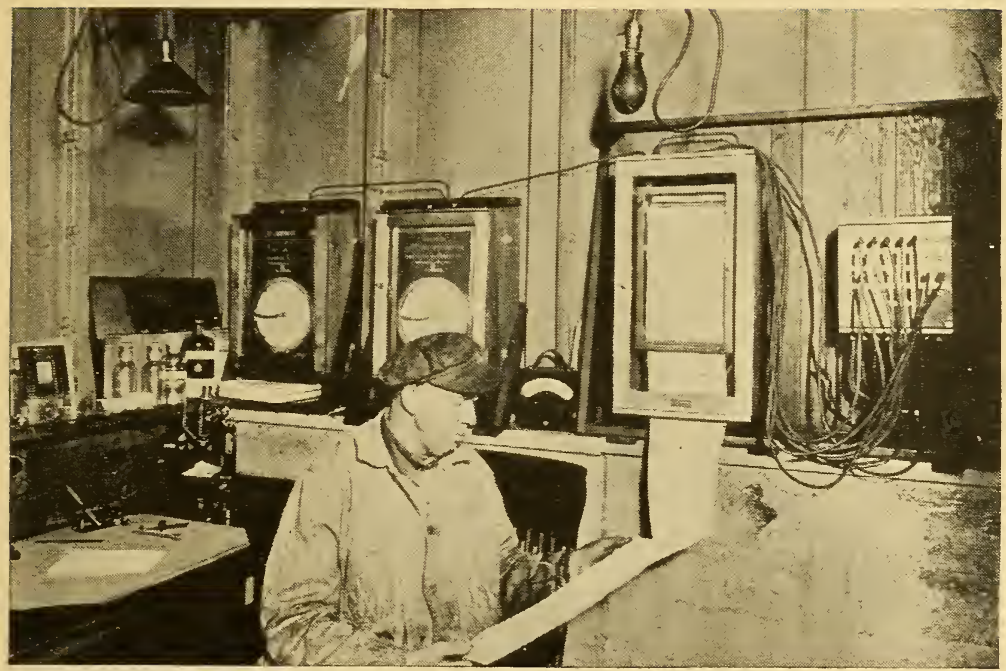

FIG. I84.-The foreman's office in a pottery

The foreman has in hand the complete record of the firing of a kiln. Close study of such records in correlation with the quality of the ware produced is doing much to replace art by science in the ceramic industries

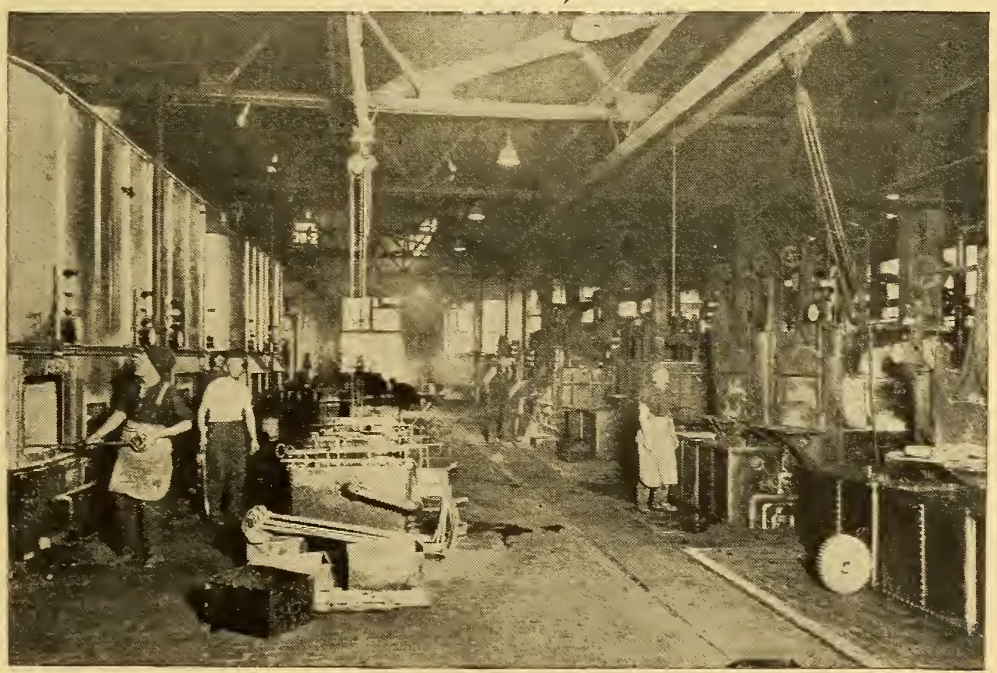

FIG. I85.-Heat treating department of a large plant

Each one of the many furnaces is equipped with an indicator and with colored signaling lamps. Such control results in the production of a uniform and high grade product 



\section{INDEX}

Page

Absorption devices. ................. 269-279

absorption-glass standardization........ $27 \mathrm{I}$

absorption-glass transmission........... $\quad 269$

red-glass standardization............. 272

sectored-disk standardization........... 27 I

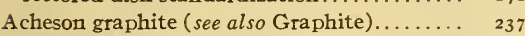

Alarm, temperature. . . . . . . . . . . . I67, I69

Alloys, melting points........... 192, 197, 2 IO

Alumel. See Chromel.

Alumina (see also Crucibles)....... 10, 212, 215, 218

Aluminum, certified samples............ ${ }_{23} 6$ graphite sheath................. 194, 238 melting point .......... I0. I92, I44, 235, 236, 268 Alundum, cement in furnace construc-

tion................. 195, 206, 240 melting point.................... $\quad 225$ protecting tubes.

Antimony, melting point.... 9, 10, 192, 194, 209, 235

Applications of pyrometry, industrial . . . . 282-299 cement kilns. . . . . . . . . . . . . . . . . . $\quad 287$

ceramic industries.................. $\quad 289$

coke industry . . . . . . . . . . . . . . . . . . . . 293

glass manufacture................ 283 steel manufacture (see also Steel manufac-

ture) ....................... 296

Arsem furnace.............. 213, 21 8, 221, 268

Atmospheres, proper, for molten materials.. I94, 2 I2

Automatic, alarm.................. 167, 169

signaling device.................. I70

temperature control. See Temperature control.

Barkley, Kreisinger and.

Benzophenone, boiling point. . . . . . . . I0, 225

Bimetallic, spring compensator.......... 55

thermometer.................... II

Black body........... I1 10, 126, 259, 267, 269, 274

Boiling points............... 9, 225, 278

Boltzmann. See Stefan.

Borax, melting point................204, 206

Bristol, automatic signaling device........ I I I

Bronze, melting range............... 201

Brooks............................ 49

Brown and Murray ................. 292

Brown, automatic temperature control..... I76 improved heatmeter....................... 248, 256 potentioneter....................... ${ }_{46} 6$ precision heatmeter................ 47 radiation pyrometer................. ${ }_{122}$

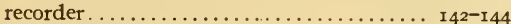
transformation-point recorder.......... I62

Burgess, G. K. .162, 200, $210,215,216,237,273,287,298$ Burgess, H. A............... 278, 282

Cadmium, meltir ${ }^{\alpha}$ point. .......... 10, 192, I94

Callendar, resistance-thermometer.......... equation .................... 209

$$
8513^{\circ}-21-21
$$

Page

Calorized iron protecting tubes........... 94

Carborundum protecting tubes............ gi

Cement, alundum, melting point......... 225

Cement kilns. ..................... $\quad{ }_{287}$

Ceramic industries.................. $\quad 289$

Ceramic materials, melting points......... 218

Chlorides, melting points.............. 203

Chromel....................... II

alumel thermocouples......... 2I, 286, 287-293

furnace heater........... I95, 242, 243, 306, 307 protecting tubes.................. 92, 287

Chromium, melting point........... 212

oxide, melting point................. 2 I 8

Chromon protecting tubes.............. 94

Clay (see also Fire clay)............. 95, 225, 294

Cleveland deflection potentiometer....... 53

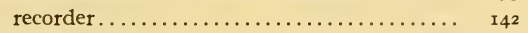

Coal ash ......................... 223

Cobalt, melting point............... 212

Coke industry ...................... 293

Cold junction (see also Thermocouple)..... 21,24 buried....................... $57,76,87$

compensation, bimetallic spring....... 55 compensating leads........... $56,76,77,83$

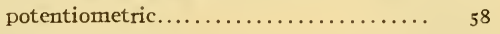
shunting method $\ldots \ldots \ldots \ldots \ldots \ldots \ldots$ 6s

Wheatstone bridge.................. 63 compensator.................... 249, 255 corrections.................. 54, 55,246

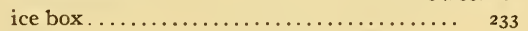
thermostated ........................ ${ }_{56}$ water-jacketed................... 56, 227 Commutating switch (see also Switchboard).. $\quad 82$ Comparator, thermometer testing........ 225 Compensating leads or cold-junction extension leads................ 56, $76,77,83$ Constantan, in thermocouples..... 21, 286, 306, 307 Conversion tables. See Tables.

Copper, certified samples.............. ${ }_{23} 6$ emissivity .............................. I 127 eutectics.................... 10, 198, 267 melting point........ 9, 192, I94, 197, 209, 234, 268 oxide..................... I $27,197,209$ Crowe......................... 2II Crucibles, acheson graphite......... 193, 237, 267 alumina ...................... I93, 2 I2 Dixon graphite................ 207, 237 duriron........................ 244 fire clay ...................... 203 for black body ................... ${ }_{26} 6_{7}$ for molten palladium.............. $2_{2} \mathrm{I}_{3}$ magnesia .................... 193, 212 magnesium aluminate. ............ 193, 212 nickel. . . . . . . . . . . . . . . . . . . . ${ }_{203}$ platinum and platinum alloys....... 203, 205 porcelain .................. I93 
Page derived differential .................. ${ }_{16}^{234}$ freezing........................... 196 inverse-rate. ..................... I6 obliquity...................... I89, 203 time-temperature................. I6r

Dana............................ ${ }_{187}$

Deflection potentiometer........... 42, 48, $26_{2}$ Depth, immersion of thermocouples. See Thermocouples.

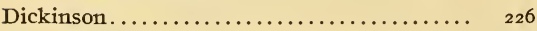

Diesselhorst potentiometer............. 233

Differential galvanometer.............. I30

Diopside, melting point............. 204, 206

Disappearing filament optical pyrometer... I0 105 ,

Dixon. See Crucibles.

$210,223,260$

Duriron. 94,244

Effective wave length................ 270

Electromotive force of thermocouples... 20, 226-236

Emissive powers.................... 95, II4 Englehard, platinum, platinum-rhodium thermocouple................ 64,306, 309

recorder........................... 145

Eutectic....................... 10, 199, 268

Extension leads. See Compensating leads.

Fairchild .................... 272,273, 289

Ferguson ........................ 219

Fery pyrometer, optical ............. 98, 266 radiation. ................... 122, 274

Field ........................... 223, 292

Fieldner........................ 223, 292

Fire bricks and clays, melting points. . 218, 220, 225

Fixed points ( points)........................

F. and F. optical pyrometer........... 104, 266

Foote........................ Io, $39,60,211,256,272,273,274,276,298$

Forsythe .......................... 216,273

Foster radiation pyrometer............. I2I

Freeman......................... 200

Freezing point. See Melting points.

Furnaces, electric, Arsem....... 213,218, 221, 268 carbon resistance................. $22 \mathrm{I}$ chromel. .................. I95, 24I, 243

for critical points . . . . . . . . . . . . . 200 for melting points, metals......... 195, 208 refractories................. $22 \mathrm{I}$ salts and silicates..............205, 274 for pyrometer standardization, optical.. $2 I_{3}$, $258,268,269$ thermocouples............... 237,240,24I

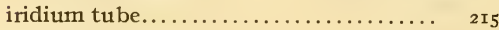
Northrup-A jax................. 2I5 gas for melting points........... 208, 222, 224 for thermocouple standardization..... 237,242 control. See Temperature control.

Fusible cones.................. 222, 289, 292

Galvanometer, differential.............. r, r30

for radiation pyrometer.............. $\quad 277$

for thermocouples............ 26-43, I38-1 56 standardization............. 248, 253, 257
Gas, furnaces for melting points (see also Furnaces)................... 208 ideal. ................................ 8 pyrometer................... II, I8, I35 thermometer. ............. 8, 9, II, I8, I35 Gauze, glowing, temperature........... II4-II6 Geophysical laboratory.................. I993, 204 Glass, absorption and transmission........ 270 annealing.......................... $\quad{ }_{285}$ manufacture, pyrometry applied . . . . . $288_{3}-287$ Gold, melting point. . . . . . . I92, I94, 208, 209, 268 Graphite. . . . . . . . . . . . . . . . . . . . Acheson ............... 193, 194, 207, 237, 238 black body ..................... 267, 274 Dixon .................... 207, 237

Hall............................ 223, 292

Harper.......................... 2 10

Harrison ......................... 239 Harrison-Foote compensated galvanometer. $\quad 39$,

Heatmeter.................. 39, 47, 248, 256

High temperature thermometry (see also

Thermometry) .............. 10-20, 225

Homogeneity test . . . . . . . . . . . . . 228, 245

Hoskins recorder. ............... I42, 145

Hot junction. See Thermocouples.

Ice box for thermocouple junctions........ 233

Ice point. . . . . . . . . . . . . . . .

Ideal gas............................ 8

Immersion of thermometers ............ 15,226

Impervite. . . . . . . . . . . . . . . . . . . I94

Indicator. See Measuring instruments.

Ingersoll, Mendenhall and ............. 216 Insulating tubes for thermocouples. See Protecting tubes.

Installations, illustrations of pyrometric. . . 310-319

Iridium . . . . . . . . . . . . . . . . . . 22, 203 tube furnace................... ${ }_{2 I_{5}}$

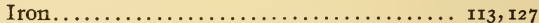
melting point....................... 212 oxide....................... II $3, \mathrm{I}_{27}$

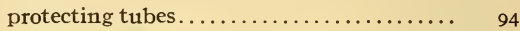
thermocouples............... 21, 286, 306

Jaeger............................. ${ }_{206}$ Johnson-Matthey thermocouples..........64, 6406 Junction box. .................... $83-87$

Kanolt................. 2II, 2I3, 2I8, 2I9, 225

Kreisinger and Barkley................. 299

Lamps, electric, calibration of optical pyrometers ..................... 260 melting-point determination........... 216 optical pyrometer................. $\quad 260$ Langmuir....................... $\quad 216$ Le Chatelier....................... ${ }_{237}$ thermocouples............. 2I, 55, 64,306, 308 Lead, certified samples................ ${ }_{236}$ freezing point..................... 10, 194 Leads, compensating............ $56,76,77,84$ thermocouples.............. 2 I resistance.................. 28-33 correction............ 33, 39, 4I 
Leakage, thermoelectric circuit........... 205

Leeds and Northrup, automatic signaling recorder...................... ${ }^{17 \mathrm{I}}$ automatic temperature control........... I77 cold-junction compensator............. 58:253 deflection potentiometer............... ${ }_{252}$ recorder....................... I56, 253 transformation-point indicator............ ${ }^{\prime} 6_{3}$ Lime, melting point .................. 218 Liquid-filled thermometers............ II , I8, I35 Luminosity........................ $\quad 270$ Lummer-Kurlbaum black body.......... $\quad 267$

Magnesia, melting point............... 218 Magnesium aluminate (see also Crucible).. I93, 212 Measuring instruments, thermoelectric

calibrated with thermocouples.......... 257 calibration................... 248, 253, 263 deflection potentiometers.......... 43, 52, 262 galvanometers... 26, $53,138,156,248,253,257,277$ heatmeter.................. $39,248,256$ indicators.......... $2 \mathrm{I}, 26,75,78, \mathrm{I} 34, \mathrm{I} 6 \mathrm{I}-\mathrm{I} 65$ multipyrograph.................. I 48 potentiometers. See Potentiometers.

pyrovolter........................ ${ }_{24}$ range control board................ 5I, 54 recorders (see also Galvanometers; Poten-

tiometers $) \ldots \ldots \ldots \ldots \ldots \ldots \ldots \ldots, 75, \mathrm{I}_{34}-\mathrm{I} 64$ tapalog......................... I53 use of, with calibrated thermocouples. $248-253$ Melting point, methods at high temperatures

by micropyrometer.

I $87-225$ by optical pyrometer.............. 210-217 by resistance thermometer............ 209 by thermocouple. . . . . . . . . . . . . . I89-209 effect of pressure.................. I 88 for optical pyrometer standardization.. $\quad 268$ for thermocouple standardization ..... I92-197, $200,206,234-236$

furnaces, electric....... 195, 206, 208, 213, 221, 237 gas................ 208, 222, 223, 237, 242 heating or cooling rate............. 213,221 obliquity of curve........... I89, 196, 199, 202 of alloys.................... 197, 198, 210 of alundum cements................... ${ }_{225}$ of ceramic materials................ 218-223 of chlorides........................ ${ }_{203}$ of coal ash.......................... 223 of clays.......................... ${ }_{225}$ of copper oxide................ 127, 197, 209 of eutectics................. I0, I98, 199, 268 of fire bricks.................. 212-223, 225 of metals......... 9, 10, 192-202, 212, 234-236, 268 of nitrates....................... 203 of refractories (see also Refractories)...... 219-225 of salts.............................. ${ }^{206}$ of sands........................... 225 of silicates.........................203, 206 of sulphates...................... ${ }_{203}$ standard samples.................... ${ }_{23}^{236}$ surfusion or undercooling............... $\quad 235$ wire method........................ 207 Melting-range determination.............. 200 Mendenhall and Ingersoll................ 216 melting points. See Melting points. molten-bath...................... 243 surface temperature ................. 298

Mercurial thermometer................. 10-20 accuracy $\ldots \ldots \ldots \ldots \ldots \ldots \ldots \ldots \ldots \ldots \ldots$, I2 filling under pressure................. I6 graduation......................... I2 industrial....................... I2 Merica........................... Mertelmeyer.......................... 60

Merwin, Ferguson and ................. 219

Meyer, Pirani and .................. Millivoltmeters. See Galvanometer; Measuring instruments.

Mricropyrometer..................... ${ }_{216}$

Mohler...................... 272, 273

Molybdenum in thermocouples........... 22 Mueller............... 10, 200, 278, 279, 282, 299 Murray, Brown and ................. 292

Naphthalene, boiling point............. I0, 225

Nela Research Laboratory.............. $\quad{ }_{260}$

Nichrome................ 2I , $92, \mathrm{II}_{3}, 243,287$

Nickel.................... 94, I27, 203, 260 melting point................ 10, 194, 212 oxide..................... II $3, \mathrm{I}_{27} 7,275$ resistance thermometer................ $28 \mathrm{I}$ strip........................... 275

Nitrates, melting points................ 203

Nitrogen thermometer................... 8

Northrup-Ajax furnace................. 215

Optical pyrometers........... 94, $98-118,258-273$ 'absorption, devices.................. 263, 269 window. ........................ II applications............ 108-1 I0, 2 IO , 213, 284-299 black body and nonblack body ......... IIO-II6 disappearing-filament type......... 105-108, $210,223,260,267,292$ F. and F..................... 104, 266 Fery ......................... 98,266

glowing gauze. $\ldots \ldots \ldots \ldots \ldots \ldots \ldots \ldots \ldots \ldots \ldots \ldots$ micropyrometer.................... 2 2I6

Scimatco......................... I03 sectered disk ....................... ${ }_{271}$ Shore pyroscope..................... 99 sighted into closed tube................ II 8 standardization ................ 95,97, $258^{-273}$ temperature-scale.................... 96 extension......................... ${ }_{263}$

theory ............................. 94

Wanner.................... 100-103, 263-266 Orton ............................. ${ }_{289}$

Palladium, melting point............ 10, 208, 212 Parasitic emfs. See Homogeneity.

Peltier emf .............................

Pirani and Meyer.................... 216

Planck, Wien-, radiation law.............. $\quad 96$

Platinum, crucibles................. 202-205 melting point.................... ro, 21 2 resistance thermometer. See Resistance thermometer

thermocouples. See Thermocouples.

Porcelain, crucibles................... I94 protecting tubes.......... 9I, 193, 227, 238, 294 
Potentiometers. .................. ${ }_{43-46}$ deflection. . . . . . . . . . . . . . 43, 48-54, 262 precision..................... 232, 253 shielding........................ ${ }_{205}$ standardization.................. 253-258 used with optical pyrometers.......... $\quad{ }_{26}$ used with standardized couples........ 249-252

Potentiometric, cold-junction compensator... 58, $249-252,255,257$ recorder I 56-I6I, I77, 255

Protecting tubes for thermocouples. $24,26,78,89-94$ alundum

calorized iron. ...................... 94 carborundum.................... 9I characteristics..................... 89 chromel...................... 92, 287

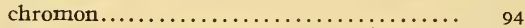
clay .......................... 92, 294

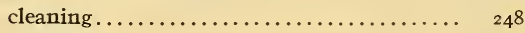
corundite........................ 92 duriron........................... 94 fused quartz or silica............... 90, I93 graphite................... 93, I94, 238 impervite....................... I94, 294 in salts and silicates................ 202, 205 iron........................... 94 nichrome..................... 92,287 nickel.......................... 94 porcelain................. 9I, I93, 238,294 precautions in use ................. ${ }_{23} 8$ Pyrex glass........................ I93 requirements...................... 89 sillimanite....................... I94 steel........................... 94 usalite...................... I94, 294 Pyrometry, industrial applications . . .... 282, 299 Pyrovolter...................... 47, 248

Quartz, melting point................ 204,219 thermocouple protecting tubes......... 90, I93

Radiation, intensity.

laws...

$9,96,97,118,270$

Radiation pyrometer....... 94,97, I I8-1 26, 273-277 advantages and disadvantages. applications................. 290, 293, 298 black-body conditions........... I26-I 27,274 emf measurement.................. 277 error sources.................. I 25, 274 Fery...................... I22, 274

Foster...................... I2I recorder........................ I35 standardization................ 95, 273-2 77 temperature scale................. 97

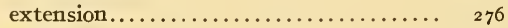

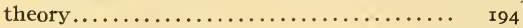

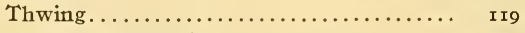

Range control board.................. 54

Raoult-Van't Hoff.................. ${ }_{197}$

Rawdon............................ ${ }_{298}$

Recording pyrometers............. I7, I34-I64 automatic signaling................ I70

Bristol. ........................ I43

Brown .................... I42, I44, I62 circular chart................... I42

Cleveland $\ldots \ldots \ldots \ldots \ldots \ldots \ldots \ldots \ldots \ldots \ldots$ I42
Recording pyrometers-Continued Page damping vibrations.................. $\quad 75$

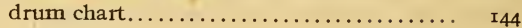

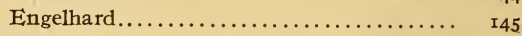
forms of records.................. 135 galvanometric..................... $\mathrm{I}_{3} 8-\mathrm{I}_{5} 6$

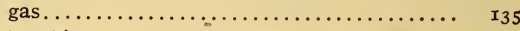
Hoskins..................... I42, I45 industrial. ............... I $8 \mathrm{I}-\mathrm{I} 87,284-289$ Leeds and Northrup........... I6 3 , I $56-16$ I, I 7 I

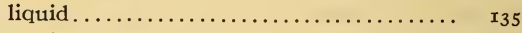
motive power.................. I40 multiple point................ I38, I68 multipyrograph.................. I48 parallel connections with indicators...... 79 potentiometric (see also Potentiometers). I56-I6I radiation $\ldots \ldots \ldots \ldots \ldots \ldots \ldots \ldots \ldots \ldots \ldots, I_{35}$ resistance....................... ${ } 35$

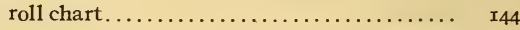
saturated vapor.................. I35 Siemens and Halske............... I 39

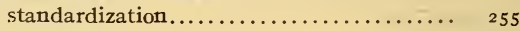
Stupakoff ...................... I44

tapalog........................ I5

Taylor........................ I47

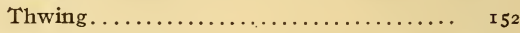

transformation point . . . . . . . . . . I6I-I65

Wilson-Maeulen................... I I $_{53}$ Refractories, melting points (see also Sili-

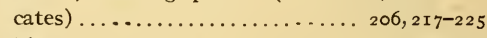
table.......................... 225

Resistance pyrometers.......... 8, 9, II , I2 7-I 34 agreement, with thermodynamic scale.... 9

with thermocouple............... 209 as standard pyrometer................ 209 compensating lead wire............... I 29 deflection galvanometer............... I32 for melting points............... 209 formula................... I 29, 209, 28I four-lead .......................... I32 industrial...................... I28 leads........................ ${ }_{28} 8_{2}$ measuring instrument .............. I 29 nickel coil .................. $28 \mathrm{n}$ operating principle............. I 27 platinum...................... 9, 209 potential terminals. . . . . . . . . . . . . . . I30 standardization................... $278^{2-282}$ temperature scale................. 9, 209 with indicator.................. 28 I Rosenhain................... 200, 202 Rhodium. See Thermocouples.

Ruthenium, in thermocouples.......... $\quad 22$

Salts, furnaces for melting points........ 206 melting points................. 204, 206

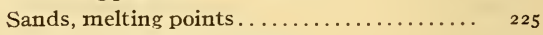
Scimatco optical pyrometer............. I03 Scott and Freeman.................. 200 Sectored disk .................... $27 \mathrm{I}$ Seebeck......................... 20 Seger cones................... 222, 289, 292 Shielding thermoelectric circuits.......... 205 Shore pyroscope................... 99 Siemens and Halske.................. I I39

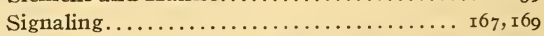
Silicates, furnace for melting points........ 206 melting points................... I9I, 202-206 
Page Silicon, melting point.................. ${ }_{212}$
Sillimanite.................. I94, 2II, 222 Silver, melting point....... I0, 192, 194, I98, 209, 268 effect of absorbed oxygen............. I9 8 Sodium chloride, for checking pyrometers. . 207, 244 Sosman........................ 222, 289 Standard cells, standardization ........... 253

Standard melting point samples.......... $\quad 236$ Standardization of pyrometers.......... 225-282 optical..................... 95, $25^{8-273}$ radiation ................. 95,97, 273-277 resistance....................... $278-282$ thermoelectric....... I92, I94, 200, 206, 207, 226-247 thermometric...................... 225 Steam point.................. 8, 9, 225, 278 Steel manufacture, pyrometry .......... 296-299 Stefan-Boltzmann radiation law..... 9, 97, II 8,2 I 7 Stem correction to mercurial thermometers. . I5, 226 Storage cells, for potentiometers.......... 233 Stupakoff recorder.................... I I44 Sulphates, melting points.............. 203 Sulphur boiling point............ 9, 10, 225, 278 Switches, commutating.............. 82

Tables, annealing temperatures of optical glasses........................ ${ }_{286}$ conversion centigrade and Fahrenheit... 301, 302 cold-junction correction factors........... $\quad 55$ emissive powers. $\ldots \ldots \ldots \ldots \ldots \ldots \ldots \ldots \ldots$ II 4 logarithms of tangents.............. 304 melting points, metals............ Io, I94, 2 I 2 refractories................. 206.2 2 I8, 225 salts.......................... 206 silicates....................... 206 softening temperatures of fusible cones... 292 thermoelectric.................... 306.307 true temperatures verses apparent tempera-

tures ................... II $3, I_{1}, I_{2} 7$

Tapalog......................... I53

Taylor recorder................... I I $_{4}$

Temperature coefficient of galvanometers... 29,4I

Temperature control............ 96, I $34,165-187$ automatic........................ I factors increasing difficulty ............ I6 glass manufacture.................. $\quad{ }_{283}$ location of pyrometer in furnace......... I66 necessity of pyrometers............ I6 $\quad 16$ signaling devices................. I67, I69

Temperature scales.................. 8 optical pyrometer.................. 96 radiation pyrometer ................ 9, 97 resistance pyrometer................ 9.209 thermodynamic.................... 8 thermoelectric pyrometer.......... 9, 209, 236

Test set. . . . . . . . . . . . . . . . . . . 33

Thermocouples, base-metal.............. 24 chromel-alumel....... 2I, 286, 287, 293, 295, 306 compensating leads................ 76 copper-constantan . . . . . . . . . . . . 21,306 iron-constantan............... 21, 286, 306 method of making junctions.......... 247 nichrome-constantan .............. 2 I standardization.................... $242-246$ use.................. 286, 287, 291, 296 calibration tables................ 306-308
Thermocouples-Continued Page

cold junctions .................. $2 \mathrm{I}, 24$

buried........................ 56,76

compensation, bimetallic spring ....... 55 compensating leads......... $56-58,76,77,84$ potentiometric................. $5^{8} 8-60,248$ shunt....................... 60

Wheatstone bridge ............... $6_{3}$

correction factor ............... 54, 55, 246 ice box....................... ${ }_{233}$ junction box.................... $88_{3}-87$ mounted couples................. 227 temperature................. 54-64,87, 24 I thermostated ...................... ${ }_{56} 6$ depth of immersion............. 88, 196, 229 fused junctions................... 25, 247 homogeneity.................... 22, 227 hot junction............... 21, 25, 240, 247 installations ........................ $74-9$ common return................. 76,78 damping vibrations................ 75 location, couples in furnaces........ 293,295 precautions......................

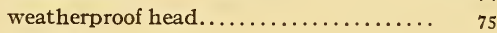
wiring......................... 74 wiring diagrams................... $77-83$ insulation (see also Protecting tubes) ....... 24 iridium......................... 22 Le Chatelier............... 21, 55, 64, 306, 308 lead wires (see also Compensating leads)... 2 i

location, furnace............... 75,8 I, I66 measuring instruments used . . . . . . . . $248-258$ melting point, determinations. ........ I89-209 metals used ...................... 2I mounted......................24, 227 platinum, platinum-rhodium. . . . . . . 22 , $55,64,226,285,290,29 \mathrm{I}, 293$ agreement, with resistance pyrometer... 209 with thermodynamic scale ......... 9 annealing...................... 227 calibration data. . . . . . . . . . 306-308 contaminated .................... 229 for precision work ................ I92 for primary standards. . . . . . . . . . . . $\quad 239$ homogeneity test. . . . . . . . . . . . 228-232 protection ...................... ${ }_{23} 8$ standardization.... I92, I94, 200, 206, 207, 227-242 Peltier emf........................ 20 protecting tubes. See Protecting tubes.

reproducibility ................... ${ }_{23}, 6_{4}-74$ resistance of leads and errors due to....... $28-33$ correction...................... 39 measurements.................... 33 seat of emf................. 20, 227, 245 size of wires...................... 22, 24 standardization. . . . . . . . . . . . . 227-246 for industrial laboratories....... 227, 236, 240 furnaces.................. 237, 240, 243 with measuring instrument. .......... 254

Thomson emf ...................... 20 Thermoelectric circuits, leakage, and shield-

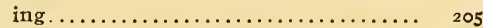
emfs....................... 20, 227, 228 measuring instruments. See Measuring instruments.

recorder. See Recorder. 


\begin{tabular}{|c|c|}
\hline & Pag \\
\hline $\begin{array}{l}\text { hermometers, high-temperature... } \\
\text { accuracy of high-grade........... }\end{array}$ & $\begin{array}{l}\text { Tungsten (see also Lamps). } \\
\text { melting point............... }\end{array}$ \\
\hline$\ldots 17,226$ & \\
\hline$\ldots$ & Indercooling \\
\hline$\ldots \ldots \ldots \ldots$ & I \\
\hline$\cdots \cdots \cdots \cdots$ & alasek \\
\hline$\cdots \quad 8,9$ & I9 \\
\hline . II, I8, I35 & pressure thermometcr........... II, I $7, I_{35}$ \\
\hline$\cdots$ & rings................... $\quad 289$ \\
\hline 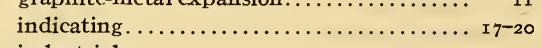 & . \\
\hline$\ldots \ldots \ldots \ldots \quad$ I2 & 9,2 Io, $2 \mathrm{I}_{5}, 2 \mathrm{I}$ \\
\hline II $, I 8, I 35$ & $\ldots \ldots \ldots \ldots \ldots \ldots \ldots$ \\
\hline n....... & $\mathrm{r} \ldots \ldots \ldots$ 100-103, $263-266$ \\
\hline$\cdots \cdots$ & $\ldots \ldots \quad 2 I 6$ \\
\hline$\ldots \ldots$ II $, I_{7}, I_{35}$ & $\ldots \ldots \ldots$ \\
\hline 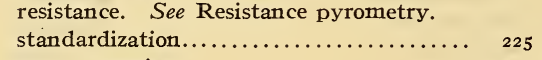 & 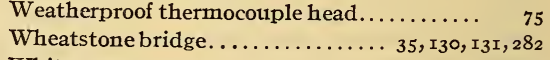 \\
\hline 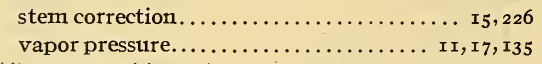 & 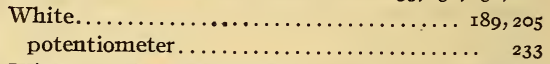 \\
\hline $\begin{array}{lr}\ldots \ldots \cdots \cdots \cdots & \text { 130 } \\
\cdots \cdots \cdots \cdots \cdots & 20\end{array}$ & 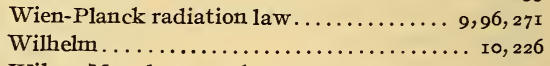 \\
\hline ter........... & $\ldots \ldots \ldots \ldots \cdots \cdots \cdots$ \\
\hline$\ldots \ldots \ldots \ldots$ & $\cdots \cdots \cdots \cdots \cdots \cdots \cdot 7^{8-83}$ \\
\hline 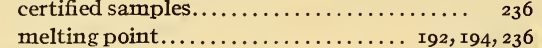 & s... \\
\hline$\ldots \ldots \ldots \ldots \ldots$ & \\
\hline $\begin{array}{l}\text { lation-point indicators and re- } \\
\text { ers } \ldots \ldots \ldots \ldots \ldots \ldots \ldots \ldots \ldots \ldots \ldots \ldots \ldots \ldots \text { I6I-I }\end{array}$ & $\begin{array}{r}497,236 \\
1 \quad 236\end{array}$ \\
\hline ransmission of absorption glass............ & 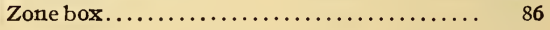 \\
\hline
\end{tabular}

